REPORT ON THE U.S. GEOLOGICAL SURVEY'S EVALUATION PROGRAM FOR STANDARD REFERENCE SAMPLES DISTRIBUTED IN SEPTEMBER 1996:

T-143 (TRACE CONSTITUENTS), T-145 (TRACE CONSTITUENTS), M-140 (MAJOR CONSTITUENTS), N-51 (NUTRIENT CONSTITUENTS), N-52 (NUTRIENT CONSTITUENTS), P-27 (LOW IONIC STRENGTH CONSTITUENTS), AND Hg-23 (MERCURY)

by Jerry W. Farrar and H. Keith Long

U.S. GEOLOGICAL SURVEY

Open-File Report 97-20

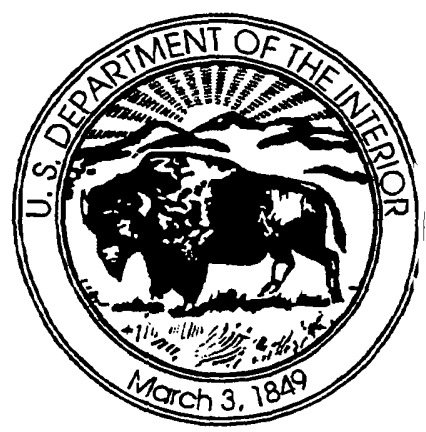

Lakewood, Colorado

1997 


\section{DEPARTMENT OF THE INTERIOR \\ BRUCE BABBITT, Secretary}

U.S. GEOLOGICAL SURVEY

Gordon P. Eaton, Director

For additional information

write to:

William J. Shampine

U.S. Geological Survey

Water Resources Division

Box 25046, Mail Stop 414

Denver Federal Center

Denver, Colorado 80225-0046
Copies of this report can be purchased from:

U.S. Geological Survey

Branch of Information Services

Box 25286

Denver, Colorado 80225-0286 
Abstract .

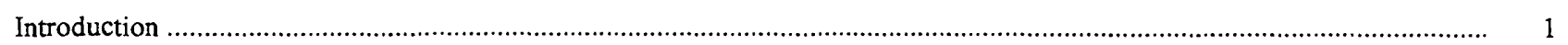

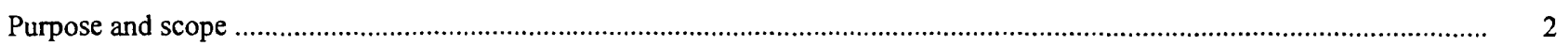

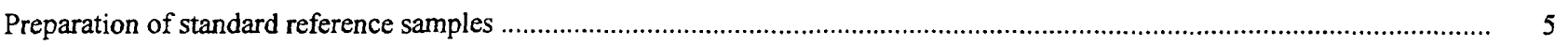

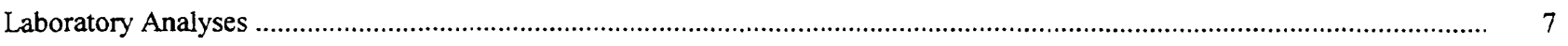

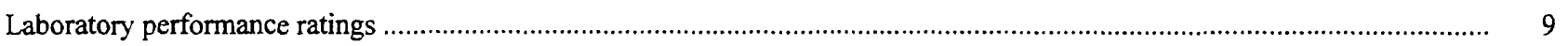

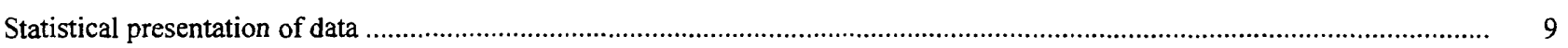

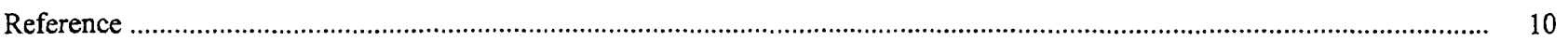

FIGURE

Figure 1. Statistical parameters shown on reported-data graphs

\section{TABLES}

Table 1. Laboratory participants in the analyses of standard reference samples distributed in September 1996 ..................... 3

2. Analytes determined in standard reference samples distributed in September 1996................................................ 7

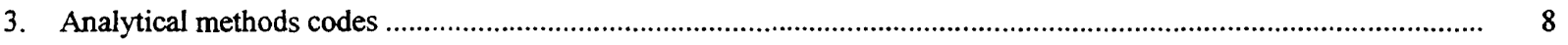

4. Overall laboratory performance ratings for standard reference water samples distributed in September 1996............. 11

5. Laboratory performance ratings for standard reference water sample $T-143$ (trace constituents) ..................................13

6. Laboratory performance ratings for standard reference water sample T-145 (trace constituents) ................................. 21

7. Laboratory performance ratings for standard reference water sample M-140 (major constituents) ............................ 29.

8. Laboratory performance ratings for standard reference water sample $\mathrm{N}-51$ (nutrient constituents) .................................35

9. Laboratory performance ratings for standard reference water sample $\mathrm{N}-52$ (nutrient constituents) ................................37

10. Laboratory performance ratings for standard reference water sample P-27 (low ionic strength constituents) ............... 39

11. Laboratory performance ratings for standard reference water sample $\mathrm{Hg}-23$ (mercury) .............................................43

12. Statistical summary of reported data for standard reference water sample T-143 (trace constituents) ...........................44

13. Statistical summary of reported data for standard reference water sample T-145 (trace constituents) ...........................73

14. Statistical summary of reported data for standard reference water sample M-140 (major constituents) ..................... 102

15. Statistical summary of reported data for standard reference water sample N-51 (nutrient constituents) ...................... 119

16. Statistical summary of reported data for standard reference water sample N-52 (nutrient constituents) ..................... 125

17. Statistical summary of reported data for standard reference water sample P-27 (low ionic strength constituents) ......... 131

18. Statistical summary of reported data for standard reference water sample $\mathrm{Hg}-23$ (mercury) ......................................143

19. Most probable values for constituents and properties in standard reference samples distributed in September $1996 \ldots 145$ 


\title{
REPORT ON THE U.S. GEOLOGICAL SURVEY'S EVALUATION PROGRAM FOR \\ STANDARD REFERENCE SAMPLES DISTRIBUTED IN SEPTEMBER 1996: \\ T-143 (TRACE CONSTITUENTS), T-145 (TRACE CONSTITUENTS), M-140 (MAJOR CONSTITUENTS), N-51 (NUTRIENT CONSTITUENTS), N-52 (NUTRIENT CONSTITUENTS), P-27 (LOW IONIC STRENGTH CONSTITUENTS), AND Hg-23 (MERCURY)
}

\author{
By Jerry W. Farrar and $H$. Keith Long
}

\begin{abstract}
This report presents the results of the U.S. Geological Survey's analytical evaluation program for 7 standard reference samples - T-143 (trace constituents), T-145 (trace constituents), M-140 (major constituents), N-51 (nutrient constituents), N-52 (nutrient constituents), P-27 (low ionic strength constituents), and Hg-23 (mercury) - that were distributed in September 1996 to 167 laboratories registered in the U.S. Geological Survey sponsored interlaboratory testing program. Analytical data that were received from 140 of the laboratories were evaluated with respect to: overall laboratory performance and relative laboratory performance for each analyte in the seven reference samples. Results of these evaluations are presented in tabular form. Also presented are tables and graphs summarizing the analytical data provided by each laboratory for each analyte in the seven standard reference samples. The most probable value for each analyte was determined using nonparametric statistics.
\end{abstract}

\section{INTRODUCTION}

The U.S. Geological Survey (USGS) conducts an interlaboratory evaluation program semiannually. This program provides a variety of reference materials to accomplish quality assurance testing of laboratories and to provide an adequate supply of samples that contribute to quality control programs of participating laboratories. Natural-matrix reference materials are preferred for use in this interlaboratory evaluation program. A series of samples are prepared and distributed each spring and fall. Occasionally, sediment samples are provided.

The program began in 1962 with a single sample containing major constituents that was prepared from distilled water and reagent grade chemicals. Twenty-three USGS laboratories participated in the 1962 determinations of six analytes in the major standard reference sample (SRS). Since that time, objectives of the program have been to:

(1) evaluate and improve the performance of USGS and other participating laboratories;

(2) provide a library of carefully prepared, homogeneous, stable reference materials for use in the quality control programs of laboratories;

(3) identify analytical problem areas;

(4) identify quality assurance needs with respect to environmental analyses and develop new reference materials to meet these needs; and

(5) ascertain the accuracy and precision of analytical methods.

Two hundred fifteen USGS and non-USGS laboratories are registered in the program, which can currently provide eight standard reference sample types: 
1. Trace constituents.

2. Major constituents.

3. Nutrient constituents.

4. Low ionic strength constituents.

5. Mercury.

6. Whole water (water with suspended sediment).

7. Acid mine drainage constituents.

8. Sediment (bed material) for major and trace constituents.

When sufficient data are available, a most probable value is statistically determined for each analyte in the SRS.

Though this is not a laboratory certification program, participation in this continuing quality assurance program is mandatory for all laboratories providing water-analyses data for USGS data storage or use (publications). Federal, State, municipal, and university laboratories can participate even though they do not provide data to the USGS. Analyses of these SRS provides the means to alert participating laboratories of possible deficiencies in their analytical operations, and also provides reference mater als for in-house quality control programs. Participating laboratories are identified only by a confidential code number.

A library of SRS, from previous evaluations, are available. Participating laboratories can purchase previous SRS for further testing, continuing quality assurance, and quality control programs by contacting:

\author{
U.S. Geological Survey \\ Branch of Technical Development and Quality Systems \\ Denver Federal Center \\ Box 25046 MS 401 \\ Denver, Colorado 80225-0046 \\ (303) 236-1870
}

\title{
Purpose and Scope
}

This report summarizes the analytical results submitted by 140 of the 167 laboratories that requested and were shipped SRS for the January 1997 evaluation (table 1). Not all SRS are requested, nor necessarily analyzed by all the laboratories; nor do all laboratories enrolled in the program participate in each evaluation. Analytical results for the following, which were mailed the week of September 9, 1996, are presented in this report:

$\begin{array}{ll}\text { T-143 } & \text { Trace constituents } \\ \text { T-145 } & \text { Trace constituents } \\ \text { M-140 } & \text { Major constituents } \\ \text { N-51 } & \text { Nutrient constituents } \\ \text { N-52 } & \text { Nutrient constituents } \\ \text { P-27 } & \text { Low ionic strength constituents (precipitation) } \\ \text { Hg-23 } & \text { Mercury }\end{array}$

The USGS requested that analytical results be returned by November 15, 1996 for evaluation and preparation of this report. Each participating laboratory is requested to perform those determinations routinely made on the respective SRS for USGS investigations and to indicate the information was provided, it has been included in the respective data table. The analytical data are represented in wrys 
that allow participants to evaluate data distribution, scatters, outliers, central tendency, bias, skewness, and method relationships.

Table 1.-Laboratory participants in the analyses of standard reference samples distributed in September 1996

\begin{tabular}{|c|c|c|}
\hline State & City & Participating Laboratory \\
\hline Alabama & Tuscaloosa & Geological Survey of Alabama \\
\hline Alaska & Soldotna & Alaska Department of Fish and Game \\
\hline Arizona & Yuma & Burns and Roe Services Corporation \\
\hline \multirow[t]{3}{*}{ Arkansas } & Arkadelphia & Ouachita Baptist University \\
\hline & Fayetteville & University of Arkansas \\
\hline & Little Rock & Arkansas Department of Pollution Control and Ecology \\
\hline \multirow[t]{12}{*}{ California } & Davis & University of California - Davis \\
\hline & La Verne & Metropolitan Water District \\
\hline & Martinez & Central Contra Costa Sanitary District \\
\hline & Oakland & East Bay Municipal Utility District \\
\hline & Perris & Eastern Municipal Water District \\
\hline & Sacramento & Anlab \\
\hline & Sacramento & US Bureau of Reclamation \\
\hline & Sacramento & USGS WRD \\
\hline & Santa Fe Springs & West Coast Analytical Service, Inc. \\
\hline & Tahoe City & Tahoe Research Group \\
\hline & West Sacramento & California Department of Water Resources \\
\hline & West Sacramento & Quanterra \\
\hline \multirow[t]{15}{*}{ Colorado } & Alamosa & Bureau of Reclamation \\
\hline & Arvada & Quanterra \\
\hline & Arvada & USGS-NWQL \\
\hline & Aurora & Core Laboratories, Inc. \\
\hline & Colorado Springs & City of Colorado Springs \\
\hline & Denver & US Bureau of Reclamation \\
\hline & Denver & USGS Colorado District Toxic Project \\
\hline & Denver & Denver Water Department \\
\hline & Fort Collins & City of Fort Collins - Water Quality \\
\hline & Fort Collins & CSU - Soil Testing Laboratory \\
\hline & Fort Collins & USDA Forest Service \\
\hline & Golden & Kaiser - Hill Rocky Flats \\
\hline & Loveland & Northern Colorado Water Conservation \\
\hline & Northglenn & Northglenn Water Treatment Plant \\
\hline & Westminster & City of Westminster \\
\hline \multirow[t]{11}{*}{ Florida } & Bradenton & Manatee County Environmental Management \\
\hline & Brooksville & SW Florida Water Management District \\
\hline & Ocala & USGS WRD QWSU \\
\hline & Orlando & Post, Bucklye, Schuh, and Jernigan, Inc. \\
\hline & Ormond Beach & Environmental Laboratory \\
\hline & Palatka & St. John's River Management District \\
\hline & Tallahassee & City of Tallahassee \\
\hline & Tallahassee & Florida Department of Environmental Regulations \\
\hline & Tallahassee & Savannah Laboratories \\
\hline & Tampa & Hillsborough County Environmental Protection Commission \\
\hline & West Palm Beach & South Florida Water Management District \\
\hline \multirow[t]{5}{*}{ Georgia } & Athens & University of Georgia \\
\hline & Atlanta & Georgia Department of Natural Resources \\
\hline & Atlanta & USGS WRD \\
\hline & Decatur & Dekalb County Water Quality Laboratory \\
\hline & Tifton & USDA - ARS \\
\hline Hawaii & Honolulu & University of Hawaii - SOEST Analytical Services \\
\hline
\end{tabular}


Table 1.-Laboratory participants in the analyses of standard reference samples distributed in September 1996-Continued

\begin{tabular}{|c|c|c|}
\hline State & City & Participating Laboratory \\
\hline \multirow[t]{2}{*}{ Idaho } & Boise & US Bureau of Reclamation \\
\hline & Pocatello & Idaho State University \\
\hline \multirow[t]{2}{*}{ Illinois } & Champaign & Hazardous Waste Research Center \\
\hline & Champaign & Illinois Environmental Protection Agency \\
\hline Indiana & Indianapolis & Indianapolis Department of Public Works \\
\hline Iowa & Des Moines & University Hygienic Laboratory, Des Moines Branch \\
\hline \multirow[t]{4}{*}{ Kansas } & Lawrence & Kansas Geological Survey \\
\hline & Topeka & City of Topeka \\
\hline & Topeka & Kansas Department of Health and Environment \\
\hline & Wichita & City of Wichita \\
\hline \multirow[t]{3}{*}{ Kentucky } & Frankfort & Division of Environmental Studies \\
\hline & Lexington & Kentucky Geological Survey \\
\hline & Louisville & Metropolitan Sewer District \\
\hline Maryland & Baltimore & Maryland Department of Health and Mental Hygiene \\
\hline \multirow[t]{2}{*}{ Michigan } & Ann Arbor & University of Michigan \\
\hline & Detroit & Detroit Water and Sewerage Department \\
\hline \multirow[t]{3}{*}{ Minnesota } & Minneapolis & University of Minnesota, Department of Geology and Geophysics \\
\hline & St. Paul & Metro Waste Control Commission \\
\hline & St. Paul & University of Minnesota \\
\hline \multirow[t]{2}{*}{ Missouri } & Columbia & University of Missouri \\
\hline & Jefferson City & Missouri Department of Health \\
\hline \multirow[t]{2}{*}{ Montana } & Butte & Montana Bureau of Mines \& Geology \\
\hline & Missoula & University of Montana \\
\hline \multirow[t]{6}{*}{ Nevada } & Boulder City & US Bureau of Reclamation \\
\hline & Las Vegas & University of Nevada - Las Vegas \\
\hline & Reno & Desert Research Institute \\
\hline & Reno & Nevada State Health Laboratory \\
\hline & Reno & Reno-Sparks Wastewater Treatment \\
\hline & Sutcliffe & Pyramid Lake Fisheries \\
\hline \multirow[t]{15}{*}{ New York } & Brewster & NYC DEP Brewster Lab \\
\hline & Brockport & SUNY - Brockport \\
\hline & Grahamsville & New York City Department of Environmental Protection \\
\hline & Hauppauge & Suffolk County Water Authority \\
\hline & Hempstead & Nassau County Department of Health \\
\hline & Ithaca & Cornell Nutrient Analysis Lab \\
\hline & Milbrook & Institute of Ecosystem Studies \\
\hline & North Babylon & Ecotest Laboratories \\
\hline & Rochester & Monroe County \\
\hline & Shokan & New York City Department of Environmental Protection \\
\hline & Syracuse & Onandaga County DDS \\
\hline & Troy & USGS-WRD \\
\hline & Valhalla & Department of Environmental Protection \\
\hline & Wantagh & Cedar Creeks Projects laboratory \\
\hline & Yorktown & New York City Department of Environmental Protection \\
\hline \multirow[t]{2}{*}{ North Carolina } & Chapel Hill & City of Durham Water Resources \\
\hline & Charlotte & Mecklenburg County \\
\hline North Carolina & Greensboro & City of Greensboro \\
\hline \multirow[t]{3}{*}{ North Dakota } & Bismarck & North Dakota State Health Department \\
\hline & Bismarck & North Dakota State Water Commission \\
\hline & Bismarck & US BOR \\
\hline \multirow[t]{4}{*}{ Ohio } & Cincinnati & US EPA \\
\hline & Cuyahoga Heights & Northeast Ohio Regional Sewer District \\
\hline & Tiffin & Heidelberg College \\
\hline & Wooster & The Ohio State University \\
\hline
\end{tabular}


Table 1.-Laboratory participants in the analyses of standard reference samples distributed in September 1996--Continued

\begin{tabular}{|c|c|c|}
\hline State & City & Participating Laboratory \\
\hline \multirow[t]{2}{*}{ Oklahoma } & Norman & Oklahoma Geological Survey \\
\hline & Oklahoma City & Oklahoma Department of Environmental Quality \\
\hline \multirow[t]{2}{*}{ Oregon } & Corvallis & USDA - CCAL \\
\hline & Tigard & Unified Sewerage Agency \\
\hline \multirow[t]{2}{*}{ Pennsylvania } & Harrisburg & Pennsylvania Department of Environmental Resources \\
\hline & Somerset & Geochemical Testing \\
\hline Puerto Rico & San Juan & Department of Natural Resources \\
\hline South Dakota & Brookings & SDSU - Water Quality Laboratory \\
\hline Tennessee & Chattanooga & TVA Environmental Chemistry \\
\hline \multirow[t]{3}{*}{ Texas } & Austin & Lower Colorado River Authority \\
\hline & College Station & Texas A \& M \\
\hline & Seguin & Guadalupe-Blanco River Authority \\
\hline Vermont & Waterbury & Vermont Agency of Natural Resources \\
\hline \multirow[t]{3}{*}{ Virginia } & Culpepper & ESS Labs \\
\hline & Manassas & Occoquan Watershed Monitoring Laboratory \\
\hline & Richmond & Consolidated Laboratory Services \\
\hline \multirow[t]{2}{*}{ Washington } & Seattle & Frontier Geoscience \\
\hline & Seattle & Brooks-Rand, Ltd. \\
\hline \multirow[t]{2}{*}{ Wisconsin } & Madison & University of Wisconsin, Department of Hygiene \\
\hline & Milwaukee & Milwaukee Metro Sewerage District \\
\hline \multicolumn{3}{|c|}{ Middle East Laboratories } \\
\hline Location & & Participating Laboratory \\
\hline \multirow[t]{4}{*}{ Gaza } & & Al-Azar University, Water Research Center Laboratory \\
\hline & & Islamic University, Environmental \& Rural Research Center Laboratory \\
\hline & & Ministry of Agriculture Laboratory \\
\hline & & Ministry of Health, Public Health Laboratory \\
\hline \multirow[t]{4}{*}{ Israel } & & Geological Survey of Israel Laboratory \\
\hline & & Israeli Hydrological Service Laboratory \\
\hline & & Mekeroth Water Company, Central Laboratory \\
\hline & & Water Resources Research Center, Institute for Desert Research \\
\hline \multirow[t]{2}{*}{ Jordan } & & Royal Scientific Society of Jordan, Environmental Research Center Laboratory \\
\hline & & Water Authority of Jordan, Central Laboratory \\
\hline \multirow[t]{4}{*}{ West Bank } & & Al-Quds University, College of Science \& Technology, Water Research Center \\
\hline & & Bethlehem University, Water and Soil Environmental Research Unit \\
\hline & & Birzeit University, Center for Environmental \& Occupational Health Services \\
\hline & & Najah-WESC \\
\hline
\end{tabular}

\section{Preparation of Standard Reference Samples}

All of the SRS used in this evaluation were prepared by personnel of the USGS in Lakewood, Colorado and were analyzed for analyte concentrations and physical property values prior to mailing. A library of reference samples is maintained and can be requested by participating laboratories for use in their quality control programs.

Trace constituent sample T-143 was prepared using water collected from the North Platte River near Windover, Wyoming. The water was pumped through $0.45,0.2-$ and $0.1-\mu \mathrm{m}$ filters, in series, into a $3500-\mathrm{L}$ polypropylene drum. The water was continuously circulated and passed through a $0.1-\mu \mathrm{m}$ filter and ultraviolet sterilizer for 24 hours. Following this circulation, the water was acidified to $\mathrm{pH} 1.3$ with nitric acid and chlorinated to $5 \mathrm{ppm}$ free chlorine. The trace constituent concentrations were adjusted by adding reagent grade chemicals. The sample was circulated an additional 24 hours prior to bottling. 
polypropylene bottles used were acid leached, deionized-water rinsed, and autoclave sterilized.

Trace constituent sample T-145 was prepared using water collected from the Yampa River near Steamboat Springs, Colorado. The water was pumped through $0.45,0.2-$ and $0.1-\mu \mathrm{m}$ filters, in series, into a $3500-\mathrm{L}$ polypropylene drum. The water was continuously circulated and passed through a $0.1-\mu \mathrm{m}$ filter and ultraviolet sterilizer for 24 hours. Following this circulation, the water was acidified to $\mathrm{pH} 1.3$ with nitric acid and chlorinated to $5 \mathrm{ppm}$ free chlorine. The trace constituent concentrations vrere adjusted by adding reagent grade chemicals. The sample was circulated an additional 24 hours prior to bottling. During bottling the sample was pumped through an ultraviolet sterilizer and a $0.1-\mu \mathrm{m}$ filter. The 500-mL polypropylene bottles used were acid leached, deionized-water rinsed, and autoclave sterilized.

Major constituent sample M-140 was prepared using water collected from the Arkansas River near Avondale, Colorado. The water was pumped through $0.45,0.2-$ and $0.1-\mu \mathrm{m}$ filters, in series, into a 1300-L polypropylene drum. The water was chlorinated to 5-ppm free chlorine with sod"rum hypochlorite, continuously circulated, and passed through a $0.1-\mu \mathrm{m}$ filter and ultraviolet sterilizer for 24 hours prior to bottling. During bottling the sample was pumped through an ultraviolet sterilizer ard a $0.1-\mu \mathrm{m}$ filter. The 500-mL polypropylene bottles used were acid leached, deionized-water rinsed, and autoclave sterilized.

Nutrient sample N-51 was prepared using water collected from the West Chicago Creek near Idaho Springs, Colorado. These samples were prepared the week prior to the mailing for this SRS evaluation. The water was pumped through $0.45,0.2-$ and $0.1-\mu \mathrm{m}$ filters, in series, into a $600-\mathrm{L}$ polypropylene drum and continuously circulated and passed through a $0.1-\mu \mathrm{m}$ filter for 24 hours. The desired nutr: $\mathrm{nt}$ concentrations were obtained by adding reagent-grade chemicals. The sample was continuously circulated for 24 hours prior to being bottled. The $250-\mathrm{mL}$ polyethylene bottles used were new, amT' ar, acid leached, and deionized-water rinsed.

Nutrient sample N-52 was prepared using deionized water. These samples were prepared the week prior to the mailing for this SRS evaluation. The water was pumped through $0.45,0.2-$ and $0.1-\mu \mathrm{m}$ filters, in series, into a $600-\mathrm{L}$ polypropylene drum and continuously circulated and passed through a $0.1-\mu \mathrm{m}$ filter for 24 hours. The desired nutrient concentrations were obtained by adding reagent-grade chemicals. The sample was continuously circulated for 24 hours prior to being bottled. The $30-\mathrm{mL}$ glass vials used were new, amber, acid leached, and deionized-water rinsed.

Sample P-27 was prepared in a 400-L polypropylene drum using water collected from the West Chicago Creek near Idaho Springs, Colorado. The water was pumped into the drum through $0.45,0.2-$ and $0.1-\mu \mathrm{m}$ filters in series. Desired phosphate and fluoride concentrations were obtained by adding reagent-grade chemicals. Prior to bottling, the sample was continuously mixed for 24 hours while being circulated through a $0.1-\mu \mathrm{m}$ filter and an ultraviolet sterlizer. During bottling the sample was pumped through an ultraviolet sterilizer and a $0.1-\mu \mathrm{m}$ filter. The $500-\mathrm{mL}$ polypropylene bottles used were acid leached, deionized-water rinsed, and autoclave sterilized.

Sample Hg-23 was prepared using water collected from the Fall River near Idaho Springs, Colorado. The sample was prepared in a 190-L polypropylene drum. The river water was pumped into this drum through $0.45,0.2-$ and $0.1-\mu \mathrm{m}$ filters in series. The water was continuously circulated and passed through a $0.1-\mu \mathrm{m}$ filter and ultraviolet sterilizer for 48 hours. Nitric acid (5-percent, v/v) and dichromate ion (0.05-percent, $\mathrm{w} / \mathrm{w}$ ) were added to stabilize the sample. The desired mercury concentration was obtained by a dding a mercury standard solution. Following an additional 24 hours of circulation, the sample was bottled. The $250-$ $\mathrm{mL}$ glass bottles and tetrafluoroethylene fluorocarbon resin caps used were new, acid leached, and de:onizedwater rinsed. 


\section{LABORATORY ANALYSES}

The participating laboratories were asked to determine analytes which are summarized in table 2 . The number of analytes varied from 28 in T-143 \& T-145 (trace constituents) to $1 \mathrm{in} \mathrm{Hg-23} \mathrm{(mercury).}$

Table 2.-Analytes determined in standard reference samples distributed in September 1996

\begin{tabular}{|c|c|c|c|c|c|c|c|}
\hline Analyte or propert & & Units & $T-143, T-145$ & $5 \quad M-140$ & $\mathrm{~N}-51, \mathrm{~N} 52$ & P-27 & $\mathrm{Hg}-23$ \\
\hline Acidity & Acidity as $\mathrm{CaCO}_{3}$ & $\mathrm{mg} / \mathrm{L}$ & & & & $\mathrm{x}$ & \\
\hline Alk & Alkalinity as $\mathrm{CaCO}_{3}$ & $\mathrm{mg} / \mathrm{L}$ & & $\mathrm{x}$ & & & \\
\hline Ag & Silver & $\mu \mathrm{g} / \mathrm{L}$ & $\mathrm{x}$ & & & & \\
\hline Al & Aluminum & $\mu g / L$ & $\mathrm{x}$ & & & & \\
\hline As & Arsenic & $\mu g / L$ & $\mathrm{x}$ & & & & \\
\hline B & Boron & $\mu g / L$ & $\mathrm{x}$ & & & & \\
\hline $\mathrm{Ba}$ & Barium & $\mu \mathrm{g} / \mathrm{L}$ & $\mathrm{x}$ & & & & \\
\hline $\mathrm{Be}$ & Beryllium & $\mu \mathrm{g} / \mathrm{L}$ & $\mathrm{x}$ & & & & \\
\hline $\mathrm{Ca}$ & Calcium & $\mathrm{mg} / \mathrm{L}$ & $\mathrm{x}$ & $\mathrm{x}$ & & $\mathrm{x}$ & \\
\hline$\underline{\mathrm{Cd}}$ & Cadmium & $\mu \mathrm{g} / \mathrm{L}$ & $\mathrm{x}$ & & & & \\
\hline $\mathrm{Cl}$ & Chloride & $\mathrm{mg} / \mathrm{L}$ & & $\mathrm{x}$ & & & \\
\hline Co & Cobalt & $\mu \mathrm{g} / \mathrm{L}$ & $\mathrm{x}$ & & & & \\
\hline $\mathrm{Cr}_{\mathrm{r}}$ & Chromium, total & $\mu g / L$ & $\mathrm{x}$ & & & & \\
\hline $\mathrm{Cu}$ & Copper & $\mu g / L$ & $\mathrm{x}$ & & & & \\
\hline DSRD & Dissolved solids & $\mathrm{mg} / \mathrm{L}$ & & $\mathrm{x}$ & & & \\
\hline $\mathrm{F}$ & Fluoride & $\mathrm{mg} / \mathrm{L}$ & & $\mathrm{x}$ & & $\mathrm{x}$ & \\
\hline Fe & Iron & $\mu \mathrm{g} / \mathrm{L}$ & $\mathrm{x}$ & & & & \\
\hline $\mathrm{Hg}$ & Mercury & $\mu \mathrm{g} / \mathrm{L}$ & & & & & $\mathrm{x}$ \\
\hline $\mathrm{K}$ & Potassium & $\mathrm{mg} / \mathrm{L}$ & $\mathrm{x}$ & $\mathrm{x}$ & & $\mathrm{x}$ & \\
\hline$\underline{\mathrm{Li}}$ & Lithium & $\mu g / L$ & $\mathrm{x}$ & & & & \\
\hline $\mathrm{Mg}$ & Magnesium & $\mathrm{mg} / \mathrm{L}$ & $\mathrm{x}$ & $\mathrm{x}$ & & $\mathrm{x}$ & \\
\hline Mn & Manganese & $\mu \mathrm{g} / \mathrm{L}$ & $\mathrm{x}$ & & & & \\
\hline Mo & Molybdenum & $\mu \mathrm{g} / \mathrm{L}$ & $\mathrm{x}$ & & & & \\
\hline $\mathrm{Na}$ & Sodium & $\mathrm{mg} / \mathrm{L}$ & $\mathrm{x}$ & $\mathrm{x}$ & & $\mathrm{x}$ & \\
\hline $\mathrm{NH}_{3} \underline{\text { as N }}$ & Ammonia & $\mathrm{mg} / \mathrm{L}$ & & & $x$ & & \\
\hline $\mathrm{NH}_{3}+\mathrm{Org} \mathrm{N}$ as $\mathrm{N}$ & Ammonia + Organic $\mathrm{N}$ & $\mathrm{mg} / \mathrm{L}$ & & & $\mathrm{x}$ & & \\
\hline $\mathrm{Ni}$ & Nickel & $\mu g / L$ & $\mathrm{x}$ & & & & \\
\hline $\mathrm{NO}_{3}+\mathrm{NO}_{2}$ as $\mathrm{N}$ & Nitrate + Nitrite & $\mathrm{mg} / \mathrm{L}$ & & & $\mathrm{x}$ & & \\
\hline $\mathrm{Pb}$ & Lead & $\mu \mathrm{g} / \mathrm{L}$ & $\mathrm{x}$ & & & & \\
\hline $\mathrm{pH}$ & & unit & & $\mathrm{x}$ & & $\mathrm{x}$ & \\
\hline $\mathrm{PO}_{4}$ as $\mathrm{P}$ & Orthophosphate & $\mathrm{mg} / \mathrm{L}$ & & & $\mathrm{x}$ & & \\
\hline total $\mathrm{P}$ as $\mathrm{P}$ & Phosphorus & $\mathrm{mg} / \mathrm{L}$ & & $\mathrm{x}$ & $\mathrm{x}$ & $\mathrm{x}$ & \\
\hline $\mathrm{Sb}$ & Antimony & $\mu \mathrm{g} / \mathrm{L}$ & $\mathrm{x}$ & & & & \\
\hline $\mathrm{Se}$ & Selenium & $\mu \mathrm{g} / \mathrm{L}$ & $\mathrm{x}$ & & & & \\
\hline$\underline{\mathrm{SiO}}_{2}$ & Silica & $\mathrm{mg} / \mathrm{L}$ & $\mathrm{x}$ & $x$ & & & \\
\hline $\mathrm{SO}_{4}$ & Sulfate & $\mathrm{mg} / \mathrm{L}$ & & $\mathrm{x}$ & & $\mathrm{x}$ & \\
\hline Sp Cond & Specific conductance & $\mu \mathrm{S} / \mathrm{cm}$ & & $\mathrm{x}$ & & $\mathrm{x}$ & \\
\hline Sr & Strontium & $\mu \mathrm{g} / \mathrm{L}$ & $\mathrm{x}$ & $\mathrm{x}$ & & & \\
\hline $\mathrm{Tl}$ & Thallium & $\mu \mathrm{g} / \mathrm{L}$ & $\mathrm{x}$ & & & & \\
\hline U & Uranium & $\mu \mathrm{g} / \mathrm{L}$ & $\mathrm{x}$ & & & & \\
\hline $\mathrm{v}$ & Vanadium & $\mu g / \mathrm{L}$ & $\mathrm{x}$ & $\mathrm{x}$ & & & \\
\hline $\mathrm{Zn}$ & Zinc & $\mu \mathrm{g} / \mathrm{L}$ & $\mathrm{x}$ & & & & \\
\hline
\end{tabular}


Laboratories were requested to identify the method used for each analyte according to table 3 analytical method codes.

Table 3. Analytical methods codes

\begin{tabular}{cl} 
Code & Method \\
\hline 0 & Other \\
1 & Atomic absorption: direct, air \\
2 & Atomic absorption: direct, nitrous oxide \\
3 & Atomic absorption: graphite furnace \\
4 & Inductively coupled plasma \\
5 & Direct current plasma \\
6 & Inductively coupled plasma/Mass spectrometry \\
7 & Ion chromatography \\
8 & Atomic absorption: cold vapor \\
9 & Atomic fluoresence \\
10 & Atomic absorption: extraction [specify chelating agents ] \\
11 & Atomic absorption: hydride [specify reducing agent ] \\
12 & Flame emission \\
20 & Titration: colorimetric [specify color reagent ] \\
21 & Titration: electrometric [specify reducing or oxidizing agent/color reagent ] \\
22 & Colorimetric: [specify reducing or oxidizing agent/color reagent ] \\
40 & Ion selective electrode \\
41 & Electrometric [pH and Specific Conductance] \\
50 & Gravimetric: [specify filtration, evaporation, and so forth] \\
51 & Turbidimetric \\
\hline
\end{tabular}

Participating laboratories were also asked to use the references listed below to further define the methods.

1. American Public Health Association and others, 1992, Standard methods for the examination of water and wastewater 18th ed: Washington, D.C., American Public Health Association, $981 \mathrm{lp}$.

2. American Society for Testing and Materials, Annual book of ASTM standards:

Philadelphia, v. 11.01, and v. 11.02.

3. Kopp, J.F., and McKee, G.F., 1979, Methods for chemical analysis of water and wastes: Cincinnati, U.S. Environmental Protection Agency, EPA 600/4-79-020, rev. 1983, $460 \mathrm{p}$.

4. Fishman, M.J., and Friedman, L.C., eds., 1989. Methods for determination of inorganic substances in water and fluvial sediments (3d ed.): U.S. Geological Survey Techniques of Water-Resources Investigations, Book 5, Chapter A1, 545 p.

5. Miscellaneous manufacturer's instrument manuals or references. 


\section{LABORATORY PERFORMANCE RATINGS}

To facilitate interlaboratory performance comparisons, laboratory performance ratings, based on the analyses reported for each SRS, are included in tables 4 through 11 in this report. Averages of the analyte ratings and the number of analyte values reported for each SRS are given for each participating laboratory. Laboratory performance for each analyte is rated on a scale 4 to 0 , based on the absolute Zvalue, as listed below:

\begin{tabular}{ll}
\hline Rating & Absolute $Z$-value \\
\hline 4 (Excellent) & 0.00 to 0.50 \\
3 (Good) & 0.51 to 1.00 \\
2 (Satisfactory) & 1.01 to 1.50 \\
1 (Questionable) & 1.51 to 2.00 \\
0 (Poor). & Greater than 2.00 \\
\hline
\end{tabular}

Overall laboratory performance ratings greater than 2.4 are considered satisfactory. Overall laboratory performance ratings between 2.0 and 2.39 are considered marginal; those less than 2.0 are considered poor.

\section{STATISTICAL PRESENTATION OF DATA}

Data in this report have been evaluated using nonparametric statistics as described by Hoaglin and others (1983). This statistical approach is a resistant statistic because the median is not influenced by outliers as is the mean in traditional statistics.

Analytical data for each analyte are presented in tabular and graphical forms in tables 12 through 18 . Tabulated data for each analyte include the laboratory code number, reported values, analytical method, most probable value (MPV), number of reported values - excluding less than values $(N)$, data range, $Z$ value, and the $F$-pseudosigma. (The $Z$-value is equivalent to the $Z$-score of traditional statistics, being the number of deviations the reported value is from the MPV. The F-pseudosigma is equivalent to the standard deviation $(\sigma)$ of traditional statistics when the data has a Gaussian distribution.) If an analyte has a sufficient number of determinations by a given method, usually 7 , the F-pseudosigma for that analytical method is reported in the block of data listed for each analyte.

The median value is considered the MPV. Reported values of "less than" are used to establish the median, but are not considered in determining the data range. The median (midpoint) divides the ordered data into halves and is designated the MPV. The hinges include the middle 50-percent of the data and are the mid-values of the upper and lower halves of the data. (The hinges are similar to quartiles, but are not mathematically equivalent.) The range of data between the upper hinge $(\mathrm{Hu})$ and the lower hinge $(\mathrm{HI})$, the hinge spread ( $\mathrm{H}$-spr), is used to calculate the F-pseudosigma, the laboratory performance rating, the upper warning level (UWL) and lower warning level (LWL), the upper control level (UCL) and the lower control level (LCL). The F-pseudosigma is calculated by comparison of the $\mathrm{H}$-spr value to the Gaussian distribution relation; 67.45 percent of the data "hinges" between plus and minus $1 \sigma$, resulting in a $\mathrm{H}$-spr of $2 \times 0.6745=1.349 \sigma$, This relation allows the calculation of the F-pseudosigma $=(\mathrm{H}-\mathrm{spr}) / 1.349$. Laboratories reporting "less than" values are not performance rated unless their reported "less than" values are greater than two $Z$-values from the MPV.

The graphical plot of the reported data is shown in figure 1. The upper and lower boundaries of the graphical plots generally are +3 and -3 F-pseudosigma deviations from the median. (Computer-program 
scaling constraints do not permit these boundaries to always be graphed at exactly these values.) The graphical plot is a box plot/control chart with reported values grouped by analytical method in ascencing order of value. Lines designate the MPV, Hu, HI, and the (UWL) and (LWL) at +2 and $-2 \mathrm{~F}-$ pseudosigma, respectively. "Less than" values are not plotted. The analytical data are presented in ways that allow participants to evaluate data distribution, scatter, outliers, central tendency, bias, skewness, and method relationships.

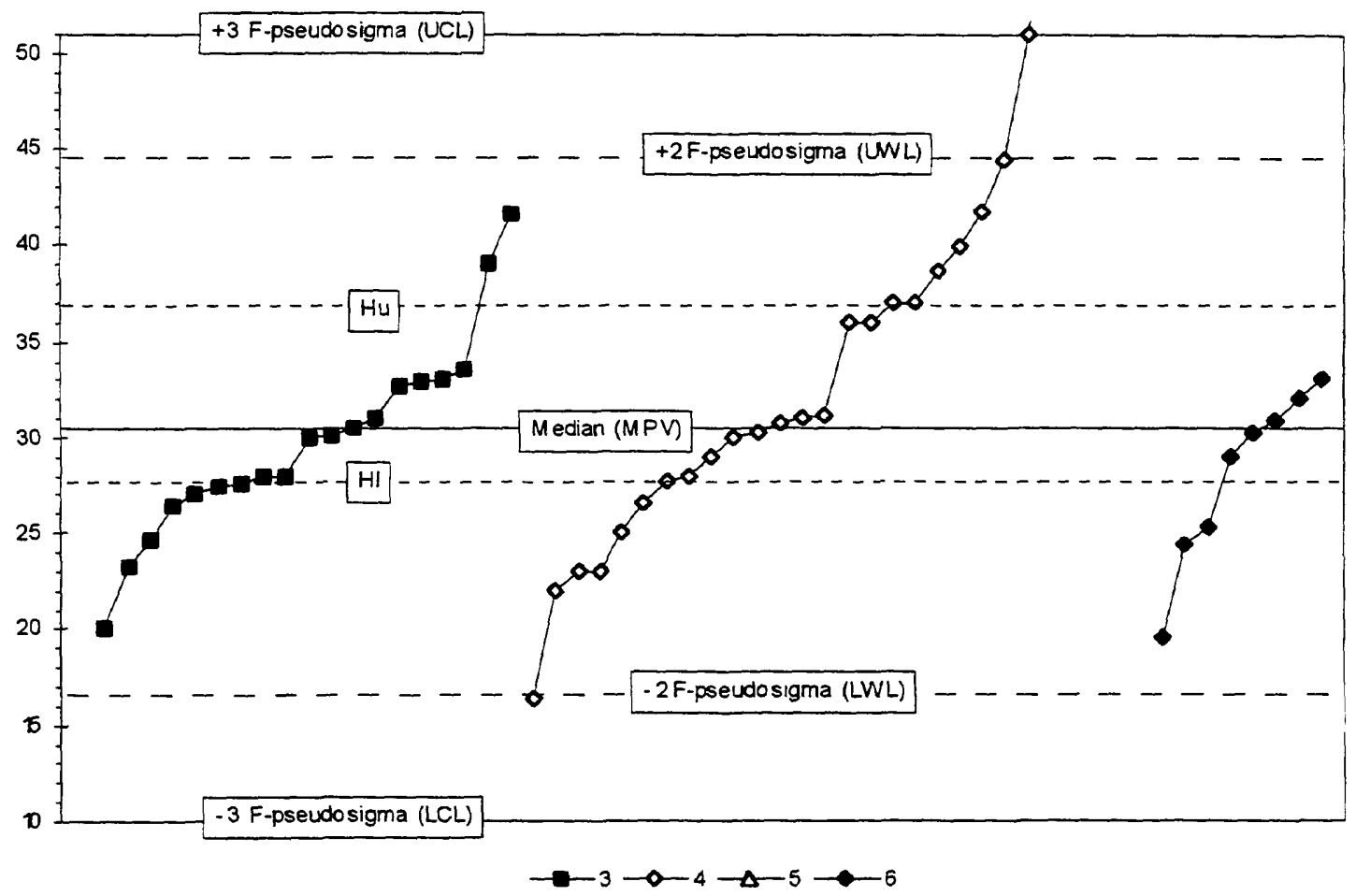

NOTE: vertical scale is the concentration value of the individual analyte in appropriate units (see table 2.) Methods shown are defined in Tables 3 and 12 through 18.

Figure 1.-Statistical parameters shown on reported-data graphs

\section{REFERENCE}

Hoaglin, D.C., Mosteller, F., and Tukey, J.W., eds., 1983, Understanding Robust and Exploratory Data Analysis: John Wiley and Sons, Inc., 447p. 
Table 4. Overall laboratory performance ratings for standard reference water samples distributed in September 1996

Lab, laboratory number, OWR, overall woighted rating for all sample types; OLR, overall laboratory rating for reported values of a sample type; V/94, number of reported values of 94 total possible values trom all sample types; V/28, V/28, V/16, V/5, V/5, V/11,V/1 are number of reported

values possible for T-143,T-145, M-140, N-51,N-52, P-27 and Hg-23 respectively]

\begin{tabular}{|c|c|c|c|c|c|c|c|c|c|c|c|c|c|c|c|c|}
\hline Standard refe & $\begin{array}{l}\text { rence s } \\
\text { OWR }\end{array}$ & $\frac{\text { iple }=}{V / 94}$ & $\frac{1-143}{\therefore \text { OLR }}$ & & $\frac{T-145}{\text { OLR }}$ & V/28 & $\frac{M-140}{O Q R}$ & $\frac{16}{\mathrm{~V} / 6}$ & $\frac{N-51}{O L R}$ & & $\frac{\mathrm{N}: 52}{\mathrm{a}: \mathrm{2}}$ & $\frac{\pi}{y / 5}$ & $\frac{P-27}{O L R}$ & & $1+2=23$ & $\mathrm{VI}$ \\
\hline$\frac{\mathrm{Lab}}{1}$ & 3.5 & $\frac{V / 94}{90}$ & $\frac{\text { ORR }}{36}$ & $\frac{y / 28}{28}$ & $\frac{O L R}{3.4}$ & $\frac{V / 28}{28}$ & QQLR & $\frac{\mathrm{V} / 6}{16}$ & OLR & $\frac{V / 5}{3}$ & Qlik & $\nabla / 5$ & & V/11 & Q4 & VI \\
\hline $\begin{array}{l}1 \\
2\end{array}$ & 1.7 & $\begin{array}{l}90 \\
18\end{array}$ & 3,6 & 288 & 3.4 & 28 & - 15 & 10 & 3.0 & 3 & 34 & 5 & $\begin{array}{l}3.8 \\
2.0\end{array}$ & $\begin{array}{l}9 \\
8\end{array}$ & 3 & 1 \\
\hline 3 & 1.8 & 86 & 17 & 26 & 1.5 & 25 & 79 & 16 & 2.5 & 4 & 30 & 5 & 2.0 & 9 & 0 & 1 \\
\hline 4 & 3.1 & 29 & $\because \mathbf{z} 8$ & 10 & 3.2 & 17 & 40 & $\because 2$ & & & & & & & & \\
\hline 5 & 3.0 & 49 & & & 3.4 & 23 & 34 & 13 & 0.5 & 2 & 20 & 3 & 2.3 & 8 & .... & $\cdots$ \\
\hline 7 & 2.6 & 59 & 25 & 23 & 2.5 & 19 & $2: 2$ & 60 & 2.0 & 1 & 13 & 4 & 3.8 & 5 & $\therefore 4$ & $\because \div$ \\
\hline 10 & 3.3 & 37 & 28 & $\because 6$ & 2.8 & 8 & 35 & 13 & 3.8 & 4 & 36 & 5 & & & $\because 4$ & 1 \\
\hline 11 & 2.8 & 72 & 30 & 25 & 2.9 & 26 & 17 & 12 & 2.7 & 3 & 32 & 5 & & & 3 & 1 \\
\hline 13 & 2.8 & 61 & 26 & 23 & 2.8 & 22 & 27 & 12 & & & 38 & 4 & & & & \\
\hline 15 & 1.9 & 70 & 22 & 21 & 1.6 & 20 & 18 & 12 & 0.0 & 2 & 26 & 5 & 1.9 & 9 & 3 & 1 \\
\hline 16 & 2.8 & 80 & $\because 3.2$ & 27 & 2.7 & 27 & 2.5 & 15 & 2.2 & 5 & 166 & 5 & & & $\therefore$ & $\because$ \\
\hline 18 & 3.3 & 66 & 3.5 & 22 & 3.6 & 20 & 29 & 44 & 2.8 & 4 & 26 & 5 & & & 3 & 1 \\
\hline 19 & 3.2 & 33 & 32 & 13 & 3.3 & 6 & 30 & 10 & & & 35 & 4 & & & & \\
\hline 21 & 3.0 & 6 & 00 & 1 & & & m & & 3.6 & 5 & & & & & & \\
\hline 22 & 3.3 & 3 & & & & & 40 & $\mathrm{t}$ & 2.0 & 1 & 40 & 1 & & & $\ldots$ & $\because \because$ \\
\hline 23 & 2.7 & 53 & 2.9 & 75 & 2.4 & 14 & 27 & 44 & 0.0 & 1 & 13 & 3 & 3.7 & 6 & & \\
\hline 24 & 2.9 & 47 & 26 & 20 & 2.8 & 14 & 37 & 13 & & & & & & & & \\
\hline 25 & 2.5 & 55 & $\because 21$ & 15 & 2.2 & 11 & 25 & 12 & 3.0 & 4 & 24 & 5 & 3.1 & 8 & & \\
\hline 26 & 3.4 & 73 & 3.3 & 24 & 3.5 & 24 & 3.4 & 13 & & & 20 & 2 & 3.4 & 9 & 4 & 1 \\
\hline 28 & 1.5 & 46 & 15 & 16 & 1.5 & 16 & 2.3 & 7 & 0.0 & 2 & $m$ & $\ldots$ & 1.5 & 4 & 0 & 4 \\
\hline 30 & 3.1 & 49 & 32 & 27 & 2.9 & 21 & 32 & 5 & & & 40 & 2 & & & & \\
\hline 32 & 3.4 & 67 & 36 & 26 & 3.6 & 26 & 29 & 14 & & & & & & & 3 & 1 \\
\hline 33 & 3.0 & 42 & 27 & 10 & 2.4 & 10 & 34 & 17 & 3.0 & 1 & 25 & 2 & 3.6 & 8 & & \\
\hline 34 & 3.6 & 7 & 3 & 3 & 3.7 & 3 & $\because$ & 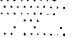 & & & & $\therefore \div$ & & & 4 & 1 \\
\hline 35 & 3.5 & 2 & 4.0 & 1 & 3.0 & 1 & ऑू & 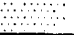 & & & $\cdots$ & $\because \cdots$ & & & $\ldots$ & $\cdots$ \\
\hline 36 & 1.4 & 59 & 13 & 18 & 0.8 & 20 & 18 & 17 & & & 20 & $3 \therefore$ & 2.5 & 4 & 3 & 1 \\
\hline 38 & 3.4 & 27 & & & & & 3.3 & 40 & 3.0 & 5 & 36 & 5 & 3.6 & 7 & & \\
\hline 39 & 2.4 & 27 & $\therefore 2.5$ & 11. & & & 26 & 7 & 2.0 & 3 & & & 2.2 & 5 & 3 & 1 \\
\hline 40 & 2.4 & 48 & 2.2 & 19 & 2.1 & 16 & 29 & 13 & & & & m & 2.2 & & & \\
\hline 42 & 2.3 & 72 & $\begin{array}{r}3.0 \\
\end{array}$ & 25 & 2.0 & 26 & 18 & 12 & & & 0.3 & 3 & 2.4 & 5 & 4 & 1 \\
\hline 43 & 3.6 & 25 & 3.4 & 7 & 3.4 & 7 & 3.7 & $\mathrm{t1}$ & & & & & & & & \\
\hline 46 & 3.0 & 70 & 3.2 & 22 & 3.1 & 21 & 26 & 12 & 3.0 & 2 & 2.0 & 5 & 3.3 & 7 & 4 & 1 \\
\hline 48 & 2.4 & 74 & 22 & 22 & 2.8 & 21 & 22 & 12 & 3.5 & 4 & 20 & 5 & 1.9 & 9 & 4 & $\therefore$ \\
\hline 50 & 2.6 & 25 & 2.1 & 11 & 2.0 & & 3,2 & 13 & & & & 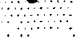 & & & 0 & 1 \\
\hline 51 & 2.7 & 15 & 2.7 & 3 & 2.7 & 3 & 28 & 8 & & & & $\because{ }^{\prime}$ & & & 3 & 1 \\
\hline 53 & 1.5 & 6 & & & & & & & $1 . \overline{0}$ & 3 & 20 & 3 & & & & \\
\hline 55 & 2.8 & 32 & 27 & 10 & & & 2.6 & 12 & 2.8 & 4 & 3.6 & 5 & & & 3 & 1 \\
\hline 56 & 2.2 & 18 & & & & & 2.3 & $\because 9$ & 3.3 & 4 & 10 & 5 & & & $\therefore$ & $\because$ \\
\hline 57 & 2.0 & 18 & & $\cdots$ & & & 2.2 & 13 & & & 14 & 5 & & & & \\
\hline 58 & 1.7 & 34 & 24 & 9 & 1.0 & 8 & $\because$ & & 1.0 & 4 & 15 & 4 & 1.9 & 9 & $\therefore$ & $\because$ \\
\hline 59 & 3.2 & 46 & & & 3.5 & 27 & 23 & 12 & 3.3 & 3 & $4: 0$ & $5 \therefore$ & 3.5 & 4 & 4 & 1 \\
\hline 64 & 3.6 & 23 & 30 & 3 & & & 3.4 & $\because 7$ & 3.5 & 2 & 40 & 3 & 3.9 & 8 & & \\
\hline 68 & 2.2 & 71 & 22 & 25 & 1.9 & 24 & 2.5 & 43 & 2.3 & 4 & 28 & 4 & & & 2 & 1 \\
\hline 69 & 2.9 & 48 & 2.9 & 18. & 2.9 & 18 & 2.6 & 10 & & & 40 & 1 & & & 4 & 1 \\
\hline 70 & 3.4 & 55 & 3.3 & 19 & 3.3 & 16 & 3.5 & 13 & 4.0 & 1 & 3.4 & 5 & & & 3 & $1 \ldots$ \\
\hline 73 & 2.6 & 22 & 2.7 & 71 & 2.5 & 11 & & & & & & & & & & \\
\hline 75 & 3.5 & 51 & 3.3 & 21. & 3.6 & 19 & 3.7 & 10 & & & 2.0 & 1 & & & & \\
\hline 76 & 2.8 & 26 & 2.7 & 10 & 2.8 & 9 & 2.6 & $\because 5$ & & & 4.0 & 1 & & & 3 & 1 \\
\hline 80 & 2.3 & 30 & 2.8 & 8 & 2.6 & 7 & 1.8 & 12. & & & 2.0 & 3 & & & & \\
\hline 81 & 2.7 & 76 & 2.2 & 21 & 2.3 & 22 & 3,4 & 14 & 3.3 & 3 & 2.6 & 5 & 3.4 & 10 & 3 & 1 \\
\hline 83 & 3.4 & 51 & 3.6 & 15 & 3.6 & 16 & $3: 0$ & 9 & 3.0 & 1 & 2.8 & 4 & 3.2 & 6 & & \\
\hline 85 & 3.1 & 52 & 3.2 & 17. & 2.8 & 13 & 3.1 & 12 & 3.4 & 5 & 3.2 & 5 & & & & \\
\hline 86 & 3.3 & 55 & $\therefore 3: 4$ & 21 & 3.2 & 21 & 3.3 & 9 & & & 3.0 & 3 & & & 3 & 1 \\
\hline 87 & 2.0 & 58 & $2 \mathbf{2 6}$ & 18 & 1.3 & 18 & 1.8 & 12 & 2.8 & 4 & 24 & 5 & & & 0 & 1 \\
\hline 88 & 1.2 & 6 & & & & & & & 1.3 & 3 & 10 & 3 & & . & & $\because$ \\
\hline 89 & 3.0 & 76 & 2.8 & 22 & 2.9 & 21 & 3.0 & 13 & 3.0 & 4 & 3.8 & 5 & 3.4 & 10 & 3 & 1 \\
\hline 90 & 1.8 & 6 & & & & & 1.0 & 4 & 4.0 & 1 & 30 & 1 & & & & \\
\hline 91 & 3.4 & 10 & 3.5 & 2. & 3.5 & 2 & & & 3.5 & 2 & 33 & 4 & & & & \\
\hline 92 & 3.6 & 14 & & & & & 3.8 & 6 & 3.7 & 3 & 37 & 3 & 3.0 & 2 & & \\
\hline 96 & 3.0 & 42 & 2.5 & 13 & 2.9 & 13 & 3.3 & 7 & 3.3 & 3 & 38 & 5 & & & 4 & 1 \\
\hline 97 & 2.9 & 72 & 2.7 & 25 & 3.1 & 24 & 2.7 & 14 & 2.8 & 4 & 3.2 & 5 & & & & \\
\hline 102 & 1.8 & 63 & 18 & 22 & 1.8 & 23 & 15 & 10 & 2.7 & 3 & 2.2 & 5 & & & & \\
\hline 104 & 3.7 & 12 & 40 & 1 & 3.0 & 1 & & & 3.4 & 5 & 4.0 & 5 & & & & \\
\hline 105 & 3.1 & 81 & 3.0 & 25 & 3.4 & 25 & 2.9 & 14 & 3.3 & 3 & 3.0 & 5 & 2.9 & 8 & 4 & 1 \\
\hline 107 & 3.1 & 44 & 2.6 & 11 & 3.1 & 11 & 3.5 & 11 & & & 3.0 & 4 & 3.6 & 7 & & \\
\hline 108 & 2.6 & 5 & & & & & & & & & 3.3 & 4 & & & 0 & 1 \\
\hline 109 & 2.5 & 43 & 2.1 & 11 . & 2.5 & 11 & $2.8^{\circ}$ & -11 & & & & $\because \because$ & 2.6 & 10 & & \\
\hline 110 & 2.9 & 11 & 2.0 & 4 & & & & & 4.0 & 1 & m & & 3.3 & 6 & $\therefore:$ & $\ldots$ \\
\hline 113 & 3.1 & 71 & 3.2 & 21 & 3.2 & 21 & 2.9 & 14 & 4.0 & 1 & 2.6 & 5 & 3.0 & 8 & $\because 3$. & 1 \\
\hline 114 & 2.4 & 31 & 21 & 12 & 1.7 & 7 & 3.6 & 8 & 4.0 & 1 & 13 & 3 & & & $\ldots \quad \therefore$ & $\cdots$ \\
\hline
\end{tabular}


Table 4. Overall laboratory performance ratings for standard reference water samples distributed in September 1996-Continued

Lab, laboratory number, OWR, overall woighted rating for all sample types; OLR, overall laboratory rating for reported values of a sample type; V/94, number of reported values of 94 total possible values from all sample types; V/28,V/28, V/16, V/5, V/5, V/11, V/1 are number of reported

values possible for T-143,T-145, M-140, N-51,N-52, P-27 and $\mathrm{Hg}-23$ respectively]

\begin{tabular}{|c|c|c|c|c|c|c|c|c|c|c|c|c|c|c|c|c|}
\hline$\frac{\text { Standard refe }}{\text { Lab }}$ & $\frac{\text { rence s }}{\text { OWR }}$ & $\frac{\text { iple }=}{\text { V/94 }}$ & $\frac{T-143}{\text { OLR }}$ & y/28 & $\frac{T-145}{\text { OLR }}$ & V/28 & M140 & VIT & $\frac{N-51}{\text { OLR }}$ & V/5 & $\frac{N: 52}{\text { OLR }}$ & V/5 & $\frac{P-27}{O L R}$ & V/11 & $\frac{\mathrm{Hg}=23}{\mathrm{dLR}}$ & $\mathrm{V}+1$ \\
\hline 118 & 1.9 & 35 & 0.6 & Ato & 2.7 & 10 & 27 & $\ldots 6.66$ & 20 & 4 & $\frac{14}{22}$ & 5 & & vili & & \\
\hline 119 & 3.0 & 85 & 30 & 26 & 2.8 & 26 & 32 & 14 & 3.0 & 4 & 26 & 5 & 3.1 & 9 & 3 & 1 \\
\hline 121 & 3.3 & 36 & 32 & 16 & 3.1 & 14 & 40 & 6 & & & $\because z: 0$ & & 3.1 & 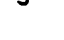 & : & $\because$ \\
\hline 127 & 3.1 & 46 & 3.0 & 24 & & & 3.4 & 14 & 3.5 & 2 & 24 & 5 & & & 4 & 1 \\
\hline 128 & 3.0 & 68 & 28 & 25 & 2.8 & 25 & 33 & 12 & 3.0 & 2 & 35 & 4 & & & $\ldots$ & $\ldots$ \\
\hline 129 & 2.3 & 41 & $2 \cdot 0$ & 9 & 1.7 & 9 & 2.6 & 74 & 3.8 & 4 & 18 & 5 & & & & \\
\hline 132 & 2.5 & 56 & 28 & 16 & 2.1 & 16 & 31 & 9 & 2.5 & 4 & 23 & 4 & 2.1 & 7 & & \\
\hline 133 & 2.8 & 45 & 31 & 14 & 2.5 & 14 & 28 & 6 & 3.0 & 5 & 24 & 5 & & & 4 & 1 \\
\hline 134 & 3.7 & 89 & 38 & 27 & 3.7 & 27 & 33 & 75 & 3.6 & 5 & 36 & 5 & 3.9 & 9 & 3 & 1 \\
\hline 138 & 3.4 & 86 & 33 & 26 & 3.3 & 26 & 38 & 75 & 3.0 & 4 & 32 & 5 & 3.9 & 9 & 4 & 1 \\
\hline 140 & 2.4 & 54 & $2:$ & $\therefore 3$ & 1.9 & 13 & $2: 5$ & mt & 3.7 & 3 & 1.8 & 5 & 2.2 & 9 & & \\
\hline 141 & 2.9 & 72 & 30 & 22 & 2.4 & 19 & $3: 3$ & 12 & 1.0 & 3 & 3.4 & 5 & 3.5 & 10 & 2 & 1 \\
\hline 142 & 2.9 & 83 & 31 & 28 & 2.9 & 28 & 28 & 16 & 2.6 & 5 & 28 & 5 & & & 2 & 1 \\
\hline 143 & 3.6 & 18 & $\because$ & & & & 36 & 5 & 3.6 & 5 & 34 & 5 & 3.7 & 3 & & \\
\hline 144 & 2.7 & 9 & 2.3 & 4 & 2.8 & 4 & a & $\because$ & & & $\because$ & $\ldots$ & & & 4 & 1 \\
\hline 145 & 1.8 & 78 & 10 & 23 & 1.2 & 23 & 29 & 15 & 3.5 & 2 & 32 & 5 & 2.8 & 9 & 2 & A \\
\hline 146 & 2.6 & 55 & 2.9 & 18 & 2.5 & 16 & 28 & 11 & & & 0,0 & 4 & 2.8 & 5 & 4 & 4 \\
\hline 149 & 3.0 & 22 & & & 3.0 & 11 & 32 & 6 & 2.5 & 4 & ato & m & & & 4 & 1 \\
\hline 151 & 3.3 & 54 & 33 & 49 & 3.5 & 19 & 30 & 13 & 3.3 & 3 & $\because \cdots$ & $n$ & & & & \\
\hline 155 & 2.9 & 23 & 40 & 1 & 1.0 & 1 & 3.3 & 8 & 3.2 & 5 & 26 & 5 & 2.0 & 3 & & $\ldots$ \\
\hline 158 & 2.4 & 56 & 25 & 16 & 1.9 & 77 & 3.5 & 8 & 3.0 & 3 & 3.8 & 4 & 1.3 & -8 & $\cdots$ & \\
\hline 180 & 3.4 & 64 & 34 & 20 & 3.6 & 18 & 28 & 12 & 3.5 & 2 & $3: 4$ & 5 & 3.9 & 7 & & a \\
\hline 183 & 1.5 & 13 & 00 & $\therefore 1$ & 0.0 & 1 & 17 & 3 & 2.0 & 2 & 20 & 3 & 1.7 & 3 & & \\
\hline 190 & 2.4 & 70 & 24 & 18 & 2.6 & 18 & 24 & 74 & 1.8 & 5 & 16 & 5 & 3.1 & 10 & $\therefore$ & $\therefore$ \\
\hline 191 & 3.2 & 70 & 33 & 25 & 3.4 & 25 & 30 & 11 & 4.0 & 1 & 35 & 2 & 2.2 & 6 & 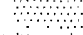 & $\because$ \\
\hline 193 & 2.3 & 35 & $\because 2,3$ & 15 & 1.9 & 12 & 30 & 3 & 3.0 & 1 & $2: 5$ & 2 & 3.0 & 1 & 2 & 1 \\
\hline 196 & 3.5 & 48 & 37 & 27 & 3.3 & 21 & 27 & 3 & & & & & 4.0 & 3 & & $\cdots$ \\
\hline 197 & 3.5 & 6 & & & & & & & 3.0 & 2 & 40 & 2 & 3.5 & 2 & & \\
\hline 203 & 2.4 & 36 & 27 & $\theta$ & 2.3 & 9 & 22 & 6 & 3.5 & 4 & 24 & 5 & 1.3 & 3 & $\because$ & $\therefore$ \\
\hline 204 & $\begin{array}{l}2.4 \\
2.5\end{array}$ & 48 & 2.3 & 14 & 2.2 & 13 & 3.0 & 8 & 3.0 & 3 & 28 & 4 & 2.7 & 6 & & 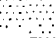 \\
\hline 212 & 2.4 & 80 & 2.1 & 27 & 2.3 & 28 & 2.7 & 16 & 3.3 & 3 & 2.8 & 5 & & & 3 & 1 \\
\hline 213 & 2.3 & 34 & 18 & .12 & 2.9 & 12 & 3,0 & 4 & 4.0 & 1 & 15 & 4 & & & $\theta$ & $\because 1$ \\
\hline 215 & 2.4 & 79 & 2.4 & 24 & 2.0 & 24 & $\therefore 28$ & 14 & 2.0 & 2 & 22 & 5 & 2.8 & 9 & 0 & 1 \\
\hline 217 & 2.6 & 69 & 3.0 & 28 & 2.7 & 27 & 16 & 14 & & & & & & & & \\
\hline 218 & 2.2 & 18 & 28 & 5 & 2.6 & 5 & 16 & 8 & & & $\therefore$ & $\because$ & & & & $\because$ \\
\hline 219 & 2.7 & 50 & 2.7 & 21. & 2.8 & 19 & 27 & 9 & & & & & & & 3 & 7 \\
\hline 220 & 3.0 & 40 & 31 & 10 & 3.3 & 10 & 33 & 7 & 2.7 & 3 & 13 & 3 & 2.9 & 7 & & 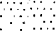 \\
\hline 221 & 3.0 & 62 & 32 & 18 & 3.3 & 18 & 3.1 & 8 & 2.8 & 5 & 24 & 5 & 2.4 & 7 & 3 & 1 \\
\hline 224 & 1.9 & 71 & 16 & 19 & 1.8 & 19 & 1.6 & 13 & 2.2 & 5 & 20 & 5 & 3.2 & 10 & & \\
\hline 234 & 3.2 & 78 & 31 & 27 & 3.1 & 27 & 3.5 & 16 & 3.3 & 3 & 3.3 & 4 & & & 4 & 1 \\
\hline 235 & 2.0 & 60 & 2.4 & 23 & 1.8 & 22 & 11 & 9 & & & تحبرب & & 2.0 & 5 & 2 & 1 \\
\hline 236 & 2.2 & 65 & 1,8 & 25 & 2.2 & 25 & 29 & 15 & & & & $\therefore$ & & & & \\
\hline 240 & 2.0 & 64 & 17 & 18 & 1.2 & 18 & 3.0 & 12 & 0.7 & 3 & 26 & 5 & 3.1 & 8 & & \\
\hline 241 & 2.1 & 80 & 117 & 23 & 1.6 & 23 & 2.3 & 14 & 3.6 & 5 & 3.4 & 5 & 2.2 & 9 & 3 & 1 \\
\hline 243 & 2.7 & 10 & & & & & 2.7 & $\because 3$ & 3.0 & 2 & 30 & 3 & 2.0 & 2 & & \\
\hline 244 & 2.9 & 7 & 0.0 & 1. & 0.0 & 1 & 4.0 & 3 & & & $\because \because$ & $\because$ & 4.0 & 2 & & \\
\hline 245 & 2.4 & 11 & $\cdots$ & & 2.3 & 10 & & & & & & & & & 3 & 1 \\
\hline 246 & 0.0 & 5 & 0.0 & 5 & & & & & & & & $\therefore$ & & & & \\
\hline 247 & 2.7 & 15 & $\therefore \quad \because$ & $\because \cdots$ & & & 23 & 6 & 4.0 & 1 & 3.0 & 2 & 2.8 & 6 & & \\
\hline 248 & 2.0 & 4 & & & & & & & & & 2.0 & 4 & & & & \\
\hline 249 & 1.6 & 38 & 1.4 & 12 & 1.8 & 12 & 18 & 9 & & & 1.4 & 5 & & & & \\
\hline 253 & 1.4 & 19 & 0.5 & 4 & 0.8 & 4 & 20 & 5 & 4.0 & 1 & $1: 4$ & 5 & & & & \\
\hline 255 & 3.4 & 71 & 3.5 & 23 & 3.5 & 23 & 3.1 & 74 & & & 3.5 & 4 & 3.7 & 6 & 4 & 1 \\
\hline 256 & 1.6 & 29 & 18 & 13 & & & 16 & 9 & & & & $\therefore$ & 1.3 & 7 & & $\therefore$ \\
\hline 257 & 1.4 & 62 & 13 & 19 & 0.7 & 19 & 20 & 13 & & & & $\because$ & 2.2 & 10 & 0 & 1 \\
\hline 258 & 1.0 & 20 & & & & & 1.4 & 11 & & & & & 0.4 & 9 & & \\
\hline 259 & 2.1 & 42 & 1.5 & 13 & 1.5 & 13 & $3: 1$ & 15 & & & & $\therefore$ & & & 3 & 1 \\
\hline 261 & 1.1 & 24 & 2.0 & 4 & 0.8 & 4 & 1.0 & 9 & & & & & 0.9 & 7 & & \\
\hline 262 & 2.2 & 20 & & : & & & 1.9 & 11 & & & & & 2.4 & 9 & & $\therefore$ \\
\hline 265 & 3.2 & 77 & 3.3: & 28 & 3.5 & 28 & $\therefore 3.3$ & 13 & & & & & 2.4 & 7 & 1 & 1 \\
\hline 268 & 2.6 & 24 & 3.3 & 4 & 2.7 & 3 & 2.0 & 9 & & & & & 3.0 & 8 & & \\
\hline 270 & 0.3 & 12 & 0.0 & 3 & 0.7 & 3 & 0.0 & 3 & & & & & 0.3 & 3 & & \\
\hline 271 & 1.3 & 24 & 2.5 & 4 & 1.0 & 4 & $\therefore 11$ & 8 & & & & & 1.1 & 8 & & \\
\hline 272 & 0.6 & 26 & 0.0 & 4 & 0.8 & 4 & $\quad 0.4$ & $\therefore 9$ & & & $\therefore \quad \because \because$ & & 1.0 & 9 & & \\
\hline 273 & 1.1 & 57 & 11 & 18 & 0.7 & 18 & 1.1 & 12 & & & & & 1.7 & 9 & & \\
\hline 274 & 0.3 & 34 & 0.5 & 11 & 0.1 & 11 & 0.4 & 12 & & & & & & & & \\
\hline 276 & 1.3 & 12 & & $\because \ldots$ & & & 0.7 & $\because 6$ & & & & & 1.8 & 6 & & \\
\hline
\end{tabular}


Table 5. Laboratory performance ratings for standard reference water sample $T-143$ (trace constituents)

\begin{tabular}{|c|c|c|c|c|c|c|c|c|c|c|c|c|c|c|c|c|}
\hline \multicolumn{3}{|c|}{\begin{tabular}{|l|} 
Rating \\
4 (Excellent) \\
3 (Good) \\
2 (Satisfactory) \\
\end{tabular}} & \multicolumn{3}{|c|}{$\begin{array}{l}\text { Absolute Z-value } \\
0.00-0.50 \\
0.51-1.00 \\
1.01-1.50 \\
\end{array}$} & \multicolumn{2}{|c|}{$\begin{array}{l}\text { Rating } \\
1 \text { (Questionable) } \\
O \text { (Poor) } \\
\text { NR (Not Rated) } \\
\end{array}$} & \multicolumn{2}{|c|}{$\begin{array}{l}\text { Absolute Z-value } \\
1.51-2.00 \\
\text { greater than } 2.00\end{array}$} & & \multirow{2}{*}{\multicolumn{2}{|c|}{$\mathrm{Ba}$ (Barium) }} & & & & \\
\hline & & alyte $=$ & Ag (Silv & & Al (Alum & nium) & As (Ars & nic) & B (Boro & & & & $\mathrm{Be}(\mathrm{Ber}$ & lium) & $\mathrm{Ca}$ (Calc & um) \\
\hline & & MPV $=$ & 19.8 & $\mu g / L$ & 22.1 & $\mu g / L$ & 15.2 & $\mu g / L$ & 35.0 & $\mu g / L$ & 81.9 & $\mu g / L$ & 8.50 & $\mu g / L$ & $53: 7$ & $\mathrm{mg} / \mathrm{L}$ \\
\hline F-ps & seudos & gma $=$ & $1: 4$ & & 8.3 & & 1.2 & & 5.2 & & 4.5 & $\because$ & 0.66 & & 2.2 & \\
\hline Lab & OLR & V/28 & RV & Rating & RV & Rating & RV & Rating & $\mathrm{RV}$ & Rating & RV & Rating & RV & Rating & RV & Ratuing \\
\hline 1 & 3.6 & 28 & 19.6 & 4 & 19.1 & 4 & 16.2 & 3 & 34.9 & 4 & 82.2 & 4 & 8.13 & 3 & 52.5 & 4 \\
\hline 3 & 1.7 & 26 & 15.0 & 0 & 206.0 & 0 & 14.3 & 3 & 30.0 & 3 & 84.4 . & 3 & 8.00 & 3 & 54.4 & 4 \\
\hline 4 & 2.8 & 10 & & & $<2000$ & NR & & & & & 87.0 & 2 & 9.00 & 3 & 53.4 & 4 \\
\hline 7 & 2.6 & 23 & 21.6 & 2 & 37.6 & 1 & $<120$ & NR & & & 83.6 & 4 & 8.10 & 3 & $55: 8$ & 3 \\
\hline 10 & 2.8 & 6 & & & & & 16.0 & 3 & & & & & & & & \\
\hline 11 & 3.0 & 25 & 21.0 & 3 & & & 15.0 & 4 & 37.0 & 4 & 81.0 & 4 & 8.40 & 4 & $56: 3$ & 2 \\
\hline 13 & 2.6 & 23 & 20.1 & 4 & 22.2 & 4 & 15.5 & 4 & & & 88.1 & .2 & 8.74 & 4 & $59: 0$ & 0 \\
\hline 15 & 2.2 & 21 & 17.6 & 1 & $<50$ & NR & $<100$ & NR & $<50$ & NR & 77.9 & 3 & 8.60 & 4 & 50.6 & 2. \\
\hline 16 & 3.2 & 27 & 19.9 & 4 & 18.5 & 4 & 15.4 & 4 & 57.1 & 0 & 82.2 & 4 & 7.70 & 2 & 54.0 & 4 \\
\hline 18 & 3.5 & 22 & 20.9 & 3 & $<100$ & NR & 14.6 & 4 & $<50$ & NR & 79.0 & 3 & 8.50 & 4 & 53.5 & 4 \\
\hline 19 & 3.2 & 13 & & & & & & & & & 82.2 & 4 & & & 53.6 & 4 \\
\hline 21 & 0.0 & 1 & & & & & & & & & & & & & & \\
\hline 23 & 2.9 & 15 & 22.2 & 1 & $<50$ & NR & 20.5 & 0 & & & & $\because$ & & & 525 & 3 \\
\hline 24 & 2.6 & 20 & $\because$ & $\cdot$ & & & & $\therefore$ & 30.7 & 3 & 78.8 & 3 & & & 53.4 & 4 \\
\hline 25 & 2.1 & 15 & & & & & & $\because$ & & & 84.4 & 3. & 9.90 & 0 & 54.8 & 3 \\
\hline 26 & 3.3 & 24 & 15.6 & 0 & 18.2 & 4 & 14.9 & 4 & 26.1 & 1 & 80.9 & 4 & 8.66 & 4 & 53.8 & 4 \\
\hline 28 & 1.5 & 16 & & & 32.3 & 2 & & & 42.2 & 2 & 85.1 & 3 & & & 55.4 & 3 \\
\hline 30 & 3.2 & 21 & 19.0 & 3 & & & 14.0 & 3 & & & 80.0 & 4 & 7.30 & 1 & 54.0 & 4 \\
\hline 32 & 3.6 & 26 & 19.9 & 4 & 22.6 & 4 & 14.9 & 4 & & & 77.9 & 3 & 9.40 & 2 & 550 & 3 \\
\hline 33 & 2.7 & 10 & & & 100.0 & 0 & & & & & 102.0 & 0 & & & 54.2 & 4. \\
\hline 34 & 3.3 & 3 & & & & & 15.6 & 4 & & & & & & & & \\
\hline 35 & 4.0 & 1 & & & & & & & & & & & & & & \\
\hline 36 & 1.3 & 18 & 13.0 & 0 & $<200$ & NR & 11.0 & 0 & & & 90.0 & 1 & 10.00 & 0 & 52.0 & 3 \\
\hline 39 & 2.5 & 11 & 20.2 . & 4 & & & 18.2 & 0 & & & $79: 1$ & 3 & 9.50 & 1 & & \\
\hline 40 & 2.2 & 19 & 19.3 & 4 & & & & & & & 78.1 & 3 & 7.90 & 3 & & \\
\hline 42 & 3.0 & 25 & 19.0 & 3 & 23.0 & 4 & 15.0 & 4 & 36.0 & 4 & & & & & 567 & 2 \\
\hline 43 & 3.4 & 7 & & & & & & & & & & & & & 540 & 4 \\
\hline 46 & 3.2 & 22 & 20.0 & 4 & & & 13.5 & 2 & 22.7 & 0 & 85.0 & 3 & 8.76 & 4 & 56.0 & 2 \\
\hline 48 & 2.2 & 22 & 20.0 & 4 & 18.5 & 4 & 14.2 & 3. & $<100$ & NR & 97.8 & 0 & 10.00 & 0 & $.55 ; 9$ & 2 \\
\hline 50 & 2.1 & 11 & & & & & 14.0 & 3 & & & 900 & 1 & & & & \\
\hline 51 & 2.7 & 3 & & & & & & & & & & & & & & \\
\hline 55 & 2.7 & 10 & . & & & & & & & & 78.2 & 3 & & & 549 & 3 \\
\hline 58 & 2.4 & 9 & 21.0 & 3 & 17.0 & 3 & $19.0^{\circ}$ & 0 & & & & & & & & \\
\hline 64 & 3.0 & 3 & & & & & & & & & & & & & & \\
\hline 68 & 2.2 & 25 & 40.0 & 0. & 54.5 & 0 & 9.6. & 0 & 140.0 & 0 & 83.0 & 4 & 8.90 & 3 & $56: 5$ & 2 \\
\hline 69 & 2.9 & 18 & 19.8 & 4 & 16.5 & 3 & 16.2 & 3 & & & & & 7.32 & 1 & 52.4 & 3 \\
\hline 70 & 3.3 & 19 & 18.7 & 3 & $<100$ & NR & .15 .3 & 4 & $<50$ & NR & 82.3 & 4 & 8.67 & 4 & 55.4 & 3 \\
\hline 73 & 2.7 & 11 & 17.0 & 1 & & & 220 & 0. & & & & & & & & \\
\hline 75 & 3.3 & 21 & 20.1 & 4 & $<30$ & NR & 14.6 & 4 & & & 81.2 & 4 & 8.50 & 4 & 53.3 & 4 \\
\hline 76 & 2.7 & 10 & 19.4 & 4 & 20.2 & 4 & 16.5 & 2 & & & & & 9.68 & 1 & & $\therefore$ \\
\hline 80 & 2.8 & 8 & & & & & 16.3 & 3 & & & & & & & & \\
\hline 81 & 2.2 & 21. & & & $<6$ & NR & $16: 0$ & 3 & & & 78.0 & 3 & 7.00 & 0 & 530 & 4 \\
\hline 83 & 3.6 & 15 & & $\because$ & $<25$ & NR & & & & & $\pi 7.8$ & 3 & 8.20 & 4 & 53.1 & 4 \\
\hline 85 & 3.2 & 17 & 20.0 & 4 & $<100$ & NR & & & 35.6 & 4 & 83.2 & 4 & 9.17 & 2 & 55.4 & 3 \\
\hline 86 & 3.4 & 21 & & & & & 15.3 & 4 & 36.9 & 4 & 81.5 & 4 & 8.25 & 4 & 55.5 & 3 \\
\hline 87 & 2.6 & 18 & 26.0 & 0 & & & 14.9 & 4 & & & 90.7 & 1 & & & 51.3 & 2 \\
\hline 89 & 2.8 & 22 & $19: 1$ & 4 & 23.1 & 4 & 16.4: & 2 & & & 981 & 0 & 7.40 & 1 & 52.6 & 4 \\
\hline 91 & 3.5 & 2 & & & & & & & & & & & & & & \\
\hline 96 & 2.5 & 13 & 20.4 & 4 & & & 14.6 & 4 & & & $<100$ & NR & 10.00 & 0 & & \\
\hline 97 & 2.7 & 25 & 19.0 & 3. & 25.7 & 4 & 17.0 & $i$ & & & 69.6 & 0 & 8.99 & 3 & 51.8 & 3 \\
\hline 102 & 1.8 & 22 & 143.0 & 0 & 20.0 & 4 & 15.8 & 3 & & & 94.0 & 0 & 8.00 & 3 & 58.0 & 1 \\
\hline 104 & 4.0 & 1 & & & & & & & & & & & & & & \\
\hline 105 & 3.0 & 25 & 17.9 & 2 & 21.1 & 4 & 15.3 & 4 & & & 76.2 & 2 & 7.30 & 1 & 52.8 & 4 \\
\hline 107 & 2.6 & 11 & 20.0 & 4 & 23.0 & 4 & & & & & $\pi 7.0$ & 2 & & & 520 & 3 \\
\hline 109 & 2.1 & 11 & & & & & 13.1 & 1 & & & & & & & 526 & 4 \\
\hline 110 & 2.0 & 4 & & & & & & & & & & & & & 55.0 & 3 \\
\hline 113 & 3.2 & 21 & 18.1 & 2 & 20.2 & 4 & -17.5 & 1 & & & 80.2 & 4 & 8.78 & 4 & .57 .9 & 1 \\
\hline 114 & 2.1 & 12 & 18.0 & 2 & & & & & & & & & & & 44.0 & 0 \\
\hline 118 & 0.6 & 10 & 6.7 & 0 & $<2000$ & NR & 17.9 & 0 & & & 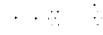 & & & & & \\
\hline 119 & 3.0 & 26 & 16.5 & 0 & 19.5 & 4 & 15.0 & 4 & 32.0 & 3 & 84.0 & 4 & 7.72 & 2 & 53.7 & 4 \\
\hline 121 & 3.2 & 16 & & & & & & & & & 81.0 & 4 & 8.00 & 3 & 52.2 & 3 \\
\hline 127 & 3.0 & 24 & 18.8 & 3 & $<30$ & NR & 14.4 & 3 & 35.7 & 4 & 75.8 & 2 & 6.53 & 0 & $\theta$ & $\because$ \\
\hline 128 & 2.8 & 25 & 19.5 & 4 & 18.7 & 4 & 14.8 & 4 & 30.0 & 3 & 70.1 & 0 & 7.54 & 2 & $55: 7$ & 3 \\
\hline 129 & 2.0 & 9 & & & & & & & 95.0 & 0 & & & & & $58: 0$ & 1 \\
\hline 132 & 2.8 & 16 & & & 49.0 & 0 & & & 26.5 & 1 & & & & & 53.4 & 4 \\
\hline
\end{tabular}


Table 5. Laboratory performance ratings for standard reference water sample T-143 (trace constituents)-Continued

\begin{tabular}{|c|c|c|c|c|c|c|c|c|c|c|c|c|c|c|c|c|}
\hline \multicolumn{3}{|c|}{\begin{tabular}{|l|} 
Rating \\
4 (Excellent) \\
3 (Good) \\
2 (Satisfactory) \\
\end{tabular}} & \multicolumn{3}{|c|}{$\begin{array}{l}\text { Absolute Z-value } \\
0.00-0.50 \\
0.51-1.00 \\
1.01-1.50 \\
\end{array}$} & \multicolumn{2}{|c|}{$\begin{array}{l}\text { Rating } \\
1 \text { (Questionable) } \\
O \text { (Poor) } \\
\text { NR (Not Rated) } \\
\end{array}$} & \multicolumn{2}{|c|}{$\begin{array}{l}\text { Absolute Z-value } \\
1.51-2.00 \\
\text { greater than } 2.00\end{array}$} & & & & & & & \\
\hline & \multicolumn{3}{|c|}{ Analyte $=A g \cdot($ Silver $)$} & ver) & \multicolumn{2}{|c|}{ Al (Aluminium) } & \multicolumn{2}{|c|}{ As (Arsenic) } & \multicolumn{2}{|c|}{ B (Boron) } & \multicolumn{2}{|c|}{ Ba. (Barium) } & \multicolumn{2}{|c|}{ Be (Beryllium) } & \multicolumn{2}{|c|}{$\mathrm{Ca}$ (Calcium) } \\
\hline & & $M P V=$ & 19.8 & $\therefore \quad \mu g / L$ & 22.1 & $\mu g / L$ & 15.2 & $\mu g / L$ & 35.0 & $\mu g / L$ & $81: 9$ & $\mu g / L$ & 8.50 & $\mu g / L$ & 53.7 & $\mathrm{mg} / \mathrm{L}$ \\
\hline F-ps & seudosi & igma = & 1.4 & & 8.3 & & 1.2 & & 5.2 & & 4.5 & & 0.66 & & 2.2 & \\
\hline Lab & OLR & $V / 28$ & $\mathrm{RV}$ & Rating & RV & Rating & RV & Rating & RV & Rating & RV & Rating & $\mathrm{RV}$ & Rating & R I & Rating \\
\hline 133 & 3.1 & 14 & 19.6 & 4 & & & 16.5 & 2 & & & 77.7 & 3 & 8.70 & 4 & 53.0 & 4 \\
\hline 134 & 3.8 & 27 & 19.0 & 3 & 22.9 & 4 & 14.0 & 3 & 35.8 & 4 & $78: 9$ & 3 & 8.51 & 4 & 54.5 & 4 \\
\hline 138 & 3.3 & 26 & 20.6 & 3 & 20.0 & 4 & 14.0 & 3 & 28.8 & 2 & 80.5 & 4 & 8.65 & 4 & 54.9 & 3 \\
\hline 140 & 2.9 & 13 & & . & & & & & & & & & & & 54.5 & 4 \\
\hline 141 & 3.0 & 22 & 29.0 & 0 & $<100$ & NR & .15 .0 & 4 & 24.1 & 0 & 84.8 & 3 & 8.15 & 3 & 56.2 & 2 \\
\hline 142 & 3.1 & 28 & 19.3 & 4 & 27.3 & 3 & 15.2 & 4 & 35.7 & 4 & 81.8 & 4 & 8.87 & 3 & 53.3 & 4 \\
\hline 144 & 2.3 & 4 & & & & & 14.5 & 3 & & & & & $<0.01$ & 0 & & \\
\hline 145 & 1.0 & 23 & & . & 58.3 & 0 & 27.4 & 0 & 35.4 & 4 & 90.9 & 1 & 9.50 & 1 & 59.1 & 0 \\
\hline 146 & 2.9 & 18 & $<10$ & 0 & $<200$ & NR & 15.1 & 4 & & & 82.2 & 4 & 7.23 & 1 & 50.0 & 3 \\
\hline 151 & 3.3 & 19 & 17.9 & 2 & 18.1 & 4 & 14.1 & 3 & & & 84.2 & 3 & 8.16 & 3 & & $\because$ \\
\hline $15 \overline{5}$ & 4.0 & 1 &.$\cdots$ & & & & & & & & & & & & & $\cdots$ \\
\hline 158 & 2.5 & 16 & $\because$ & & & & & & 38.0 & 3 & 82.0 & 4 & 9.30 & 2 & 54.7 & 4 \\
\hline 180 & 3.4 & 20 & 19.9 & 4 & $<40.6$ & NR & $<37.1$ & NR & 32.4 & 4 & 79.0 & 3 & 8.60 & 4 & 54.4 & 4 \\
\hline 183 & 0.0 & 1 & & & & & & & & & & & & & & \\
\hline 190 & 2.4 & 18 & 22.4 & 1 & 16.7 & 3 & 15.4 & 4 & & & & $\therefore$ & & & 53.3 & 4 \\
\hline 191 & 3.3 & 25 & & & 22.0 & 4 & 17.0 & 1 & 34.0 & 4 & 41.0 & 0 & 8.89 & 3 & 53.1 & 4 \\
\hline 193 & 2.3 & 15 & 19.0 & 3 & & & 13.0 & 1 & & & & & 8.00 & 3 & 51.8 & 3 \\
\hline 196 & 3.7 & 21 & 19.5 & 4 & 22.0 & 4 & 15.5 & 4 & & & 82.2 & 4 & 8.85 & 3 & & \\
\hline 203 & 2.7 & 9 & & . & 18.6 & 4 & & & & & & : & & & 47.8 & 0 \\
\hline 204 & 2.3 & 14 & & & 24.6 & 4 & 15.1 & 4 & & & 87.6 & 2 & & & 532 & 4 \\
\hline 212 & 2.1 & 27 & 14,0 & 0 & 21.0 & 4 & 16.0 & 3 & $<50$ & NR & 92.0 & 0 & 9.80 & 1 & 56.5 & 2 \\
\hline 213 & 1.8 & 12 & 19.2 & 4 & & & 13.9 & 2 & & & & & 8.87 & 3 & $\because \quad$ & $\because$ \\
\hline 215 & 2.4 & 24 & 28.0 & 0 & 137.0 & 0 & 15.0 & 4 & 106.0 & 0 & 82.0 & 4 & 8.10 & 3 & 55.3 & 3 \\
\hline 217 & 3.0 & 28 & 20.7 & 3 & 53.1 & 0 & $15: 7$ & 4 & 40.2 & 2 & 82.4 & 4 & 8.40 & 4 & 502 & 1 \\
\hline 218 & 2.8 & 5 & & & & & & & & & & & & & 520 & 3 \\
\hline 219 & 2.7 & 21 & & 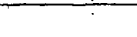 & 29.0 & 3 & & & 35.0 & 4 & 80.0 & 4 & & & 50.0 & 1 \\
\hline 220 & 3.1 & 10 & $\therefore \quad \therefore$ & $\cdot$ & & & 44.6 & 4 & & & & & & & 55.0 & 3 \\
\hline 221 & 3.2 & 18. & 22.1 & 1 & 27.8 & 3 & 14.7 & 4 & & & $\therefore$ & & & & 55.1 & 3 \\
\hline 224 & 1.6 & 19 & & & 0.0 & 0 & $15: 5$ & 4 & & & 64.5 & 0 & 12.60 & 0 & $51: 3$ & 2 \\
\hline 234 & 3.1 & 27 & 20.6 & 3 & 17.9 & 3 & $17: 2$ & 1 & 33.2 & 4 & 84.0 & 4 & 8.38 & 4 & 55.5 & 3 \\
\hline 235 & 2.4 & 23 & 20.0 & 4 & 15.0 & 3 & & & 30.0 & 3 & 88.0 & 2 & 8.00 & 3 & $59: 0$ & 0 \\
\hline 236 & 1.8 & 25 & 14.5: & 0 & 29.7 & 3 & $<35$ & NR & 29.2 & 2 & .79 .6 & 3 & 7.90 & 3 & 52.5 & 4 \\
\hline 240 & 1.7 & 18 & . $\quad \because$ & & 181.0 & 0 & $\therefore$ & & 28.0 & 2 & 72.0 & 0 & & & 47.6 & 0 \\
\hline 241 & 1.7 & 23 & 18.3 & 2 & 15.2 & 3 & 14.4 & 3 & & & 92.3 & 0 & 6.70 & 0 & 44.8 & 0 \\
\hline 244 & 0.0 & 1 & & & & & & & & & & & & &.$\quad \therefore \quad$ & \\
\hline 246 & 0.0 & 5 & & & & & & & & & & & & & 32.7 & 0 \\
\hline 249 & 1.4 & 12 & 16.0 & 0 & 45.5 & 0 & 19.1 & 0 & & & . & . & & & & \\
\hline 253 & 0.5 & 4 & & & & & & & & & & & & & $\therefore 3$ & \\
\hline 255 & 3.5 & 23 & $19.9^{\circ}$ & 4 & 15.0 & 3 & 14.6 & 4 & 36.0 & 4 & 80.5 & 4 & 8.27 & 4 & 52.8 & 4 \\
\hline 256 & 1.8 & 13 & 25.4 & 0 & & & & & & & & $\therefore$ & & & & \\
\hline 257 & 1.3 & 19 & 17.0 & 1 & 110.0 & 0 & & & & & & & & & 51.0 & 2 \\
\hline 259 & 1.5 & 13 & 21.0 & 3 & & & & & & & $74: 0$ & 1 & & & $\because \ldots$ & $\therefore$ \\
\hline 261 & 2.0 & 4 & $\ldots$. & & & & & & & & & & & & 57.1 & 1 \\
\hline 265 & 3.3 & 28 & 20.2 & . 4 & 26.9 & 3 & 16.7 & 2 & 34.7 & 4 & 77.0 & 2 & 8.70 & 4 & 54.1 & 4 \\
\hline 268 & 3.3 & 4 & & & & & . & & & &. & & & & 53.3 & 4 \\
\hline 270 & 0.0 & 3 &. & & & & & & & & . & & & & 48.3 & 0 \\
\hline 271 & 2.5 & 4 & & & & & & & & & & & & & 53.9 & 4 \\
\hline 272 & 0.0 & 4 & & & & & & & & & & & & & 23.7 & 0 \\
\hline 273 & 1.1 & 18 & 33.0 & 0 & 6.0 & 1 & & & 58.0 & 0 & & & & & $56: 9$ & 2 \\
\hline 274 & 0.5 & 11 & & 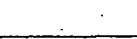 & & & & & & & & & & & 0.0 & $\begin{array}{r}0 \\
\end{array}$ \\
\hline
\end{tabular}


Table 5. Laboratory performance ratings for standard reference water sample T-143 (trace constituents)-Continue 1

\begin{tabular}{|c|c|c|c|c|c|c|c|c|c|c|c|c|c|c|}
\hline \multirow{4}{*}{$\begin{array}{r}\text { Analyte }= \\
\text { MPV }= \\
\text {-pseudosigma }=\end{array}$} & \multirow{2}{*}{\multicolumn{2}{|c|}{$\begin{array}{l}\text { Rating } \\
4 \text { (Excellent) } \\
3 \text { (Good) } \\
2 \text { (Satisfactory) } \\
\text { Cd (Cadmium) }\end{array}$}} & \multirow{2}{*}{\multicolumn{2}{|c|}{$\begin{array}{l}\text { Absolute Z-value } \\
0.00-0.50 \\
0.51-1.00 \\
1.01-1.50 \\
\text { Co (Cobalt) }\end{array}$}} & \multicolumn{2}{|c|}{$\begin{array}{l}\text { Rating } \\
1 \text { (Questionable) } \\
O \text { (Poor) } \\
\text { NR (Not Rated) }\end{array}$} & \multicolumn{2}{|c|}{$\begin{array}{l}\text { Absolute Z-value } \\
1.51-2.00 \\
\text { greater than } 2.00\end{array}$} & \multirow[b]{2}{*}{$\mathrm{Fe}$ (1ron) } & \multirow{4}{*}{$\mu g / L$} & & & & \\
\hline & & & & & $\mathrm{Cr}$ (Chr & nium) & $\mathrm{Cu}$ (Cop & & & & K (Potas & ium) & tif / Lithiur & \\
\hline & 19.1 & $\mu g / L$ & 17.0 & $\mu g / L$ & 37.0 & $\mu g / L$ & 22.3 & $\mu g / L$ & 222 & & 2.50 & $\mathrm{mg} / \mathrm{L}$ & $\quad 18.0$ & $\mu g / L$ \\
\hline & 1.5 & & 1.2 & & 26. & & 1.9 & & 14 & & 0.21 & & 21 & \\
\hline Lab & RV & Rating & $\mathrm{RV}$ & Rating & RV & Rating & RV & Rating & RV & Rating & $\mathrm{RV}$ & Rating & RV & Ratin? \\
\hline 1 & 20.1 & 3 & 17.4 & 4 & $35: 5$ & 3 & 22.2 & 4 & 214 & 3 & 2.55 & 4 & 16.6 & 3 \\
\hline 3 & $\because 19.5$ & 4 & 15.0 & 1 & 39.0 & 3 & 24.0 & 3 & 261 & 0 & 5.40 & 0 & 260 & 0 \\
\hline 4 & $<100$ & NR & $<100$ & NR & $<100$ & NR & 23.0 & 4 & 235 & 3 & & & $<100$ & NR: \\
\hline 7 & 21.0 & 2 & 19.9 & 0 & 38.1 & 4 & 23.4 & 3 & 225 & 4 & 2.84 & 1 & $\therefore \quad 19.4$ & 3 \\
\hline 10 & & & & & & & 22.8 & 4 & 246 & 1 & & & & \\
\hline 11 & 19.0 & 4 & 18.0 & 3 & 36.0 & 4 & 24.0 & 3 & 202 & 2. & 2.50 & 4 & & \\
\hline 13 & 17.4 & 2 & 12.6 & 0 & 38.5 & 3 & 10.4 & 0 & 207 & 2 & 2.38 & 3 & & \\
\hline 15 & 15.0 & 0 & $<20$ & NR & 35.1 & 3 & 21.3 & 4 & 208 & 2 & 2.28 & 2 & & \\
\hline 16 & 19.7 & 4 & 16.9 & 4 & 34.2 & 2 & 21.8 & 4 & 200 & 1 & 2.10 & 1 & 14.5 & 1 \\
\hline 18 & 18.7 & 4 & 16.1 & 3 & 36.3 & 4 & 22.3 & 4 & 222 & 4 & 2.40 & 4 & & \\
\hline 19 & 19.8 & 4 & & & 37.8 & 4 & 16.0 & 0 & 227 & 4 & 2.50 & 4 & & \\
\hline 21 & & & & & & & & & 152 & 0 & & & & \\
\hline 23 & 18.8 & 4 & & & 35.0 & 3 & 23.9 & 3 & 218. & 4 & 2.44 & 4 & & \\
\hline 24 & 17.7 & 3 & 12.3 & 0 & 31.2 & 0 & 16.2 & 0 & 222. & 4. & 2.34 & 3 & 24.4 & 0 \\
\hline 25 . & 21.0 & 2 & & & 37.0 & 4 & 21.0 & 3 & 202. & 2 & 2.64 & 3 & 18.0 & 4 \\
\hline 26. & 20.4 & 3 & 16.4 & 3 & 37.5 & 4 & 23.4 & 3 & 222 & 4 & 2.49 & 4 & 17.7 & 4 \\
\hline 28 & 13.6 & 0 & & & & & 7.8 & 0 & & & 2.32 & 3 & & \\
\hline 30 . & 19.5 & 4 & 17.0 & 4 & 37.0 & 4 & 22.5 & 4 & 47.0 & 0 & & & 16.0 & 3 \\
\hline 32 & 18.6 & 4 & 17.2 & 4 & 37.3 & 4 & 23.2 & 4 & $365^{\circ}$ & 0. & 2.50 & 4 & 17.2 & 4 \\
\hline 33 & & & & & & & & & 250 & 1 & 2.54 & 4 & & \\
\hline 34 & $\therefore$ & & & & & & & & $\therefore$ & & & & & \\
\hline 35 & & & & & & & & & 216 & 4 & & & & \\
\hline 36 & 19.0 & 4 & & & 37.0 & 4 & 10.0 & 0 & 250 & 1 & 1.80 & 0 & & $\because$ \\
\hline 39 & 18.0 & 3 & & & 28.0 & 0 & & & & & & & & \\
\hline 40 & 18.6 & 4 & 15.2 & 1 & 32.5 & 1 & 21.3 & 4 & 21 & 0 & 2.32 & 3 & 16.7 & 3 \\
\hline 42 & 18.0 & 3 & 20.0 & 0 & 38.0 & 4 & 22.0 & 4 & 224 & 4 & 2.50 & 4 & $<6$ & 0 \\
\hline 43 & & & & & & & & & 230 & 3 & 2.30 & 3 & & \\
\hline 46 & 18.6 & 4 & 17.4 & 4 & 35.8 & 4 & 22.4 & 4 & 222 & 4 & 2.62 & 3 & & \\
\hline 48 & 18.0 & 3 & $<50$ & $\begin{array}{c}\text { NR } \\
3\end{array}$ & 41.8 & 1. & 19.7 & 2 & 160 & $\begin{array}{l}0 \\
0\end{array}$ & 2.51 & 4 & $\therefore$ & 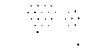 \\
\hline$\frac{50}{51}$ & & & 16.0 & 3 & 36.0 & 4 & 24.0 & & $25 t$. & & 2.48 & 4 & & \\
\hline $\begin{array}{l}51 \\
55\end{array}$ & & & & & & & 20.8 & 3 & 222 & 4 & & & & \\
\hline 58 & 20.0 & 3 & & & 37.0 & 4 & $<50$ & NR & 210 & 3 & & & & \\
\hline 64 & & & & & & & & & & & 2.57 & 4 & 22.2 & $1:$ \\
\hline 68 & 19.5 & 4 & 18.0 & 3 & 38.5 & 3 & 27.0 & 0 & .225 & 4 & 2.80 & 2 & 18.0 & 4 \\
\hline 69 & 20.0 & 3 & & & 36.0 & 4 & 21.4 & 4 & 226 & 4 & 2.72 & 2 & 19.2 & 3 \\
\hline 70 & 18.2 & 3 & $<50$ & NR & 37.1: & 4 & 22.8 & 4 & 214 & 3. & 2.43 & 4 & & \\
\hline 73 & 19.0 & 4 & & & 33.0 & 1 & 22.0 & 4 & $22 t$ & 4 & & & $\therefore \therefore$ & $\cdots$ \\
\hline 75 & 192 & 4 & 18.9 & 1 & 37.1 & 4 & 22.2 & 4 & 214 & 3 & & & 19.7 & 3 \\
\hline 76 & & & & & 36.3 & 4 & & & & & & & 18.3 & 4 \\
\hline 80 & 13.9 & 0 & & & & & 22.0 & 4 & 223 & 4 & & & & \\
\hline 81 & 20.0 & 3 & & & 41.0 & 1 & 22.0 & 4 & 220 & 4 & 2.37 & 3 & & \\
\hline 83 & 18.5 & 4 & & & 37.2 & 4 & 23.0 & 4 & 219 & 4 & 2.50 & 4 & & $\therefore$ \\
\hline 85 & 19.6 & 4 & & & 38.4 & 3 & 25.2 & 1 & 224 & 4 & 2.82 & 2 & 17.5 & 4 \\
\hline 86 & 17.7 & 3 & 16.8 & 4 & 29.2 & 0 & 23.1 & 4 & 213 & 3 & 2.58 & 4 & & \\
\hline 87 & .21 .0 & 2 & & & 38.2 & 4 & 21.0 & 3 & 212 & 3 & 2.42 & 4 & & \\
\hline 89. & 20.3 & 3 & 17.8 & 3 & 36.0 & 4 & 21.4 & 4 & 244 & 1. & 2.35 & 3 & & \\
\hline 91 & & & & & & & & & 212 & 3 & & & & $\because$ \\
\hline $96^{\circ}$ & 17.5 & 2 & & & 38.9 & 3 & 23.4 & 3 & 248 & 1. & & & & \\
\hline 97 & 19.3 & 4 & 17.2 & 4 & 39.0 & 3 & 21.2 & 3 & 233 & 3 & 2.54 & 4 & & \\
\hline 102 & 21.0 & 2 & 20.0 & 0 & 41.0 & 1 & 22.0 & 4 & 244 & 1 & 2.50 & 4 & & \\
\hline 104 & & & & & & & & & & & & & & \\
\hline 105 & 19.7 & 4. & 15.5 & 2 & 32.9 & 1 & 22.4 & 4 & 213 & 3 & 2.47 & 4 & 15.7 & 2 \\
\hline 107 & & & & & & . & 24.8 & 2 & 200 & 1. & 2.63 & 3 & & \\
\hline 109 & & & & & & & & & 256 & 0 & 2.51 & 4 & 19.4 & 3 \\
\hline 110 & & & & & & & & & & & & & & \\
\hline 113 & 19.1 & 4 & & & 38.1 & 4 & 23.5 & 3 & 223 & 4 & 2.40 & 4 & & \\
\hline 114 & 19.0 & 4 & & & & & 21.0 & 3 & 204 & 2 & 3.00 & 0 & & . \\
\hline 118 & 6.4 & 0 & & & 44.8 & 0 & 7.9 & 0 & & & & & & \\
\hline 119 & 18.4 & 4 & 15.9 & 3 & 35.0 & 3 & 26.0 & 1 & 226 & 4 & 3.20 & 0 & & \\
\hline 121 & 18.0 & 3 & 16.0 & 3 & & & 18.0 & 0 & 210 & 3 & 2.55 & 4 & & \\
\hline 127 & 17.4 & 2 & 17.3 & 4 & 39.2 & 3 & 22.4 & 4 & 220 & 4 & 2.68 & 3 & 16.5 & 3 \\
\hline 128 & 19.5 & 4 & 17.0 & 4 & 32.4 & 1 & 19.2 & 1 & 205 & 2 & 2.11 & 1 & & \\
\hline 129 & & & & & & & 30.0 & 0 & 185 & .0 & 2.40 & 4 & & \\
\hline 132 & 18.5 & 4 & 17.0 & 4 & 40.5 & 2 & 42.0 & 0 & 228 & 4 & 2.64 & 3 & & \\
\hline
\end{tabular}


Table 5. Laboratory performance ratings for standard reference water sample T-143 (trace constituents)-Contin:led

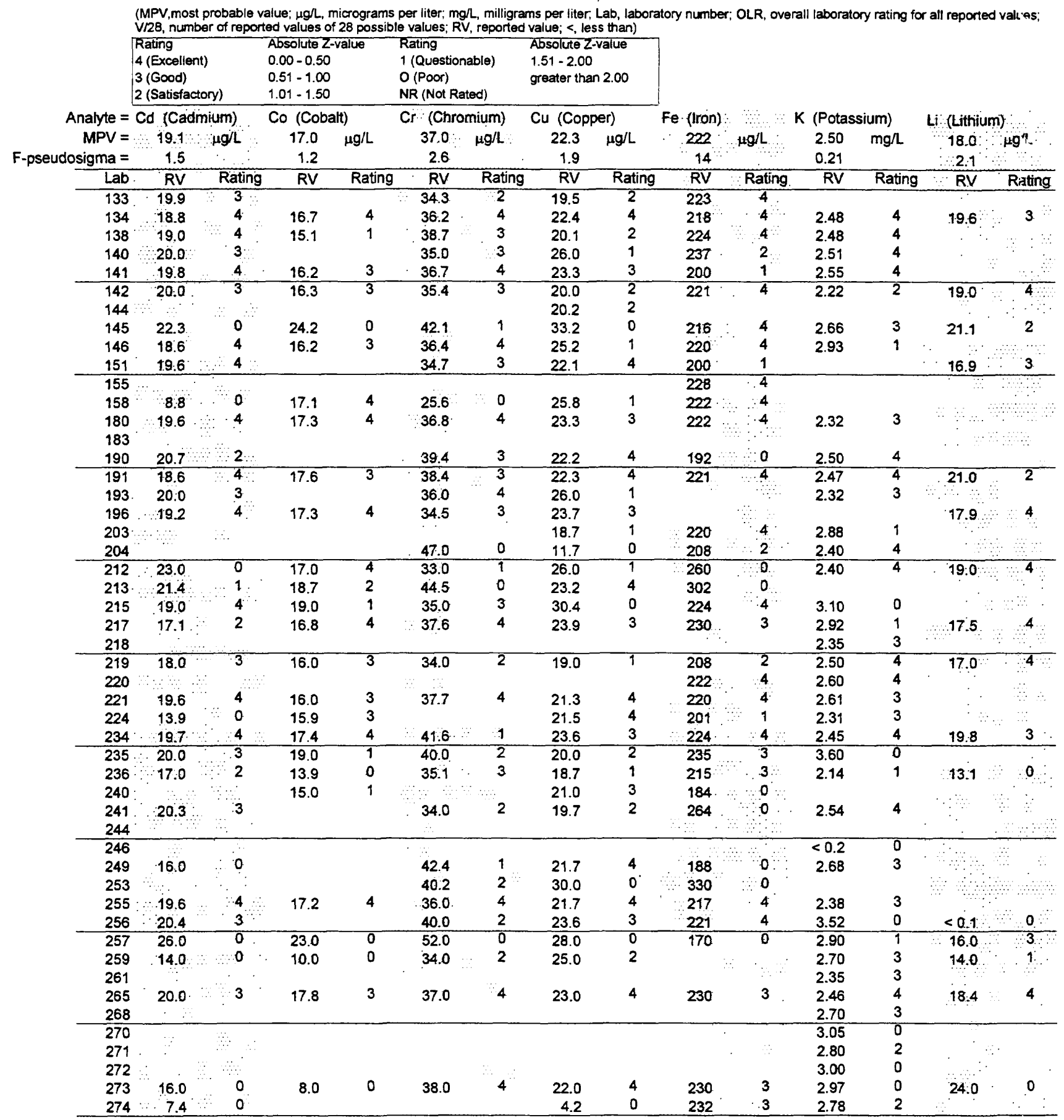


Table 5. Laboratory performance ratings for standard reference water sample T-143 (trace constituents)-Continued

\begin{tabular}{|c|c|c|c|c|c|c|c|c|c|c|c|c|c|c|}
\hline \multirow{3}{*}{$\begin{array}{r}\text { Analyte }= \\
\text { MPV }=\end{array}$} & \multirow{2}{*}{\multicolumn{2}{|c|}{$\begin{array}{l}\text { Rating } \\
4 \text { (Excellent) } \\
3 \text { (Good) } \\
2 \text { (Satisfactory) } \\
\text { Mg (Magnesium) }\end{array}$}} & \multirow{2}{*}{\multicolumn{2}{|c|}{$\begin{array}{l}\text { Absolute 2-value } \\
0.00-0.50 \\
0.51-1.00 \\
1.01-1.50 \\
\text { Mn (Manganese) }\end{array}$}} & \multicolumn{2}{|c|}{$\begin{array}{l}\text { Rating } \\
1 \text { (Questionable) } \\
\text { O(Poor) } \\
\text { NR (Not Rated) }\end{array}$} & \multicolumn{2}{|c|}{$\begin{array}{l}\text { Absolute Z-value } \\
1.51-2.00 \\
\text { greater than } 2.00\end{array}$} & \multirow{2}{*}{\multicolumn{2}{|c|}{ Ni (Nickel) }} & \multirow{2}{*}{\multicolumn{2}{|c|}{$\mathrm{Pb}$ (Lead) }} & & \\
\hline & & & & & Mo (Moly & denum) & $\mathrm{Na}$ (Sodi & & & & & & Sb (Antin & ony): \\
\hline & 10.4 & $\mathrm{mg} / \mathrm{L}$ & 18.2 & $\mu g / L$ & 36.1 & $\mu g / L$ & 34.0 & $m g / L$ & 71.0 & $\mu g / L$ & 83.4 & $\mu g / L$ & $16: 6$ & $\mu \mathrm{g} t !$ \\
\hline F-pseudosigma $=$ & 0.5 & & 1.9 & & 4.3 & & 1.6 & & 5.0 & & 7.1 & & $1: 5$ & \\
\hline Lab & $R V$ & Rating & $\mathrm{RV}$ & Rating & RV & Rating & RV & Rating & RV & Rating & RV & Rating & RV & Rating \\
\hline 1 & 10.1 & 3 & 17.8 & 4 & 34.4 & 4 & 33.6 & 4 & 71.1 & 4 & 83.6 & 4 & 15:9. & 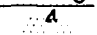 \\
\hline 3 & 10.3 & 4 & 14.7 & 1 & 27.0 & 0 & 55.0 & 0 & 57.0 & 0 & 88.6 & 3 & 19.0 & 1. \\
\hline 4 & 10.9 & 3. & 19.0 & 4 & $<500$ & NR & 34.9 & 3 & $<200$ & NR & $<400$ & NR & & \\
\hline 7 & 10.8 & 3 & 18.6 & 4 & 29.4 & 1 & 35.6 & 2 & 79.5 & 1 & 86.5 & 4 & 31.3 & 0 \\
\hline 10 & & & 22.0 & 1 & & & & & & & & & & \\
\hline 11 & 11.1 & 2 & 18.0 & 4 & 37.0 & 4 & 33.8 & 4 & 73.0 & 4 & 86.4 & 4 & 16.8 & $A$ \\
\hline 13 & 10.7 & 3 & 16.7 & 3 & & & 35.0 & 3 & 64.4 & 2 & 89.5 & 3 & 15.4 & 3 \\
\hline 15 . & 9.8 & 2 & 28.3 & 0 & 38.3 & 3 & 31.8 & 2 & 75.3 & 3 & 95.6 & 1 & 17.2 & 4 \\
\hline 16 & 10.5 & 4 & 18.2 & 4 & 37.7 & 4 & 32.8 & 3 & 72.9 & 4 & 86.7 & 4 & 16.6 & 4 \\
\hline 18 & 10.2 & 4 & 16.5 & 3 & 37.0 & 4 & 33.2 & 4 & 71.1 & 4 & 83.5 & 4 & 14.6 . & 2 \\
\hline 19 & 10.5 & 4 & 18.8 & 4 & & & 34.3 & 4 & 84.7 & 0 & 93.9 & 2 & & \\
\hline 21 & & & & & & & & & & & & & & \\
\hline 23 & 10.2 & 4 & 17.8 & 4 & $<100$ & NR & 33.4 & 4 & 74.0 & 3 & 78.3 & 3 & & \\
\hline 24 & 10.3 & 4 & 17.4 & 4 & 34.0 & 4 & 33.3 & 4 & 68.0 & 3. & 59.8 & 0 & & \\
\hline 25 & 10.0 & 3 & 22.0 & 1 & & & 37.8 & 0 & & $\therefore$ & & & 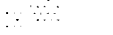 & \\
\hline 26 & $10.9^{\circ}$ & 3 & 14.7 & 1 & 37.0 & 4 & 34.6 & 4 & 74.6 & 3 & 80.7 & 4 & $<20$ & $N^{7}$ \\
\hline 28 & 10.8 & 3. & 25.1 & 0 & 26.8 & 0 & 37.3 & 0 & 31.8 & 0 & 17.9 & 0 & & \\
\hline 30 & 10.0 & 3 & 17.0 & 3 & 36.0 & 4 & & & 70.0 & 4 & 82.0 & 4 & 17.0 & 2 \\
\hline 32 & 10.8 & 3 & 17.7 & 4 & 36.4 & 4 & 33.1 & 3 & 71.0 & 4 & 81.3 & 4 & $160:$ & 4 \\
\hline 33 & 10.3 & 4 & 20.0 & 3 & & & 33.7 & 4 & & & & & & \\
\hline 34 & & & & & & & & & & & 74.1 & 2 & & $\therefore$ \\
\hline 35 & & & & & & & & & & & & & & \\
\hline 36 & 8.7 & 0 & 19.0 & 4 & & & 38.0 & 0 & 62.0 & 1 & 78.0 & 3 & 10.0 & $c$ \\
\hline 39 & & & & & & & & & 66.0 & 3. & 81.0 & 4 & 16.7 & 4 \\
\hline 40 & 10.3 & 4 & 14.4 & 1 & 37.9 & 4 & 32.9 & 3 & 51.3 & 0 & & & 5.7 & c. \\
\hline 42 & 11.1 & 2 & 21.0 & 2 & 36.0 & 4 & 33.9 & 4 & 70.0 & 4 & 83.0 & 4 & 160. & 4 \\
\hline 43 & 10.5 & 4. & 20.0 & 3 & & & 35.0 & 3 & & & & & & \\
\hline 46 & 10.5 & 4 & 16.2 & 2 & 36.0 & 4 & 35.6 & 2 & 74.2 & 3 & 77.0 & 3 & 17.2 & 4 \\
\hline 48 & 10.8 & 3 & 20.0 & 3 & 37.6 & 4 & 35.2 & 3 & 76.4 & 2 & 84.4 & 4 & 12.8 & $f:$ \\
\hline 50 & & & 21.0 & 2 & 32.0 & 3 & & & 56.0 & 0 & & & & \\
\hline 51 & 8.8 & 0 & & & & & 34.3 & 4 & & & & & & \\
\hline 55 & 11.3 & 1 & 13.0 & 0 & 30.0 & 2 & & & & & & & & \\
\hline 58 & & & $<50$ & NR & & & & & 110.0 & 0 & 92.0 & 2 & & \\
\hline 64 & & & & & & & 33.8 & 4 & & & & & & \\
\hline 68 & 11.0 & 2 & 18.5 & 4 & 37.0 & 4 & 34.5 & 4 & 74.0 & 3 & 183.0 & 0 & 9.5 & 6 \\
\hline 69. & 10.1. & 3 & $<20$ & NR & & & 32.6 & 3 & 58.5 & 0 & 80.4 & 4 & 15.0 & 2 \\
\hline 70 & 10.5 & 4. & $<20$ & NR & $<50$ & NR & 34.2 & 4 & 70.9 & 4 & 82.2 & 4 & 21.2 . & c. \\
\hline 73 & & & 18.0 & 4 & & & & & 73.0 & 4 & 87.0 & 4 & & \\
\hline 75 & 10.6 & 4 & 18.1 & 4 & 31.7 & 2 & 34.5 & 4 & 71.1 & 4 & 97.6 & 1 & 22.9 & c: \\
\hline 76 & & & & & & & & & $74: 8$ & $3:$ & 83.3 & 4 & & \\
\hline 80 & & & 15.3 & 2 & & & & & & & 83.5 & 4 & & \\
\hline 81 & 10.3 & 4 & 17.0 & 3 & 28.0 & 1 & 32.7 & 3 & 50.0 & 0 & 87.0 & 4 & $<6$ & $c$ \\
\hline 83 & 10.1 & 3 & 17.4 & 4 & & & 32.9 & 3 & 70.6 & 4 & 83.6 & 4 & & \\
\hline 85 & 10.8 & 3 & & & & & 34.5 & 4 & 74.4 & 3 & 76.5 & 3 & & \\
\hline 86 & 10.4 & 4 & 18.0 & 4 & 40.5 & 2 & 35.0 & 3 & 64.5 & 2 & 81.0 & 4 & & \\
\hline 87 & 10.2 & 4 & 20.5 & 2 & 35.1 & 4 & 32.8 & 3 & 78.0 & 2 & 78.0 & 3 & & \\
\hline 89 & 104 & 4 & 17.6 & 4 & & & 33.6 & 4 & 67.5 & 3. & 85.2 & 4 & 18.7 & $\hat{2}$ \\
\hline 91 & & $\therefore$ & 17.6 & 4 & & & & & & & & & & \\
\hline 96 & 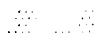 & & 22.0 & 1 & & & & & 67.6 & 3 & 92.8 & 2 & 15.1 & 2 \\
\hline 97 & 10.4 & 4 & 22.0 & 1 & 35.4 & 4 & 34.5 & 4 & 69.0 . & 4 & 81.9 & 4 & 16.6 & 4 \\
\hline 102 & 12.5 & 0 & 20.0 & 3 & & & 30.9 & 1 & 80.0 & 1 & 95.0 & 1 & 17.0 & 4 \\
\hline 104 & & & & & & & & & & & & & & \\
\hline 105 & 10.3 & 4 & 17.5 & 4 & 40.2 & 3 & 30.8 & 1 & 66.7 & 3. & 87.9 & 3 & 17.0 & 4 \\
\hline 107 & 10.7 & 3 & 20.0 & 3 & & & 32.8 & 3 & & & & & & \\
\hline 109 & 10.4 & 4 & 19.3 & 3 & 12.5 & 0 & 34.1 & 4 & & & 32.0 & 0 & & \\
\hline 110 & 8.3 & 0 & & & & & 33.0 & 3 & & & & & & \\
\hline 113 & 10.7 & 3 & 18.6 & 4 & & & 29.7 & 0 & 70.6 & 4. & 81.1 & 4 & & \\
\hline 114 & 10.0 & 3 & 20.0 & 3 & & & 28.0 & 0 & 70.0 & $4:$ & 72.0 & 1 & & \\
\hline 118 & & & & & & & & & 30.0 & 0 & 27.9 & 0 & & \\
\hline 119 & 10.6 & 4 & 20.0 & 3 & 35.1 & 4 & 34.2 & 4 & 104.0 & 0 & 82.0 & 4 & 15.2 & 3 \\
\hline 121 & 10.0 & 3. & 18.0 & 4 & & & 32.0 & 2 & $70: 0$ & 4 & & & & \\
\hline 127 & 10.4 & 4. & 16.7 & 3 & 32.1 & 3 & 33.2 & 4 & 71.0 & 4 & 87.2 & 3 & 15.6 & $\because$ \\
\hline 128 & 10.0 & 3 & 15.7 & 2 & 31.3 & 2 & 32.9 & 3 & 64.6 & 2 & 82.8 & 4 & 16.3 & 4 \\
\hline 129 & 10.0 & 3 & 20.0 & 3 & & & 35.0 & 3 & & & & & & \\
\hline 132 & 10.5 & 4 & 17.0 & 3 & 26.0 & 0 & 34.4 & 4 & 75.0 & 3 & 80.5 & 4 & & \\
\hline
\end{tabular}


Table 5. Laboratory performance ratings for standard reference water sample $T-143$ (trace constituents)-Continued

\begin{tabular}{|c|c|c|c|c|c|c|c|c|c|c|c|c|c|c|}
\hline \multirow{3}{*}{$\begin{array}{r}\text { Analyte }= \\
\text { MPV }=\end{array}$} & \multicolumn{2}{|c|}{$\begin{array}{l}\text { Rating } \\
4 \text { (Excellent) } \\
3 \text { (Good) } \\
2 \text { (Satisfactory) }\end{array}$} & \multicolumn{2}{|c|}{$\begin{array}{l}\text { Absolute Z-value } \\
0.00-0.50 \\
0.51-1.00 \\
1.01-1.50 \\
\end{array}$} & \multicolumn{2}{|c|}{$\begin{array}{l}\text { Rating } \\
1 \text { (Questionable) } \\
\text { O (Poor) } \\
\text { NR (Not Rated) }\end{array}$} & \multicolumn{2}{|c|}{$\begin{array}{l}\text { Absolute Z-value } \\
1.51-2.00 \\
\text { greater than } 2.00\end{array}$} & \multirow{2}{*}{\multicolumn{2}{|c|}{ Ni (Nickel) }} & \multirow{2}{*}{\multicolumn{2}{|c|}{$\mathrm{Pb}$ (Lead) }} & \multirow{2}{*}{\multicolumn{2}{|c|}{ Sb (Antimor y) }} \\
\hline & \multicolumn{2}{|c|}{ Mg (Magnesium) } & \multicolumn{2}{|c|}{ Mn (Manganese) } & \multicolumn{2}{|c|}{ Mo (Molybdenum) } & \multicolumn{2}{|c|}{$\mathrm{Na}$ (Sodium) } & & & & & & \\
\hline & 10.4 & $\mathrm{mg} / \mathrm{L}$ & 18.2 & $\mu g / L$ & 36.1 & $\mu g / L$ & 34.0 & $\mathrm{mg} / \mathrm{L}$ & 71.0 & $\mu g / L$ & 83.4 & $\mu g / L$ & 16.6 & $\mu g / L$ \\
\hline F-pseudosigma = & 0.5 & & 1.9 & & 4.3 & & 1.6 & & 5.0 & & 7.1 & & 1.5 & \\
\hline$\frac{L a b}{L}$ & RV & Rating & RV & Rating & RV & Rating & RV & Rating & $R V$ & Rating & RV & Rating & $\mathrm{RV}$ & Rating \\
\hline 133 & 9.9 & .3 & & & & & & & 74.3 & 3 & 92.0 & 2 & & \\
\hline 134 & 10.2 & 4 & 18.1 & 4 & 37.0 & 4 & 33.7 & 4 & 69.4 & 4 & 83.4 & 4 & 16.4 & 4 \\
\hline 138 & 10.7 & 3 & 18.0 & 4 & 35.9 & 4 & 34.1 & 4 & 70.0 & 4 & 77.1 & 3 & 16.8 & 4 \\
\hline 140 & 11.0 & 2 & 17.0 & 3 & $\because \cdots$ & & 42.5 & 0 & 71.0 & 4 & 86.0 & 4 & & \\
\hline 141 & 10.9 & 3 & 18.2 & 4 & 37.3 & 4 & 35.4 & 3 & 69.0 & 4 & 101.0 & 0 & 16.4 & 4 \\
\hline 142 & 10.0 & 3 & 19.0 & 4 & 38.3 & 3 & 34.2 & 4 & 68.3 & 3 & 87.0 & 4 & 19.9 & 0 \\
\hline 144 & $\therefore \therefore$ & & & & & & & & & & & & & \\
\hline 145 & 11.4 & 1 & 20.7 & 2 & 30.6 & 2 & 37.1 & 1 & 86.3 & 0 & 498.9 & 0 & & \\
\hline 146 & 10.1 & 3 & 17.8 & 4 & 36.2 & 4 & 35.5 & 3 & 72.4 & 4 & 84.2 & 4 & $<20$. & NR \\
\hline 151 & & & 16.5 & 3 & 38.6 & 3 & & & 69.0 & 4 & 82.8 & 4 & 17.2 & 4 \\
\hline 155 & & & & & & & & & & & & & & \\
\hline 158 & 11.1 & 2 & 19.5 & 3 & & & & & 76.0 & 3 & 30.1 & 0 & $\cdot$ & . \\
\hline 180 & .10 .6 & 4 & 18.1 & 4 & 41.0 & 2 & 34.0 & 4 & 76.0 & 3 & 92.1 & 2 & 31.6 & 0 \\
\hline 183 & $\therefore$ & & 22.7 & 0 & . & & & & & . & & & $\because$ & . \\
\hline 190 & 10.4 & 4 & 19.3 & 3 & & & 34.0 & 4 & 59.4 & 0 & 60.8 & 0 & & \\
\hline 191 & 10.7 & 3 & 19.0 & 4 & $39: 7$ & 3 & 33.2 & 4 & 72.2 & 4 & 82.0 & 4 & & \\
\hline 193 & 9.8 & 2 & & & & : & 31.6 & 2 & 66.0 & 3 & 88.0 & 3 & 150 & 2 \\
\hline 196 & $\therefore$ & & 19.0 & 4 & 36.4 & 4 & & & 70.5 & 4 & 85.4 & 4 & $\therefore 15.8$ & 3 \\
\hline 203 & 10.6 & 4 & 20.0 & 3 & & & 32.5 & 3 & $\cdots$ & & & & & \\
\hline 204 & 7.6 & 0 & 14.0 & 0 & & & 31.2 & 1 & & $\because$ & 82.2 & 4 & & \\
\hline 212 & $\because 11.2$ & 1 & 19.0 & 4 & 38.0 & 4 & 36.7 & 1 & 72.0 & 4 & 120.0 & 0 & 16.0 & 4 \\
\hline 213 & & & & & & & & & 91.2 & 0 & 90.0 & 3 & $\because$ & \\
\hline 215 & 10.6 & 4 & 18.1 & 4 & 37.0 & 4 & 34.5 & 4 & 71.0 & 4 & 82.0 & 4 & 8.0 & 0 \\
\hline 217 & $\therefore \quad 10.0$ & 3 & 18.2 & 4 & 37.8 & 4 & 33.9 & 4 & 74.1 & 3 & 90.1 & 3 & $17: 9$ & 3 \\
\hline 218 & 10.4 & 4 & & & & & 31.0 & 1 & & & & & & \\
\hline 219 & 10.0 & 3 & 17.0 & 3 & 27.0 & 0 & 33.0 & 3 & 66.0 & 3 & 70.0 & 1 & & \\
\hline 220 & $10.0^{\circ}$ & 3 & 19.2 & 3 & & & 31.0 & 1 & & & 78.4 & 3 & & . \\
\hline 221 & 9.7 & 2 & 19.7 & 3 & 35.4 & 4 & 34.1 & 4 & 71.2 & 4 & 84.5 & 4 & & \\
\hline 224 & 10.0 & 3 & 19.0 & 4 & 48.0 & $\theta$ & 31.2 & 1 & 59.9 & 0 & 140.3 & 0 & & \\
\hline 234 & 9.9 & 3 & 18.4 & 4 & 34.3 & 4 & 33.1 & 3 & 74.6 & 3 & 89.3 & 3 & 17.0 & 4 \\
\hline 235 & 10.5 & 4 & 18.0 & 4 & 39.0 & 3 & 39.0 & 0 & 74.0 & 3 & 87.0 & 4 & $<50$ & NR \\
\hline 236 & $10: 7$ & 4 & 16.4 & 3 & 19.7 & 0 & 32.8 & 3 & 65.5 & 2 & 62.4 & 0 & 49.7 & $\therefore \quad 0$ \\
\hline 240 & 10.2 & 4. & 18.0 & 4 & 11.0 & 0 & & & 75.0 & 3 & 131.0 & 0 & $18: 0$ & 3 \\
\hline 241. & 9.4 & 1. & 17.0 & 3 & 52.0 & 0 & 31.0 & 1 & 67.6 & 3 & 99.2 & 0 & 15.0 & 2 \\
\hline 244 & $\because$ & & 31.5 & 0 & & . & & & & & & & $\ldots \quad \ldots$ & $\therefore$ \\
\hline 246 & 8.7 & 0 & & & & & 41.0 & 0 & & & & & & \\
\hline 249 & $\cdots$ & & & & & & 35.6 & 2 & 69.2 & 4 & 79.2 & 3 & & \\
\hline 253 & & & & & & & & & & & & & & \\
\hline 255 & 10.3 & 4 & 18.0 & 4 & 36.3 & 4 & 32.8 & 3 & 70.9 & 4 & 83.4 & 4 & 20.4 & 0 \\
\hline 256 & & & 19.0 & 4 & 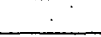 & & 31.2 & 1 & 76.6 & 2 & 155.0 & 0 & & \\
\hline 257 & 8.7 & 0 & 18.0 & 4 & 44.0 & 1 & 33.0 & 3 & 68.0 & 3 & 91.0 & 2 & 2.0 & 0 \\
\hline 259 & $\therefore \quad \cdots$ & & & & & & 34.0 & 4 & 125.0 & 0 & 54.0 & 0 & & \\
\hline 261 & 10.5 & 4 & & & & & 40.2 & 0 & & & & & & \\
\hline 265 & 10.1 & 3 & 19.0 & 4 & 35.0 & 4 & 33.0 & 3 & 73.0 & 4 & 79.6 & 3 & 16.8 & 4 \\
\hline 268 & 10.2 & 4 & & & & & 35.8 & 2 & & & & & & \\
\hline 270 & & & & & & & 42.2 & 0 & & & & & & \\
\hline $271^{\circ}$ & 25.4 & 0 & & & & & 34.0 & 4 & & $\therefore$ & & & & \\
\hline 272 & 90 & 0 & & & & & 50.0 & 0 & & & & & $\therefore \ldots$ & \\
\hline 273 & 11.2 & 1 & 21.0 & 2 & & & 36.2 & 2 & 82.0 & 0 & 65.0 & 0 & & \\
\hline $274^{\circ}$ & 17.7 & 0 & 7.4 & 0 & & & 18.4 & 0 & & & 1.2 & 0 & . & \\
\hline
\end{tabular}


Table 5. Laboratory performance ratings for standard reference water sample T-143 (trace constituents)-Continued

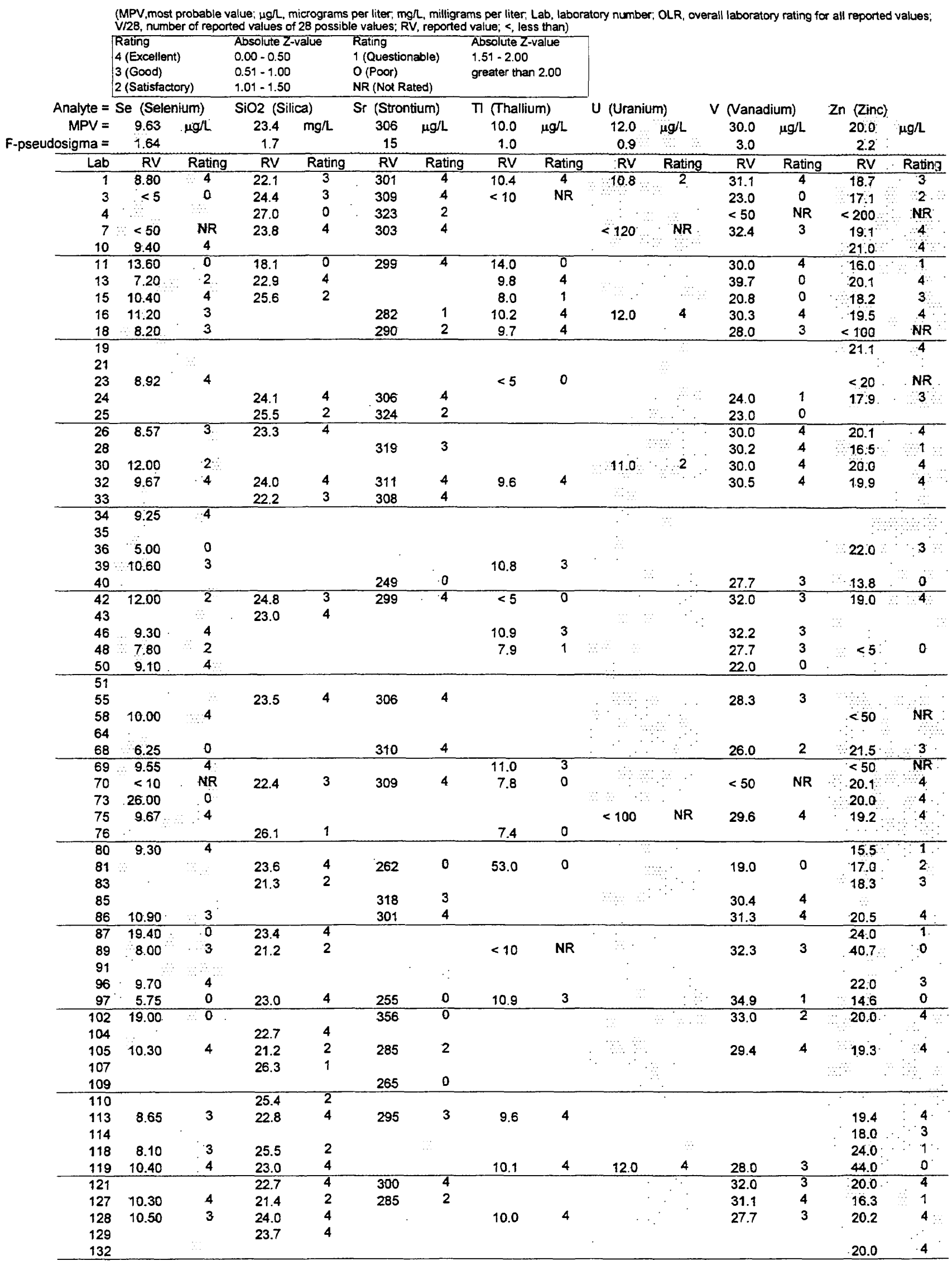


Table 5. Laboratory performance ratings for standard reference water sample T-143 (trace constituents)-Contirued

\begin{tabular}{|c|c|c|c|c|c|c|c|c|c|c|c|c|c|c|}
\hline \multirow{3}{*}{$\begin{array}{r}\text { Analyte }= \\
\text { MPV }=\end{array}$} & \multicolumn{2}{|c|}{$\begin{array}{l}\text { Rating } \\
4 \text { (Excellent) } \\
3 \text { (Good) } \\
2 \text { (Satisfactory) }\end{array}$} & \multicolumn{2}{|c|}{$\begin{array}{l}\text { Absolute Z-value } \\
0.00-0.50 \\
0.51-1.00 \\
1.01-1.50\end{array}$} & \multicolumn{2}{|c|}{$\begin{array}{l}\text { Rating } \\
1 \text { (Questionable) } \\
\text { O (Poor) } \\
\text { NR (Not Rated) }\end{array}$} & \multicolumn{2}{|c|}{$\begin{array}{l}\text { Absolute Z-value } \\
1.51-2.00 \\
\text { greater than } 2.00\end{array}$} & \multirow{2}{*}{\multicolumn{2}{|c|}{ U (Uranium) }} & \multirow{2}{*}{\multicolumn{2}{|c|}{$\checkmark$ (Vanadium) }} & \multirow[b]{2}{*}{ Zn (Zinc) } & \multirow{4}{*}{$\mu \varsigma \Omega$} \\
\hline & Se (Sele & llum) & \multicolumn{2}{|c|}{$\mathrm{SiO} 2$ (Silica) } & \multicolumn{2}{|c|}{ Sr (Strontium) } & \multicolumn{2}{|c|}{ TI (Thallium) } & & & & & & \\
\hline & 9.63 & $\mu \mathrm{g} / \mathrm{L}$ & 23.4 & $\mathrm{mg} / \mathrm{L}$ & 306 & $\mu g / L$ & 10.0 & $\mu g / L$ & 12.0 & $\mu \mathrm{g} / \mathrm{L}$ & 30.0 & $\mu g / L$ & 20.0 & \\
\hline F-pseudosigma $=$ & 1.64 & & 1.7 & & 15 & & 1.0 & & 0.9 & & 3.0 & & 2.2 & \\
\hline$L a b$ & RV & Rating & RV & Rating & RV & Rating & RV & Rating & RV & Rating & RV & Rating & RV & Rating \\
\hline 133 & 8.20 & 3 & & & & & & & & & & & 20.5 & 4 \\
\hline 134 & 10.65: & 3 & 22.9 & 4 & 295 . & 3 & 10.2 & 4 & & & 29.7 & 4 & 20.4 & 4 \\
\hline 138 & 9.58 & 4 & 21.5 & 2 & 297 & 3 & 9.4 & 3 & . & & 29.5 & 4 & 17.3 & 2 \\
\hline 140 & & & 22.8 & 4 & & & & & & & & & 20.0 & 4 \\
\hline 141 & 10.30 . & 4 & & & & & $<50$ & NR & & & 30.8 & 4 & 19.7 & 4 \\
\hline 142 & 10.50 & 3 & 26.7 & 1 & 315 & 3 & 10.4 & 4 & 12.2 & 4 & 29.6 & 4 & 15.7 & 1 \\
\hline 144 & 9.30 & 4 & & & & & & & & & & & & \\
\hline 145 & & & 26.0 & 1 & 333 & 1 & & & & & 37.3 & 0 & 25.1 & 0 \\
\hline 146 & $<10$ & NR & & & & & $<10$ & NR & & & 30.0 & 4 & $<20$ & NR \\
\hline 151 & 10.80 & 3 & & & 316 & 3 & 10.3 & 4 & & $\ldots$ & & & 20.2 & 4 \\
\hline 155 & & $\therefore$ & & & & & & & & & & & & \\
\hline 158 & & & 24.6 & 3 & & & & & & & 30.6 & 4 & 18.6 & 3 \\
\hline 180. & $<50.1$ & NR: & & & & & $<32.1$ & NR & & & 30.7 & 4 & $20: 4$ & 4 \\
\hline 183 & & & & & & & & & & & & & & \\
\hline 190 & $31: 50$ & 0 & 23.5 & 4 & 613 & 0 & & & & $\ldots$ & & & 21.3: & 3 \\
\hline 191 & $\$ 1.40$ & 2 & 23.3 & 4 & 305 & 4 & 9.9 & 4 & & & 32.2 & 3 & 18.8 & 3 \\
\hline 193 & 7.00 & 1 & & & & & 7.0 & 0 & & & & & $<50$ & NR \\
\hline 196 & 10.80 & 3 & & & 312 & 4 & 10.1 & 4 & 11.5 & 3 & 29.9 & 4 & 19.8 & 4 \\
\hline 203 & & & 23.7 & 4 & & & & & & & & & & \\
\hline 204 & . & & 23.4 & 4 & & & & & & & & & 18.0 . & 3 \\
\hline 212 & 12.00 & 2 & 24.3 & 3 & 330 & 1 & 10.0 & 4 & 14.0 & 0 & 32.0 & 3 & 22.0 & 3 \\
\hline 213 & & & & & & & 9.0 & 3 & & & & & 29.3 & 0 \\
\hline 215 & 9.10 & 4 & 45.9 & 0 & & & $<7$ & 0 & & & & & 20.0 & 4 \\
\hline 217 & 9.77 & 4 & 20.7 & 1 & 295 & 3 & 10.9 & 3 & 13.0 & 2 & 28.6 & 4 & 17.9 & 3 \\
\hline 218 & $\therefore$ & & & & 318 & 3 & & & & & & & & \\
\hline 219 & & & 23.0 & 4 & 310 & 4 & & & & & 28.0 & 3 & 17.0 & 2 \\
\hline 220 & $7: 80$ & 2 & & & & & & & & & & & 20.0 & 4 \\
\hline 221 & 9.16 & 4 & & & & & & & & & & & 34.8 & 0 \\
\hline 224 & 6.10 & 0 & & & & & & & & & 34.5 & 1 & 19.0 & 4 \\
\hline 234 & 9.56 & 4 & 22.5 & 4 & 310 & 4 & 7.6 & 0 & $\cdot$ & & 27.6 & 3 & 15.4 & 0 \\
\hline 235 & & & 25.9 & 2 & 311 & 4 & 5.0 & 0 & & & 36.0 & 1 & 19.5 & 4 \\
\hline 236 & 174.10 & 0. & 14.3 & 0 & 297 & 3 & & & & & 31.1 & 4 & 15.5 & 1 \\
\hline 240 . & 15.00 & 0 & 21.0 & 2 & 294 & 3 & 9.0 & 3 & & & & & 23.0 & 2 \\
\hline 241 & $6: 44$ & 1 & 24.7 & 3 & & & 10.5 & 4 & & . & 34.7 & 1 & 16.0 & 1 \\
\hline 244 & & & & & & & & & & & & & & \\
\hline 246 & $3 \quad$ & & 5.8 & 0 & & & & & & $\therefore$ & & & & \\
\hline 249 & $\therefore$ & & & & & & & & & r & & & $184: 0$ & 0 \\
\hline 253 & & & & & & & & & & & & & 50.0 & 0 \\
\hline 255 & 5.33 & 0 & & & & & & & & & 29.7 & 4 & 18.8 & 3 \\
\hline 256 & & & 20.6 & 1 & & & & & . & & & & 20.4 & 4 \\
\hline 257 & & & & & & . & & & & & 93.0 & 0 & 21.0 & 4 \\
\hline 259 . & 8.60 & 3 & & & & & & & & 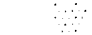 & & & 50.0 & 0 \\
\hline 261 & & & & & & & & & & & & & & \\
\hline $\begin{array}{l}265 \\
268\end{array}$ & 11.85 & 2 & 21.2 & 2 & 300 & 4 & 8.9 & 2 & 11.0 & 2 & 30.0 & 4 & 22.0 & 3 \\
\hline $\begin{array}{l}270 \\
271\end{array}$ & $\because \quad$ & & & & & & & & & & & & & \\
\hline 272 & & & & & & & & & $\therefore$ & & & & $\ldots$ & \\
\hline 273 & & & & & 336 & 1 & & & & & & & $15: 0$ & 0. \\
\hline 274 & & & 31.3 & 0 & & & & & & & & & 5.8 & 0 \\
\hline
\end{tabular}


Table 6. Laboratory performance ratings for standard reference water sample T-145 (trace constituents)

\begin{tabular}{|c|c|c|c|c|c|c|c|c|c|c|c|c|c|c|c|c|}
\hline & \multicolumn{2}{|c|}{$\begin{array}{l}\text { Rating } \\
4 \text { (Excellent) } \\
3 \text { (Good) } \\
2 \text { (Satisfactory) } \\
\end{array}$} & ed values & \multicolumn{2}{|c|}{$\begin{array}{l}\text { Absolute Z-value } \\
0.00-0.50 \\
0.51-1.00 \\
1.01-1.50 \\
\end{array}$} & \multicolumn{2}{|c|}{$\begin{array}{l}\text { Rating } \\
1 \text { (Questionable) } \\
O \text { (Poor) } \\
\text { NR (Not Rated) }\end{array}$} & \multicolumn{2}{|c|}{$\begin{array}{l}\text { Absolute Z-value } \\
1.51-2.00 \\
\text { greater than } 2.00\end{array}$} & & & & & & & \\
\hline & \multicolumn{4}{|c|}{ Analyte $=\mathrm{Ag}$ (Silver) } & \multicolumn{2}{|c|}{ Al (Aluminium) } & \multicolumn{2}{|c|}{ As (Arsenic) } & B (Boron) & & Ba (Bari & & Be (Bery & um) & Ca (Cale & um) \\
\hline & & MPV $=$ & 7.55 & $\mu \quad \mu g / L$ & 67.6 & $\mu g / L$ & 9.88 & $\mu g / L$ & 45.6 & $\mu g / L$ & 37.1 & $\mu g / L$ & 9.04 & $\mu g / L$ & $30 . \%$ & $\dot{m g h}$ \\
\hline & F-pseud & igma $=$ & 0.92 & $\therefore$ & 11.0 & & 1.04 & & 5.8 & & 1.9 & & 0.70 & & 1,3 & \\
\hline Lab & OLR & $\mathrm{V} / 28$ & $R V$ & Rating & RV & Rating & RV & Rating & RV & Rating & RV & Rating & RV & Rating & $\mathrm{RV}^{\prime}$ & Rating \\
\hline 1 & 3.4 & 28 & 7.55 & 4 & 60.1 & 3 & 9.90 & 4 & 45.7 & 4 & 36.8 & 4 & 8.94 & 4 & 29.0 & -2 \\
\hline 3 & 1.5 & 25 & 6.00 & 1 & 98.0 & 0 & 10.00 & 4 & 40.0 & 3 & 36.5 & 4 & 9.20 & 4 & 31.1 & 4 \\
\hline 4 & 3.2 & 17 & 8.00 & 4 & 68.0 & 4 & 13.00 & 0 & & & 37.0 & 4 & & & 30.5 & 4 \\
\hline 5 & 3.4 & 23 & 6.55 & 2 & 74.2 & 3 & 9.86 & 4 & 49.2 & 3 & 36.0 & 3 & 8.84 & 4 & 28.2 & 1. \\
\hline 7 & 2.5 & 19 & 9.90 & 0 & 83.8 & 2 & $<120$. & NR & & & 38.5 & 3 & 8.70 & 4 & $34 \varepsilon$ & 3 \\
\hline 10 & 2.8 & 8 & & & & & 10.00 & 4 & & & $\because$ & & & & & \\
\hline 11 & 2.9 & 26 & 8.00 & 4. & 60.0 & 3 & 10.00 & 4 & 50.0 & 3 & 36.0 & 3 & 9.00 & 4 & 31,7 & 3 \\
\hline 13 & 2.8 & 22 & 8.13 & 3 & 79.8 & 2 & 10.50 & 3 & & & 39.8 & 2 & 9.17 & 4 & 33.2 & 1 \\
\hline 15 & 1.6 & 20 & $<10$ & NR. & 82.1 & 2 & $<100$ & NR & $<50$ & NR & 34.2 & 1 & 8.70 & 4 & 28.6 & 1 \\
\hline 16 & 2.7 & 27 & 7.60 & .4 & 59.1 & 3 & 10.40 & 4 & 77.0 & 0 & 38.0 & 4 & 8.40 & 3 & 301 & 4 \\
\hline 18 & 3.6 & 20 & 8.40 & 3 & $<100$ & NR & 9.20 & 3 & $<50$ & NR & 36.2 & 4 & 9.20 & 4 & 306 & 4 \\
\hline 19 & 3.3 & 6 & & & & & & & & & & & & & 31.6 & 4 \\
\hline 23 & 2.4 & 14 & 7.79 & 4 & $<50$ & NR & 12.80 & 0 & & & & : & & & 12.4 & 0 \\
\hline 24 & 2.8 & 14 & $\because$ & & & & & & 36.9 & 2 & 34.8 & 2 & & & $30 . \mathrm{C}$ & 3 \\
\hline 25 & 2.2 & 11 & & $\therefore$ & & & & & & & 38.3 & 3 & 8.10 & 2 & 31.1 & 4 \\
\hline 26 & 3.5 & 24 & 7.76 & 4 & 65.8 & 4 & 9.62 & 4 & 37.6 & 2 & $37: 6$ & 4 & 9.17 & 4 & 30.6 & 4 \\
\hline 28 & 1.5 & 16 & & & 37.5 & 0 & & & 45.5 & 4 & 38.6 & 3. & & & 31: 5 & 3 \\
\hline 30 & 2.9 & 21 & 7.60 & 4 & & & 9.00 & 3 & & & 36.0 & 3 & 7.60 & 0 & $32 . \mathrm{C}$. & 2 \\
\hline 32 & 3.6 & 26 & 7.40 & 4 & 69.1 & 4 & 9.77 & 4 & & & 35.4 & 3 & 9.65 & 3 & 31.5 & 3 \\
\hline 33 & 2.4 & 10 & & & 100.0 & 0 & & & & & 41.0 & 0 & & & $30: 2$ & 4 \\
\hline 34 & 3.7 & 3 & & & & & 9.60 & 4 & & & & & & & & $\because$ \\
\hline 35 & 3.0 & 1 & $\because \quad \therefore$ & & & & & . & & & & & & & $\because$ & $\because$ \\
\hline 36 & 0.8 & 20 & 15.00 & 0 & 300.0 & 0 & 8.00 & 1 & & & $<0.05$ & 0 & 11.00 & 0 & 30.0 & 3 \\
\hline 40 & 2.1 & 16 & & & 58.8 & 3 & & & & & $\because 35.3$ & 3 & 2.30 & 0 & & \\
\hline 42 & 2.0 & 26 & 7.50 & 4 & 67.0 & 4 & 11.00 & 2 & 47.0 & 4 & & & 13.00 & 0 & 12.1 & 0 \\
\hline 43 & 3.4 & 7 & & & & & & & & & & & & & $3 t . C$ & 4 \\
\hline 46 & 3.1 & 21 & 710 & 4 & 86.8 & 1 & 9.10 & 3 & 22.4 & 0 & 37.6 & 4 & 8.96 & 4 & $32 . C$ & 2 \\
\hline 48 & 2.8 & 21 & 7.60 & 4 & 49.2 & 1 & 9.80 & 4 & $<100$ & NR & 43.2 & 0 & 10.00 & 2 & 31.6 & 3 \\
\hline 51 & 2.7 & 3 & & . & & & & & & & & & & & & \\
\hline 58 & 1.0 & 8 & 9.00 & 1 & 117.0 & 0 & 12.00 & 1 & & & & & & & & \\
\hline 59 & 3.5 & 21 & 7.60 & 4 & 74.4 & 3 & 10.00 & 4 & & & 37.0 & 4 & 9.80 & 2 & 30.0 & 3 \\
\hline 68 & 1.9 & 24 & 10.10 & 0 & 98.5 & 0 & 6.90 & 0 . & 125.0 & 0 & 38.5 & 3 & 9.30 & 4 & & \\
\hline 69 & 2.9 & 18 & 6.97 & 3 & 50.0 & 1 & 10.20 & 4 & & & $\because \quad \therefore \quad$ & & 7.94 & 1 & 30.2 & 4 \\
\hline 70 & 3.3 & 16 & $<10$ & NR & $<100$ & NR & $<10$ & NR & $<50$ & NR & $<50$ & NR & 9.17 & 4 & 316 & 3 \\
\hline 73 & 2.5 & 11 & & : & 62.0 & 3 & 13.00 & 0 & & & . & & & & & $\because$ \\
\hline 75 & 3.6 & 19 & 6.12 & 1 & 67.2 & 4 & 9.44 & 4 & & & 37.2 & 4 & 9.10 & 4 & 300 & 3 \\
\hline 76 & 2.8 & 9 & & & 66.7 & 4 & 10.60 & 3 & & & & & 10.20 & 1 & & \\
\hline 80 & 2.6 & 7 & & & & & 10.60 & 3 & . & & $\because$ & & & & . & \\
\hline 81 & 2.3 & 22 & $\cdots$ & $\because$ & 51.0 & 1 & 10.00 & 4 & & & 35.0 & 2. & 8.00 & 2 & 30.1 & 4 \\
\hline 83 & 3.6 & 16 & & $\cdots$ & 57.0 & 3 & & . & & & 35.5 & 3 & 8.80 & 4 & 30.0 & 3 \\
\hline 85 & 2.8 & 13 & 8.20 & 3 & $<100$ & NR & & & 42.4 & 3 & 37.7 & 4 & 9.64 & 3 & $31: 0$ & 4 \\
\hline 86 & 3.2 & 21 & & & & & 9.95 & 4 & 45.7 & 4 & 36.8 & 4 & 8.79 & 4 & 318 & 3 \\
\hline 87 & 1.3 & 18 & 13.00 & 0 & & & 9.40 & 4 & & & 40.0 & 1 & & & $29: 2$ & 2 \\
\hline 89 & 2.9 & 21 & 7.60 & 4 & 64.6 & 4 & 9.00 & 3 & & & $<50$. & NR & 8.20 & 2 & 29.5 & 3 \\
\hline 91 & 3.5 & 2 & & & & & & & & & & & & & & \\
\hline 96 & 2.9 & 13 & 8.20 & 3 & & & 9.70 & 4 & & & $<100$ & NR: & 10.00 & 2 & & \\
\hline 97 & 3.1 & 24 & 6.64 & 3. & 72.7 & 4 & 10.40 & 4 & & & 29.2 & $: 0$ & 9.43 & 3 & 29.1 & 2 \\
\hline 102 & 1.8 & 23 & 52.00 & 0 & 69.0 & 4 & 6.10 & 0 & & & 42.0 & 0 & 8.80 & 4 & 330 & 1. \\
\hline 104 & 3.0 & 1 & & & & & & & & & & & & & & \\
\hline 105 & 3.4 & 25 & 6.78 & 3 & 66.0 & 4 & 10.30 & 4 & & & 34.8 & 2 & 10.00 & 2 & 30.3 & 4 \\
\hline 107 & 3.1 & 11 & 7.40 & 4 & 65.0 & 4 & 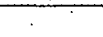 & & & & 39.0 & 2 & & & 30.9 & 4 \\
\hline 109 & 2.5 & 11 & $\therefore$ & $\because$ & & & 8.50 & 2 & & & & & & & 28.7 & 1 \\
\hline 113 & 3.2 & 21 & 6.80 & 3 & 68.1 & 4 & 11.50 & 1 & & & 37.0 & $4 \vdots$ & 10.10 & 1 & 33.2 & 1 \\
\hline 114 & 1.7 & 7 & $<10$ & NR & & & & & & & & $\because$ & $<10$ & NR & 24.0 & 0 \\
\hline 118 & 2.7 & 10 & 6.90 & 3 & $<2000$ & NR & 10.60 & 3 & & & & & & & 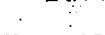 & \\
\hline 119 & 2.8 & 26 & 6.90 & 3 & 62.0 & 3 & 9.00 & 3 & 42.0 & 3 & 39.0 & 2. & 8.92 & 4 & 31.7 & 3 \\
\hline 121 & 3.1 & 14 & & & & & & & & & 36.0 & 3 & 10.00 & 2 & $30 . C$ & 3 \\
\hline 128 & 2.8 & 25 & 7.50 & 4 & 71.0 & 4 & 9.95 & 4 & 40.9 & 3 & 31.9 & 0 & 8.40 & 3 & 32.0 & 2 \\
\hline 129 & 1.7 & 9 & & . & & & & & 125.0 & 0 & & & & & $34: C$ & 0 \\
\hline 132 & 2.1 & 16 & $\because$ & : & 73.0 & 4 & & & 28.0 & 0 & & & & & 30.8 & 4 \\
\hline
\end{tabular}


Table 6. Laboratory performance ratings for standard reference water sample T-145 (trace constituents)--Continued

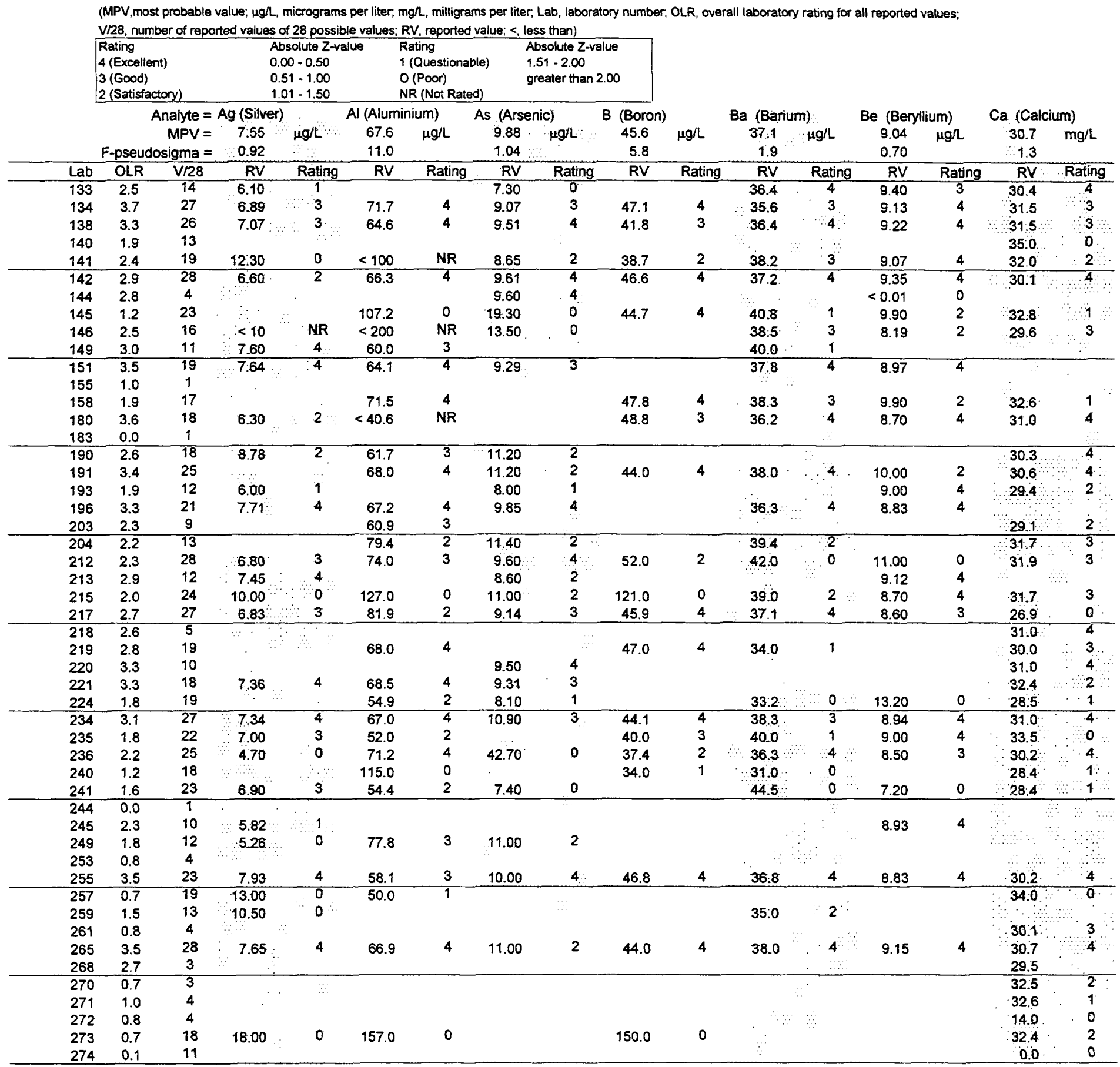


Table 6. Laboratory performance ratings for standard reference water sample T-145 (trace constituents)-Continued

\begin{tabular}{|c|c|c|c|c|c|c|c|c|c|c|c|c|c|c|}
\hline \multirow{3}{*}{$\begin{array}{r}\text { Analyte }= \\
\text { MPV }=\end{array}$} & \multicolumn{2}{|c|}{$\begin{array}{l}\text { Rating } \\
4 \text { (Excellent) } \\
3 \text { (Good) } \\
2 \text { (Satisfactory) }\end{array}$} & \multicolumn{2}{|c|}{$\begin{array}{l}\text { Absolute Z-value } \\
0.00-0.50 \\
0.51-1.00 \\
1.01-1.50\end{array}$} & \multicolumn{2}{|c|}{$\begin{array}{l}\text { Rating } \\
1 \text { (Questionable) } \\
\text { O (Poor) } \\
\text { NR (Not Rated) }\end{array}$} & \multicolumn{2}{|c|}{$\begin{array}{l}\text { Absolute Z-value } \\
1.51-2.00 \\
\text { greater than } 2.00\end{array}$} & \multirow{2}{*}{\multicolumn{2}{|c|}{ Fe (Iron) }} & \multirow{2}{*}{\multicolumn{2}{|c|}{ K (Potassium) }} & \multirow{2}{*}{\multicolumn{2}{|c|}{ Li (Lithium) }} \\
\hline & \multicolumn{2}{|c|}{$=\mathrm{Cd}$ (Cadmium) } & \multicolumn{2}{|c|}{ Co (Cobalt) } & $\mathrm{Cr}:(\mathrm{Chr}$ & nium) & Cu (Cop & & & & & & & \\
\hline & 9.33 & $\mu g / 1$ & 10.0 & $\mu g / L$ & 15.3 & $\mu g / L$ & 11.0 & $\mu g / L$ & 1.01 & $\mu g / L$ & 2.13 & $\mathrm{mg} / \mathrm{L}$ & 27.3 & $\mu g / L$ \\
\hline F-pseudosigma = & 0.82 & & 0.9 & & 1.4 & & 1.4 & & 8 & & 0.16 & & 2.5 & \\
\hline Lab & RV & Rating & $\mathrm{RV}$ & Rating & RV & Rating & $R V$ & Rating & $\mathrm{RV}$ & Rating & RV & Rating & $\mathrm{RV}$ & Rating \\
\hline 1 & 9.79 & 3 & 10.0 & 4 & 15.5 & 4 & 11.1 & 4 & 96 & $\therefore 3$ & 2.17 & 4 & 25.5 & 3 \\
\hline 3 & 10.10 & $3:$ & 6.0 & 0 & 17.0 & 2 & 8.0 & 0 & 120 & 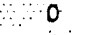 & 4.50 & 0 & 33.0 & 0 \\
\hline 4 & 9.50 & 4 & 10.6 & 3 & 15.0 & 4 & & & 102 & 4. & & & 36.0 & 0 \\
\hline 5 & 8.90 & 3 & 10.1 & 4 & 15.5 & 4 & 11.8 & 3 & 100 & 4 & 2.12 & 4 & 26.2 & 4. \\
\hline 7 & 13.00 & 0 & 11.4 & 1 & 18.0 & 1 & 13.9 & 1 & 104 & 4 & 2.46 & 1 & 27.5 & 4 \\
\hline 10 & & & & & 16.0 & 4. & 10.8 & 4 & 105 & 3 & & & & \\
\hline 11 & 9.00 & 4 & 11.0 & 2 & 15.0 & 4 & 12.0 & 3 & 90 & 2 & 2.12 & 4 & 44.3 & 0 \\
\hline 13 & 9.44 & 4 & 7.6 & 0 & 16.1 & 3 & 11.3 & 4 & 117 & 1 & 2.08 & 4 & & \\
\hline 15 & 17.20 & 0 & $<20$ & NR & 16.1 & 3 & 11.1 & 4 & 92 & 2 & 38.30 & 0 & & \\
\hline 16 & 9.10 & 4 & 10.4 & 4 & 13.8 & 2 & 11.4 & 4 & 77 & 0 & 1.60 & 0 & 22.8 & 1 \\
\hline 18 & 9.20 & 4 & 9.9 & 4 & 14.3 & 3 & 12.3 & 3 & 100 & 4 & 2.10 & 4 & & \\
\hline 19 & & & & & & & 8.0 & 0 & & & 2.10 & 4 & & \\
\hline 23 & 9.16 & 4 & & & 12.3 & 0 & 11.5 & 4 & $<500$ & NR & 2.09 & 4 & & \\
\hline 24 & 7.60 & 0 & 4.8 & 0 & & & & & 98 & 4 & 1.94 & 2 & 27.6 & 4 \\
\hline 25 & & & & & & & & & 83 & 0 & 2.31 & 2 & $29.0^{\circ}$ & 3 \\
\hline 26 . & 10.40 & 2 & 10.4 & 4 & 15.4 & 4 & 11.7 & 4 & 102 & 4 & 2.13 & 4 & 25.5 & 3 \\
\hline 28 & 8.10 & 1 & & & & & 4.6 & 0 & & & 2.32 & 2 & & \\
\hline 30 & 9.60 & 4 & 10.2 & 4 & 14.3 & 3 & 11.0 & 4 & 230 & 0 & & & 24.0 & 2 \\
\hline 32 & 9.18 & 4 & 10.2 & 4 & 14.5 & 3 & 11.3 & 4 & 183 & 0 & 2.10 & 4 & 27.3 & 4 \\
\hline 33. & & & & & & & & & 120. & 0 & 2.18 & 4 & & \\
\hline 34 & & & & & & & & & & & & & & \\
\hline 35 & & & & & & & & & 97 & 3 & & & 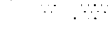 & \\
\hline 36 & 8.60 & 3 & & & 10.0 & 0 & 19.0 & 0 & 140 & 0 & 1.60 & 0 & & \\
\hline 40 & 8.50 & 2 & 7.1 & 0 & 9.5 & 0 & 10.0 & 3 & 95 & 3 & 1.99 & 3 & 25.3 & 3 \\
\hline 42 & 9.00 & 4 & 14.0 & 0 & 18.0 & 1 & 13.0 & 2 & 107 & 3. & 2.30 & 2 & 6.0 & 0 \\
\hline 43 & & & & & & & & & 110. & 2 & 2.20 & 4 & & . \\
\hline 46 & 8.84 & 3 & 10.7 & 3 & 16.4 & 3 & 11.3 & 4 & 101 & 4 & 2.26 & 3 & & \\
\hline 48 & 9.20 & 4 & $<50$ & NR & 17.4 & 2 & 11.3 & 4 & 30 & 0 & 2.13 & 4 & & : \\
\hline 51 & & & & & & & & & & & 2.16 & 4 & & \\
\hline 58 & 3700 & 0 & & & 17.0 & 2 & $<50$ & NR & 100 & 4 & & & & $\because$ \\
\hline 59 & 9.00 & 4 & & & 16.0 & 4 & 10.8 & 4 & 100 & 4 & 1.70 & 0 & & \\
\hline 68 & 10.00 & 3 & 11.5 & 1 & 16.5 & 3 & 15.0 & 0 & 105 & 3 & 2.10 & 4 & 2.8 & 0 \\
\hline 69 & 10.40 & 2 & & & 14.2 & 3 & 10.2 & 3 & 108 & 3 & 2.34 & 2 & 28.7 & 3 \\
\hline 70 & 8.60 & 3 & $<50$ & NR & 15.2 & 4 & 11.8 & 3 & 102 & 4 & 1.96 & 2 & & \\
\hline 73 & 9.00 & 4 & & & 16.0 & 4 & 11.0 & 4 & 101 & 4 & & & & \\
\hline 75 & 8.99 & 4 & 10.8 & 3 & 16.1 & 3 & 10.2 & 3 & 98 & 4 & & & 27.3 & \\
\hline 76 & & & & & 14.8 & 4 & & & & & & & 27.6 & 4 \\
\hline 80 & $<10$ & NR & & & & & 10.7 & 4 & $99:$ & 4 & & & & \\
\hline 81 & 10.00. & 3. & 8.0 & 0 & 16.0. & 4 & 11.0 & 4 & 85 & 1 & 2.03 & 3 & & \\
\hline 83 & 8.80 & 3 & & & 15.2 & 4 & 11.8 & 3 & 100 & 4 & 2.20 & 4 & & \\
\hline 85 & 7.60 & 0 & & & $<10$ & NR & 12.6 & 2 & 103 & 4 & 2.50 & 0 & $26: 2$ & 4 \\
\hline 86 & 9.20 & 4 & 10.5 & 3 & 14.7 & 4 & 10.9 & 4 & 1.03 & 4 & 2.21 & 4 & & \\
\hline 87 & 12.00 & 0 & & & 14.4 & 3 & 8.3 & 1 & 128 & 0 & 5.15 & 0 & & $\cdots$ \\
\hline $\begin{array}{l}89 . \\
91\end{array}$ & 9.46 & 4. & 10.0 & 4 & 17.5 & 1 & 10.2 & 3 & $\begin{array}{r}126 \\
96\end{array}$ & $\begin{array}{l}0 \\
3\end{array}$ & 2.01 & 3 & & $\therefore:$ \\
\hline 96 & 8.90 & 3 & & & 16.2 & 3 & 12.9 & 2 & 112 & 2 & & & & \\
\hline 97 & 9.20 & 4 & 9.7 & 4 & 16.2 & 3 & 10.2 & 3 & .102 & 4 & 2.14 & 4 & & \\
\hline 102 & 9.90 & 3 & 11.0 & 2 & 16.0 & 4 & 11.0 & 4 & 111 & 2 & 2.00 & 3 & & \\
\hline 104 & & & & & & & & & & & & & & \\
\hline 105 & 9.85 & 3 & 9.6 & 4 & $15: 4$ & 4 & 11.4 & 4 & 103 & 4 & 2.08 & 4 & 23.2 & 1. \\
\hline 107 & & & & & & & 10.2 & 3 & 90 & $2:$ & 2.28 & 3 & & \\
\hline 109 & & & & & & & & & 97 & 4 & 2.20 & 4 & 26.6 & 4 \\
\hline 113 & $9.71^{\circ}$ & 4 & & & 15.6 & 4 & 12.2 & 3 & 102 & 4 & 2.12 & 4 & & \\
\hline 114 & $<10$ & NR & & & 20.0 & 0 & $<10$ & NR & 100 & 4. & 2.00 & 3 & & \\
\hline 118 & 10.60 & 1 & & & 16.5 & 3 & 10.9 & 4 & & & & & & \\
\hline 119 & 9.20 & 4 & 9.4 & 3 & 13.0 & 1 & 10.0 & 3 & 104 & 4 & 1.60 & 0 & & \\
\hline 121 & 8.00 & 1 & 10.0 & 4 & & & 11.0 & 4 & 101 & 4 & 2.15 & 4 & & \\
\hline 128 & 10.10 & 3 & 11.0 & 2 & 13.0 & 1 & 9.3 & 2 & 91 & 2 & 1.99 & 3 & & \\
\hline 129 & & & & & & & 30.0 & 0 & 78 & 0 & 2.10 & 4 & $\therefore$ & \\
\hline 132 & 8.50 & 2 & 9.5 & 3 & 15.5 & 4 & 29.0 & 0 & 129 & 0 & 2.29 & 3 & & \\
\hline
\end{tabular}


Table 6. Laboratory performance ratings for standard reference water sample T-145 (trace constituents)-Continued'

\begin{tabular}{|c|c|c|c|c|c|c|c|c|c|c|c|c|c|c|}
\hline \multicolumn{15}{|c|}{ (MPV,most probable value; $\mu \mathrm{g}$, , micrograms per liter, mgh, milligrams per liter, Lab, laboratory number, OLR, overall laboratory rating for all reported values; } \\
\hline & \multicolumn{2}{|c|}{$\begin{array}{l}\text { Rating } \\
4 \text { (Excellent) } \\
3 \text { (Good) } \\
2 \text { (Satisfactory) }\end{array}$} & \multicolumn{2}{|c|}{$\begin{array}{l}\text { Absolute Z-value } \\
0.00-0.50 \\
0.51-1.00 \\
1.01-1.50\end{array}$} & \multicolumn{2}{|c|}{$\begin{array}{l}\text { Rating } \\
1 \text { (Questionable) } \\
O \text { (Poor) } \\
\text { NR (Not Rated) }\end{array}$} & \multicolumn{2}{|c|}{$\begin{array}{l}\text { Absolute Z-value } \\
1.51-2.00 \\
\text { greater than } 2.00\end{array}$} & \multirow{2}{*}{\multicolumn{2}{|c|}{ Fe (tron). }} & \multirow{2}{*}{\multicolumn{2}{|c|}{ K (Potassium) }} & \multirow{2}{*}{\multicolumn{2}{|c|}{ Li (Lithium) }} \\
\hline Analyte $=$ & \multicolumn{2}{|c|}{ Cd (Cadmiurn) } & \multicolumn{2}{|c|}{ Co (Cobalt) } & \multicolumn{2}{|c|}{$\mathrm{Cr}$ (Chromium) } & \multicolumn{2}{|c|}{ Cu (Copper) } & & & & & & \\
\hline$M P V=$ & 9.33 & $\mu \mathrm{gh}$ & 10.0 & $\mu \mathrm{g} / \mathrm{L}$ & 15.3 & $\mu \mathrm{g} / \mathrm{L}$ & $11.0^{\circ}$ & $\mu \mathrm{g} / \mathrm{L}$ & 101 & $\mu \mathrm{g} / \mathrm{L}$ & 2.13 & $\mathrm{mg} / \mathrm{L}$ & 27.3 & $\mu g / \mathrm{L}$ \\
\hline F-pseudosigma = & 0.82 & & 0.9 & & 1.4 & & 1.4 & & 8 & & 0.16 & & 2.5 & \\
\hline Lab & RV & Rating & RV & Rating & RV & Rating & $\mathrm{RV}$ & Rating & RV & Rating & $\mathrm{RV}$ & Rating & RV & Rating \\
\hline 133 & 10.60 & 1 & & & 15.3 & 4 & 8.9 & 1 & 104 & 4 & & & & \\
\hline 134 & 9.26 & 4 & 10.2 & 4 & 14.6 & 4 & 11.0 & 4 & 100 & 4 & 2.13 & 4 & $27: 7$ & 4. \\
\hline 138 & 9.08 & 4. & 8.6 & 1 & 10.9 & 0 & 9.8 & 3 & 104 & 4 & 2.10 & 4 & & \\
\hline 140 & 9.00 & 4 & & & 16.0 & 4 & 14.0 & 0 & 107 & 3. & 2.25 & 3 & & \\
\hline 141 & 8.98 & 4 & $<10$ & NR & 12.0 & 0 & 12.7 & 2 & 85 & 1 & 2.17 & 4 & & \\
\hline 142 & 9.82 & 3 & 9.7 & 4 & 13.9 & 3 & 10.1 & 3 & 106 & 3 & 1.86 & 1 & 28.0 & 4 \\
\hline 144 & & & & & & & 10.3 & 3 & & & & & & \\
\hline 145 & $11.00:$ & 1 & 14.6 & 0 & 19.3 & 0 & 19.3 & 0 & 102 & 4 & 2.22 & 3 & 31.3 & 1 \\
\hline 146 & 9.03 & 4 & 9.2 & 3 & 14.5 & 3 & 13.7 & 1 & 99. & 4 & 2.61 & 0 & & \\
\hline 149 & 10.00 & 3 & & & 17.0 & 2 & & & 100 & 4 & & & & \\
\hline 151 & 9.39 & 4 & & & 14.5 & 3 & 11.8 & 3 & 90 & 2 & & & 25.5 & 3 \\
\hline 155 & & & & & & & & & 113 & 1 & & & & \\
\hline 158 & 4.60 & 0 & 10.6 & 3 & 13.1 & 1 & 13.8 & 1 & 109 & 2. & & & & \\
\hline $\begin{array}{l}180 \\
183\end{array}$ & 10.00 & 3 & 9.7 & 4 & 14.3 & 3 & 10.6 & 4 & 99 & 4 & 2.00 & 3 & $\because$ & \\
\hline 190 & 10.30 & 2 & & & 19.1 & 0 & 11.0 & 4 & 88 & 1 & 2.13 & 4 & & \\
\hline 191 & 9.31 & 4 & 10.8 & 3 & 15.4 & 4 & 11.0 & 4 & 98 & 4 & 2.11 & 4 & 31.1 & 1. \\
\hline 193 & 11.00 & 1 & & & 15.0 & 4 & $<25$ & NR & & & 2.04 & 3 & & \\
\hline 196 & 9.39 & 4 & 9.4 & 3 & 13.6 & 2 & 11.2 & 4 & & & & & 26.6 & 4 \\
\hline 203 & & & & & & & 7.7 & 0 & 96 & 3 & 2.53 & 0 & & \\
\hline 204 & & & & & 19.8 & 0 . & 5.4 & 0 & 93 & 2 & 2.12 & 4 & & \\
\hline 212 & 1.1 .00 & 1 & 10.0 & 4 & 12.0 & 0 & 14.0 & 0 & 110 & 2 & 2.00 & 3 & 29.0 & 3 \\
\hline 213 & 8.19 & 2 & 10.6 & 3 & 15.4 & 4 & 11.2 & 4 & 105 & 3 & & & & \\
\hline 215 & to.00 & 3 & 9.0 & 2 & 14.0 & 3 & 15.0 & 0 & 106 & 3 & 2.20 & 4 & & \\
\hline 217 & 7.10 & 0 & 9.5 & 3 & 12.7 & 1 & 11.0 & 4 & 106 & 3 & 1.84 & 1 & 26.3 & 4 \\
\hline 218 & & & & & & & & & & & 2.07 & 4 & . & \\
\hline 219 & 8.80 & 3 & 9.3 & 3 & 13.0 & 1 & 9.4 & 2 & 92 & 2 & 2.20 & 4 & 25.0 & 3 \\
\hline 220 & & & & & & & & & .98 & 4 & 2.20 & 4 & & \\
\hline 221 & 9.63 & 4 & 10.0 & 4 & 15.0 & 4 & 10.6 & 4 & 117 & 1. & 2.22 & 3 & & . \\
\hline 224 & $5: 90$ : & 0. & 10.1 & 4 & & & 13.2 & 1 & 95 & 3 & 1.95 & 2 & & \\
\hline 234 & 9.60 & 4 & 10.6 & 3 & 16.9 & 2 & 12.4 & 3 & 102 & 4 & 2.12 & 4 & 30.4 & 2 \\
\hline 235 & 9.80 & 3 & 13.0 & 0 & 15.0 & 4 & 8.5 & 1 & 110 & 2 & 3.50 & 0 & & \\
\hline 236 . & 7.40 & 0 & 5.6 & 0 & 14.7 & 4 & 8.8 & 1 & 98 & 4 & 2.13 & 4 & 20.6 & 0 \\
\hline 240 & 1200 & 0 & 5.8 & 0 & & & 12.0 & 3 & 83 & 0 & & & & \\
\hline 241 & $9: 80$ & 3 & & & 14.4 & 3 & 14.9 & 0 & 121 & 0 & 2.06 & 4 & . & \\
\hline 244 & & & & & & & & & & & & & & \\
\hline 245 & 9.35 & 4 & & & 13.0 & 1 & 11.2 & 4 & & & & & & \\
\hline 249 & 8.00 & 1 & & & 17.6 & 1 & 10.4 & 4 & 41 & 0 & 2.36 & 2 & & \\
\hline 253 & & & & & 20.1 & 0 & 10.0 & 3 & 180 & 0 & & & & \\
\hline 255 & 9.87 & 3. & 10.5 & 3 & 14.6 & 3 & 10.3 & 4 & 99 & 4 & 2.08 & 4 & & \\
\hline 257 & 16.00 & 0 & 19.0 & 0 & 30.0 & 0 & 13.0 & 2 & 81 & 0 & 2.60 & 0 & 28.0 & 4 \\
\hline 259 & 6.00 & 0 & 9.0 & 2 & $14: 0$ & 3 & 11.0 & 4 & & & 2.30 & 2 & 21.0 & 0. \\
\hline 261 & & & & & & & & & & & 1.76 & 0 & & \\
\hline 265 & 10.00 & 3 & 9.5 & 3 & 16.0 & 4 & 11.8 & 3 & 97 & 3 & 2.09 & 4 & 27.4 & 4 \\
\hline 268 & & & & & & & & & & & 2.55 & 0 & & \\
\hline 270 & & & & & & & & & & & 2.66 & 0 & & \\
\hline 271 & & & & & & & & & $\ldots$ & & 2.80 & 0 & & \\
\hline 272 & & & & & & & & & & & 2.00 & 3 & & \\
\hline 273 & 17.20 & 0 & 11.0 & 2 & 17.0 & 2 & 5.0 & 0 & 96 & 3 & 2.98 & 0 & 46.0 & 0 \\
\hline 274 & 6.90 & 0 & & & & & 2.6 & 0 & 147 & 0 & 2.68 & 0 & & \\
\hline
\end{tabular}


Table 6. Laboratory performance ratings for standard reference water sample T-145 (trace constituents)-Continued

(MPV,most probable value; $\mu g / L$, micrograms per liter, $m g / L$, milligrams per liter, Lab, laboratory number, OLR, overall laboratory rating for all reported values: V/28, number of reported values of 28 possible values; RV, reported value; <, less than)

\begin{tabular}{llll|}
\hline Rating & Absolute Z-value & Rating & Absolute Z-value \\
4 (Excellent) & $0.00-0.50$ & 1 (Questionable) & $1.51-2.00$ \\
3 (Good) & $0.51-1.00$ & $O$ (Poor) & greater than 2.00 \\
2 (Satisfactory) & $1.01-1.50$ & NR (Not Rated) &
\end{tabular}

Analyte $=\mathrm{Mg}$ (Magnesium) Mn (Manganese) Mo (Molybdenum) $\mathrm{Na}$ (Sodium) Ni (Nickel) . Pb (Lead) $\quad$ Sb (Antimony)

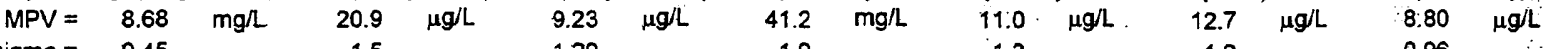

:-pseudosigma =

\begin{tabular}{|c|c|c|c|c|c|c|c|c|c|c|c|c|c|c|}
\hline Lab & $\mathbf{R V}$ & Rating & $\mathrm{RV}$ & Rating & $\mathbf{R V}$ & Rating & RV & Rating & $\mathrm{RV}$ & Rating & $\mathrm{RV}$ & Rating & RV & Rating \\
\hline 1 & 8.17 & 2 & 20.3 & 4 & 8.67 & 4 & 40.2 & 3 & 10.9 & 4 & 12.6 & 4 & 8.65 & 4 \\
\hline 3 & 8.52 & 4 & 17.0 & 0 & 6.00 & 0 & 43.6 & 2 & 7.8 & 0 & 6.0 & 0 & 1200 & 0 \\
\hline 4 & 8.70 & 4 & 21.0 & 4 & & & & & 100 & 3 & 13.0 & 4 & $\therefore$ & \\
\hline 5 & 8.10 & 2 & 21.0 & 4 & $<10$ & NR & 41.4 & 4 & $<10$ & NR & 13.5 & 3 & $\because 20$. & NR \\
\hline 7 & 9.06 & 3 & 21.4 & 4 & $<15$. & NR & 43.3 & 2 & $<20$ & NR & $<55$ & NR & $<30$ & $\mathbf{N R}$ \\
\hline 10 & & & 25.0 & 0 & & & & & & & 12.7 & 4 & & \\
\hline 11 & 9.20 & 2 & 21.0 & 4 & 10.00 & 3 & 40.7 & 4 & 10.0 & 3 & 13.0 & 4 & 9.70 & 3 \\
\hline 13 & 9.07 & 3 & 19.5 & 3 & & & 43.7 & 2 & 10.5 & 4 & 13.5 & 3 & 7.80 & 2 \\
\hline 15 & 8.10 & 2. & 24.0 & 0 & 20.10 & 0 & 38.3 & 1 & 13.0 & 2 & 15.2 & 1 & 9.25 & 4 \\
\hline 16 & 8.60 & 4 & 21.5 & 4 & $8.90^{\circ}$ & 4 & 40.2 & 3 & 13.4 & 1 & 13.4 & 3 & 8.80 & 4 \\
\hline 18 & 8.60 & 4 & 20.3 & 4 & $<20$ & NR & 40.0 & 3 & $<25$. & NR & 12.7 & 4 & 7.20 & 1 \\
\hline 19 & 8.80 & 4 & & & & & 41.9 & 4 & & . & 13.2 & 4 & & \\
\hline 23 & 8.72 & 4 & 21.8 & 3 & $<100$ & NR & 38.6 & 2 & 11.8 & 3 & 11.1 & 2 & $\because \quad \therefore$ & \\
\hline 24 & 8.44 & 3 & 19.8 & 3 & & & 40.5 & 4 & & & & & & \\
\hline 25 & 8.40 & 3 & 21.0 & 4 & & & 46.0 & 0 & & . & & & $\cdot$ & \\
\hline 26 & 9.10 & 3 & 21.5 & 4 & 9.90 & 3 & 42.8 & 3 & 10.8 & 4 & 10.8 & 1 & $<20$ & NR \\
\hline 28 & 8.90 & 4 & 23.3 & 1 & 6.40 & 0 & 53.1 & 0 & 17.3 & 0 & 9.5 & 0 & & \\
\hline 30 & 9.00 & 3 & 21.0 & 4 & 9.40 & 4 & & & 11.5 & 4 & 11.5 & 2 & 920 & 4 \\
\hline 32 & 9.11 & 3 & 20.9 & 4 & 8.18 & 3 & 40.5 & 4 & 11.0 & 4 & 12.6 & 4 & 8.43 & 4. \\
\hline 33 & 8.95 & 3 & 20.0 & 3 & & & 42.2 & 3 & & . & & & & \\
\hline 34 & & & & & & & & & & & 13.4 & 3 & & \\
\hline 35 & & & & & & & & & & & & & & \\
\hline 36 & 7.60 & 0 & 22.0 & 3 & . & & 38.0 & 1 & 9.0 & 2 & 15.0 & 1 & 6.00 & 0 \\
\hline 40 & 8.48 & 4 & 18.1 & 1 & 9.90 & 3 & 40.0 & 3 & & & & & & \\
\hline 42 & 9.30 & 2 & 23.0 & 2 & 10.00 & 3 & 41.0 & 4 & 13.0 & 2 & 6.0 & 0 & 10.00 & 2 \\
\hline 43 & 8.80 & 4 & 20.0 & 3 & & & 43.0 & 3 & & & & & & \\
\hline 46 & 8.67 & 4 & 20.4 & 4 & & & 42.6 & 3 & & 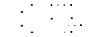 & 13.7 & 3 & 9.30 & 3 \\
\hline 48 & 8.89 & 4 & 20.0 & 3 & 9.30 & 4 & 42.7 & 3 & 11.4 & 4 & 13.0 & 4 & 7.60 & 2 \\
\hline 51 & 16.47 & 0. & & & & & 41.0 & 4 & $\therefore$ & & & & & \\
\hline 58 & & & $<50$ & NR & & & & & 70.0 & 0 & 42.0 & 0 & & . \\
\hline 59 & 8.50 & 4 & 21.0 & 4 & & & 40.0 & 3 & 11.4 & 4 & 12.4 & 4 & 9.30 & 3 \\
\hline 68 & 9.05 & 3 & 21.5 & 4 & 7.30 & 2 & 43.0 & 3 & $8: 5$ & 1 & 16.6 & 0 & 7.85 & 3 \\
\hline 69 & 8.37 & 3 & $<20$ & NR & & & 41.2 & 4 & 9.6 & 2 & 12.0 & 3 & 8.15 & 3 \\
\hline 70 & 8.73 & 4 & 20.6 & 4 & $<50$ & NR & 41.8 & 4 & $<50$ & NR & 12.8 & 4 & 11.40 & 0 \\
\hline 73 & & & 21.0 & 4 & & & & & 10.0 & 3 & 16.0 & 0 & & \\
\hline 75 & 8.90 & 4 & 20.8 & 4 & $<10$ & NR & 42.5 & 3 & $<20$ & NR & 12.5 & 4 & $<50$ & NR \\
\hline 76 & & & & & & & & & 117 & 3 & 12.1 & 3 & & \\
\hline 80 & $\cdots$ & $\because$ & 18.5 & 1 & . & & & & $\because$ & $\cdots$ & 11.7 & 3 & & . \\
\hline 81 & 8.58 & 4 & 20.0 & 3 & & & 40.0 & 3 & 7.0 & 0 & 13.0 & 4 & $<6$ & 0 \\
\hline 83 & 8.45: & 4 & 20.3 & 4 & & & 40.4 & 4 & 10.5 & 4 & 12.4 & 4 & & \\
\hline 85 & 8.92 & 3 & & & & & 42.1 & 4 & $<10$ & NR & $<50$ & NR & & $\therefore$ \\
\hline 86 & 8.67 & 4 & 20.3 & 4 & $17: 70$ & 0 & 42.8 & 3 & 9.0 & 1 & 13.4 & 3 & $\therefore$ & \\
\hline 87 & 8.36 & 3 & 27.0 & 0 & 11.00 & 2 & 39.6 & 3 & 15.3 & 0 & 11.2 & 2 & & \\
\hline 89 & 8.29 & 3. & 20.4 & 4 & & & 40.1 & 3 & 11.3 & 4 & 12.1 & 3 & 8.30 & 3 \\
\hline 91 & & & 20.3 & 4 & & $\cdots$ & & & & $\therefore$ & & & & \\
\hline 96 & & & 27.0 & 0 & & & & & $10.5:$ & 4 & 13.1 & 4 & 8.20 & 3 \\
\hline 97 & 8.55 & 4 & 24.2 & 0 & 9.58 & 4 & 41.8 & 4 & 10.5 & 4 & 12.7 & 4 & 9.06 & 4 \\
\hline 102 & 10.30 & 0 & 23.0 & 2 & & & 37.0 & 0 & 11.0 & 4 & 15.0 & 1 & $<7$ & 0 \\
\hline 104 & & & & & & & & & & & & & & \\
\hline 105 & 8.68 & 4 & 19.6 & 3 & 9.38 & 4 & 42.2 & 3 & 10.8 & 4 & 13.8 & 3 & 9.03 & 4 \\
\hline 107 & 8.91 & 3 & 20.0 & 3 & & & 40.2 & 3 & & & & & & \\
\hline 109 & 8.50 & 4 & 19.3 & 2 & 8.20 & 3 & 41.2 & 4 & & & 10.0 & 0 & & \\
\hline 113 & 8.84 & 4 & 21.5 & 4 & & & 35.5 & 0 & 11.5 & 4 & 12.2 & 4 & &. \\
\hline 114 & 8.00 & 1 & 21.0 & 4 & & ? & 34.0 & 0 & $<10$ & NR & $<10$ & NR & & \\
\hline 118 & & & & & & & & & 13.4 & 1 & 12.5 & 4 & $\therefore$ & \\
\hline 119 & 9.10 & 3 & 21.0 & 4 & 8.33 & 3 & 43.1 & 2 & 14.3 & 0 & 11.5 & 2 & 8.10 & 3 \\
\hline 121 & 8.30 & 3 & 22.0 & 3 & & & 39.0 & 2 & & & & & & \\
\hline 128 & 8.28 & 3 & 18.7 & 2 & 5.50 & 0 & 40.4 & 4 & 11.0 & 4 & 12.9 & 4 & 9.15 & $\therefore 4$ \\
\hline 129 & 9.00 & 3 & 10.0 & 0 & & & 42.0 & 4 & & & & & & $\because$ \\
\hline 132 & 8.79 & 4 & 19.0 & 2 & 12.00 & 0 & 42.4 & 3 & 14.0 & 0 & 22.5 & 0 & & \\
\hline
\end{tabular}


Table 6. Laboratory performance ratings for standard reference water sample T-145 (trace constituents)-Continued

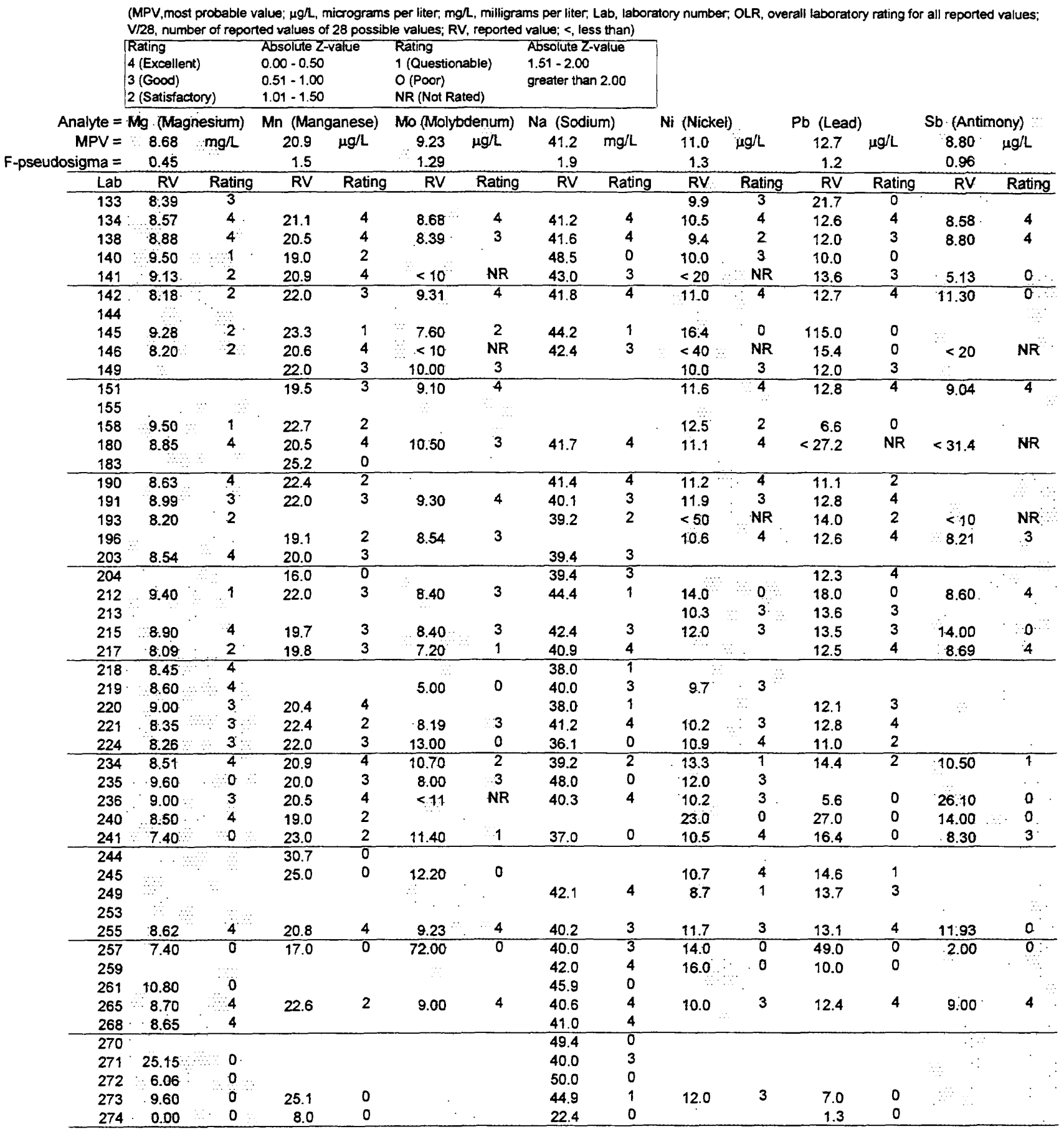


Table 6. Laboratory performance ratings for standard reference water sample T-145 (trace constituents)-Continued

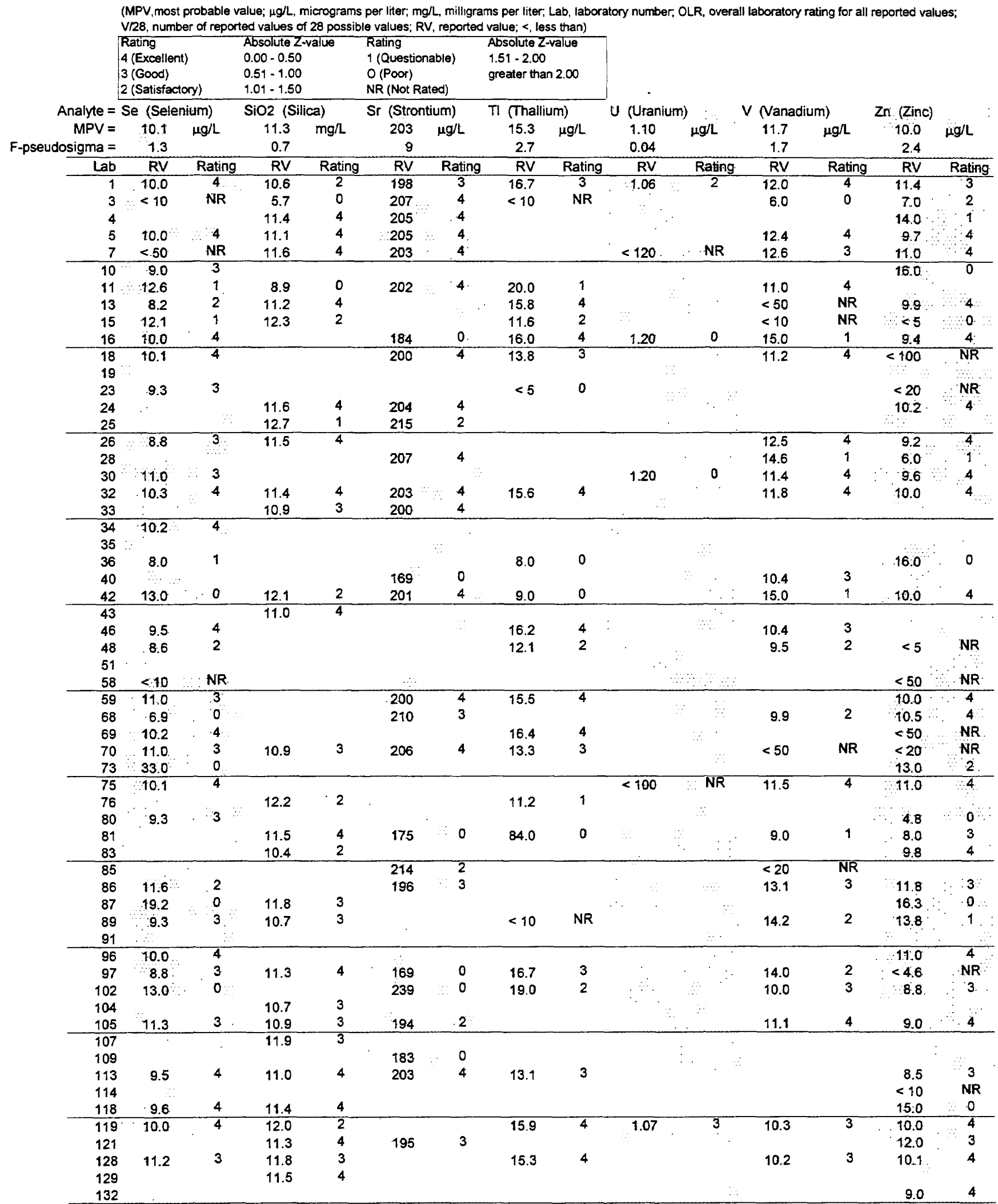


Table 6. Laboratory performance ratings for standard reference water sample $T-145$ (trace constituents)-Continued

\begin{tabular}{|c|c|c|c|c|c|c|c|c|c|c|c|c|c|c|}
\hline \multirow{4}{*}{$\begin{array}{r} \\
\text { Analyte }= \\
\text { MPV }= \\
\text { F-pseudosigma }=\end{array}$} & \multicolumn{2}{|c|}{$\begin{array}{l}\text { Rating } \\
4 \text { (Excellent) } \\
3 \text { (Good) } \\
2 \text { (Satisfactory) } \\
\end{array}$} & \multicolumn{2}{|c|}{$\begin{array}{l}\text { Absolute Z-value } \\
0.00-0.50 \\
0.51-1.00 \\
1.01-1.50 \\
\end{array}$} & \multicolumn{2}{|c|}{$\begin{array}{l}\text { Rating } \\
1 \text { (Questionable) } \\
\text { O (Poor) } \\
\text { NR (Not Rated) } \\
\end{array}$} & \multicolumn{2}{|c|}{$\begin{array}{l}\text { Absolute Z-value } \\
1.51-2.00 \\
\text { greater than } 2.00\end{array}$} & \multirow{2}{*}{\multicolumn{2}{|c|}{ U (Uranium) }} & \multirow{2}{*}{\multicolumn{2}{|c|}{$\therefore$ Vanadium) }} & \multirow[b]{2}{*}{$\operatorname{Zn~(Zinc)}$} & \multirow{4}{*}{$\mu g / l$} \\
\hline & Se (Sele & ium) & $\mathrm{SiO} 2(\mathrm{~S}$ & & Sr. (Stro: & um) & TI (Thal & & & & & & & \\
\hline & 10.1 & $\mu g / L$ & 11.3 & $\mathrm{mg} / \mathrm{L}$ & 203 & $\mu \mathrm{g} / \mathrm{L}$ & 15.3 & $\mu g / L$ & 1.10 & $\mu g / L$ & $\quad 11.7$ & $\mu \mathrm{g} / \mathrm{L}$ & 10:0 & \\
\hline & 1.3 & & 0.7 & & 9 & & 2.7 & & 0.04 & & 1.7 & & 2.4 & \\
\hline Lab & RV & Rating & RV & Rating & RV. & Rating & RV & Rating & $\mathrm{RV}$ & Rating & RV & Rating & RV & Rating \\
\hline 133 & 10.8 & 3 & & & & & & & & & & & 10.1 & 4 \\
\hline 134 & .10 .7 & 4 & 11.2 & 4 & 196 & 3 & 16.9 & 3 & & & 10.7 & 3 & 11.7 & 3 \\
\hline 138 & 9.6 & 4 & 10.8 & 3 & 198 & 3 & 15.0 & 4 & & & 11.0 & 4 & 80 & 3 \\
\hline 140 & & & 11.2 & 4 & & & & & & & & & 14.0 & 1 \\
\hline 141 & 9.3 & 3 & & & & & $<50$ & NR & & & 12.7 & 3 & 9.9 & 4 \\
\hline 142 & 10.8 & 3 & 12.8 & 0 & 212 & 2 & 16.7 & 3 & 0.86 & 0 & 10.7 & 3 & 8.4 & 3 \\
\hline 144 & 10.0 & 4 & & & & & & & & & & & & \\
\hline 145 & & & 12.5 & 1 & 216 & 1 & & & & & 17.0 & 0 & 13.0 & 2 \\
\hline 146 & $<10$ & NR & & & & & 14.0 & 4 & & & 11.5 & 4 & $<20$ & NF \\
\hline 149 & 10.0 & 4 & & & & & & & & & & & & \\
\hline 151 & 11.2 & 3 & & & 208 & 3 & 16.0 & 4 & & & & & 8.9 & 4. \\
\hline 155 & & & & & & & & & & & & & & \\
\hline 158. & & & 12.4 & 1 & & & & & & & 12.5 & 4 & 7.2 & 2 \\
\hline $\begin{array}{l}180 \\
183\end{array}$ & $<50.1$ & NR & & & . & & $<32.1$ & NR & & . & 12.0 & 4 & 9.5 & 4 \\
\hline 190 & 11.0 & 3 & 11.9 & 3 & 390 & 0 & & & & & & & 13.3 & 2 \\
\hline 191 & 12.0 & 2 & 11.1 & 4 & 203 & 4 & 15.8 & 4 & & & 12.0 & 4 & 8.5 & 3 \\
\hline 193 & 8.0 & 1 & & & & & 9.0 & 0 & & & & & $<50$ & NF \\
\hline $\begin{array}{l}196 \\
203\end{array}$ & 14.1 & 3 & 10.8 & 3 & 194 & 2 & 15.3 & 4 & 1.12 & 3 & 10.2 & 3 & 14.5 & 1 \\
\hline$\frac{203}{204}$ & & & $\frac{0.0}{11.4}$ & 4 & & & & & & & & & 7.2 & 2 \\
\hline 212 . & 11.0 & 3 & 11.8 & 3 & 210 & 3 & 16.0 & 4 & 1.10 & 4 & 12.0 & 4 & 12.0 & 3 \\
\hline 213 & & & & & & & 12.4 & 2 & & & & & 14.0 & 1. \\
\hline 215 & 5.0 & 0 & 11.3 & 4 & & & $<7$ & 0 & & & & & 0.0 & 0 \\
\hline 217 & 10.2 & 4 & 9.9 & 1 & 193 & 2 & 15.2 & 4 & 1.10 & 4 & 8.9 & 1 & 7.9 & 3 \\
\hline 218 & & $\because$ & & & 229. & 0 & & & & & & & & \\
\hline 219 & & & 10.8 & 3 & 210 & 3 & & & & & 10.0 & 3 & 11.0 & 4 \\
\hline 220 & 8.6 & 2 & & & & & & & $\therefore$ & & & & to. & 4 \\
\hline 221 & 9.7 & 4 & & & & & & & $\because$ & & & & 11.3 & 3 \\
\hline 224 & 10.5 & 4 & & & & & & & & & 18.8 & 0 & 10.2 & 4 \\
\hline 234 & 96 & 4 & 19.0 & 4 & 206 & 4 & 10.9 & 9 & & & 11.0 & 4 & 6.7 & 2 \\
\hline 235 & & & 12.6 & 1 & 208 & 3 & 7.9 & 0 & & & 15.0 & 1 & 8.3 & 3 \\
\hline 236 & 128.3 & 0. & 7.0 & 0 & 199 & 4 & & & & & 12.1 & 4 & .8 .6 & 3 \\
\hline $240^{\circ}$ & $18: 0$ & 0 & 10.5 & 2 & 197 & 3 & 10.0 & 1 & & & & & 11.0 & 4 \\
\hline 241 & 70 & 0 & 11.8 & 3 & & & 15.9 & 4 & & $\cdot$ & 13.0 & 3 & 5.0 & 0 \\
\hline 244 & $\therefore$ & & & & . & & & & & & & & & \\
\hline 245 & & & & & & & & & & & & & 9.3 & 4 \\
\hline 249 & $\because \quad \therefore$ & & & & & & & & & & & & 0.6 & 0 \\
\hline 253 & & & & & & & & & & & & & 20.0 & 0 \\
\hline 255 & 81. & 2 & & & & & & & & & 11.6 & 4 & 9.7 & 4 \\
\hline 257 & & & & & & & & & & & 39.0 & 0 & 9.0 & 4 \\
\hline 259 & 8.7 & 2 & & & & & & & & & & & 66.0 & 0 \\
\hline 261 & & & & & & & & & & & & & & \\
\hline 265 & 12.4 & 1 & 10.1 & 1 & 200 & 4 & 15.0 & 4 & 1.10 & 4 & 12.0 & 4 & 10.5 & 4 \\
\hline 268 & & & & & & & & & & & & & & \\
\hline 270 & & $\therefore$ & & & & & & & & & & & & $\therefore$ \\
\hline 271 & & & & & & & & & & & & & & \\
\hline 272 & & $\therefore \approx$ & & & & & & & & & & & & \\
\hline $273^{\circ}$ & $\therefore$ & & 227.0 & 0 & & & & & $\therefore$ & & & & 20.0 & 0 \\
\hline 274 & & & 19.2 & 0 & & & & & & & & & 5.8 & 1 \\
\hline
\end{tabular}


Table 7. Laboratory performance ratings for standard reference water sample M-140 (major constituents)

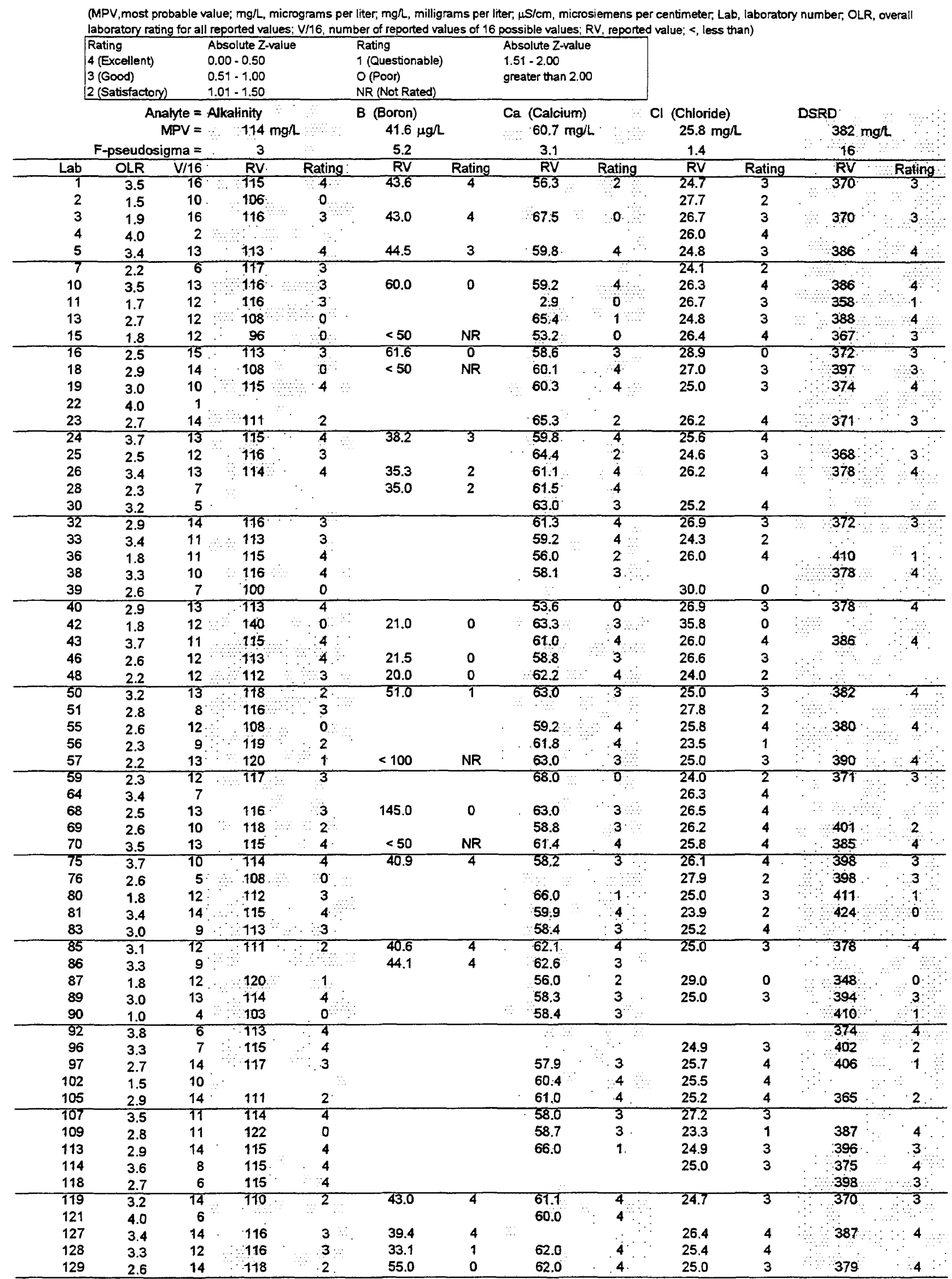


Table 7. Laboratory performance ratings for standard reference water sample M-140 (major constituents)-Continued

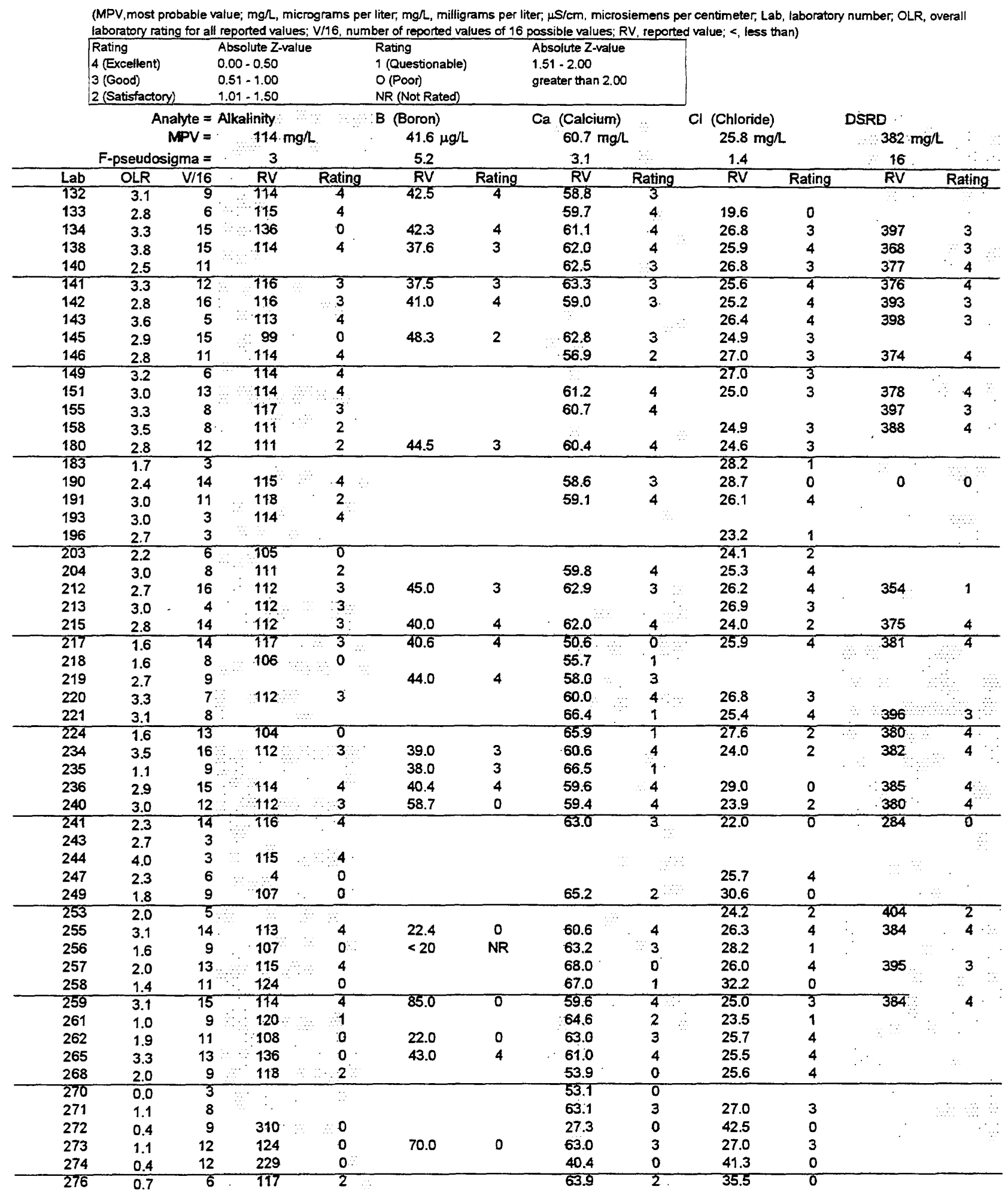


Table 7. Laboratory performance ratings for standard reference water sample $M-140$ (major constituents)-Continued

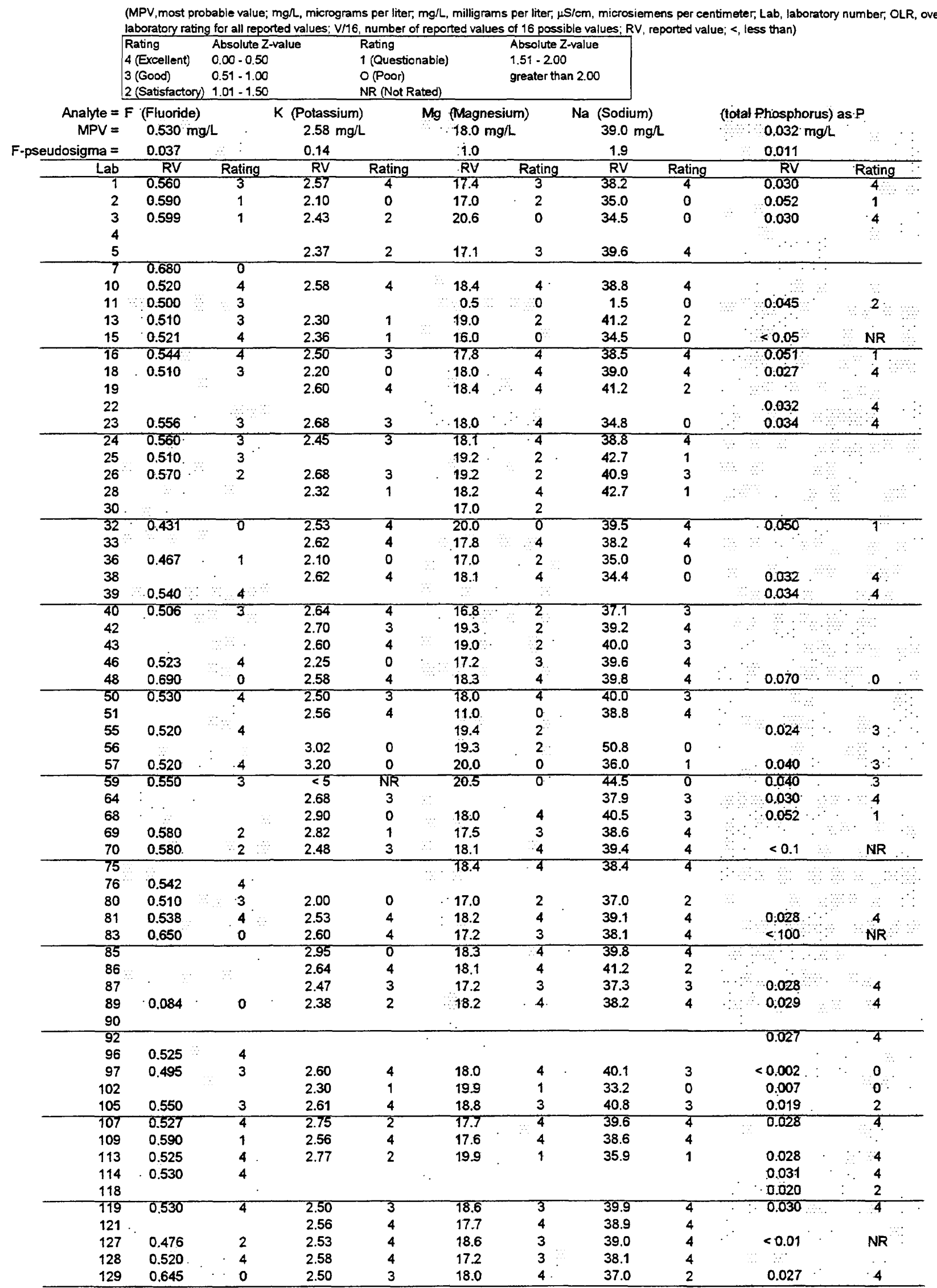


Table 7. Laboratory performance ratings for standard reference water sample $M-140$ (major constituents)-Continued

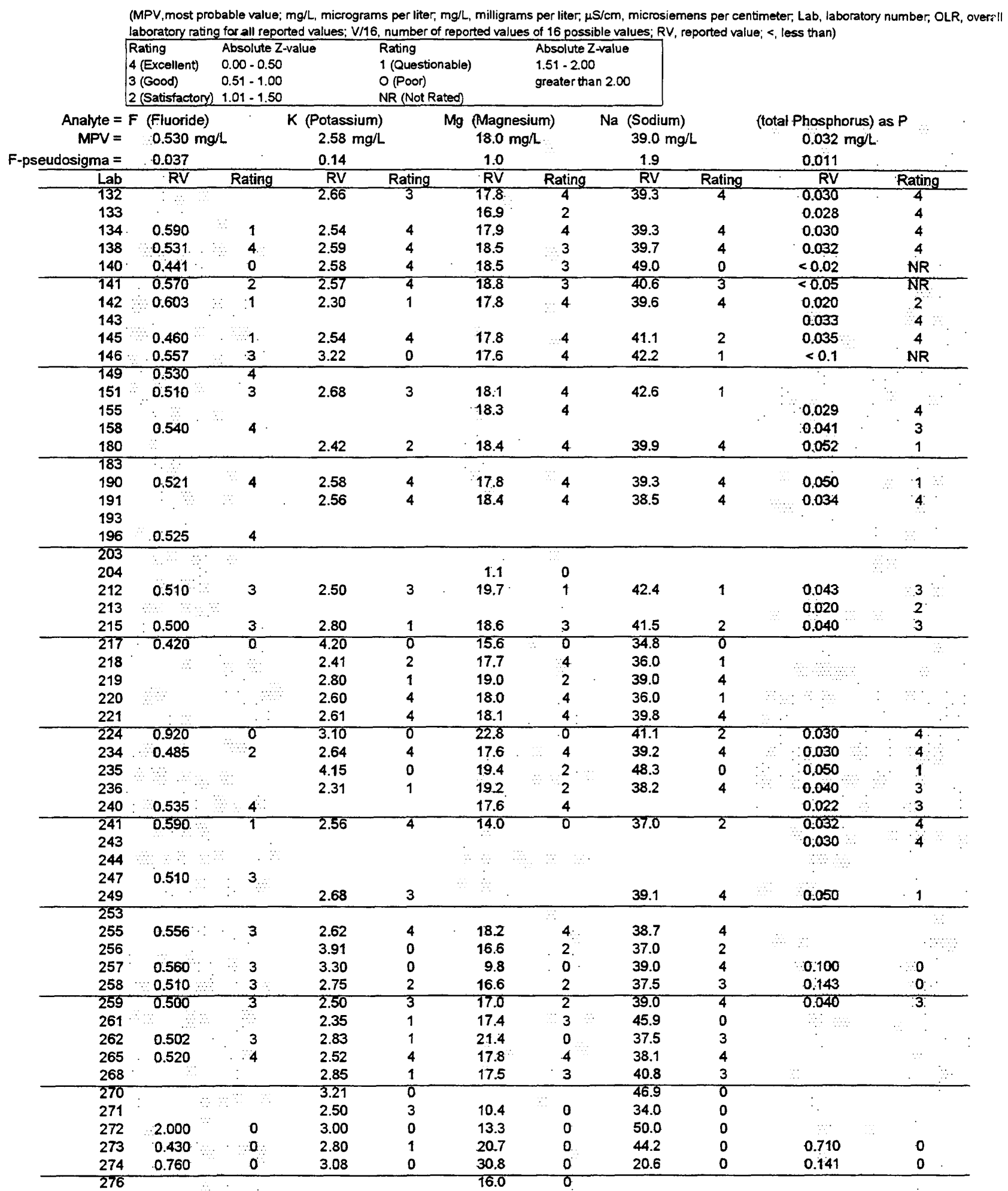


Table 7. Laboratory performance ratings for standard reference water sample $M-140$ (major constituents)-Continued

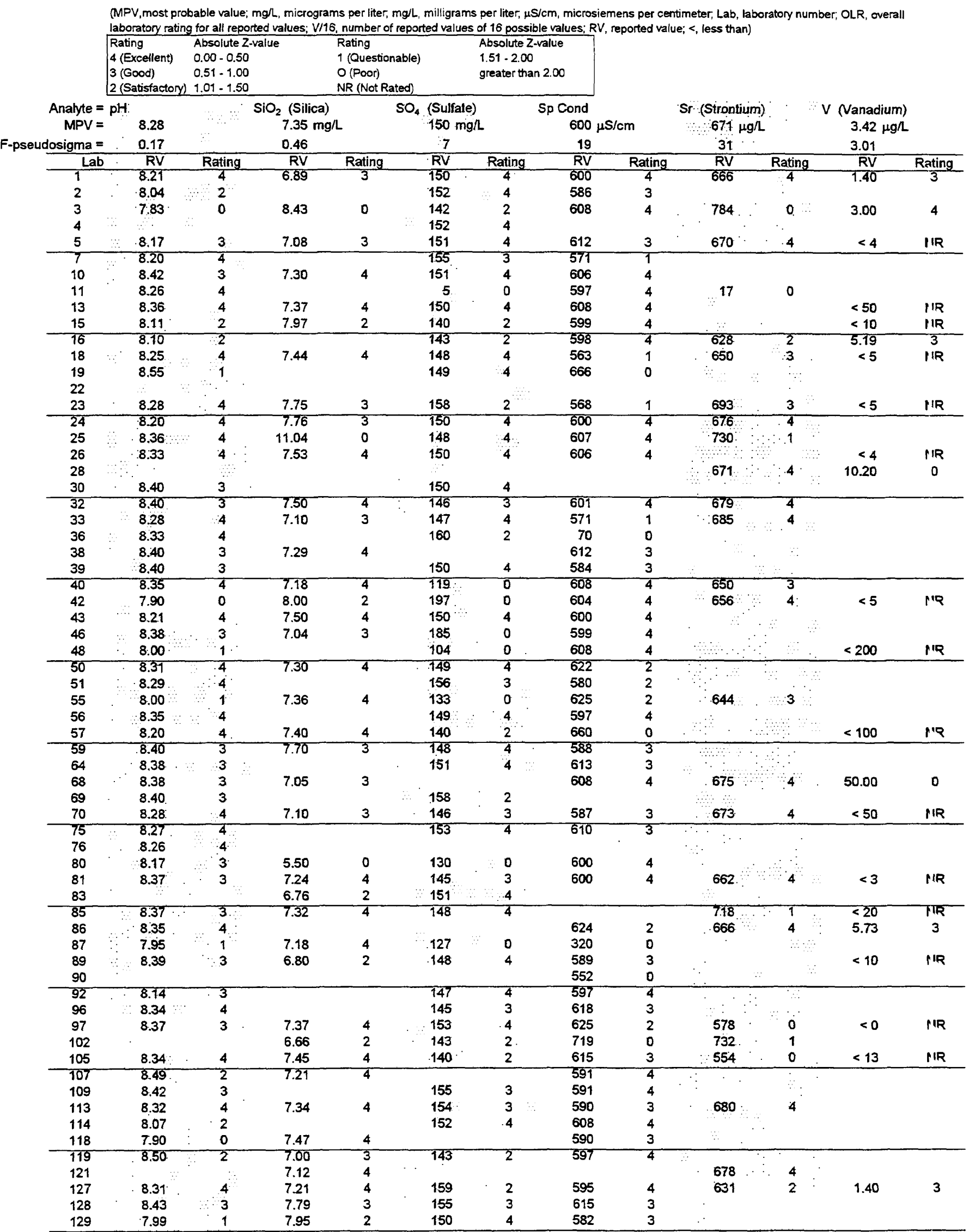


Table 7. Laboratory performance ratings for standard reference water sample $M-140$ (major constituents)-Continued

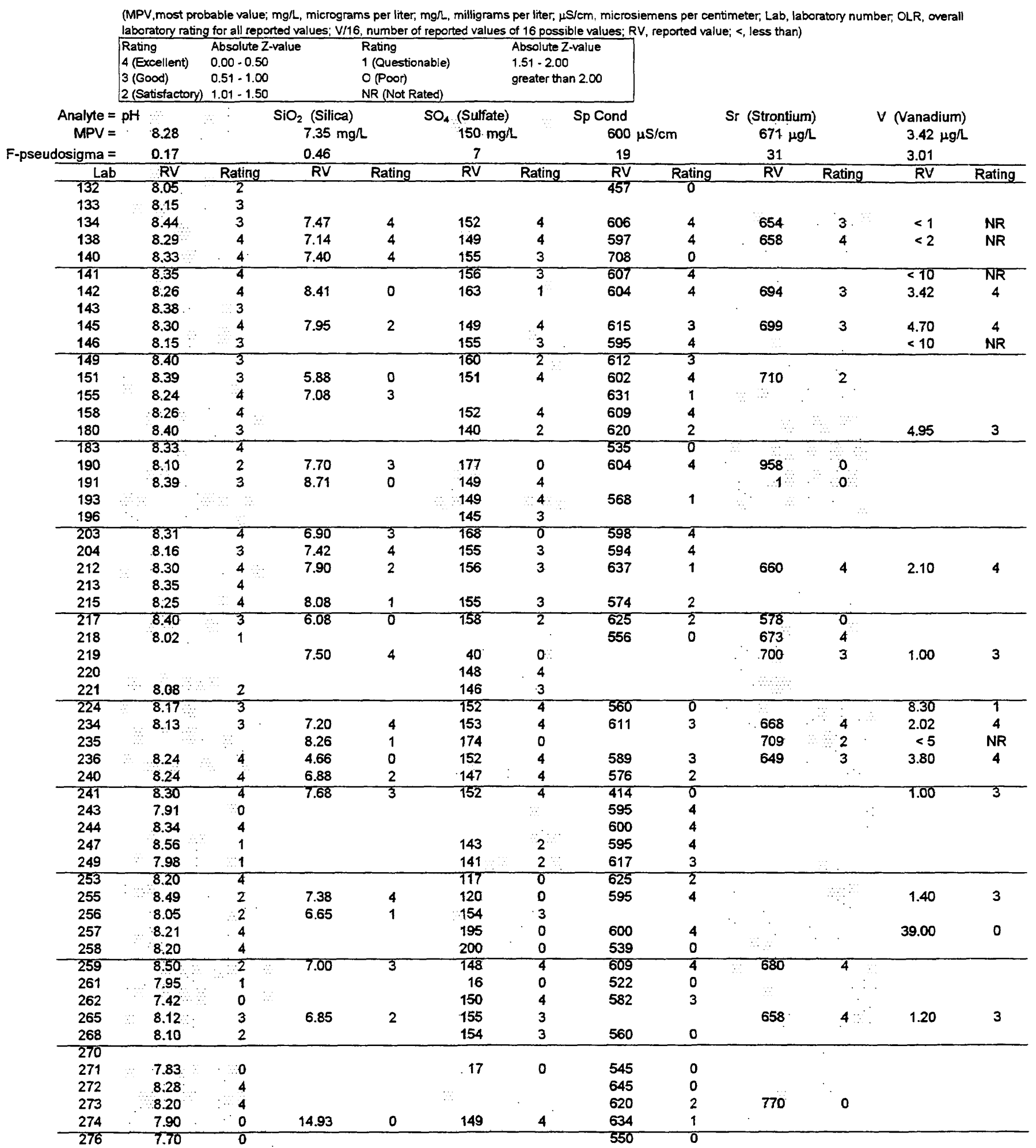


Table 8. Laboratory performance ratings for standard reference water sample N-51 (nutrient constituents)

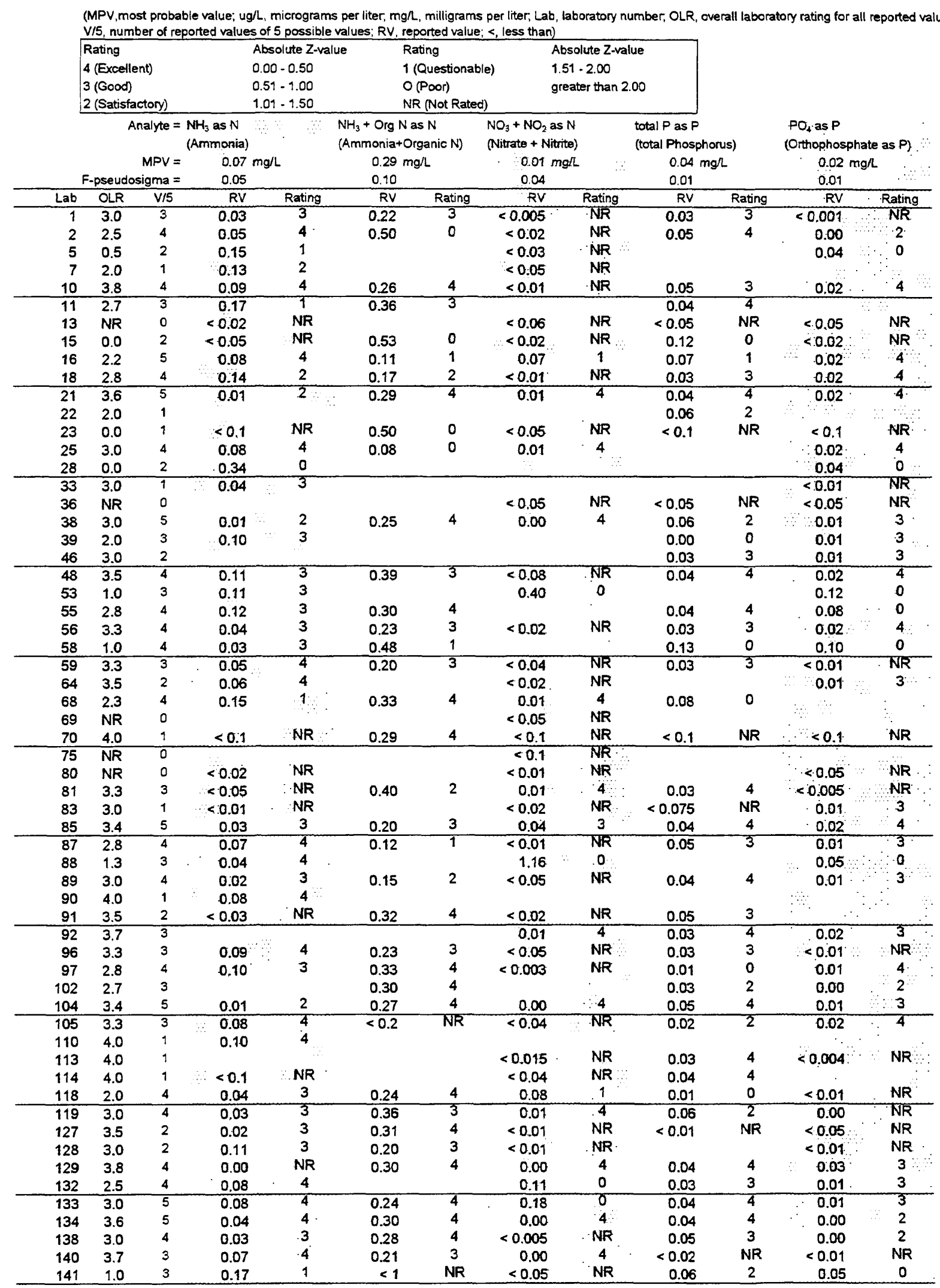


Table 8. Laboratory performance ratings for standard reference water sample N-51 (nutrient constituents)-Continued

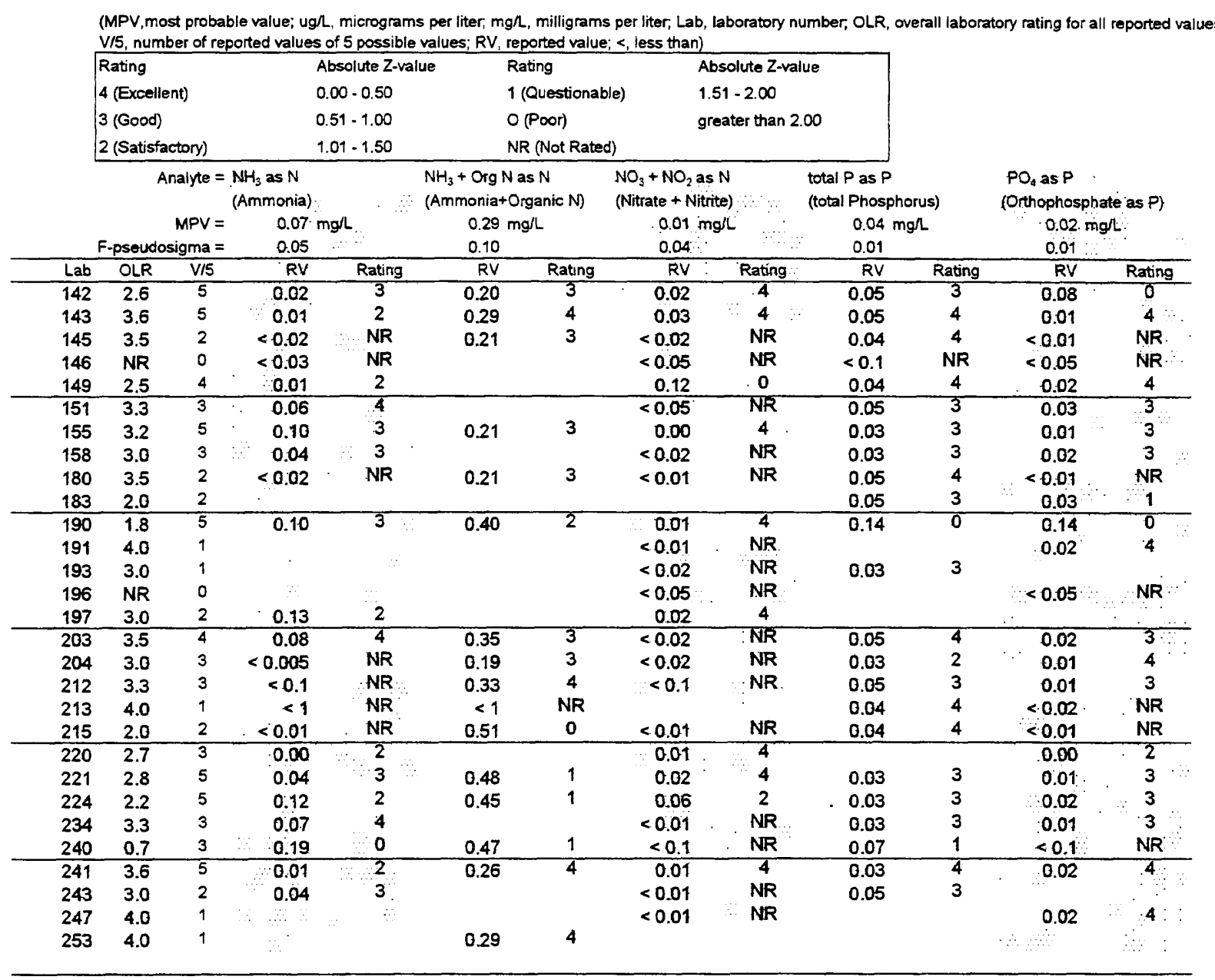


Table 9. Laboratory performance ratings for standard reference water sample N-52 (nutrient constituents)

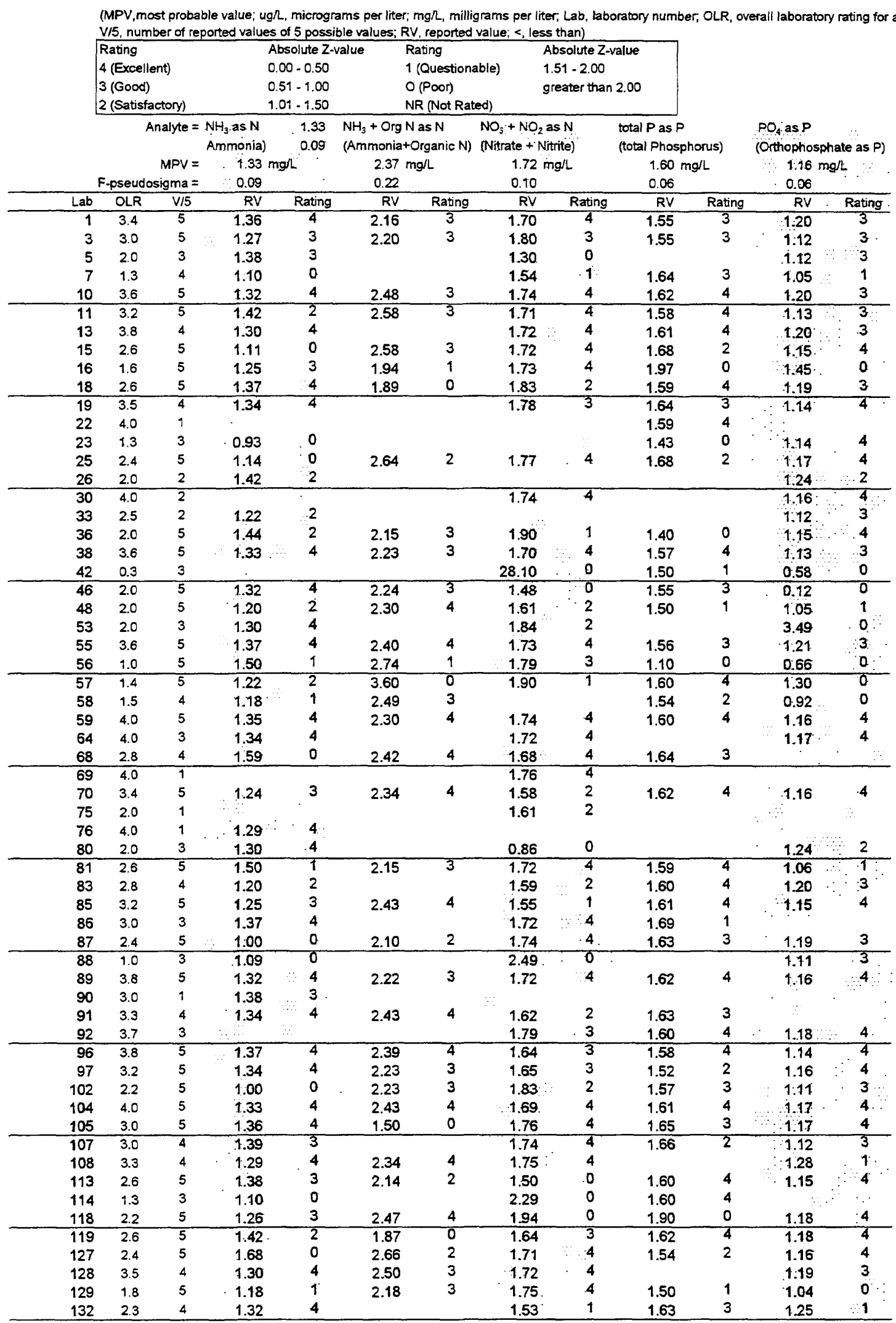


Table 9. Laboratory performance ratings for standard reference water sample N-52 (nutrient constituents)-Continußd

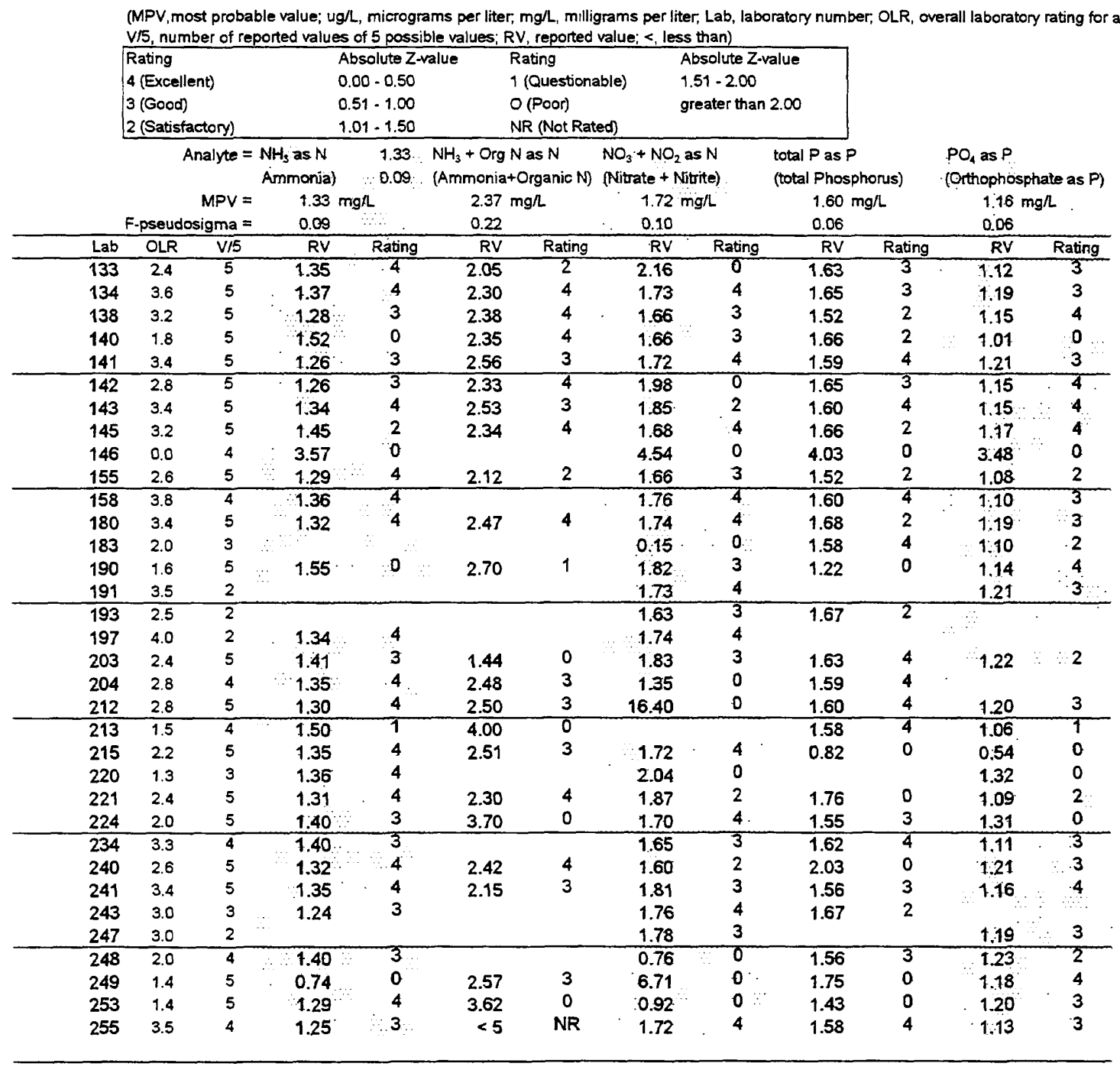


Table 10. Laboratory performance ratings for standard reference water sample P-27 (low ionic strength)

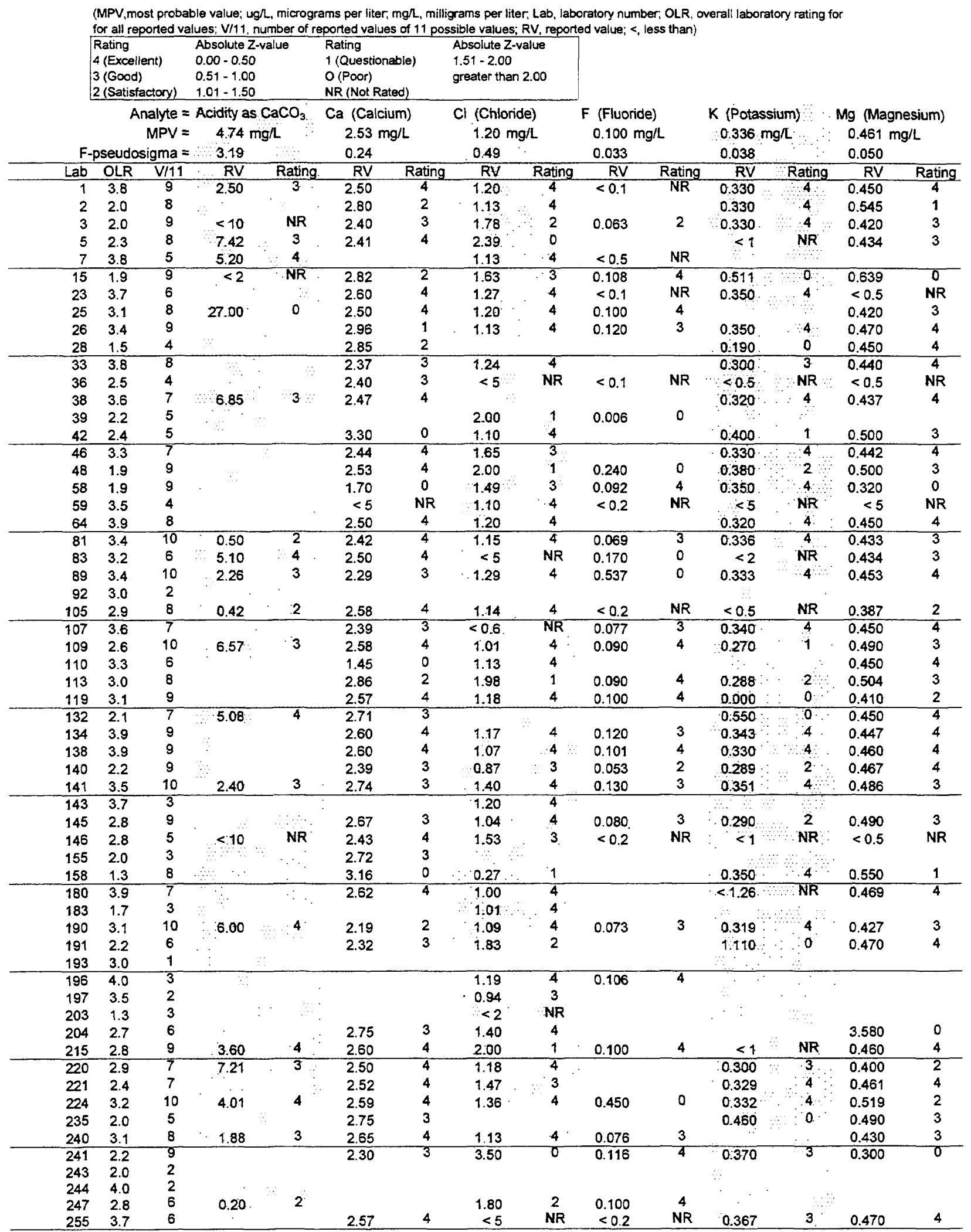


Table 10. Laboratory performance ratings for standard reference water sample P-27 (low ionic strength)-Continued

\begin{tabular}{|c|c|c|c|c|c|c|c|c|c|c|c|c|c|c|}
\hline \multicolumn{3}{|c|}{$\begin{array}{l}\text { Rating } \\
4 \text { (Excellent) } \\
3 \text { (Good) } \\
2 \text { (Satisfactory) }\end{array}$} & \multicolumn{2}{|c|}{$\begin{array}{l}\text { Absolute Z-value } \\
0.00-0.50 \\
0.51-1.00 \\
1.01-1.50 \\
\end{array}$} & \multicolumn{2}{|c|}{$\begin{array}{l}\text { Rating } \\
1 \text { (Questionable) } \\
O \text { (Poor) } \\
\text { NR (Not Rated) } \\
\end{array}$} & \multicolumn{2}{|c|}{$\begin{array}{l}\text { Absolute Z-value } \\
1.51-2.00 \\
\text { greater than } 2.00\end{array}$} & & & & \multirow{2}{*}{\multicolumn{2}{|c|}{ Mg (Magnesium) }} \\
\hline & \multicolumn{2}{|c|}{ Analyte $=$} & \multicolumn{2}{|c|}{$=$ Acidity as $\mathrm{CaCO}_{3}$} & \multicolumn{2}{|c|}{ Ca (Calcium) } & \multicolumn{2}{|c|}{ Cl (Chionide) } & \multicolumn{2}{|c|}{ F (Fluoride) } & K $\cdot$ (Potassium) & & & \\
\hline & & MPV $=$ & $=\quad 4.74 \mathrm{~m}$ & & $2.53 \mathrm{~m}$ & & $1.20 \mathrm{~m}$ & & $0.100 n$ & & $0.336 \cdot n$ & & \multicolumn{2}{|c|}{$0.461 \mathrm{mg} / \mathrm{L}$} \\
\hline $\mathrm{F}$ & pseudo & igma = & 3.19 & & 0.24 & & 0.49 & & 0.033 & & 0.038 & & 0.050 & \\
\hline Lab & OLR & $V / 11$ & RV & Rating & RV & Rating & RV & Rating & RV & Rating & RV & Rating & RV & Rating \\
\hline 256 & 1.3 & 7 & & & 2.50 & 4 & 1.95 & 1 & & & 0.000 & 0 & 1.500 & 0 \\
\hline 257 & 2.2 & 10 & 1.25 & 2. & 2.80 & 2 & 1.63 & 3 & 0.085 & 4 & 0.200 & 0 & 0.630 & 0 \\
\hline 258 & 0.4 & 9 & & & 1.60 & 0 & 8.40 & 0 & 0.170 & 0 & 0.500 & 0 & 1.940 & 0 \\
\hline 261 & 0.9 & 7 & & & 3.26 & 0 & 0.00 & 0 & & & $<0.39$ & NR & 1.950 & 0 \\
\hline 262 & 2.4 & 9 & & & 2.50 & 4 & 130 & 4 & 0.082 & 3 & 0.350 & 4 & 0.440 & 4 \\
\hline 265 & 2.4 & 7 & & & 2.90 & 1 & 0.95 & 3 & $<0.1$ & NR & 0.370 & 3 & 0.720 & 0 \\
\hline 268 & 3.0 & 8 & . & & 1.42 & 0 & 1.11 & 4 & & & 0.365 & 3 & 0.480 & 4 \\
\hline 270 & 0.3 & 3 & & & 1.06 & 0 & & & & & 0.410 & 1 & & \\
\hline 271 & 1.1 & 8 & . $\quad \because$ & & 4.28 & 0 & 2.00 & 1 & & & 0.900 & 0 & 10.370 & 0 \\
\hline 272 & 1.0 & 9 & 15.00 & 0 & 2.41 & 3 & 7.09 & 0 & 0.405 & 0 & 0.000 & 0 & 0.000 & 0 \\
\hline 273 & 1.7 & 9 & 5.20 & 4 & 2.80 & 2 & 13.00 & 0 & 0.400 & 0 & 0.412 & 1 & 0.521 & 2 \\
\hline 276 & 1.8 & 6 & 4.40 & 4 & 1.80 & 0 & 3.50 & 0 & & & $\therefore$ & $\therefore$ & 1.400 & 0 \\
\hline
\end{tabular}


Table 10. Laboratory performance ratings for standard reference water sample P-27 (low ionic strength)-Continued

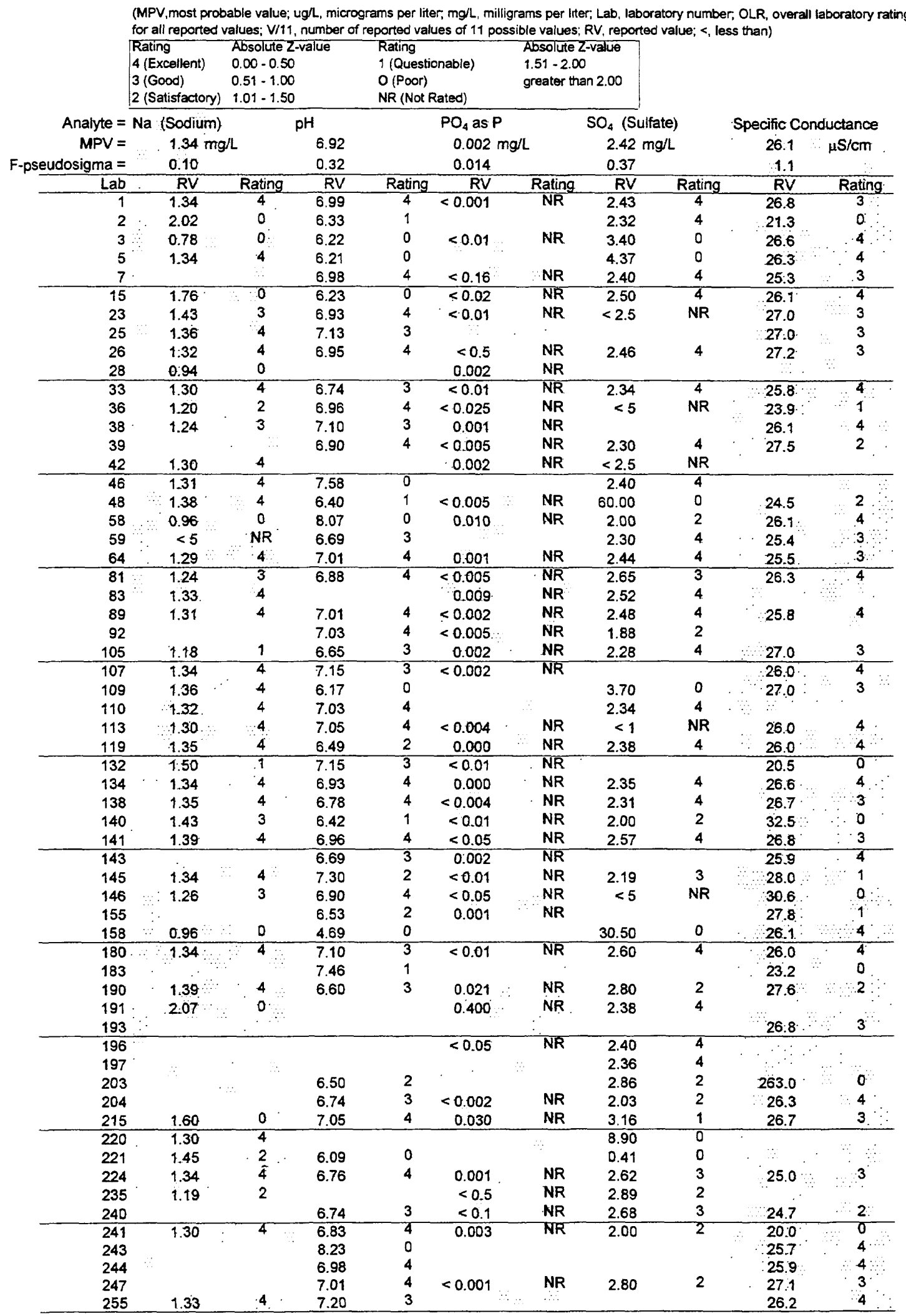


Table 10. Laboratory performance ratings for standard reference water sample P-27 (low ionic strength)-Continued

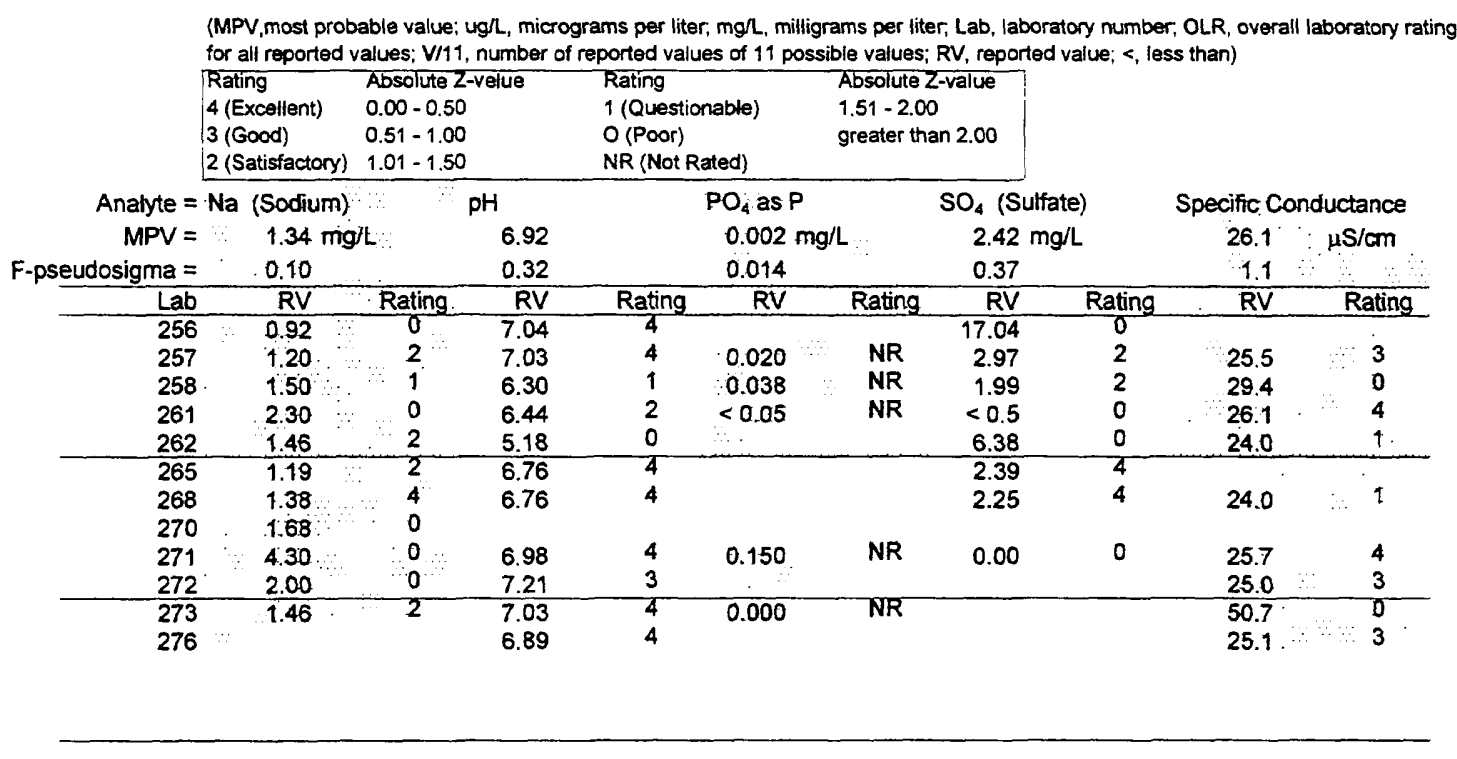


Table 11. Laboratory performance ratings for standard reference water sample Hg-23 (mercury)

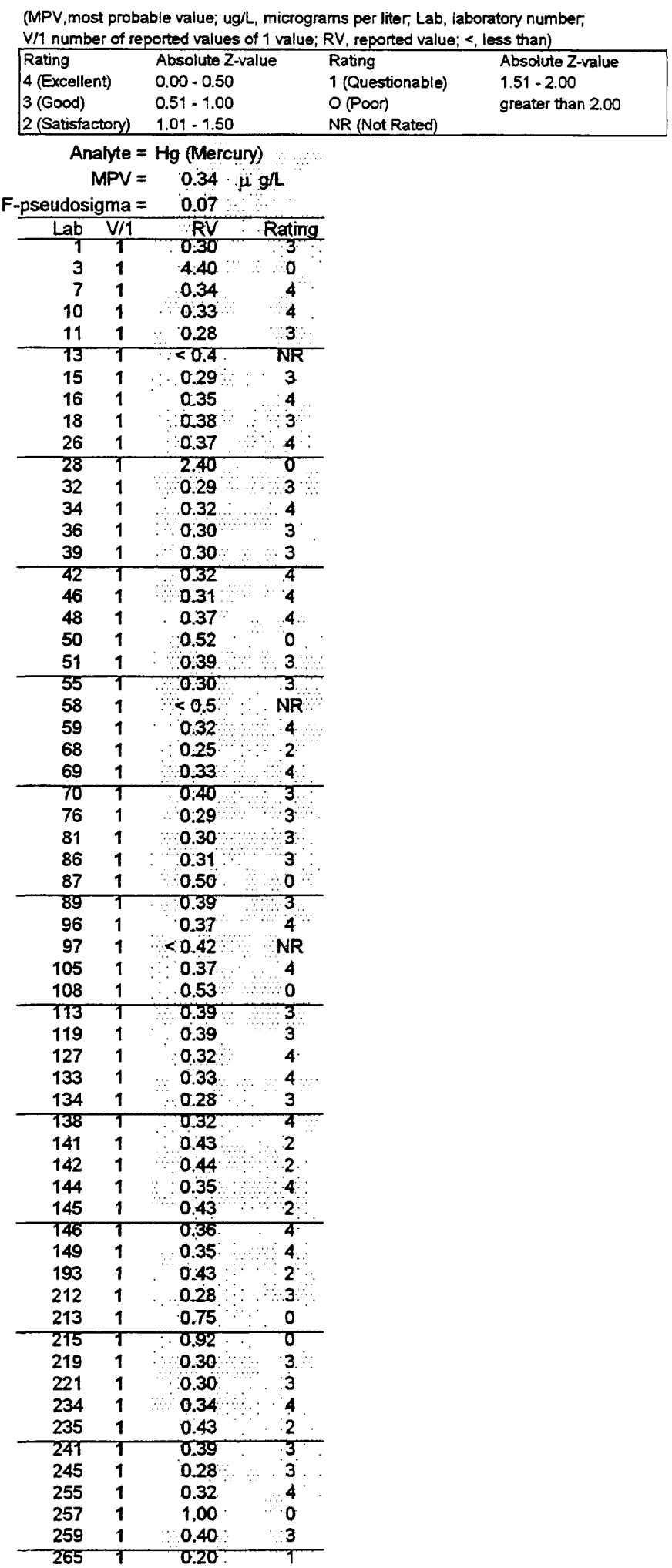




Analutical methods
0. Other/Not reported
1. AA: direct, air
2. AA: direct, $\mathrm{N}_{2} \mathrm{O}$
3. AA: graphite furnace
4. ICP
5. DCP
6. ICPMS
7. IC
10. AA: extraction
11. AA: hydride
12. AA: flame emission
22. Color:

$=$ atomic absorption: direct,air
$=\quad$ atomic absorption: direct,nitrous oxide
$=\quad$ atomic absorption: graphite furnace
$=\quad$ inductively coupled plasma
$=\quad$ direct current plasma
$=\quad$ inductively coupled plasma/mass spectrometry
$=\quad$ ion chromatography
$=\quad$ atomic absorption: extraction [chelating agent(s) specified]
$=\quad$ atomic absorption: hydride [reducing agent specified]
$=\quad$ atomic absorption: flame emission
$=\quad$ colorimetric [color reagent specified]

\begin{tabular}{|c|c|c|c|c|c|}
\hline \multicolumn{2}{|c|}{ Constituent } & page & \multicolumn{2}{|c|}{ Constituent } & \multirow{2}{*}{$\frac{\text { page }}{59}$} \\
\hline$\overline{\mathrm{Ag}}$ & Silver & 45 & $\mathrm{Mg}$ & Magnesium & \\
\hline $\mathrm{Al}$ & Aluminium & 46 & $\mathrm{Mn}$ & Manganese & 60 \\
\hline As & Arsenic & 47 & Mo & Molybdenum & 61 \\
\hline Cd & Cadmium & 52 & Se & Selenium & 66 \\
\hline Co & Cobalt & 53 & $\mathrm{SiO} 2$ & Silica & 67 \\
\hline $\mathrm{Cr}$ & Chromium & 54 & $\mathrm{Sr}$ & Strontium & 68 \\
\hline $\mathrm{Cu}$ & Copper & 55 & $\mathrm{TI}$ & Thallium & 69 \\
\hline $\mathrm{Fe}$ & Iron & 56 & $u$ & Uranium & 70 \\
\hline $\mathrm{K}$ & Potassium & 57 & $v$ & Vanadium & 71 \\
\hline $\mathrm{Li}$ & Lithium & 58 & $\mathrm{Zn}$ & Zinc & 72 \\
\hline
\end{tabular}


Table 12. Statistical summary of reported data for standard reference water sample T-143 (trace constituents)-Continued $\mathrm{Ag}$ (Silver)

$\mu \mathrm{g} / \mathrm{L}$

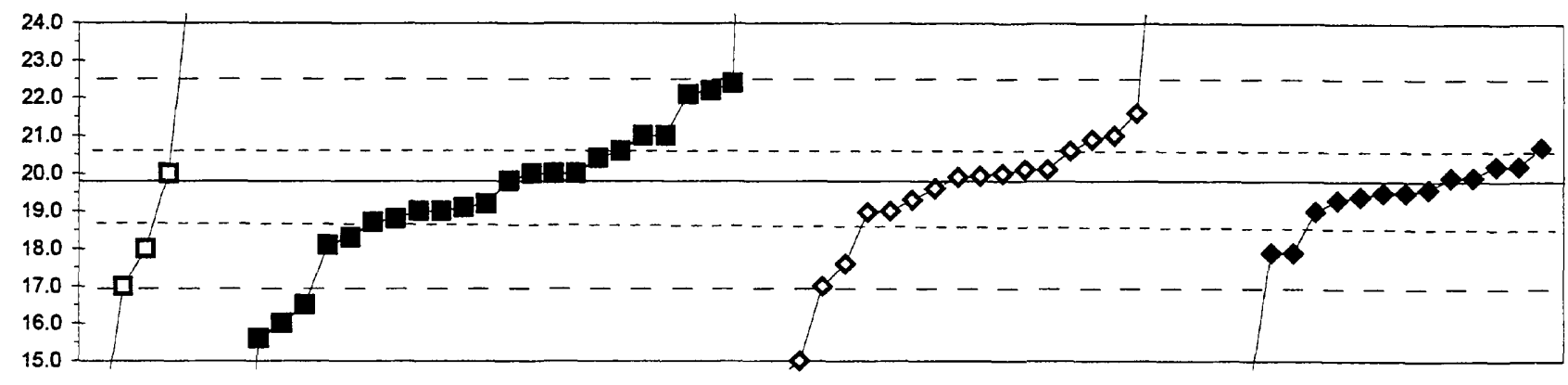

$-\square-1-a-3-4 \multimap 6$

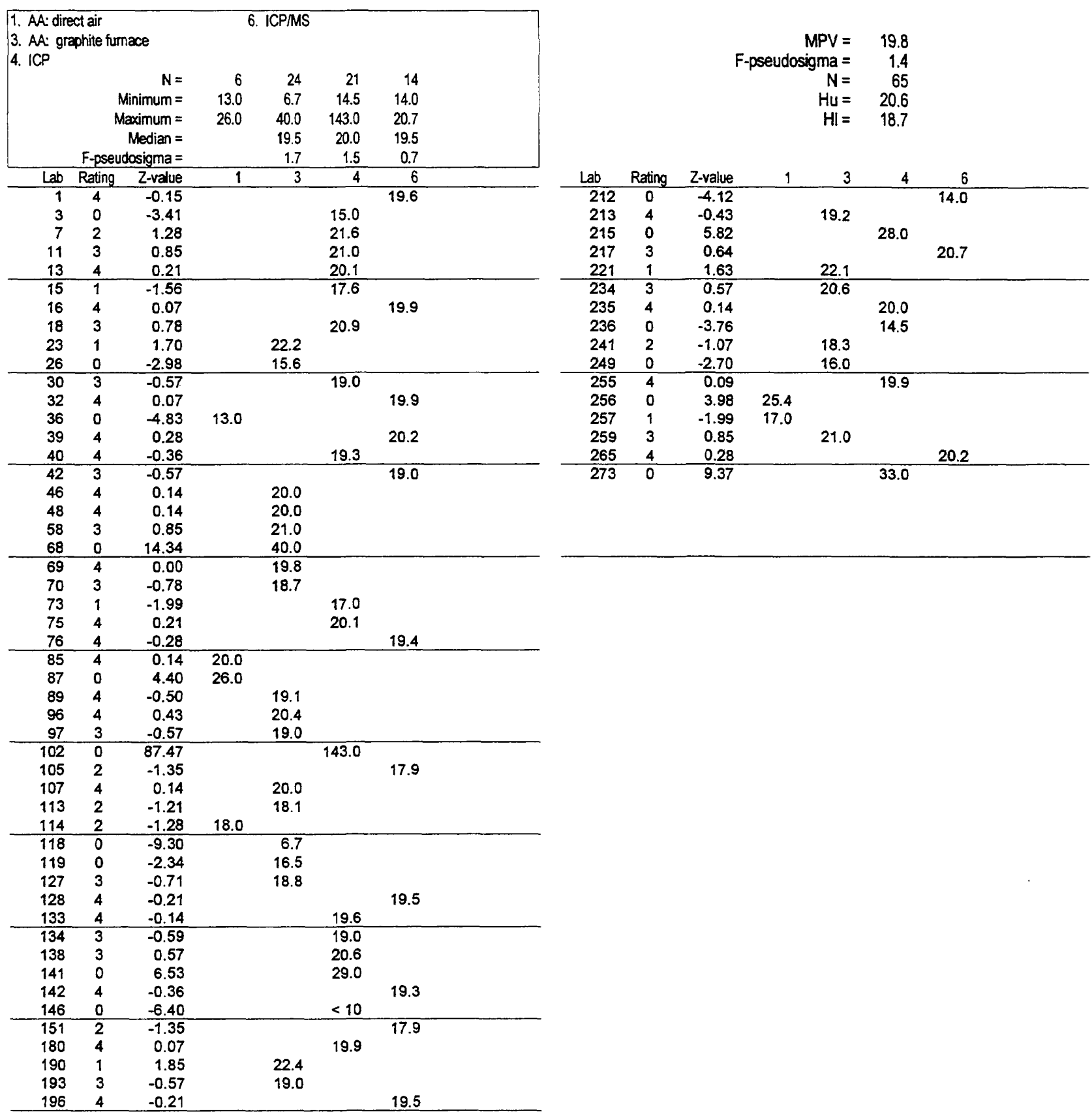


Table 12. Statistical summary of reported data for standard reference water sample T-143 (trace constituents)-Continued Al (Aluminum) $\mu g / L$

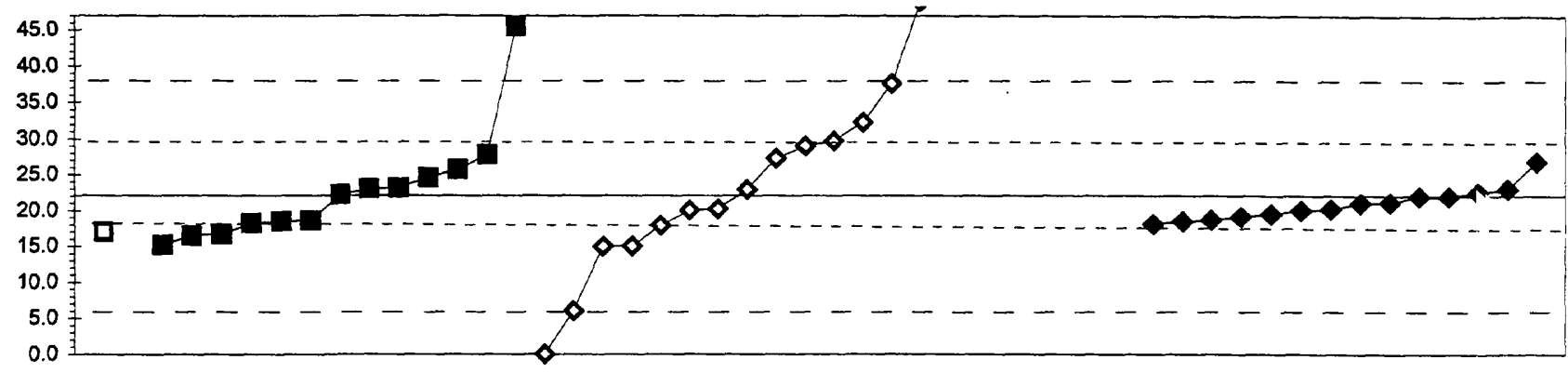

$-\square-1-0-2-3-\Delta-4-\Delta-5-6$

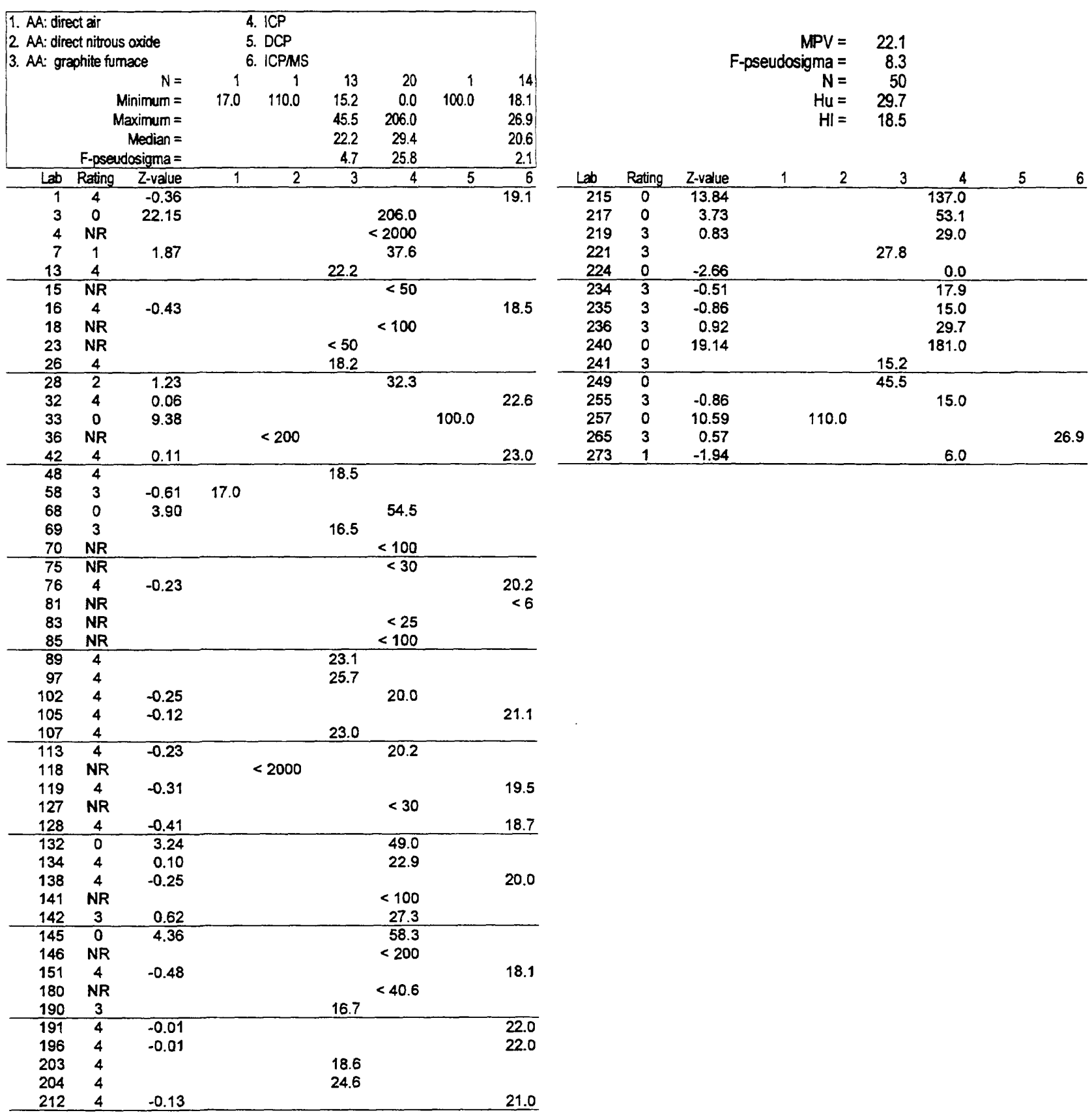


Table 12. Statistical summary of reported data for standard reference water sample T-143 (trace constituents)-Continued As (Arsenic)

$\mu \mathrm{g} / \mathrm{L}$

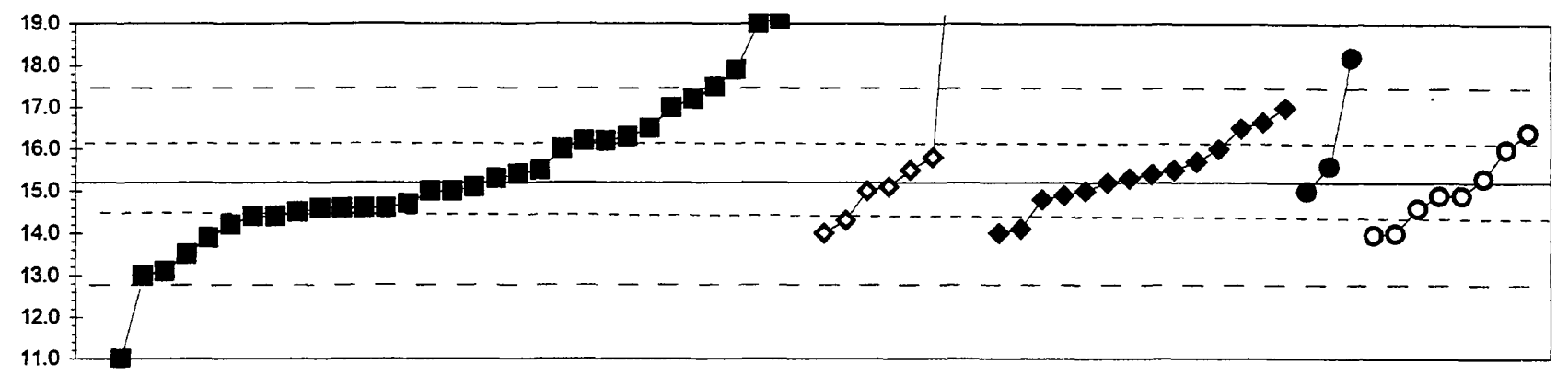

$-3 \multimap-4 \multimap 6-11-0-11 \mathrm{na}$

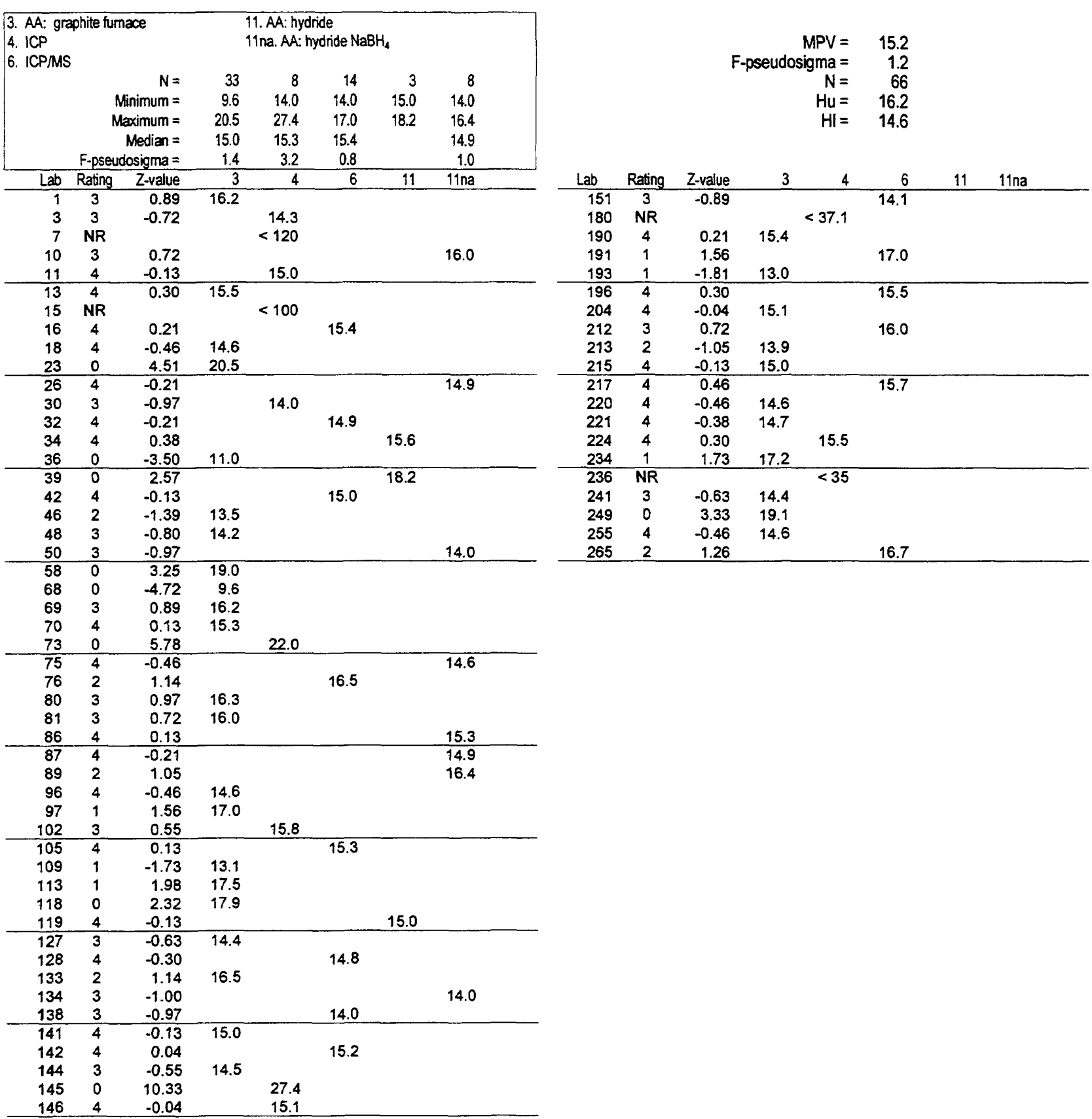


Table 12. Statistical summary of reported data for standard reference water sample T-143 (trace constituents)-Continued B (Boron)

$\mu \mathrm{g} / \mathrm{L}$

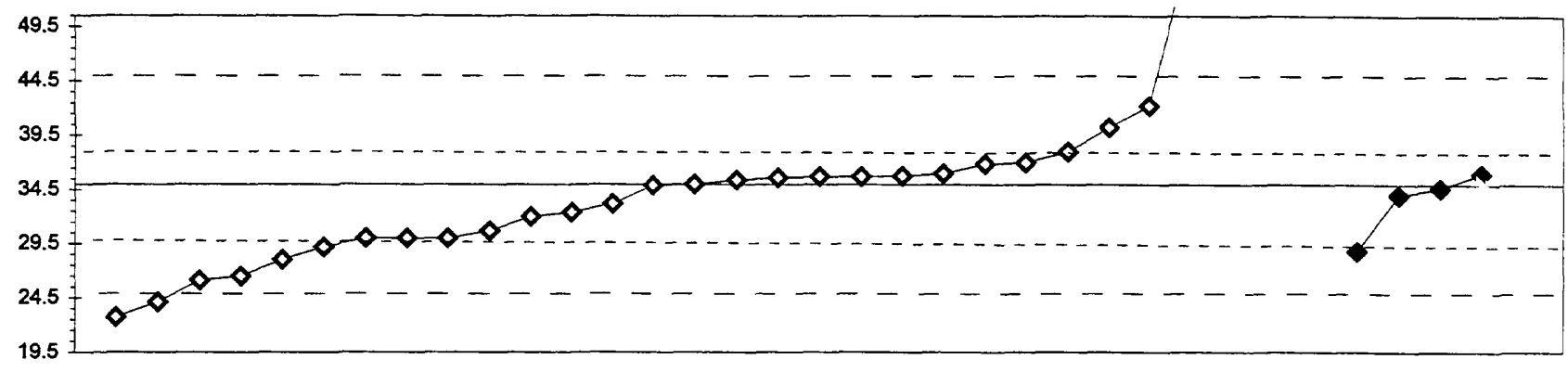

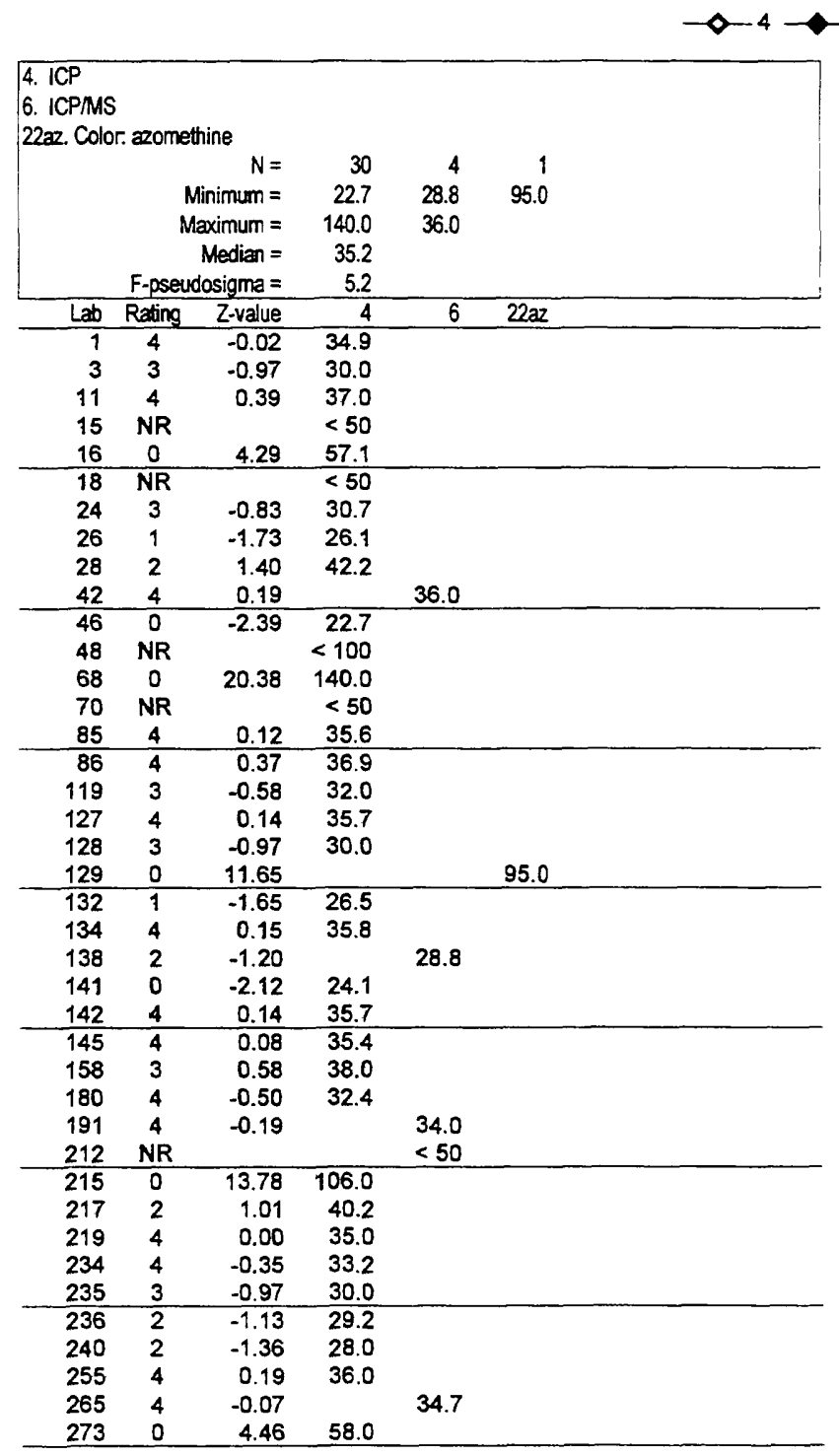


Table 12. Statistical summary of reported data for standard reference water sample T-143 (trace constituents)-Continued Ba (Barium) $\mu \mathrm{g} / \mathrm{L}$

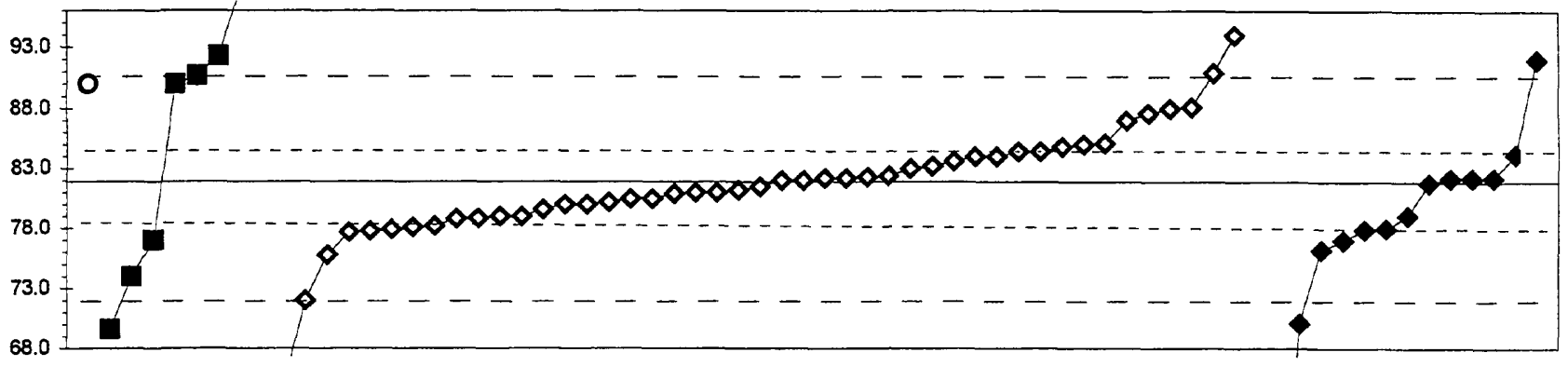

$-0-2-3-0-4-5 \multimap 6$

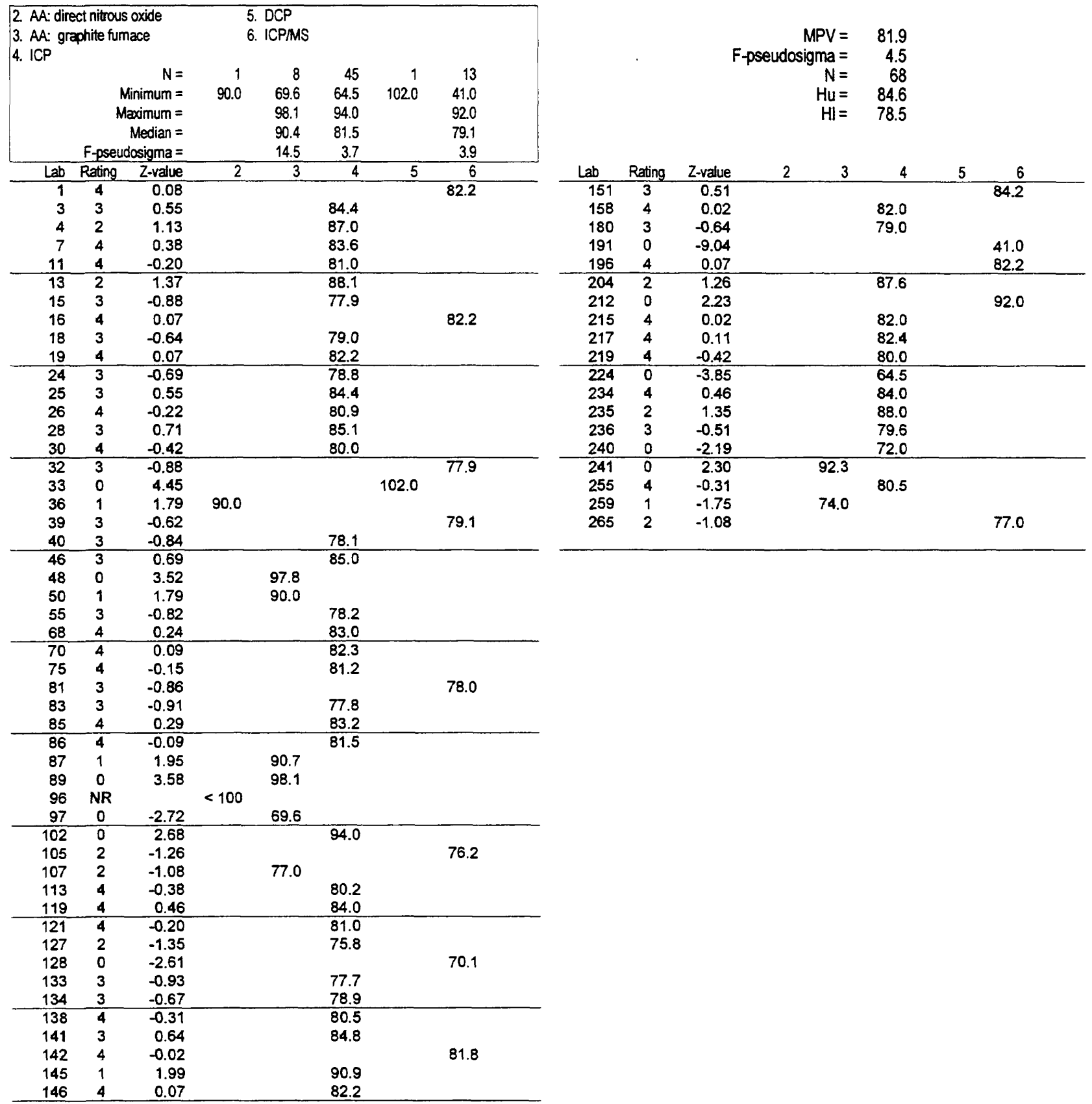


Table 12. Statistical summary of reported data for standard reference water sample T-143 (trace constituents)-Continued Be (Berlyium) $\mu \mathrm{g} / \mathrm{L}$

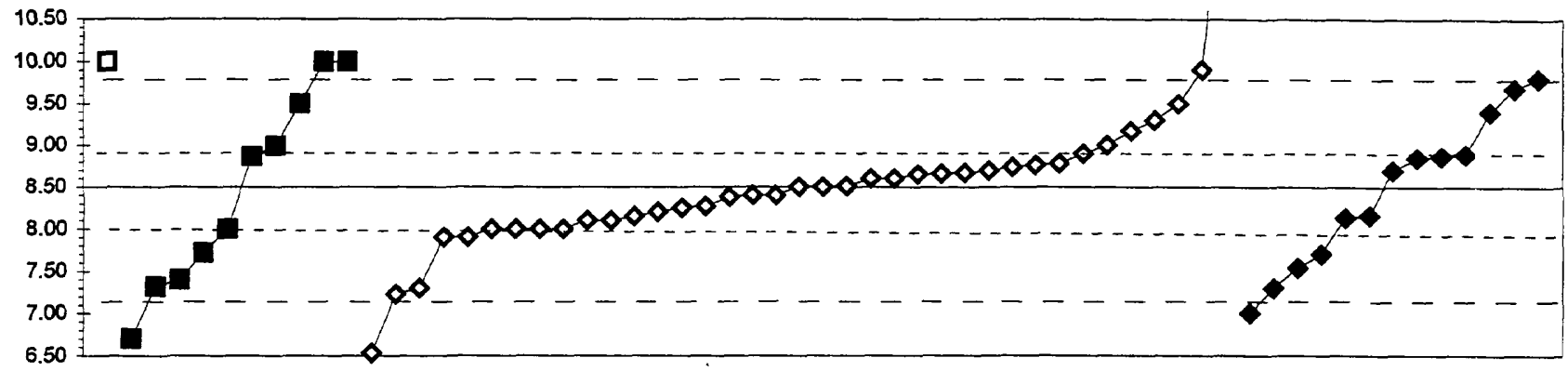

$-a-1-2-3-4-6$

\begin{tabular}{|c|c|c|c|c|c|c|c|c|c|c|c|c|c|}
\hline $\begin{array}{l}\text { 1. AA: dire } \\
\text { 3. AA: gra } \\
\text { 4. ICP }\end{array}$ & aphite fur & $\begin{array}{r}N= \\
\text { inimum }= \\
\text { 2ximum }= \\
\text { Median }= \\
\text { osigma }=\end{array}$ & 10.00 & $\begin{array}{r}10 \\
6.70 \\
10.00 \\
8.44 \\
1.56 \\
\end{array}$ & $\begin{array}{r}37 \\
6.53 \\
12.60 \\
8.50 \\
0.47 \\
\end{array}$ & $\begin{array}{r}13 \\
7.00 \\
9.80 \\
8.70 \\
0.88 \\
\end{array}$ & & & & do & $\begin{aligned} P V & = \\
m a & = \\
N & = \\
H u & = \\
H I & =\end{aligned}$ & $\begin{array}{r}8.50 \\
0.66 \\
61 \\
8.89 \\
8.00\end{array}$ & \\
\hline $\mathrm{Lab}$ & Rating & Z-value & 1 & 3 & 4 & 6 & Lab & Rating & Z-value & 1 & 3 & 4 & 6 \\
\hline 3 & 3 & -0.76 & & & 8.00 & & 212 & 1 & 1.97 & & & & 9.80 \\
\hline 4 & 3 & 0.76 & & & 9.00 & & 213 & 3 & 0.56 & & 8.87 & & \\
\hline 7 & 3 & -0.61 & & & 8.10 & & 215 & 3 & -0.61 & & & 8.10 & \\
\hline 11 & 4 & -0.15 & & & 8.40 & & 217 & 4 & -0.15 & & & 8.40 & \\
\hline 13 & 4 & 0.36 & & & 8.74 & & 224 & 0 & 6.21 & & & 12.60 & \\
\hline 15 & 4 & 0.15 & & & 8.60 & & 234 & 4 & -0.18 & & & 8.38 & \\
\hline 16 & 2 & -1.21 & & & & 7.70 & 235 & 3 & -0.76 & & & 8.00 & \\
\hline 18 & 4 & 0.00 & & & 8.50 & & 236 & 3 & -0.91 & & & 7.90 & \\
\hline 25 & 0 & 2.12 & & & 9.90 & & 241 & 0 & -2.73 & & 6.70 & & \\
\hline 30 & 1 & -1.82 & & & 7.30 & & 265 & 4 & 0.30 & & & & 8.70 \\
\hline 32 & 2 & 1.36 & & & & 9.40 & & & & & & & \\
\hline 36 & 0 & 2.27 & & 10.00 & & & & & & & & & \\
\hline 39 & 1 & 1.52 & & 9.50 & & & & & & & & & \\
\hline 40 & 3 & -0.91 & & & 7.90 & & & & & & & & \\
\hline 46 & 4 & 0.39 & & & 8.76 & & & & & & & & \\
\hline 48 & 0 & 2.27 & & 10.00 & & & & & & & & & \\
\hline 68 & 3 & 0.61 & & & 8.90 & & & & & & & & \\
\hline 69 & 1 & -1.79 & & 7.32 & & & & & & & & & \\
\hline 70 & 4 & 0.26 & & & 8.67 & & & & & & & & \\
\hline 75 & 4 & 0.00 & & & 8.50 & & & & & & & & \\
\hline 76 & 1 & 1.79 & & & & 9.68 & & & & & & & \\
\hline 81 & 0 & -2.27 & & & & 7.00 & & & & & & & \\
\hline 83 & 4 & -0.45 & & & 8.20 & & & & & & & & \\
\hline 85 & 2 & 1.02 & & & 9.17 & & & & & & & & \\
\hline 86 & 4 & -0.38 & & & 8.25 & & & & & & & & \\
\hline 89 & 1 & -1.67 & & 7.40 & & & & & & & & & \\
\hline 96 & 0 & 2.27 & 10.00 & & & & & & & & & & \\
\hline 113 & 4 & 0.42 & & & 8.78 & & & & & & & & \\
\hline 119 & 2 & -1.18 & & 7.72 & & & & & & & & & \\
\hline 121 & 3 & -0.76 & & & 8.00 & & & & & & & & \\
\hline 127 & 0 & -2.99 & & & 6.53 & & & & & & & & \\
\hline 128 & 2 & -1.46 & & & & 7.54 & & & & & & & \\
\hline 133 & 4 & 0.30 & & & 8.70 & & & & & & & & \\
\hline 134 & 4 & 0.02 & & & 8.51 & & & & & & & & \\
\hline 138 & 4 & 0.23 & & & 8.65 & & & & & & & & \\
\hline 141 & 3 & -0.53 & & & 8.15 & & & & & & & & \\
\hline 142 & 3 & 0.56 & & & & 8.87 & & & & & & & \\
\hline 144 & 0 & -12.86 & $<0.01$ & & & & & & & & & & \\
\hline 145 & 1 & 1.52 & & & 9.50 & & & & & & & & \\
\hline 146 & 1 & -1.92 & & & 7.23 & & & & & & & & \\
\hline 151 & 3 & -0.52 & & & & 8.16 & & & & & & & \\
\hline 158 & 2 & 1.21 & & & 9.30 & & & & & & & & \\
\hline 180 & 4 & 0.15 & & & 8.60 & & & & & & & & \\
\hline 191 & 3 & 0.59 & & & & 8.89 & & & & & & & \\
\hline 193 & 3 & -0.76 & & 8.00 & & & & & & & & & \\
\hline
\end{tabular}


Table 12. Statistical summary of reported data for standard reference water sample T-143 (trace constituents)-Continued $\mathrm{Ca}$ (Calcium) $\mathrm{mg} / \mathrm{L}$

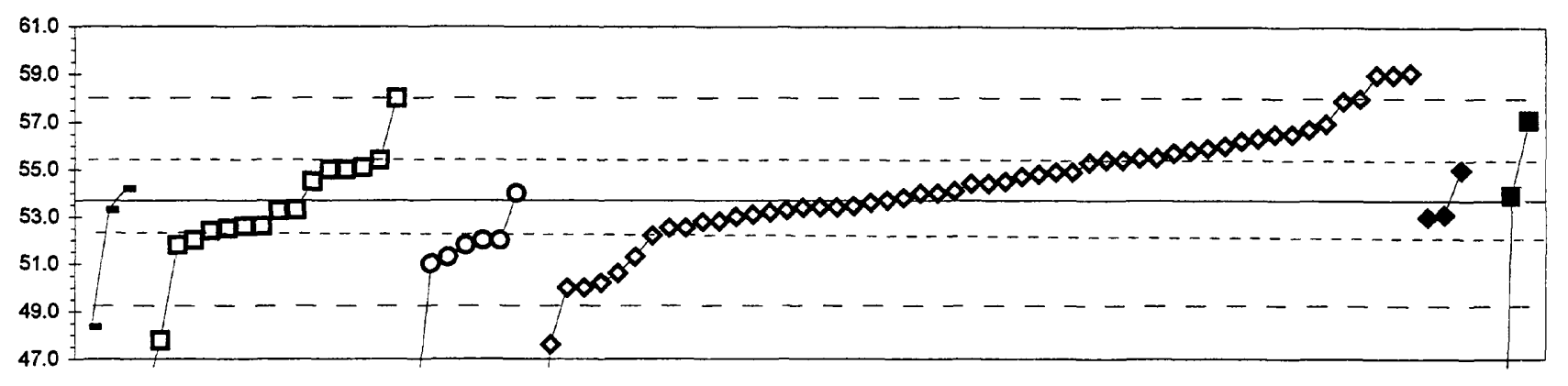

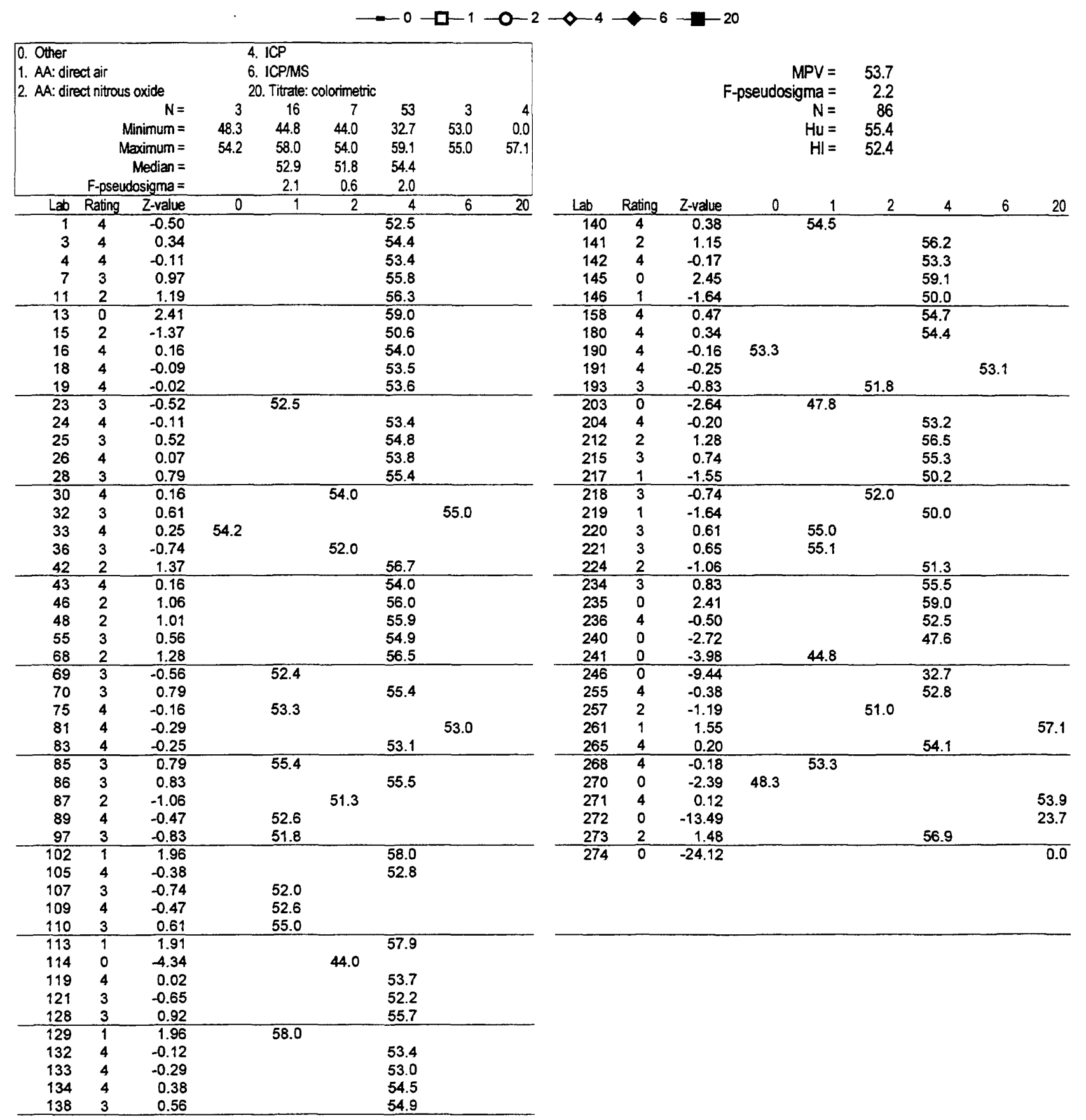


Table 12. Statistical summary of reported data for standard reference water sample T-143 (trace constituents)-Continued Cd (Cadmium) $\mu \mathrm{g} / \mathrm{L}$

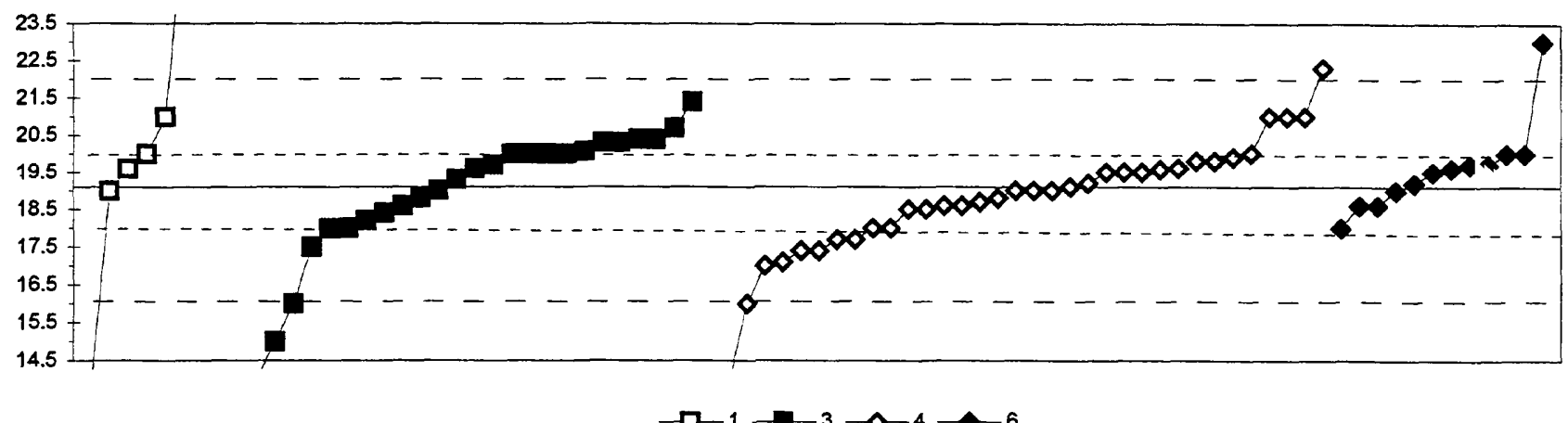

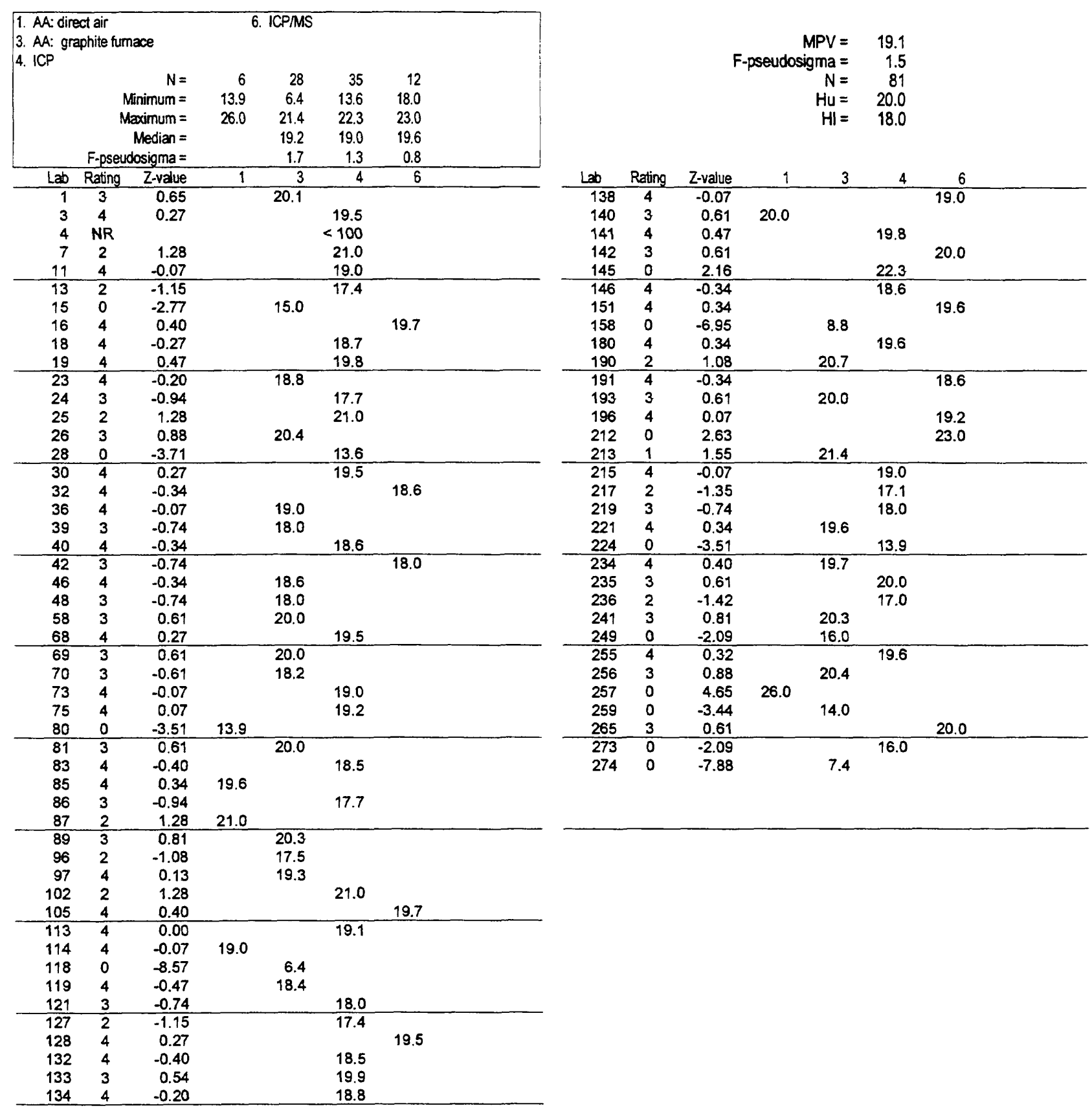


Table 12. Statistical summary of reported data for standard reference water sample T-143 (trace constituents)-Continued Co (Cobalt) $\mu \mathrm{g} / \mathrm{L}$

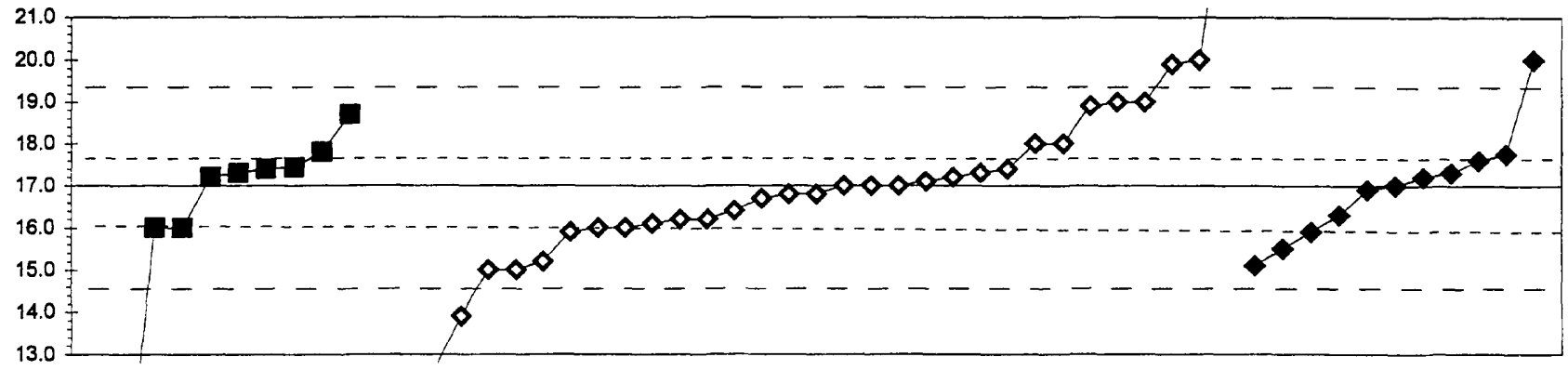

$\rightarrow-1-0-3-4 \multimap-6$

\begin{tabular}{|c|c|c|c|c|c|c|}
\hline $\begin{array}{l}\text { 1. } A \text { : dire } \\
\text { 3. } A A: \text { gre } \\
\text { 4. ICP }\end{array}$ & $\begin{array}{l}\text { ct air } \\
\text { phite fur }\end{array}$ & & & $\overline{\mathrm{CP} M S}$ & & \\
\hline & & $N=$ & 1 & 9 & 32 & 11 \\
\hline & & nimum $=$ & 23.0 & 10.0 & 8.0 & 15.1 \\
\hline & & ximum = & & 18.7 & 24.2 & 20.0 \\
\hline & & Median = & & 17.3 & 16.8 & 17.0 \\
\hline & F-pseu & ssigma $=$ & & 1.1 & 1.3 & 1.0 \\
\hline Lab & Rating & Z-value & 1 & 3 & 4 & 6 \\
\hline 1 & 4 & 0.36 & & 17.4 & & \\
\hline 3 & 1 & -1.69 & & & 15.0 & \\
\hline 4 & NR & & & & $<100$ & \\
\hline 7 & 0 & 2.45 & & & 19.9 & \\
\hline 11 & 3 & 0.84 & & & 18.0 & \\
\hline 13 & 0 & -3.71 & & & 12.6 & \\
\hline 15 & NR & & & & $<20$ & \\
\hline 16 & 4 & -0.08 & & & & 16.9 \\
\hline 18 & 3 & -0.76 & & & 16.1 & \\
\hline 24 & 0 & -3.96 & & & 12.3 & \\
\hline 26 & 3 & -0.51 & & & 16.4 & \\
\hline 30 & 4 & 0.00 & & & 17.0 & \\
\hline 32 & 4 & 0.17 & & & & 17.2 \\
\hline 40 & 1 & -1.52 & & & 15.2 & \\
\hline 42 & 0 & 2.53 & & & & 20.0 \\
\hline 46 & 4 & 0.34 & & & 17.4 & \\
\hline 48 & NR & & & & $<50$ & \\
\hline 50 & 3 & -0.84 & & 16.0 & & \\
\hline 68 & 3 & 0.84 & & & 18.0 & \\
\hline 70 & NR & & & & $<50$ & \\
\hline 75 & 1 & 1.60 & & & 18.9 & \\
\hline 86 & 4 & -0.17 & & & 16.8 & \\
\hline 89 & 3 & 0.67 & & 17.8 & & \\
\hline 97 & 4 & 0.17 & & 17.2 & & \\
\hline 102 & 0 & 2.53 & & & 20.0 & \\
\hline 105 & 2 & -1.26 & & & & 15.5 \\
\hline 119 & 3 & -0.93 & & & & 15.9 \\
\hline 121 & 3 & -0.84 & & & 16.0 & \\
\hline 127 & 4 & 0.25 & & 17.3 & & \\
\hline 128 & 4 & 0.00 & & & 17.0 & \\
\hline 132 & 4 & 0.00 & & & 17.0 & \\
\hline 134 & 4 & -0.25 & & & 16.7 & \\
\hline 138 & 1 & -1.60 & & & & 15.1 \\
\hline 141 & 3 & -0.67 & & & 16.2 & \\
\hline 142 & 3 & -0.59 & & & & 16.3 \\
\hline 145 & 0 & 6.07 & & & 24.2 & \\
\hline 146 & 3 & -0.67 & & & 16.2 & \\
\hline 158 & 4 & 0.08 & & & 17.1 & \\
\hline 180 & 4 & 0.25 & & & 17.3 & \\
\hline 191 & 3 & 0.51 & & & & 17.6 \\
\hline 196 & 4 & 0.25 & & & & 17.3 \\
\hline 212 & 4 & 0.00 & & & & 17.0 \\
\hline 213 & 2 & 1.43 & & 18.7 & & \\
\hline 215 & 1 & 1.69 & & & 19.0 & \\
\hline 217 & 4 & -0.17 & & & 16.8 & \\
\hline 219 & 3 & -0.84 & & & 16.0 & \\
\hline 221 & 3 & -0.84 & & 16.0 & & \\
\hline 224 & 3 & -0.93 & & & 15.9 & \\
\hline 234 & 4 & 0.34 & & 17.4 & & \\
\hline 235 & 1 & 1.69 & & & 19.0 & \\
\hline
\end{tabular}


Table 12. Statistical summary of reported data for standard reference water sample T-143 (trace constituents)-Continued $\mathrm{Cr}$ (Chromium) $\mu \mathrm{g} / \mathrm{L}$

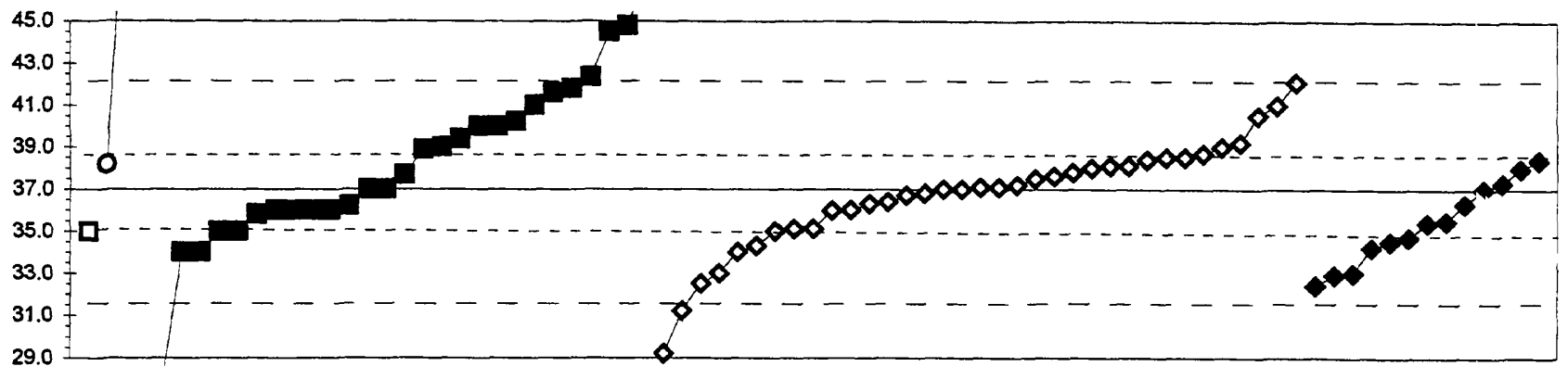

$-\square-1-0-2 \rightarrow-3-\diamond-4$

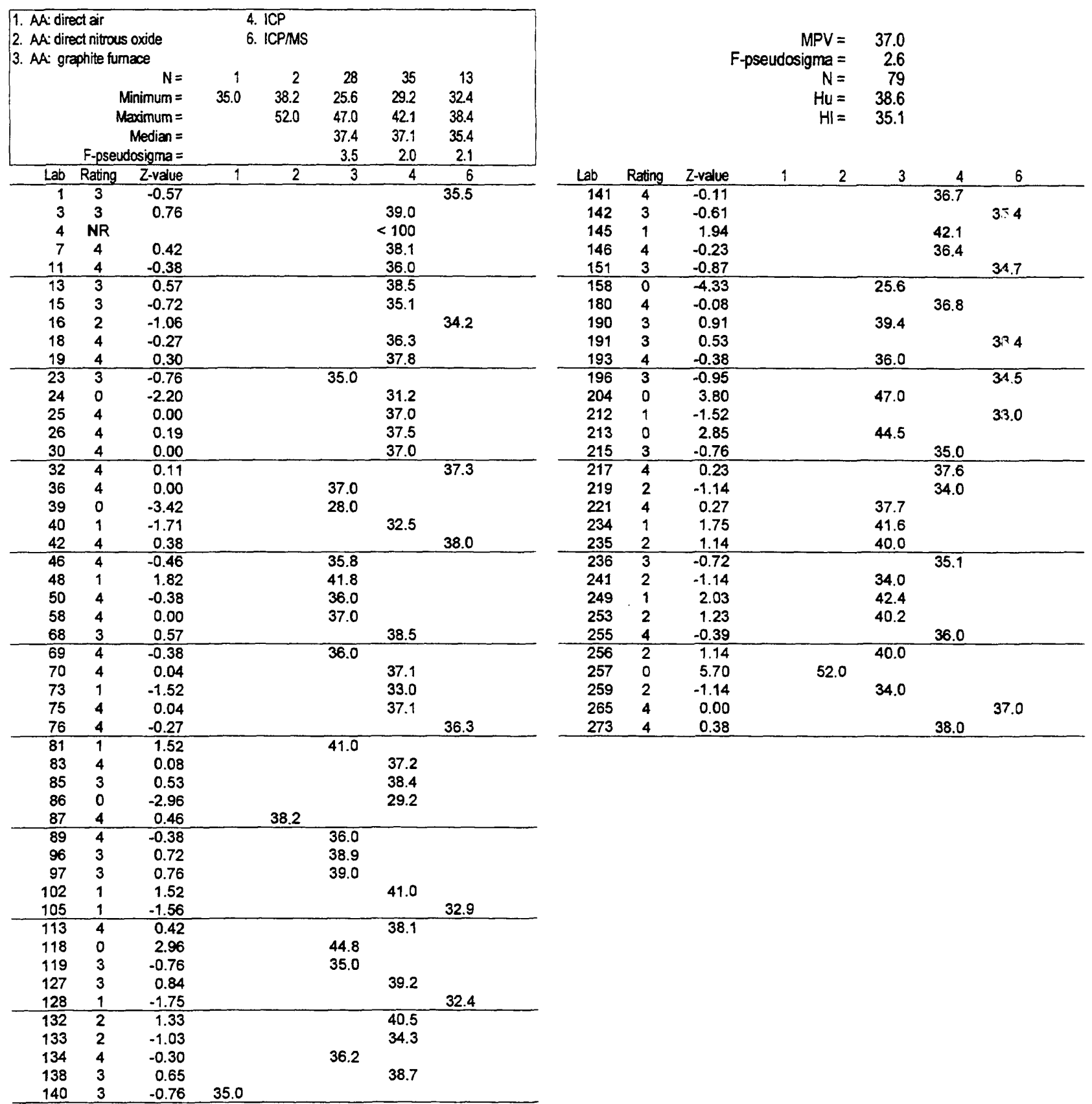


Table 12. Statistical summary of reported data for standard reference water sample T-143 (trace constituents)-Continued $\mathrm{Cu}$ (Copper) $\mu \mathrm{g} / \mathrm{L}$

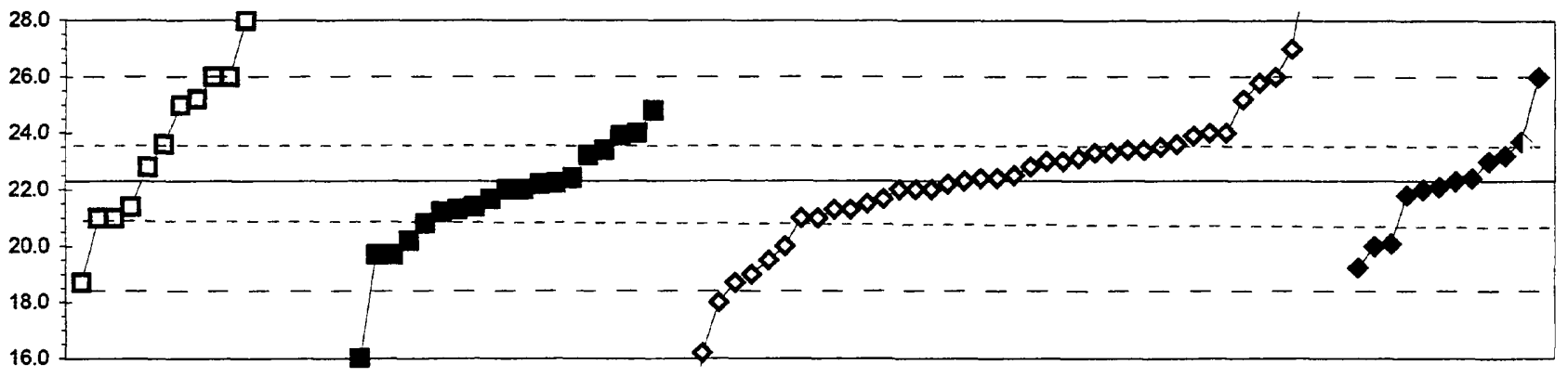

$-\square-1-3-\Delta-4-6$

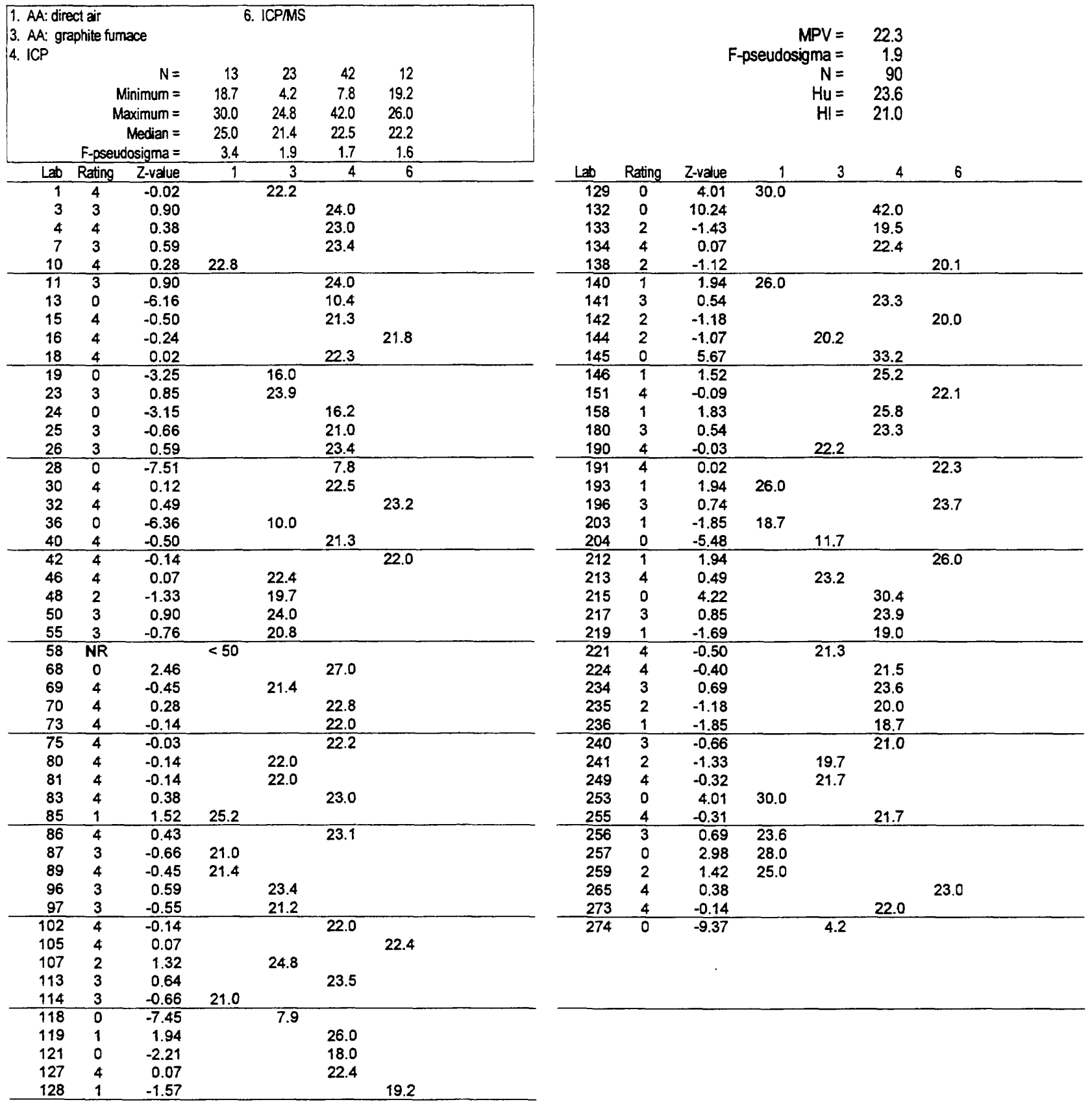


Table 12. Statistical summary of reported data for standard reference water sample T-143 (trace constituents)-Continued $\mathrm{Fe}$ (Iron) $\mu \mathrm{g} / \mathrm{L}$

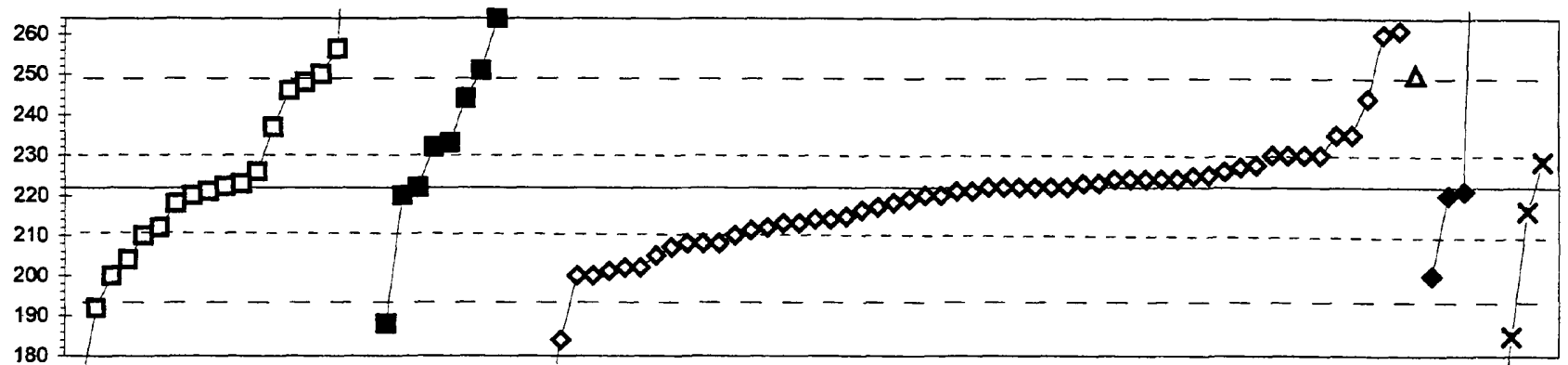

$-\square-1-6-3-4-\Delta-5-6-x-22$

\begin{tabular}{|c|c|c|c|c|c|c|c|c|}
\hline \multicolumn{3}{|c|}{$\begin{array}{l}\text { 1. AA: direct air } \\
\text { 3. AA: graphite fumace } \\
\text { 4. ICP }\end{array}$} & \multicolumn{3}{|c|}{$\begin{array}{l}\text { 5. DCP } \\
\text { 6. ICPMS } \\
\text { 22. Colonimetric }\end{array}$} & \multirow{6}{*}{$\begin{array}{r}1 \\
250\end{array}$} & \multirow{6}{*}{$\begin{array}{r}4 \\
200 \\
365\end{array}$} & \multirow{6}{*}{$\begin{array}{r}4 \\
152 \\
228\end{array}$} \\
\hline & & $N=$ & 19 & 9 & 56 & & & \\
\hline & & Ainimum = & 170 & 188 & 21 & & & \\
\hline & & laximum = & 470 & 302 & 261 & & & \\
\hline & & Median = & 222 & 233 & 221 & & & \\
\hline & F-psed & tosigma $=$ & 27 & 22 & 10 & & & \\
\hline Lab & Rating & Z-value & 1 & 3 & 4 & 5 & 6 & 22 \\
\hline 1 & 3 & -0.77 & & & 211 & & & \\
\hline 3 & 0 & 2.81 & & & 261 & & & \\
\hline 4 & 3 & 0.94 & & & 235 & & & \\
\hline 7 & 4 & 0.20 & & & 225 & & & \\
\hline 10 & 1 & 1.73 & 246 & & & & & \\
\hline 11 & $\frac{1}{2}$ & -1.44 & & & 202 & & & \\
\hline 13 & 2 & -1.08 & & & 207 & & & \\
\hline 15 & 2 & -1.01 & & & 208 & & & \\
\hline 16 & 1 & -1.59 & & & 200 & & & \\
\hline 18 & 4 & 0.00 & & & 222 & & & \\
\hline 19 & 4 & 0.36 & & & 227 & & & \\
\hline 21 & 0 & -5.05 & & & & & & 152 \\
\hline 23 & 4 & -0.29 & 218 & & & & & \\
\hline 24 & 4 & 0.00 & & 222 & & & & \\
\hline 25 & 2 & -1.44 & & & 202 & & & \\
\hline 26 & 4 & 0.00 & & & 222 & & & \\
\hline 30 & 0 & 17.89 & 470 & & & & & \\
\hline 32 & 0 & 10.32 & & & & & 365 & \\
\hline 33 & 1 & 2.02 & & & & 250 & & \\
\hline 35 & 4 & -0.43 & & & & & & 216 \\
\hline 36 & 1 & 2.02 & 250 & & & & & \\
\hline 40 & 0 & -14.52 & & & 21 & & & \\
\hline 42 & 4 & 0.14 & & & 224 & & & \\
\hline 43 & 3 & 0.58 & & & 230 & & & \\
\hline 46 & 4 & 0.00 & & & 222 & & & \\
\hline 48 & 0 & -4.47 & & & 160 & & & \\
\hline 50 & 0 & 2.09 & & 251 & & & & \\
\hline 55 & 4 & 0.00 & & & 222 & & & \\
\hline 58 & 3 & -0.87 & 210 & & & & & \\
\hline 68 & 4 & 0.22 & & & 225 & & & \\
\hline 69 & 4 & 0.29 & 226 & & & & & \\
\hline 70 & 3 & -0.58 & & & 214 & & & \\
\hline 73 & 4 & -0.07 & & & 221 & & & \\
\hline 75 & 3 & -0.58 & & & 214 & & & \\
\hline 80 & 4 & 0.07 & 223 & & & & & \\
\hline 81 & 4 & -0.14 & & & & & 220 & \\
\hline 83 & 4 & -0.22 & & & 219 & & & \\
\hline 85 & 4 & 0.14 & & & 224 & & & \\
\hline 86 & 3 & -0.65 & & & 213 & & & \\
\hline 87 & 3 & -0.72 & 212 & & & & & \\
\hline 89 & $\frac{4}{1}$ & 1.59 & & 244 & & & & \\
\hline 91 & 3 & -0.72 & & & 212 & & & \\
\hline 96 & 1 & 1.88 & 248 & & & & & \\
\hline 97 & 3 & 0.79 & & 233 & & & & \\
\hline 102 & 1 & 1.59 & & & 244 & & & \\
\hline 105 & 3 & -0.65 & & & 213 & & & \\
\hline 107 & 1 & -1.59 & 200 & & & & & \\
\hline 109 & 0 & 2.48 & 256 & & & & & \\
\hline 113 & 4 & 0.07 & & & 223 & & & \\
\hline 114 & 2 & -1.30 & 204 & & & & & \\
\hline
\end{tabular}

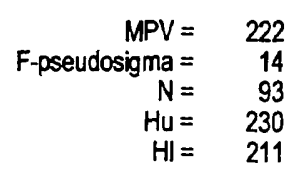

\begin{tabular}{|c|c|c|c|c|c|c|c|c|}
\hline Lab & Rating & Z-value & 1 & 3 & 4 & 5 & 6 & 22 \\
\hline 119 & 4 & 0.29 & & & 226 & & & \\
\hline 121 & 3 & -0.87 & & & 210 & & & \\
\hline 127 & 4 & -0.14 & & & 220 & & & \\
\hline 128 & 2 & -1.23 & & & 205 & & & \\
\hline 129 & 0 & -2.67 & & & & & & 185 \\
\hline
\end{tabular}

$\begin{array}{llll}132 & 4 & 0.40 & 228 \\ 133 & 4 & 0.07 & 223\end{array}$

$\begin{array}{llll}134 & 4 & -0.28 & 223\end{array}$

\begin{tabular}{lllll}
138 & 4 & 0.14 & & 224 \\
140 & 2 & 1.08 & 237 & \\
\hline
\end{tabular}

\begin{tabular}{rrrrrrr}
140 & 2 & 1.08 & 237 & & & \\
\hline 141 & 1 & -1.59 & & 200 & & \\
142 & 4 & -0.07 & 221 & & \\
145 & 4 & -0.42 & 216 & & \\
146 & 4 & -0.14 & 220 & & & \\
151 & 1 & -1.59 & & & 200 & \\
\hline 155 & 4 & 0.46 & & & & 228
\end{tabular}

$\begin{array}{llll}158 & 4 & 0.00 & 222\end{array}$

\begin{tabular}{|c|c|c|c|c|c|c|}
\hline $\begin{array}{l}180 \\
190 \\
191 \\
\end{array}$ & $\begin{array}{l}4 \\
0 \\
4 \\
\end{array}$ & $\begin{array}{r}0.00 \\
-2.16 \\
-0.07 \\
\end{array}$ & 192 & & 222 & 221 \\
\hline 203 & 4 & -0.14 & 220 & & & \\
\hline 204 & 2 & -1.01 & & & 208 & \\
\hline 212 & 0 & 2.74 & & & 260 & \\
\hline 213 & 0 & 5.77 & & 302 & & \\
\hline 215 & 4 & 0.14 & & & 224 & \\
\hline 217 & 3 & 0.58 & & & 230 & \\
\hline 219 & 2 & -1.01 & & & 208 & \\
\hline 220 & 4 & 0.02 & 222 & & & \\
\hline 221 & 4 & -0.14 & & 220 & & \\
\hline 224 & 1 & -1.51 & & & 201 & \\
\hline 234 & 4 & 0.14 & & & 224 & \\
\hline 235 & 3 & 0.94 & & & 235 & \\
\hline 236 & 3 & -0.53 & & & 215 & \\
\hline 240 & 0 & -2.74 & & & 184 & \\
\hline 241 & 0 & 3.03 & & 264 & & \\
\hline 249 & 0 & -2.45 & & 188 & & \\
\hline 253 & 0 & 7.79 & 330 & & & \\
\hline 255 & 4 & -0.35 & & & 217 & \\
\hline 256 & 4 & -0.07 & 221 & & & \\
\hline 257 & 0 & -3.75 & 170 & & & \\
\hline 265 & 3 & 0.58 & & & 230 & \\
\hline 273 & 3 & 0.58 & & & 230 & \\
\hline 274 & 3 & 0.73 & & 232 & & \\
\hline
\end{tabular}


Table 12. Statistical summary of reported data for standard reference water sample T-143 (trace constituents)-Continued $\mathrm{K}$ (Potassium) $\mathrm{mg} / \mathrm{L}$

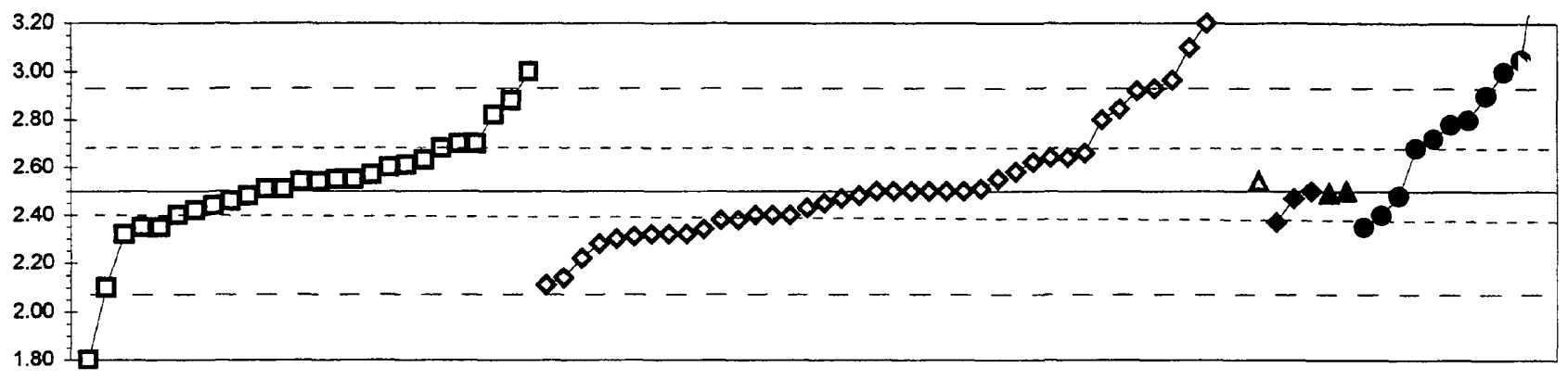

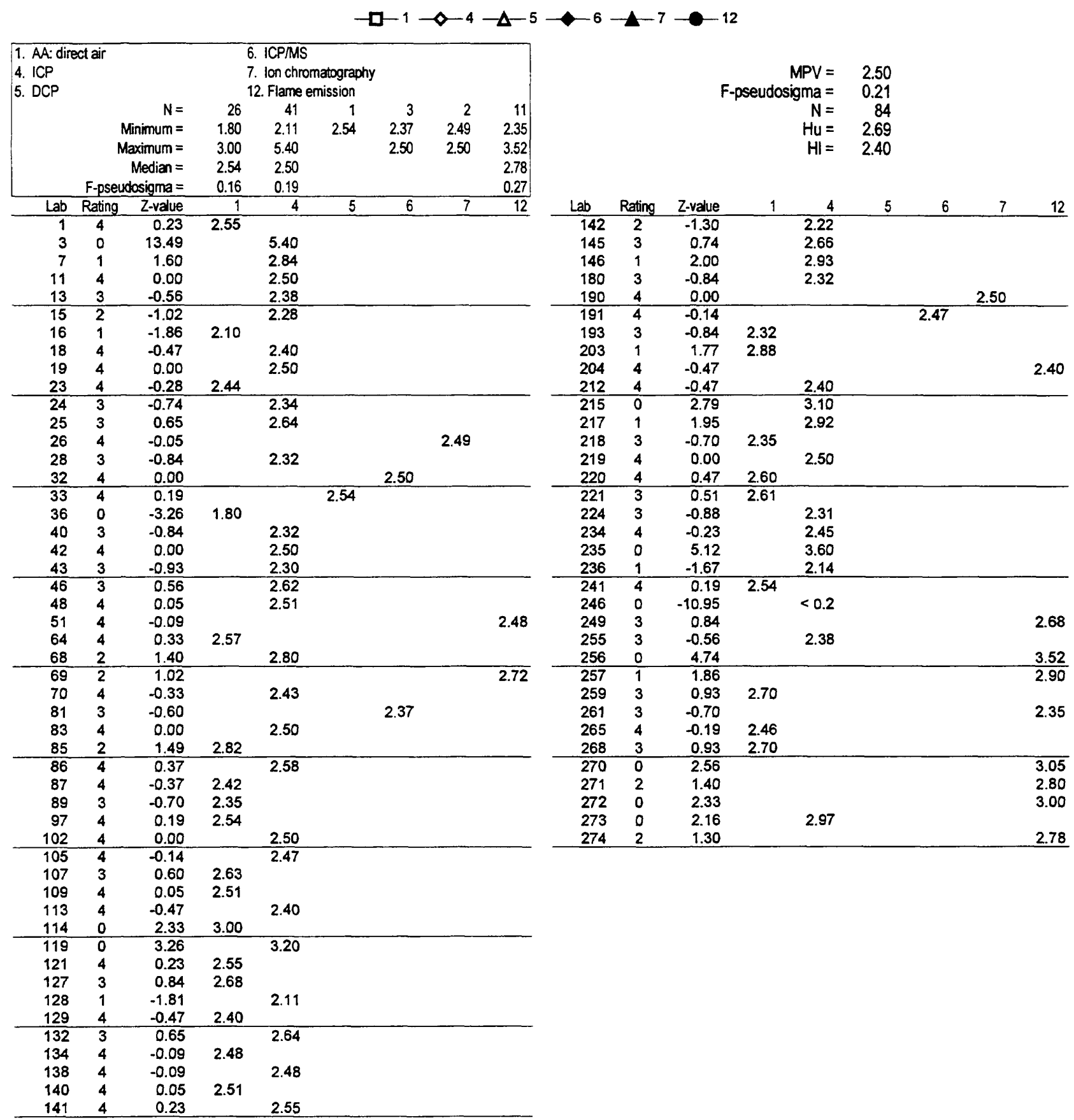


Table 12. Statistical summary of reported data for standard reference water sample T-143 (trace constituents)-Continued Li (Lithium) $\mu \mathrm{g} / \mathrm{L}$

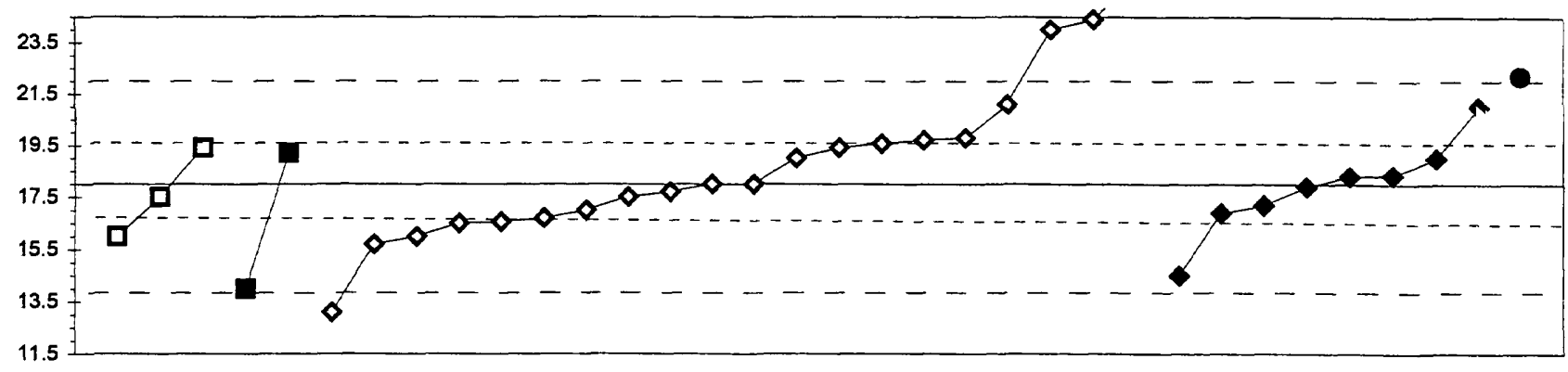

$-\square-1 \multimap-3-4-6-6-12$

\begin{tabular}{|c|c|c|c|c|c|c|c|}
\hline $\begin{array}{l}\text { 1. } A \mathrm{~A}: \mathrm{dir} \\
\text { 3. } A \mathrm{~A}: \mathrm{gr} \\
\text { 4. ICP }\end{array}$ & phite fur & & & $\begin{array}{l}\text { ICP/MS } \\
\text { Flame }\end{array}$ & mission & & \\
\hline & & $N=$ & 3 & 2 & 20 & 8 & 1 \\
\hline & & nimum = & 16.0 & 14.0 & 13.1 & 14.5 & 22.2 \\
\hline & & ximum = & 19.4 & 19.2 & 26.0 & 21.0 & \\
\hline & & Median = & & & 18.0 & 18.1 & \\
\hline & F-pseu & osigma = & & & 2.4 & 1.2 & \\
\hline Lab & Rating & Z-value & 1 & 3 & 4 & 6 & 12 \\
\hline 1 & 3 & -0.67 & & & 16.6 & & \\
\hline 3 & 0 & 3.77 & & & 26.0 & & \\
\hline 4 & NR & & & & $<100$ & & \\
\hline 7 & 3 & 0.66 & & & 19.4 & & \\
\hline 16 & 1 & -1.65 & & & & 14.5 & \\
\hline 24 & 0 & 3.02 & & & 24.4 & & \\
\hline 25 & 4 & 0.00 & & & 18.0 & & \\
\hline 26 & 4 & -0.14 & & & 17.7 & & \\
\hline 30 & 3 & -0.94 & & & 16.0 & & \\
\hline 32 & 4 & -0.38 & & & & 17.2 & \\
\hline 40 & 3 & -0.61 & & & 16.7 & & \\
\hline 42 & 0 & -5.71 & & & $<6$ & & \\
\hline 64 & 1 & 1.98 & & & & & 22.2 \\
\hline 68 & 4 & 0.00 & & & 18.0 & & \\
\hline 69 & 3 & 0.57 & & 19.2 & & & \\
\hline 75 & 3 & 0.80 & & & 19.7 & & \\
\hline 76 & 4 & 0.14 & & & & 18.3 & \\
\hline 85 & 4 & -0.24 & 17.5 & & & & \\
\hline 105 & 2 & -1.08 & & & 15.7 & & \\
\hline 109 & 3 & 0.66 & 19.4 & & & & \\
\hline 127 & 3 & -0.71 & & & 16.5 & & \\
\hline 134 & 3 & 0.74 & & & 19.6 & & \\
\hline 142 & 4 & 0.47 & & & 19.0 & & \\
\hline 145 & 2 & 1.46 & & & 21.1 & & \\
\hline 151 & 3 & -0.52 & & & & 16.9 & \\
\hline 191 & 2 & 1.41 & & & & 21.0 & \\
\hline 196 & 4 & -0.05 & & & & 17.9 & \\
\hline 212 & 4 & 0.47 & & & & 19.0 & \\
\hline 217 & 4 & -0.24 & & & 17.5 & & \\
\hline 219 & 4 & -0.47 & & & 17.0 & & \\
\hline 234 & 3 & 0.85 & & & 19.8 & & \\
\hline 236 & 0 & -2.31 & & & 13.1 & & \\
\hline 256 & 0 & -8.52 & & & & & $<0.1$ \\
\hline 257 & 3 & -0.94 & 16.0 & & & & \\
\hline 259 & 1 & -1.89 & & 14.0 & & & \\
\hline 265 & 4 & 0.16 & & & & 18.4 & \\
\hline 273 & 0 & 2.83 & & & 24.0 & & \\
\hline
\end{tabular}


Table 12. Statistical summary of reported data for standard reference water sample $T$-143 (trace constituents)-Continued Mg (Magnesium) $\mathrm{mg} / \mathrm{L}$

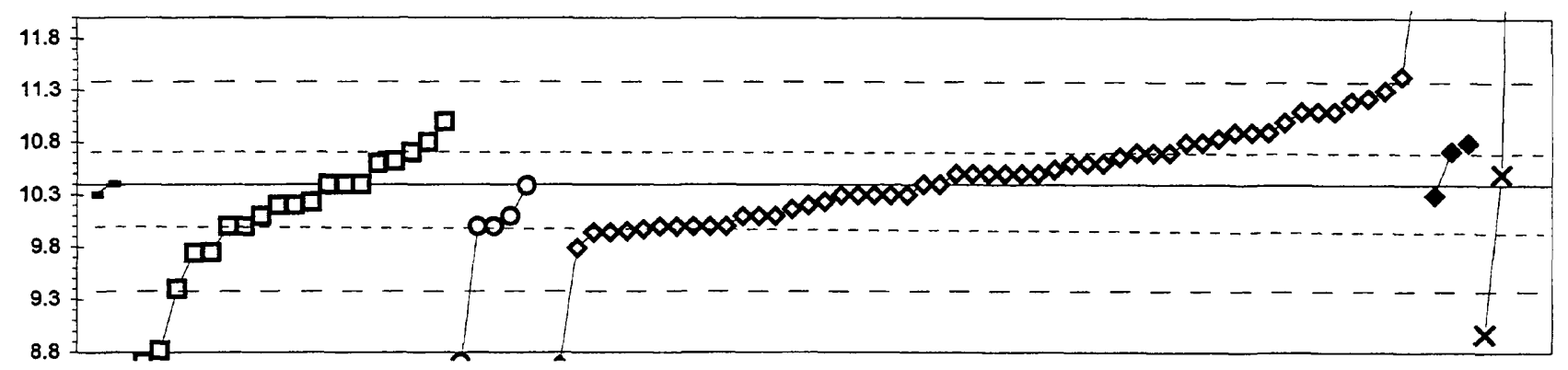

$=0-\square-1-0-2 \diamond-4-6-x-20$

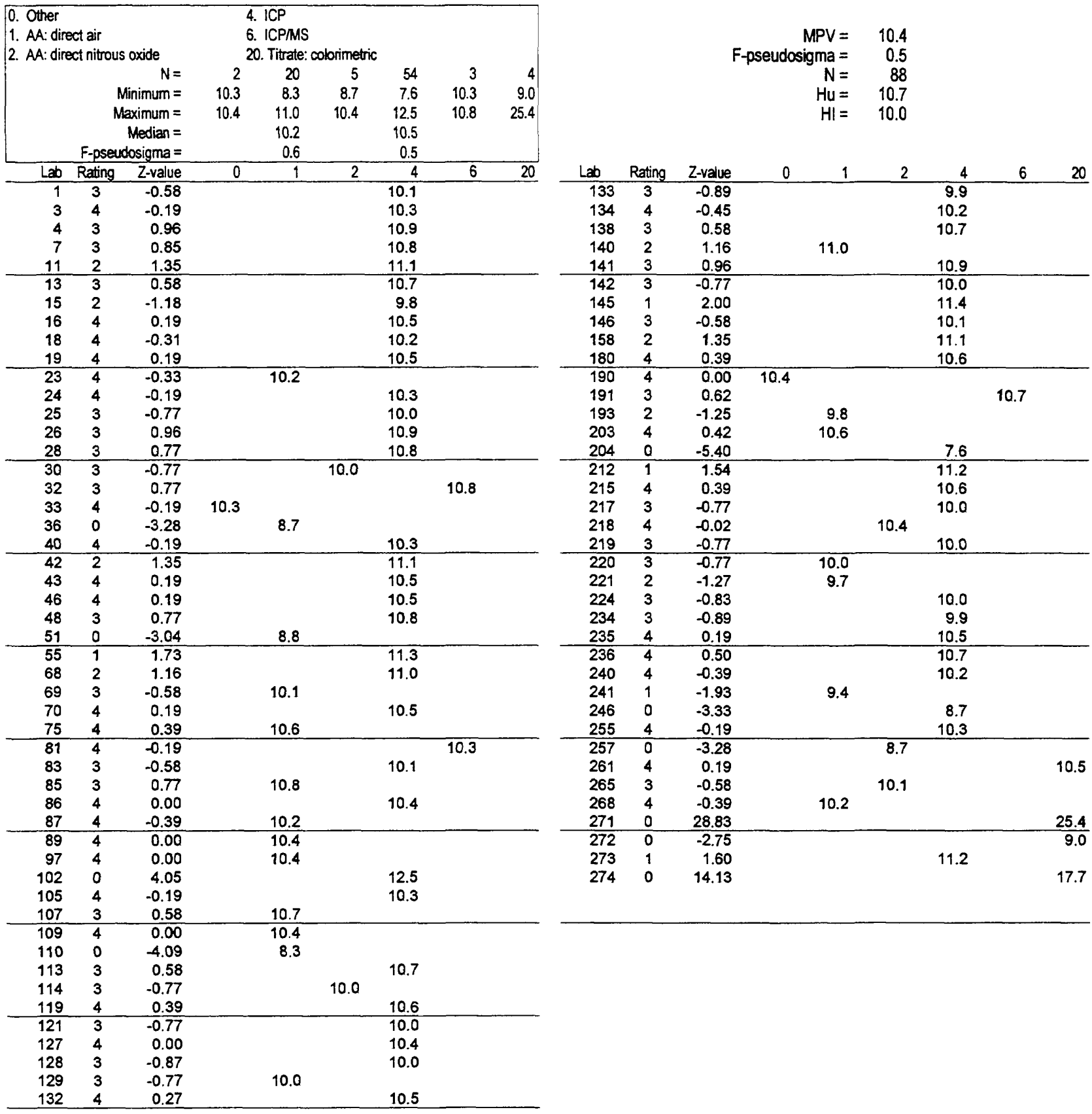


Table 12. Statistical summary of reported data for standard reference water sample T-143 (trace constituents)-Continued Mn (Manganese) $\mu \mathrm{g} / \mathrm{L}$

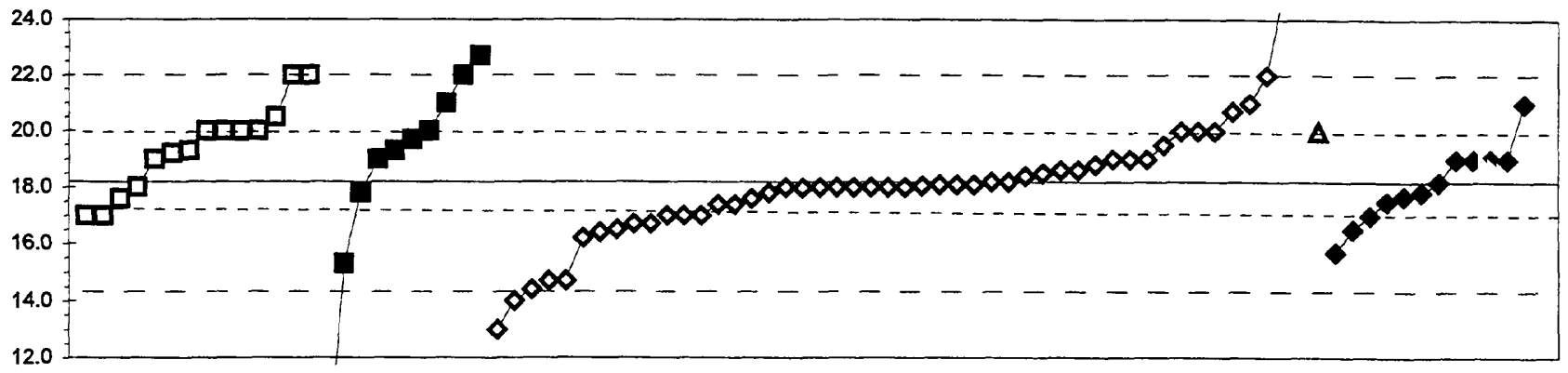

$-\square-1-3-4-\Delta-5 \multimap 6-X-22$

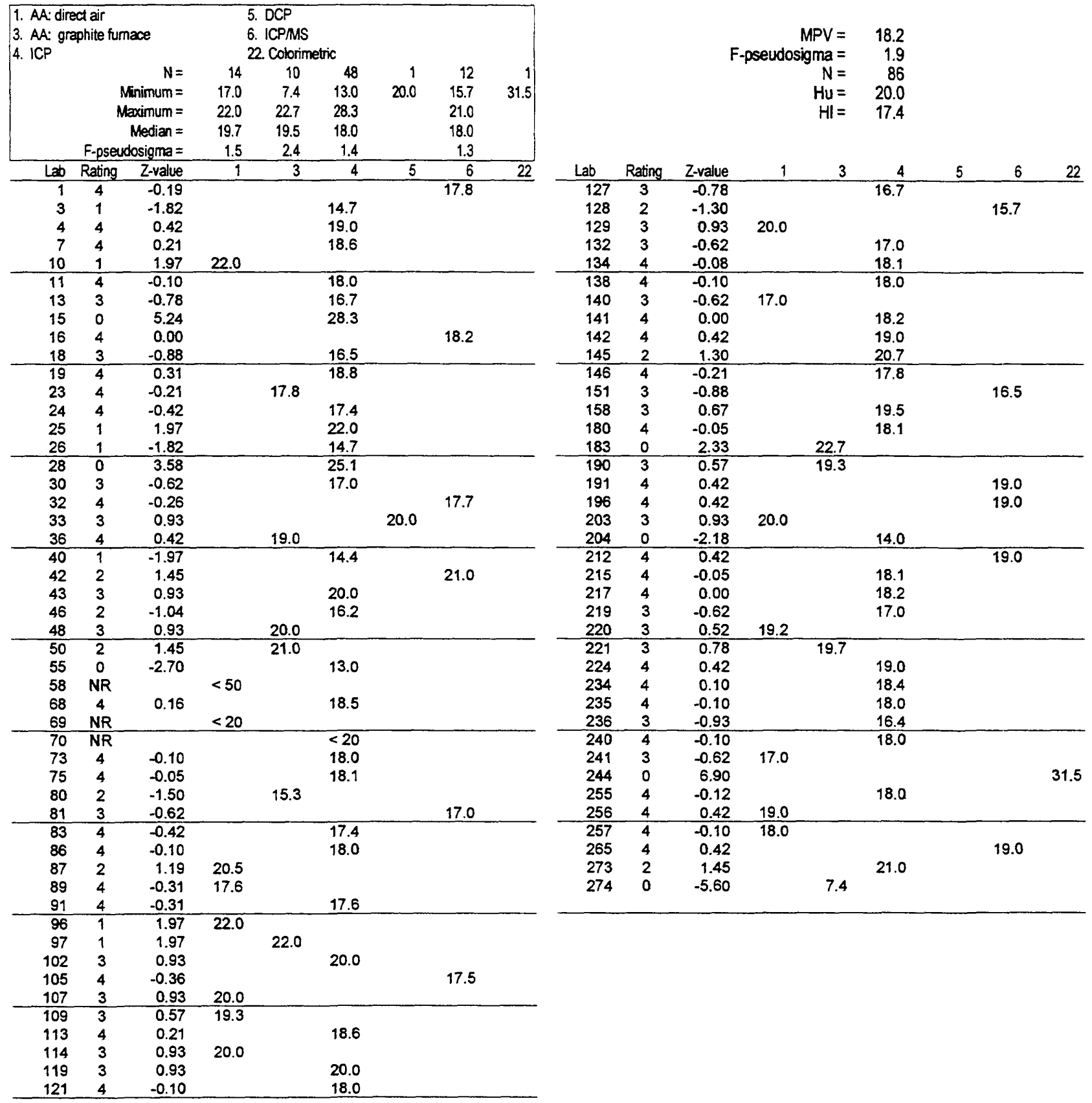


Table 12. Statistical summary of reported data for standard reference water sample T-143 (trace constituents)--Continued Mo (Molybdenum)

$\mu \mathrm{g} / \mathrm{L}$

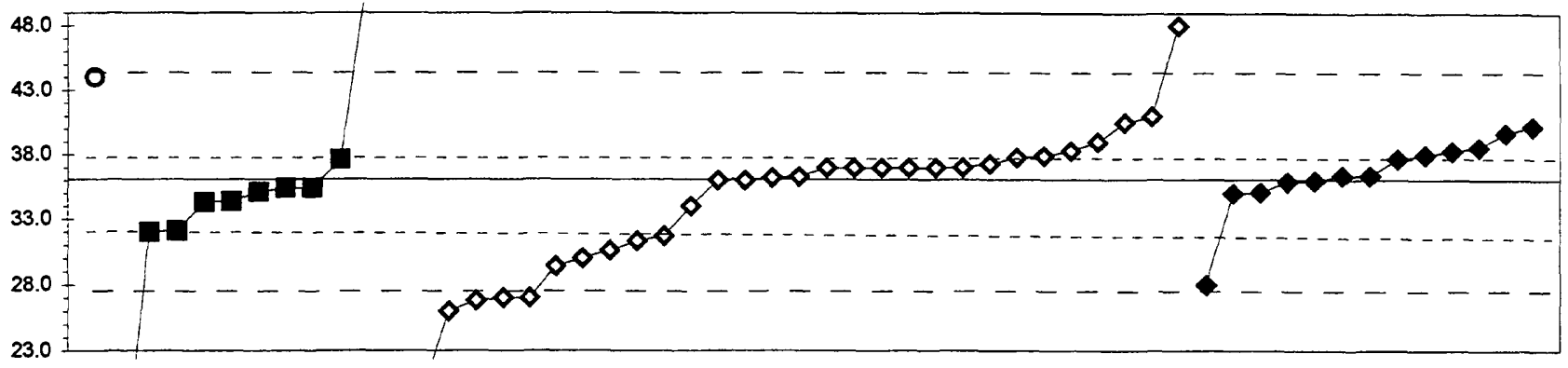

$-\square-2-3-\diamond-4 \multimap 6$

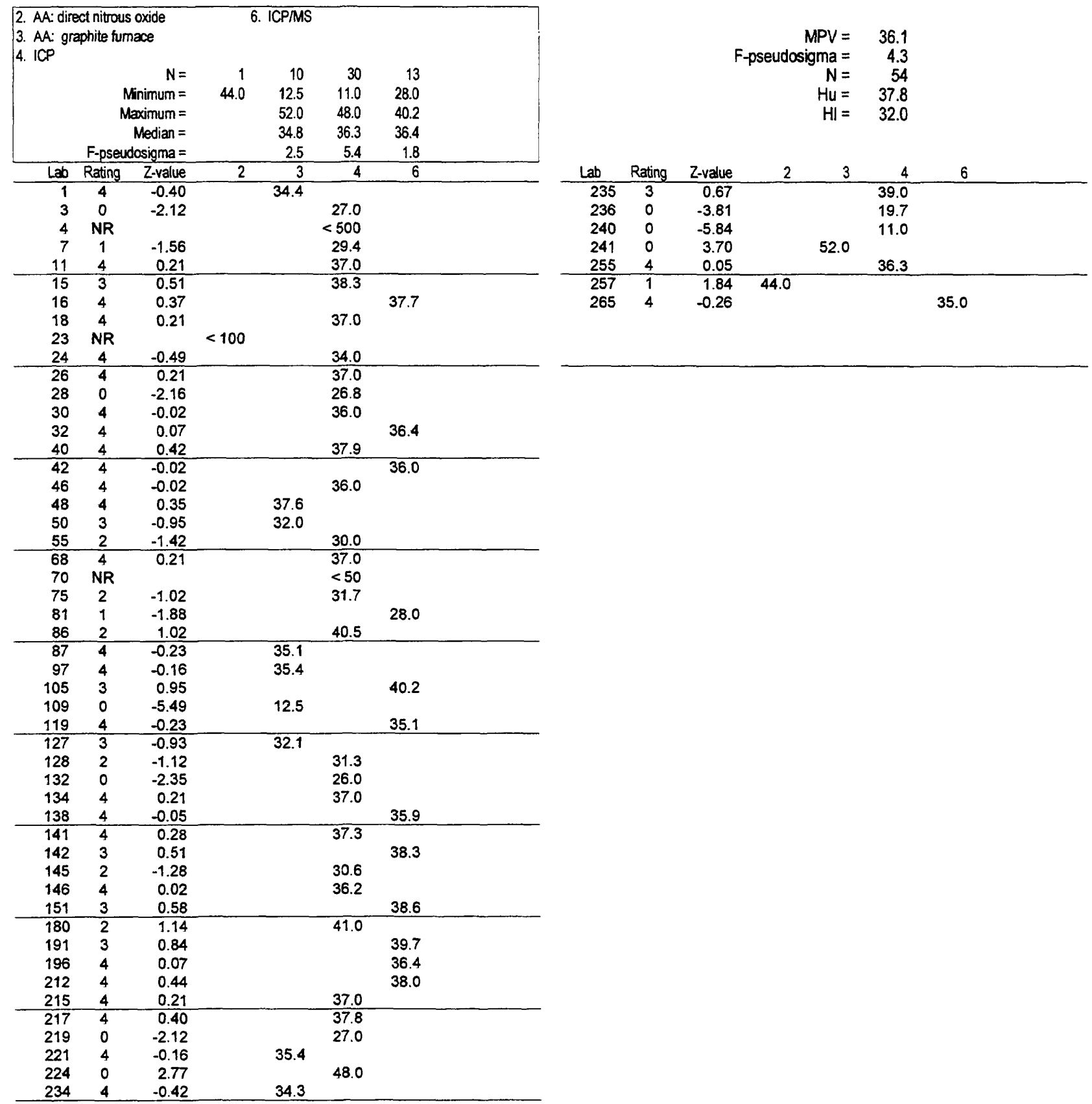


Table 12. Statistical summary of reported data for standard reference water sample T-143 (trace constituents)-Continued $\mathrm{Na}$ (Sodium) $\mathrm{mgl}$

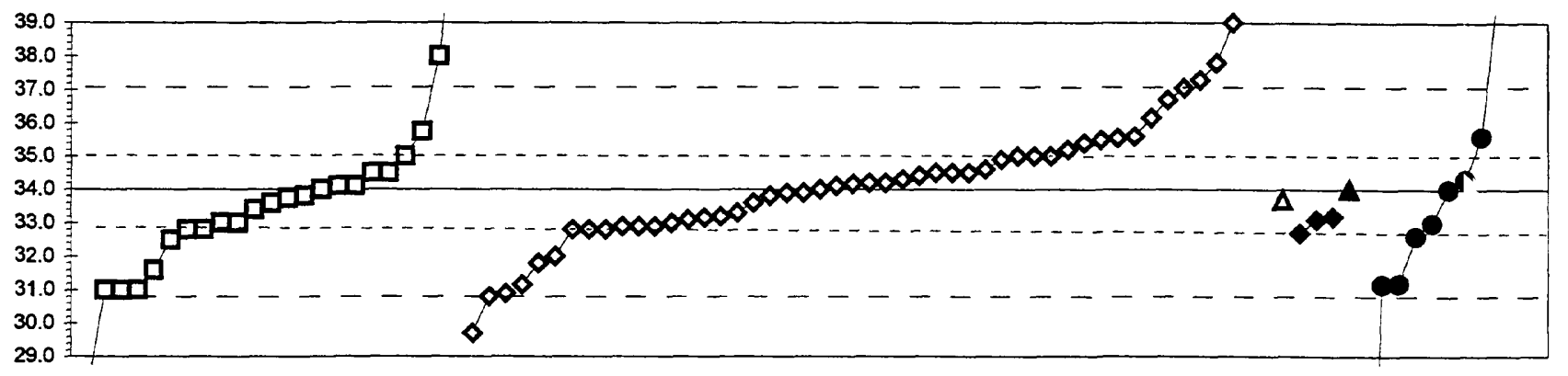

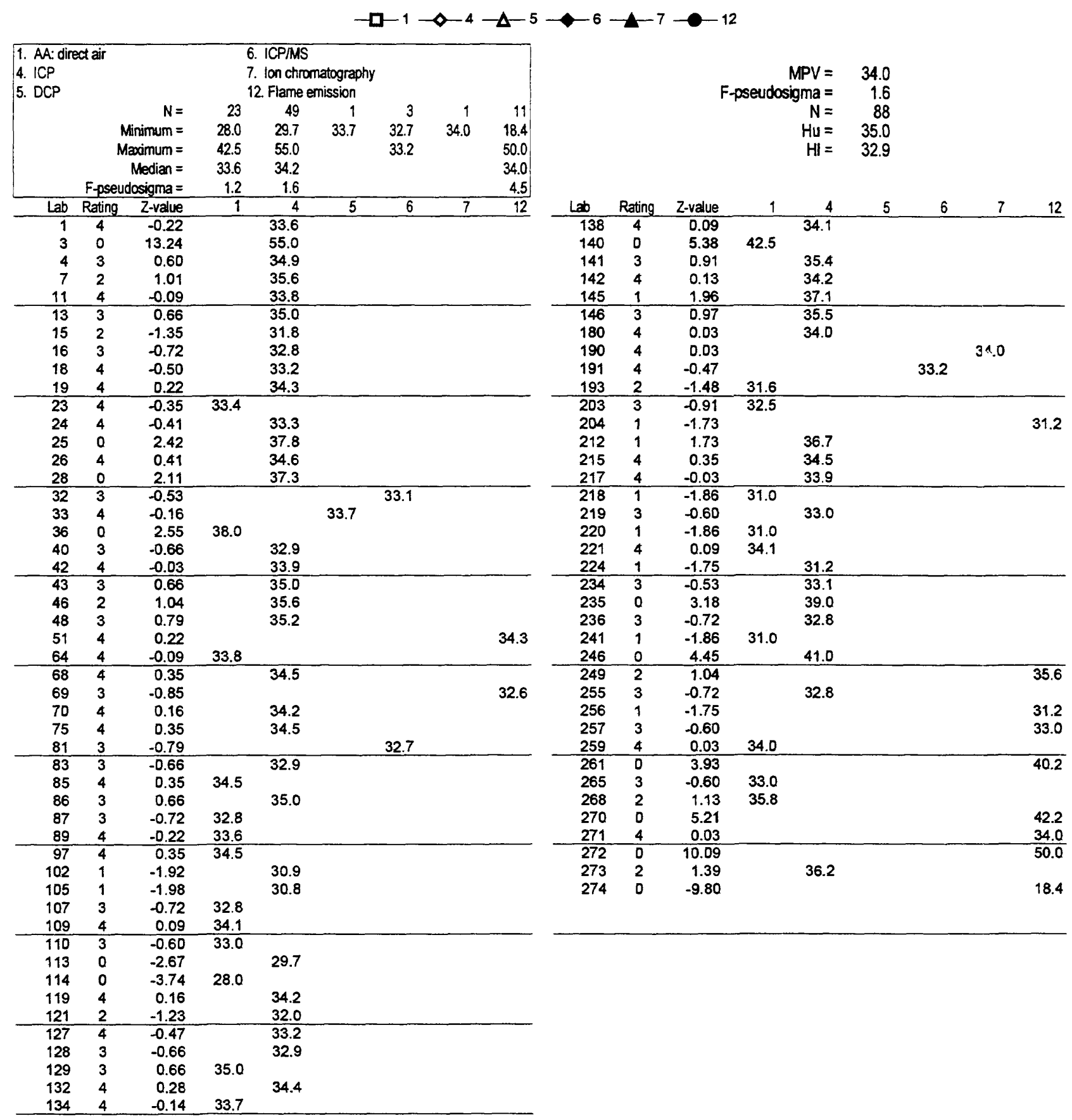


Table 12. Statistical summary of reported data for standard reference water sample T-143 (trace constituents)-Continued $\mathrm{Ni}$ (Nickel)

$\mu \mathrm{g} / \mathrm{L}$

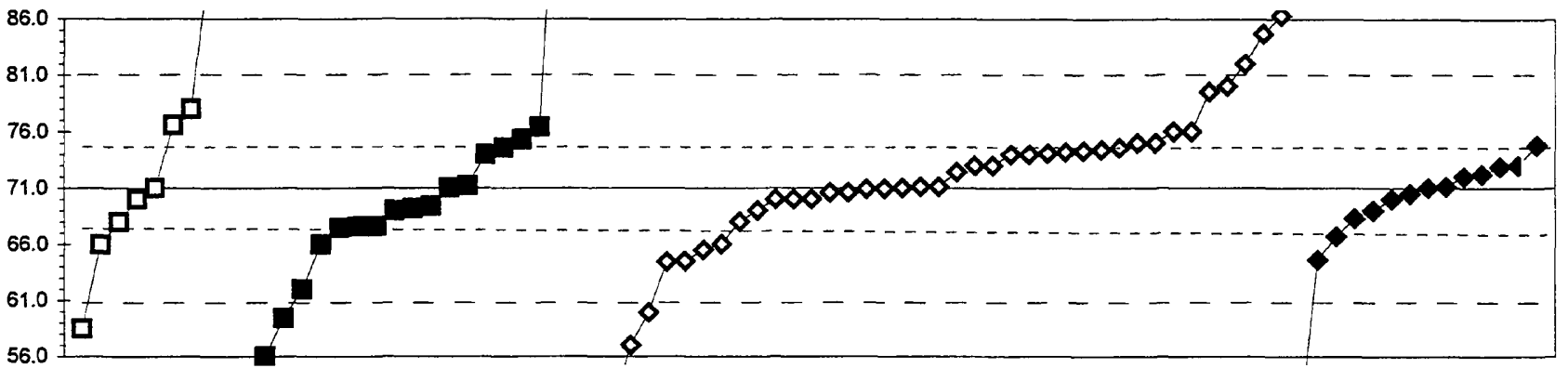

$-\square-1-3 \multimap-4 \multimap-6$

\begin{tabular}{|c|c|c|c|c|c|c|c|c|c|c|c|c|c|}
\hline $\begin{array}{l}\text { 1. } \mathrm{AA}: \text { dire } \\
\text { 3. } \mathrm{AA}: \text { gre } \\
\text { 4. ICP }\end{array}$ & aphite fur & $\begin{array}{r}N= \\
\text { inimum }= \\
\text { aximum }= \\
\text { Median }= \\
\text { osigma }=\end{array}$ & $\begin{array}{r}9 \\
58.5 \\
110.0 \\
71.0 \\
7.4 \\
\end{array}$ & $\begin{array}{r}19 \\
30.0 \\
125.0 \\
69.2 \\
5.6 \\
\end{array}$ & $\begin{array}{r}39 \\
31.8 \\
86.3 \\
71.1 \\
3.7 \\
\end{array}$ & $\begin{array}{r}14 \\
50.0 \\
74.8 \\
70.8 \\
2.9 \\
\end{array}$ & & & & seudos & $\begin{aligned} \text { MPV } & = \\
\text { gma } & = \\
N & = \\
H u & = \\
H l & =\end{aligned}$ & $\begin{array}{r}71.0 \\
5.0 \\
81 \\
74.4 \\
67.6\end{array}$ & \\
\hline Lab & Rating & Z-value & 1 & 3 & 4 & 6 & Lab & Rating & Z-value & 1 & 3 & 4 & 6 \\
\hline 1 & 4 & 0.02 & & & & 71.1 & 138 & 4 & -0.20 & & & 70.0 & \\
\hline 3 & 0 & -2.78 & & & 57.0 & & 140 & 4 & 0.00 & 71.0 & & & \\
\hline 4 & NR & & & & $<200$ & & 141 & 4 & -0.40 & & & 69.0 & \\
\hline 7 & 1 & 1.69 & & & 79.5 & & 142 & 3 & -0.54 & & & & 68.3 \\
\hline 11 & 4 & 0.40 & & & 73.0 & & 145 & 0 & 3.04 & & & 86.3 & \\
\hline 13 & 2 & -1.31 & & & 64.4 & & 146 & 4 & 0.28 & & & 72.4 & \\
\hline 15 & 3 & 0.85 & & 75.3 & & & 151 & 4 & -0.40 & & & & 69.0 \\
\hline 16 & 4 & 0.38 & & & & 72.9 & 158 & 3 & 0.99 & & & 76.0 & \\
\hline 18 & 4 & 0.02 & & & 71.1 & & 180 & 3 & 0.99 & & & 76.0 & \\
\hline 19 & 0 & 2.72 & & & 84.7 & & 190 & 0 & -2.30 & & 59.4 & & \\
\hline 23 & 3 & 0.60 & & 74.0 & & & 191 & 4 & 0.24 & & & & 72.2 \\
\hline 24 & 3 & -0.60 & & & 68.0 & & 193 & 3 & -0.99 & 66.0 & & & \\
\hline 26 & 3 & 0.71 & & & 74.6 & & 196 & 4 & -0.10 & & & & 70.5 \\
\hline 28 & 0 & -7.78 & & & 31.8 & & 212 & 4 & 0.20 & & & & 72.0 \\
\hline 30 & 4 & -0.20 & & & 70.0 & & 213 & 0 & 4.01 & 91.2 & & & \\
\hline 32 & 4 & 0.00 & & & & 71.0 & 215 & 4 & 0.00 & & & 71.0 & \\
\hline 36 & 1 & -1.79 & & 62.0 & & & 217 & 3 & 0.61 & & & 74.1 & \\
\hline 39 & 3 & -0.99 & & 66.0 & & & 219 & 3 & -0.99 & & & 66.0 & \\
\hline 40 & 0 & -3.91 & & & 51.3 & & 221 & 4 & 0.04 & & 71.2 & & \\
\hline 42 & 4 & -0.20 & & & & 70.0 & 224 & 0 & -2.20 & & & 59.9 & \\
\hline 46 & 3 & 0.63 & & & 74.2 & & 234 & 3 & 0.71 & & 74.6 & & \\
\hline 48 & 2 & 1.07 & & 76.4 & & & 235 & 3 & 0.60 & & & 74.0 & \\
\hline 50 & 0 & -2.98 & & 56.0 & & & 236 & 2 & -1.09 & & & 65.5 & \\
\hline 58 & 0 & 7.74 & 110.0 & & & & 240 & 3 & 0.79 & & & 75.0 & \\
\hline 68 & 3 & 0.60 & & & 74.0 & & 241 & 3 & -0.67 & & 67.6 & & \\
\hline 69 & 0 & -2.48 & 58.5 & & & & 249 & 4 & -0.36 & & 69.2 & & \\
\hline 70 & 4 & -0.02 & & & 70.9 & & 255 & 4 & -0.01 & & & 70.9 & \\
\hline 73 & 4 & 0.40 & & & 73.0 & & 256 & 2 & 1.11 & 76.6 & & & \\
\hline 75 & 4 & 0.02 & & & 71.1 & & 257 & 3 & -0.60 & 68.0 & & & \\
\hline 76 & 3 & 0.75 & & & & 74.6 & 259 & 0 & 10.71 & & 125.0 & & \\
\hline 81 & 0 & -4.17 & & & & 50.0 & 265 & 4 & 0.40 & & & & 73.0 \\
\hline 83 & 4 & -0.08 & & & 70.6 & & 273 & 0 & 2.18 & & & 82.0 & \\
\hline 85 & 3 & 0.67 & & & 74.4 & & & & & & & & \\
\hline 86 & 2 & -1.29 & & & 64.5 & & & & & & & & \\
\hline 87 & 2 & 1.39 & 78.0 & & & & & & & & & & \\
\hline 89 & 3 & -0.69 & & 67.5 & & & & & & & & & \\
\hline 96 & 3 & -0.67 & & 67.6 & & & & & & & & & \\
\hline 97 & 4 & -0.40 & & 69.0 & & & & & & & & & \\
\hline 102 & 1 & 1.79 & & & 80.0 & & & & & & & & \\
\hline 105 & 3 & -0.85 & & & & 66.7 & & & & & & & \\
\hline 113 & 4 & -0.08 & & & 70.6 & & & & & & & & \\
\hline 114 & 4 & -0.20 & 70.0 & & & & & & & & & & \\
\hline 118 & 0 & -8.13 & & 30.0 & & & & & & & & & \\
\hline 119 & 0 & 6.55 & & 104.0 & & & & & & & & & \\
\hline 121 & 4 & -0.20 & & & 70.0 & & & & & & & & \\
\hline 127 & 4 & 0.00 & & 71.0 & & & & & & & & & \\
\hline 128 & 2 & -1.27 & & & & 64.6 & & & & & & & \\
\hline 132 & 3 & 0.79 & & & 75.0 & & & & & & & & \\
\hline 133 & 3 & 0.65 & & & 74.3 & & & & & & & & \\
\hline 134 & 4 & -0.32 & & 69.4 & & & & & & & & & \\
\hline
\end{tabular}


Table 12. Statistical summary of reported data for standard reference water sample T-143 (trace constituents)-Continued $\mathrm{Pb}$ (Lead) $\mu \mathrm{g} / \mathrm{L}$

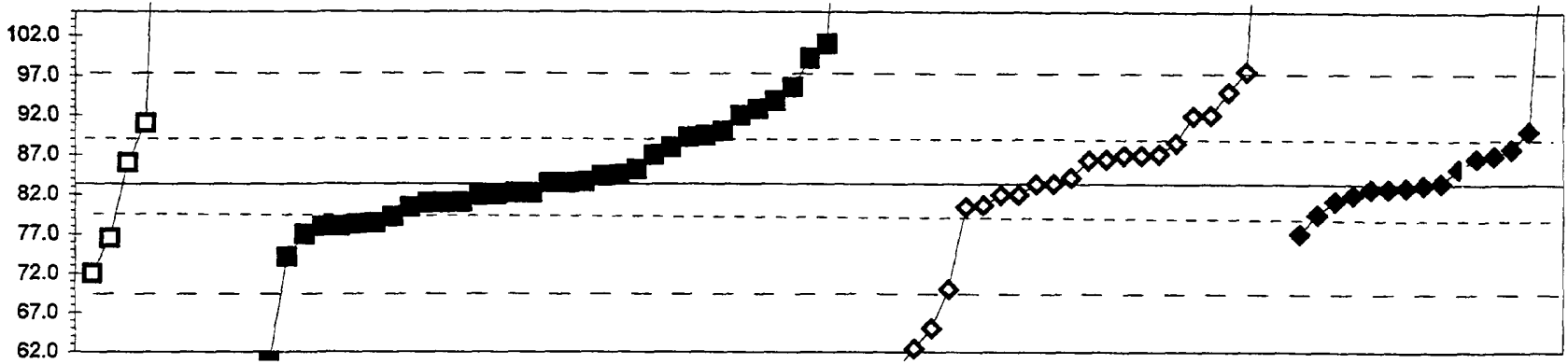

$-\square-1-0-3-4-6$

\begin{tabular}{|c|c|c|c|c|c|c|}
\hline \multicolumn{3}{|c|}{$\begin{array}{l}\text { 1. AA: direct air } \\
\text { 3. AA: graphite fumace } \\
\text { 4. ICP }\end{array}$} & \multicolumn{2}{|c|}{ 6. ICPMMS } & \multirow[b]{2}{*}{24} & \multirow[b]{2}{*}{15} \\
\hline & & $N=$ & & 40 & & \\
\hline & & linimum = & 72.0 & 1.2 & 17.9 & 77.1 \\
\hline & & aximum = & 155.0 & 183.0 & 498.9 & 120.0 \\
\hline & & Median = & & 82.2 & 85.3 & 83.3 \\
\hline & F-pset & losigma $=$ & & 8.3 & 7.2 & 3.3 \\
\hline Lab & Rating & Z-value & 1 & 3 & 4 & 6 \\
\hline 1 & 4 & 0.02 & & & & 83.6 \\
\hline 3 & 3 & 0.73 & & & 88.6 & \\
\hline 4 & NR & & & & $<400$ & \\
\hline 7 & 4 & 0.43 & & & 86.5 & \\
\hline 11 & 4 & 0.42 & & & 86.4 & \\
\hline 13 & 3 & 0.85 & & 89.5 & & \\
\hline 15 & 1 & 1.71 & & 95.6 & & \\
\hline 16 & 4 & 0.46 & & & & 86.7 \\
\hline 18 & $4^{\circ}$ & 0.01 & & 83.5 & & \\
\hline 19 & 2 & 1.47 & & 93.9 & & \\
\hline 23 & 3 & -0.72 & & 78.3 & & \\
\hline 24 & 0 & -3.33 & & & 59.8 & \\
\hline 26 & 4 & -0.39 & & & 80.7 & \\
\hline 28 & 0 & -9.23 & & & 17.9 & \\
\hline 30 & 4 & -0.20 & & & 82.0 & \\
\hline 32 & $\overline{4}$ & -0.30 & & & & 81.3 \\
\hline 34 & 2 & -1.32 & & 74.1 & & \\
\hline 36 & 3 & -0.77 & & 78.0 & & \\
\hline 39 & 4 & -0.34 & & 81.0 & & \\
\hline 42 & 4 & -0.06 & & & & 83.0 \\
\hline 46 & 3 & -0.91 & & 77.0 & & \\
\hline 48 & 4 & 0.14 & & 84.4 & & \\
\hline 58 & 2 & 1.21 & & 92.0 & & \\
\hline 68 & 0 & 14.03 & & 183.0 & & \\
\hline 69 & 4 & -0.43 & & 80.4 & & \\
\hline 70 & 4 & -0.17 & & 82.2 & & \\
\hline 73 & 4 & 0.50 & & & 87.0 & \\
\hline 75 & 1 & 1.99 & & & 97.6 & \\
\hline 76 & 4 & -0.02 & & & & 83.3 \\
\hline 80 & 4 & 0.01 & & 83.5 & & \\
\hline 81 & 4 & 0.50 & & 87.0 & & \\
\hline 83 & 4 & 0.02 & & 83.6 & & \\
\hline 85 & 3 & -0.98 & 76.5 & & & \\
\hline 86 & 4 & -0.34 & & 81.0 & & \\
\hline 87 & 3 & -0.77 & & 78.0 & & \\
\hline 89 & 4 & 0.25 & & 85.2 & & \\
\hline 96 & 2 & 1.32 & & 92.8 & & \\
\hline 97 & 4 & -0.22 & & 81.9 & & \\
\hline 102 & 1 & 1.63 & & & 95.0 & \\
\hline 105 & 3 & 0.63 & & & & 87.9 \\
\hline 109 & 0 & -7.25 & & 32.0 & & \\
\hline 113 & 4 & -0.33 & & 81.1 & & \\
\hline 114 & 1 & -1.61 & 72.0 & & & \\
\hline 118 & 0 & -7.82 & & 27.9 & & \\
\hline 119 & 4 & -0.20 & & 82.0 & & \\
\hline 127 & 3 & 0.53 & & & 87.2 & \\
\hline 128 & 4 & -0.09 & & & & 82.8 \\
\hline 132 & 4 & -0.41 & & & 80.5 & \\
\hline 133 & 2 & 1.21 & & & 92.0 & \\
\hline 134 & 4 & -0.01 & & & 83.4 & \\
\hline
\end{tabular}
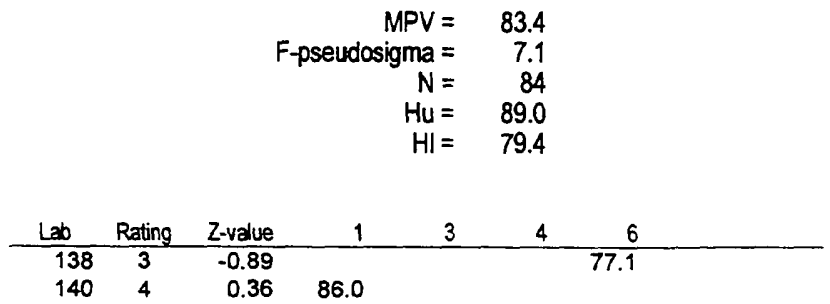

\begin{tabular}{rrrrrrr}
140 & 4 & 0.36 & 86.0 & & & \\
141 & 0 & 2.47 & & 101.0 & & 87.0 \\
142 & 4 & 0.50 & & & & \\
145 & 0 & 58.53 & & & 498.9 & \\
\hline
\end{tabular}

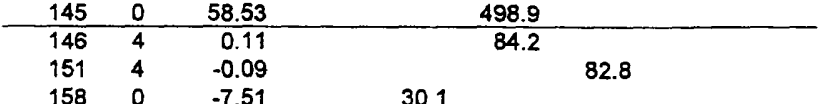

190

\begin{tabular}{rrrrr}
180 & 2 & 1.22 & & 92.1 \\
190 & 0 & -3.19 & 60.8 & \\
\hline 191 & 4 & -0.20 & & 82.0 \\
193 & 3 & 0.64 & 88.0 &
\end{tabular}

$\begin{array}{rrrrr}193 & 3 & 0.64 & 88.0 & 85.4 \\ 196 & 4 & 0.28 & & 85.2 \\ 204 & 4 & -0.17 & 82.2 & \end{array}$

\begin{tabular}{|c|c|c|c|c|c|c|}
\hline 212 & 0 & 5.15 & & (2. & & 120.0 \\
\hline 213 & 3 & 0.92 & & 90.0 & & \\
\hline 215 & 4 & -0.20 & & & 82.0 & \\
\hline 217 & 3 & 0.94 & & & & 90.1 \\
\hline 219 & 1 & -1.89 & & & 70.0 & \\
\hline 220 & 3 & -0.71 & & 78.4 & & \\
\hline 221 & 4 & 0.15 & & 84.5 & & \\
\hline 224 & 0 & 8.00 & & 140.3 & & \\
\hline 234 & 3 & 0.83 & & 89.3 & & \\
\hline 235 & 4 & 0.50 & & & 87.0 & \\
\hline 236 & 0 & -2.96 & & & 62.4 & \\
\hline 240 & 0 & 6.70 & & & 131.0 & \\
\hline 241 & 0 & 2.22 & & 99.2 & & \\
\hline 249 & 3 & -0.60 & & 79.2 & & \\
\hline 255 & 4 & -0.01 & & & 83.4 & \\
\hline 256 & 0 & 10.08 & 155.0 & & & \\
\hline 257 & 2 & 1.07 & 91.0 & & & \\
\hline 259 & 0 & -4.15 & & 54.0 & & \\
\hline 265 & 3 & -0.55 & & & & 79.6 \\
\hline 273 & 0 & -2.60 & & & 65.0 & \\
\hline 274 & 0 & -11.59 & & 1.2 & & \\
\hline
\end{tabular}


Table 12. Statistical summary of reported data for standard reference water sample T-143 (trace constituents)-Continued $\mathrm{Sb}$ (Antimony)

$\mu g / L$

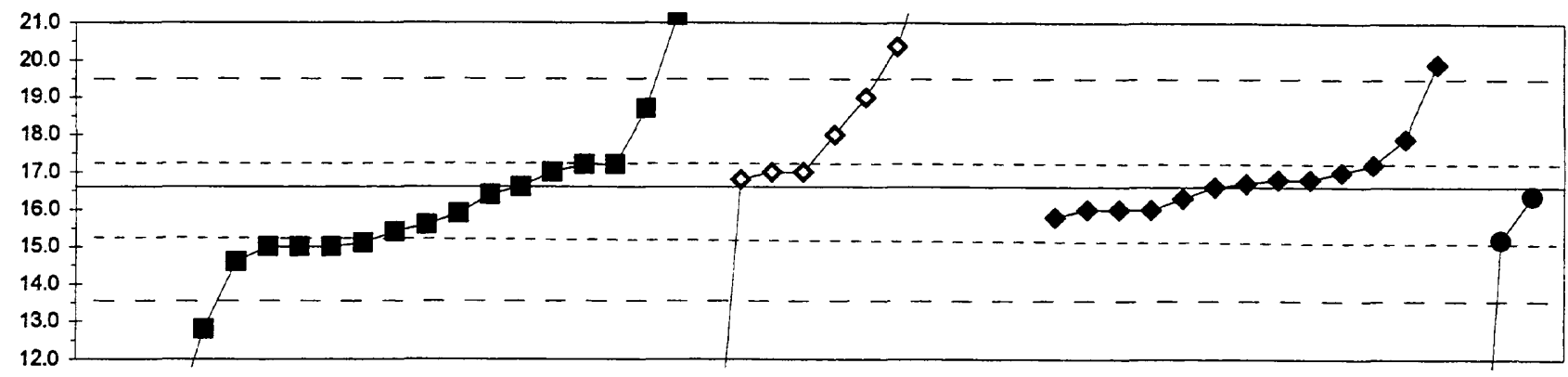

$-3-\diamond-4 \multimap 6-11 \mathrm{na}$

\begin{tabular}{|c|c|c|c|c|c|c|}
\hline $\begin{array}{l}\text { 3. AA: gra } \\
\text { 4. ICP } \\
\text { 6. ICPMS }\end{array}$ & aphite fur & $a c e$ & & กa. $A \mathrm{~A}:$ & dride $\mathrm{Na}$ & \\
\hline & & $N=$ & 19 & 11 & 13 & 3 \\
\hline & & inimum $=$ & 8.0 & 5.7 & 15.8 & 2.0 \\
\hline & & ximum = & 21.2 & 49.7 & 19.9 & 16.4 \\
\hline & & Median = & 15.4 & 19.0 & 16.7 & \\
\hline & F-pseu & osigma $=$ & 1.5 & 7.5 & 0.7 & \\
\hline Lab & Rating & Z-value & 3 & 4 & 6 & $11 \mathrm{na}$ \\
\hline 1 & 4 & -0.47 & 15.9 & & & \\
\hline 3 & 1 & 1.62 & & 19.0 & & \\
\hline 7 & 0 & 9.92 & & 31.3 & & \\
\hline 11 & 4 & 0.13 & & 16.8 & & \\
\hline 13 & 3 & -0.81 & 15.4 & & & \\
\hline 15 & 4 & 0.40 & 17.2 & & & \\
\hline 16 & 4 & 0.00 & & & 16.6 & \\
\hline 18 & 2 & -1.35 & 14.6 & & & \\
\hline 26 & NR & & & $<20$ & & \\
\hline 30 & 4 & 0.27 & & 17.0 & & \\
\hline 32 & 4 & -0.40 & & & 16.0 & \\
\hline 36 & 0 & -4.45 & 10.0 & & & \\
\hline 39 & 4 & 0.07 & & & 16.7 & \\
\hline 40 & 0 & -7.35 & & 5.7 & & \\
\hline 42 & 4 & -0.40 & & & 16.0 & \\
\hline 46 & 4 & 0.40 & 17.2 & & & \\
\hline 48 & 0 & -2.56 & 12.8 & & & \\
\hline 68 & 0 & -4.79 & 9.5 & & & \\
\hline 69 & 2 & -1.08 & 15.0 & & & \\
\hline 70 & 0 & 3.10 & 21.2 & & & \\
\hline 75 & 0 & 4.25 & & 22.9 & & \\
\hline 81 & 0 & -7.07 & & & $<6$ & \\
\hline 89 & 2 & 1.42 & 18.7 & & & \\
\hline 96 & 2 & -1.01 & 15.1 & & & \\
\hline 97 & 4 & 0.00 & 16.6 & & & \\
\hline 102 & 4 & 0.27 & & 17.0 & & \\
\hline 105 & 4 & 0.27 & & & 17.0 & \\
\hline 119 & 3 & -0.94 & & & & 15.2 \\
\hline 127 & 3 & -0.67 & 15.6 & & & \\
\hline 128 & 4 & -0.20 & & & 16.3 & \\
\hline 134 & 4 & -0.15 & & & & 16.4 \\
\hline 138 & 4 & 0.13 & & & 16.8 & \\
\hline 141 & 4 & -0.13 & 16.4 & & & \\
\hline 142 & 0 & 2.23 & & & 19.9 & \\
\hline 146 & NR & & & $<20$ & & \\
\hline 151 & 4 & 0.40 & & & 17.2 & \\
\hline 180 & 0 & 10.12 & & 31.6 & & \\
\hline 193 & 2 & -1.08 & 15.0 & & & \\
\hline 196 & 3 & -0.54 & & & 15.8 & \\
\hline 212 & 4 & -0.40 & & & 16.0 & \\
\hline 215 & 0 & -5.80 & 8.0 & & & \\
\hline 217 & 3 & 0.88 & & & 17.9 & \\
\hline 234 & 4 & 0.27 & 17.0 & & & \\
\hline 235 & NR & & & $<50$ & & \\
\hline 236 & 0 & 22.33 & & 49.7 & & \\
\hline 240 & 3 & 0.94 & & 18.0 & & \\
\hline 241 & 2 & -1.08 & 15.0 & & & \\
\hline 255 & 0 & 2.54 & & 20.4 & & \\
\hline 257 & 0 & -9.85 & & & & 2.0 \\
\hline 265 & 4 & 0.13 & & & 16.8 & \\
\hline
\end{tabular}


Table 12. Statistical summary of reported data for standard reference water sample T-143 (trace constituents)-Continued $\mathrm{Se}$ (Selenium)

$\mu \mathrm{g} / \mathrm{L}$

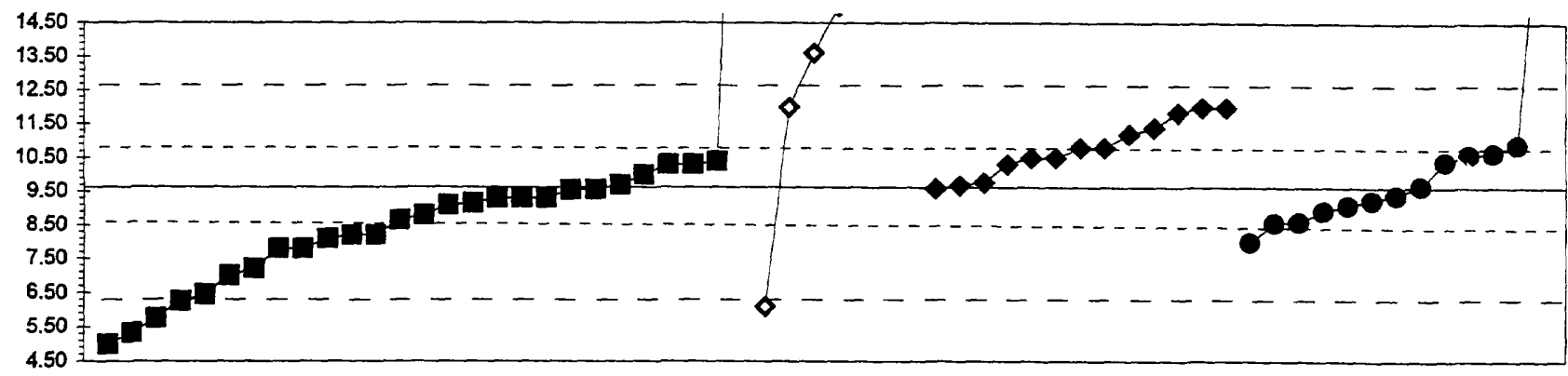

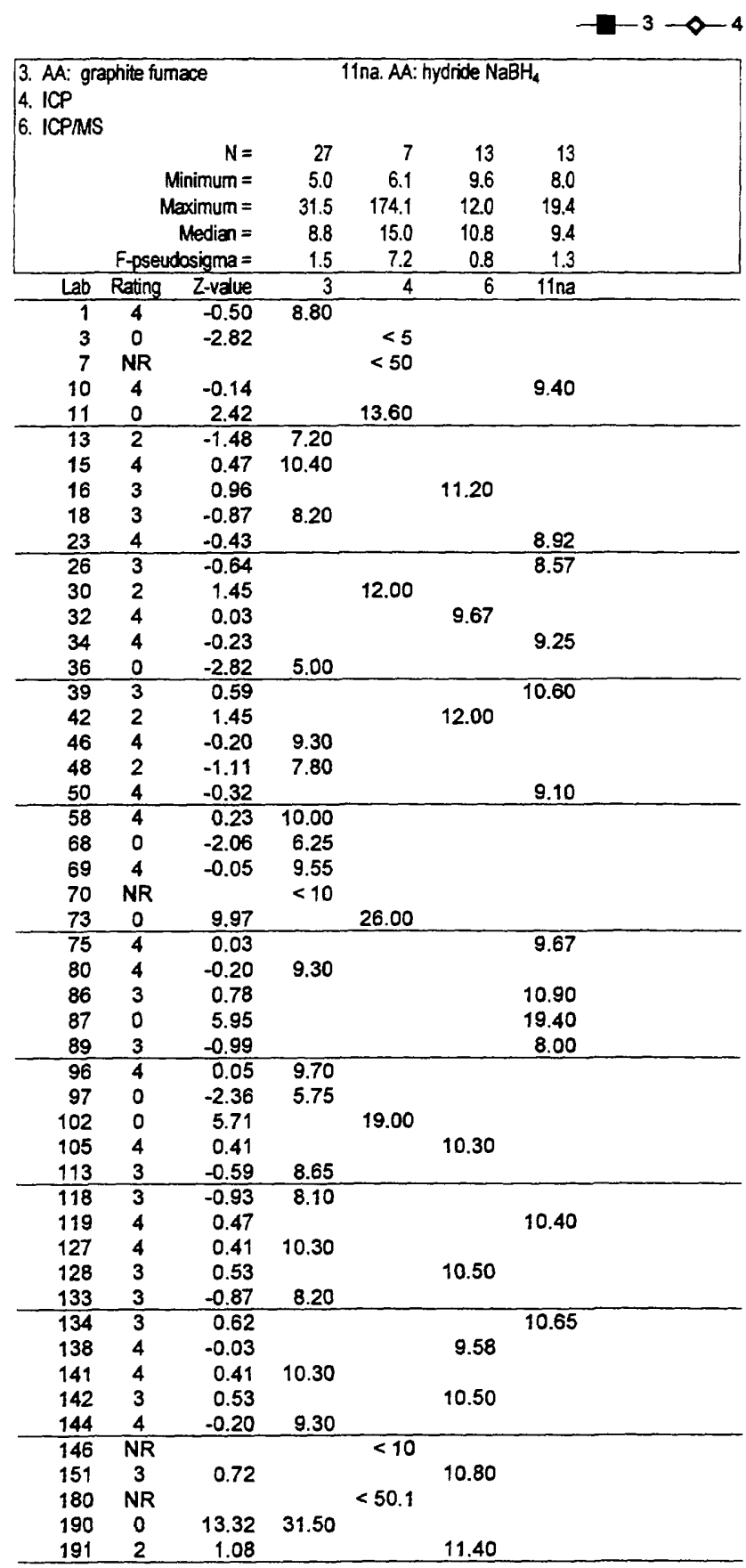

\begin{tabular}{|c|c|c|c|c|c|c|}
\hline Lab & Rating & Z-yalue & 3 & 4 & 6 & l1na \\
\hline 193 & 1 & -1.60 & 7.00 & & & \\
\hline 196 & 3 & 0.72 & & & 10.80 & \\
\hline 212 & 2 & 1.45 & & & 12.00 & \\
\hline 215 & 4 & -0.32 & 9.10 & & & \\
\hline 217 & 4 & 0.09 & & & 9.77 & \\
\hline 220 & 2 & -1.11 & 7.80 & & & \\
\hline 221 & 4 & -0.28 & 9.16 & & & \\
\hline 224 & 0 & -2.15 & & 6.10 & & \\
\hline 234 & 4 & -0.04 & 9.56 & & & \\
\hline 236 & 0 & 100.17 & & 174.10 & & \\
\hline 240 & 0 & 3.27 & & 15.00 & & \\
\hline 241 & 1 & -1.94 & 6.44 & & & \\
\hline 255 & 0 & -2.62 & 5.33 & & & \\
\hline 259 & 3 & -0.62 & & & & 8.60 \\
\hline 265 & 2 & 1.36 & & & 11.85 & \\
\hline
\end{tabular}


Table 12. Statistical summary of reported data for standard reference water sample T-143 (trace constituents)-Continued $\mathrm{SiO}_{2}$ (Silica) $\mathrm{mg} / \mathrm{L}$

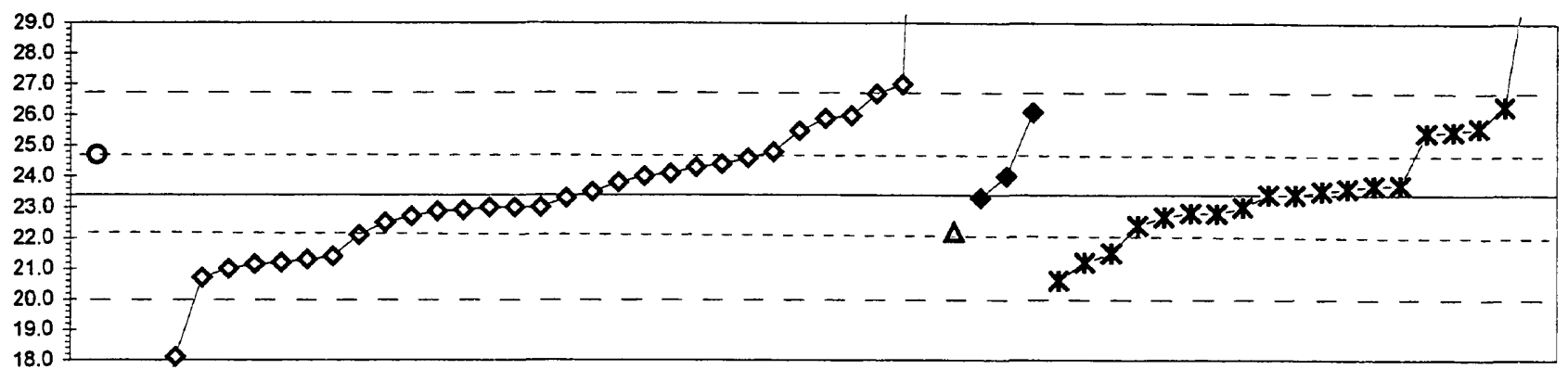

$-0-2-4-\Delta-5 \multimap-6-x-22 \mathrm{mb}$

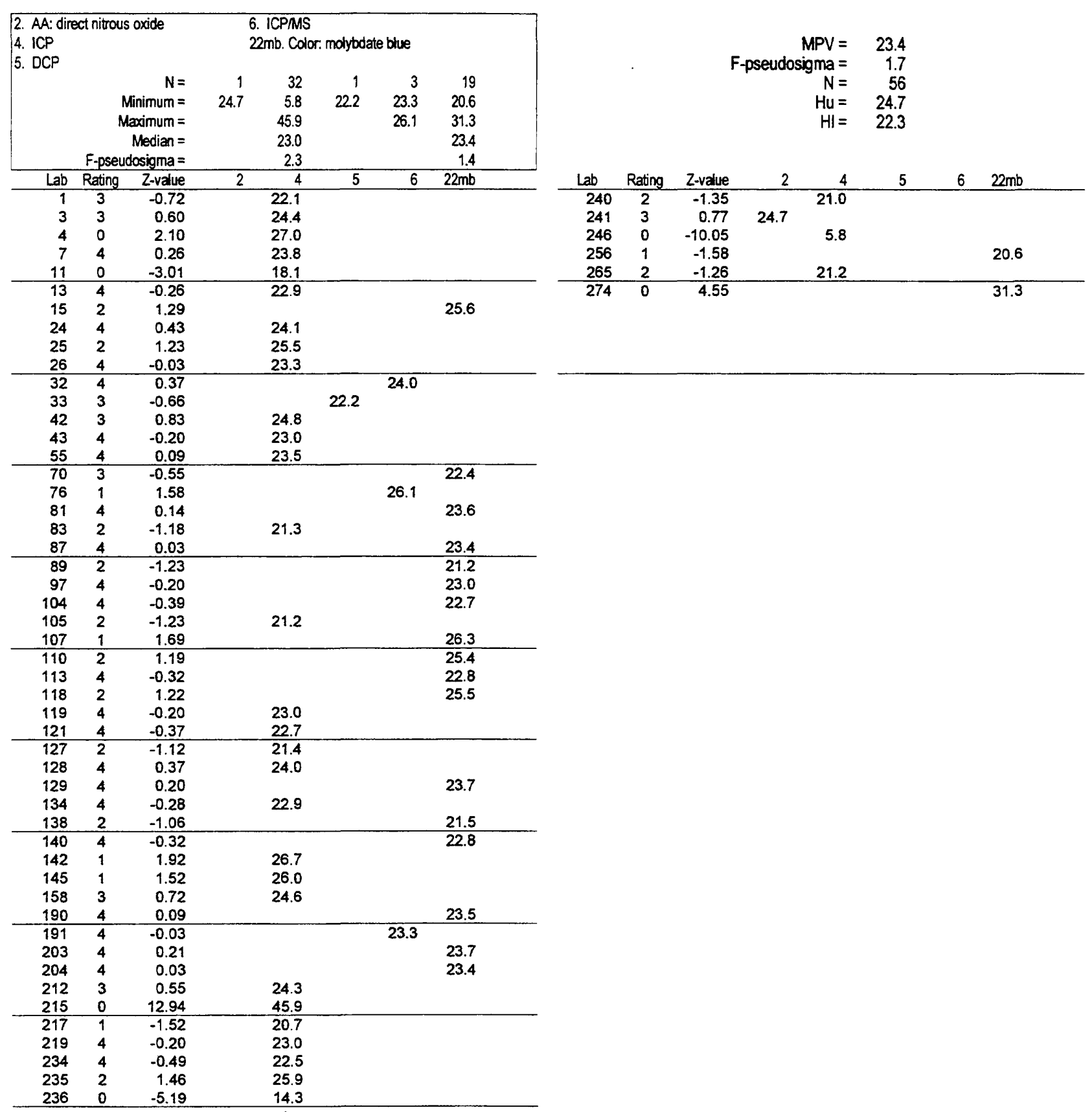


Table 12. Statistical summary of reported data for standard reference water sample T-143 (trace constituents)-Continued $\mathrm{Sr}$ (Strontium)

$\mu \mathrm{g} h$

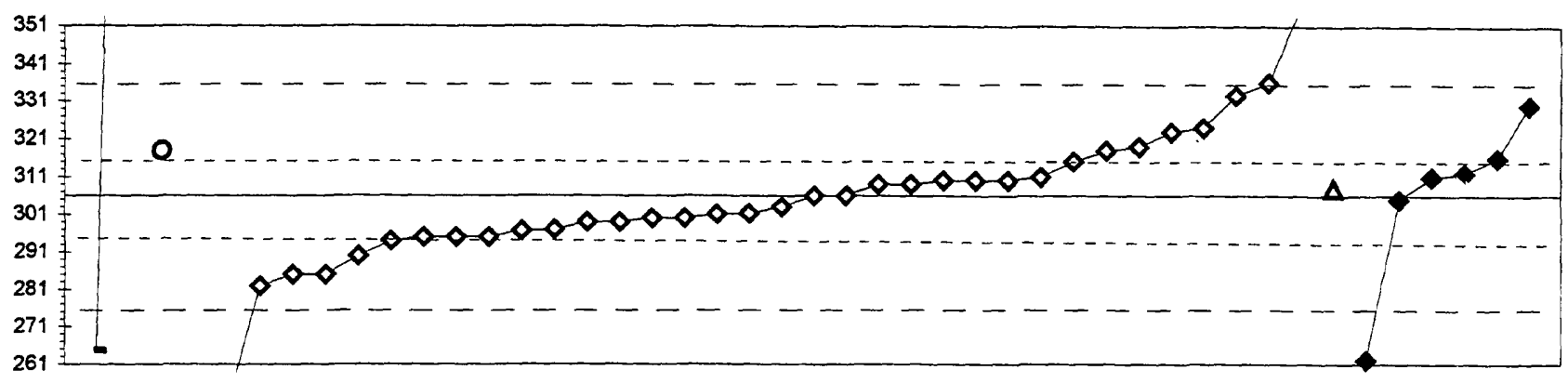

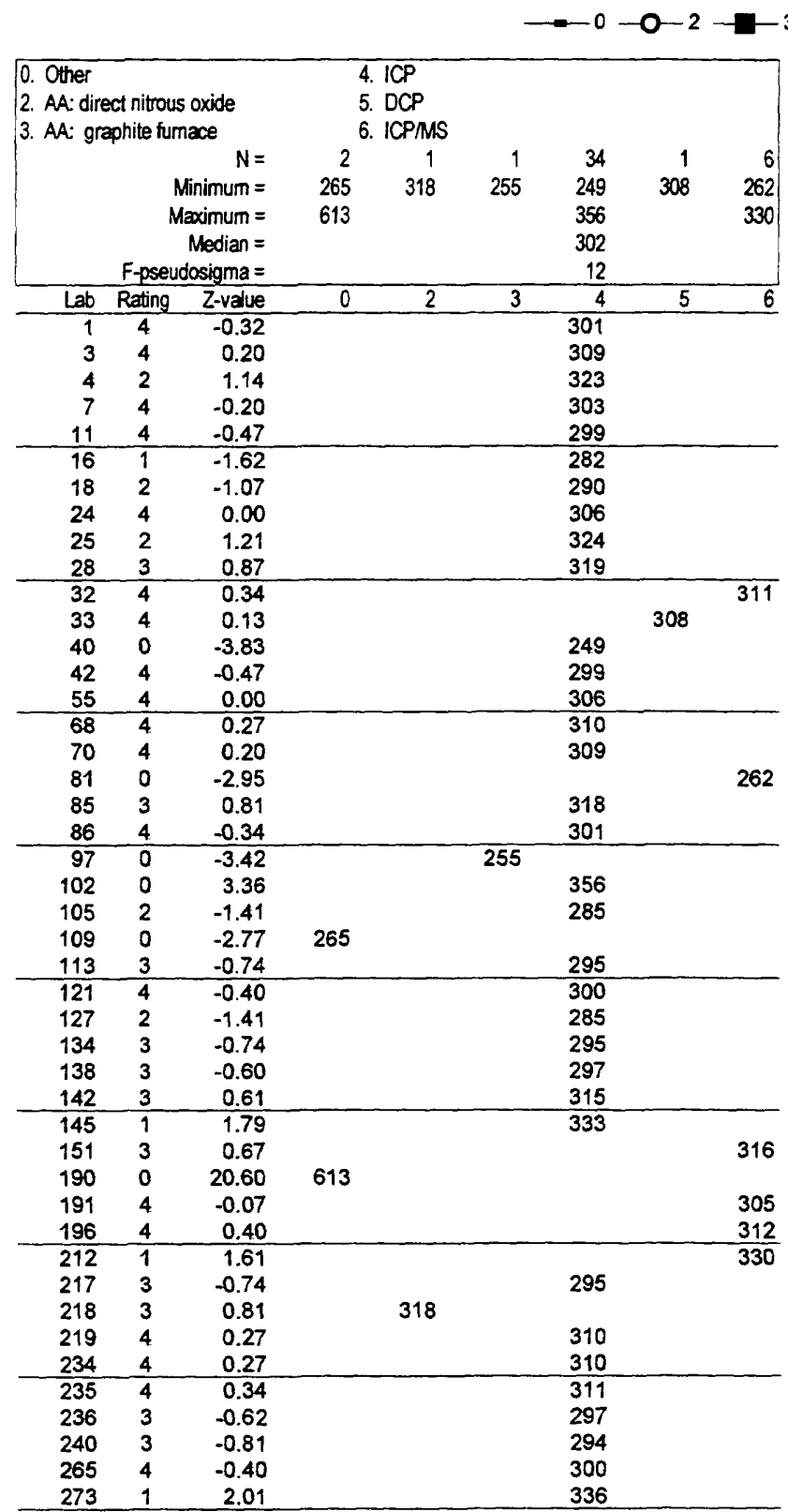


Table 12. Statistical summary of reported data for standard reference water sample T-143 (trace constituents)-Continued $\mathrm{TI}$ (Thallium) $\mu \mathrm{g} / \mathrm{L}$

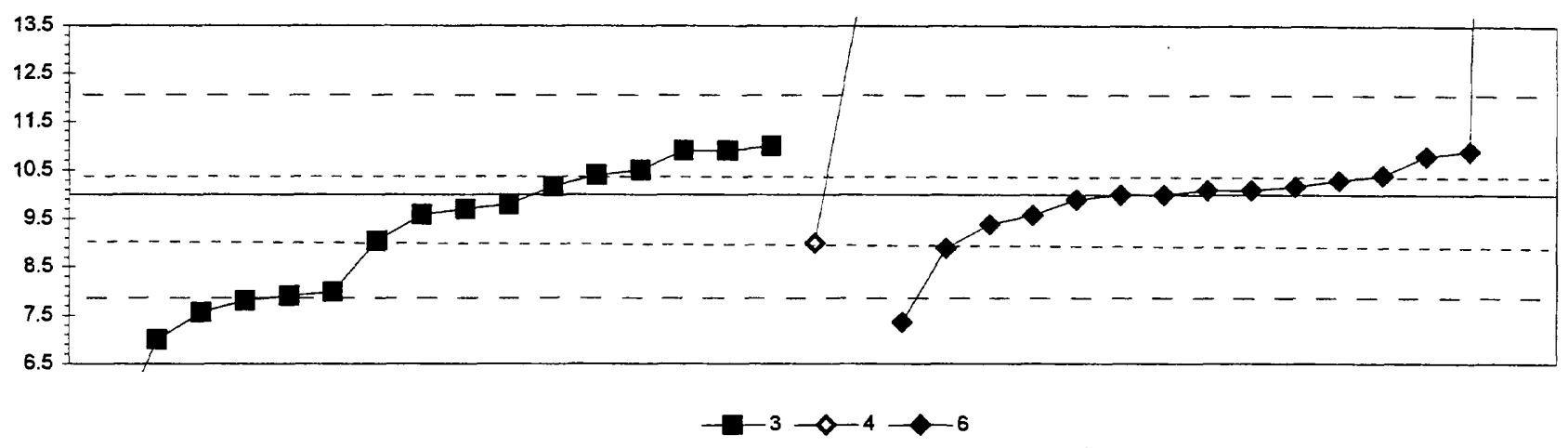

\begin{tabular}{|c|c|c|c|c|c|}
\hline $\begin{array}{l}\text { 3. AA: gra } \\
\text { 4. ICP } \\
\text { 6. ICPMS }\end{array}$ & aphite fur & & & & \\
\hline & & $N=$ & 16 & 2 & 15 \\
\hline & & inimum = & 5.0 & 9.0 & 7.4 \\
\hline & & sximum = & 11.0 & 14.0 & 53.0 \\
\hline & & Median = & 9.6 & & 10.1 \\
\hline & F-pseu & losigma $=$ & 1.9 & & 0.5 \\
\hline Lab & Rating & Z-value & 3 & 4 & 6 \\
\hline 1 & 4 & 0.39 & 10.4 & & \\
\hline 3 & NR & & & $<10$ & \\
\hline 11 & 0 & 3.85 & & 14.0 & \\
\hline 13 & 4 & -0.19 & 9.8 & & \\
\hline 15 & 1 & -1.95 & 8.0 & & \\
\hline 16 & 4 & 0.16 & & & 10.2 \\
\hline 18 & 4 & -0.29 & 9.7 & & \\
\hline 23 & 0 & -5.00 & $<5$ & & \\
\hline 32 & 4 & -0.40 & & & 9.6 \\
\hline 39 & 3 & 0.77 & & & 10.8 \\
\hline 42 & 0 & -5.00 & & & $<5$ \\
\hline 46 & 3 & 0.87 & 10.9 & & \\
\hline 48 & 1 & -2.02 & 7.9 & & \\
\hline 69 & 3 & 0.96 & 11.0 & & \\
\hline 70 & 0 & -2.12 & 7.8 & & \\
\hline 76 & 0 & -2.54 & & & 7.4 \\
\hline 81 & 0 & 41.43 & & & 53.0 \\
\hline 89 & NR & & $<10$ & & \\
\hline 97 & 3 & 0.87 & 10.9 & & \\
\hline 113 & 4 & -0.40 & 9.6 & & \\
\hline 119 & 4 & 0.10 & & & 10.1 \\
\hline 128 & 4 & 0.00 & & & 10.0 \\
\hline 134 & 4 & 0.16 & 10.2 & & \\
\hline 138 & 3 & -0.59 & & & 9.4 \\
\hline 141 & NR & & & $<50$ & \\
\hline 142 & 4 & 0.39 & & & 10.4 \\
\hline 146 & NR & & & $<10$ & \\
\hline 151 & 4 & 0.29 & & & 10.3 \\
\hline 180 & NR & & & $<32.1$ & \\
\hline 191 & 4 & -0.10 & & & 9.9 \\
\hline 193 & 0 & -2.89 & 7.0 & & \\
\hline 196 & 4 & 0.10 & & & 10.1 \\
\hline 212 & 4 & 0.00 & & & 10.0 \\
\hline 213 & 3 & -0.93 & 9.0 & & \\
\hline 215 & 0 & -3.00 & $<7$ & & \\
\hline 217 & 3 & 0.87 & & & 10.9 \\
\hline 234 & 0 & -2.35 & 7.6 & & \\
\hline 235 & 0 & -4.87 & 5.0 & & \\
\hline 240 & 3 & -0.96 & & 9.0 & \\
\hline 241 & 4 & 0.48 & 10.5 & & \\
\hline 265 & 2 & -1.06 & & & 8.9 \\
\hline
\end{tabular}


Table 12. Statistical summary of reported data for standard reference water sample T-143 (trace constituents)-Continued $\cup$ (Uranium) $\mu \mathrm{g} / \mathrm{L}$

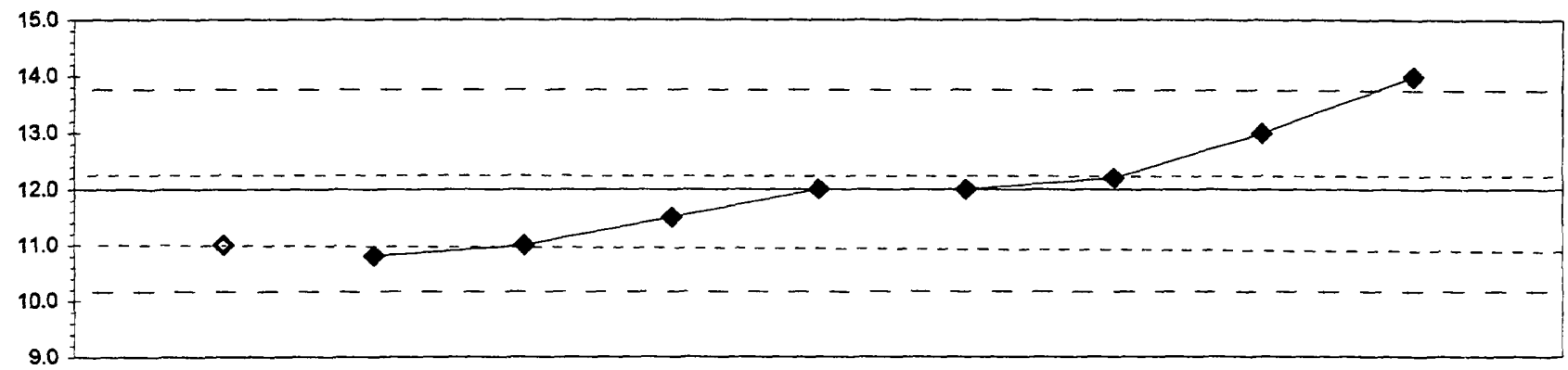

$$
-4 \rightarrow 6
$$

\begin{tabular}{|c|c|c|c|c|}
\hline $\begin{array}{l}\text { 4. ICP } \\
\text { 6. ICPMS } \\
\text { 0. Other }\end{array}$ & & & & \\
\hline & & $N=$ & 1 & 8 \\
\hline & & nimum = & 11.0 & 10.8 \\
\hline & & ximum = & & 14.0 \\
\hline & & Median = & & 12.0 \\
\hline & F-pseu & sigma = & & 1.0 \\
\hline $\mathrm{Lab}$ & Rating & Z-value & 4 & 6 \\
\hline 1 & 2 & -1.34 & & 10.8 \\
\hline 7 & NR & & $<120$ & \\
\hline 16 & 4 & 0.00 & & 12.0 \\
\hline $\begin{array}{l}30 \\
75\end{array}$ & $\stackrel{2}{N R}$ & -1.12 & $\begin{array}{r}11.0 \\
<100\end{array}$ & \\
\hline 119 & 4 & 0.00 & & 12.0 \\
\hline 142 & 4 & 0.22 & & 12.2 \\
\hline 196 & 3 & -0.56 & & 11.5 \\
\hline 212 & 0 & 2.25 & & 14.0 \\
\hline 217 & 2 & 1.12 & & 13.0 \\
\hline 265 & 2 & -1.12 & & 11.0 \\
\hline
\end{tabular}


Table 12. Statistical summary of reported data for standard reference water sample T-143 (trace constituents)-Continued $V$ (Vanadium)

$\mu \mathrm{g} / \mathrm{L}$

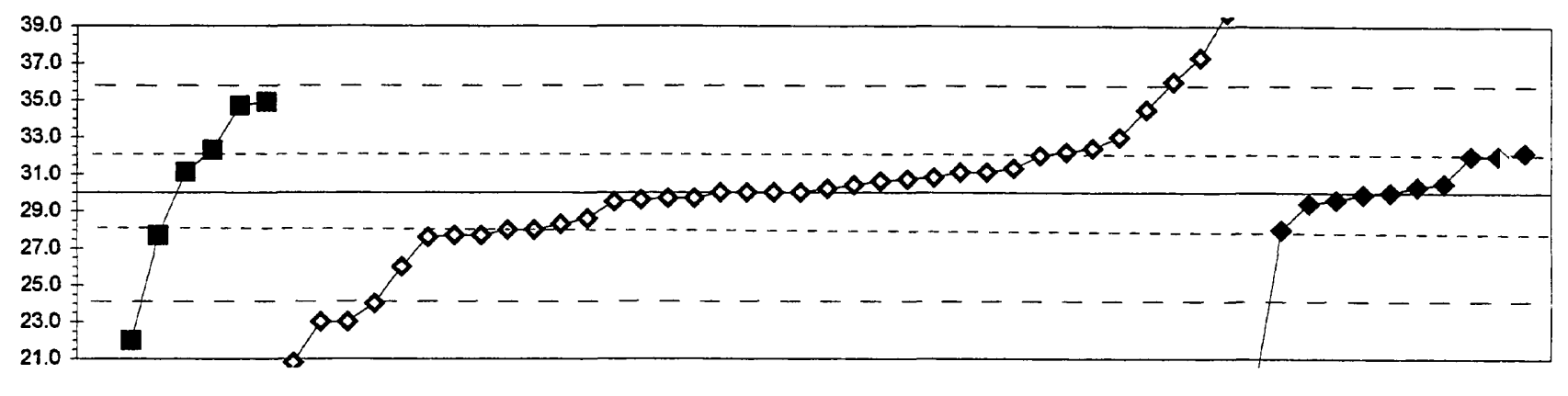

$-0-2 \div-3-4-6$

\begin{tabular}{|c|c|c|c|c|c|c|}
\hline $\begin{array}{l}\text { 2. AA: dire } \\
\text { 3. AA: gre } \\
\text { 4. ICP }\end{array}$ & $\begin{array}{l}\text { ct nitrou } \\
\text { phite fur }\end{array}$ & & & ICPMS & & \\
\hline & & $N=$ & 1 & 6 & 36 & 11 \\
\hline & & nimum = & 93.0 & 22.0 & 20.8 & 19.0 \\
\hline & & ximum = & & 34.9 & 39.7 & 32.2 \\
\hline & & Median = & & & 30.0 & 30.0 \\
\hline & F-pses & sigma = & & & 2.5 & 1.3 \\
\hline Lab & Rating & Z-value & 2 & 3 & 4 & 6 \\
\hline 1 & 4 & 0.37 & & & 31.1 & \\
\hline 3 & 0 & -2.36 & & & 23.0 & \\
\hline 4 & NR & & & & $<50$ & \\
\hline 7 & 3 & 0.81 & & & 32.4 & \\
\hline 11 & 4 & 0.00 & & & 30.0 & \\
\hline 13 & 0 & 3.27 & & & 39.7 & \\
\hline 15 & 0 & -3.10 & & & 20.8 & \\
\hline 16 & 4 & 0.10 & & & & 30.3 \\
\hline 18 & 3 & -0.67 & & & 28.0 & \\
\hline 24 & 1 & -2.02 & & & 24.0 & \\
\hline 25 & 0 & -2.36 & & & 23.0 & \\
\hline 26 & 4 & 0.00 & & & 30.0 & \\
\hline 28 & 4 & 0.07 & & & 30.2 & \\
\hline 30 & 4 & 0.00 & & & 30.0 & \\
\hline 32 & 4 & 0.17 & & & & 30.5 \\
\hline 40 & 3 & -0.78 & & & 27.7 & \\
\hline 42 & 3 & 0.67 & & & & 32.0 \\
\hline 46 & 3 & 0.74 & & & 32.2 & \\
\hline 48 & 3 & -0.78 & & 27.7 & & \\
\hline 50 & 0 & -2.70 & & 22.0 & & \\
\hline 55 & 3 & -0.57 & & & 28.3 & \\
\hline 68 & 2 & -1.35 & & & 26.0 & \\
\hline 70 & NR & & & & $<50$ & \\
\hline 75 & 4 & -0.13 & & & 29.6 & \\
\hline 81 & 0 & -3.71 & & & & 19.0 \\
\hline 85 & 4 & 0.13 & & & 30.4 & \\
\hline 86 & 4 & 0.44 & & & 31.3 & \\
\hline 89 & 3 & 0.78 & & 32.3 & & \\
\hline 97 & 1 & 1.65 & & 34.9 & & \\
\hline 102 & 2 & 1.01 & & & 33.0 & \\
\hline 105 & 4 & -0.20 & & & & 29.4 \\
\hline 119 & 3 & -0.67 & & & & 28.0 \\
\hline 121 & 3 & 0.67 & & & 32.0 & \\
\hline 127 & 4 & 0.37 & & 31.1 & & \\
\hline 128 & 3 & -0.78 & & & 27.7 & \\
\hline 134 & 4 & -0.11 & & & 29.7 & \\
\hline 138 & 4 & -0.17 & & & 29.5 & \\
\hline 141 & 4 & 0.27 & & & 30.8 & \\
\hline 142 & 4 & -0.13 & & & & 29.6 \\
\hline 145 & 0 & 2.46 & & & 37.3 & \\
\hline 146 & 4 & 0.00 & & & 30.0 & \\
\hline 158 & 4 & 0.20 & & & 30.6 & \\
\hline 180 & 4 & 0.24 & & & 30.7 & \\
\hline 191 & 3 & 0.74 & & & & 32.2 \\
\hline 196 & 4 & -0.03 & & & & 29.9 \\
\hline 212 & 3 & 0.67 & & & & 32.0 \\
\hline 217 & 4 & -0.47 & & & 28.6 & \\
\hline 219 & 3 & -0.67 & & & 28.0 & \\
\hline 224 & 1 & 1.52 & & & 34.5 & \\
\hline 234 & 3 & -0.81 & & & 27.6 & \\
\hline
\end{tabular}

\begin{tabular}{crrrrrr} 
Lab & Rating & Z-value & 2 & 3 & 4 & 6 \\
\hline 235 & 1 & 2.02 & & & 36.0 & \\
236 & 4 & 0.37 & & & 31.1 & \\
241 & 1 & 1.59 & & 34.7 & & \\
255 & 4 & -0.10 & & & 29.7 & \\
257 & 0 & 21.25 & 93.0 & & & \\
\hline 265 & 4 & 0.00 & & & & 30.0
\end{tabular}

MPV $=30.0$

F-pseudosigma $=3.0$

$\mathrm{N}=54$

$\mathrm{Hu}=32.0$

$H I=28.0$ 
Table 12. Statistical summary of reported data for standard reference water sample T-143 (trace constituents)-Continued Zn (Zinc) $\mu \mathrm{g} / \mathrm{L}$

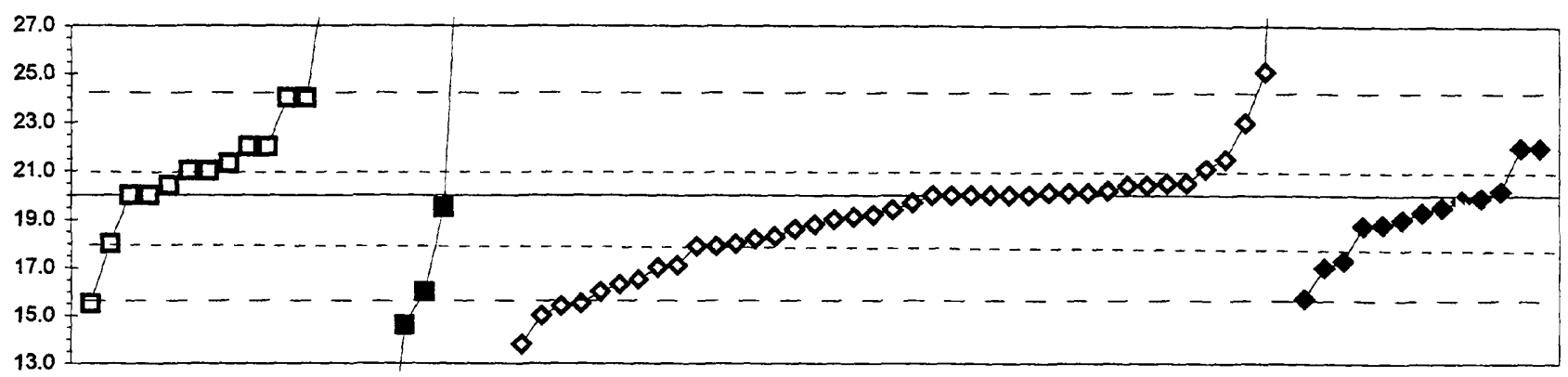

$-\square-1-3-\diamond-4 \multimap 6$

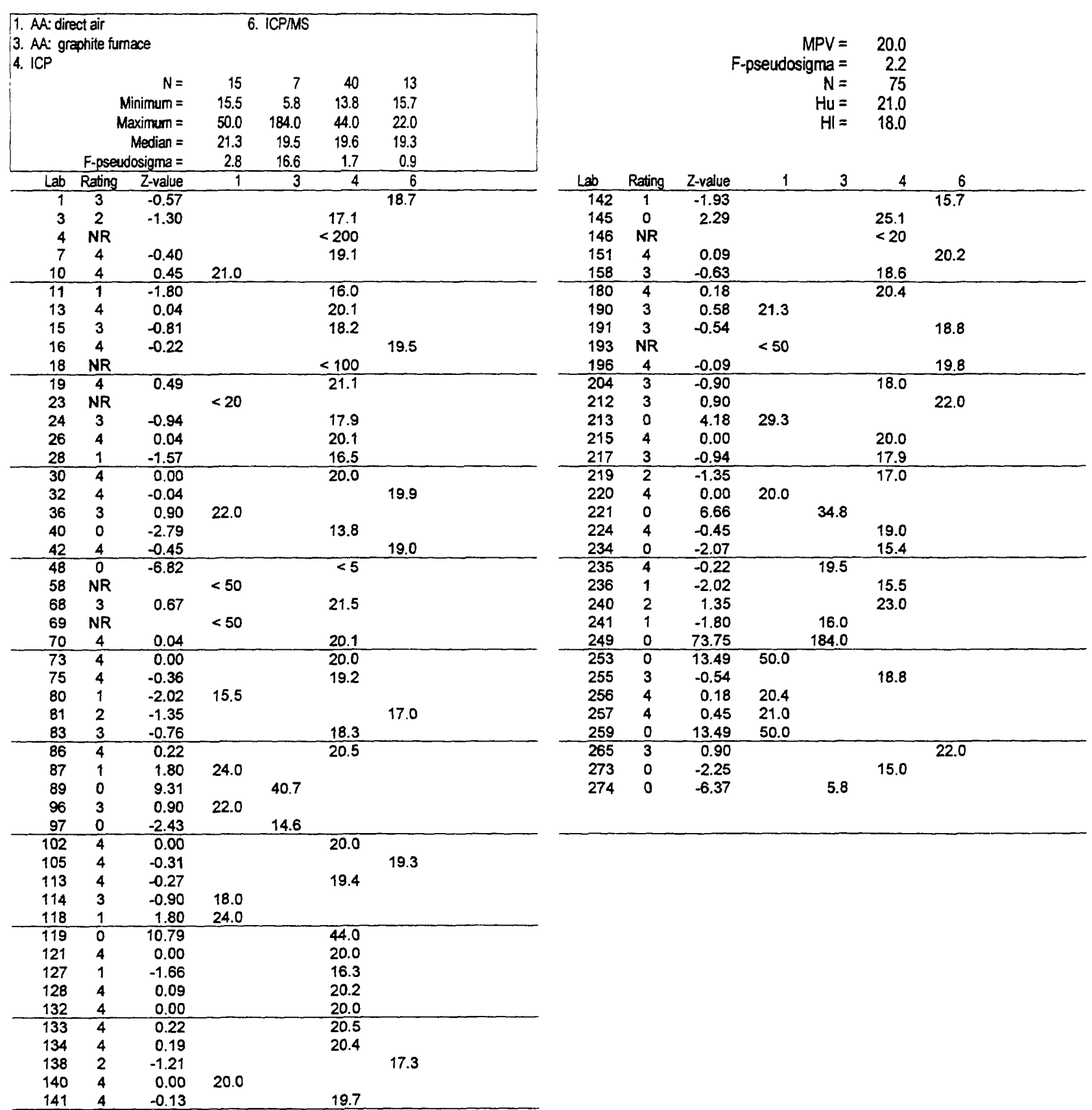


Table 13. Statistical summary of reported data for standard reference water sample T-145 (trace constituents)

Definition of analytical methods, abbreviations, and symbols

Analytical methods

0. Other/Not reported

1. AA: direct, air

2. AA: direct, $\mathrm{N}_{2} \mathrm{O}$

3. AA: graphite furnace

4. ICP

5. DCP

6. ICPMS

10. AA: extraction

11. AA: hydride

12. Flame emission

22. Color:

= atomic absorption: direct,air

= atomic absorption: direct,nitrous oxide

= atomic absorption: graphite furnace

$=\quad$ inductively coupled plasma

= direct current plasma

$=\quad$ inductively coupled plasma/mass spectrometry

$=\quad$ atomic absorption: extraction [chelating agent(s) specified]

= atomic absorption: hydride [reducing agent specified]

= flame emission

= colorimetric [color reagent specified]

$\begin{array}{rlrl}\text { Abbreviations and symbols } & & & \\ \text { MPV } & = & & \text { number of samples } \\ & & \text { most probable value } \\ \text { F-pseudosigma } & = & & \text { nonparametric statistic deviation } \\ H u & = & & \text { upper hinge value } \\ H I & = & & \text { lower hinge value } \\ \mu g / L & = & \text { micrograms per liter } \\ \mathrm{mg} / \mathrm{L}= & & \text { milligrams per liter } \\ \text { Lab } & = & & \text { laboratory code number } \\ \mathrm{NR} & = & & \text { not rated, less than value reported } \\ & = & & \text { less than }\end{array}$

Constituent

$\mathrm{Ag} \quad$ Silver

Al Aluminium

As Arsenic

B Boron

Ba Barium

Be Beryllium

$\mathrm{Ca}$ Calcium

cd Cadmium

Co Cobalt

$\mathrm{Cr} \quad$ Chromium

Cu Copper

$\mathrm{Fe}$ Iron

K Potassium

Li Lithium

\begin{tabular}{r} 
page \\
\hline 74 \\
75 \\
76 \\
77 \\
78 \\
79 \\
80 \\
81 \\
82 \\
83 \\
84 \\
85 \\
86 \\
87
\end{tabular}

\begin{tabular}{ll}
\multicolumn{2}{l}{ Constituent } \\
\hline $\mathrm{Mg}$ & Magnesium \\
$\mathrm{Mn}$ & Manganese \\
$\mathrm{Mo}$ & Molybdenum \\
$\mathrm{Na}$ & Sodium \\
$\mathrm{Ni}$ & Nickel \\
$\mathrm{Pb}$ & Lead \\
$\mathrm{Sb}$ & Antimony \\
$\mathrm{Se}$ & Selenium \\
$\mathrm{SiO} 2$ & Silica \\
$\mathrm{Sr}$ & Strontium \\
$\mathrm{TI}$ & Thallium \\
$\mathrm{U}$ & Uranium \\
$\mathrm{V}$ & Vanadium \\
$\mathrm{Zn}$ & Zinc
\end{tabular}

\begin{tabular}{r} 
page \\
\hline 88 \\
89 \\
90 \\
91 \\
92 \\
93 \\
94 \\
95 \\
96 \\
97 \\
98 \\
99 \\
100 \\
101
\end{tabular}


Table 13. Statistical summary of reported data for standard reference water sample T-145 (trace constituents)-Continued $\mathrm{Ag}$ (Silver) $\mu \mathrm{g} / \mathrm{L}$

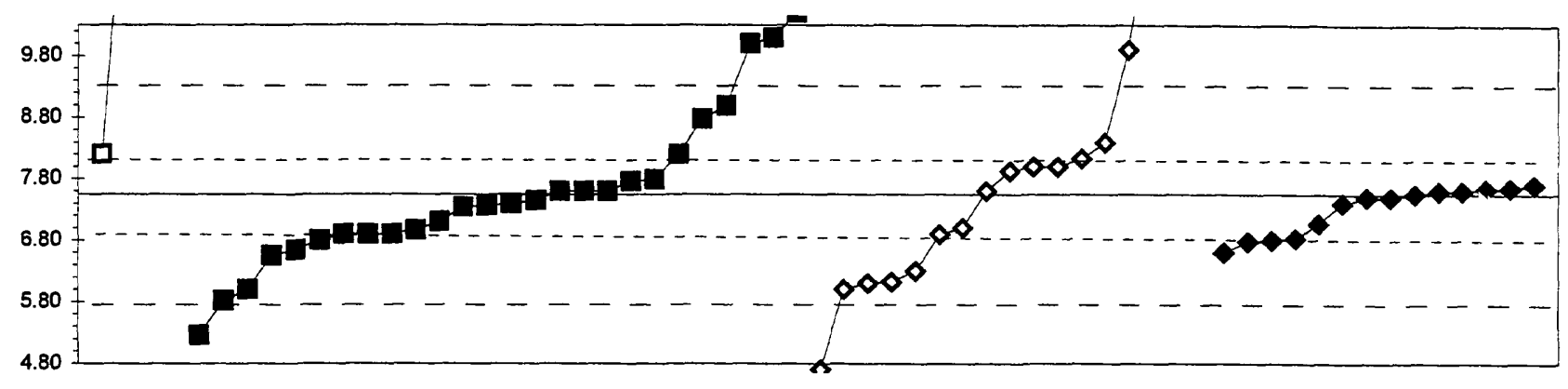

$-a-1-n-3-4-6$

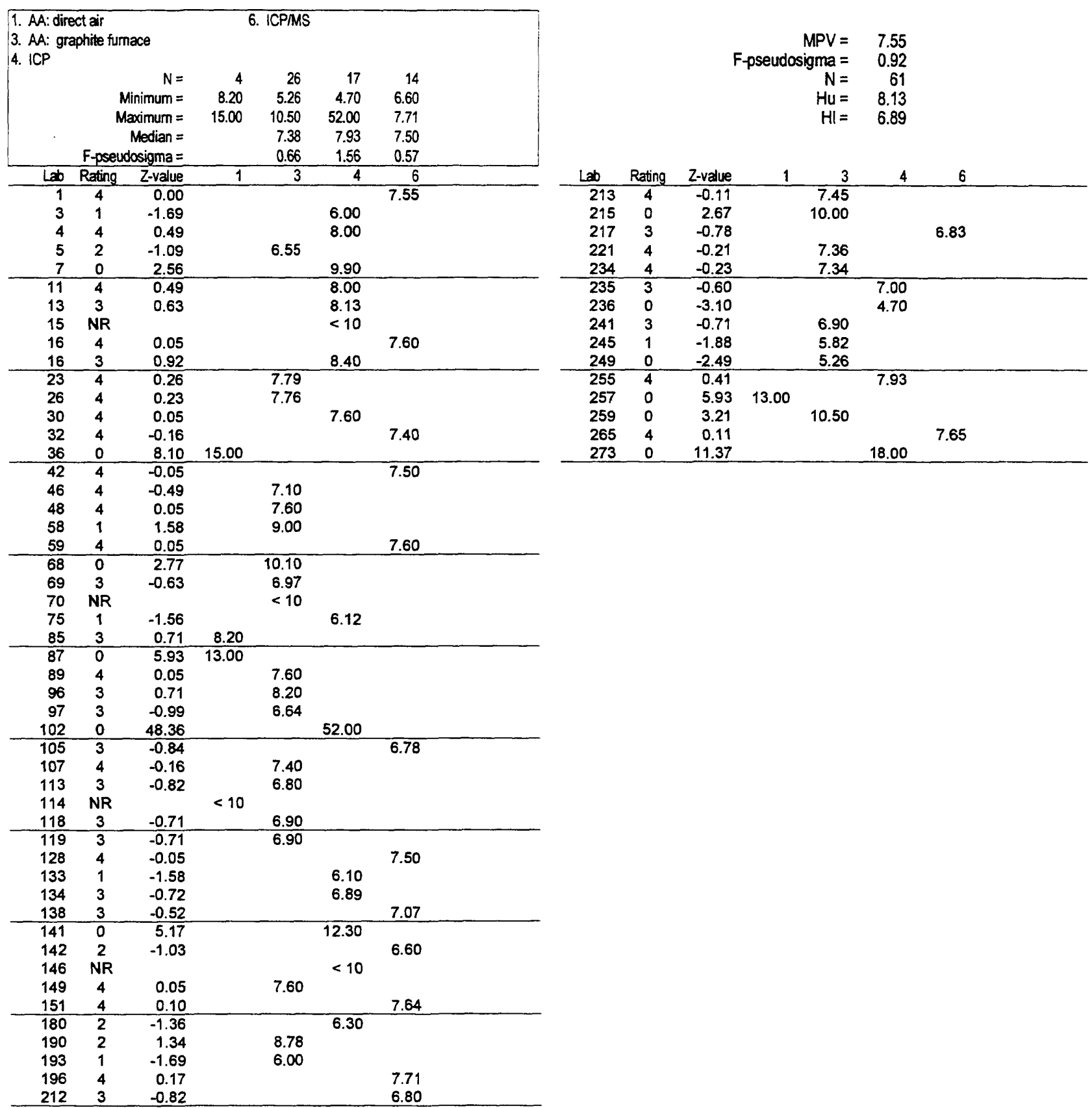


Table 13. Statistical summary of reported data for standard reference water sample T-145 (trace constituents)-Continued Al (Aluminum)

$\mu \mathrm{g} / \mathrm{L}$

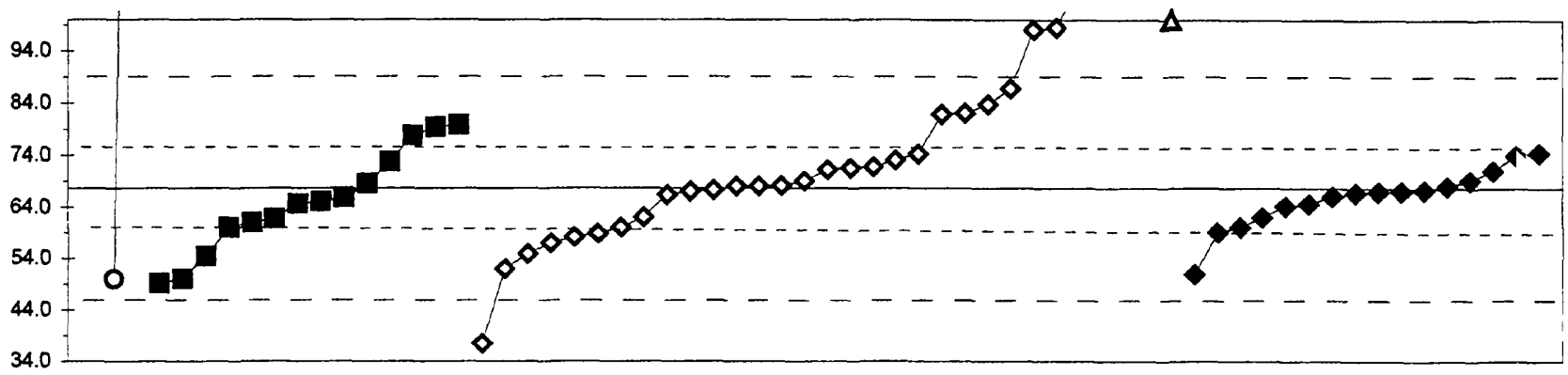

$-\square-1-0-2-3-4-\Delta-5 \multimap-6$

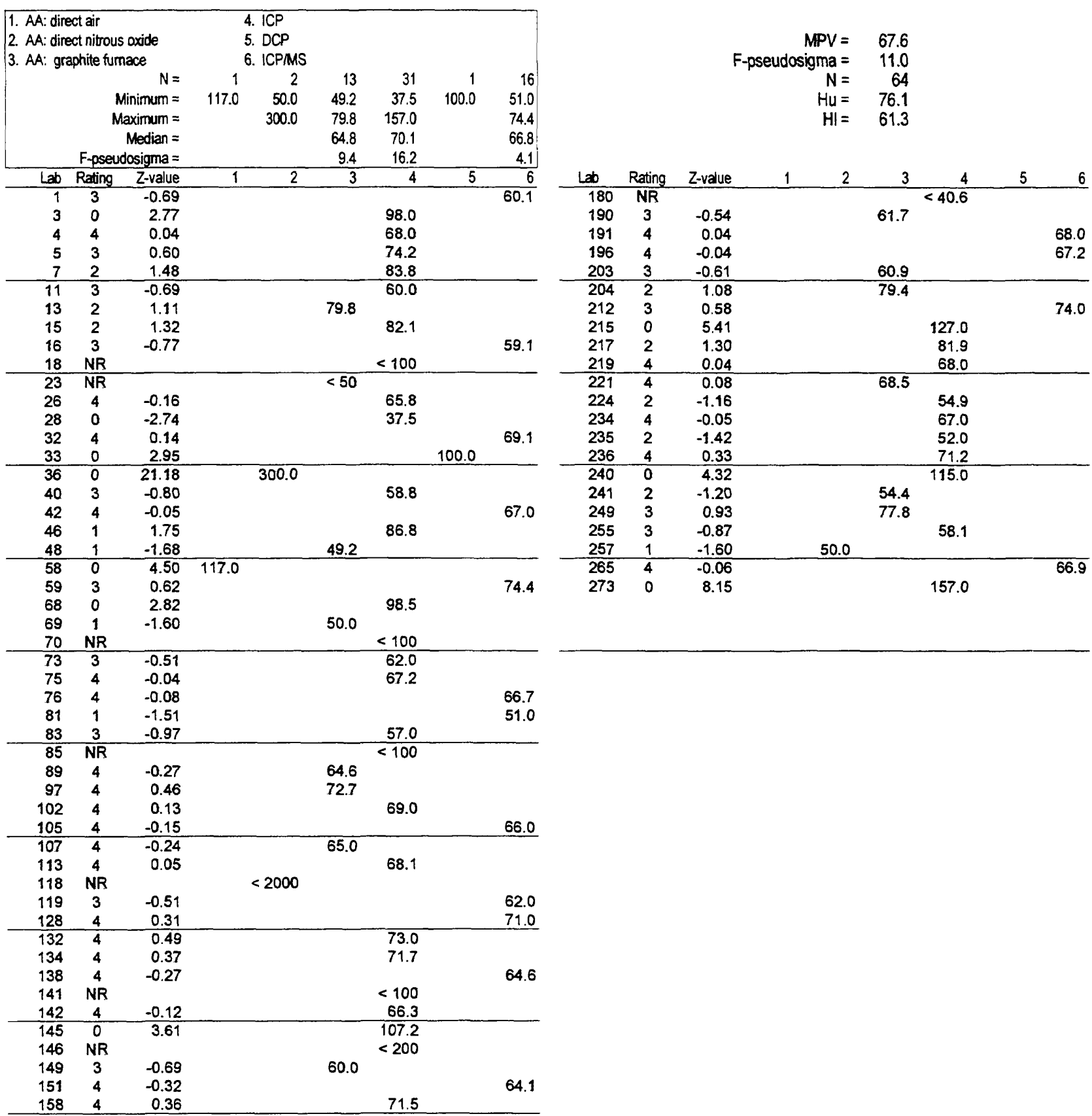


Table 13. Statistical summary of reported data for standard reference water sample T-145 (trace constituents)-Continued As (Arsenic) $\mu \mathrm{g} / \mathrm{L}$

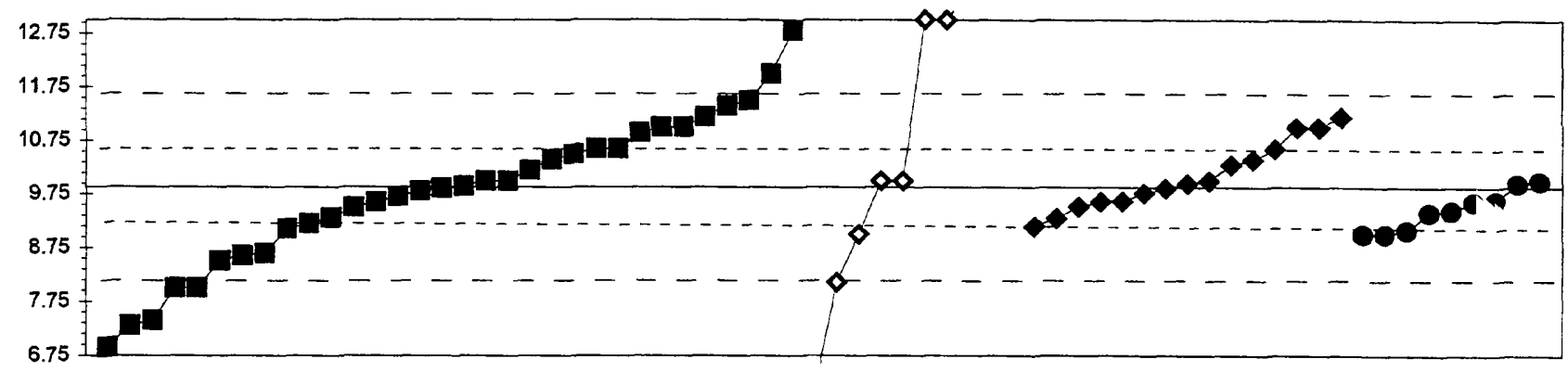

$\rightarrow-3-4-4-6-11$ na

\begin{tabular}{|c|c|c|c|c|c|c|}
\hline $\begin{array}{l}\text { 3. AA: gra } \\
\text { 4. ICP } \\
\text { 6. ICPMS }\end{array}$ & aphite fur & & & 11na. AA: & ydride $\mathrm{N}$ & \\
\hline & & $N=$ & 32 & 10 & 15 & 9 \\
\hline & & inimum = & 6.90 & 6.10 & 9.14 & 9.00 \\
\hline & & ximum = & 12.80 & 42.70 & 11.20 & 10.00 \\
\hline & & Median = & 9.88 & 11.50 & 9.95 & 9.44 \\
\hline & F-pseu & osigma = & 1.22 & 3.34 & 0.66 & 0.41 \\
\hline Lab & Rating & Z-value & 3 & 4 & 6 & 11na \\
\hline 1 & 4 & 0.02 & 9.90 & & & \\
\hline 3 & 4 & 0.12 & & 10.00 & & \\
\hline 4 & 0 & 3.01 & & 13.00 & & \\
\hline 5 & 4 & -0.02 & 9.86 & & & \\
\hline 7 & NR & & & $<120$ & & \\
\hline 10 & 4 & 0.12 & & & & 10.00 \\
\hline 11 & 4 & 0.12 & & 10.00 & & \\
\hline 13 & 3 & 0.60 & 10.50 & & & \\
\hline 15 & NR & & & $<100$ & & \\
\hline 16 & 4 & 0.50 & & & 10.40 & \\
\hline 18 & 3 & -0.66 & 9.20 & & & \\
\hline 23 & 0 & 2.81 & 12.80 & & & \\
\hline 26 & 4 & -0.25 & & & & 9.62 \\
\hline 30 & 3 & -0.85 & & 9.00 & & \\
\hline 32 & 4 & -0.11 & & & 9.77 & \\
\hline 34 & 4 & -0.27 & & & & 9.60 \\
\hline 36 & 1 & -1.81 & 8.00 & & & \\
\hline 42 & 2 & 1.08 & & & 11.00 & \\
\hline 46 & 3 & -0.75 & 9.10 & & & \\
\hline 48 & 4 & -0.08 & 9.80 & & & \\
\hline 58 & $\frac{7}{1}$ & 2.04 & 12.00 & & & \\
\hline 59 & 4 & 0.12 & & & 10.00 & \\
\hline 68 & 0 & -2.87 & 6.90 & & & \\
\hline 69 & 4 & 0.31 & 10.20 & & & \\
\hline 70 & NR & & $<10$ & & & \\
\hline 73 & $\frac{0}{0}$ & 3.01 & & 13.00 & & \\
\hline 75 & 4 & -0.42 & & & & 9.44 \\
\hline 76 & 3 & 0.69 & & & 10.60 & \\
\hline 80 & 3 & 0.69 & 10.60 & & & \\
\hline 81 & 4 & 0.12 & 10.00 & & & \\
\hline 86 & 4 & 0.07 & & & & 9.95 \\
\hline 87 & 4 & -0.46 & & & & 9.40 \\
\hline 89 & 3 & -0.85 & & & & 9.00 \\
\hline 96 & 4 & -0.17 & 9.70 & & & \\
\hline 97 & 4 & 0.50 & 10.40 & & & \\
\hline 102 & 0 & -3.64 & & 6.10 & & \\
\hline 105 & 4 & 0.40 & & & 10.30 & \\
\hline 109 & 2 & -1.33 & 8.50 & & & \\
\hline 113 & 1 & 1.56 & 11.50 & & & \\
\hline 118 & 3 & 0.69 & 10.60 & & & \\
\hline 119 & 3 & -0.85 & & & & 9.00 \\
\hline 128 & 4 & 0.07 & & & 9.95 & \\
\hline 133 & 0 & -2.49 & 7.30 & & & \\
\hline 134 & 3 & -0.78 & & & & 9.07 \\
\hline 138 & 4 & -0.36 & & & 9.51 & \\
\hline 141 & 2 & -1.19 & 8.65 & & & \\
\hline 142 & 4 & -0.26 & & & 9.61 & \\
\hline 144 & 4 & -0.27 & 9.60 & & & \\
\hline 145 & 0 & 9.08 & & 19.30 & & \\
\hline 146 & 0 & 3.49 & & 13.50 & & \\
\hline
\end{tabular}

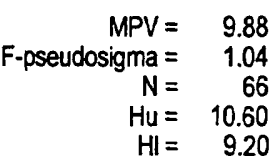

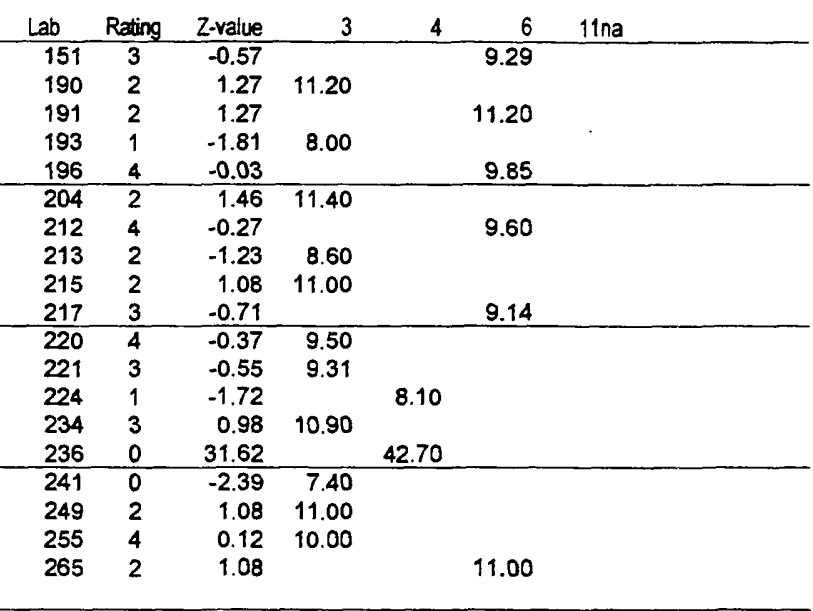


Table 13. Statistical summary of reported data for standard reference water sample T-145 (trace constituents)-Continued B (Boron) $\mu \mathrm{g} / \mathrm{L}$

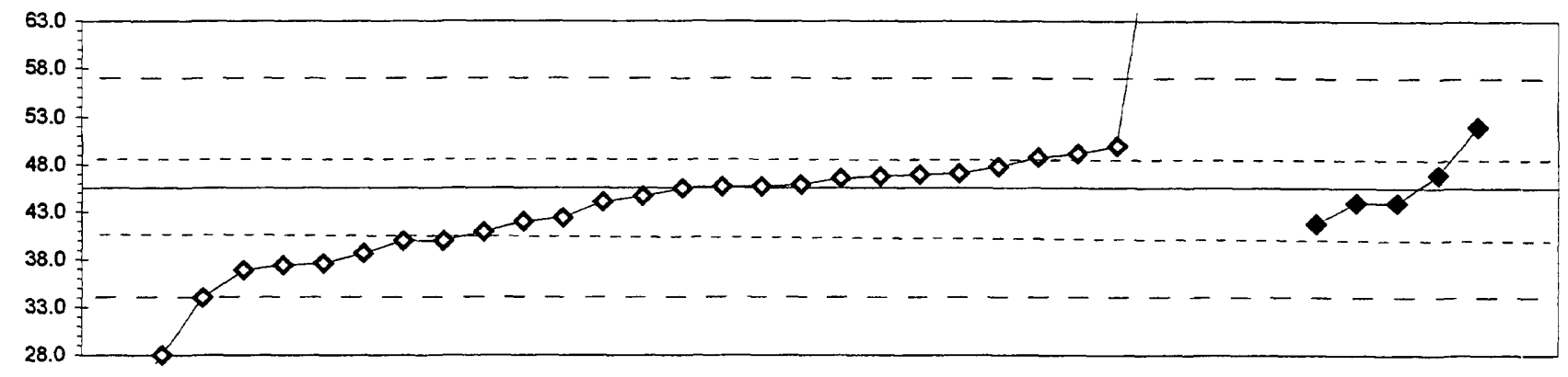

$-4-6-x-22 a z$

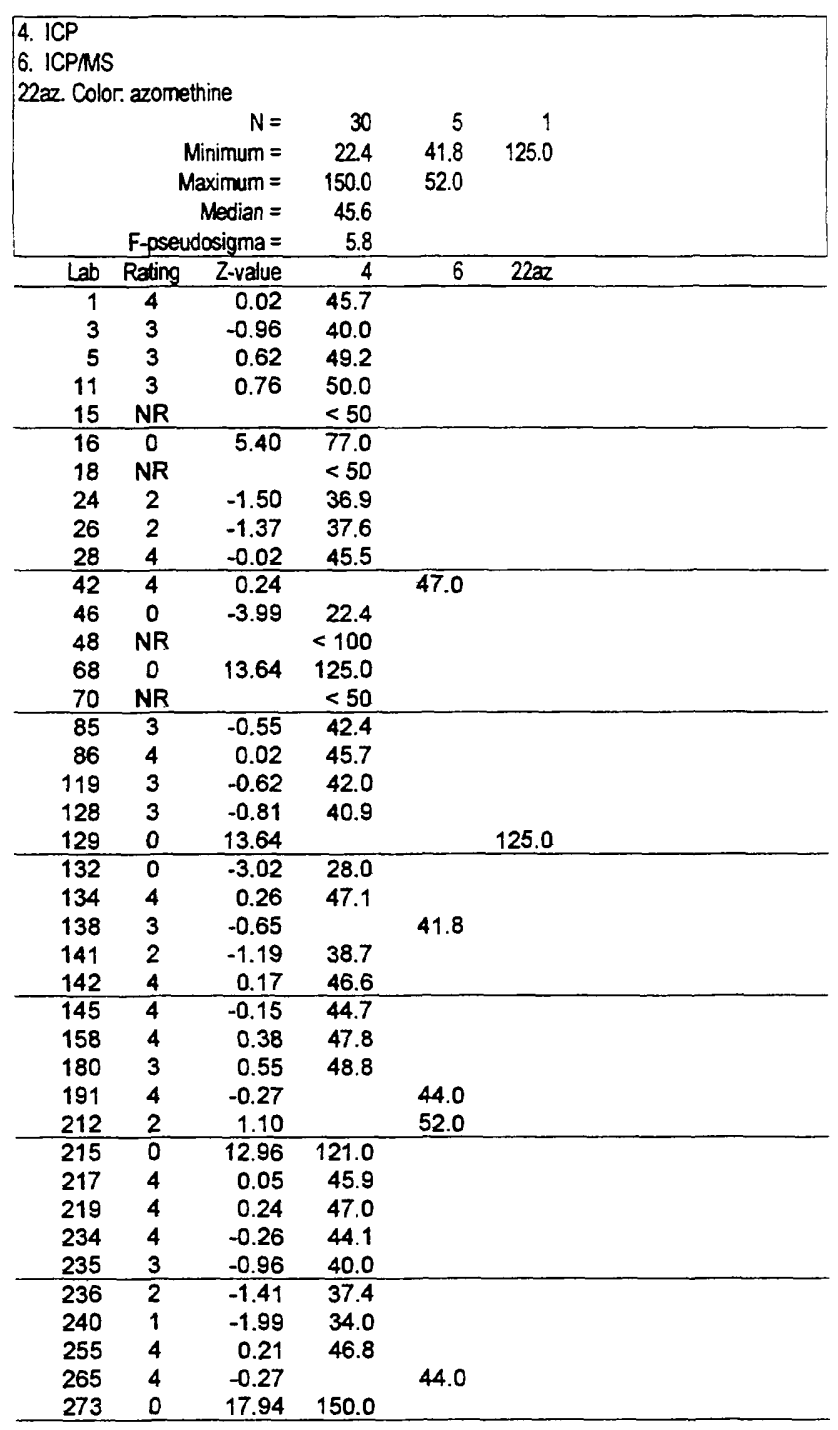


Table 13. Statistical summary of reported data for standard reference water sample T-145 (trace constituents)-Continued Ba (Barium)

$\mu \mathrm{g} / \mathrm{L}$

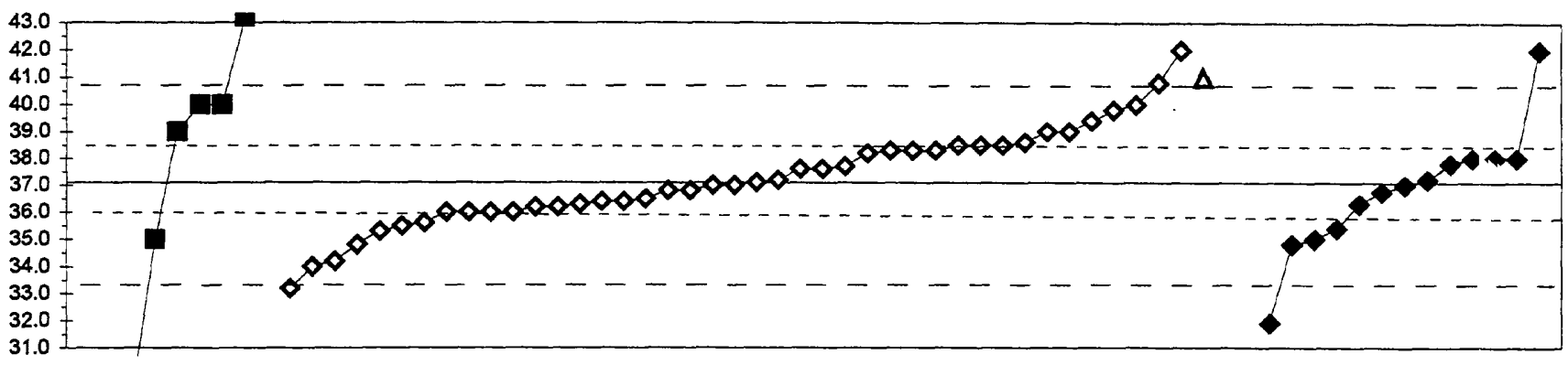

$-\square-1-0-2-0-3-4-\Delta-5 \multimap-6$

\begin{tabular}{|c|c|c|c|c|c|c|c|c|}
\hline \multicolumn{3}{|c|}{$\begin{array}{l}\text { 1. AA: direct air } \\
\text { 2. AA: direct nitrous oxide } \\
\text { 3. AA: graphite furnace }\end{array}$} & \multicolumn{3}{|c|}{$\begin{array}{l}\text { 4. ICP } \\
\text { 5. DCP } \\
\text { 6. ICPMS }\end{array}$} & \multirow[b]{2}{*}{42} & \multirow[b]{2}{*}{1} & \multirow[b]{2}{*}{13} \\
\hline & & $N=$ & 0 & 0 & 7 & & & \\
\hline & & linimum $=$ & $<0.05$ & $<100$ & 29.2 & 31.0 & 41.0 & 31.9 \\
\hline & & aximum = & & & 44.5 & 42.0 & & 42.0 \\
\hline & & Median = & & & 40.0 & 37.0 & & 37.0 \\
\hline & F-pseu & losigma = & & & 3.4 & 1.9 & & 1.9 \\
\hline Lab & Rating & Z-value & 1 & 2 & 3 & 4 & 5 & 6 \\
\hline 1 & 4 & -0.19 & & & & & & 36.8 \\
\hline 3 & 4 & -0.32 & & & & 36.5 & & \\
\hline 4 & 4 & -0.05 & & & & 37.0 & & \\
\hline 5 & 3 & -0.59 & & & & 36.0 & & \\
\hline 7 & 3 & 0.76 & & & & 38.5 & & \\
\hline 11 & 3 & -0.59 & & & & 36.0 & & \\
\hline 13 & 2 & 1.46 & & & & 39.8 & & \\
\hline 15 & 1 & -1.56 & & & & 34.2 & & \\
\hline 16 & 4 & 0.49 & & & & & & 38.0 \\
\hline 18 & 4 & -0.49 & & & & 36.2 & & \\
\hline 24 & 2 & -1.24 & & & & 34.8 & & \\
\hline 25 & 3 & 0.65 & & & & 38.3 & & \\
\hline 26 & 4 & 0.27 & & & $\cdot$ & 37.6 & & \\
\hline 28 & 3 & 0.81 & & & & 38.6 & & \\
\hline 30 & 3 & -0.59 & & & & 36.0 & & \\
\hline 32 & 3 & -0.92 & & & & & & 35.4 \\
\hline 33 & 0 & 2.10 & & & & & 41.0 & \\
\hline 36 & 0 & -20.02 & $<0.05$ & & & & & \\
\hline 40 & 3 & -0.97 & & & & 35.3 & & \\
\hline 46 & 4 & 0.27 & & & & 37.6 & & \\
\hline 48 & 0 & 3.29 & & & 43.2 & & & \\
\hline 59 & 4 & -0.05 & & & & & & 37.0 \\
\hline 68 & 3 & 0.76 & & & & 38.5 & & \\
\hline 70 & NR & & & & & $<50$ & & \\
\hline 75 & 4 & 0.05 & & & & 37.2 & & \\
\hline 81 & 2 & -1.13 & & & & & & 35.0 \\
\hline 83 & 3 & -0.86 & & & & 35.5 & & \\
\hline 85 & 4 & 0.32 & & & & 37.7 & & \\
\hline 86 & 4 & -0.16 & & & & 36.8 & & \\
\hline 87 & 1 & 1.56 & & & 40.0 & & & \\
\hline 89 & NR & & & & $<50$ & & & \\
\hline 96 & NR & & & $<100$ & & & & \\
\hline 97 & 0 & -4.26 & & & 29.2 & & & \\
\hline 102 & 0 & 2.64 & & & & 42.0 & & \\
\hline 105 & 2 & -1.24 & & & & & & 34.8 \\
\hline 107 & 2 & 1.03 & & & 39.0 & & & \\
\hline 113 & 4 & -0.05 & & & & 37.0 & & \\
\hline 119 & 2 & 1.03 & & & & 39.0 & & \\
\hline 121 & 3 & -0.59 & & & & 36.0 & & \\
\hline 128 & 0 & -2.81 & & & & & & 31.9 \\
\hline 133 & 4 & -0.38 & & & & 36.4 & & \\
\hline 134 & 3 & -0.81 & & & & 35.6 & & \\
\hline 138 & 4 & -0.38 & & & & 36.4 & & \\
\hline 141 & 3 & 0.59 & & & & 38.2 & & \\
\hline 142 & 4 & 0.05 & & & & & & 37.2 \\
\hline 145 & 1 & 2.00 & & & & 40.8 & & \\
\hline 146 & 3 & 0.76 & & & & 38.5 & & \\
\hline 149 & 1 & 1.56 & & & 40.0 & & & \\
\hline 151 & 4 & 0.38 & & & & & & 37.8 \\
\hline 158 & 3 & 0.65 & & & & 38.3 & & \\
\hline
\end{tabular}

MPV $=\quad 37.1$

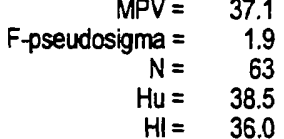

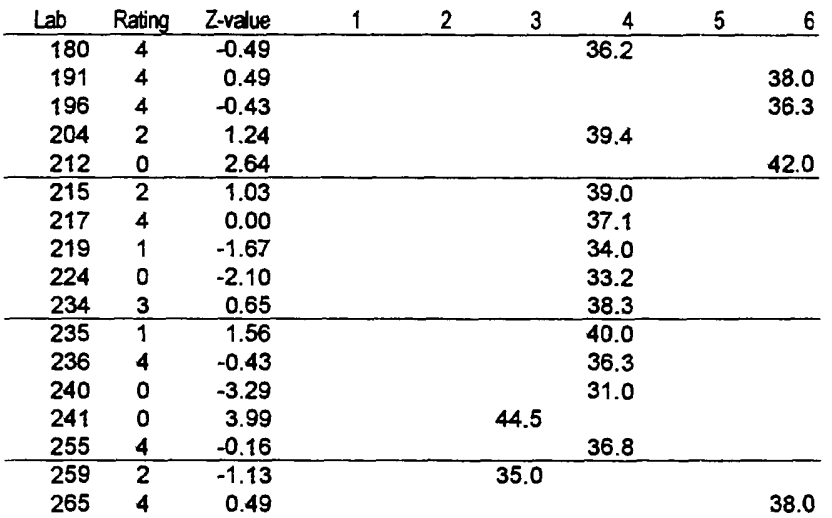

\section{.0}


Table 13. Statistical summary of reported data for standard reference water sample T-145 (trace constituents)-Continued Be (Berylium)

$\mu \mathrm{g} / \mathrm{L}$

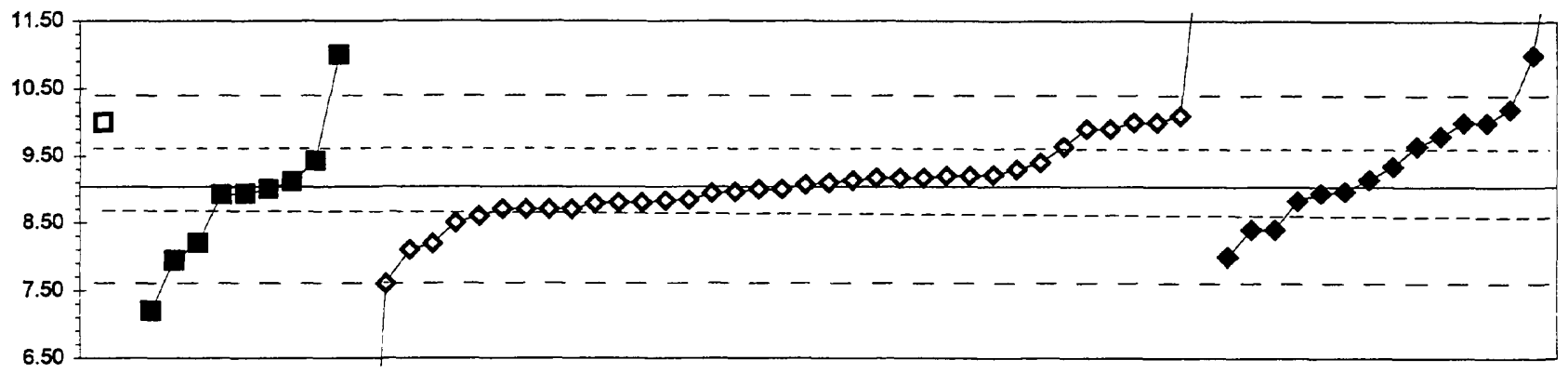

$-\square-1-0-2-3-3-4-6$

\begin{tabular}{|c|c|c|c|c|c|c|c|}
\hline $\begin{array}{l}\text { 1. AA: dir } \\
\text { 2. AA: dir } \\
\text { 3. AA: gr }\end{array}$ & $\begin{array}{l}\text { ect air } \\
\text { ect nitrou } \\
\text { aphite fu }\end{array}$ & $\begin{array}{l}\text { axide } \\
\text { ace }\end{array}$ & & $\begin{array}{l}\text { ICP } \\
\text { ICP/MS } \\
\text { Other }\end{array}$ & & & \\
\hline & & $N=$ & 1 & 0 & 9 & 37 & 15 \\
\hline & & inimum = & 10.00 & $<10$ & 7.20 & 2.30 & 8.00 \\
\hline & & aximum $=$ & & & 11.00 & 13.20 & 13.00 \\
\hline & & Median $=$ & & & 8.93 & 9.00 & 9.35 \\
\hline & F-ose & osigma $=$ & & & 0.68 & 0.39 & 0.83 \\
\hline Lab & Rating & Z-value & 1 & 2 & 3 & 4 & 6 \\
\hline 1 & 4 & -0.14 & & & & & 8.94 \\
\hline 3 & 4 & 0.24 & & & & 9.20 & \\
\hline 5 & 4 & -0.28 & & & & 8.84 & \\
\hline 7 & 4 & -0.48 & & & & 8.70 & \\
\hline 11 & 4 & -0.05 & & & & 9.00 & \\
\hline 13 & 4 & 0.19 & & & & 9.17 & \\
\hline 15 & 4 & -0.48 & & & & 8.70 & \\
\hline 16 & 3 & -0.91 & & & & & 8.40 \\
\hline 18 & 4 & 0.24 & & & & 9.20 & \\
\hline 25 & 2 & -1.34 & & & & 8.10 & \\
\hline 26 & 4 & 0.19 & & & & 9.17 & \\
\hline 30 & 0 & -2.06 & & & & 7.60 & \\
\hline 32 & 3 & 0.88 & & & & & 9.65 \\
\hline 36 & 0 & 2.82 & & & 11.00 & & \\
\hline 40 & 0 & -9.67 & & & & 2.30 & \\
\hline 42 & 0 & 5.69 & & & & & 13.00 \\
\hline 46 & 4 & -0.11 & & & & 8.96 & \\
\hline 48 & 2 & 1.38 & & & & 10.00 & \\
\hline 59 & 2 & 1.10 & & & & & 9.80 \\
\hline 68 & 4 & 0.38 & & & & 9.30 & \\
\hline 69 & 1 & -1.57 & & & 7.94 & & \\
\hline 70 & 4 & 0.19 & & & & 9.17 & \\
\hline 75 & 4 & 0.09 & & & & 9.10 & \\
\hline 76 & 1 & 1.67 & & & & & 10.20 \\
\hline 81 & 2 & -1.49 & & & & & 8.00 \\
\hline 83 & 4 & -0.34 & & & & 8.80 & \\
\hline 85 & 3 & 0.87 & & & & 9.64 & \\
\hline 86 & 4 & -0.35 & & & & 8.79 & \\
\hline 89 & 2 & -1.20 & & & 8.20 & & \\
\hline 96 & 2 & 1.38 & 10.00 & & & & \\
\hline 97 & 3 & 0.57 & & & 9.43 & & \\
\hline 102 & 4 & -0.34 & & & & 8.80 & \\
\hline 105 & 2 & 1.38 & & & & & 10.00 \\
\hline 113 & $\overline{1}$ & 1.53 & & & & 10.10 & \\
\hline 114 & NR & & & $<10$ & & & \\
\hline 119 & 4 & -0.17 & & & 8.92 & & \\
\hline 121 & 2 & 1.38 & & & & 10.00 & \\
\hline 128 & 3 & -0.91 & & & & & 8.40 \\
\hline 133 & 3 & 0.52 & & & & 9.40 & \\
\hline 134 & 4 & 0.14 & & & & 9.13 & \\
\hline 138 & 4 & 0.27 & & & & 9.22 & \\
\hline 141 & 4 & 0.05 & & & & 9.07 & \\
\hline 142 & 4 & 0.45 & & & & & 9.35 \\
\hline 144 & 0 & -12.96 & $<0.01$ & & & & \\
\hline 145 & 2 & 1.24 & & & & 9.90 & \\
\hline 146 & 2 & -1.21 & & & & 8.19 & \\
\hline 151 & 4 & -0.09 & & & & & 8.97 \\
\hline 158 & 2 & 1.24 & & & & 9.90 & \\
\hline 180 & 4 & -0.48 & & & & 8.70 & \\
\hline 191 & 2 & 1.38 & & & & & 10.00 \\
\hline
\end{tabular}

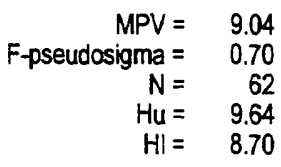

\begin{tabular}{|c|c|c|c|c|c|c|c|}
\hline \multirow[b]{2}{*}{ Lab } & \multirow[b]{2}{*}{ Rating } & \multirow[b]{2}{*}{ Z-value } & \multicolumn{2}{|c|}{$\begin{aligned} \text { MPV } & = \\
\text { F-pseudosigma } & = \\
N & = \\
H u & = \\
H I & =\end{aligned}$} & \multicolumn{2}{|l|}{$\begin{array}{r}9.04 \\
0.70 \\
62 \\
9.64 \\
8.70\end{array}$} & \multirow[b]{2}{*}{6} \\
\hline & & & 1 & 2 & 3 & 4 & \\
\hline 193 & 4 & -0.05 & & & 9.00 & & \\
\hline 196 & 4 & -0.29 & & & & & 8.83 \\
\hline 212 & 0 & 2.82 & & & & & 11.00 \\
\hline 213 & 4 & 0.12 & & & 9.12 & & \\
\hline 215 & 4 & -0.48 & & & & 8.70 & \\
\hline 217 & 3 & -0.62 & & & & 8.60 & \\
\hline 224 & 0 & 5.98 & & & & 13.20 & \\
\hline 234 & 4 & -0.14 & & & & 8.94 & \\
\hline 235 & 4 & -0.05 & & & & 9.00 & \\
\hline 236 & 3 & -0.77 & & & & 8.50 & \\
\hline 241 & 0 & -2.63 & & & 7.20 & & \\
\hline 245 & 4 & -0.15 & & & 8.93 & & \\
\hline 255 & 4 & -0.29 & & & & 8.83 & \\
\hline 265 & 4 & 0.17 & & & & & 9.15 \\
\hline
\end{tabular}


Table 13. Statistical summary of reported data for standard reference water sample T-145 (trace constituents)-Continued Ca (Calcium) $\mathrm{mg} / \mathrm{L}$

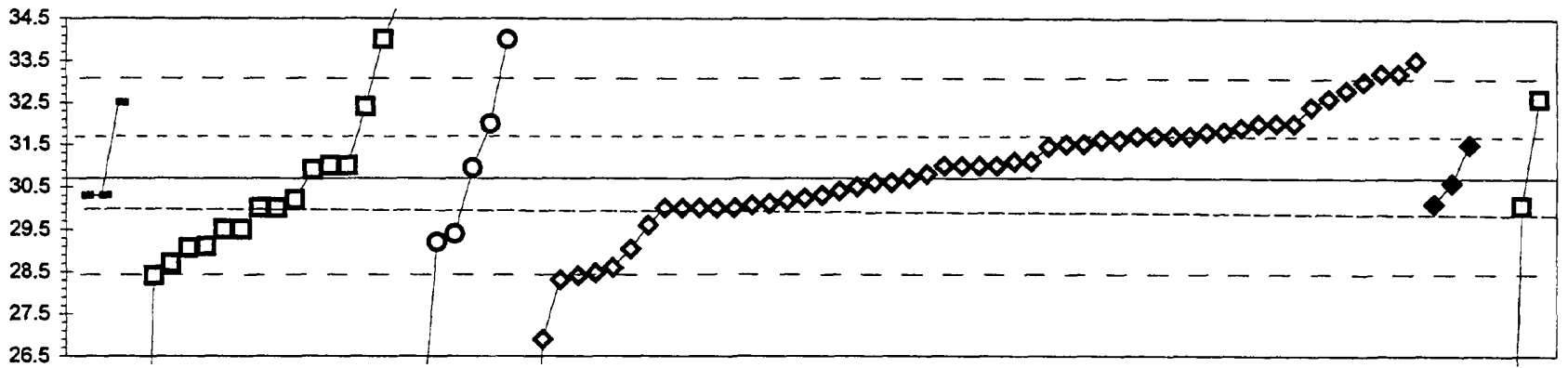

$=-0-\square-1-0-2 \diamond-4 \neg 6-\square-20$

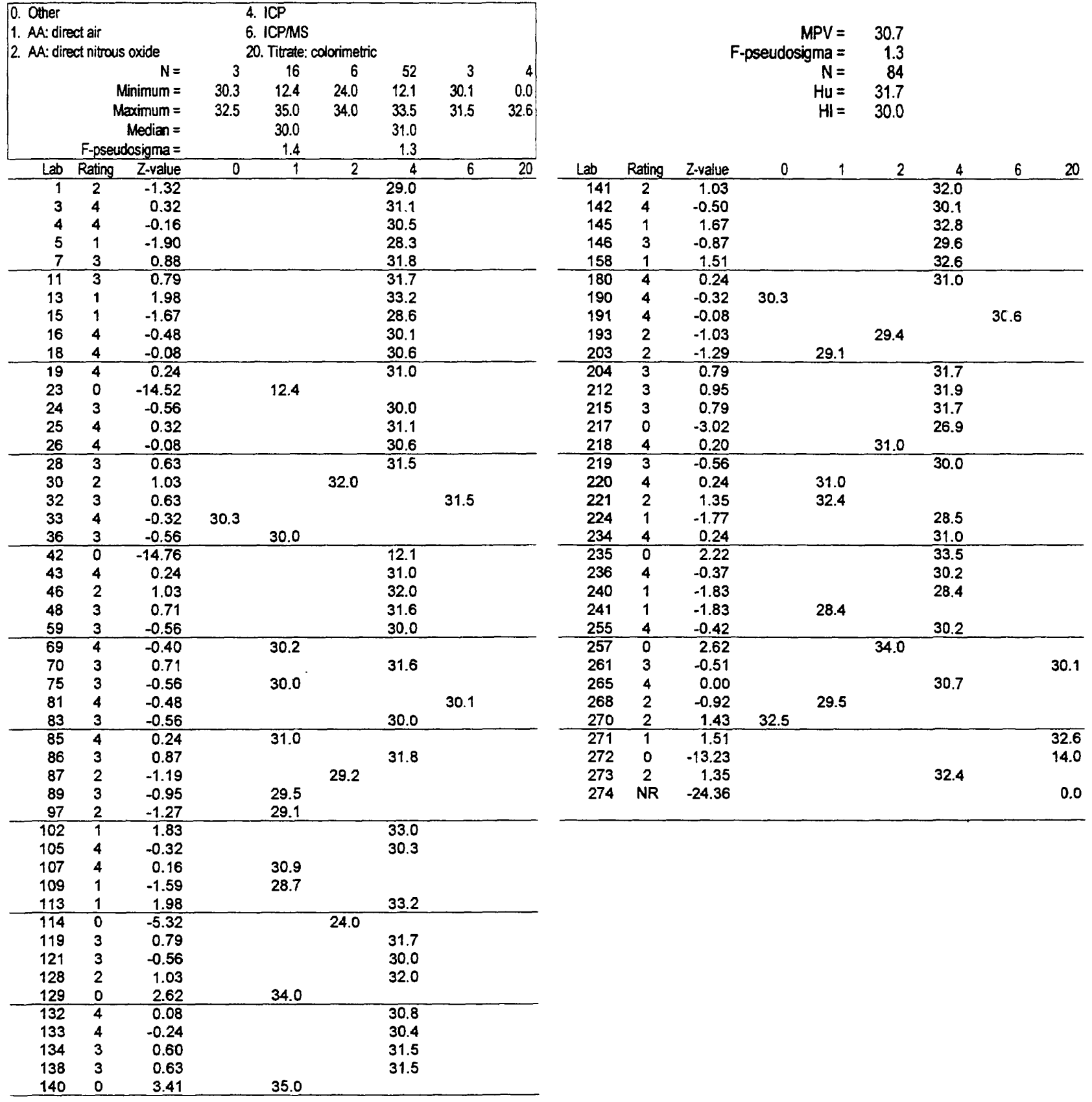


Table 13. Statistical summary of reported data for standard reference water sample T-145 (trace constituents)-Continued Cd (Cadmium)

$\mu \mathrm{g} / \mathrm{L}$

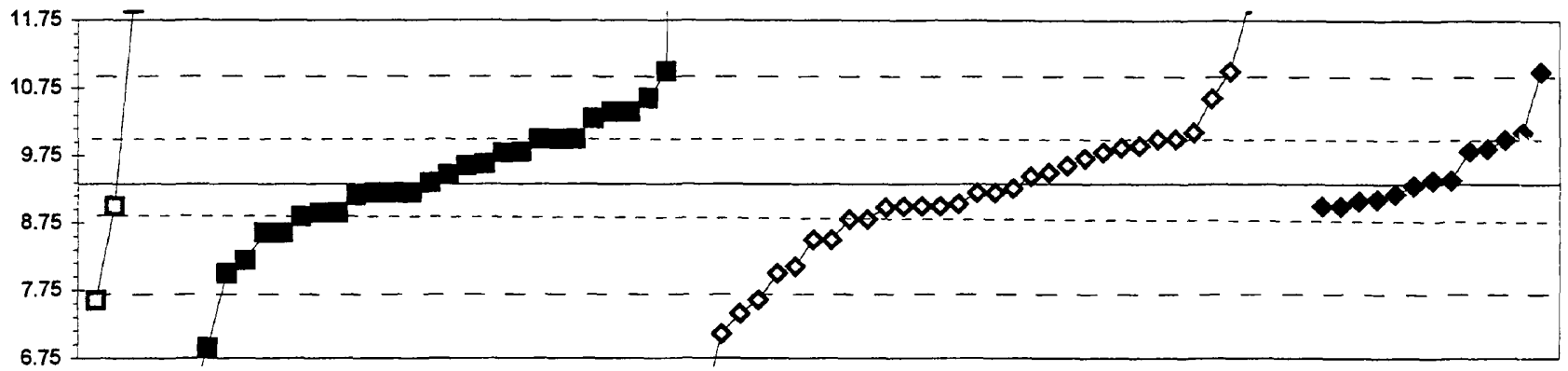

$-\square-1-0-3-4-6$

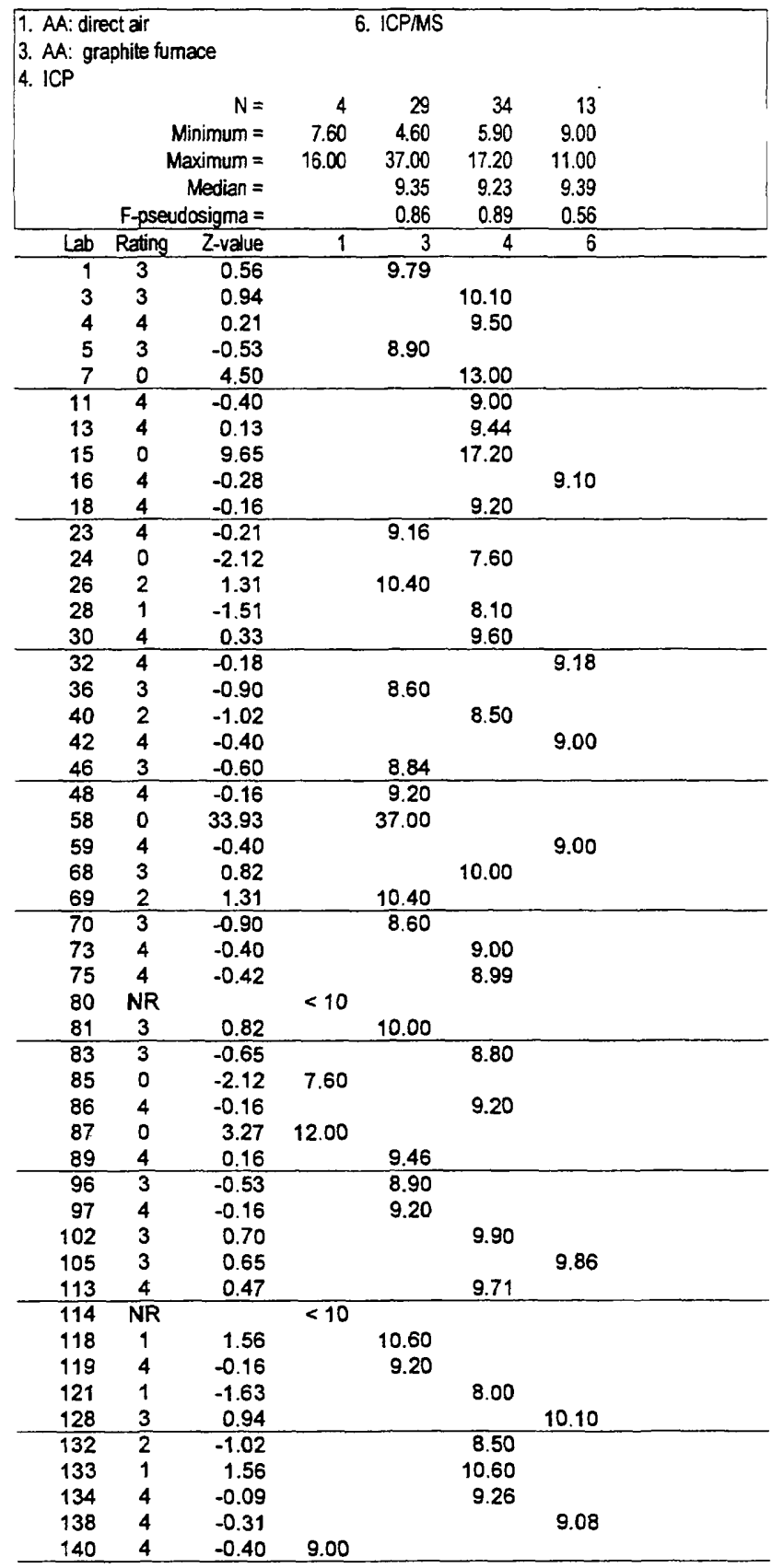

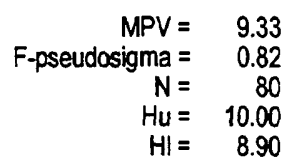

\begin{tabular}{|c|c|c|c|c|c|c|}
\hline Lab & Rating & Z-value & 1 & 3 & 4 & 6 \\
\hline 141 & 4 & -0.43 & & & 8.98 & \\
\hline 142 & 3 & 0.60 & & & & 9.82 \\
\hline 145 & 1 & 2.05 & & & 11.00 & \\
\hline 146 & 4 & -0.37 & & & 9.03 & \\
\hline 149 & 3 & 0.82 & & 10.00 & & \\
\hline 151 & 4 & 0.07 & & & & 9.39 \\
\hline 158 & 0 & -5.80 & & 4.60 & & \\
\hline 180 & 3 & 0.82 & & & 10.00 & \\
\hline 190 & 2 & 1.19 & & 10.30 & & \\
\hline 191 & 4 & -0.02 & & & & 9.31 \\
\hline 193 & 1 & 2.05 & & 11.00 & & \\
\hline 196 & 4 & 0.07 & & & & 9.39 \\
\hline 212 & 1 & 2.05 & & & & 11.00 \\
\hline 213 & 2 & -1.40 & & 8.19 & & \\
\hline 215 & 3 & 0.82 & & 10.00 & & \\
\hline 217 & 0 & -2.73 & & & 7.10 & \\
\hline 219 & 3 & -0.65 & & & 8.80 & \\
\hline 221 & 4 & 0.37 & & 9.63 & & \\
\hline 224 & 0 & -4.21 & & & 5.90 & \\
\hline 234 & 4 & 0.33 & & 9.60 & & \\
\hline 235 & 3 & 0.58 & & & 9.80 & \\
\hline 236 & 0 & -2.37 & & & 7.40 & \\
\hline 240 & 0 & 3.27 & & & 12.00 & \\
\hline 241 & 3 & 0.58 & & 9.80 & & \\
\hline 245 & 4 & 0.02 & & 9.35 & & \\
\hline 249 & 1 & -1.63 & & 8.00 & & \\
\hline 255 & 3 & 0.66 & & & 9.87 & \\
\hline 257 & 0 & 8.18 & 16.00 & & & \\
\hline 259 & 0 & -4.08 & & 6.00 & & \\
\hline 265 & 3 & 0.82 & & & & 10.00 \\
\hline 273 & 0 & 9.65 & & & 17.20 & \\
\hline 274 & 0 & -2.98 & & 6.90 & & \\
\hline
\end{tabular}

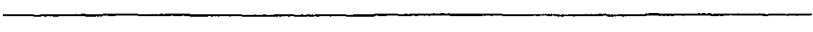


Table 13. Statistical summary of reported data for standard reference water sample T-145 (trace constituents)-Continued Co (Cobalt) $\mu \mathrm{g} / \mathrm{L}$

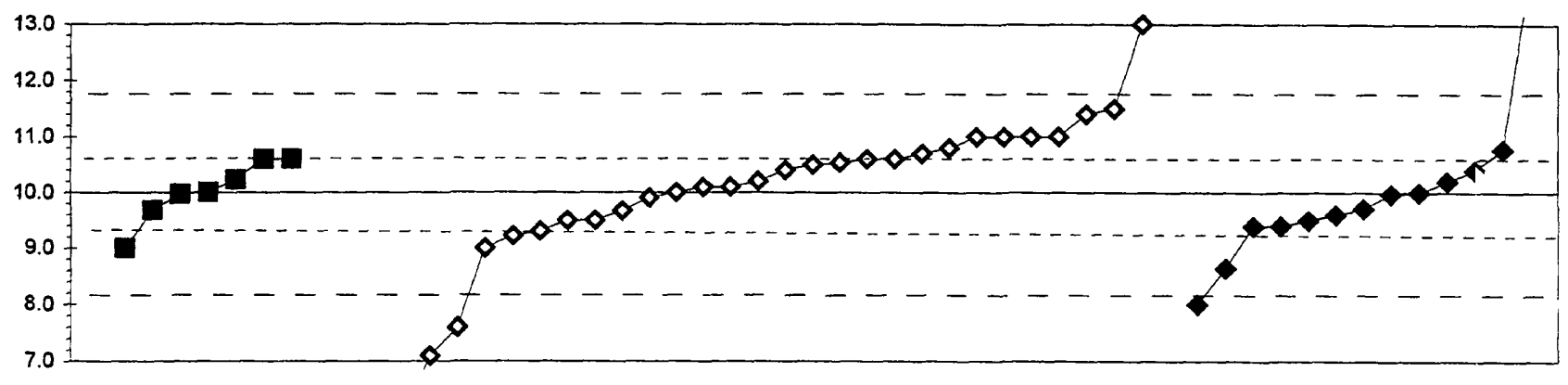

$-\square-1-a-3 \multimap-4 \multimap 6$

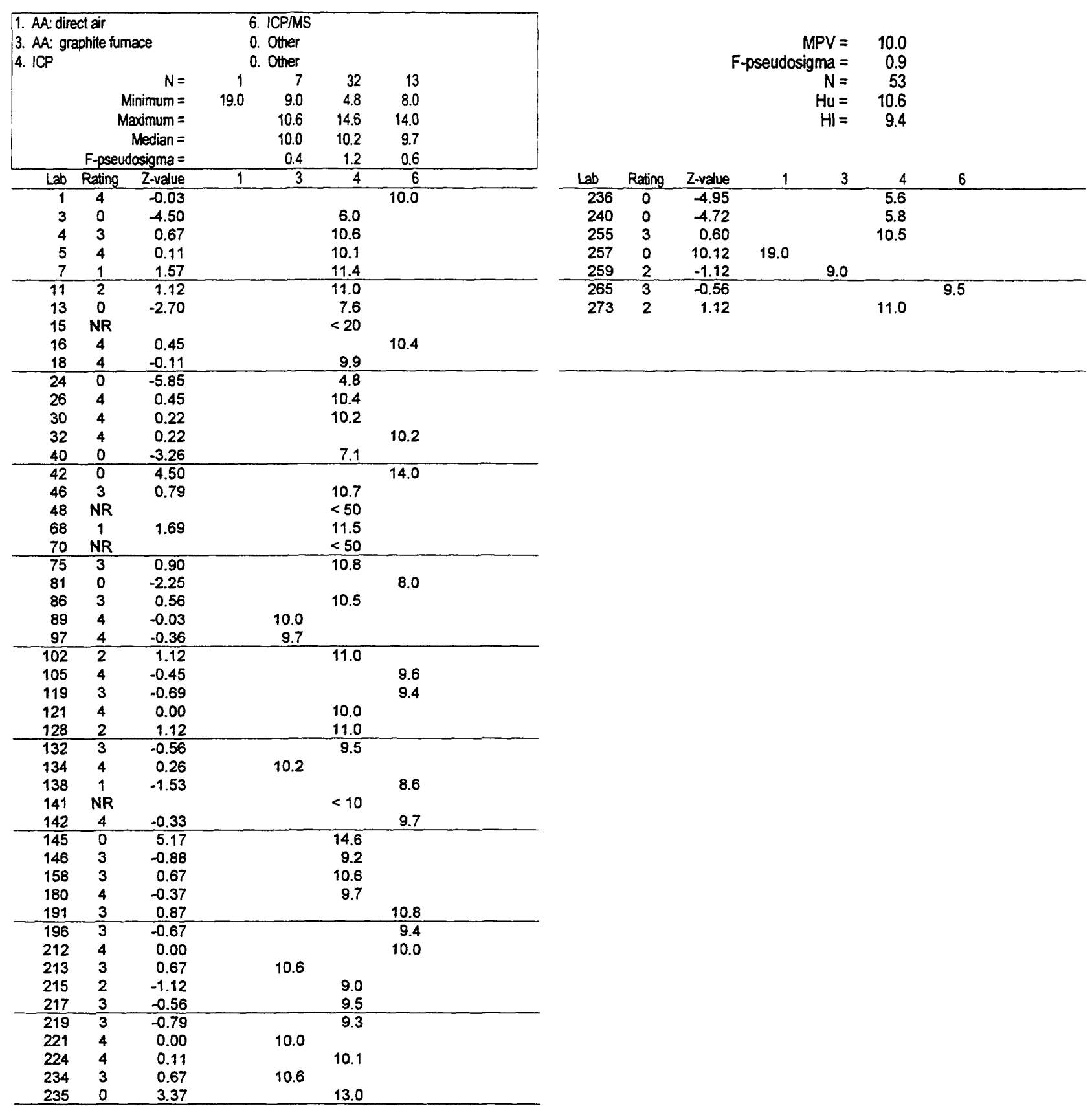


Table 13. Statistical summary of reported data for standard reference water sample $T$ - 145 (trace constituents)-Continued $\mathrm{Cr}$ (Chromium) $\mu \mathrm{g} / \mathrm{L}$

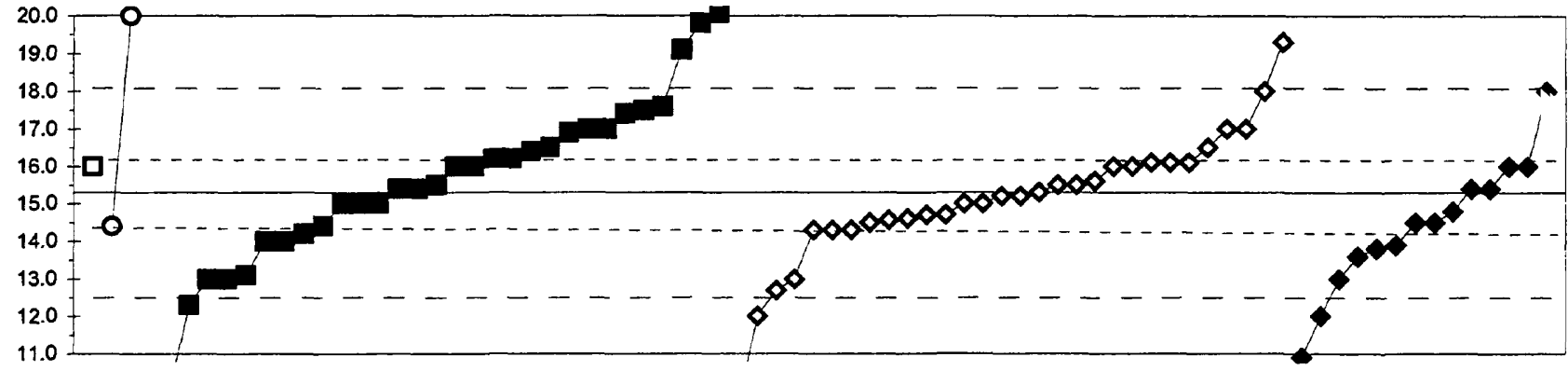

$-\square-1-0-2-a-3 \multimap-4-6$

\begin{tabular}{|c|c|c|c|c|c|c|c|}
\hline \multicolumn{3}{|c|}{$\begin{array}{l}\text { 1. AA: direct air } \\
\text { 2. AA: direct nitrous oxide } \\
\text { 3. AA: graphite fumace }\end{array}$} & & $\begin{array}{l}\text { CP } \\
\text { CP/MS }\end{array}$ & & & \\
\hline & & $N=$ & 1 & 3 & 30 & 30 & 14 \\
\hline & & nimum = & 16.0 & 14.4 & 10.0 & 9.5 & 10.9 \\
\hline & & ximum = & & 30.0 & 20.1 & 19.3 & 18.0 \\
\hline & & Median = & & & 15.8 & 15.2 & 14.5 \\
\hline & F-pset & osigma $=$ & & & 2.1 & 1.2 & 1.3 \\
\hline Lab & Rating & Z-value & 1 & 2 & 3 & 4 & 6 \\
\hline 1 & 4 & 0.14 & & & 15.5 & & \\
\hline 3 & 2 & 1.21 & & & & 17.0 & \\
\hline 4 & 4 & -0.21 & & & & 15.0 & \\
\hline 5 & 4 & 0.14 & & & & 15.5 & \\
\hline 7 & 1 & 1.92 & & & & 18.0 & \\
\hline 10 & 4 & 0.50 & & & 16.0 & & \\
\hline 11 & 4 & -0.21 & & & & 15.0 & \\
\hline 13 & 3 & 0.57 & & & & 16.1 & \\
\hline 15 & 3 & 0.57 & & & & 16.1 & \\
\hline 16 & 2 & -1.07 & & & & & 13.8 \\
\hline 18 & 3 & -0.71 & & & & 14.3 & \\
\hline 23 & 0 & -2.13 & & & 12.3 & & \\
\hline 26 & 4 & 0.07 & & & 15.4 & & \\
\hline 30 & 3 & -0.71 & & & & 14.3 & \\
\hline 32 & 3 & -0.57 & & & & & 14.5 \\
\hline 36 & 0 & -3.76 & & & 10.0 & & \\
\hline 40 & 0 & -4.12 & & & & 9.5 & \\
\hline 42 & 1 & 1.92 & & & & & 18.0 \\
\hline 46 & 3 & 0.78 & & & 16.4 & & \\
\hline 48 & 2 & 1.49 & & & 17.4 & & \\
\hline 58 & 2 & 1.21 & & & 17.0 & & \\
\hline 59 & 4 & 0.50 & & & & & 16.0 \\
\hline 68 & 3 & 0.85 & & & & 16.5 & \\
\hline 69 & 3 & -0.78 & & & 14.2 & & \\
\hline 70 & 4 & -0.07 & & & & 15.2 & \\
\hline 73 & 4 & 0.50 & & & & 16.0 & \\
\hline 75 & 3 & 0.57 & & & & 16.1 & \\
\hline 76 & 4 & -0.36 & & & & & 14.8 \\
\hline 81 & 4 & 0.50 & & & 16.0 & & \\
\hline 83 & 4 & -0.07 & & & & 15.2 & \\
\hline 85 & NR & & & & & $<10$ & \\
\hline 86 & 4 & -0.43 & & & & 14.7 & \\
\hline 87 & 3 & -0.64 & & 14.4 & & & \\
\hline 89 & 1 & 1.56 & & & 17.5 & & \\
\hline 96 & 3 & 0.64 & & & 16.2 & & \\
\hline 97 & 3 & 0.64 & & & 16.2 & & \\
\hline 102 & 4 & 0.50 & & & & 16.0 & \\
\hline 105 & 4 & 0.07 & & & & & 15.4 \\
\hline 113 & 4 & 0.21 & & & & 15.6 & \\
\hline 114 & 0 & 3.34 & & 20.0 & & & \\
\hline 118 & 3 & 0.85 & & & 16.5 & & \\
\hline 119 & 1 & -1.63 & & & 13.0 & & \\
\hline 128 & 1 & -1.63 & & & & & 13.0 \\
\hline 132 & 4 & 0.14 & & & & 15.5 & \\
\hline 133 & 4 & 0.00 & & & & 15.3 & \\
\hline 134 & 4 & -0.49 & & & & 14.6 & \\
\hline 138 & 0 & -3.12 & & & & & 10.9 \\
\hline 140 & 4 & 0.50 & 16.0 & & & & \\
\hline 141 & 0 & -2.34 & & & & 12.0 & \\
\hline 142 & 3 & -0.99 & & & & & 13.9 \\
\hline
\end{tabular}

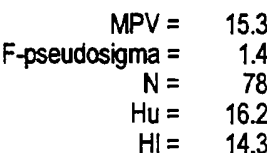

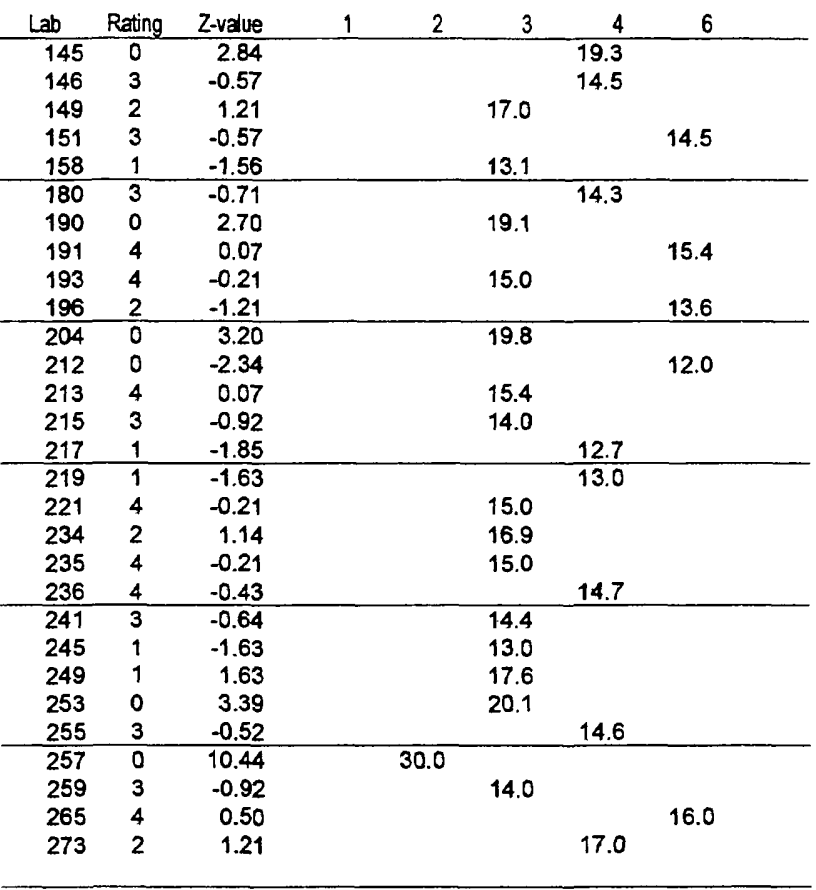


Table 13. Statistical summary of reported data for standard reference water sample T-145 (trace constituents)-Continued $\mathrm{Cu}$ (Copper) $\mu \mathrm{g} / \mathrm{L}$

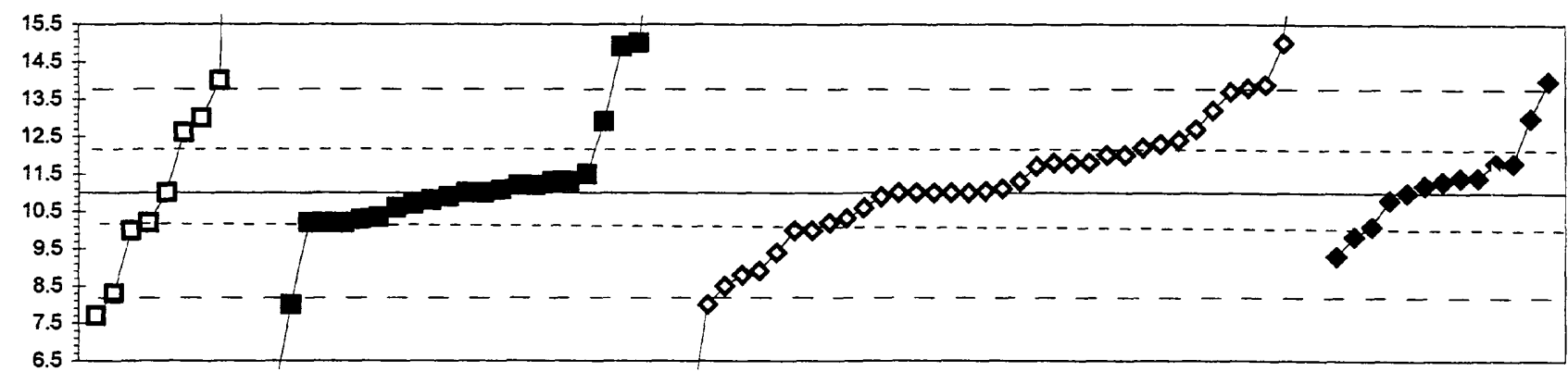

$-\square-1-3-0-4-6$

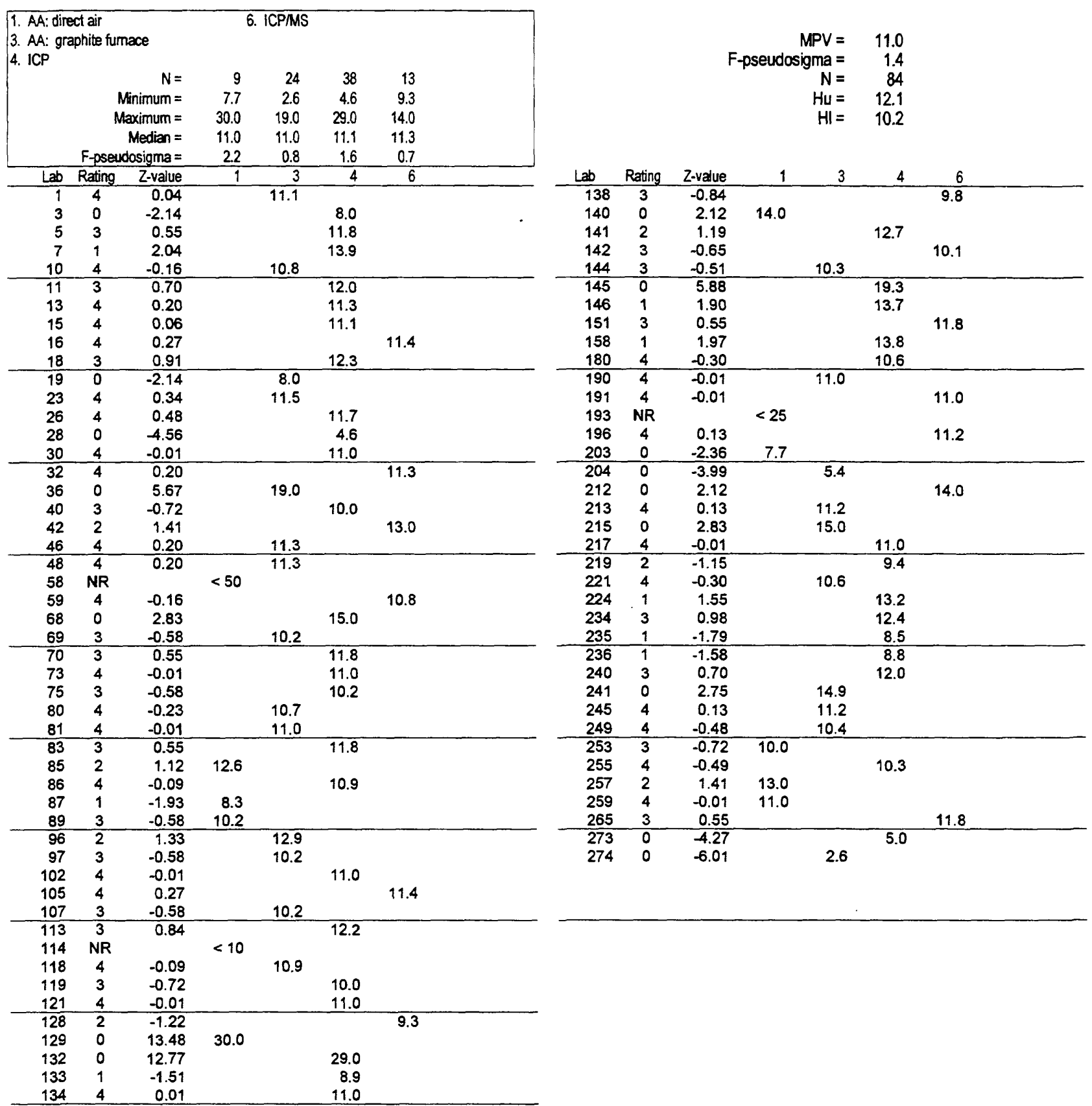


Table 13. Statistical summary of reported data for standard reference water sample T-145 (trace constituents)-Continued Fe (Iron) $\mu \mathrm{g} / \mathrm{L}$

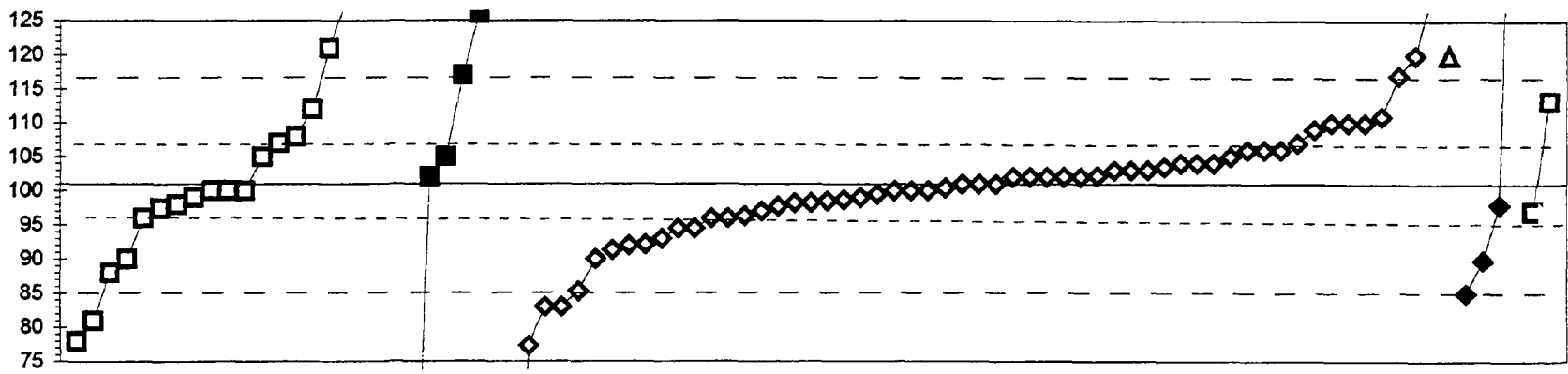

$-\square-1-\square-3-4 \Delta-5-6-\square-22$

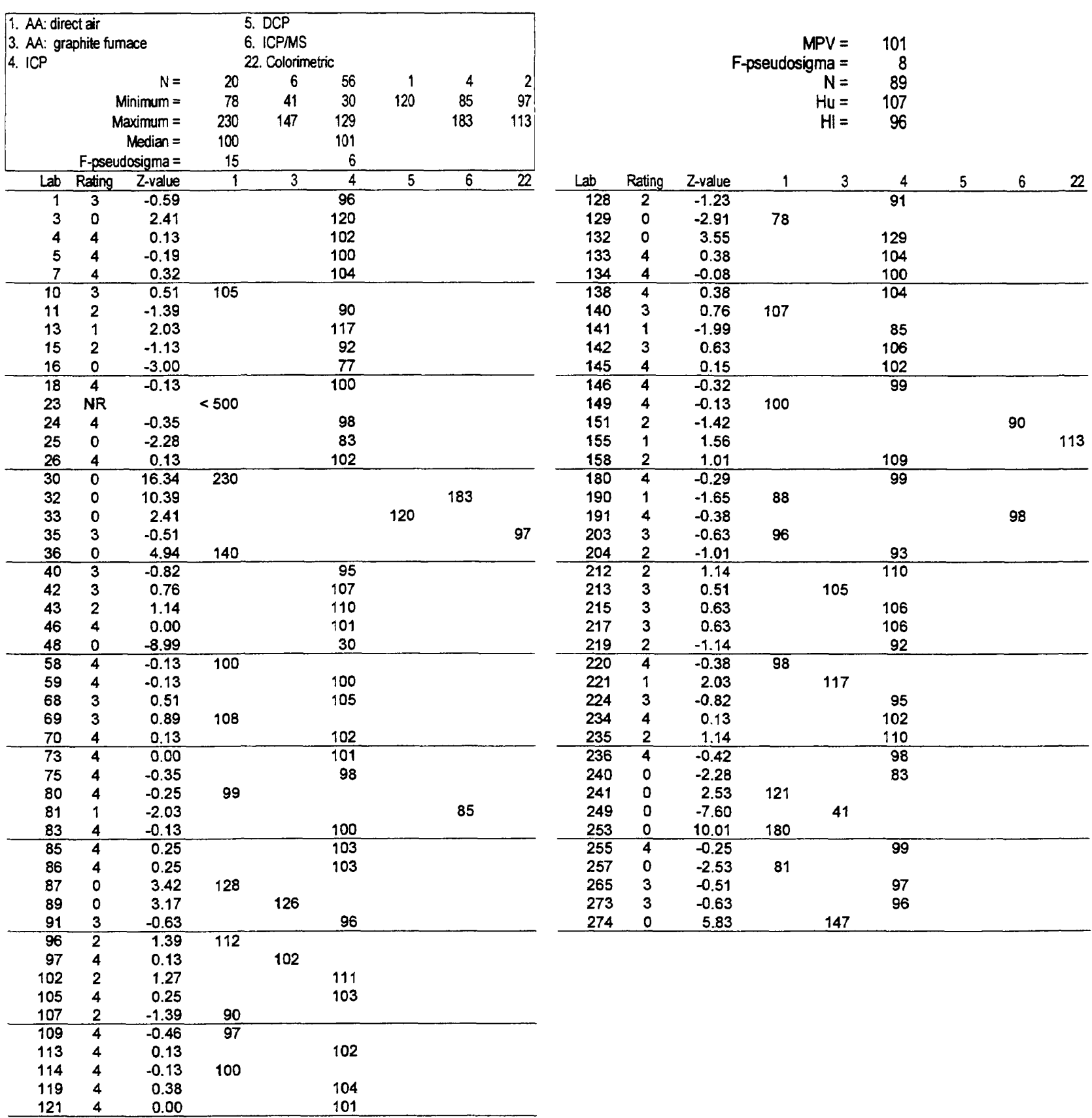


Table 13. Statistical summary of reported data for standard reference water sample T-145 (trace constituents)-Continued $\mathrm{K}$ (Potassium) $\mathrm{mg} / \mathrm{L}$

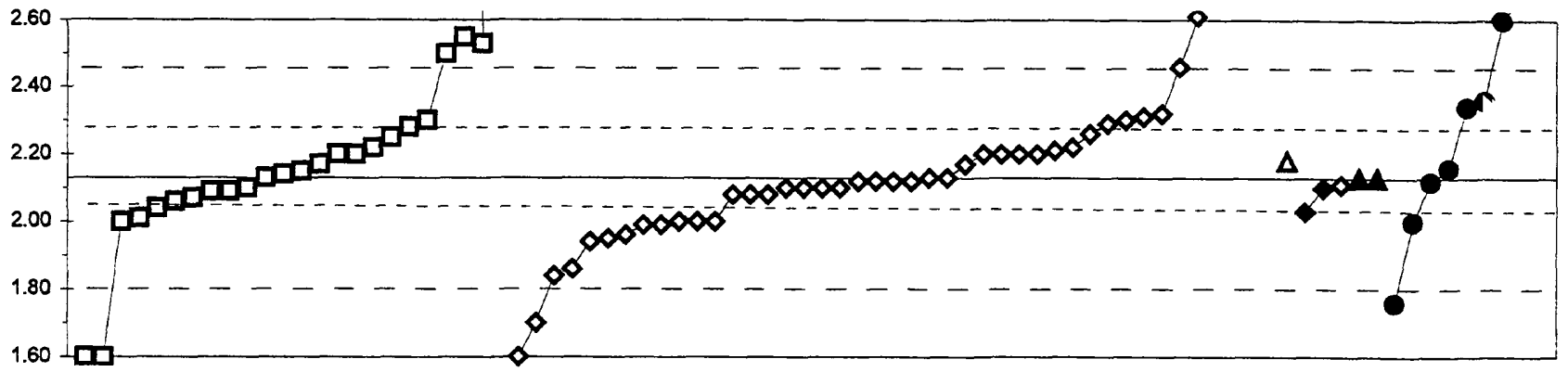

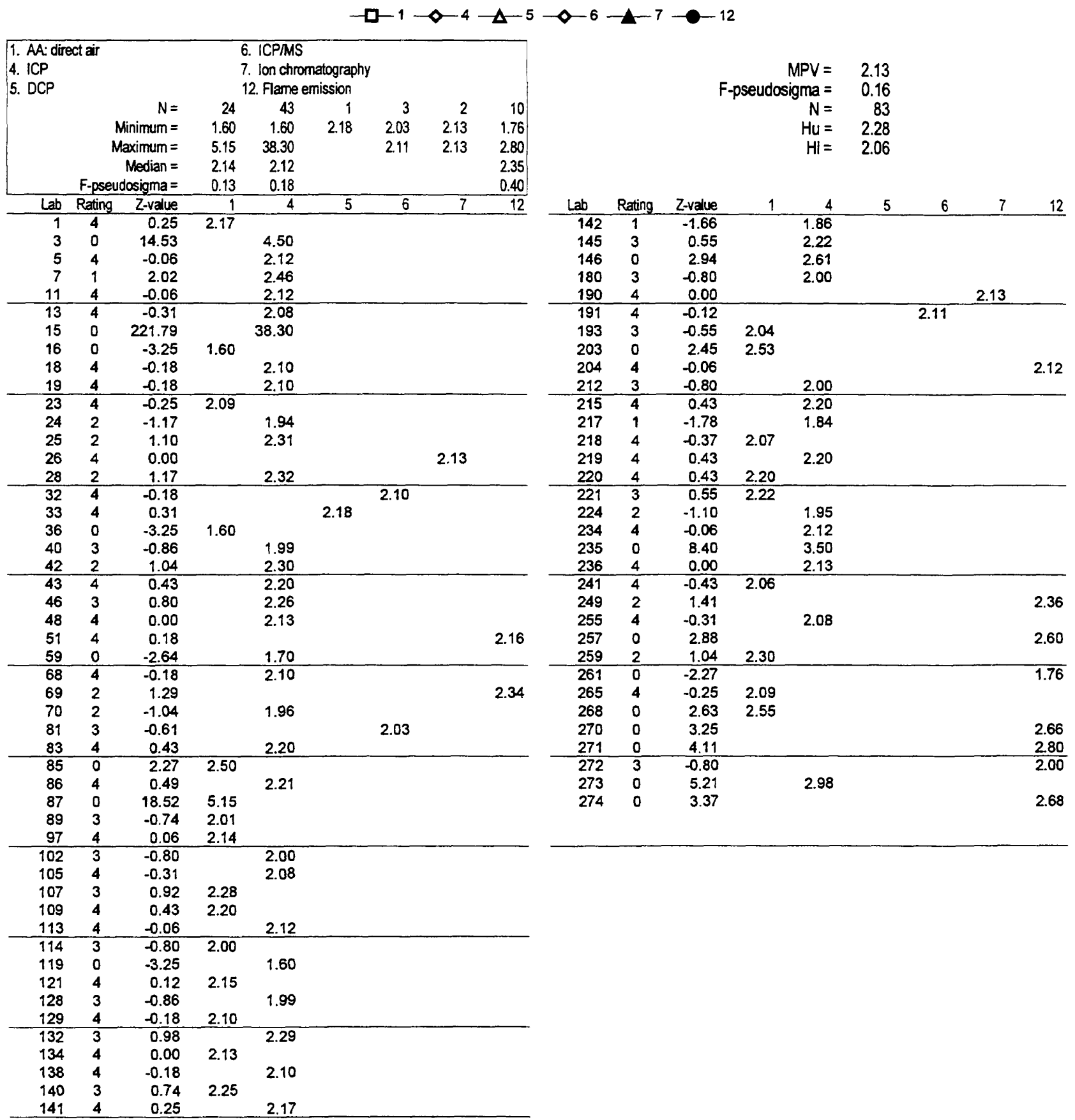


Table 13. Statistical summary of reported data for standard reference water sample T-145 (trace constituents)-Continued Li (Lithium)

$\mu \mathrm{gh}$

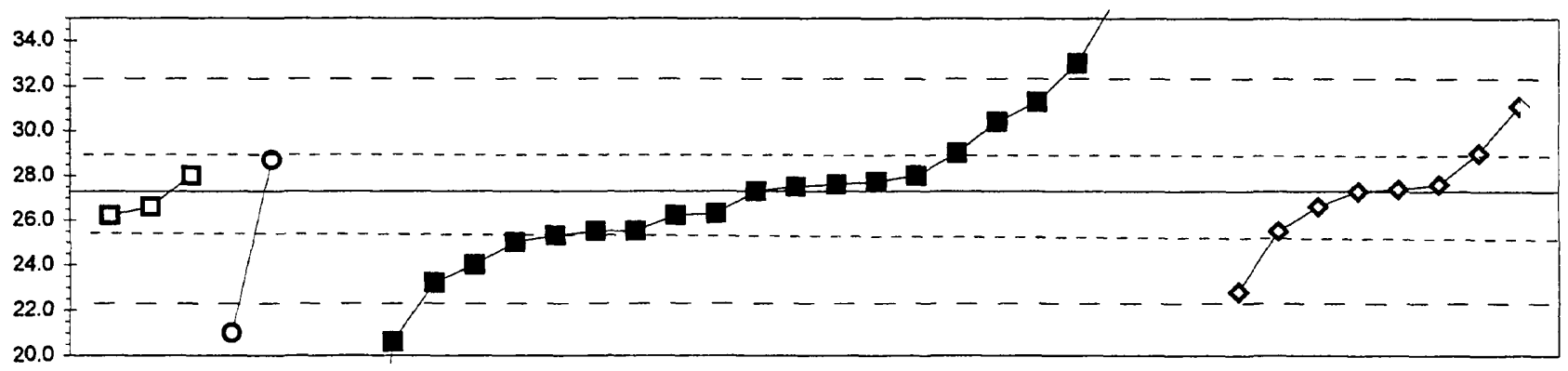

$-\square-1-0-3-4-4-6$

\begin{tabular}{|c|c|c|c|c|c|c|}
\hline $\begin{array}{l}\text { 1. AA: dire } \\
\text { 3. } A A: \text { gre } \\
\text { 4. ICP }\end{array}$ & phite fur & & & $\begin{array}{l}\text { ICP/MS } \\
\text { Other } \\
\text { Other }\end{array}$ & & \\
\hline & & $N=$ & 3 & 2 & 23 & 8 \\
\hline & & nimum = & 26.2 & 21.0 & 2.8 & 228 \\
\hline & & ximum = & 28.0 & 28.7 & 46.0 & 31.1 \\
\hline & & Median = & & & 27.3 & 27.4 \\
\hline & F-pseu & ssigma $=$ & & & 3.4 & 1.7 \\
\hline Lab & Rating & Z-value & 1 & 3 & 4 & 6 \\
\hline 1 & 3 & -0.72 & & & 25.5 & \\
\hline 3 & 0 & 2.30 & & & 33.0 & \\
\hline 4 & 0 & 3.50 & & & 36.0 & \\
\hline 5 & 4 & -0.44 & & & 26.2 & \\
\hline 7 & 4 & 0.08 & & & 27.5 & \\
\hline 11 & 0 & 6.85 & & & 44.3 & \\
\hline 16 & 1 & -1.81 & & & & 22.8 \\
\hline 24 & 4 & 0.12 & & & 27.6 & \\
\hline 25 & 3 & 0.68 & & & 29.0 & \\
\hline 26 & 3 & -0.72 & & & 25.5 & \\
\hline 30 & 2 & -1.33 & & & 24.0 & \\
\hline 32 & 4 & 0.00 & & & & 27.3 \\
\hline 40 & 3 & -0.81 & & & 25.3 & \\
\hline 42 & 0 & -8.58 & & & 6.0 & \\
\hline 68 & 0 & -9.89 & & & 2.8 & \\
\hline 69 & 3 & 0.56 & & 28.7 & & \\
\hline 75 & 4 & 0.00 & & & 27.3 & \\
\hline 76 & 4 & 0.12 & & & & 27.6 \\
\hline 85 & 4 & -0.44 & 26.2 & & & \\
\hline 105 & 1 & -1.65 & & & 23.2 & \\
\hline 109 & 4 & -0.28 & 26.6 & & & \\
\hline 134 & 4 & 0.17 & & & 27.7 & \\
\hline 142 & 4 & 0.28 & & & 28.0 & \\
\hline 145 & 1 & 1.61 & & & 31.3 & \\
\hline 151 & 3 & -0.72 & & & & 25.5 \\
\hline 191 & 1 & 1.53 & & & & 31.1 \\
\hline 196 & 4 & -0.28 & & & & 26.6 \\
\hline 212 & 3 & 0.68 & & & & 29.0 \\
\hline 217 & 4 & -0.40 & & & 26.3 & \\
\hline 219 & 3 & -0.93 & & & 25.0 & \\
\hline 234 & 2 & 1.25 & & & 30.4 & \\
\hline 236 & 0 & -2.70 & & & 20.6 & \\
\hline 257 & 4 & 0.28 & 28.0 & & & \\
\hline 259 & 0 & -2.54 & & 21.0 & & \\
\hline 265 & 4 & 0.04 & & & & 27.4 \\
\hline 273 & 0 & 7.53 & & & 46.0 & \\
\hline
\end{tabular}


Table 13. Statistical summary of reported data for standard reference water sample T-145 (trace constituents)-Continued Mg (Magnesium) $\mathrm{mgh}$

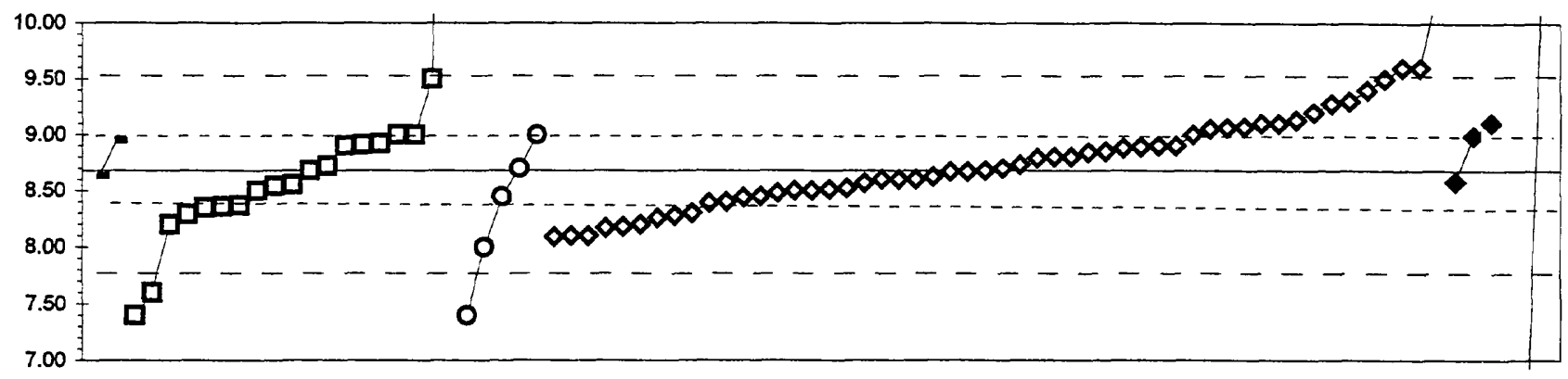

$=0-\square-1-0-2-\Delta-4-6-\square-20$

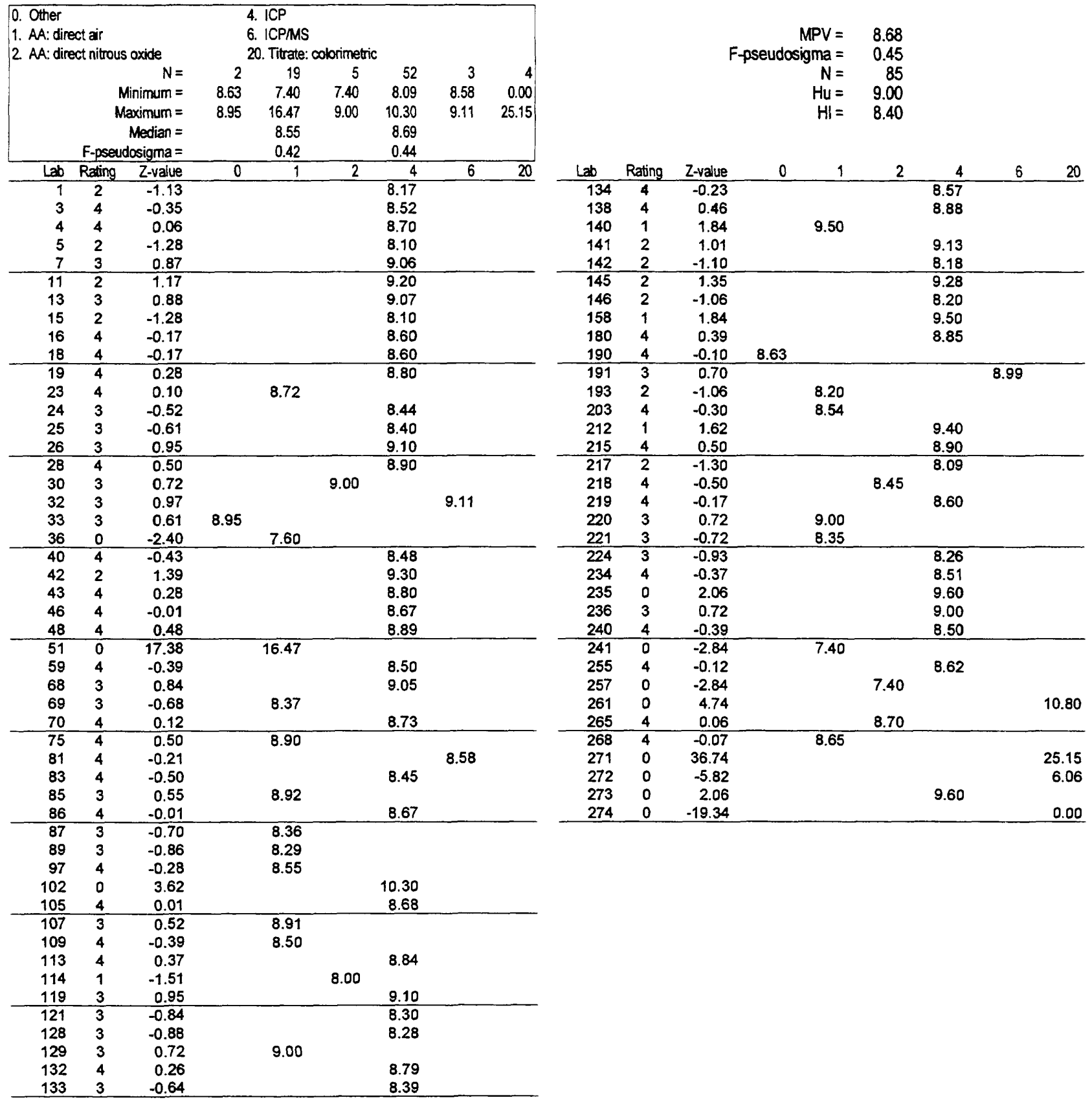


Table 13. Statistical summary of reported data for standard reference water sample T-145 (trace constituents)-Continued $\mathrm{Mn}$ (Manganese) $\mu \mathrm{g} / \mathrm{L}$

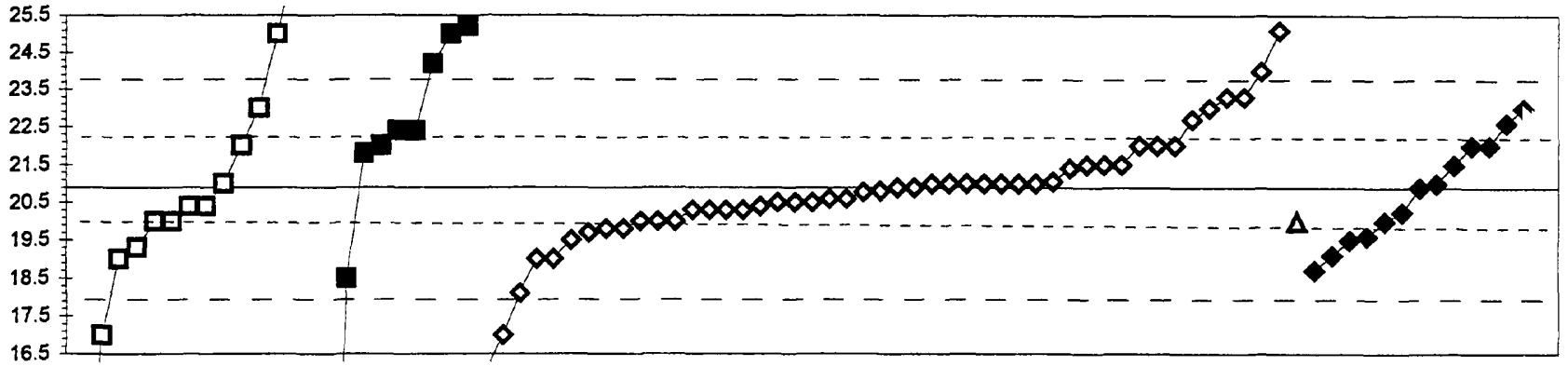

$-[-1 \multimap-3-4-\Delta-5 \multimap 6-x-22$

\begin{tabular}{|c|c|c|c|c|c|c|c|c|c|c|c|c|c|c|c|c|c|}
\hline \multicolumn{3}{|c|}{$\begin{array}{l}\text { 1. } \mathrm{AA} \text { : direct air } \\
\text { 3. } \mathrm{AA} \text { : graphite furnace } \\
\text { 4. ICP }\end{array}$} & 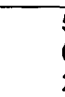 & $\begin{array}{l}\text { DCP } \\
\text { ICPMS } \\
\text { Colorin }\end{array}$ & & & & & \multicolumn{5}{|c|}{$\begin{array}{r}\text { MPV }= \\
\text { F-pseudosigma }=\end{array}$} & $\begin{array}{r}20.9 \\
15\end{array}$ & & & \\
\hline & & $N=$ & 14 & 9 & 47 & 1 & 13 & 1 & & & & & $N=$ & 85 & & & \\
\hline & & inimum = & 10.0 & 8.0 & 16.0 & 20.0 & 18.7 & 30.7 & & & & & $H u=$ & 22.0 & & & \\
\hline & & aximum = & 27.0 & 25.2 & 25.1 & & 23.0 & & & & & & $H I=$ & 20.0 & & & \\
\hline & & Median = & 20.4 & 22.4 & 20.8 & & 20.9 & & & & & & & & & & \\
\hline & F-pseu & osigma $=$ & 2.7 & 1.8 & 1.0 & & 1.8 & & & & & & & & & & \\
\hline Lab & Rating & Z-value & 1 & 3 & 4 & 5 & 6 & 22 & Lab & Rating & Z-value & 1 & 3 & 4 & 5 & 6 & 22 \\
\hline 3 & 0 & -2.63 & & & 17.0 & & & & 132 & 2 & -1.28 & & & 19.0 & & & \\
\hline 4 & 4 & 0.07 & & & 21.0 & & & & 134 & 4 & 0.10 & & & 21.1 & & & \\
\hline 5 & 4 & 0.07 & & & 21.0 & & & & 138 & 4 & -0.27 & & & 20.5 & & & \\
\hline 7 & 4 & 0.34 & & & 21.4 & & & & 140 & 2 & -1.28 & 19.0 & & & & & \\
\hline 10 & 0 & 2.77 & 25.0 & & & & & & 141 & 4 & 0.00 & & & 20.9 & & & \\
\hline 11 & 4 & 0.07 & & & 21.0 & & & & 142 & 3 & 0.74 & & & 22.0 & & & \\
\hline 13 & 3 & -0.94 & & & 19.5 & & & & 145 & 1 & 1.62 & & & 23.3 & & & \\
\hline 15 & 0 & 2.09 & & & 24.0 & & & & 146 & 4 & -0.20 & & & 20.6 & & & \\
\hline 16 & 4 & 0.40 & & & & & 21.5 & & 149 & 3 & 0.74 & 22.0 & & & & & \\
\hline 23 & 3 & 0.61 & & 21.8 & & & & & 158 & 2 & 1.21 & & & 22.7 & & & \\
\hline 24 & 3 & -0.74 & & & 19.8 & & & & 180 & 4 & -0.27 & & & 20.5 & & & \\
\hline 25 & 4 & 0.07 & & & 21.0 & & & & 183 & 0 & 2.90 & & 25.2 & & & & \\
\hline 26 & 4 & 0.40 & & & 21.5 & & & & 190 & 2 & 1.01 & & 22.4 & & & & \\
\hline 28 & 1 & 1.62 & & & 23.3 & & & & 191 & 3 & 0.74 & & & & & 22.0 & \\
\hline 30 & 4 & 0.07 & & & 21.0 & & & & 196 & 2 & -1.21 & & & & & 19.1 & \\
\hline 32 & 4 & 0.00 & & & & & 20.9 & & 203 & 3 & -0.61 & 20.0 & & & & & \\
\hline 33 & 3 & -0.61 & & & & 20.0 & & & 204 & 0 & -3.31 & & & 16.0 & & & \\
\hline 36 & 3 & 0.74 & & 22.0 & & & & & 212 & 3 & 0.74 & & & & & 22.0 & \\
\hline 40 & 1 & -1.89 & & & 18.1 & & & & 215 & 3 & -0.81 & & & 19.7 & & & \\
\hline 42 & 2 & 1.42 & & & & & 23.0 & & 217 & 3 & -0.74 & & & 19.8 & & & \\
\hline 43 & 3 & -0.61 & & & 20.0 & & & & 220 & 4 & -0.34 & 20.4 & & & & & \\
\hline 46 & 4 & -0.34 & & & 20.4 & & & & 221 & 2 & 1.01 & & 22.4 & & & & \\
\hline 48 & 3 & -0.61 & & & 20.0 & & & & 224 & 3 & 0.74 & & & 22.0 & & & \\
\hline 58 & NR & & $<50$ & & & & & & 234 & 4 & 0.00 & & & 20.9 & & & \\
\hline 59 & 4 & 0.07 & & & & & 21.0 & & 235 & 3 & -0.61 & & & 20.0 & & & \\
\hline 68 & 4 & 0.40 & & & 21.5 & & & & 236 & 4 & -0.27 & & & 20.5 & & & \\
\hline 69 & NR & & $<20$ & & & & & & 240 & 2 & -1.28 & & & 19.0 & & & \\
\hline 70 & 4 & -0.20 & & & 20.6 & & & & 241 & 2 & 1.42 & 23.0 & & & & & \\
\hline 80 & 1 & -1.62 & & 18.5 & & & & & 255 & 4 & -0.09 & & & 20.8 & & & \\
\hline 81 & 3 & -0.61 & & & & & 20.0 & & 257 & $\overrightarrow{0}$ & -2.63 & 17.0 & & & & & \\
\hline 83 & 4 & -0.40 & & & 20.3 & & & & 265 & 2 & 1.15 & & & & & 22.6 & \\
\hline 86 & 4 & -0.40 & & & 20.3 & & & & 273 & 0 & 2.83 & & & 25.1 & & & \\
\hline 87 & 0 & 4.11 & 27.0 & & & & & & 274 & 0 & -8.73 & & 8.0 & & & & \\
\hline 89 & 4 & -0.34 & 20.4 & & & & & & & & & & & & & & \\
\hline 91 & 4 & -0.40 & & & 20.3 & & & & & & & & & & & & \\
\hline 96 & 0 & 4.11 & 27.0 & & & & & & & & & & & & & & \\
\hline 97 & 0 & 2.23 & & 24.2 & & & & & & & & & & & & & \\
\hline 102 & 2 & 1.42 & & & 23.0 & & & & & & & & & & & & \\
\hline 105 & 3 & -0.88 & & & & & 19.6 & & & & & & & & & & \\
\hline 107 & 3 & -0.61 & 20.0 & & & & & & & & & & & & & & \\
\hline 109 & 2 & -1.08 & 19.3 & & & & & & & & & & & & & & \\
\hline 113 & 4 & 0.40 & & & 21.5 & & & & & & & & & & & & \\
\hline 114 & 4 & 0.07 & 21.0 & & & & & & & & & & & & & & \\
\hline 119 & 4 & 0.07 & & & 21.0 & & & & & & & & & & & & \\
\hline 121 & 3 & 0.74 & & & 22.0 & & & & & & & & & & & & \\
\hline 128 & 2 & -1.48 & & & & & 18.7 & & & & & & & & & & \\
\hline
\end{tabular}


Table 13. Statistical summary of reported data for standard reference water sample T-145 (trace constituents)-Continued Mo (Molybdenum) $\mu \mathrm{g} / \mathrm{L}$

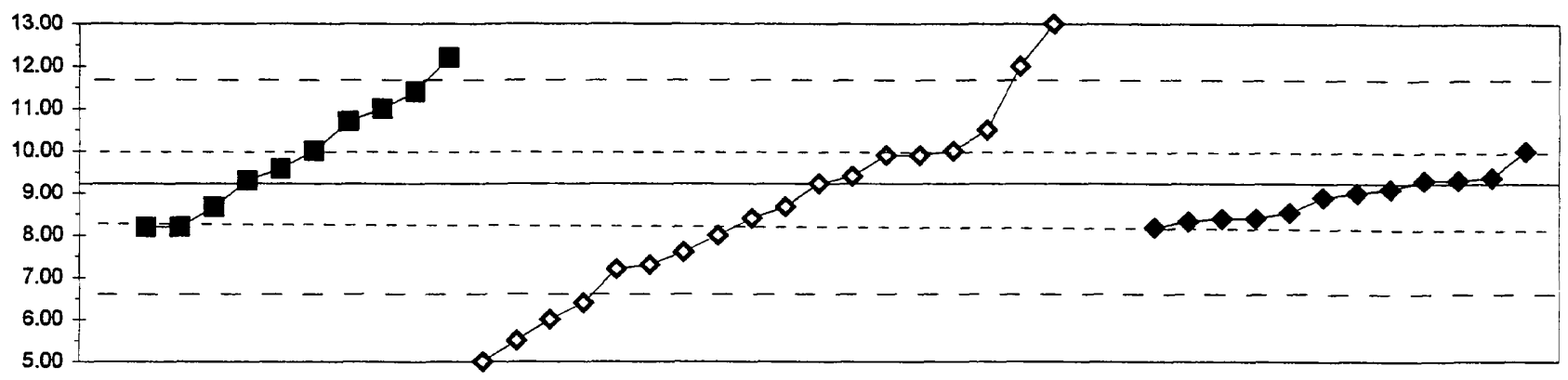

$-0-2-1-3-0-4-6$

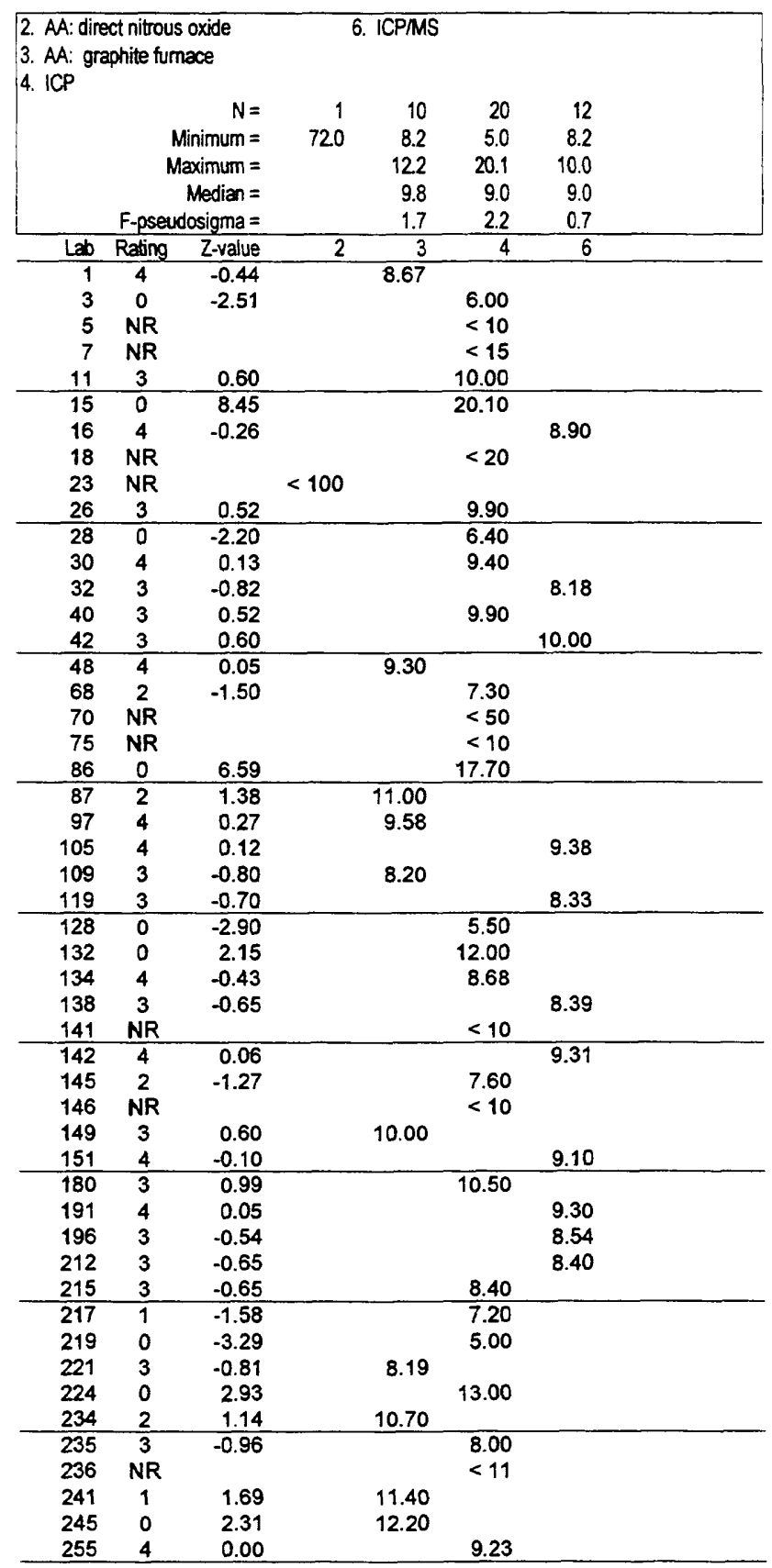

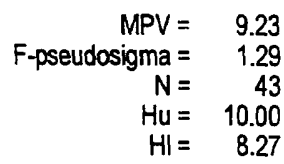

\begin{tabular}{rcrrrrr} 
Lab & Rating & Z-value & 2 & 3 & 4 & 6 \\
\hline 257 & 0 & 48.81 & 72.00 & & & 9.00 \\
265 & 4 & -0.18 & & &
\end{tabular}


Table 13. Statistical summary of reported data for standard reference water sample T-145 (trace constituents)--Continued $\mathrm{Na}$ (Sodium) $\mathrm{mg} / \mathrm{L}$

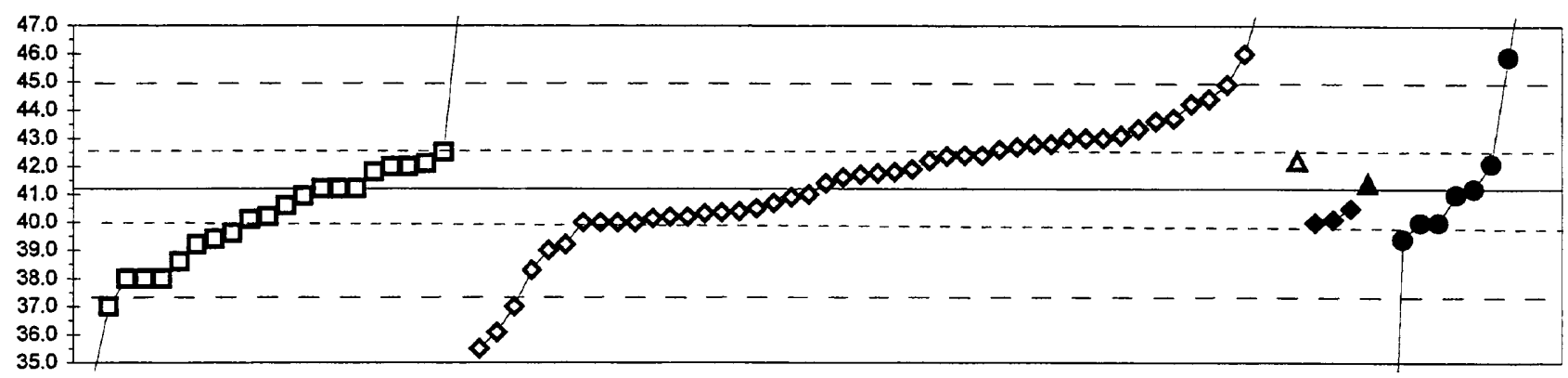

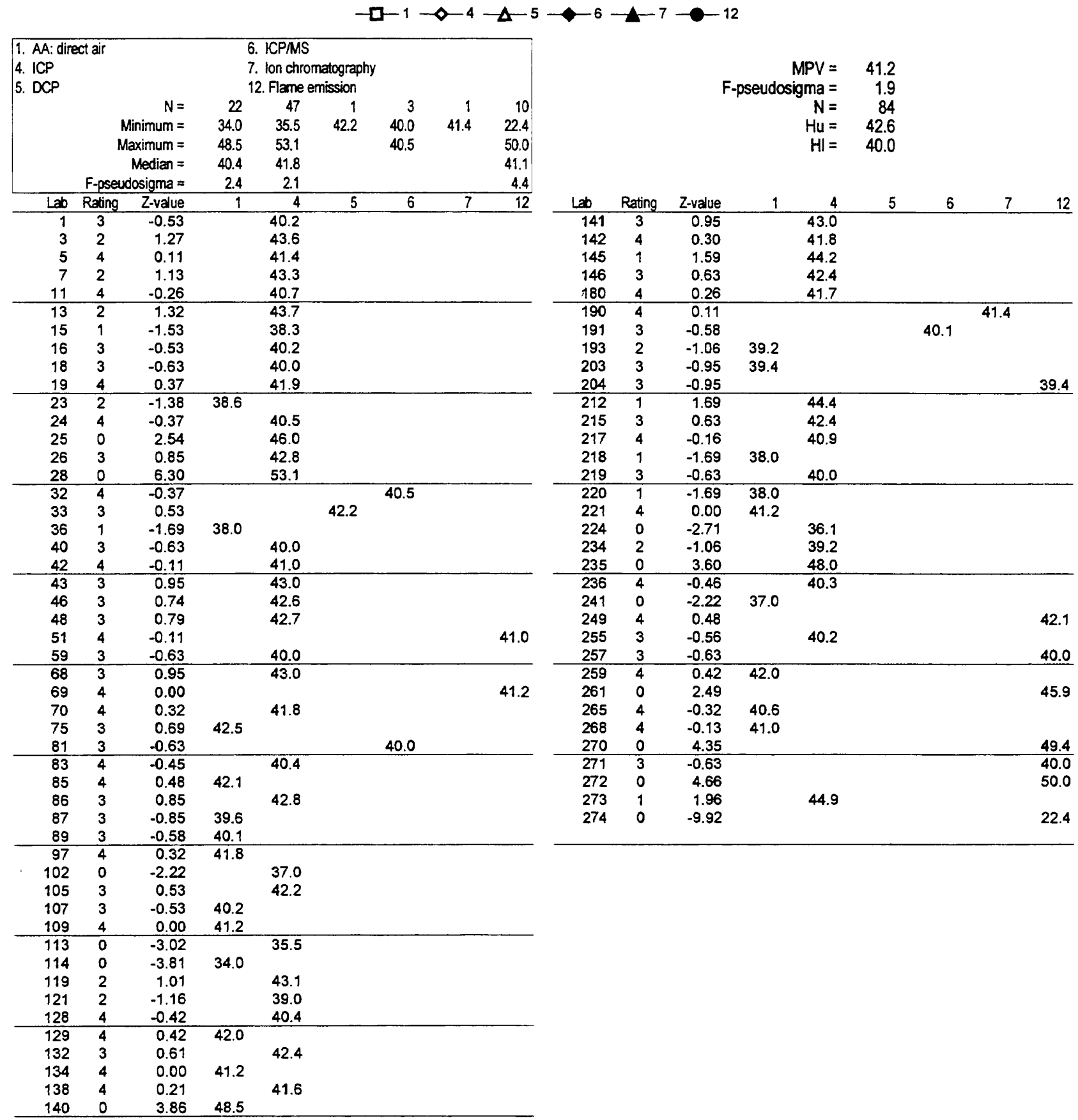


Table 13. Statistical summary of reported data for standard reference water sample T-145 (trace constituents)-Continued $\mathrm{Ni}$ (Nickel) $\mu \mathrm{g} / \mathrm{L}$

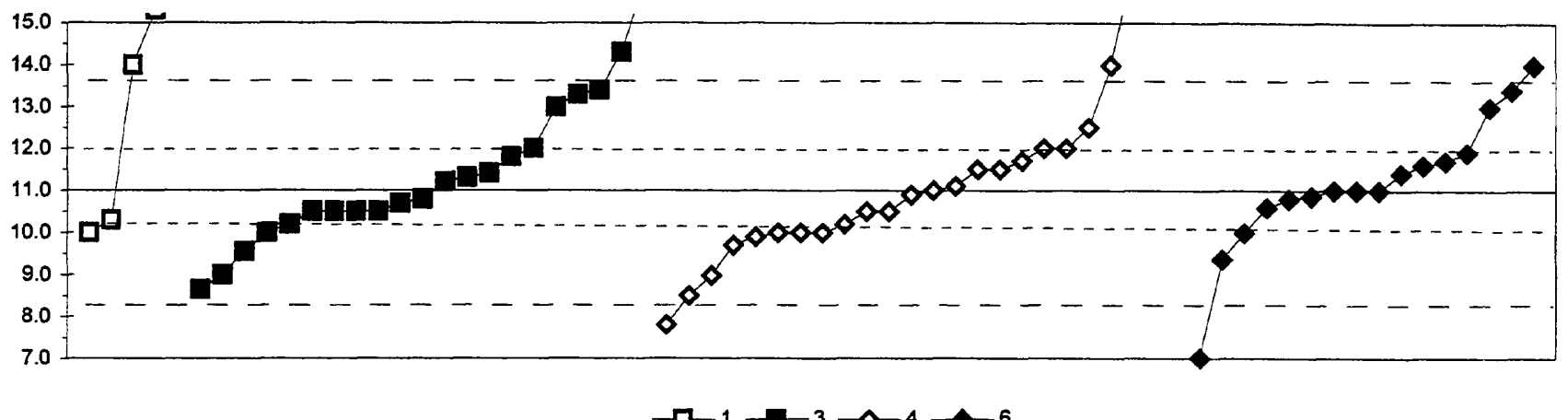

\begin{tabular}{|c|c|c|c|c|c|c|}
\hline \multicolumn{3}{|c|}{$\begin{array}{l}\text { 1. AA: direct air } \\
\text { 3. AA: graphite fumace } \\
\text { 4. ICP }\end{array}$} & \multicolumn{3}{|c|}{ 6. ICPMS } & \multirow{3}{*}{16} \\
\hline & & $N=$ & 5 & 21 & 24 & \\
\hline \multicolumn{3}{|c|}{ Minimum = } & 10.0 & 8.7 & 7.8 & \\
\hline \multicolumn{3}{|c|}{ Maximum = } & 70.0 & 16.0 & 23.0 & 14.0 \\
\hline \multicolumn{3}{|c|}{ Median = } & & 10.8 & 11.0 & 11.0 \\
\hline \multicolumn{3}{|c|}{ F-pseudosigma $=$} & & 1.1 & 1.5 & 0.8 \\
\hline Lab & Rating & Z-value & 1 & 3 & 4 & 6 \\
\hline 1 & 4 & -0.10 & & & & 10.9 \\
\hline 3 & 0 & -2.40 & & & 7.8 & \\
\hline 4 & 3 & -0.75 & & & 10.0 & \\
\hline 5 & NR & & & & $<10$ & \\
\hline 7 & NR & & & & $<20$ & \\
\hline 11 & 3 & -0.75 & & & 10.0 & \\
\hline 13 & 4 & -0.37 & & & 10.5 & \\
\hline 15 & 2 & 1.50 & & 13.0 & & \\
\hline 16 & 1 & 1.80 & & & & 13.4 \\
\hline 18 & NR & & & & $<25$ & \\
\hline 23 & 3 & 0.60 & & 11.8 & & \\
\hline 26 & 4 & -0.15 & & 10.8 & & \\
\hline 28 & 0 & 4.72 & & & 17.3 & \\
\hline 30 & 4 & 0.37 & & & 11.5 & \\
\hline 32 & 4 & 0.00 & & & & 11.0 \\
\hline 36 & 2 & -1.50 & & 9.0 & & \\
\hline 42 & 2 & 1.50 & & & & 13.0 \\
\hline 48 & 4 & 0.30 & & 11.4 & & \\
\hline 58 & 0 & 44.22 & 70.0 & & & \\
\hline 59 & 4 & 0.30 & & & & 11.4 \\
\hline 68 & 1 & -1.87 & & & 8.5 & \\
\hline 69 & 2 & -1.09 & & 9.6 & & \\
\hline 70 & NR & & & & $<50$ & \\
\hline 73 & 3 & -0.75 & & & 10.0 & \\
\hline 75 & NR & & & & $<20$ & \\
\hline 76 & 3 & 0.52 & & & & 11.7 \\
\hline 81 & 0 & -3.00 & & & & 7.0 \\
\hline 83 & 4 & -0.37 & & & 10.5 & \\
\hline 85 & NR & & & & $<10$ & \\
\hline 86 & 1 & -1.51 & & & 9.0 & \\
\hline 87 & 0 & 3.22 & 15.3 & & & \\
\hline 89 & 4 & 0.22 & & 11.3 & & \\
\hline 96 & 4 & -0.37 & & 10.5 & & \\
\hline 97 & 4 & -0.37 & & 10.5 & & \\
\hline 102 & 4 & 0.00 & & & 11.0 & \\
\hline 105 & 4 & -0.15 & & & & 10.8 \\
\hline 113 & 4 & 0.37 & & & 11.5 & \\
\hline 114 & NR & & $<10$ & & & \\
\hline 118 & 1 & 1.80 & & 13.4 & & \\
\hline 119 & 0 & 2.47 & & 14.3 & & \\
\hline 128 & 4 & 0.00 & & & & 11.0 \\
\hline 132 & 0 & 2.25 & & & 14.0 & \\
\hline 133 & 3 & -0.82 & & & 9.9 & \\
\hline 134 & 4 & -0.37 & & 10.5 & & \\
\hline 138 & 2 & -1.23 & & & & 9.4 \\
\hline 140 & 3 & -0.75 & 10.0 & & & \\
\hline 141 & NR & & & & $<20$ & \\
\hline 142 & 4 & 0.00 & & & & 11.0 \\
\hline 145 & 0 & 4.05 & & & 16.4 & \\
\hline 146 & NR & & & & $<40$ & \\
\hline
\end{tabular}

MPV $=110$

F-pseudosigma $=1.3$

$N=66$

12.0

$H I=10.2$

\begin{tabular}{|c|c|c|c|c|c|c|}
\hline Lab & Rating & Z-value & 1 & 3 & 4 & 6 \\
\hline 149 & 3 & -0.75 & & 10.0 & & \\
\hline 151 & 4 & 0.45 & & & & 11.6 \\
\hline 158 & 2 & 1.12 & & & 12.5 & \\
\hline 180 & 4 & 0.07 & & & 11.1 & \\
\hline 190 & 4 & 0.15 & & 11.2 & & \\
\hline 191 & 3 & 0.67 & & & & 11.9 \\
\hline 193 & NR & & $<50$ & & & \\
\hline 196 & 4 & -0.30 & & & & 10.6 \\
\hline 212 & 0 & 2.25 & & & & 14.0 \\
\hline 213 & 3 & -0.52 & 10.3 & & & \\
\hline 215 & 3 & 0.75 & & 12.0 & & \\
\hline 219 & 3 & -0.97 & & & 9.7 & \\
\hline 221 & 3 & -0.60 & & 10.2 & & \\
\hline 224 & 4 & -0.07 & & & 10.9 & \\
\hline 234 & 1 & 1.72 & & 13.3 & & \\
\hline 235 & 3 & 0.75 & & & 12.0 & \\
\hline 236 & 3 & -0.60 & & & 10.2 & \\
\hline 240 & 0 & 8.99 & & & 23.0 & \\
\hline 241 & 4 & -0.37 & & 10.5 & & \\
\hline 245 & 4 & -0.22 & & 10.7 & & \\
\hline 249 & 1 & -1.76 & & 8.7 & & \\
\hline 255 & 3 & 0.52 & & & 11.7 & \\
\hline 257 & 0 & 2.25 & 14.0 & & & \\
\hline 259 & 0 & 3.75 & & 16.0 & & \\
\hline 265 & 3 & -0.75 & & & & 10.0 \\
\hline 273 & 3 & 0.75 & & & 12.0 & \\
\hline
\end{tabular}


Table 13. Statistical summary of reported data for standard reference water sample T-145 (trace constituents)--Continued $\mathrm{Pb}$ (Lead) $\mu \mathrm{g} / \mathrm{L}$

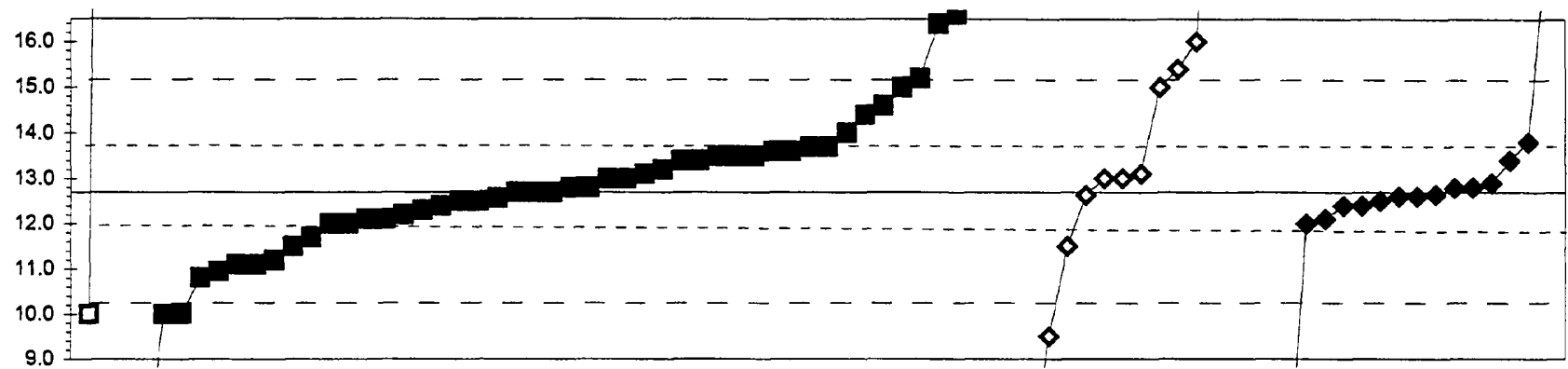

$-\square-1-\square-3-4-6$

\begin{tabular}{|c|c|c|c|c|c|c|}
\hline $\begin{array}{l}\text { 1. AA: dire } \\
\text { 3. AA: gra } \\
\text { 4. ICP }\end{array}$ & ahite fun & ace & & ICPIMS & & \\
\hline & & $N=$ & 2 & 47 & 16 & 15 \\
\hline & & inimum = & 10.0 & 1.3 & 5.6 & 6.0 \\
\hline & & ximum = & 49.0 & 42.0 & 115.0 & 18.0 \\
\hline & & Median = & & 12.7 & 13.1 & 12.6 \\
\hline & F-pseu & osigma = & & 1.2 & 6.2 & 0.3 \\
\hline Lab & Rating & Z-value & 1 & 3 & 4 & 6 \\
\hline 1 & 4 & -0.14 & & 12.6 & & \\
\hline 3 & 0 & -5.51 & & & 6.0 & \\
\hline 4 & 4 & 0.21 & & & 13.0 & \\
\hline 5 & 3 & 0.62 & & 13.5 & & \\
\hline 7 & NR & & & & $<55$ & \\
\hline 10 & 4 & -0.04 & & 12.7 & & \\
\hline 11 & 4 & 0.21 & & & 13.0 & \\
\hline 13 & 3 & 0.62 & & 13.5 & & \\
\hline 15 & 1 & 2.01 & & 15.2 & & \\
\hline 16 & 3 & 0.54 & & & & 13.4 \\
\hline 18 & 4 & -0.04 & & 12.7 & & \\
\hline 19 & 4 & 0.37 & & 13.2 & & \\
\hline 23 & 2 & -1.34 & & 11.1 & & \\
\hline 26 & 1 & -1.59 & & 10.8 & & \\
\hline 28 & 0 & -2.65 & & & 9.5 & \\
\hline 30 & 2 & -1.02 & & & 11.5 & \\
\hline 32 & 4 & -0.12 & & & & 12.6 \\
\hline 34 & 3 & 0.54 & & 13.4 & & \\
\hline 36 & 1 & 1.84 & & 15.0 & & \\
\hline 42 & 0 & -5.51 & & & & 6.0 \\
\hline 46 & 3 & 0.78 & & 13.7 & & \\
\hline 48 & 4 & 0.21 & & 13.0 & & \\
\hline 58 & 0 & 23.92 & & 42.0 & & \\
\hline 59 & 4 & -0.28 & & & & 12.4 \\
\hline 68 & 0 & 3.15 & & 16.6 & & \\
\hline 69 & 3 & -0.61 & & 12.0 & & \\
\hline 70 & 4 & 0.04 & & 12.8 & & \\
\hline 73 & 0 & 2.66 & & & 16.0 & \\
\hline 75 & 4 & -0.20 & & 12.5 & & \\
\hline 76 & 3 & -0.53 & & & & 12.1 \\
\hline 80 & 3 & -0.85 & & 11.7 & & \\
\hline 81 & 4 & 0.21 & & 13.0 & & \\
\hline 83 & 4 & -0.28 & & 12.4 & & \\
\hline 85 & NR & & $<50$ & & & \\
\hline 86 & 3 & 0.54 & & 13.4 & & \\
\hline 87 & 2 & -1.26 & & 11.2 & & \\
\hline 89 & 3 & -0.53 & & 12.1 & & \\
\hline 96 & 4 & 0.29 & & 13.1 & & \\
\hline 97 & 4 & -0.04 & & 12.7 & & \\
\hline 102 & 1 & 1.84 & & & 15.0 & \\
\hline 105 & 3 & 0.86 & & & & 13.8 \\
\hline 109 & 0 & -2.24 & & 10.0 & & \\
\hline 113 & 4 & -0.45 & & 12.2 & & \\
\hline 114 & NR & & $<10$ & & & \\
\hline 118 & 4 & -0.20 & & 12.5 & & \\
\hline 119 & 2 & -1.02 & & 11.5 & & \\
\hline 128 & 4 & 0.13 & & & & 12.9 \\
\hline 132 & 0 & 7.98 & & & 22.5 & \\
\hline 133 & 0 & 7.32 & & & 21.7 & \\
\hline 134 & 4 & -0.09 & & & 12.6 & \\
\hline
\end{tabular}

$\begin{array}{rr}\text { MPV }= & 12.7 \\ \text { F-pseudosigma } & =1.2 \\ N= & 80 \\ H U= & 13.7 \\ H I= & 12.0\end{array}$

\begin{tabular}{|c|c|c|c|c|c|c|}
\hline Lab & Rating & Z-value & 1 & 3 & 4 & 6 \\
\hline 138 & 3 & -0.61 & & & & 12.0 \\
\hline 140 & 0 & -2.24 & 10.0 & & & \\
\hline 141 & 3 & 0.70 & & 13.6 & & \\
\hline 142 & 4 & -0.08 & & & & 12.7 \\
\hline 145 & 0 & 83.60 & & & 115.0 & \\
\hline 146 & 0 & 2.17 & & & 15.4 & \\
\hline 149 & 3 & -0.61 & & 12.0 & & \\
\hline 151 & 4 & 0.04 & & & & 12.8 \\
\hline 158 & 0 & -5.02 & & 6.6 & & \\
\hline 180 & NR & & & & $<27.2$ & \\
\hline 190 & 2 & -1.34 & & 11.1 & & \\
\hline 191 & 4 & 0.04 & & & & 12.8 \\
\hline 193 & 2 & 1.03 & & 14.0 & & \\
\hline 196 & 4 & -0.12 & & & & 12.6 \\
\hline 204 & 4 & -0.36 & & 12.3 & & \\
\hline 212 & 0 & 4.30 & & & & 18.0 \\
\hline 213 & 3 & 0.70 & & 13.6 & & \\
\hline 215 & 3 & 0.62 & & 13.5 & & \\
\hline 217 & 4 & -0.20 & & & & 12.5 \\
\hline 220 & 3 & -0.53 & & 12.1 & & \\
\hline 221 & 4 & 0.04 & & 12.8 & & \\
\hline 224 & 2 & -1.47 & & 11.0 & & \\
\hline 234 & 2 & 1.35 & & 14.4 & & \\
\hline 236 & 0 & -5.84 & & & 5.6 & \\
\hline 240 & 0 & 11.65 & & & 27.0 & \\
\hline 241 & 0 & 2.99 & & 16.4 & & \\
\hline 245 & 1 & 1.52 & & 14.6 & & \\
\hline 249 & 3 & 0.78 & & 13.7 & & \\
\hline 255 & 4 & 0.29 & & & 13.1 & \\
\hline 257 & 0 & 29.64 & 49.0 & & & \\
\hline 259 & 0 & -2.24 & & 10.0 & & \\
\hline 265 & 4 & -0.28 & & & & 12.4 \\
\hline 273 & 0 & -4.70 & & & 7.0 & \\
\hline 274 & 0 & -9.35 & & 1.3 & & \\
\hline
\end{tabular}


Table 13. Statistical summary of reported data for standard reference water sample T-145 (trace constituents)-Continued $\mathrm{Sb}$ (Antimony) $\mu \mathrm{g} / \mathrm{L}$

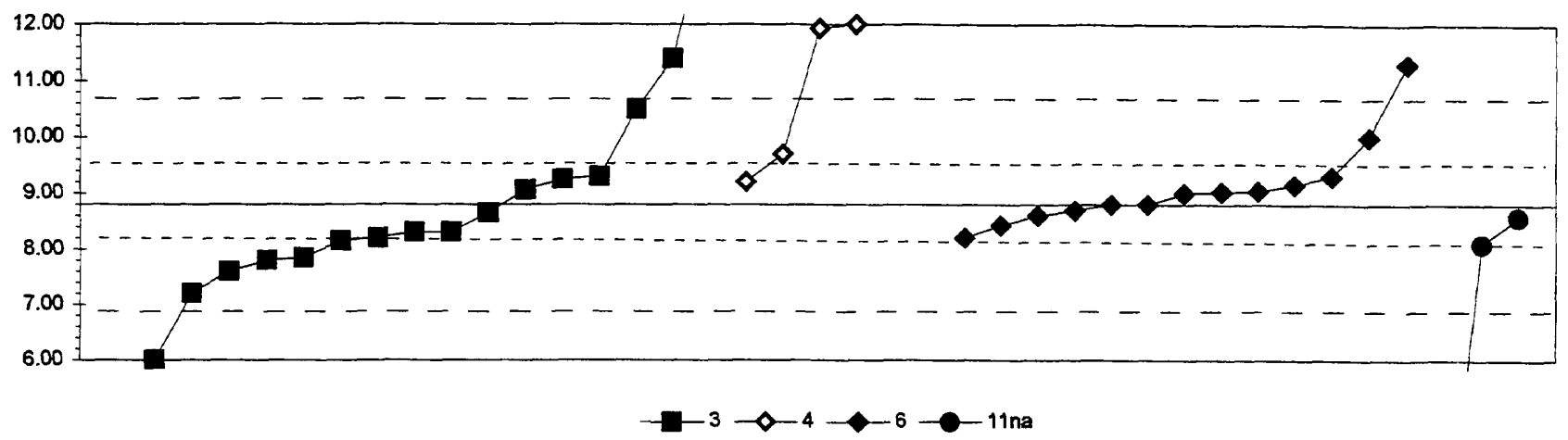

\begin{tabular}{|c|c|c|c|c|c|c|}
\hline $\begin{array}{l}\text { 3. AA: gr } \\
\text { 4. ICP } \\
\text { 6. ICPMS }\end{array}$ & phite fur & ace & & Ina AA: & lydride $\mathrm{Ne}$ & \\
\hline & & $N=$ & 17 & 6 & 13 & 3 \\
\hline & & inimum = & 5.13 & 9.20 & 8.21 & 200 \\
\hline & & sximum = & 14.00 & 26.10 & 11.30 & 8.58 \\
\hline & & Median = & 8.30 & & 9.00 & \\
\hline & F-pseu & osigma = & 1.07 & & 0.34 & \\
\hline Lab & Rating & Z-value & 3 & 4 & 6 & 11 na \\
\hline 1 & 4 & -0.16 & 8.65 & & & \\
\hline 3 & 0 & 3.33 & & 12.00 & & \\
\hline 5 & NR & & & $<20$ & & \\
\hline 7 & NR & & & $<30$ & & \\
\hline 11 & 3 & 0.94 & & 9.70 & & \\
\hline 13 & 2 & -1.04 & 7.80 & & & \\
\hline 15 & 4 & 0.47 & 9.25 & & & \\
\hline 16 & 4 & 0.00 & & & 8.80 & \\
\hline 18 & 1 & -1.67 & 7.20 & & & \\
\hline 26 & NR & & & $<20$ & & \\
\hline 30 & 4 & 0.42 & & 9.20 & & \\
\hline 32 & 4 & -0.39 & & & 8.43 & \\
\hline 36 & 0 & -2.92 & 6.00 & & & \\
\hline 42 & 2 & 1.25 & & & 10.00 & \\
\hline 46 & 3 & 0.52 & 9.30 & & & \\
\hline 48 & 2 & .1 .25 & 7.60 & & & \\
\hline 59 & 3 & 0.52 & & & 9.30 & \\
\hline 68 & 3 & -0.99 & 7.85 & & & \\
\hline 69 & 3 & -0.68 & 8.15 & & & \\
\hline 70 & 0 & 2.71 & 11.40 & & & \\
\hline 75 & NR & & & $<50$ & & \\
\hline 81 & 0 & -2.92 & & & $<6$ & \\
\hline 89 & 3 & -0.52 & 8.30 & & & \\
\hline 96 & 3 & -0.63 & 8.20 & & & \\
\hline 97 & 4 & 0.27 & 9.06 & & & \\
\hline 102 & 0 & -8.13 & & $<1$ & & \\
\hline 105 & 4 & 0.24 & & & 9.03 & \\
\hline 119 & 3 & -0.73 & & & & 8.10 \\
\hline 128 & 4 & 0.36 & & & 9.15 & \\
\hline 134 & 4 & -0.23 & & & & 8.58 \\
\hline 138 & 4 & 0.00 & & & 8.80 & \\
\hline 141 & 0 & -3.82 & 5.13 & & & \\
\hline 142 & 0 & 2.60 & & & 11.30 & \\
\hline 146 & NR & & & $<20$ & & \\
\hline 151 & 4 & 0.25 & & & 9.04 & \\
\hline 180 & NR & & & $<31.4$ & & \\
\hline 193 & NR & & $<10$ & & & \\
\hline 196 & 3 & -0.61 & & & 8.21 & \\
\hline 212 & 4 & -0.21 & & & 8.60 & \\
\hline 215 & 0 & 5.42 & 14.00 & & & \\
\hline 217 & 4 & -0.11 & & & 8.69 & \\
\hline 234 & 1 & 1.77 & 10.50 & & & \\
\hline 236 & 0 & 18.02 & & 26.10 & & \\
\hline 240 & 0 & 5.42 & & 14.00 & & \\
\hline 241 & 3 & -0.52 & 8.30 & & & \\
\hline 255 & 0 & 3.26 & & 11.93 & & \\
\hline 257 & 0 & -7.08 & & & & 2.00 \\
\hline 265 & 4 & 0.21 & & & 9.00 & \\
\hline
\end{tabular}


Table 13. Statistical summary of reported data for standard reference water sample T-145 (trace constituents)-Continued $\mathrm{Se}$ (Selenium) $\mu \mathrm{g} / \mathrm{L}$

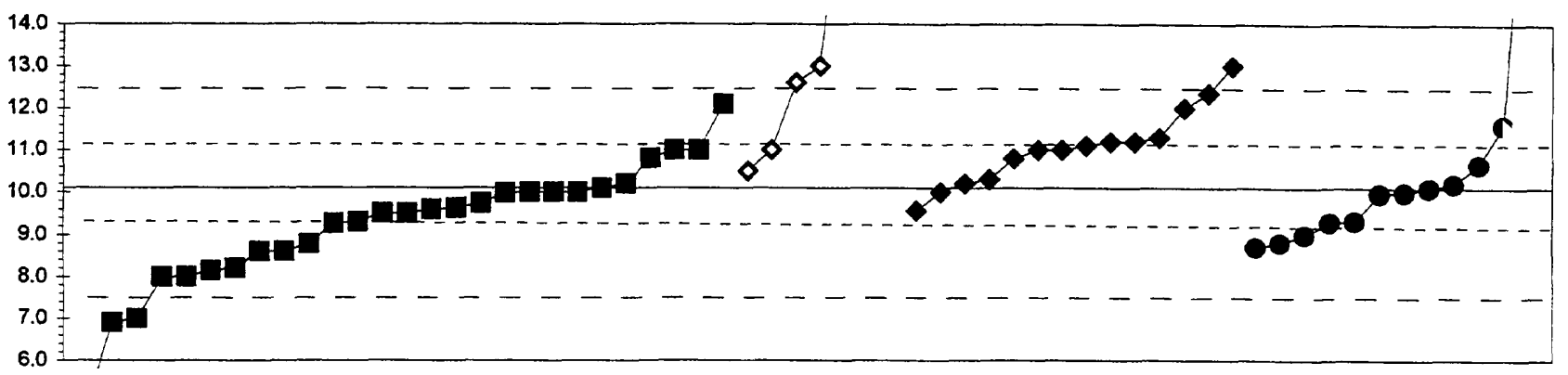

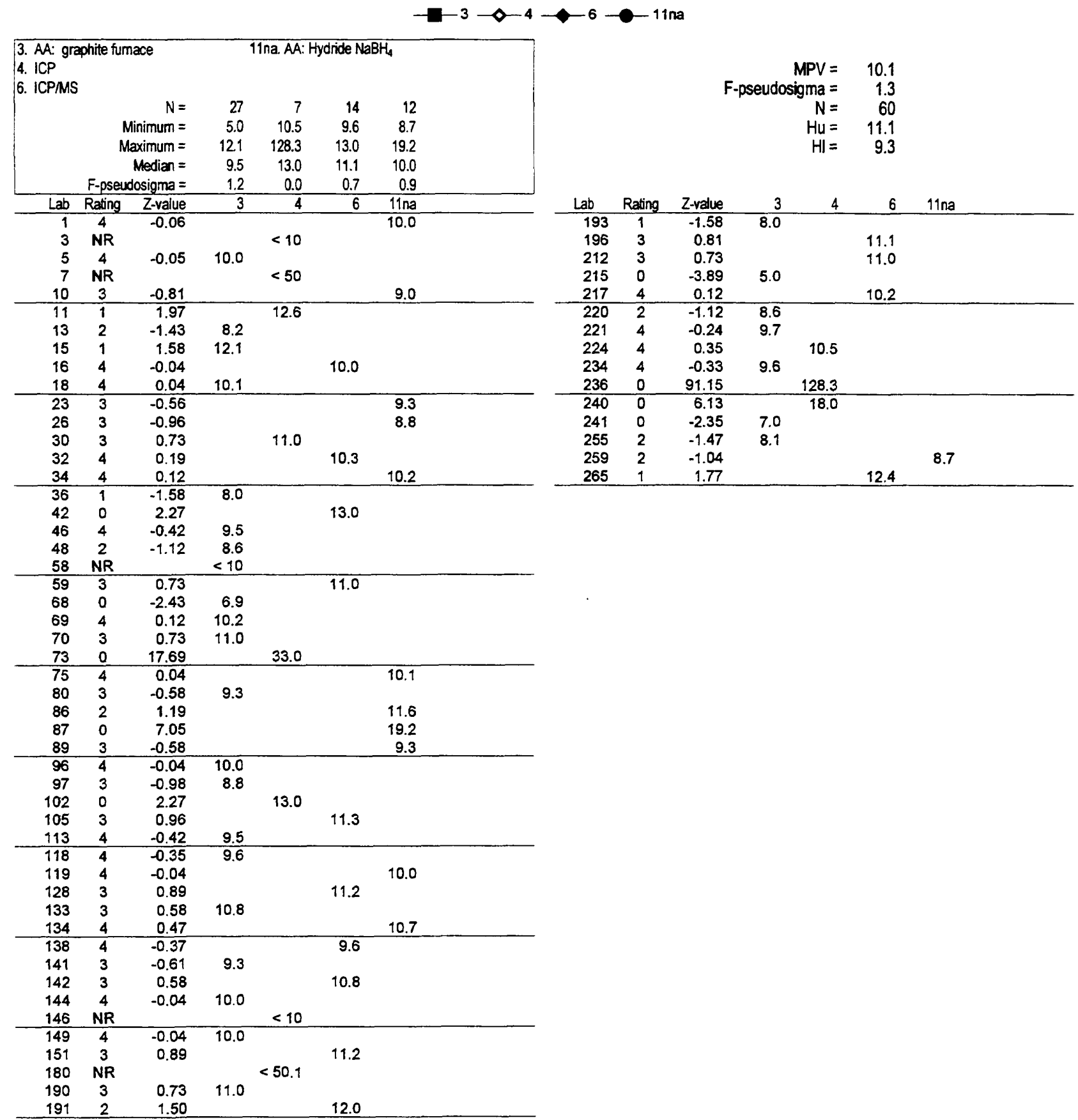


Table 13. Statistical summary of reported data for standard reference water sample T-145 (trace constituents)-Continued $\mathrm{SiO}_{2}$ (Silica) $\mathrm{mg} / \mathrm{L}$

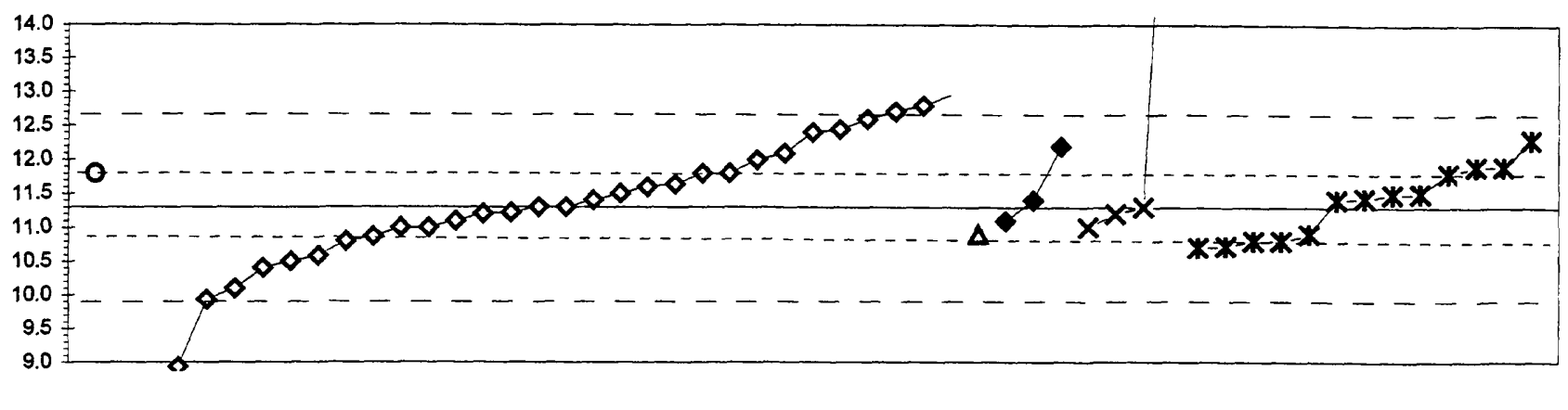

$-0-2-4-\Delta-5 \multimap-6-x-22-x-22 m b$

\begin{tabular}{|c|c|c|c|c|c|c|c|c|c|c|c|c|c|c|c|c|c|}
\hline \multicolumn{3}{|c|}{$\begin{aligned} \text { 2. AA: direct nitrous oxide } \\
\text { 4. ICP } \\
\text { 5. DCP } \\
\mathrm{N}= \\
\text { Minimum }= \\
\text { Maximum }= \\
\text { Median }= \\
\text { F-pseudosigma }=\end{aligned}$} & $\begin{array}{r}1 \\
11.8\end{array}$ & $\begin{array}{r}\text { ICPIMS } \\
\text { Colorin } \\
\text { mb. Cok } \\
31 \\
5.7 \\
227.0 \\
11.3 \\
0.9 \\
\end{array}$ & $\begin{array}{l}\text { etric } \\
\text { molybde } \\
1 \\
10.9\end{array}$ & $\begin{array}{r}\text { oblue } \\
3 \\
11.1 \\
12.2\end{array}$ & $\begin{array}{r}4 \\
11.0 \\
19.2\end{array}$ & \begin{tabular}{r|r|}
13 & 10.7 \\
12.3 \\
11.4 \\
0.7 \\
\end{tabular} & \multirow[b]{2}{*}{ Lab } & \multirow[b]{2}{*}{ Rating } & \multirow[t]{2}{*}{ Z-value } & \multirow{2}{*}{\multicolumn{2}{|c|}{$\begin{array}{r}M P V= \\
\text { seudosigma }= \\
N= \\
H u= \\
H I= \\
24\end{array}$}} & \multirow[t]{2}{*}{$\begin{array}{r}11.3 \\
0.7 \\
53 \\
11.8 \\
10.9 \\
\\
\\
\\
5\end{array}$} & \multirow[b]{2}{*}{6} & \multirow[b]{2}{*}{22} & \multirow[b]{2}{*}{$22 \mathrm{mb}$} \\
\hline Lab & Rating & Z-value & 2 & 4 & 5 & 6 & 22 & $22 \mathrm{mb}$ & & & & & & & & & \\
\hline 1 & 2 & -1.04 & & 10.6 & & & & & 265 & 1 & -1.74 & & 10.1 & & & & \\
\hline $\begin{array}{l}3 \\
4\end{array}$ & $\begin{array}{l}0 \\
4\end{array}$ & $\begin{array}{r}-8.12 \\
0.15\end{array}$ & & 5.7 & & & & & 273 & 0 & 312.88 & & 227.0 & & & & \\
\hline 5 & 4 & $\begin{array}{l}-0.29 \\
-0.10\end{array}$ & & $\begin{array}{l}11.4 \\
11.1\end{array}$ & & & & & & & & & & & & 19.2 & \\
\hline 7 & 4 & 0.49 & & 11.6 & & & & & & & & & & & & & \\
\hline 11 & 0 & -3.44 & & 8.9 & & & & & & & & & & & & & \\
\hline 13 & 4 & -0.15 & & 11.2 & & & & & & & & & & & & & \\
\hline 15 & 2 & 1.45 & & & & & & 12.3 & & & & & & & & & \\
\hline 24 & 4 & 0.44 & & 11.6 & & & & & & & & & & & & & \\
\hline 25 & 1 & 2.05 & & 12.7 & & & & & & & & & & & & & \\
\hline 26 & 4 & 0.29 & & 11.5 & & & & & & & & & & & & & \\
\hline 32 & 4 & 0.15 & & & & 11.4 & & & & & & & & & & & \\
\hline 33 & 3 & -0.58 & & & 10.9 & & & & & & & & & & & & \\
\hline 42 & 2 & 1.16 & & 12.1 & & & & & & & & & & & & & \\
\hline 43 & 4 & -0.44 & & 11.0 & & & & & & & & & & & & & \\
\hline 70 & 3 & -0.58 & & & & & & 10.9 & & & & & & & & & \\
\hline 76 & 2 & 1.31 & & & & 12.2 & & & & & & & & & & & \\
\hline 81 & 4 & 0.29 & & & & & & 11.5 & & & & & & & & & \\
\hline 83 & 2 & -1.31 & & 10.4 & & & & & & & & & & & & & \\
\hline 87 & 3 & 0.73 & & & & & & 11.8 & & & & & & & & & \\
\hline 89 & 3 & -0.87 & & & & & & 10.7 & & & & & & & & & \\
\hline 97 & 4 & 0.00 & & & & & 11.3 & & & & & & & & & & \\
\hline 104 & 3 & -0.84 & & & & & & 10.7 & & & & & & & & & \\
\hline 105 & 3 & -0.62 & & 10.9 & & & & & & & & & & & & & \\
\hline 107 & 3 & 0.87 & & & & & & 11.9 & & & & & & & & & \\
\hline 113 & 4 & -0.44 & & & & & 11.0 & & & & & & & & & & \\
\hline 118 & 4 & 0.19 & & & & & & 11.4 & & & & & & & & & \\
\hline 119 & 2 & 1.02 & & 12.0 & & & & & & & & & & & & & \\
\hline 121 & 4 & 0.00 & & 11.3 & & & & & & & & & & & & & \\
\hline 128 & 3 & 0.73 & & 11.8 & & & & & & & & & & & & & \\
\hline 129 & 4 & 0.26 & & & & & & 11.5 & & & & & & & & & \\
\hline 134 & 4 & -0.12 & & 11.2 & & & & & & & & & & & & & \\
\hline 138 & 3 & -0.73 & & & & & & 10.8 & & & & & & & & & \\
\hline 140 & 4 & -0.15 & & & & & 11.2 & & & & & & & & & & \\
\hline 142 & 0 & 2.18 & & 12.8 & & & & & & & & & & & & & \\
\hline 945 & 1 & 1.67 & & 12.5 & & & & & & & & & & & & & \\
\hline 158 & 1 & 1.60 & & 12.4 & & & & & & & & & & & & & \\
\hline 190 & 3 & 0.87 & & & & & & 11.9 & & & & & & & & & \\
\hline 191 & 4 & -0.29 & & & & 11.1 & & & & & & & & & & & \\
\hline 203 & 3 & -0.73 & & & & & & 10.8 & & & & & & & & & \\
\hline 204 & 4 & 0.15 & & & & & & 11.4 & & & & & & & & & \\
\hline 212 & 3 & 0.73 & & 11.8 & & & & & & & & & & & & & \\
\hline 215 & 4 & 0.00 & & 11.3 & & & & & & & & & & & & & \\
\hline 217 & $i$ & -1.99 & & 9.9 & & & & & & & & & & & & & \\
\hline 219 & 3 & -0.73 & & 10.8 & & & & & & & & & & & & & \\
\hline 234 & 4 & -0.44 & & 11.0 & & & & & & & & & & & & & \\
\hline 235 & 1 & 1.89 & & 12.6 & & & & & & & & & & & & & \\
\hline 236 & 0 & -6.18 & & 7.0 & & & & & & & & & & & & & \\
\hline 240 & 2 & -1.16 & & 10.5 & & & & & & & & & & & & & \\
\hline 241 & 3 & 0.73 & 11.8 & & & & & & & & & & & & & & \\
\hline
\end{tabular}


Table 13. Statistical summary of reported data for standard reference water sample T-145 (trace constituents)-Continued Sr (Strontium) $\mu \mathrm{g} / \mathrm{L}$

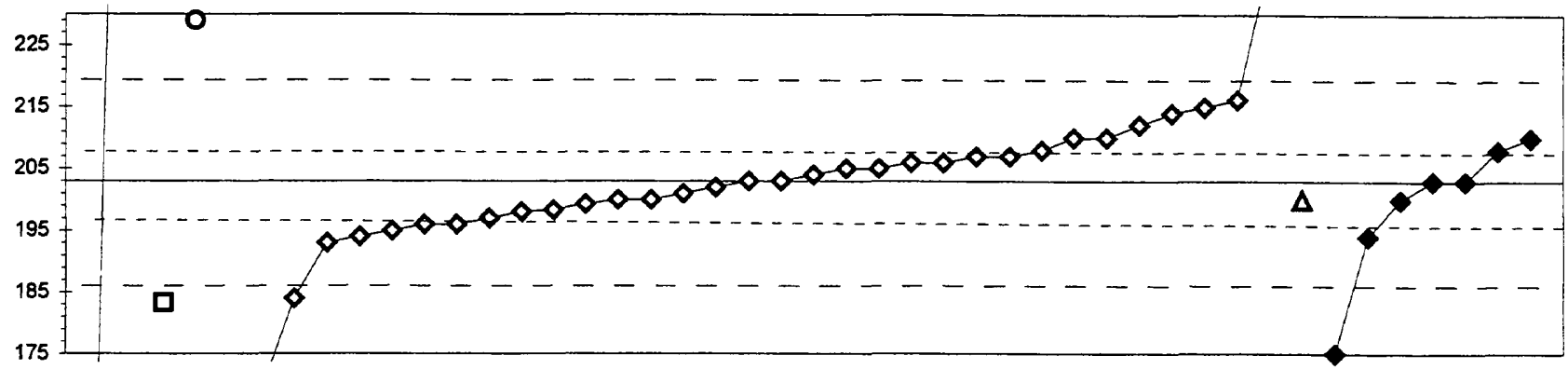

\begin{tabular}{|c|c|c|c|c|c|c|c|c|}
\hline \multicolumn{3}{|c|}{$\begin{array}{l}\text { 0. Other } \\
\text { 1. AA: direct air } \\
\text { 2. AA: direct nitrous oxide }\end{array}$} & \multicolumn{2}{|c|}{$\begin{array}{l}\text { 4. ICP } \\
\text { 5. DCP } \\
\text { 6. ICPIMS }\end{array}$} & \multirow[b]{2}{*}{1} & \multirow[b]{2}{*}{32} & \multirow[b]{2}{*}{1} & \multirow[b]{2}{*}{7} \\
\hline & & $N=$ & 2 & 1 & & & & \\
\hline & & linimum = & 169 & 183 & 229 & 169 & 200 & 175 \\
\hline & & aximum = & 390 & & & 239 & & 210 \\
\hline & & Median $=$ & & & & 203 & & 203 \\
\hline & F-pseu & losigma = & & & & 7 & & 6 \\
\hline Lab & Rating & Z-value & 0 & 1 & 2 & 4 & 5 & 6 \\
\hline 1 & 3 & -0.55 & & & & 198 & & \\
\hline 3 & 4 & 0.47 & & & & 207 & & \\
\hline 4 & 4 & 0.23 & & & & 205 & & \\
\hline 5 & 4 & 0.23 & & & & 205 & & \\
\hline 7 & 4 & 0.00 & & & & 203 & & \\
\hline$\overline{11}$ & 4 & -0.12 & & & & 202 & & \\
\hline 16 & 0 & -2.23 & & & & 184 & & \\
\hline 18 & 4 & -0.35 & & & & 200 & & \\
\hline 24 & 4 & 0.12 & & & & 204 & & \\
\hline 25 & 2 & 1.41 & & & & 215 & & \\
\hline 28 & 4 & 0.47 & & & & 207 & & \\
\hline 32 & 4 & 0.00 & & & & & & 203 \\
\hline 33 & 4 & -0.35 & & & & & 200 & \\
\hline 40 & 0 & -3.99 & & & & 169 & & \\
\hline 42 & 4 & -0.23 & & & & 201 & & \\
\hline 59 & 4 & -0.35 & & & & 200 & & \\
\hline 68 & 3 & 0.82 & & & & 210 & & \\
\hline 70 & 4 & 0.35 & & & & 206 & & \\
\hline 81 & 0 & -3.28 & & & & & & 175 \\
\hline 85 & 2 & 1.29 & & & & 214 & & \\
\hline 86 & 3 & -0.82 & & & & 196 & & \\
\hline 97 & 0 & -3.99 & 169 & & & & & \\
\hline 102 & 0 & 4.22 & & & & 239 & & \\
\hline 105 & 2 & -1.06 & & & & 194 & & \\
\hline 109 & 0 & -2.31 & & 183 & & & & \\
\hline 113 & 4 & 0.00 & & & & 203 & & \\
\hline 121 & 3 & -0.94 & & & & 195 & & \\
\hline 134 & 3 & -0.82 & & & & 196 & & \\
\hline 138 & 3 & -0.59 & & & & 198 & & \\
\hline 142 & 2 & 1.07 & & & & 212 & & \\
\hline 145 & 1 & 1.55 & & & & 216 & & \\
\hline 151 & 3 & 0.59 & & & & & & 208 \\
\hline 190 & 0 & 21.94 & 390 & & & & & \\
\hline 191 & 4 & 0.00 & & & & & & 203 \\
\hline 196 & 2 & -1.06 & & & & & & 194 \\
\hline 212 & 3 & 0.82 & & & & & & 210 \\
\hline 217 & 2 & -1.17 & & & & 193 & & \\
\hline 218 & 0 & 3.05 & & & 229 & & & \\
\hline 219 & 3 & 0.82 & & & & 210 & & \\
\hline 234 & 4 & 0.35 & & & & 206 & & \\
\hline 235 & 3 & 0.59 & & & & 208 & & \\
\hline 236 & 4 & -0.43 & & & & 199 & & \\
\hline 240 & 3 & -0.70 & & & & 197 & & \\
\hline 265 & 4 & -0.35 & & & & & & 200 \\
\hline
\end{tabular}


Table 13. Statistical summary of reported data for standard reference water sample T-145 (trace constituents)-Continued $\mathrm{TI}$ (Thallium) $\mu \mathrm{g} / \mathrm{L}$

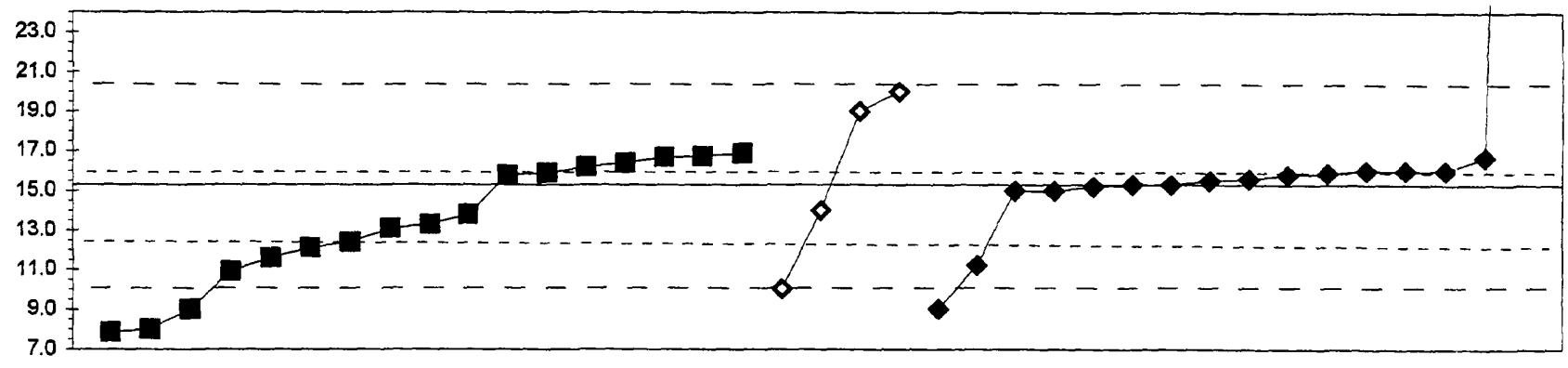

\begin{tabular}{|c|c|c|c|c|c|}
\hline \multicolumn{6}{|c|}{$\begin{array}{l}\text { 3. AA: graphite fumace } \\
\text { 4. ICP } \\
\text { 6. ICPMS }\end{array}$} \\
\hline & & $N=$ & 17 & 4 & 16 \\
\hline & & inimum = & 7.9 & 10.0 & 9.0 \\
\hline & & aximum = & 16.9 & 20.0 & 84.0 \\
\hline & & Median = & 13.3 & & 15.6 \\
\hline & F-pseu & osigma = & 3.4 & & 0.7 \\
\hline Lab & Rating & Z-value & 3 & 4 & 6 \\
\hline 1 & 3 & 0.54 & 16.7 & & \\
\hline 3 & NR & & & $<10$ & \\
\hline 11 & 1 & 1.76 & & 20.0 & \\
\hline 13 & 4 & 0.19 & 15.8 & & \\
\hline 15 & 2 & -1.39 & 11.6 & & \\
\hline 16 & 4 & 0.26 & & & 16.0 \\
\hline 18 & 3 & -0.56 & 13.8 & & \\
\hline 23 & 0 & -3.81 & $<5$ & & \\
\hline 32 & 4 & 0.11 & & & 15.6 \\
\hline 36 & 0 & -2.74 & 8.0 & & \\
\hline 42 & 0 & -2.36 & & & 9.0 \\
\hline 46 & 4 & 0.34 & 16.2 & & \\
\hline 48 & 2 & -1.20 & 12.1 & & \\
\hline 59 & 4 & 0.07 & & & 15.5 \\
\hline 69 & 4 & 0.41 & 16.4 & & \\
\hline 70 & 3 & -0.75 & 13.3 & & \\
\hline 76 & 1 & -1.54 & & & 11.2 \\
\hline 81 & 0 & 25.74 & & & 84.0 \\
\hline 89 & NR & & $<10$ & & \\
\hline 97 & 3 & 0.52 & 16.7 & & \\
\hline 102 & 2 & 1.39 & & 19.0 & \\
\hline 113 & 3 & -0.82 & 13.1 & & \\
\hline 119 & 4 & 0.22 & & & 15.9 \\
\hline 128 & 4 & 0.00 & & & 15.3 \\
\hline 134 & 3 & 0.60 & 16.9 & & \\
\hline 138 & 4 & -0.11 & & & 15.0 \\
\hline 141 & NR & & & $<50$ & \\
\hline 142 & 3 & 0.52 & & & 16.7 \\
\hline 146 & 4 & -0.49 & & 14.0 & \\
\hline 151 & 4 & 0.26 & & & 16.0 \\
\hline 180 & NR & & & $<32.1$ & \\
\hline 191 & 4 & 0.19 & & & 15.8 \\
\hline 193 & 0 & -2.36 & 9.0 & & \\
\hline 196 & 4 & 0.00 & & & 15.3 \\
\hline 212 & 4 & 0.26 & & & 16.0 \\
\hline 213 & 2 & -1.09 & 12.4 & & \\
\hline 215 & 0 & -3.07 & $<7$ & & \\
\hline 217 & 4 & -0.04 & & & 15.2 \\
\hline 234 & 1 & -1.65 & 10.9 & & \\
\hline 235 & 0 & -2.79 & 7.9 & & \\
\hline 240 & 1 & -1.99 & & 10.0 & \\
\hline 241 & 4 & 0.22 & 15.9 & & \\
\hline 265 & 4 & -0.11 & & & 15.0 \\
\hline
\end{tabular}


Table 13. Statistical summary of reported data for standard reference water sample T-145 (trace constituents)-Continued $U$ (Uranium) $\mu \mathrm{g} / \mathrm{L}$

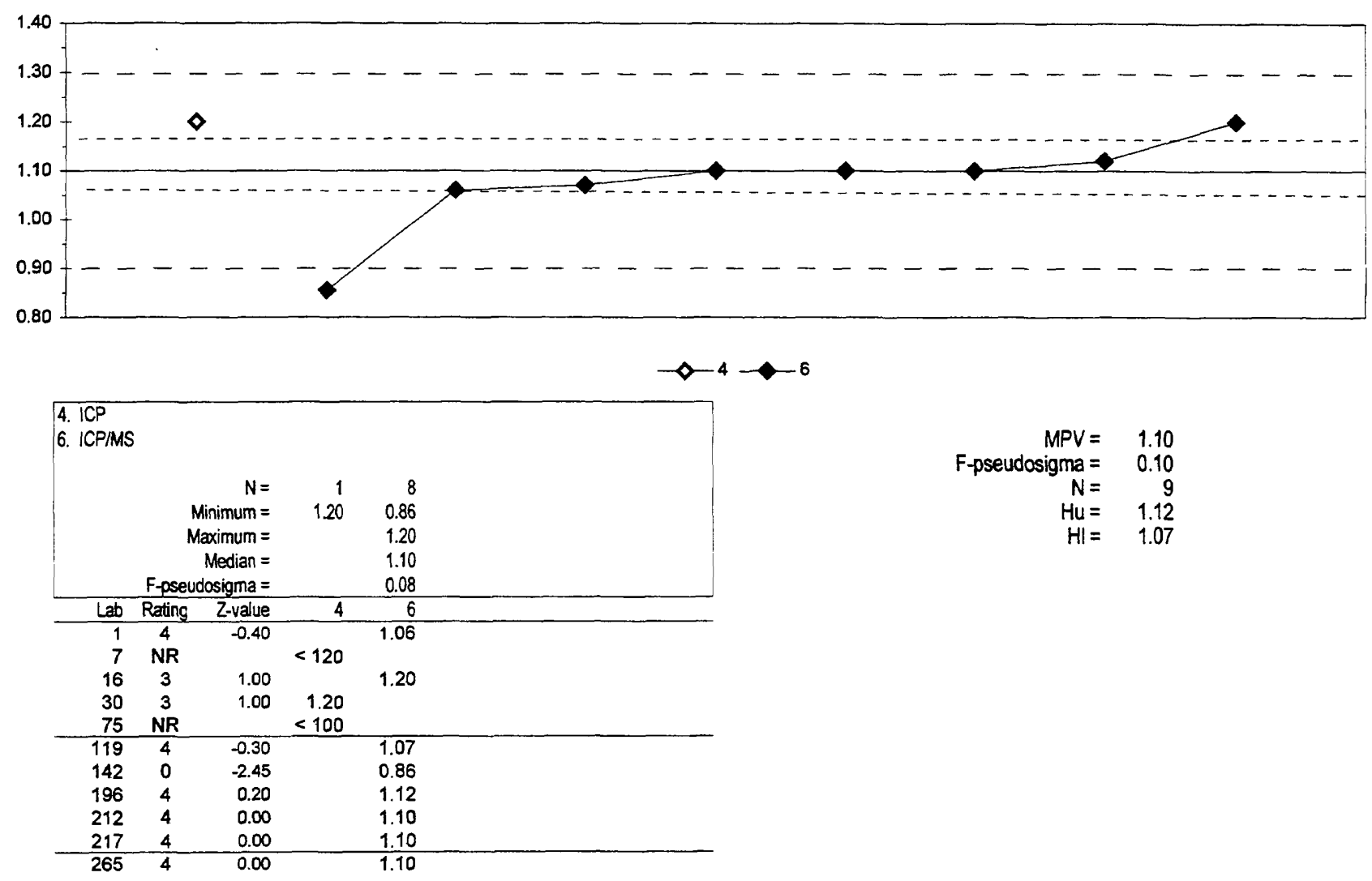


Table 13. Statistical summary of reported data for standard reference water sample T-145 (trace constituents)-Continued $V$ (Vanadium)

$\mu \mathrm{g} / \mathrm{L}$

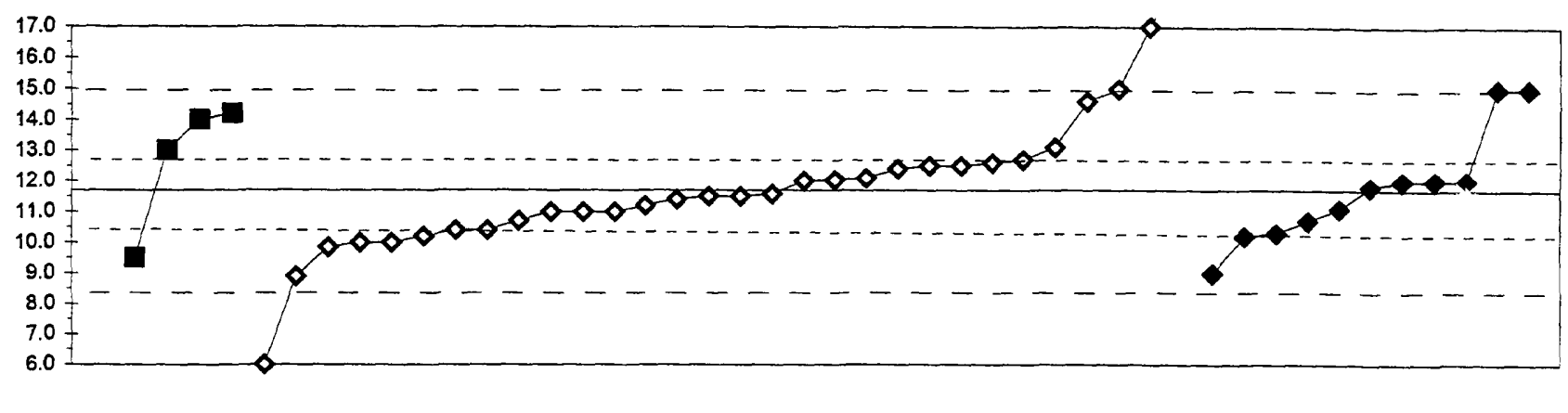

$-0-2 \rightarrow-3-0-4-6$

\begin{tabular}{|c|c|c|c|c|c|c|}
\hline \multicolumn{3}{|c|}{$\begin{array}{l}\text { 2. AA: direct nitrous oxide } \\
\text { 3. AA: graphite fumace } \\
\text { 4. ICP }\end{array}$} & \multicolumn{2}{|c|}{ 6. ICPMS } & \multirow[b]{2}{*}{30} & \multirow[b]{2}{*}{11} \\
\hline & & $\mathrm{N}=$ & 1 & 4 & & \\
\hline & & nimum $=$ & 39.0 & 9.5 & 6.0 & 9.0 \\
\hline & & ximum $=$ & & 14.2 & 18.8 & 15.0 \\
\hline & & Vedian = & & & 11.5 & 11.8 \\
\hline & F-pse & sigma $=$ & & & 1.6 & 1.1 \\
\hline Lab & Rating & Z-value & 2 & 3 & 4 & 6 \\
\hline 1 & 4 & 0.20 & & & 12.0 & \\
\hline 3 & 0 & -3.33 & & & 6.0 & \\
\hline 5 & 4 & 0.42 & & & 12.4 & \\
\hline 7 & 3 & 0.54 & & & 12.6 & \\
\hline 11 & 4 & -0.40 & & & 11.0 & \\
\hline 13 & NR & & & & $<50$ & \\
\hline 15 & NR & & & & $<10$ & \\
\hline 16 & 1 & 1.94 & & & & 15.0 \\
\hline 18 & 4 & -0.28 & & & 11.2 & \\
\hline 26 & 4 & 0.48 & & & 12.5 & \\
\hline 28 & 1 & 1.71 & & & 14.6 & \\
\hline 30 & 4 & -0.17 & & & 11.4 & \\
\hline 32 & 4 & 0.07 & & & & 11.8 \\
\hline 40 & 3 & -0.75 & & & 10.4 & \\
\hline 42 & 1 & 1.94 & & & & 15.0 \\
\hline 46 & 3 & -0.75 & & & 10.4 & \\
\hline 48 & 2 & -1.28 & & 9.5 & & \\
\hline 68 & 2 & -1.08 & & & 9.9 & \\
\hline 70 & NR & & & & $<50$ & \\
\hline 75 & 4 & -0.11 & & & 11.5 & \\
\hline 81 & 1 & -1.57 & & & & 9.0 \\
\hline 85 & NR & & & & $<20$ & \\
\hline 86 & 3 & 0.83 & & & 13.1 & \\
\hline 89 & 2 & 1.48 & & 14.2 & & \\
\hline 97 & 2 & 1.36 & & 14.0 & & \\
\hline 102 & 3 & -0.99 & & & 10.0 & \\
\hline 105 & 4 & -0.34 & & & & 11.1 \\
\hline 119 & 3 & -0.81 & & & & 10.3 \\
\hline 128 & 3 & -0.87 & & & 10.2 & \\
\hline 134 & 3 & -0.58 & & & 10.7 & \\
\hline 138 & 4 & -0.40 & & & 11.0 & \\
\hline 141 & 3 & 0.60 & & & 12.7 & \\
\hline 142 & 3 & -0.58 & & & & 10.7 \\
\hline 145 & 0 & 3.12 & & & 17.0 & \\
\hline 146 & 4 & -0.11 & & & 11.5 & \\
\hline 158 & 4 & 0.48 & & & 12.5 & \\
\hline 180 & 4 & 0.18 & & & 12.0 & \\
\hline 191 & 4 & 0.18 & & & & 12.0 \\
\hline 196 & 3 & -0.87 & & & & 10.2 \\
\hline 212 & 4 & 0.18 & & & & 12.0 \\
\hline 217 & 1 & -1.63 & & & 8.9 & \\
\hline 219 & 3 & -0.99 & & & 10.0 & \\
\hline 224 & 0 & 4.17 & & & 18.8 & \\
\hline 234 & 4 & -0.40 & & & 11.0 & \\
\hline 235 & 1 & 1.94 & & & 15.0 & \\
\hline 236 & 4 & 0.24 & & & 12.1 & \\
\hline 241 & 3 & 0.77 & & 13.0 & & \\
\hline 255 & 4 & -0.07 & & & 11.6 & \\
\hline 257 & 0 & 16.02 & 39.0 & & & \\
\hline 265 & 4 & 0.18 & & & & 12.0 \\
\hline
\end{tabular}


Table 13. Statistical summary of reported data for standard reference water sample T-145 (trace constituents)-Continued $\mathrm{Zn}$ (Zinc) $\mu \mathrm{g} / \mathrm{L}$

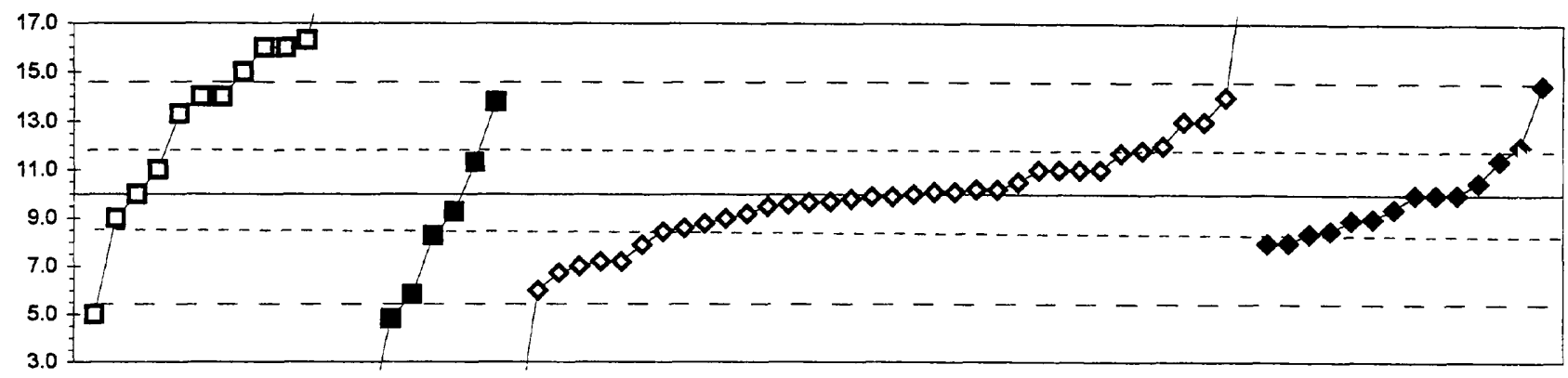

$-\square-1-3 \multimap-4 \multimap 6$

\begin{tabular}{|c|c|c|c|c|c|c|}
\hline $\begin{array}{l}\text { 1. AA: dir } \\
\text { 3. } \mathrm{AA}: \mathrm{gr} \\
\text { 4. ICP }\end{array}$ & $\begin{array}{l}\text { ect air } \\
\text { aphite fu }\end{array}$ & lace & & ICP/MS & & \\
\hline & & $N=$ & 13 & 7 & 36 & 14 \\
\hline & & linimum = & 5.0 & 0.6 & 0.0 & 8.0 \\
\hline & & aximum = & 66.0 & 13.8 & 20.0 & 14.5 \\
\hline & & Median $=$ & 14.0 & 8.3 & 9.9 & 9.7 \\
\hline & F-psel & losigma $=$ & 3.7 & 3.7 & 1.7 & 1.5 \\
\hline Lab & Rating & Z-value & 1 & 3 & 4 & 6 \\
\hline 1 & 3 & 0.59 & & & & 11.4 \\
\hline 3 & 2 & -1.26 & & & 7.0 & \\
\hline 4 & 1 & 1.69 & & & 14.0 & \\
\hline 5 & 4 & -0.13 & & & 9.7 & \\
\hline 7 & 4 & 0.42 & & & 11.0 & \\
\hline 10 & 0 & 2.53 & 16.0 & & & \\
\hline 13 & 4 & -0.04 & & & 9.9 & \\
\hline 15 & $\begin{array}{l}4 \\
0\end{array}$ & -2.11 & & & $<5$ & \\
\hline 16 & 4 & -0.25 & & & & 9.4 \\
\hline 18 & NR & & & & $<100$ & \\
\hline 23 & NR & & $<20$ & & & \\
\hline 24 & 4 & 0.08 & & & 10.2 & \\
\hline 26 & 4 & -0.34 & & & 9.2 & \\
\hline 28 & 1 & -1.69 & & & 6.0 & \\
\hline 30 & 4 & -0.17 & & & 9.6 & \\
\hline 32 & 4 & 0.00 & & & & 10.0 \\
\hline 36 & 0 & 2.53 & 16.0 & & & \\
\hline 42 & 4 & 0.00 & & & & 10.0 \\
\hline 48 & NR & & & & $<5$ & \\
\hline 58 & NR & & $<50$ & & & \\
\hline 59 & 4 & 0.00 & & & & 10.0 \\
\hline 68 & 4 & 0.21 & & & 10.5 & \\
\hline 69 & NR & & $<50$ & & & \\
\hline 70 & NR & & & & $<20$ & \\
\hline 73 & 2 & 1.26 & & & 13.0 & \\
\hline 75 & 4 & 0.42 & & & 11.0 & \\
\hline 80 & 0 & -2.19 & & 4.8 & & \\
\hline 81 & 3 & -0.84 & & & & 8.0 \\
\hline 83 & 4 & -0.08 & & & 9.8 & \\
\hline 86 & 3 & 0.76 & & & 11.8 & \\
\hline 87 & 0 & 2.66 & 16.3 & & & \\
\hline 89 & 1 & 1.60 & & 13.8 & & \\
\hline 96 & 4 & 0.42 & 11.0 & & & \\
\hline 97 & NR & & & $<4.6$ & & \\
\hline 102 & 3 & -0.51 & & & 8.8 & \\
\hline 105 & 4 & -0.42 & & & & 9.0 \\
\hline 113 & 3 & -0.65 & & & 8.5 & \\
\hline 114 & NR & & $<10$ & & & \\
\hline 118 & 0 & 2.11 & 15.0 & & & \\
\hline 119 & 4 & 0.00 & & & 10.0 & \\
\hline 121 & 3 & 0.84 & & & 12.0 & \\
\hline 128 & 4 & 0.04 & & & 10.1 & \\
\hline 132 & 4 & -0.42 & & & 9.0 & \\
\hline 133 & 4 & 0.04 & & & 10.1 & \\
\hline 134 & 3 & 0.71 & & & 11.7 & \\
\hline 138 & 3 & -0.85 & & & & 8.0 \\
\hline 140 & 1 & 1.69 & 14.0 & & & \\
\hline 141 & 4 & -0.04 & & & 9.9 & \\
\hline 142 & 3 & -0.68 & & & & 8.4 \\
\hline 145 & 2 & 1.26 & & & 13.0 & \\
\hline
\end{tabular}

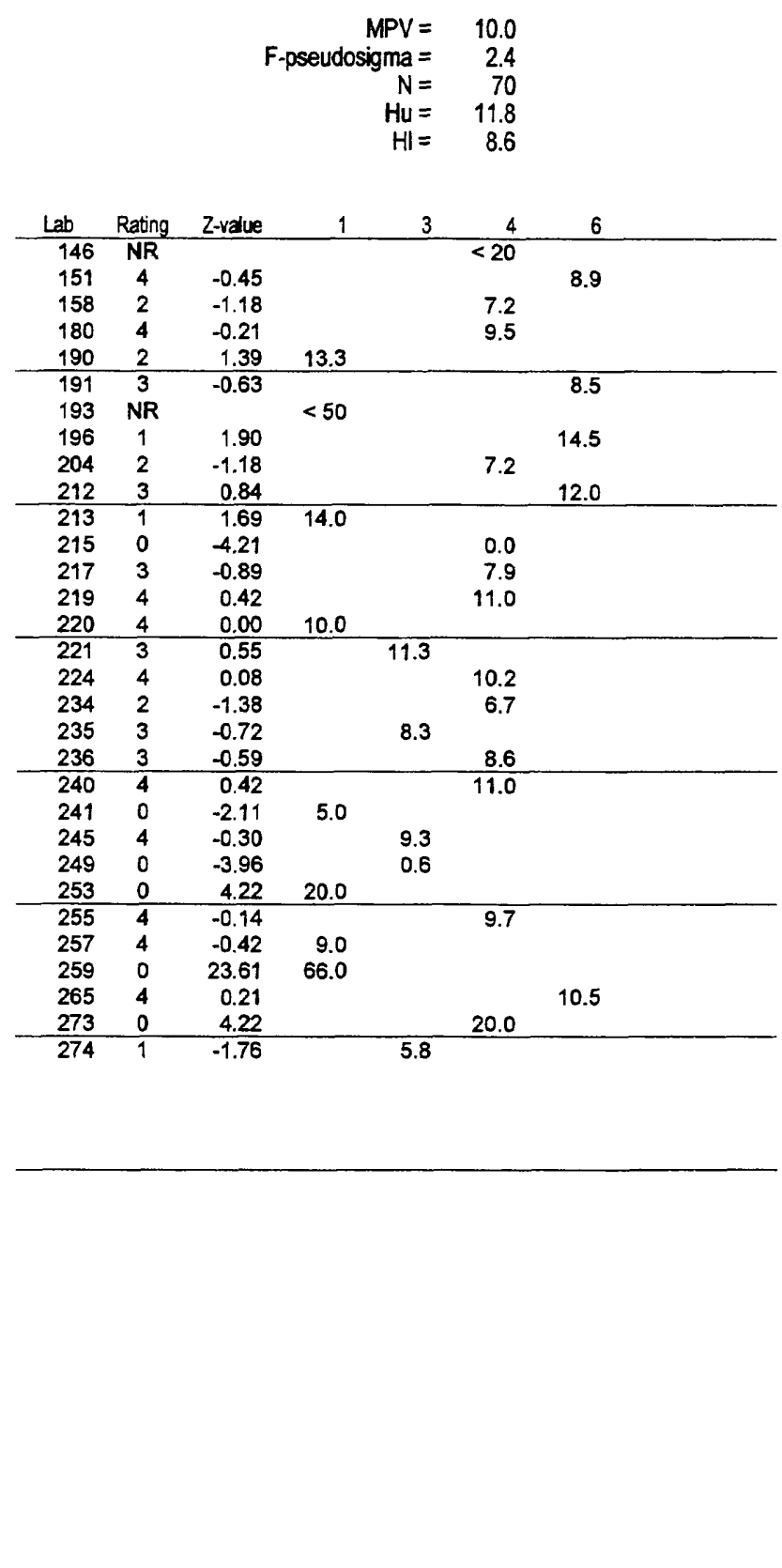


Definition of analytical methods, abbreviations, and symbols

\begin{tabular}{l} 
Analytical methods \\
\hline 0 Other/Not reported \\
1 AA: direct, air \\
2 AA: direct, $\mathrm{N}_{2} \mathrm{O}$ \\
3 AA: graphite furnace \\
4 ICP \\
5 DCP \\
6 ICPMS \\
7 IC \\
12 Flame emission \\
20 Titrate: color \\
21 Titrate: electro \\
22 Color: \\
40 lon electrode \\
41 Electro \\
50 Gravimetric \\
51 Turbidimetric
\end{tabular}

= atomic absorption: direct,air

= atomic absorption: direct, nitrous oxide

= atomic absorption: graphite fumace

= inductively coupled plasma

= direct current plasma

= mass spectrometryfinductively coupled plasma

$=$ ion chromatography

= flame emission

$=$ titration: colorimetric [color reagent specified]

$=$ titration: electrometric

$=$ colorimetric [color reagent specified]

$=$ ion selective electrode

$=$ electrometric: [type meter specified]

= gravimetric: [precipitate specified]

$=$ turbidimetric: [precipitate specified]

\begin{tabular}{|c|c|c|c|}
\hline \multicolumn{2}{|c|}{ ADorevialions and symous } & 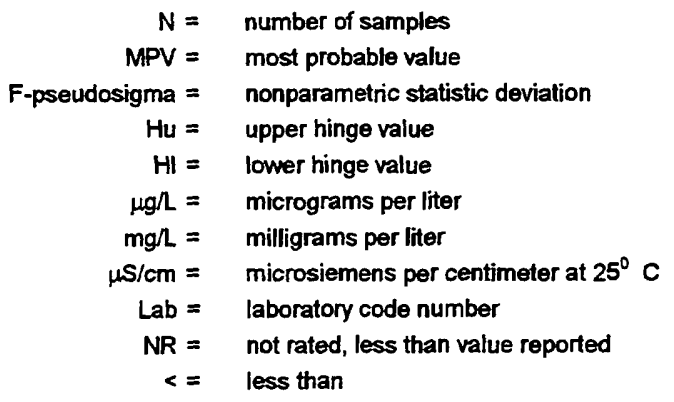 & \\
\hline \multicolumn{2}{|c|}{ Constituent } & page & \\
\hline Alk & Alkalinity as $\mathrm{CaCO}_{3}$ & 103 & \\
\hline B & Boron & 104 & \\
\hline $\mathrm{Ca}$ & Calcium & 105 & \\
\hline $\mathrm{Cl}$ & Chloride & 106 & \\
\hline DSRD & Dissolved solids & 107 & \\
\hline $\mathbf{F}$ & Fluoride & 108 & \\
\hline $\mathbf{K}$ & Potassium & 109 & \\
\hline $\mathrm{Mg}$ & Magnesium & 110 & \\
\hline $\mathrm{Na}$ & Sodium & 111 & \\
\hline total P & Phosphorus & 112 & \\
\hline $\mathrm{pH}$ & & 113 & \\
\hline $\mathrm{SiO}_{2}$ & Silica & 114 & \\
\hline $\mathrm{SO}_{4}$ & Sulfate & 115 & \\
\hline Sp Con & Specific Conductance & 116 & \\
\hline Sr & Strontium & 117 & \\
\hline $\mathbf{V}$ & Vanadium & 118 & \\
\hline
\end{tabular}




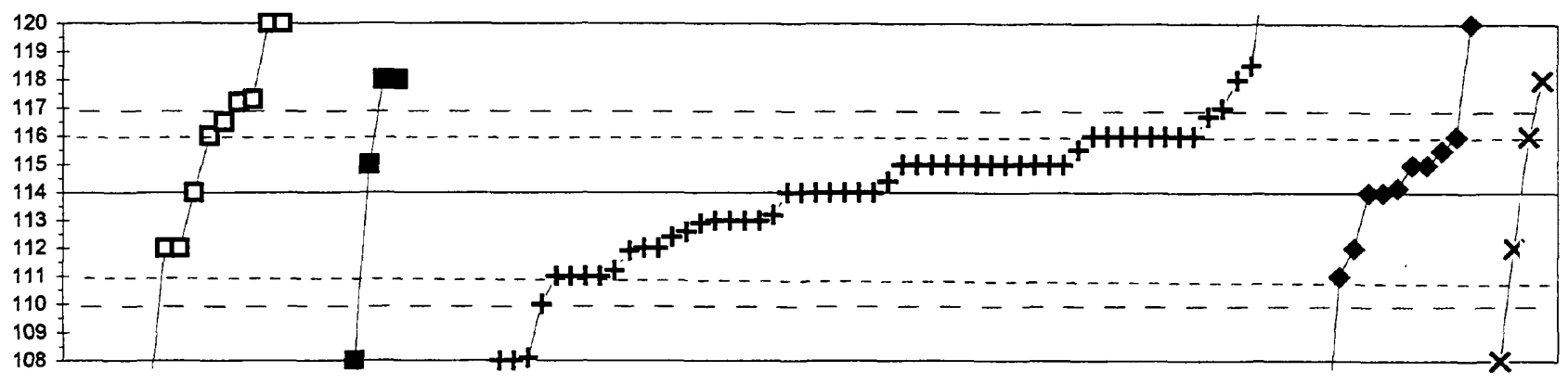

$\rightarrow-\square-20 \rightarrow-20 \mathrm{br}+21 \longrightarrow 21 \mathrm{~h} 2 \mathrm{so4}-\mathrm{X}-22$

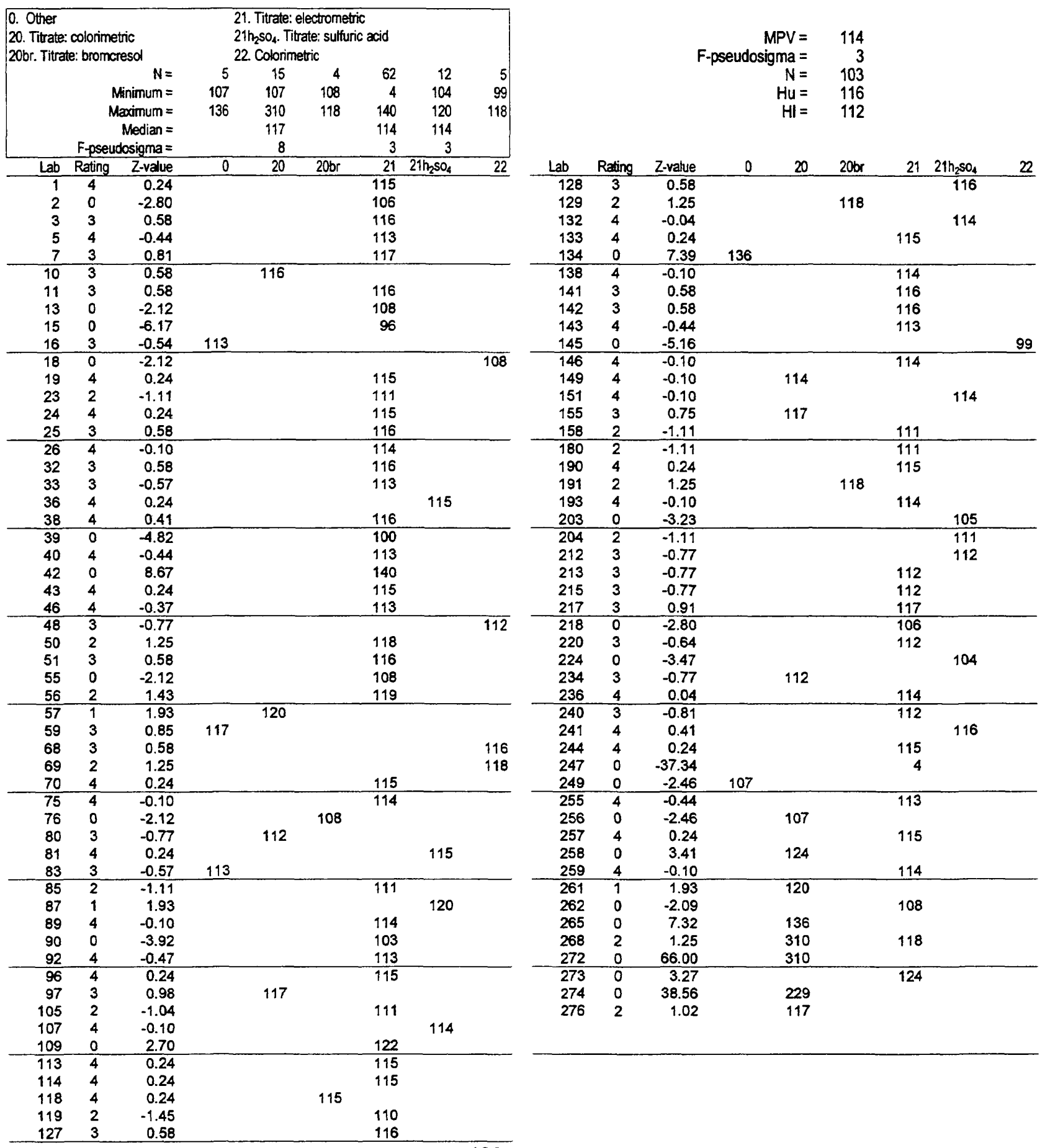


Table 14. Statistical summary of reported data for standard reference water sample M-140 (major constituents)-Continued B (Boron) $\mu \mathrm{g} / \mathrm{L}$

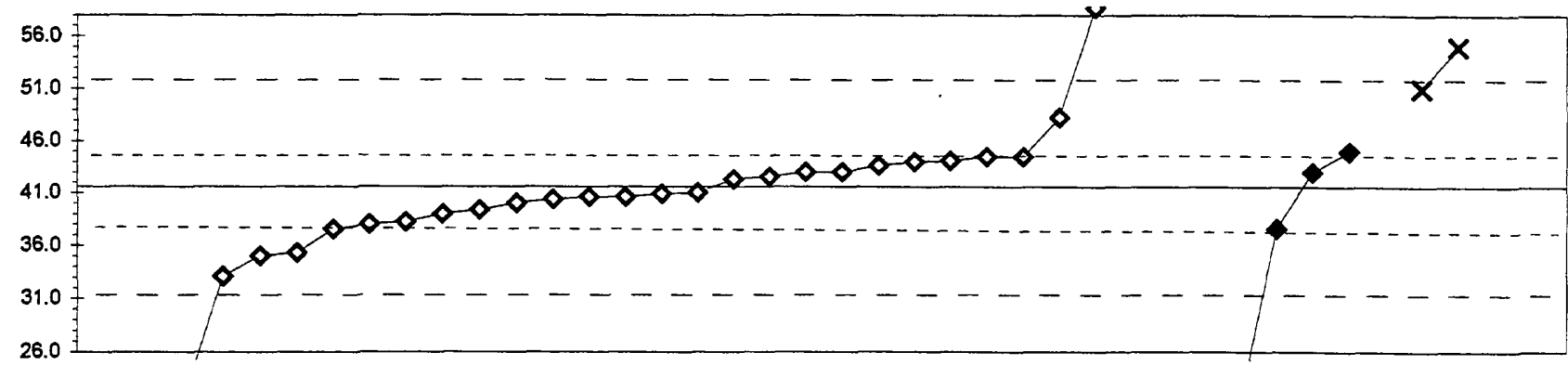

$\checkmark-4-6-6-22-x-22 a z-x-22 \mathrm{cu}$

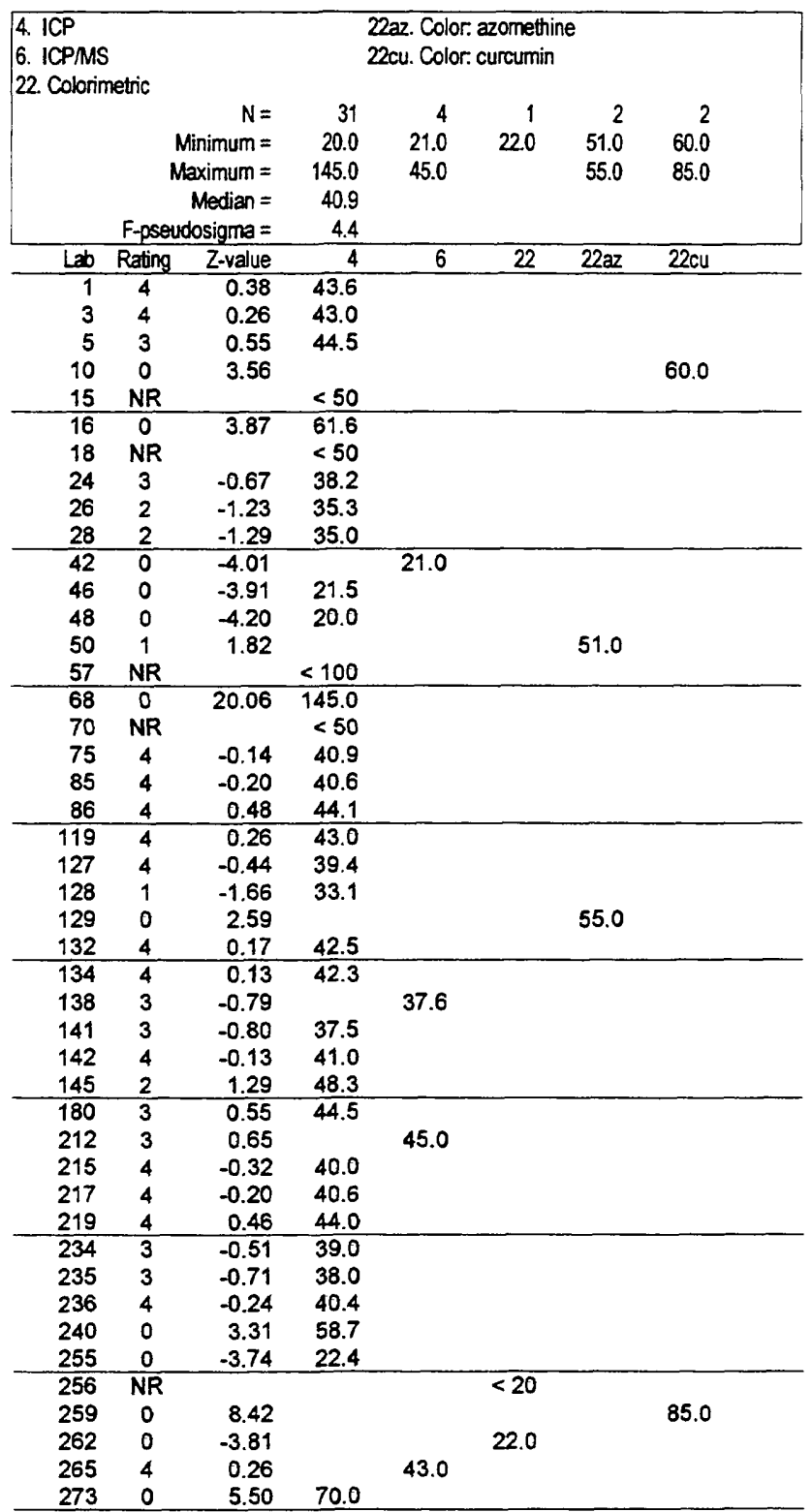

MPV $=41.6$

F-pseudosigma $=5.2$

$\mathrm{N}=40$

$\mathrm{Hu}=44.8$

$\mathrm{HI}=\quad 37.8$ 
Table 14. Statistical summary of reported data for standard reference water sample $M-140$ (major constituents)-Continued Ca (Calcium) $\mathrm{mgh}$

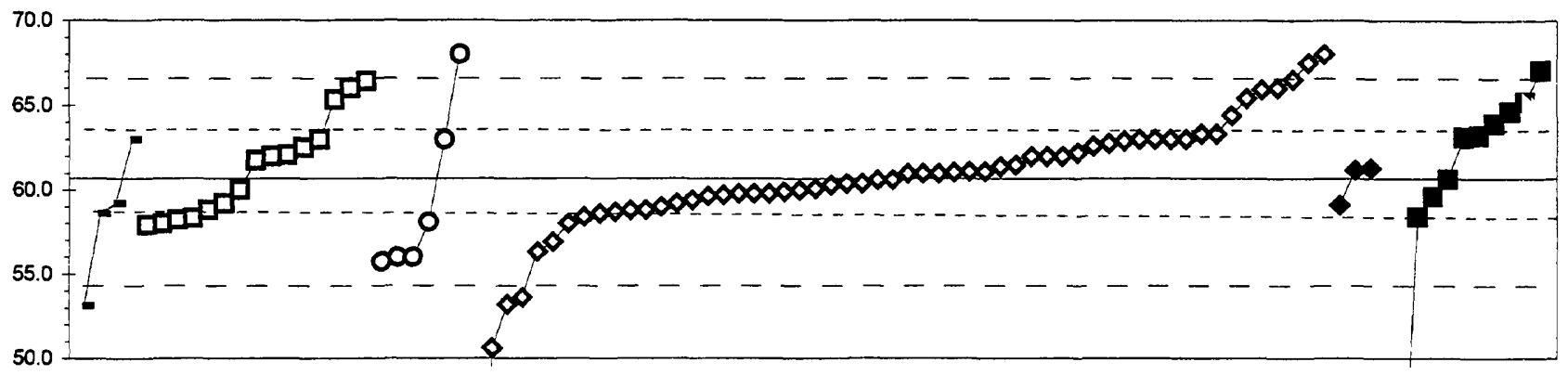

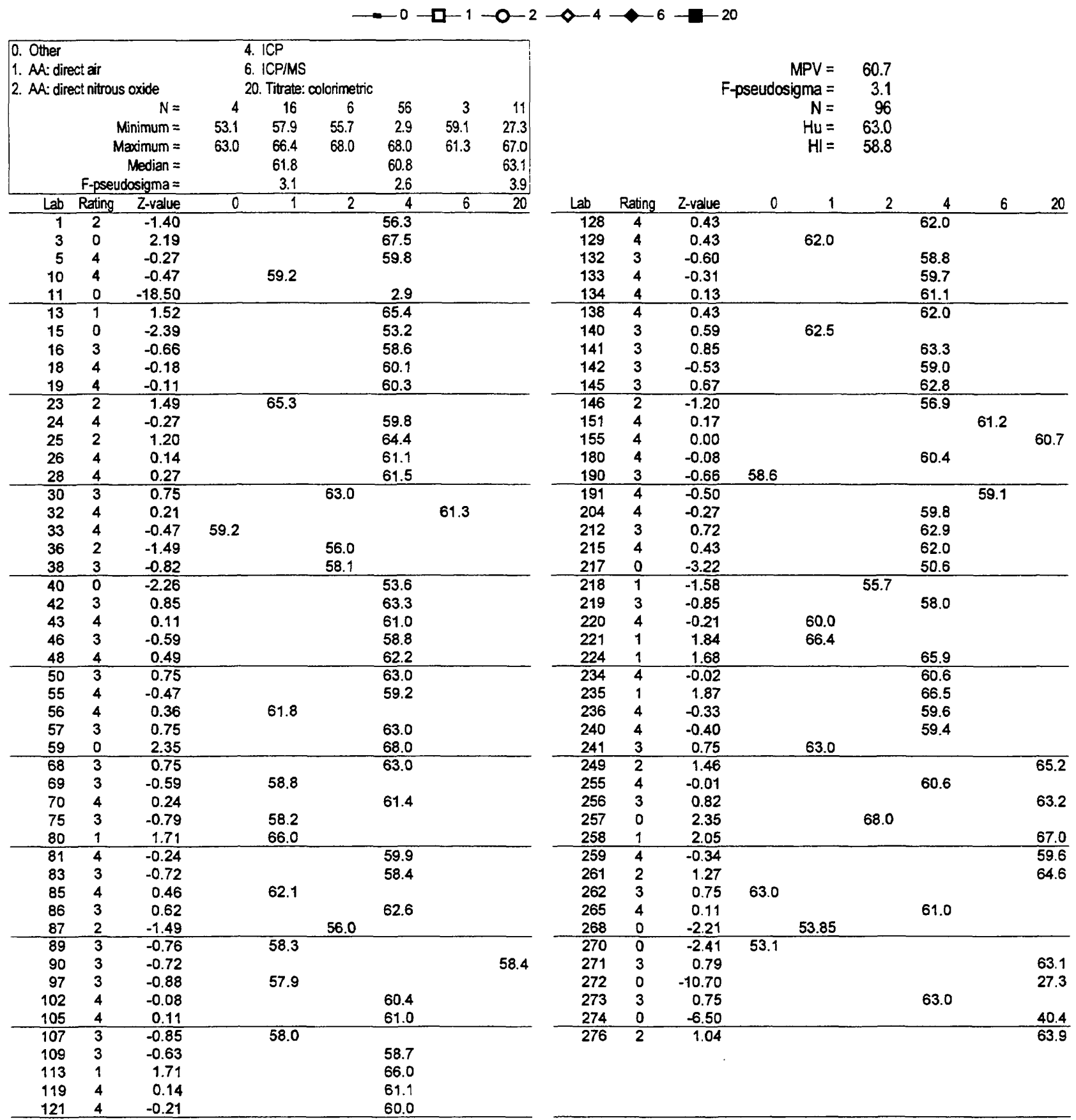


Table 14. Statistical summary of reported data for standard reference water sample $M-140$ (major constituents)-Continued $\mathrm{Cl}$ (Chloride) $\mathrm{mg} / \mathrm{L}$

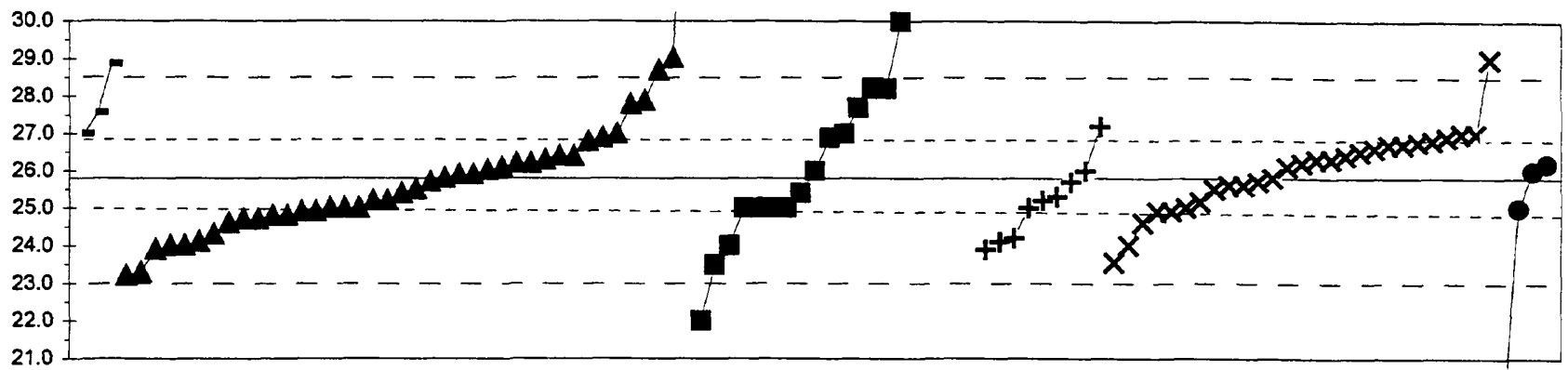

$\rightarrow-0-1-7-20+-21-x-22$

40

\begin{tabular}{|c|c|c|c|c|c|c|c|c|}
\hline \multicolumn{3}{|c|}{$\begin{array}{l}\text { 0. Other } \\
\text { 7. Ion chromatography } \\
\text { 20. Titrate: colorimetric }\end{array}$} & & $\begin{array}{l}\text { Titrate: } \\
\text { Colonin } \\
\text { lon sel }\end{array}$ & $\begin{array}{l}\text { ectrome } \\
\text { ric } \\
\text { ive elec }\end{array}$ & & \multirow[b]{2}{*}{27} & \multirow[t]{2}{*}{4} \\
\hline & & $N=$ & 3 & 41 & 20 & 9 & & \\
\hline & & inimum = & 27.0 & 23.2 & 22.0 & 23.9 & 23.5 & 19.6 \\
\hline & & ximum = & 27.6 & 35.8 & 42.5 & 27.2 & 29.0 & 26.2 \\
\hline & & Median = & & 25.5 & 27.0 & 25.2 & 26.2 & \\
\hline & F-pseu & osigma = & & 1.2 & 3.9 & 0.8 & 0.6 & \\
\hline Lab & Rating & Z-value & 0 & 7 & 20 & 21 & 22 & 40 \\
\hline 1 & 3 & -0.78 & & 24.7 & & & & \\
\hline 2 & 2 & 1.35 & & & 27.7 & & & \\
\hline 3 & 3 & 0.64 & & & & & 26.7 & \\
\hline 4 & 4 & 0.16 & & 26.0 & & & & \\
\hline 5 & 3 & -0.71 & & 24.8 & & & & \\
\hline 7 & 2 & -1.21 & & 24.1 & & & & \\
\hline 10 & 4 & 0.36 & & & & & 26.3 & \\
\hline 11 & 3 & 0.64 & & & & & 26.7 & \\
\hline 13 & 3 & -0.71 & & 24.8 & & & & \\
\hline 15 & 4 & 0.43 & & 26.4 & & & & \\
\hline 16 & 0 & 2.18 & 28.9 & & & & & \\
\hline 18 & 3 & 0.85 & & & & & 27.0 & \\
\hline 19 & 3 & -0.57 & & & 25.0 & & & \\
\hline 23 & 4 & 0.28 & & & & & & 26.2 \\
\hline 24 & 4 & -0.14 & & & & & 25.6 & \\
\hline 25 & 3 & -0.85 & & 24.6 & & & & \\
\hline 26 & 4 & 0.28 & & 26.2 & & & & \\
\hline 30 & 4 & -0.43 & & 25.2 & & & & \\
\hline 32 & 3 & 0.78 & & 26.9 & & & & \\
\hline 33 & 2 & -1.07 & & 24.3 & & & & \\
\hline 36 & 4 & 0.14 & & & 26.0 & & & \\
\hline 39 & 0 & 2.99 & & & 30.0 & & & \\
\hline 40 & 3 & 0.78 & & & & & 26.9 & \\
\hline 42 & 0 & 7.12 & & 35.8 & & & & \\
\hline 43 & 4 & 0.14 & & & & & & 26.0 \\
\hline 46 & 3 & 0.57 & & & & & 26.6 & \\
\hline 48 & 2 & -1.28 & & & & & 24.0 & \\
\hline 50 & 3 & -0.57 & & & & & 25.0 & \\
\hline 51 & 2 & 1.42 & & 27.8 & & & & \\
\hline 55 & 4 & 0.00 & & & & & 25.8 & \\
\hline 56 & 1 & -1.61 & & & & & 23.5 & \\
\hline 57 & 3 & -0.57 & & & 25.0 & & & \\
\hline 59 & 2 & -1.28 & & 24.0 & & & & \\
\hline 64 & 4 & 0.36 & & 26.3 & & & & \\
\hline 68 & 4 & 0.50 & & & & & 26.5 & \\
\hline 69 & 4 & 0.28 & & & & & 26.2 & \\
\hline 70 & 4 & 0.00 & & 25.8 & & & & \\
\hline 75 & 4 & 0.21 & & & & & 26.1 & \\
\hline 76 & 2 & 1.49 & & 27.9 & & & & \\
\hline 80 & 3 & -0.57 & & & 25.0 & & & \\
\hline 81 & 2 & -1.35 & & & & 23.9 & & \\
\hline 83 & 4 & -0.43 & & & & 25.2 & & \\
\hline 85 & 3 & -0.57 & & 25.0 & & & & \\
\hline 87 & 0 & 2.28 & & & & & 29.0 & \\
\hline 89 & 3 & -0.57 & & & 25.0 & & & \\
\hline 96 & 3 & -0.64 & & & & & 24.9 & \\
\hline 97 & 4 & -0.07 & & & & & 25.7 & \\
\hline 102 & 4 & -0.21 & & & & & 25.5 & \\
\hline 105 & 4 & -0.43 & & 25.2 & & & & \\
\hline 107 & 3 & 1.00 & & & & 27.2 & & \\
\hline 109 & 1 & -1.78 & & 23.3 & & & & \\
\hline 113 & 3 & -0.64 & & 24.9 & & & & \\
\hline 114 & 3 & -0.57 & & & & & & 25.0 \\
\hline 119 & 3 & -0.78 & & 24.7 & & & & \\
\hline 127 & 4 & 0.43 & & 26.4 & & & & \\
\hline
\end{tabular}

$\begin{aligned} \text { MPV } & =25.8 \\ \text { F-pseudosigma } & =1.4 \\ \mathrm{~N} & =103 \\ \mathrm{Hu} & =26.9 \\ \mathrm{HI} & =25.0\end{aligned}$

\begin{tabular}{ccrrrrrrr} 
Lab & Rating & Z-value & 0 & 7 & 20 & 21 & 22 & 40 \\
\hline 128 & 4 & -0.28 & & 25.4 & & & &
\end{tabular}

$\begin{array}{lllll}129 & 3 & -0.57 & 25.0 & 19.6\end{array}$

$\begin{array}{rrrr}133 & 0 & -4.41 & \\ 134 & 3 & 0.70 & 26.8\end{array}$

\begin{tabular}{rrrrl}
134 & 3 & 0.70 & 26.8 & \\
138 & 4 & 0.07 & 25.9 & 26.8 \\
\hline 140 & 3 & 0.68 & & 25.6 \\
141 & 4 & -0.14 & & 25.2 \\
142 & 4 & -0.46 & & 25.4 \\
143 & 4 & 0.43 & &
\end{tabular}

\begin{tabular}{rrrrr}
143 & 4 & 0.43 & & 26.4 \\
145 & 3 & -0.63 & 24.9 & 27.0 \\
\hline 146 & 3 & 0.85 & & 27.0
\end{tabular}

6.2

180

$\begin{array}{llrr}151 & 3 & -0.57 & 25\end{array}$

\begin{tabular}{llll}
158 & 3 & -0.64 & 2.9 .9 \\
180 & 3 & -0.85 & 21.6 \\
\hline
\end{tabular}

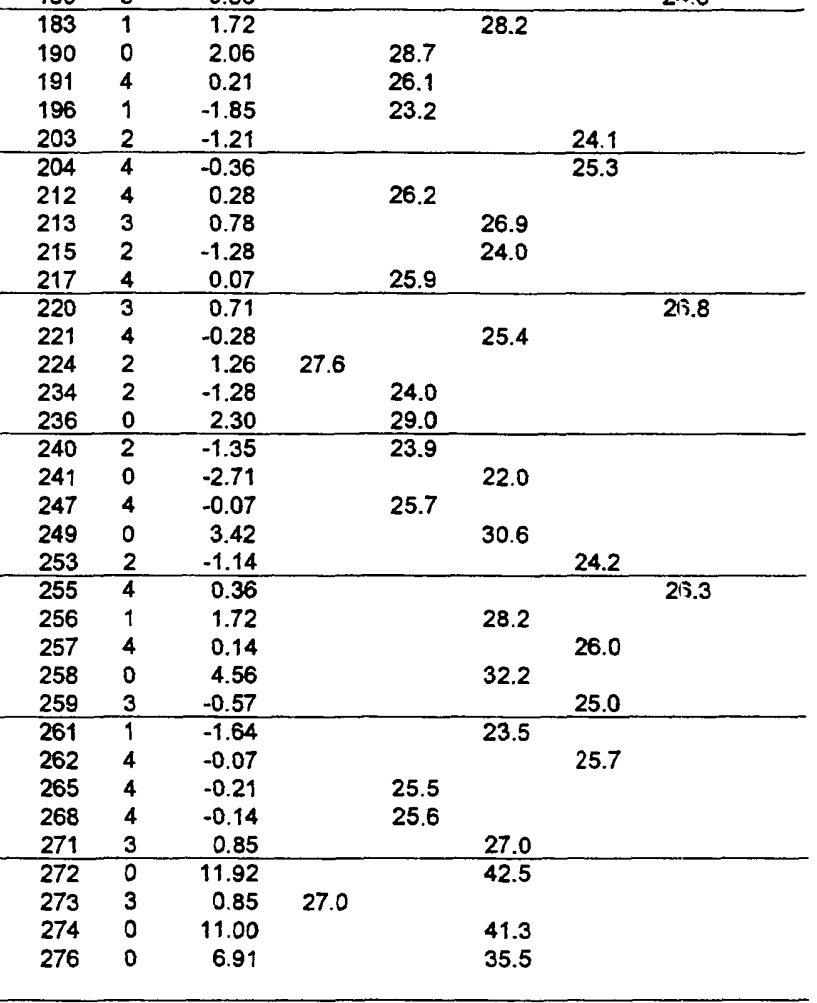


Table 14. Statistical summary of reported data for standard reference water sample M-140 (major constituents)-Continued DSRD (Dissolved solids) $\mathrm{mg} / \mathrm{L}$

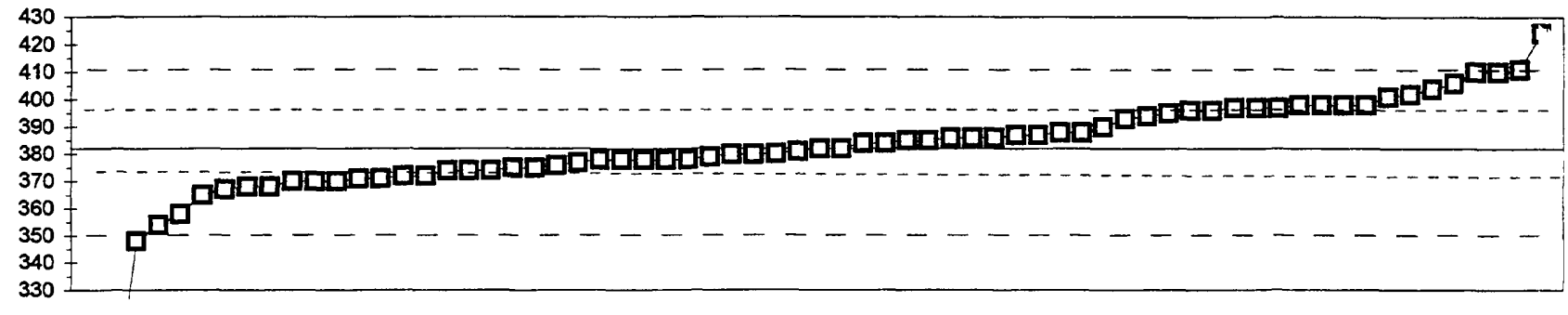

$-\square-50$

\begin{tabular}{|c|c|c|c|}
\hline \multicolumn{4}{|c|}{ 50. Gravimetric } \\
\hline & F-pseu & $\begin{array}{r}N= \\
\text { inimum }= \\
\text { iximum }= \\
\text { Median }= \\
\text { osigma }=\end{array}$ & $\begin{array}{r}67 \\
0 \\
424 \\
382 \\
0 \\
\end{array}$ \\
\hline Lab & Rating & Z-vatue & 50 \\
\hline 1 & 3 & -0.75 & 370 \\
\hline 3 & 3 & -0.75 & 370 \\
\hline 5 & 4 & 0.25 & 386 \\
\hline 10 & 4 & 0.25 & 386 \\
\hline 11 & 1 & -1.51 & 358 \\
\hline 13 & 4 & 0.38 & 388 \\
\hline 15 & 3 & -0.94 & 367 \\
\hline 16 & 3 & -0.63 & 372 \\
\hline 18 & 3 & 0.94 & 397 \\
\hline 19 & 4 & -0.50 & 374 \\
\hline 23 & 3 & -0.69 & 371 \\
\hline 25 & 3 & -0.88 & 368 \\
\hline 26 & 4 & -0.25 & 378 \\
\hline 32 & 3 & -0.63 & 372 \\
\hline 36 & 1 & 1.76 & 410 \\
\hline 38 & 4 & -0.25 & 378 \\
\hline 40 & 4 & -0.25 & 378 \\
\hline 43 & 4 & 0.25 & 386 \\
\hline 50 & 4 & 0.00 & 382 \\
\hline 55 & 4 & -0.13 & 380 \\
\hline 57 & 4 & 0.50 & 390 \\
\hline 59 & 3 & -0.69 & 371 \\
\hline 69 & 2 & 1.19 & 401 \\
\hline 70 & 4 & 0.19 & 385 \\
\hline 75 & 3 & 1.00 & 398 \\
\hline 76 & 3 & 1.00 & 398 \\
\hline 80 & 1 & 1.82 & 411 \\
\hline 81 & 0 & 2.64 & 424 \\
\hline 85 & 4 & -0.25 & 378 \\
\hline 87 & 0 & -2.13 & 348 \\
\hline 89 & 3 & 0.75 & 394 \\
\hline 90 & 1 & 1.76 & 410 \\
\hline 92 & 4 & -0.50 & 374 \\
\hline 96 & 2 & 1.25 & 402 \\
\hline 97 & 1 & 1.51 & 406 \\
\hline 105 & 2 & -1.07 & 365 \\
\hline 109 & 4 & 0.31 & 387 \\
\hline 113 & 3 & 0.88 & 396 \\
\hline 114 & 4 & -0.44 & 375 \\
\hline 118 & 3 & 1.00 & 398 \\
\hline 119 & 3 & -0.75 & 370 \\
\hline 127 & 4 & 0.31 & 387 \\
\hline 129 & 4 & -0.19 & 379 \\
\hline 134 & 3 & 0.94 & 397 \\
\hline 138 & 3 & -0.88 & 368 \\
\hline 140 & 4 & -0.31 & 377 \\
\hline 141 & 4 & -0.38 & 376 \\
\hline 142 & 3 & 0.69 & 393 \\
\hline 143 & 3 & 1.00 & 398 \\
\hline 146 & 4 & -0.50 & 374 \\
\hline
\end{tabular}

\begin{tabular}{ccrr} 
Lab & Rating & Z-value & 50 \\
\hline 151 & 4 & -0.25 & 378 \\
155 & 3 & 0.95 & 397 \\
158 & 4 & 0.38 & 388 \\
190 & 0 & -23.94 & 0 \\
212 & 1 & -1.76 & 354 \\
\hline 215 & 4 & -0.44 & 375 \\
217 & 4 & -0.06 & 381 \\
221 & 3 & 0.88 & 396 \\
224 & 4 & -0.11 & 380 \\
234 & 4 & 0.00 & 382 \\
\hline 236 & 4 & 0.19 & 385 \\
240 & 4 & -0.13 & 380 \\
241 & 0 & -6.15 & 284 \\
253 & 2 & 1.38 & 404 \\
255 & 4 & 0.13 & 384 \\
\hline 257 & 3 & 0.82 & 395 \\
259 & 4 & 0.13 & 384
\end{tabular}


Table 14. Statistical summary of reported data for standard reference water sample $M-140$ (major constituents)-Continued $F$ (Fluoride) $\mathrm{mg} / \mathrm{L}$

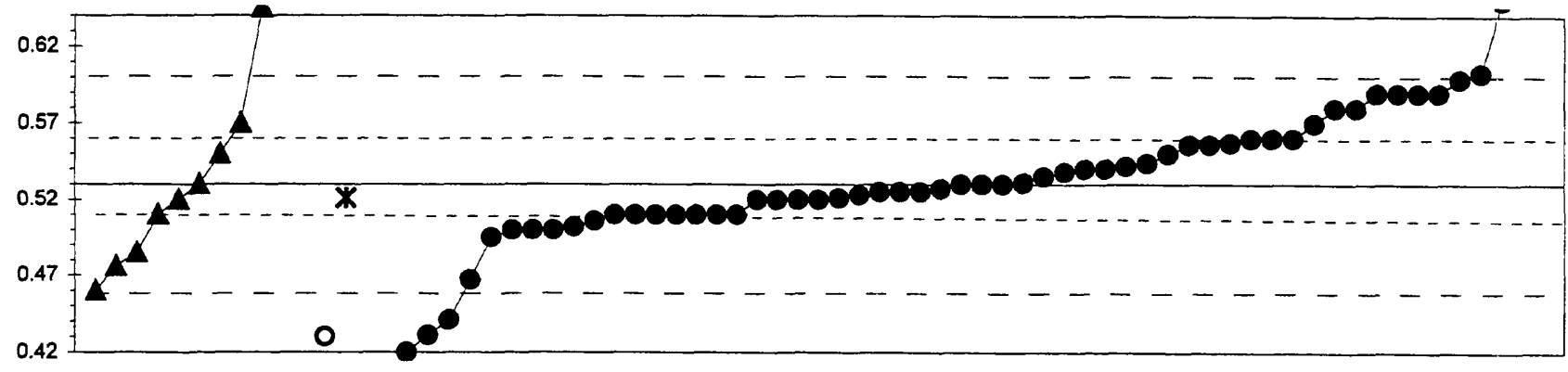

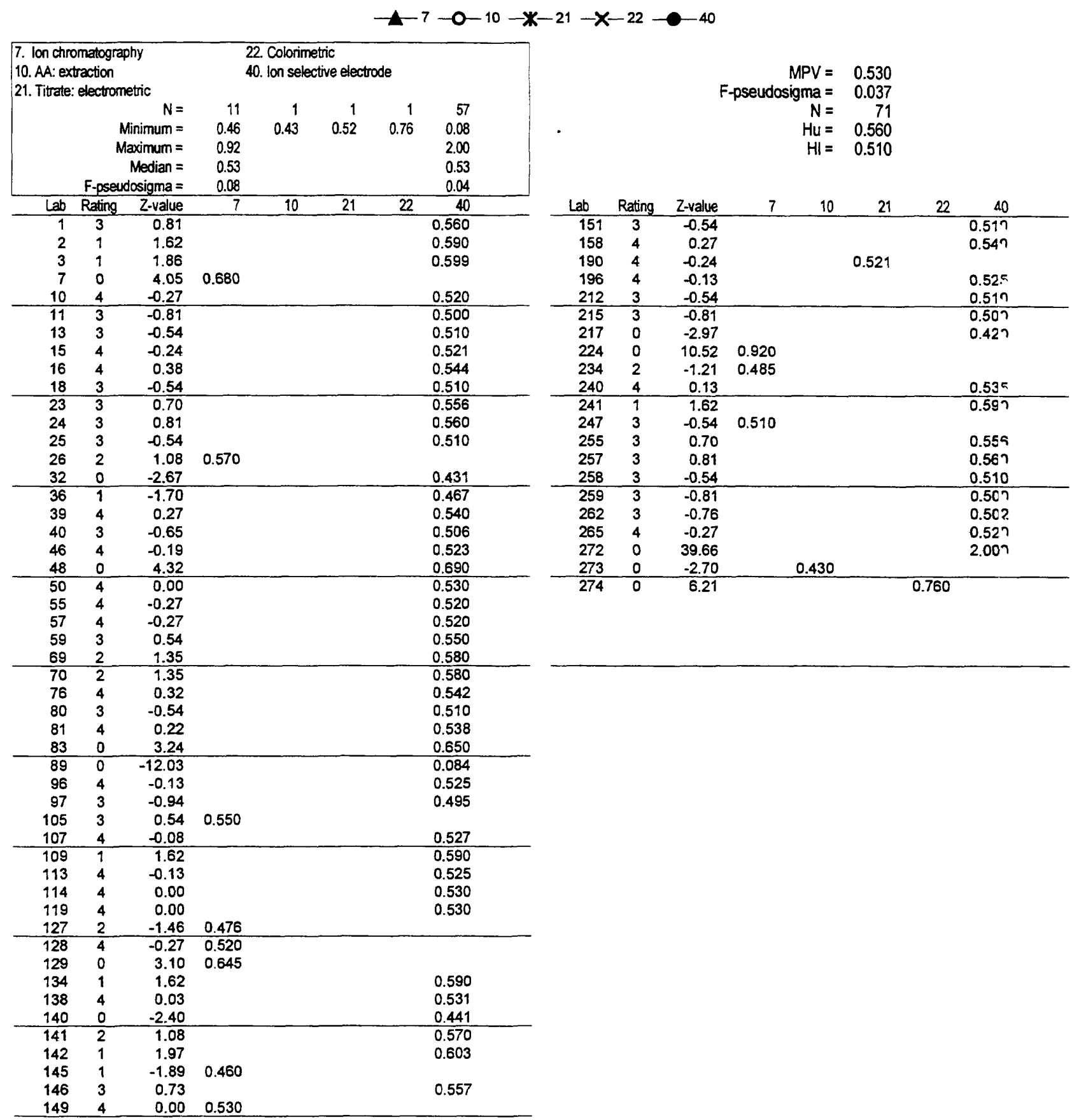


Table 14. Statistical summary of reported data for standard reference water sample M-140 (major constituents)-Continued $\mathrm{K}$ (Potassium)

$\mathrm{mg} / \mathrm{L}$

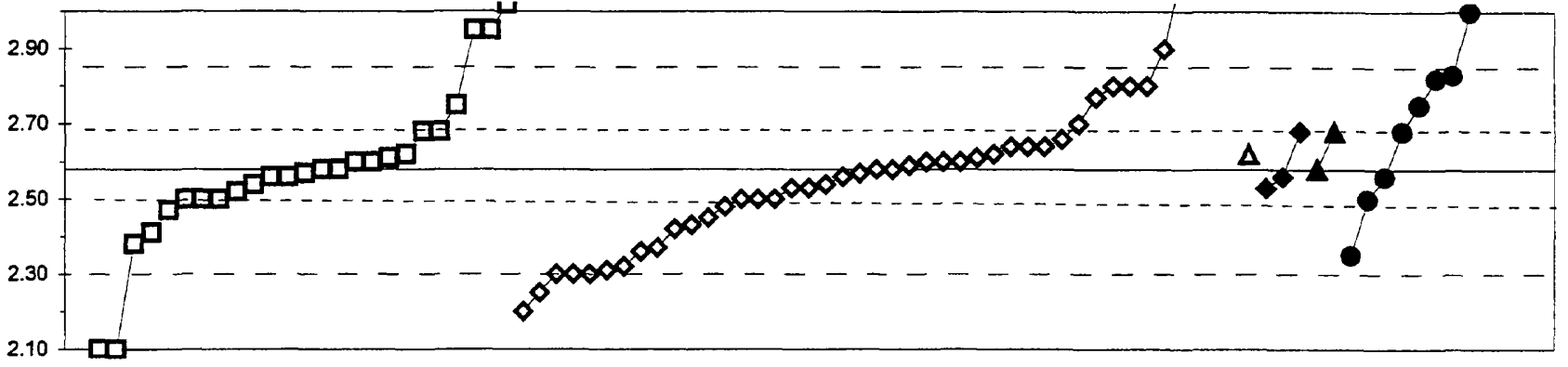

\begin{tabular}{|c|c|c|c|c|c|c|c|c|c|c|c|c|c|c|c|c|c|}
\hline \multicolumn{18}{|c|}{$-\square-1-4-\Delta-5-6-\Delta-7-12$} \\
\hline 1. AA: dire & ect air & & & ICP/MS & & & & & & & & & & & & & \\
\hline 4. ICP & & & & Ion chro & atograph & & & & & & & & $M P V=$ & 2.58 & & & \\
\hline 5. DCP & & & & Flane & ission & & & & & & & oseudos & igma $=$ & 0.14 & & & \\
\hline & & $N=$ & 26 & 43 & 1 & 3 & 2 & 12 & & & & & $N=$ & 87 & & & \\
\hline & & finimum $=$ & 2.00 & 2.20 & 2.62 & 2.53 & 2.58 & 2.35 & & & & & $\mathrm{Hu}=$ & 2.69 & & & \\
\hline & & aximum $=$ & 3.20 & 4.20 & & 2.68 & 2.68 & 3.91 & & & & & $H I=$ & 2.50 & & & \\
\hline & & Median = & 2.57 & 2.58 & & & & 283 & & & & & & & & & \\
\hline & F-pseu & losigma $=$ & 0.09 & 0.16 & & & & 0.39 & & & & & & & & & \\
\hline Lab & Rating & Z-value & 1 & 4 & 5 & 6 & 7 & 12 & Lab & Rating & Z-value & 1 & 4 & 5 & 6 & 7 & 12 \\
\hline 1 & 4 & -0.07 & 2.57 & & & & & & 132 & 3 & 0.57 & & 2.66 & & & & \\
\hline 2 & 0 & -3.41 & 2.10 & & & & & & 134 & 4 & -0.28 & 2.54 & & & & & \\
\hline 3 & 2 & -1.07 & & 2.43 & & & & & 138 & 4 & 0.07 & & 2.59 & & & & \\
\hline 5 & 2 & -1.49 & & 2.37 & & & & & 140 & 4 & 0.00 & 2.58 & & & & & \\
\hline 10 & 4 & 0.00 & 2.58 & & & & & & 141 & 4 & -0.07 & & 2.57 & & & & \\
\hline 13 & 1 & -1.99 & & 2.30 & & & & & 142 & 1 & -1.99 & & 2.30 & & & & \\
\hline 15 & 1 & -1.56 & & 2.36 & & & & & 145 & 4 & -0.28 & & 2.54 & & & & \\
\hline 16 & 3 & -0.57 & 2.50 & & & & & & 146 & 0 & 4.54 & & 3.22 & & & & \\
\hline 18 & 0 & -2.70 & & 2.20 & & & & & 151 & 3 & 0.71 & & & & 2.68 & & \\
\hline 19 & 4 & 0.14 & & 2.60 & & & & & 180 & 2 & -1.14 & & 2.42 & & & & \\
\hline 23 & 3 & 0.71 & 2.68 & & & & & & 190 & 4 & 0.00 & & & & & 2.58 & \\
\hline 24 & 3 & -0.92 & & 2.45 & & & & & 191 & 4 & -0.14 & & & & 2.56 & & \\
\hline 26 & 3 & 0.71 & & & & & 2.68 & & 212 & 3 & -0.57 & & 2.50 & & & & \\
\hline 28 & 1 & -1.85 & & 2.32 & & & & & 215 & 1 & 1.56 & & 2.80 & & & & \\
\hline 32 & 4 & -0.36 & & & & 2.53 & & & 217 & 0 & 11.50 & & 4.20 & & & & \\
\hline 33 & 4 & 0.28 & & & 2.62 & & & & 218 & 2 & -1.21 & 2.41 & & & & & \\
\hline 36 & 0 & -3.41 & 2.10 & & & & & & 219 & 1 & 1.56 & & 2.80 & & & & \\
\hline 38 & 4 & 0.28 & 2.62 & & & & & & 220 & 4 & 0.14 & 2.60 & & & & & \\
\hline 40 & 4 & 0.43 & & 2.64 & & & & & 221 & 4 & 0.21 & 2.61 & & & & & \\
\hline 42 & 3 & 0.85 & & 2.70 & & & & & 224 & 0 & 3.69 & & 3.10 & & & & \\
\hline 43 & 4 & 0.14 & & 2.60 & & & & & 234 & 4 & 0.43 & & 2.64 & & & & \\
\hline 46 & 0 & -2.34 & & 2.25 & & & & & 235 & 0 & 11.15 & & 4.15 & & & & \\
\hline 48 & 4 & 0.00 & & 2.58 & & & & & 236 & 1 & -1.92 & & 2.31 & & & & \\
\hline 50 & 3 & -0.57 & & 2.50 & & & & & 241 & 4 & -0.14 & 2.56 & & & & & \\
\hline 51 & 4 & -0.14 & & & & & & 2.56 & 249 & 3 & 0.71 & & & & & & 2.68 \\
\hline 56 & 0 & 3.12 & 3.02 & & & & & & 255 & 4 & 0.28 & & 2.62 & & & & \\
\hline 57 & 0 & 4.40 & 3.20 & & & & & & 256 & 0 & 9.44 & & & & & & 3.91 \\
\hline 59 & NR & & & $<5$ & & & & & 257 & 0 & 5.11 & & & & & & 3.30 \\
\hline 64 & 3 & 0.71 & 2.68 & & & & & & 258 & 2 & 1.21 & & & & & & 2.75 \\
\hline 68 & 0 & 2.27 & & 2.90 & & & & & 259 & 3 & -0.57 & 2.50 & & & & & \\
\hline 69 & 1 & 1.70 & & & & & & $2 . \overline{82}$ & 261 & 1 & -1.63 & & & & & & 2.35 \\
\hline 70 & 3 & -0.71 & & 2.48 & & & & & 262 & 1 & 1.78 & & & & & & 2.83 \\
\hline 80 & 0 & -4.12 & 2.00 & & & & & & 265 & 4 & -0.43 & 2.52 & & & & & \\
\hline 81 & 4 & -0.36 & & 2.53 & & & & & 268 & 1 & 1.93 & 2.85 & & & & & \\
\hline 83 & 4 & 0.14 & & 2.60 & & & & & 270 & 0 & 4.47 & & & & & & 3.21 \\
\hline 85 & 0 & 2.63 & 2.95 & 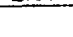 & & & & & 271 & 3 & -0.57 & & & & & & 2.50 \\
\hline 86 & 4 & 0.43 & & 2.64 & & & & & 272 & 0 & 2.98 & & & & & & 3.00 \\
\hline 87 & 3 & -0.78 & 2.47 & & & & & & 273 & 1 & 1.56 & & 2.80 & & & & \\
\hline 89 & 2 & -1.42 & 2.38 & & & & & & 274 & 0 & 3.55 & & & & & & 3.08 \\
\hline 97 & 4 & 0.14 & 2.60 & & & & & & & & & & & & & & \\
\hline 102 & 1 & -1.99 & & 2.30 & & & & & & & & & & & & & \\
\hline 105 & 4 & 0.21 & & 2.61 & & & & & & & & & & & & & \\
\hline 107 & 2 & 1.21 & 2.75 & & & & & & & & & & & & & & \\
\hline 109 & 4 & -0.14 & & 2.56 & & & & & & & & & & & & & \\
\hline 113 & 2 & 1.35 & & 2.77 & & & & & & & & & & & & & \\
\hline 119 & 3 & -0.57 & & 2.50 & & & & & & & & & & & & & \\
\hline 121 & 4 & -0.14 & 2.56 & & & & & & & & & & & & & & \\
\hline 127 & 4 & -0.36 & & 2.53 & & & & & & & & & & & & & \\
\hline 128 & 4 & 0.00 & & 2.58 & & & & & & & & & & & & & \\
\hline 129 & 3 & -0.57 & 2.50 & & & & & & & & & & & & & & \\
\hline
\end{tabular}


Table 14. Statistical summary of reported data for standard reference water sample M-140 (major constituents)-Continued $\mathrm{Mg}$ (Magnesium) $\mathrm{mg} / \mathrm{L}$

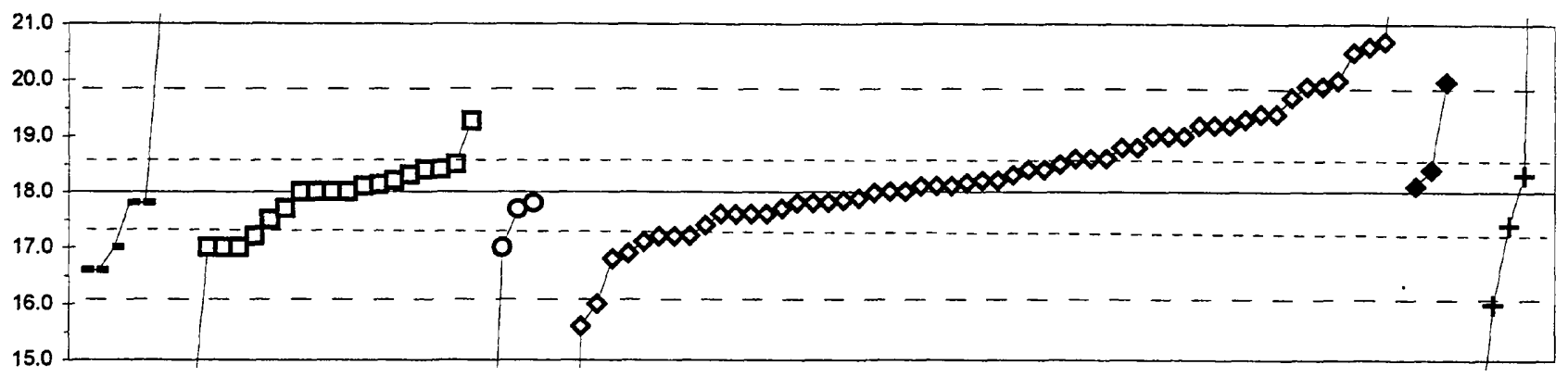

$\rightarrow 0-\square-1-0-2 \multimap 4-6+20$

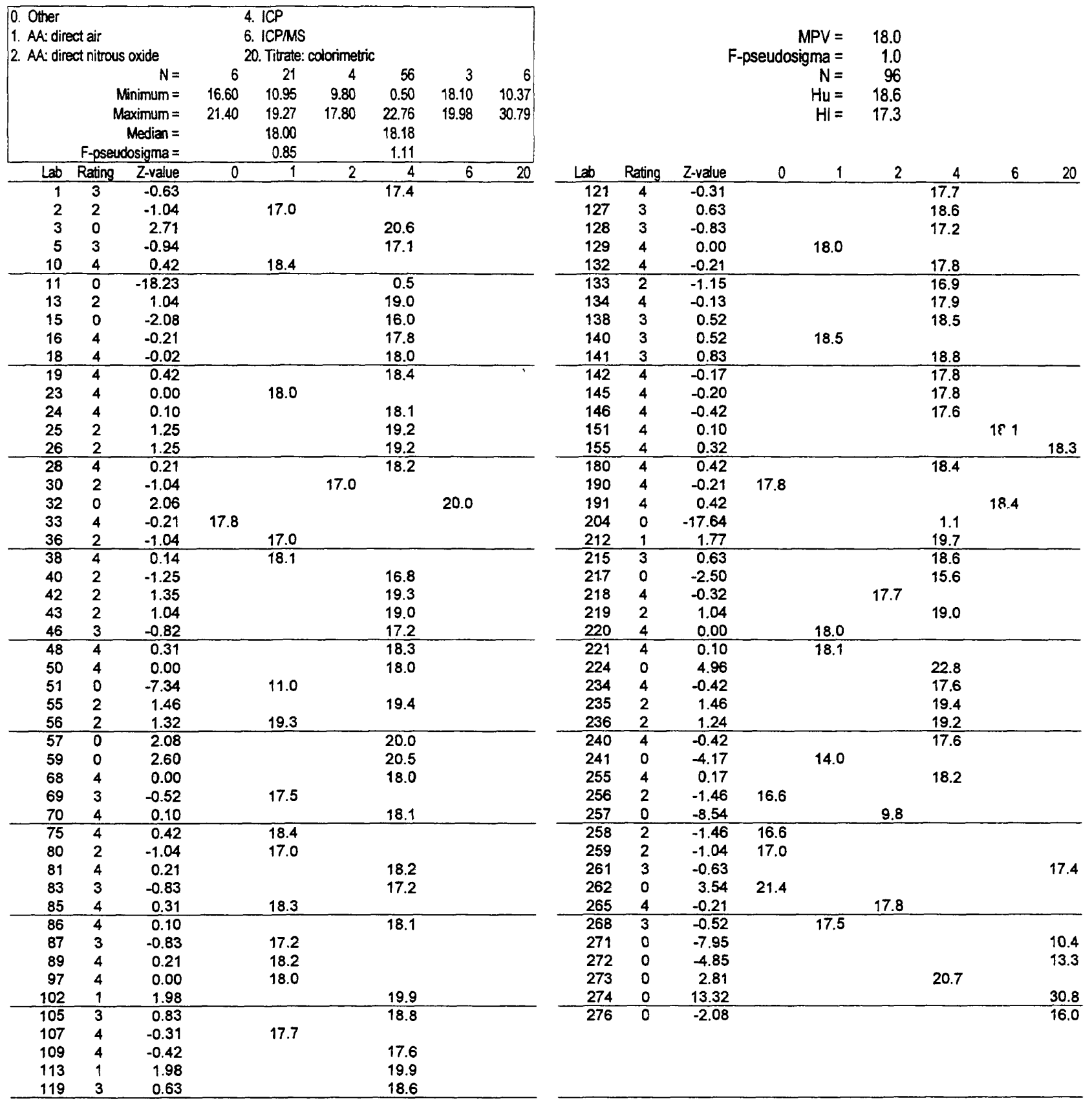


Table 14. Statistical summary of reported data for standard reference water sample M-140 (major constituents)-Continued $\mathrm{Na}$ (Sodium) $\mathrm{mg} / \mathrm{L}$

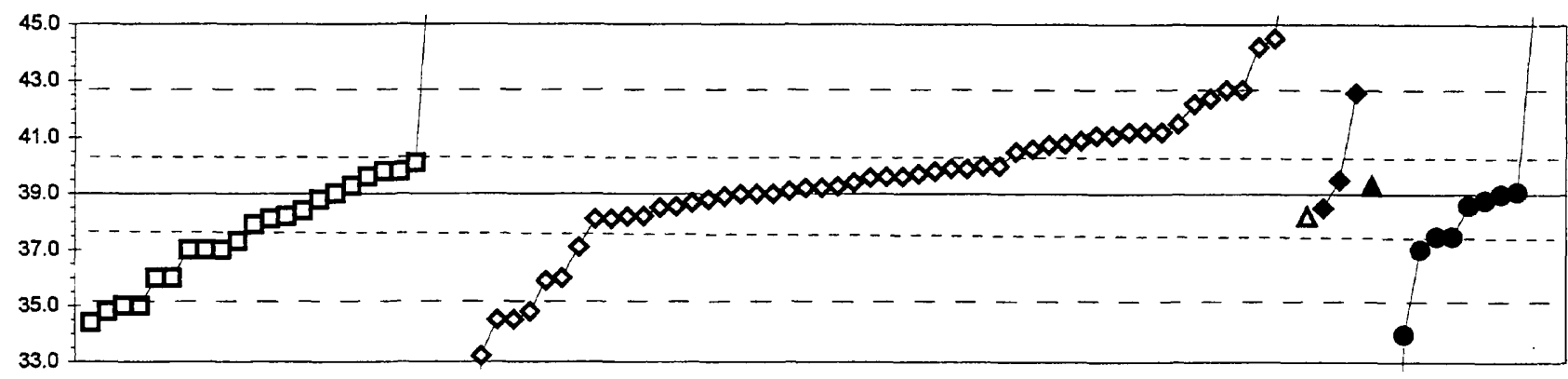

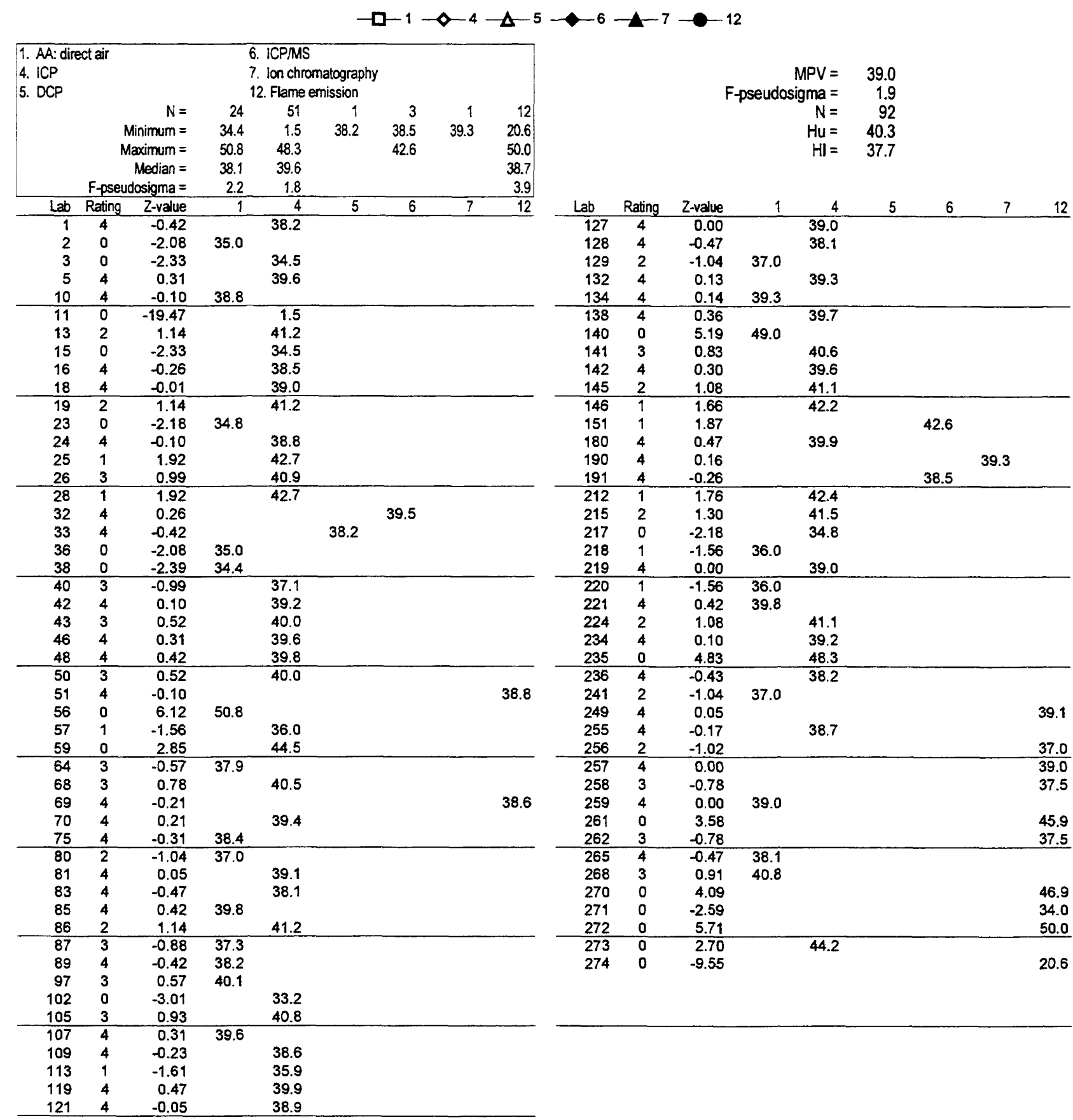


Table 14. Statistical summary of reported data for standard reference water sample $M-140$ (major constituents)-Continued total P as P (total Phosphorus as Phosphorus) $\mathrm{mg} / \mathrm{L}$

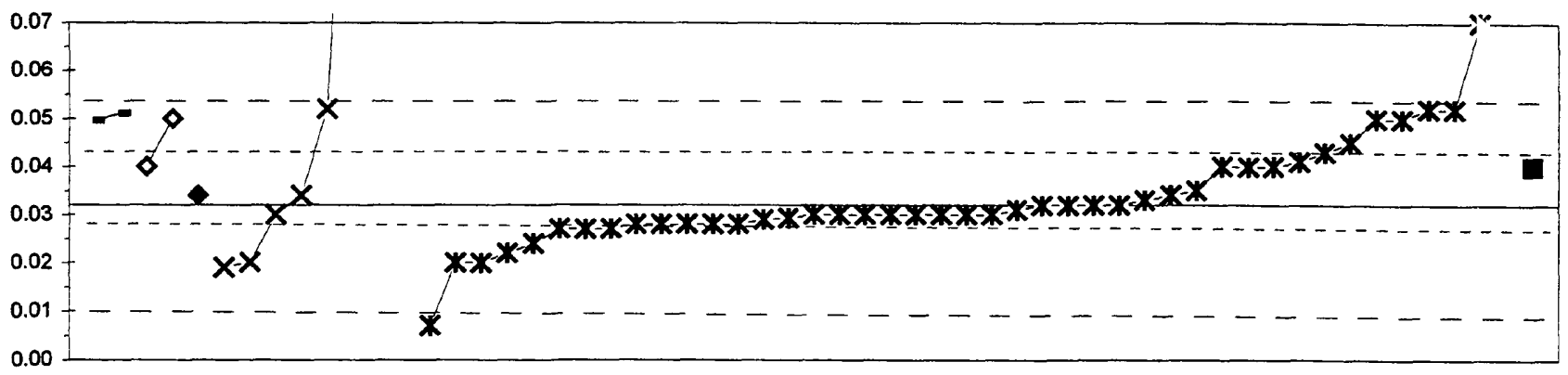

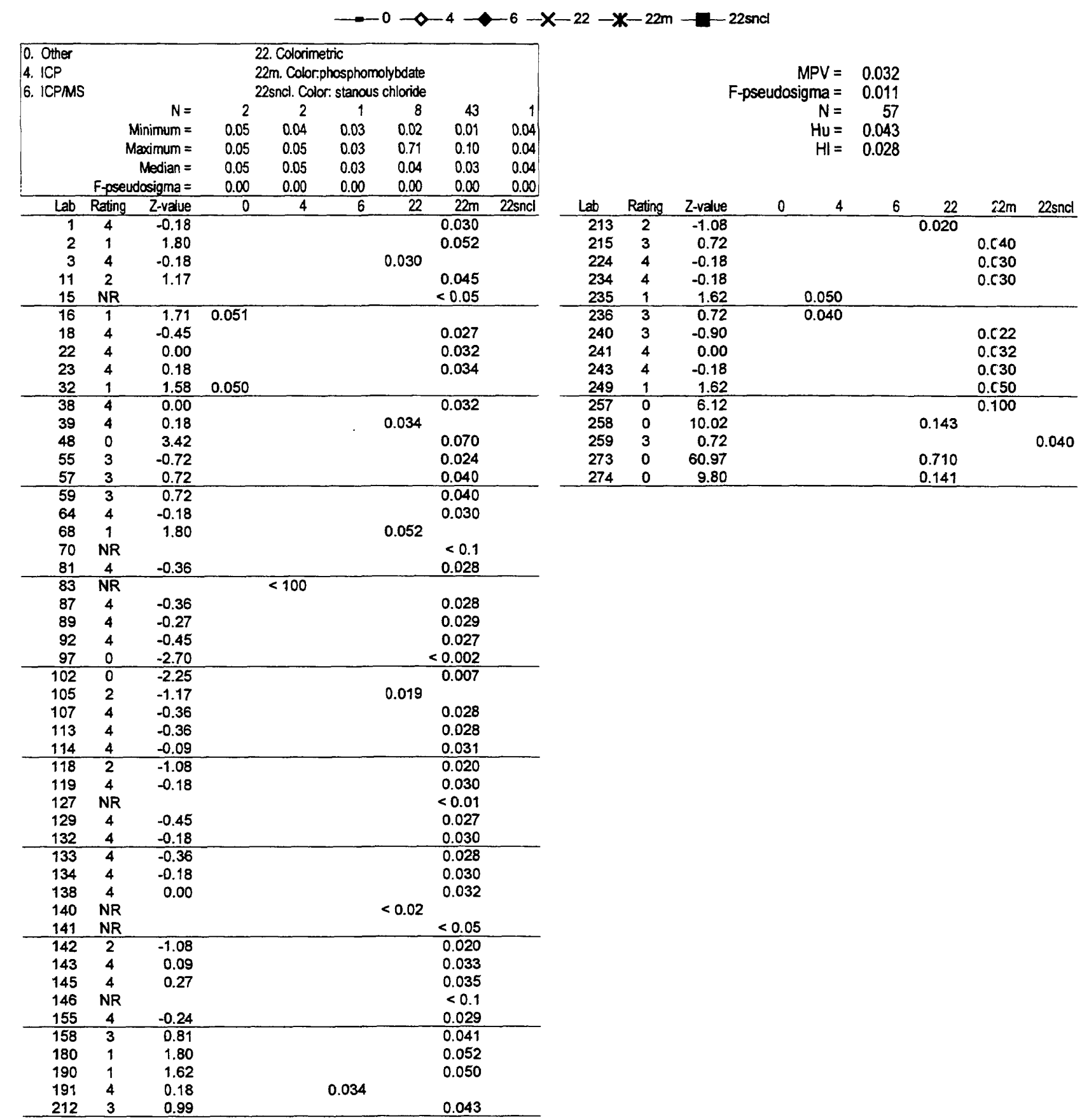


Table 14. Statistical summary of reported data for standard reference water sample $M-140$ (major constituents)-Continued $\mathrm{pH}$

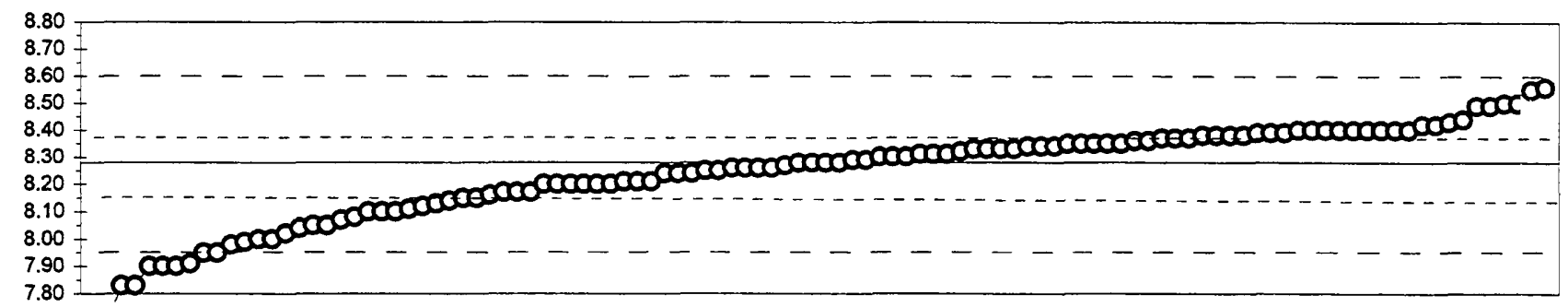

$-0-41$

\begin{tabular}{|c|c|c|c|}
\hline \multicolumn{4}{|c|}{ 41. Direct reading } \\
\hline & F-pseu & $\begin{aligned} \mathrm{N} & = \\
\text { Ainimum } & = \\
\text { laximum } & = \\
\text { Median } & = \\
\text { dosigma } & \end{aligned}$ & $\begin{array}{r}108 \\
7.42 \\
8.56 \\
8.28 \\
0.17\end{array}$ \\
\hline Lab & Rating & Z-value & $\frac{0.77}{41}$ \\
\hline 1 & 4 & -0.42 & 8.21 \\
\hline 2 & 2 & -1.44 & 8.04 \\
\hline 3 & 0 & -2.70 & 7.83 \\
\hline 5 & 3 & .0 .66 & 8.17 \\
\hline 7 & 4 & $\begin{array}{r}-0.48 \\
-0.48\end{array}$ & 8.20 \\
\hline 10 & 3 & 0.84 & 8.42 \\
\hline 11 & 4 & -0.12 & 8.26 \\
\hline 13 & 4 & 0.48 & 8.36 \\
\hline 15 & 2 & -1.02 & 8.11 \\
\hline 16 & 2 & -1.08 & 8.10 \\
\hline 18 & 4 & -0.18 & 8.25 \\
\hline 19 & 1 & 1.62 & 8.55 \\
\hline 23 & 4 & 0.00 & 8.28 \\
\hline 24 & 4 & -0.48 & 8.20 \\
\hline 25 & 4 & 0.48 & 8.36 \\
\hline 26 & 4 & 0.30 & 8.33 \\
\hline 30 & 3 & 0.72 & 8.40 \\
\hline 32 & 3 & 0.72 & 8.40 \\
\hline 33 & 4 & 0.00 & 8.28 \\
\hline 36 & 4 & 0.30 & 8.33 \\
\hline 38 & 3 & 0.72 & 8.40 \\
\hline 39 & 3 & 0.72 & 8.40 \\
\hline 40 & 4 & 0.42 & 8.35 \\
\hline 42 & 0 & -2.28 & 7.90 \\
\hline 43 & 4 & -0.42 & 8.21 \\
\hline 46 & 3 & 0.60 & 8.38 \\
\hline 48 & 1 & -1.68 & 8.00 \\
\hline 50 & 4 & 0.18 & 8.31 \\
\hline 51 & 4 & 0.06 & 8.29 \\
\hline 55 & 1 & -1.68 & 8.00 \\
\hline 56 & 4 & 0.42 & 8.35 \\
\hline 57 & 4 & -0.48 & 8.20 \\
\hline 59 & 3 & 0.72 & 8.40 \\
\hline 64 & 3 & 0.60 & 8.38 \\
\hline 68 & 3 & 0.60 & 8.38 \\
\hline 69 & 3 & 0.72 & 8.40 \\
\hline 70 & 4 & 0.00 & 8.28 \\
\hline 75 & 4 & -0.06 & 8.27 \\
\hline 76 & 4 & -0.12 & 8.26 \\
\hline 80 & 3 & -0.66 & 8.17 \\
\hline 81 & 3 & 0.54 & 8.37 \\
\hline 85 & 3 & 0.54 & 8.37 \\
\hline 86 & 4 & 0.42 & 8.35 \\
\hline 87 & 1 & -1.98 & 7.95 \\
\hline 89 & 3 & 0.66 & 8.39 \\
\hline 92 & 3 & -0.84 & 8.14 \\
\hline 96 & 4 & 0.36 & 8.34 \\
\hline 97 & 3 & 0.54 & 8.37 \\
\hline 105 & 4 & 0.36 & 8.34 \\
\hline 107 & 2 & 1.26 & 8.49 \\
\hline 109 & 3 & 0.84 & 8.42 \\
\hline 113 & 4 & 0.24 & 8.32 \\
\hline 114 & 2 & -1.26 & 8.07 \\
\hline 118 & 0 & -2.28 & 7.90 \\
\hline 119 & 2 & 1.32 & 8.50 \\
\hline
\end{tabular}

\begin{tabular}{|c|c|c|c|c|}
\hline \multirow[b]{2}{*}{ Lab } & \multirow[b]{2}{*}{ Rating } & \multicolumn{2}{|c|}{$\begin{aligned} M P V & = \\
\text { F-pseudosigma } & = \\
N & = \\
H u & = \\
H I & =\end{aligned}$} & \multirow[t]{2}{*}{$\begin{array}{l}8.28 \\
0.17 \\
108 \\
8.37 \\
8.15\end{array}$} \\
\hline & & Z-value & 41 & \\
\hline 127 & 4 & 0.18 & 8.31 & \\
\hline 128 & 3 & 0.90 & 8.43 & \\
\hline 129 & 1 & -1.74 & 7.99 & \\
\hline 132 & 2 & -1.38 & 8.05 & \\
\hline 133 & 3 & -0.78 & 8.15 & \\
\hline 134 & 3 & 0.96 & 8.44 & \\
\hline 138 & 4 & 0.06 & 8.29 & \\
\hline 140 & 4 & 0.30 & 8.33 & \\
\hline 141 & 4 & 0.42 & 8.35 & \\
\hline 142 & 4 & -0.12 & 8.26 & \\
\hline 143 & 3 & 0.60 & 8.38 & \\
\hline 145 & 4 & 0.12 & 8.30 & \\
\hline 146 & 3 & -0.78 & 8.15 & \\
\hline 149 & 3 & 0.72 & 8.40 & \\
\hline 151 & 3 & 0.66 & 8.39 & \\
\hline 155 & 4 & -0.24 & 8.24 & \\
\hline 158 & 4 & -0.12 & 8.26 & \\
\hline 180 & 3 & 0.72 & 8.40 & \\
\hline 183 & 4 & 0.30 & 8.33 & \\
\hline 190 & 2 & -1.08 & 8.10 & \\
\hline 191 & 3 & 0.66 & 8.39 & \\
\hline 203 & 4 & 0.18 & 8.31 & \\
\hline 204 & 3 & -0.72 & 8.16 & \\
\hline 212 & 4 & 0.12 & 8.30 & \\
\hline 213 & 4 & 0.42 & 8.35 & \\
\hline 215 & 4 & -0.18 & 8.25 & \\
\hline 217 & 3 & 0.72 & 8.40 & \\
\hline 218 & 1 & -1.56 & 8.02 & \\
\hline 221 & 2 & -1.20 & 8.08 & \\
\hline 224 & 3 & $\begin{array}{l}-0.66 \\
\end{array}$ & 8.17 & \\
\hline 234 & 3 & -0.90 & 8.13 & \\
\hline 236 & 4 & -0.24 & 8.24 & \\
\hline 240 & 4 & -0.24 & 8.24 & \\
\hline 241 & 4 & 0.12 & 8.30 & \\
\hline 243 & 0 & -2.22 & 7.91 & \\
\hline 244 & 4 & 0.36 & 8.34 & \\
\hline 247 & 1 & 1.68 & 8.56 & \\
\hline 249 & 1 & -1.80 & 7.98 & \\
\hline 253 & 4 & -0.48 & 8.20 & \\
\hline 255 & 2 & 1.26 & 8.49 & \\
\hline 256 & 2 & -1.38 & 8.05 & \\
\hline 257 & 4 & -0.42 & 8.21 & \\
\hline 258 & 4 & -0.48 & 8.20 & \\
\hline 259 & 2 & 1.32 & 8.50 & \\
\hline 261 & 1 & -1.98 & 7.95 & \\
\hline 262 & 0 & -5.16 & 7.42 & \\
\hline 265 & 3 & -0.96 & 8.12 & \\
\hline 268 & 2 & -1.08 & 8.10 & \\
\hline 271 & 0 & -2.70 & 7.83 & \\
\hline 272 & 4 & 0.00 & 8.28 & \\
\hline 273 & 4 & -0.48 & 8.20 & \\
\hline 274 & 0 & -2.28 & 7.90 & \\
\hline 276 & 0 & -3.48 & 7.70 & \\
\hline
\end{tabular}


Table 14. Statistical summary of reported data for standard reference water sample M-140 (major constituents)-Continued $\mathrm{SiO}_{2}$ (Silca) $\mathrm{mg} / \mathrm{L}$

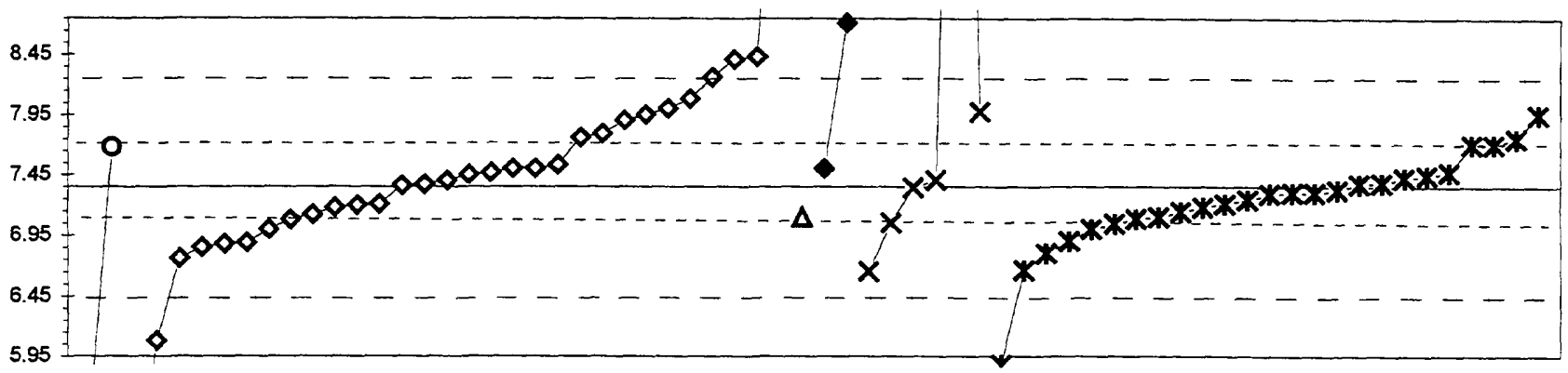

\begin{tabular}{|c|c|c|c|c|c|c|c|c|}
\hline & & & & & & $-2-$ & -4 & -5 \\
\hline $\begin{array}{l}\text { 2. } \text { AA: dire } \\
\text { 4. ICP } \\
\text { 5. DCP }\end{array}$ & ect nitrou & oxide & & $\begin{array}{l}\text { ICP/MS } \\
\text { 2. Colorir } \\
\text { 2mb. Col }\end{array}$ & $\begin{array}{l}\text { tric } \\
\text { molybod }\end{array}$ & blue & & \\
\hline & & $N=$ & 2 & 30 & 1 & 2 & 6 & 25 \\
\hline & & inimum = & 5.50 & 4.66 & 7.10 & 7.50 & 6.65 & 5.88 \\
\hline & & aximum = & 7.68 & 11.04 & & 8.71 & 14.93 & 7.95 \\
\hline & & Median = & & 7.43 & & & & 7.29 \\
\hline & F-psed & osigma $=$ & & 0.61 & & & & 0.00 \\
\hline Lab & Rating & Z-value & 2 & 4 & 5 & 6 & 22 & $22 m b$ \\
\hline 1 & 3 & -1.00 & & $6 . \overline{89}$ & & & & \\
\hline 3 & 0 & 2.35 & & 8.43 & & & & \\
\hline 5 & 3 & -0.59 & & 7.08 & & & & \\
\hline 10 & 4 & -0.11 & & & & & & 7.30 \\
\hline 13 & 4 & 0.04 & & 7.37 & & & & \\
\hline 15 & 2 & 1.35 & & & & & 7.97 & \\
\hline 18 & 4 & 0.20 & & & & & & 7.44 \\
\hline 23 & 3 & 0.87 & & & & & & 7.75 \\
\hline 24 & 3 & 0.89 & & 7.76 & & & & \\
\hline 25 & 0 & 8.03 & & 11.04 & & & & \\
\hline 26 & 4 & 0.39 & & 7.53 & & & & \\
\hline 32 & 4 & 0.33 & & & & 7.50 & & \\
\hline 33 & 3 & -0.54 & & & 7.10 & & & \\
\hline 38 & 4 & -0.13 & & & & & & 7.29 \\
\hline 40 & 4 & -0.37 & & 7.18 & & & & \\
\hline 42 & 2 & 1.41 & & 8.00 & & & & \\
\hline 43 & 4 & 0.33 & & 7.50 & & & & \\
\hline 46 & 3 & -0.67 & & & & & & 7.04 \\
\hline 50 & 4 & -0.11 & & & & & & 7.30 \\
\hline 55 & 4 & 0.02 & & 7.36 & & & & \\
\hline 57 & 4 & 0.11 & & 7.40 & & & & \\
\hline 59 & 3 & 0.76 & & & & & & 7.70 \\
\hline 68 & 3 & -0.65 & & & & & 7.05 & \\
\hline 70 & 3 & -0.54 & & & & & & 7.10 \\
\hline 80 & 0 & -4.03 & 5.50 & & & & & \\
\hline 81 & 4 & -0.24 & & & & & & 7.24 \\
\hline 83 & 2 & -1.28 & & 6.76 & & & & \\
\hline 85 & 4 & -0.07 & & & & & & 7.32 \\
\hline 87 & 4 & -0.37 & & & & & & 7.18 \\
\hline 89 & 2 & -1.20 & & & & & & 6.80 \\
\hline 97 & 4 & 0.04 & & & & & & 7.37 \\
\hline 102 & 2 & -1.50 & & & & & & 6.66 \\
\hline 105 & 4 & 0.22 & & 7.45 & & & & \\
\hline 107 & 4 & -0.30 & & & & & & 7.21 \\
\hline 113 & 4 & -0.02 & & & & & 7.34 & \\
\hline 118 & 4 & 0.26 & & & & & & 7.47 \\
\hline 119 & 3 & -0.76 & & 7.00 & & & & \\
\hline 121 & 4 & -0.50 & & 7.12 & & & & \\
\hline 127 & 4 & -0.30 & & 7.21 & & & & \\
\hline 128 & 3 & 0.96 & & 7.79 & & & & \\
\hline 129 & 2 & 1.31 & & & & & & 7.95 \\
\hline 134 & 4 & 0.25 & & 7.47 & & & & \\
\hline 138 & 4 & -0.46 & & & & & & 7.14 \\
\hline 140 & 4 & 0.11 & & & & & 7.40 & \\
\hline 142 & 0 & 2.31 & & 8.41 & & & & \\
\hline 145 & 2 & 1.31 & & 7.95 & & & & \\
\hline 151 & 0 & -3.20 & & & & & & 5.88 \\
\hline 155 & 3 & -0.58 & & & & & & 7.08 \\
\hline 190 & 3 & 0.76 & & & & & & 7.70 \\
\hline 191 & 0 & 2.96 & & & & 8.71 & & \\
\hline
\end{tabular}


Table 14. Statistical summary of reported data for standard reference water sample $M-140$ (major constituents)--Continued $\mathrm{SO}_{4}$ (Sulfate) $\mathrm{mg} / \mathrm{L}$

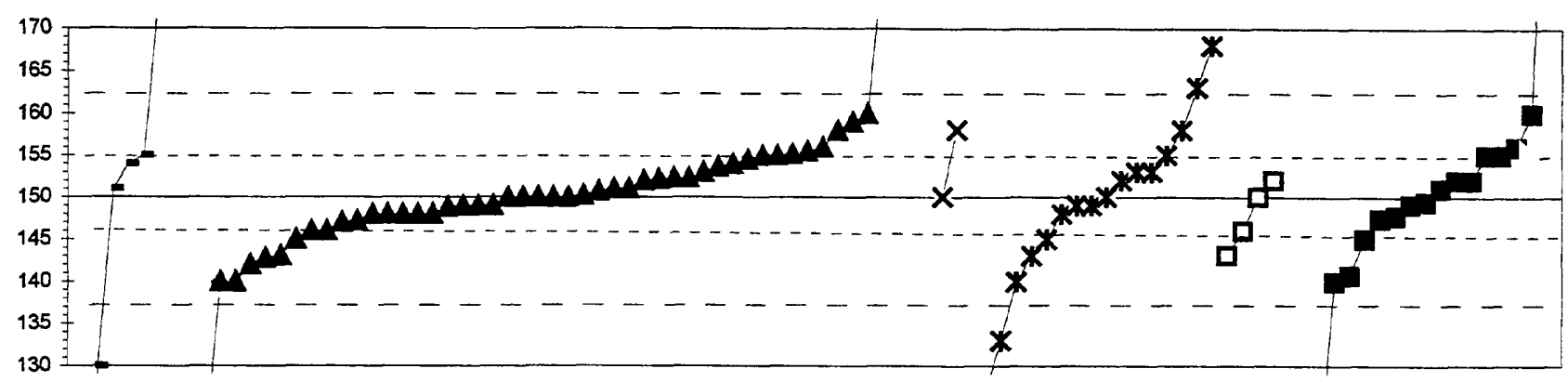

$-0-0-7-x-22-x-22 m+b-\square-50-\square-51$

\begin{tabular}{|c|c|c|c|c|c|c|c|c|c|c|c|c|c|c|c|c|c|}
\hline $\begin{array}{l}\text { 0. Other } \\
\text { 7. Ion chro } \\
\text { 22. Colonin }\end{array}$ & $\begin{array}{l}\text { matogra } \\
\text { netric }\end{array}$ & & & $\begin{array}{l}\text { th. Co } \\
\text { Gravim } \\
\text { Turbidi }\end{array}$ & $\begin{array}{l}\text { methyl } \\
\text { c } \\
\text { ric }\end{array}$ & yymol blue & & & & & & seudos & $\begin{array}{l}\mathrm{PV}= \\
\mathrm{ma}=\end{array}$ & $\begin{array}{r}150 \\
7\end{array}$ & & & \\
\hline & & iv & 0 & & 2 & 17 & & 10 & & & & & $N=$ & & & & \\
\hline & & linimum = & 16 & 5 & 150 & 120 & 143 & 17 & & & & & $\mathrm{Hu}=$ & 155 & & & \\
\hline & & aximum = & 174 & 197 & 158 & 168 & 152 & 200 & & & & & $\mathrm{HI}=$ & 146 & & & \\
\hline & & Median = & & 150 & & 149 & & 149 & & & & & & & & & \\
\hline & F-psed & losigma $=$ & & 5 & & 7 & & 11 & & & & & & & & & \\
\hline Lab & Rating & Z-value & 0 & 7 & 22 & $22 \mathrm{mtb}$ & 50 & 51 & Lab & Rating & Z-value & 0 & 7 & 22 & $22 \mathrm{mtb}$ & 50 & 51 \\
\hline 1 & 4 & 0.03 & & 150 & & & & & 114 & 4 & 0.30 & & & & & & 152 \\
\hline 2 & 4 & 0.30 & & & & & 152 & & 119 & 2 & -1.05 & & 143 & & & & \\
\hline 3 & 2 & -1.20 & & 142 & & & & & 127 & 2 & 1.35 & & 159 & & & & \\
\hline 4 & 4 & 0.34 & & 152 & & & & & 128 & 3 & 0.75 & & 155 & & & & \\
\hline 5 & 4 & 0.15 & & 151 & & & & & 129 & 4 & 0.00 & & 150 & & & & \\
\hline 7 & 3 & 0.67 & & 155 & & & & & 134 & 4 & 0.30 & & 152 & & & & \\
\hline 10 & 4 & 0.15 & & & & & & 151 & 138 & 4 & -0.15 & & 149 & & & & \\
\hline 11 & 0 & -21.75 & & 5 & & & & & 140 & 3 & 0.75 & 155 & & & & & \\
\hline 13 & 4 & 0.00 & & 150 & & & & & 141 & 3 & 0.90 & & & & & & 156 \\
\hline 15 & 2 & -1.50 & & 140 & & & & & 142 & 1 & 1.95 & & & & 163 & & \\
\hline 16 & 2 & -1.03 & & & & & 143 & & 145 & 4 & -0.18 & & 149 & & & & \\
\hline 18 & 4 & -0.30 & & & & 148 & & & 146 & 3 & 0.75 & & & & & & 155 \\
\hline 19 & 4 & -0.15 & & & & 149 & & & 149 & 2 & 1.50 & & 160 & & & & \\
\hline 23 & 2 & 1.20 & & & 158 & 等 & & & 151 & 4 & 0.15 & & 151 & & & & \\
\hline 24 & 4 & 0.00 & & & & 150 & & & 158 & 4 & 0.30 & & & & 152 & & \\
\hline 25 & 4 & -0.30 & & 148 & & & & & 180 & 2 & -1.50 & & & & 140 & & \\
\hline 26 & 4 & 0.00 & & 150 & & & & & 190 & 0 & 4.05 & & 177 & & & & \\
\hline 30 & 4 & 0.00 & & 150 & & & & & 191 & 4 & -0.15 & & 149 & & & & \\
\hline 32 & 3 & -0.60 & & 146 & & & & & 193 & 4 & -0.15 & & 149 & & & & \\
\hline 33 & 4 & -0.42 & & 147 & & & & & 196 & 3 & -0.75 & & 145 & & & & \\
\hline 36 & 2 & 1.50 & & & & & & 160 & 203 & 0 & 2.70 & & & & 168 & & \\
\hline 39 & 4 & 0.00 & & 150 & & & & & 204 & 3 & 0.75 & & & & 155 & & \\
\hline 40 & 0 & -4.65 & & 119 & & & & & 212 & 3 & 0.90 & & 156 & & & & \\
\hline 42 & 0 & 7.04 & & 197 & & & & & 215 & 3 & 0.75 & & & & & & 155 \\
\hline 43 & 4 & 0.00 & & & & & 150 & & 217 & 2 & 1.20 & & 158 & & & & \\
\hline 46 & 0 & 5.25 & & 185 & & & & & 219 & 0 & -16.49 & & 40 & & & & \\
\hline 48 & 0 & -6.89 & & & & & & 104 & 220 & 4 & -0.34 & & & & & & 148 \\
\hline 50 & 4 & -0.15 & & & & 149 & & & 221 & 3 & -0.60 & & & & & 146 & \\
\hline 51 & 3 & 0.82 & & 156 & & & & & 224 & 4 & 0.32 & & 152 & & & & \\
\hline 55 & 0 & -2.55 & & & & 133 & & & 234 & 4 & 0.45 & & 153 & & & & \\
\hline 56 & 4 & -0.13 & & & & & & 149 & 235 & 0 & 3.60 & 174 & & & & & \\
\hline 57 & 2 & -1.50 & & & & & & 140 & 236 & 4 & 0.34 & & 152 & & & & \\
\hline 59 & 4 & -0.30 & & 148. & & & & & 240 & 4 & -0.45 & & 147 & & & & \\
\hline 64 & 4 & 0.10 & & $151^{\circ}$ & & & & & 241 & 4 & 0.30 & & & & & & 152 \\
\hline 69 & 2 & 1.20 & & & & 158 & & & 247 & 2 & -1.08 & & 143 & & & & \\
\hline 70 & 3 & -0.60 & & 146 & & & & & 249 & 2 & -1.39 & & & & & & 141 \\
\hline 75 & 4 & 0.45 & & & & 153 & & & 253 & 0 & -4.95 & & & & & & 117 \\
\hline 80 & 0 & -3.00 & 130 & & & & & & 255 & 0 & -4.50 & & & & 120 & & \\
\hline 81 & 3 & -0.75 & & & & 145 & & & 256 & 3 & 0.65 & 154 & & & & & \\
\hline 83 & 4 & 0.15 & 151 & & & & & & 257 & 0 & 6.75 & & 195 & & & & \\
\hline 85 & 4 & -0.30 & & 148 & & & & & 258 & 0 & 7.46 & & & & & & 200 \\
\hline 87 & 0 & -3.45 & & & & 127 & & & 259 & 4 & -0.30 & & 148 & & & & \\
\hline 89 & 4 & -0.30 & & 148 & & & & & 261 & 0 & -20.13 & 16 & & & & & \\
\hline 92 & 4 & -0.39 & & & & & & 147 & 262 & 4 & 0.00 & & & 150 & & & \\
\hline 96 & 3 & -0.75 & & & & & & 145 & 265 & 3 & 0.75 & & 155 & & & & \\
\hline 97 & 4 & 0.45 & & & & 153 & & & 268 & 3 & 0.55 & & 154 & & & & \\
\hline 102 & 2 & -1.05 & & & & 143 & & & 271 & 0 & -20.01 & & & & & & 17 \\
\hline 105 & 2 & -1.50 & & 140 & & & & & 274 & 4 & -0.09 & & & & & & 149 \\
\hline 109 & 3 & 0.78 & & 155 & & & & & & & & & & & & & \\
\hline 113 & 3 & 0.60 & & 154 & & & & & & & & & & & & & \\
\hline
\end{tabular}


Table 14. Statistical summary of reported data for standard reference water sample M-140 (major constituents)-Continued $\mathrm{Sp}$ Cond (Specific Conductance)

$\mu \mathrm{S} / \mathrm{cm}$

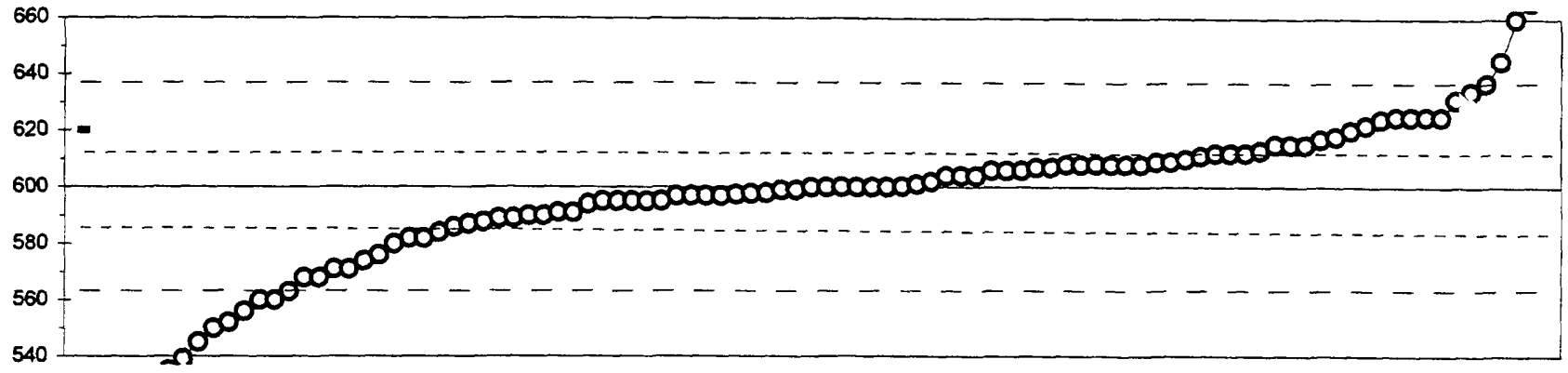

$-0-0-41$

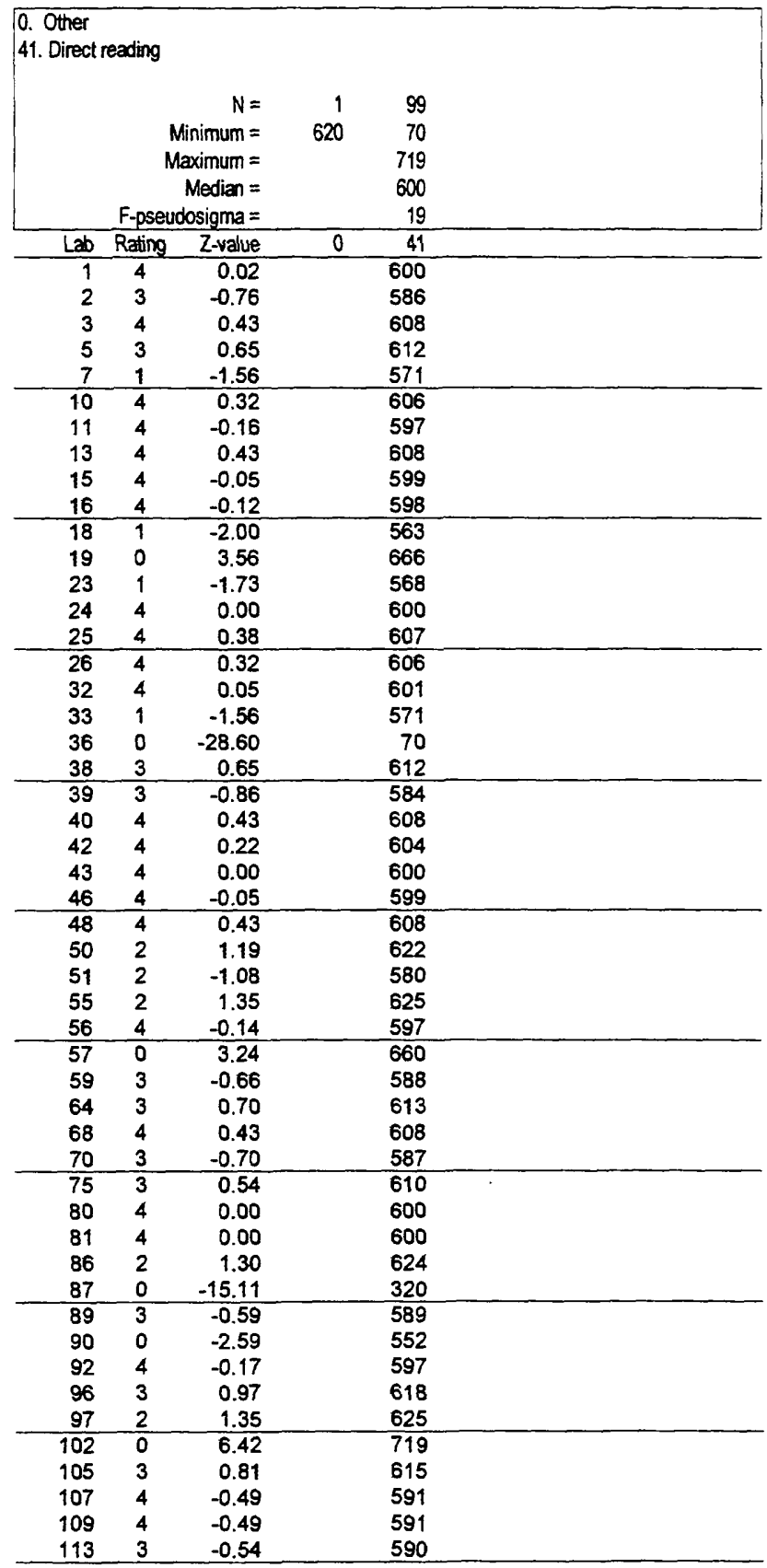

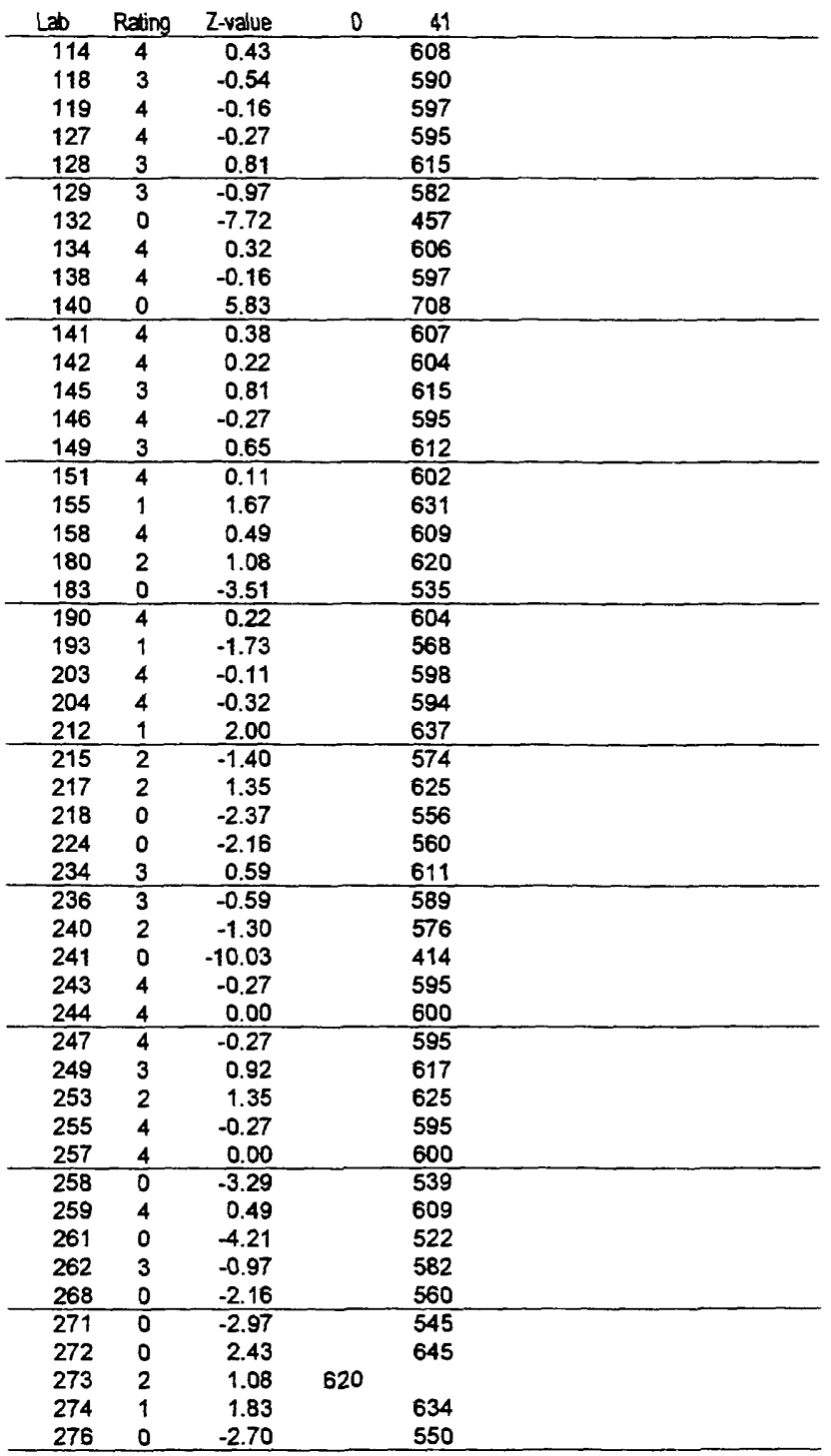


Table 14. Statistical summary of reported data for standard reference water sample $M-140$ (major constituents)-Continued $\mathrm{Sr}$ (Strontium)

$\mu \mathrm{g} / \mathrm{L}$

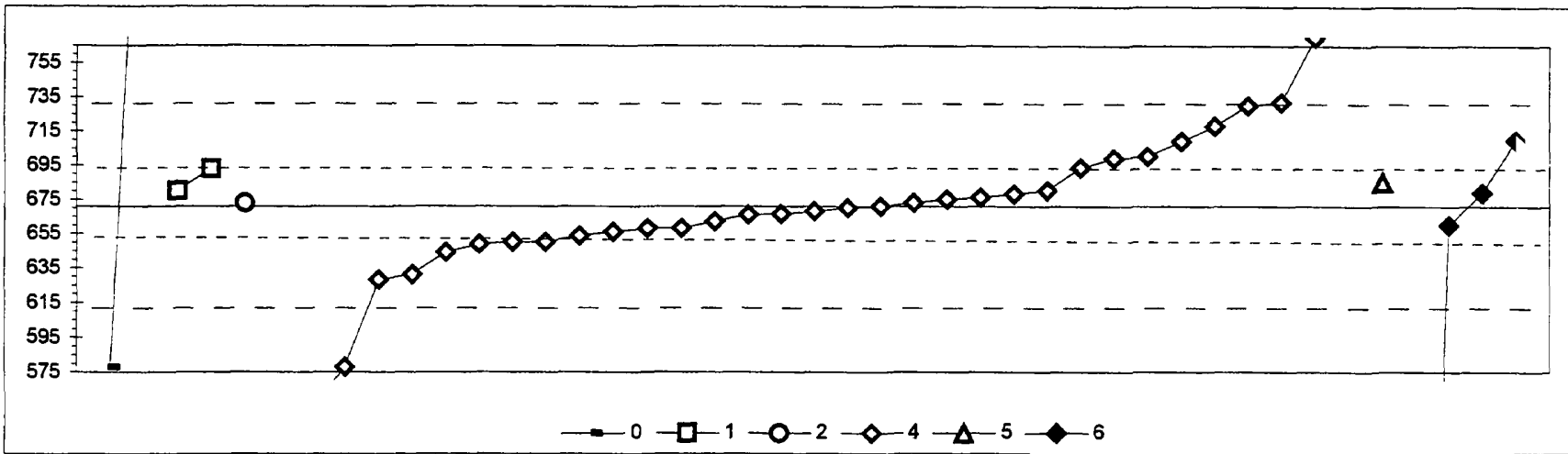

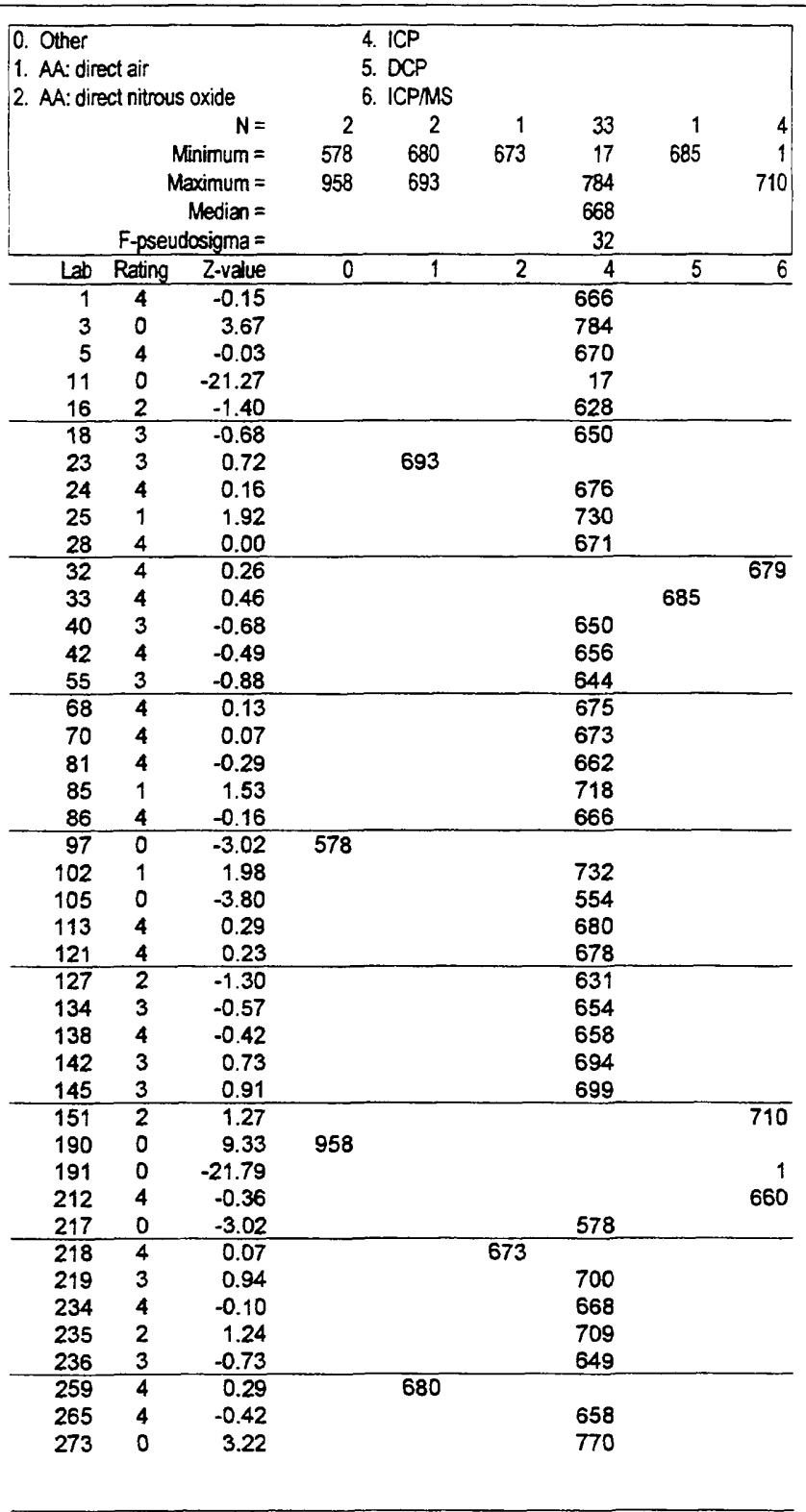


Table 14. Statistical summary of reported data for standard reference water sample $M-140$ (major constituents)-Continued $V$ (Vanadium)

$\mu \mathrm{g} / \mathrm{L}$

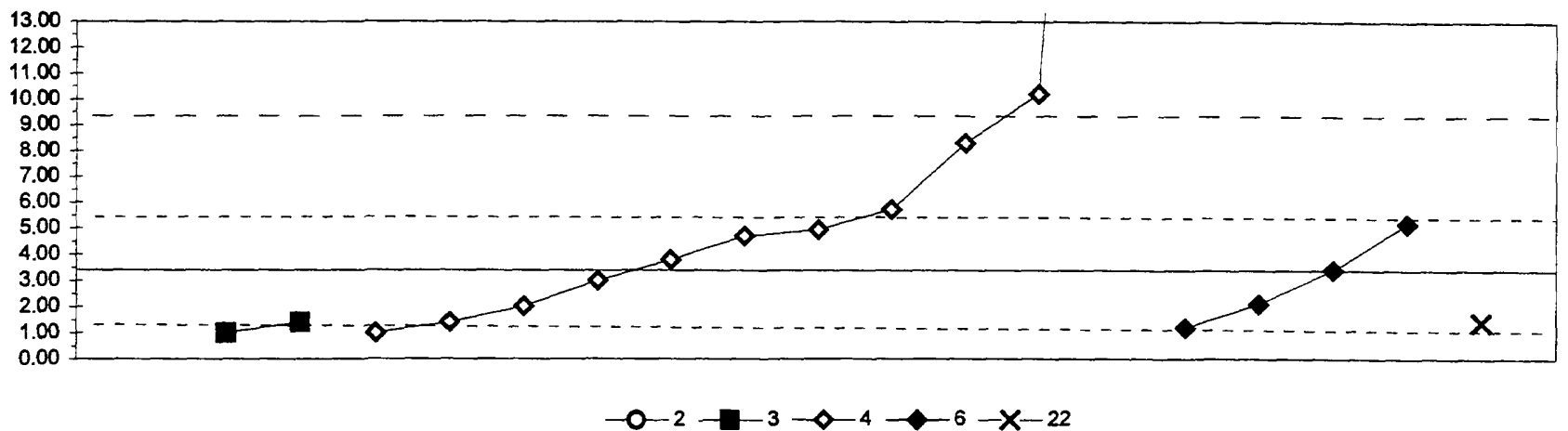

\begin{tabular}{|c|c|c|c|c|c|c|c|}
\hline \multicolumn{3}{|c|}{$\begin{array}{l}\text { 2. AA: direct nitrous oxide } \\
\text { 3. AA: graphite fumace } \\
\text { 4. ICP }\end{array}$} & \multicolumn{3}{|c|}{$\begin{array}{l}\text { 6. ICP/MS } \\
\text { 22. Colorimetric } \\
\text { 0. Other }\end{array}$} & \multirow{6}{*}{$\begin{array}{r}4 \\
1.20 \\
5.19\end{array}$} & \multirow{6}{*}{$\begin{array}{r}1 \\
1.40\end{array}$} \\
\hline & & $N=$ & 1 & 2 & 11 & & \\
\hline & & inimum $=$ & 39.00 & 1.00 & 1.00 & & \\
\hline & & ximum = & & 1.40 & 50.00 & & \\
\hline & & Median = & & & 4.70 & & \\
\hline \multicolumn{3}{|c|}{ F-pseudosigma $=$} & & & 3.34 & & \\
\hline Lab & Rating & Z-value & 2 & 3 & 4 & 6 & 22 \\
\hline 1 & 3 & -0.67 & & & & & 1.40 \\
\hline 3 & 4 & -0.14 & & & 3.00 & & \\
\hline 5 & NR & & & & $<4$ & & \\
\hline 13 & NR & & & & $<50$ & & \\
\hline 15 & NR & & & & $<10$ & & \\
\hline 16 & 3 & 0.59 & & & & 5.19 & \\
\hline 18 & NR & & & & $<5$ & & \\
\hline 23 & NR & & & $<5$ & & & \\
\hline 26 & NR & & & & $<4$ & & \\
\hline 28 & 0 & 2.25 & & & 10.20 & & \\
\hline 42 & NR & & & & & $<5$ & \\
\hline 48 & NR & & & & $<200$ & & \\
\hline 57 & NR & & & & $<100$ & & \\
\hline 68 & 0 & 15.48 & & & 50.00 & & \\
\hline 70 & NR & & & & $<50$ & & \\
\hline 81 & NR & & & & $<3$ & & \\
\hline 85 & NR & & & & $<20$ & & \\
\hline 86 & 3 & 0.77 & & & 5.73 & & \\
\hline 89 & NR & & & $<10$ & & & \\
\hline 97 & NR & & & $<0$ & & & \\
\hline 105 & NR & & & & $<13$ & & \\
\hline 127 & 3 & -0.67 & & 1.40 & & & \\
\hline 134 & NR & & & & $<1$ & & \\
\hline 138 & NR & & & & & $<2$ & \\
\hline 141 & NR & & & & $<10$ & & \\
\hline 142 & 4 & 0.00 & & & & 3.42 & \\
\hline 145 & 4 & 0.43 & & & 4.70 & & \\
\hline 146 & NR & & & & $<10$ & & \\
\hline 180 & 3 & 0.51 & & & 4.95 & & \\
\hline 212 & 4 & -0.44 & & & & 2.10 & \\
\hline 219 & 3 & -0.80 & & & 1.00 & & \\
\hline 224 & 1 & 1.62 & & & 8.30 & & \\
\hline 234 & 4 & -0.47 & & & 2.02 & & \\
\hline 235 & NR & & & & $<5$ & & \\
\hline 236 & 4 & 0.13 & & & 3.80 & & \\
\hline 241 & 3 & -0.80 & & 1.00 & & & \\
\hline 255 & 3 & -0.67 & & & 1.40 & & \\
\hline 257 & 0 & 11.82 & 39.00 & & & & \\
\hline 265 & 3 & -0.74 & & & & 1.20 & \\
\hline
\end{tabular}


Table 15. Statistical summary of reported data for standard reference water sample N-51 (nutrients)

Definition of analytical methods, abbreviations, and symbols

Analytical methods

0 . Other/Not reported

4. ICP

5. DCP

7. IC

20. Titrate: color

21. Titrate: electro

22. Color:

40. Ion electrode

$=\quad$ inductively coupled plasma

= direct coupled plasma

$=\quad$ ion chromatography

= titration: colorimetric (color reagent specified)

$=$ titration: electrmetric

$=$ colorimetric [color reagent specified]

$=\quad$ ion selective electrode

Abbreviations and symbols

$\begin{aligned} \mathrm{N}= & \text { number of samples } \\ \text { MPV }= & \text { most probable value } \\ \text { F-pseudosigma }= & \text { nonparametric statistic deviation } \\ \mathrm{Hu}= & \text { upper hinge value } \\ \mathrm{HI}= & \text { lower hinge value } \\ \mathrm{mg} / \mathrm{L}= & \text { milligrams per liter } \\ \mathrm{Lab}= & \text { laboratory code number } \\ \mathrm{NR}= & \text { not rated, less than value reported } \\ <= & \text { less than }\end{aligned}$

Constituent

$\mathrm{NH}_{3}$ as $\mathrm{N}$

Ammonia as nitrogen

$\mathrm{NH}_{3}+$ Org $\mathrm{N}$ as $\mathrm{N}$

Ammonia plus organic nitrogen

120

$\mathrm{NO}_{3}+\mathrm{NO}_{2}$ as $\mathrm{N}$

Total $P$ as $P$

Nitrate plus nitrite as nitrogen

PO as $P$

Total Phosphorus as phosphorus 
Table 15. Statistical summary of reported data for standard reference water sample N-51 (nutrient constituents)-Continued $\mathrm{NH}_{3}$ as $\mathrm{N}$ (Ammonia as nitrogen) $\mathrm{mg} / \mathrm{L}$

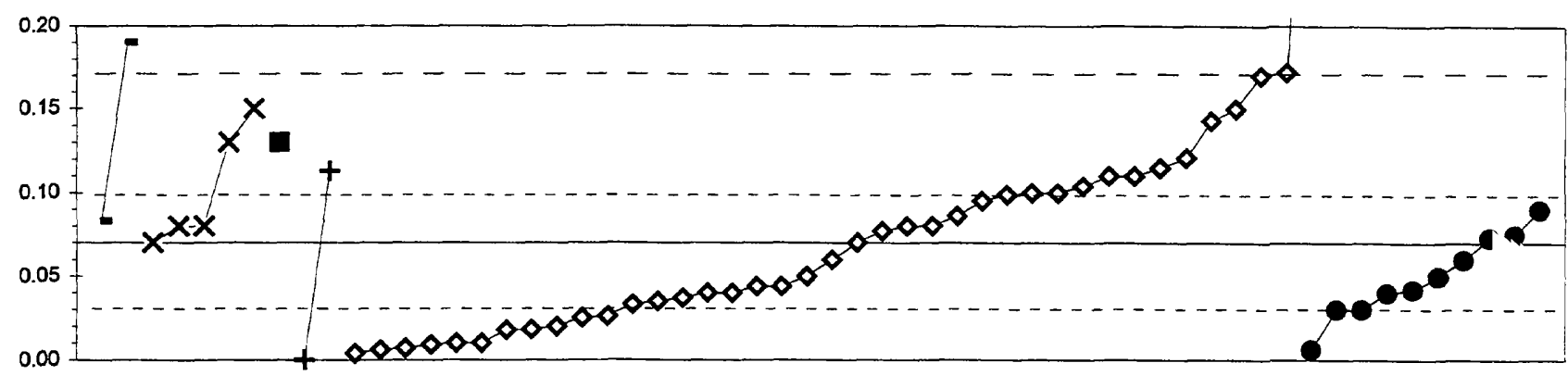

$\rightarrow 0-x-22-n-22 n i t r o+22 n-22 p$

40

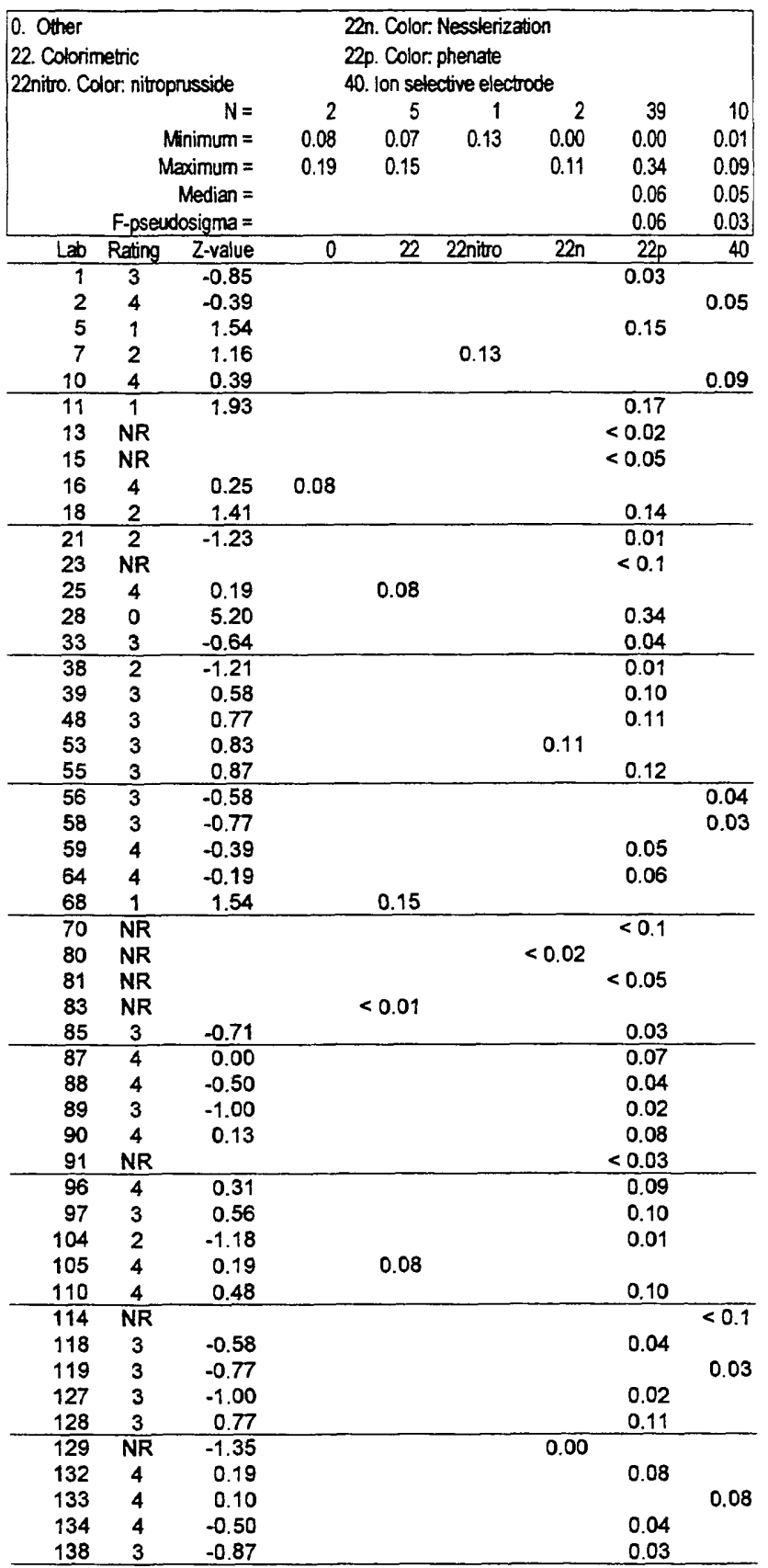

$\begin{aligned} \text { MPV } & =0.07\end{aligned}$

F-pseudosigma $=\quad 0.05$

$N=59$

$\mathrm{Hu}=0.10$

$\mathrm{HI}=0.03$

\begin{tabular}{|c|c|c|c|c|c|c|c|c|}
\hline Lab & Rating & Z-value & 0 & 22 & 22nitro & $22 n$ & $22 p$ & 40 \\
\hline 140 & 4 & 0.00 & & 0.07 & & & & \\
\hline 141 & 1 & 1.97 & & & & & 0.17 & \\
\hline 142 & 3 & -0.96 & & & & & 0.02 & \\
\hline 143 & 2 & -1.16 & & & & & 0.01 & \\
\hline 145 & $N R$ & & & & & & $<0.02$ & \\
\hline 146 & NR & & & & & & $<0.03$ & \\
\hline 149 & 2 & -1.16 & & & & & 0.01 & \\
\hline 151 & 4 & -0.19 & & & & & & 0.06 \\
\hline 155 & 3 & 0.66 & & & & & 0.10 & \\
\hline 158 & 3 & -0.67 & & & & & 0.04 & \\
\hline 180 & NR & & & & & & $<0.02$ & \\
\hline 190 & 3 & 0.58 & & & & & 0.10 & \\
\hline 197 & 2 & 1.16 & & 0.13 & & & & \\
\hline 203 & 4 & 0.19 & & & & & 0.08 & \\
\hline 204 & NR & & & & & & 0.005 & \\
\hline 212 & NR & & & & & & $<01$ & \\
\hline 213 & NR & & $<1$ & & & & & \\
\hline 215 & NR & & & & & & $<0.01$ & \\
\hline 220 & 2 & -1.27 & & & & & 0.00 & \\
\hline 221 & 3 & -0.54 & & & & & & 0.04 \\
\hline 224 & 2 & 1.02 & & & & & 0.12 & \\
\hline 234 & 4 & 0.06 & & & & & & 0.07 \\
\hline 240 & 0 & 2.31 & 0.19 & & & & & \\
\hline 241 & 2 & -1.23 & & & & & & 0.01 \\
\hline 243 & 3 & -0.58 & & & & & 0.04 & \\
\hline
\end{tabular}


Table 15. Statistical summary of reported data for standard reference water sample N-51 (nutrient constituents)-Continued $\mathrm{NH}_{3}+$ Org. $\mathrm{N}$ as $\mathrm{N}$ (Ammonia + Organic nitrogen as nitrogen) $\mathrm{mg} / \mathrm{L}$

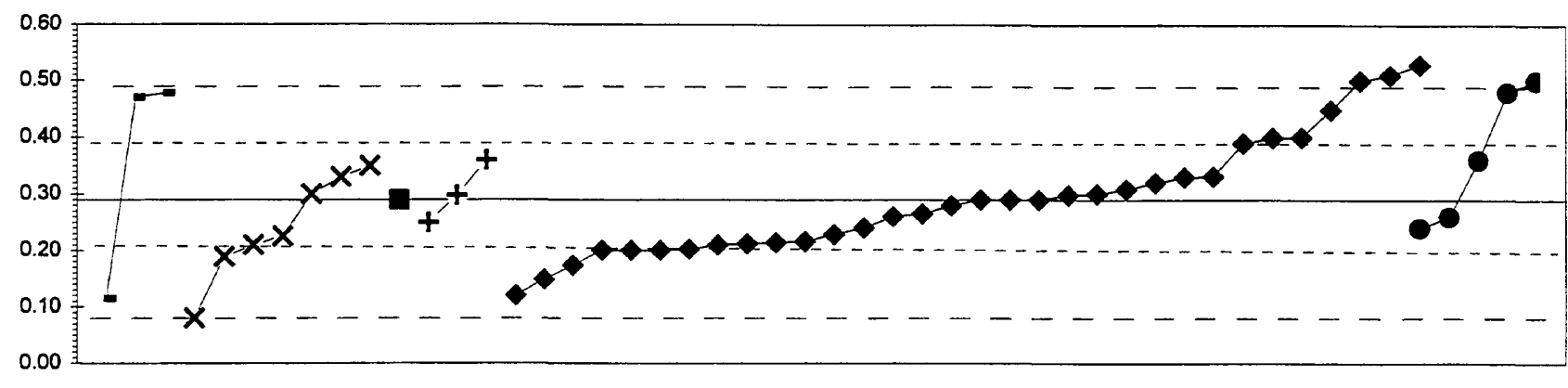

$\rightarrow-0 \rightarrow x-22 \rightarrow-22$ nitro $+22 n \rightarrow 22 p \rightarrow-40$

\begin{tabular}{|c|c|c|c|c|c|c|c|c|}
\hline $\begin{array}{l}\text { 0. Other } \\
\text { 22. Colori } \\
\text { 22nitro. Co }\end{array}$ & $\begin{array}{l}\text { etric } \\
\text { tor: nitro }\end{array}$ & usside & & $\begin{array}{l}\text { n. Color } \\
\text { p. Color } \\
\text { lon sel }\end{array}$ & $\begin{array}{l}\text { Nessleriz } \\
\text { phenate } \\
\text { ctive elec }\end{array}$ & & & \\
\hline & & $N=$ & 3 & 7 & 1 & 3 & 31 & 5 \\
\hline & & inimum = & 0.11 & 0.08 & 0.29 & 0.25 & 0.12 & 0.24 \\
\hline & & ximum = & 0.48 & 0.35 & & 0.36 & 0.53 & 0.50 \\
\hline & & Median = & & 0.23 & & & 0.28 & \\
\hline & F-pset & osigma = & & 0.09 & & & 0.09 & \\
\hline Lab & Rating & Z-value & 0 & 22 & 22nitro & $22 n$ & $22 p$ & 40 \\
\hline 1 & 3 & -0.72 & & & & & 0.22 & \\
\hline 2 & 0 & 2.07 & & & & & & 0.50 \\
\hline 10 & 4 & -0.29 & & & & & 0.26 & \\
\hline 11 & 3 & 0.69 & & & & 0.36 & & \\
\hline 15 & 0 & 2.35 & & & & & 0.53 & \\
\hline 16 & 1 & -1.73 & 0.11 & & & & & \\
\hline 18 & 2 & -1.15 & & & & & 0.17 & \\
\hline 21 & 4 & 0.00 & & & & & 0.29 & \\
\hline 23 & 0 & 2.07 & & & & & 0.50 & \\
\hline 25 & 0 & -2.06 & & 0.08 & & & & \\
\hline 38 & 4 & -0.39 & & & & 0.25 & & \\
\hline 48 & 3 & 0.99 & & & & & 0.39 & \\
\hline 55 & 4 & 0.09 & & 0.30 & & & & \\
\hline 56 & 3 & -0.64 & & 0.23 & & & & \\
\hline 58 & 1 & 1.88 & & & & & & 0.48 \\
\hline 59 & 3 & -0.88 & & & & & 0.20 & \\
\hline 68 & 4 & 0.40 & & 0.33 & & & & \\
\hline 70 & 4 & 0.00 & & & & & 0.29 & \\
\hline 81 & 2 & 1.08 & & & & & 0.40 & \\
\hline 85 & 3 & -0.88 & & & & & 0.20 & \\
\hline 87 & 1 & -1.67 & & & & & 0.12 & \\
\hline 89 & 2 & -1.39 & & & & & 0.15 & \\
\hline 91 & 4 & 0.30 & & & & & 0.32 & \\
\hline 96 & 3 & -0.61 & & & & & 0.23 & \\
\hline 97 & 4 & 0.41 & & & & & 0.33 & \\
\hline 102 & 4 & 0.10 & & & & & 0.30 & \\
\hline 104 & 4 & -0.24 & & & & & 0.27 & \\
\hline 105 & NR & & & $<0.2$ & & & & \\
\hline 118 & 4 & -0.49 & & & & & 0.24 & \\
\hline 119 & 3 & 0.69 & & & & & & 0.36 \\
\hline 127 & 4 & 0.18 & & & & & 0.31 & \\
\hline 128 & 3 & -0.88 & & & & & 0.20 & \\
\hline 129 & 4 & 0.07 & & & & 0.30 & & \\
\hline 133 & 4 & -0.49 & & & & & & 0.24 \\
\hline 134 & 4 & 0.08 & & & & & 0.30 & \\
\hline 138 & 4 & -0.10 & & & & & 0.28 & \\
\hline 140 & 3 & -0.78 & & 0.21 & & & & \\
\hline 141 & NR & & & & & $<1$ & & \\
\hline 142 & 3 & -0.86 & & & & & 0.20 & \\
\hline 143 & 4 & 0.00 & & & & & 0.29 & \\
\hline 145 & 3 & -0.78 & & & & & 0.21 & \\
\hline 155 & 3 & -0.75 & & & & & 0.21 & \\
\hline 180 & 3 & -0.76 & & & & & 0.21 & \\
\hline 190 & 2 & 1.09 & & & & & 0.40 & \\
\hline 203 & 3 & 0.59 & & 0.35 & & & & \\
\hline & 3 & -1.00 & & 0.19 & & & & \\
\hline 212 & 4 & 0.40 & & & & & 0.33 & \\
\hline 213 & NR & & $<1$ & & & & & \\
\hline 215 & 0 & 2.17 & & & & & 0.51 & \\
\hline 221 & 1 & 1.85 & 0.48 & & & & & \\
\hline
\end{tabular}


Table 15. Statistical summary of reported data for standard reference water sample N-51 (nutrient constituents)-Continued $\mathrm{NO}_{3}+\mathrm{NO}_{2}$ as $\mathrm{N}$ (Nitrate + nitrite as nitrogen) $\mathrm{mg} / \mathrm{L}$

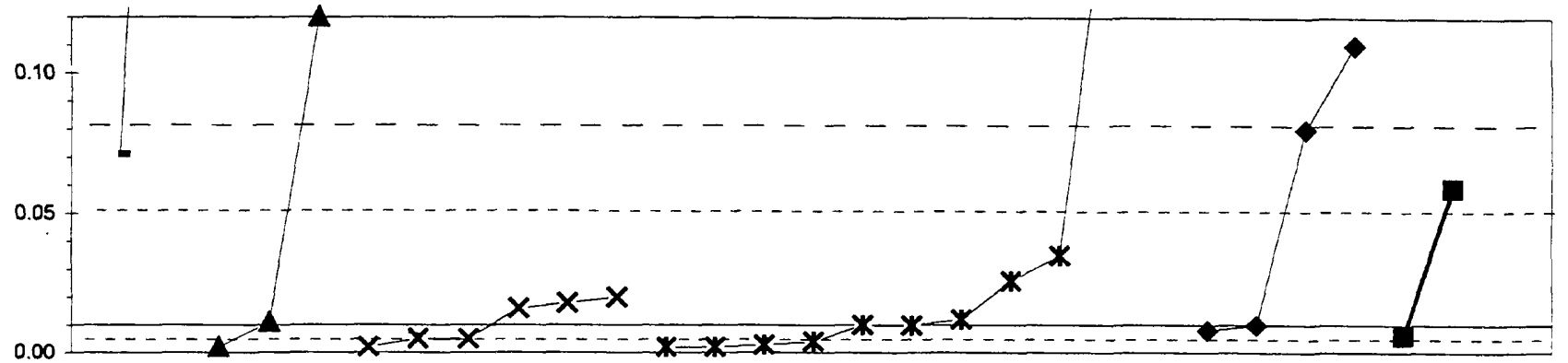

$-0 \rightarrow-7-x-22-x-220 d-22 h-22$ suff

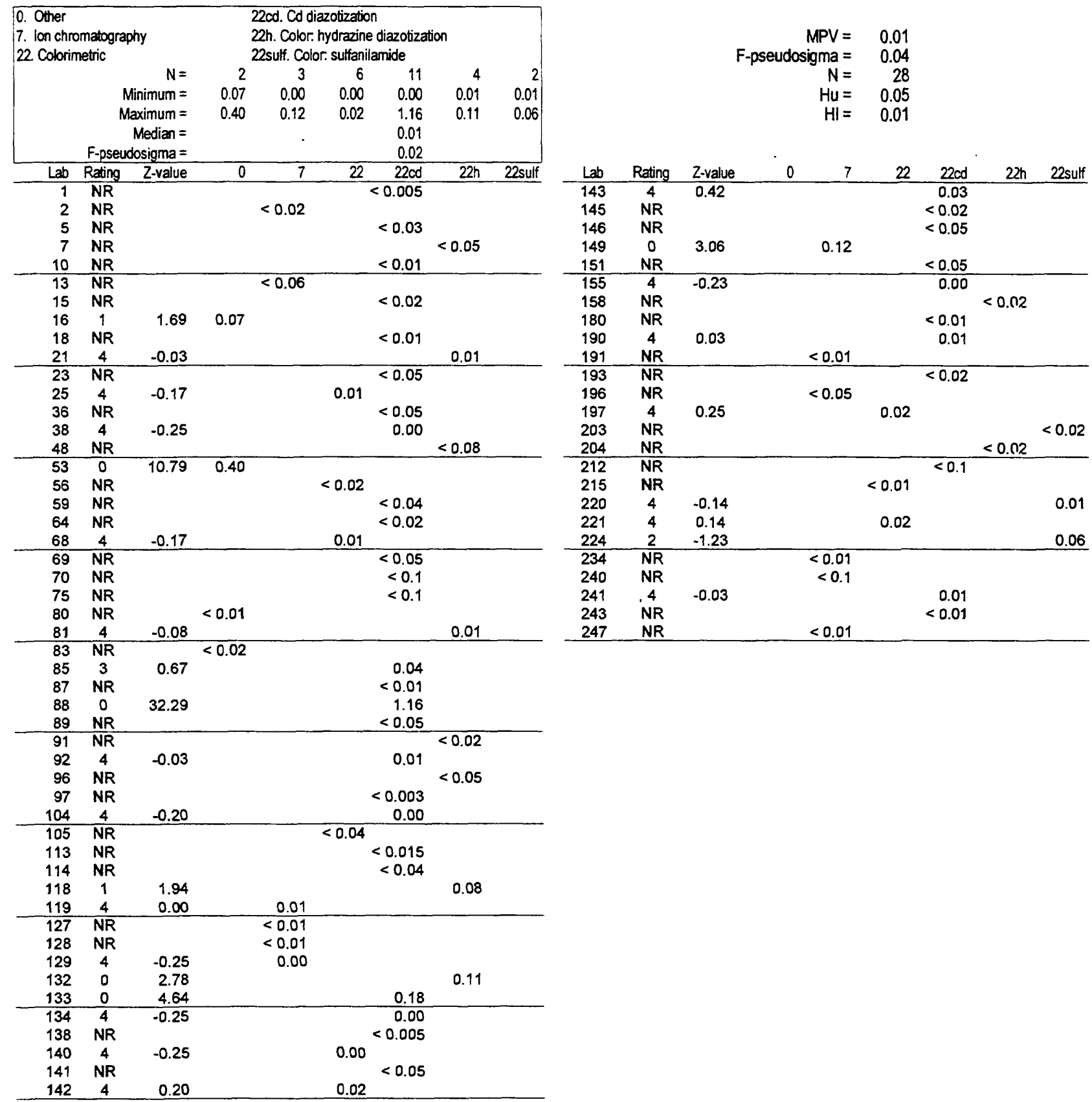


Table 15. Statistical summary of reported data for standard reference water sample N-51 (nutrient constituents)--Continued total $\mathrm{P}$ as $\mathrm{P}$ (total Phorphorus as phosphorus) $\mathrm{mg} / \mathrm{L}$

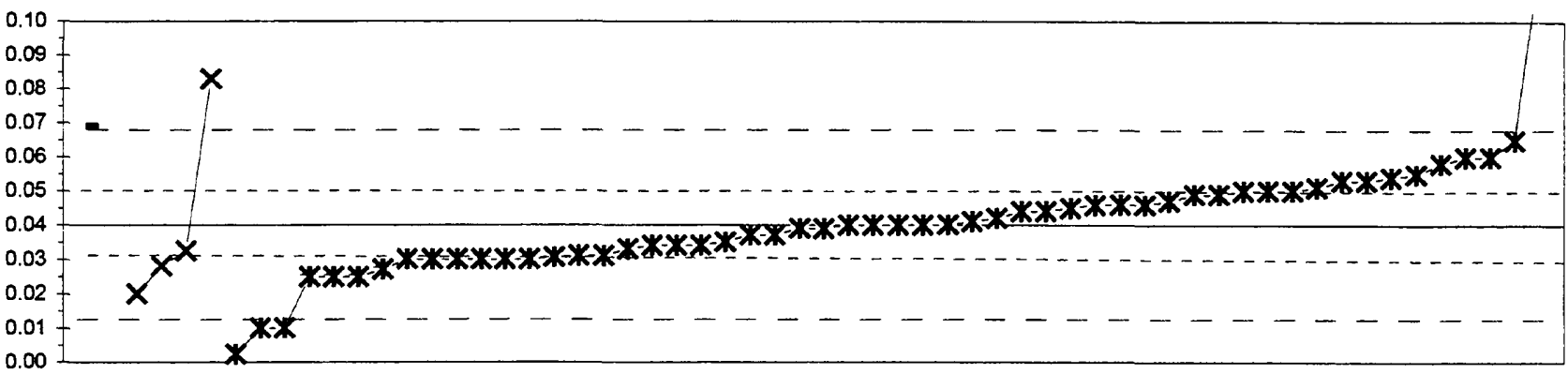

$\rightarrow 0-4-x-22-x-22 m$

\begin{tabular}{|c|c|c|c|c|c|}
\hline \multicolumn{2}{|c|}{$\begin{array}{l}\text { 0. Other } \\
\text { 4. ICP } \\
\text { 22. Colorimetric }\end{array}$} & \multicolumn{4}{|c|}{ 22m. Color.phosphomolybdate } \\
\hline \multirow{5}{*}{\multicolumn{3}{|c|}{$\begin{array}{r}N= \\
\text { Minimum }= \\
\text { Maximum }= \\
\text { Median }= \\
\text { F-pseudosigma }=\end{array}$}} & \multirow{5}{*}{$\begin{array}{rr}1 & 0 \\
0.07 & <0.075\end{array}$} & \multirow{5}{*}{$\begin{array}{r}4 \\
0.02 \\
0.08\end{array}$} & \multirow{5}{*}{$\begin{array}{r}56 \\
0.00 \\
0.14 \\
0.04 \\
0.01\end{array}$} \\
\hline & & & & & \\
\hline & & & & & \\
\hline & & & & & \\
\hline & & & & & \\
\hline Lab & Rating & Z-value & 0 & 22 & $22 m$ \\
\hline 1 & 3 & -0.70 & & & 0.03 \\
\hline 2 & 4 & 0.49 & & & 0.05 \\
\hline 10 & 3 & 0.63 & & & 0.05 \\
\hline 11 & 4 & -0.21 & & & 0.04 \\
\hline 13 & NR & & & & $<0.05$ \\
\hline 15 & 0 & 5.48 & & & 0.12 \\
\hline 16 & 1 & 2.04 & 0.07 & & \\
\hline 18 & 3 & -0.70 & & & 0.03 \\
\hline 21 & 4 & -0.21 & & & 0.04 \\
\hline 22 & 2 & 1.26 & & & 0.06 \\
\hline 23 & NR & & & & $<0.1$ \\
\hline 36 & NR & & & & $<0.05$ \\
\hline 38 & 2 & 1.05 & & & 0.06 \\
\hline 39 & 0 & -2.65 & & & 0.00 \\
\hline 46 & 3 & -0.91 & & & 0.03 \\
\hline 48 & 4 & 0.00 & & & 0.04 \\
\hline 55 & 4 & 0.07 & & & 0.04 \\
\hline 56 & 3 & -0.53 & & 0.03 & \\
\hline 58 & 0 & 6.32 & & & 0.13 \\
\hline 59 & 3 & -0.70 & & & 0.03 \\
\hline 68 & 0 & 3.02 & & 0.08 & \\
\hline 70 & NR & & & & $<0.1$ \\
\hline 81 & 4 & -0.42 & & & 0.03 \\
\hline 83 & NR & & $<0.075$ & & \\
\hline 85 & 4 & -0.07 & & & 0.04 \\
\hline 87 & 3 & 0.91 & & & 0.05 \\
\hline 89 & 4 & 0.28 & & & 0.04 \\
\hline 91 & 3 & 0.70 & & & 0.05 \\
\hline 92 & 4 & -0.49 & & & 0.03 \\
\hline 96 & 3 & -0.63 & & & 0.03 \\
\hline 97 & 0 & -2.11 & & & 0.01 \\
\hline 102 & 2 & -1.05 & & & 0.03 \\
\hline 104 & 4 & 0.42 & & & 0.05 \\
\hline 105 & 2 & -1.41 & & 0.02 & \\
\hline 113 & 4 & -0.42 & & & 0.03 \\
\hline 114 & 4 & 0.14 & & & 0.04 \\
\hline 118 & 0 & -2.11 & & & 0.01 \\
\hline 119 & 2 & 1.41 & & & 0.06 \\
\hline 127 & NR & & & & $<0.01$ \\
\hline 129 & 4 & -0.07 & & & 0.04 \\
\hline 132 & 3 & -0.70 & & & 0.03 \\
\hline 133 & 4 & 0.00 & & & 0.04 \\
\hline 134 & 4 & -0.35 & & & 0.04 \\
\hline 138 & 3 & 0.70 & & & 0.05 \\
\hline 140 & NR & & & $<0.02$ & \\
\hline 141 & 2 & 1.41 & & & 0.06 \\
\hline 142 & 3 & 0.77 & & & 0.05 \\
\hline 143 & 4 & 0.42 & & & 0.05 \\
\hline 145 & 4 & 0.00 & & & 0.04 \\
\hline 146 & NR & & & & $<0.1$ \\
\hline
\end{tabular}

F-pseudosigma $=0.01$

$\mathrm{N}=61$

$\mathrm{Hu}=0.05$

$H=0.03$

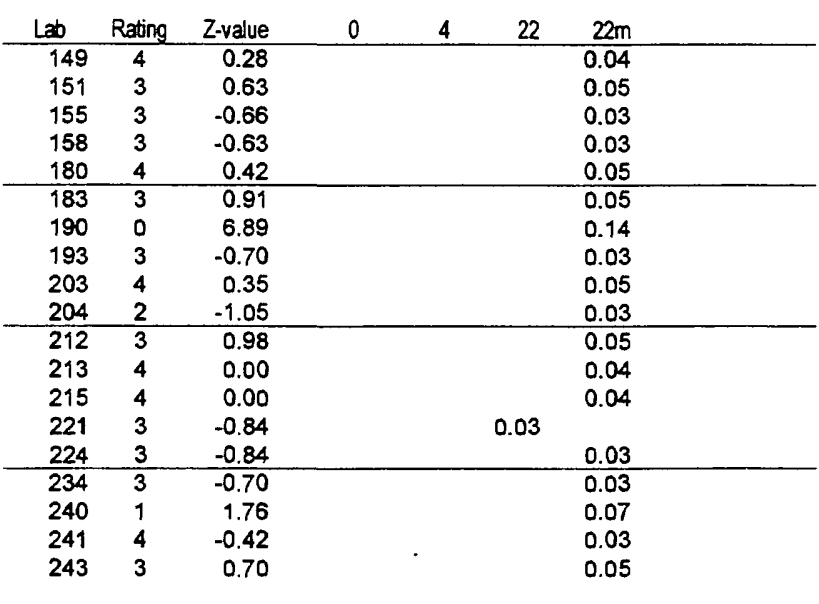


Table 15. Statistical summary of reported data for standard reference water sample N-51 (nutrient constituents)-Continued $\mathrm{PO}_{4}$ as $\mathrm{P}$ (orthophosphate as phosphorus)

$\mathrm{mg} / \mathrm{L}$

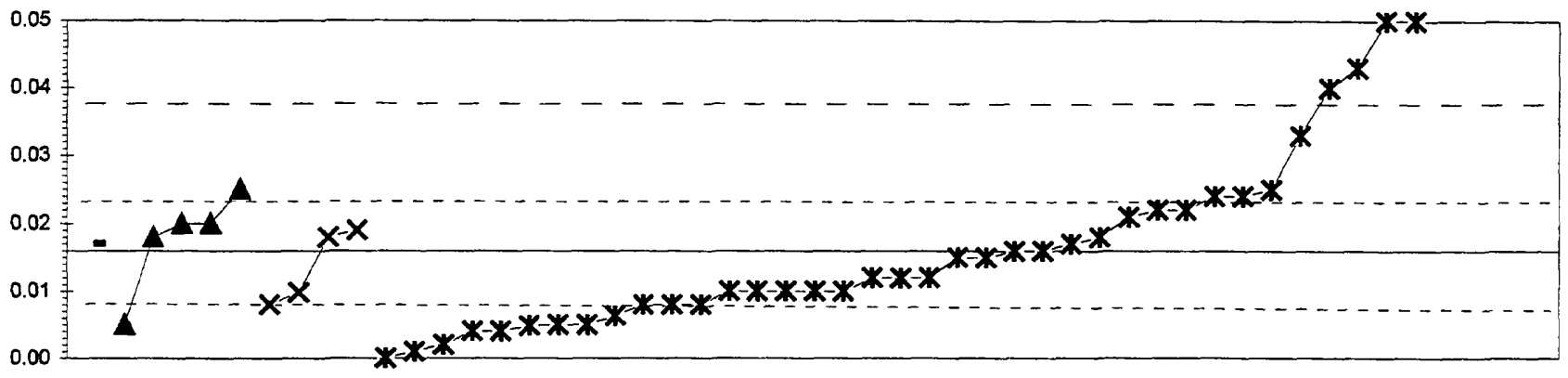

$--0-x-7-x-22-x-22 m$

\begin{tabular}{|c|c|c|c|c|c|c|}
\hline \multicolumn{3}{|c|}{$\begin{array}{l}\text { 0. Other } \\
\text { 7. Ion chromatography } \\
\text { 22. Colorimetric }\end{array}$} & \multicolumn{4}{|c|}{$22 \mathrm{~m}$. Color.phosphomolybdate } \\
\hline & & $N=$ & 1 & 5 & 4 & 42 \\
\hline & & Ainimum = & 0.02 & 0.01 & 0.01 & 0.00 \\
\hline & & aximum $=$ & & 0.03 & 0.02 & 0.14 \\
\hline \multirow{2}{*}{\multicolumn{3}{|c|}{$\begin{array}{r}\text { Median = } \\
\text { F-pseudosigma }=\end{array}$}} & & & & 0.02 \\
\hline & & & & & & 0.01 \\
\hline Lab & Rating & Z-value & 0 & 7 & 22 & $22 \mathrm{~m}$ \\
\hline 1 & $\mathrm{NR}$ & & & & & 0.001 \\
\hline 2 & 2 & -1.08 & & & & 0.00 \\
\hline 5 & 0 & 2.43 & & & & 0.04 \\
\hline 10 & 4 & 0.45 & & & & 0.02 \\
\hline 13 & NR & & & $<0.05$ & & \\
\hline 15 & NR & & & & & 0.02 \\
\hline 16 & 4 & 0.09 & 0.02 & & & \\
\hline 18 & 4 & 0.00 & & & & 0.02 \\
\hline 21 & 4 & -0.09 & & & & 0.02 \\
\hline 23 & NR & & & & & $<0.1$ \\
\hline 25 & 4 & -0.09 & & & & 0.02 \\
\hline 28 & 0 & 2.16 & & & & 0.04 \\
\hline 33 & NR & & & $<0.01$ & & \\
\hline 36 & NR & & & & & $=0.05$ \\
\hline 38 & 3 & -0.54 & & & & 0.01 \\
\hline 39 & 3 & -0.72 & & & & 0.01 \\
\hline 46 & 3 & -0.99 & & & & 0.01 \\
\hline 48 & 4 & 0.09 & & & & 0.02 \\
\hline 53 & 0 & 9.08 & & & & 0.12 \\
\hline 55 & 0 & 5.49 & & & & 0.08 \\
\hline 56 & 4 & 0.18 & & & 0.02 & \\
\hline 58 & 0 & 7.55 & & & & 0.10 \\
\hline 59 & NR & & & & & $<0.01$ \\
\hline 64 & 3 & -0.54 & & & & 0.01 \\
\hline 70 & NR & & & & & $<0.1$ \\
\hline 80 & NR & & & & & 0.05 \\
\hline 81 & NR & & & & & 0.005 \\
\hline 83 & 3 & -0.56 & & & 0.01 & \\
\hline 85 & 4 & 0.00 & & & & 0.02 \\
\hline 87 & 3 & -0.72 & & & & 0.01 \\
\hline 88 & 0 & 3.06 & & & & 0.05 \\
\hline 89 & 3 & -0.72 & & & & 0.01 \\
\hline 92 & 3 & 0.72 & & & & 0.02 \\
\hline 96 & NR & & & & & $<0.01$ \\
\hline 97 & 4 & -0.36 & & & & 0.01 \\
\hline 102 & 2 & -1.08 & & & & 0.00 \\
\hline 104 & 3 & -0.99 & & & & 0.01 \\
\hline 105 & 4 & 0.27 & & & 0.02 & \\
\hline 113 & NR & & & & & 0.004 \\
\hline 118 & NR & & & & & $<0.01$ \\
\hline 119 & NR & -1.44 & & & & 0.00 \\
\hline 127 & NR & & & $<0.05$ & & \\
\hline 128 & NR & & & $<0.01$ & & \\
\hline 129 & 3 & 0.81 & & & & 0.03 \\
\hline 132 & 3 & -0.54 & & & & 0.01 \\
\hline 133 & 3 & -0.54 & & & & 0.01 \\
\hline 134 & 2 & -1.26 & & & & 0.00 \\
\hline 138 & 2 & -1.01 & & & & 0.00 \\
\hline 140 & NR & & & & $<0.01$ & \\
\hline 141 & 0 & 3.06 & & & & 0.05 \\
\hline
\end{tabular}

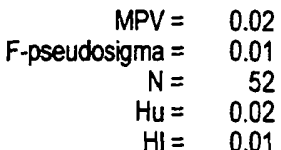

\begin{tabular}{|c|c|c|c|c|c|}
\hline Lab & Rating & $Z$-value & 0 & 22 & $22 \mathrm{~m}$ \\
\hline 142 & 0 & 6.03 & & & 0.08 \\
\hline 143 & 4 & -0.36 & & & 0.01 \\
\hline 145 & NR & & & & $<0.01$ \\
\hline 146 & NR & & & & $<0.05$ \\
\hline 149 & 4 & 0.36 & 0.02 & & \\
\hline 151 & 3 & $0 . \overline{81}$ & 0.03 & & \\
\hline 155 & 3 & -0.87 & & & 0.01 \\
\hline 158 & 3 & 0.54 & & & 0.02 \\
\hline 180 & NR & & & & $<0.01$ \\
\hline 183 & 1 & 1.53 & & & 0.03 \\
\hline 190 & 0 & 11.33 & & & 0.14 \\
\hline 191 & 4 & 0.36 & 0.02 & & \\
\hline 196 & NR & & $<0.05$ & & \\
\hline 203 & 3 & 0.54 & & & 0.02 \\
\hline 204 & 4 & -0.36 & & & 0.01 \\
\hline 212 & 3 & -0.54 & & & 0.01 \\
\hline 213 & NR & & & & $<0.02$ \\
\hline 215 & NR & & & & $<0.01$ \\
\hline 220 & 2 & -1.35 & & & 0.00 \\
\hline 221 & 3 & -0.72 & & 0.01 & \\
\hline 224 & 3 & 0.72 & & & 0.02 \\
\hline 234 & 3 & -0.99 & 0.01 & & \\
\hline 240 & NR & & $<0.1$ & & \\
\hline 241 & 4 & 0.18 & & & 0.02 \\
\hline 247 & 4 & 0.18 & 0.02 & & \\
\hline
\end{tabular}


Table 16. Statistical summary of reported data for standard reference water sample N-52 (nutrients)

Definition of analytical methods, abbreviations, and symbols

Anahtical methods

0. Other/Not reported

4. ICP

5. DCP

7. IC

20. Titrate: color

21. Titrate: electro

22. Color:

40. Ion electrode

$=\quad$ inductively coupled plasma

$=$ direct coupled plasma

$=$ ion chromatography

= titration: colorimetric (color reagent specified)

$=$ titration: electrometric

$=$ colorimetric [color reagent specified]

$=\quad$ ion selective electrode

Abbreviations and symbols

$\begin{aligned} \mathrm{N}= & \text { number of samples } \\ \text { MPV }= & \text { most probable value } \\ \text { F-pseudosigma }= & \text { nonparametric statistic deviation } \\ \mathrm{Hu}= & \text { upper hinge value } \\ \mathrm{HI}= & \text { lower hinge value } \\ \mathrm{mg} / \mathrm{L} & =\text { milligrams per liter } \\ \mathrm{Lab}= & \text { laboratory code number } \\ \mathrm{NR}= & \text { not rated, less than value reported } \\ <= & \text { less than }\end{aligned}$

Constituent

$\mathrm{NH}_{3}$ as $\mathrm{N}$

Ammonia as nitrogen

$\mathrm{NH}_{3}+$ Org $\mathrm{N}$ as $\mathrm{N}$

Ammonia plus organic nitrogen

126

$\mathrm{NO}_{3}+\mathrm{NO}_{2}$ as $\mathrm{N}$

Nitrate plus nitrite as nitrogen

Total $P$ as $P$

Total Phosphorus as phosphorus

128

$\mathrm{PO}_{4}$ as $\mathrm{P}$

Orthophosphate as phosphorus

129

130


Table 16. Statistical summary of reported data for standard reference water sample N-52 (nutrient constituents)-Continued $\mathrm{NH}_{3}$ as $\mathrm{N}$ (Ammonia as nitrogen) $\mathrm{mg} / \mathrm{L}$

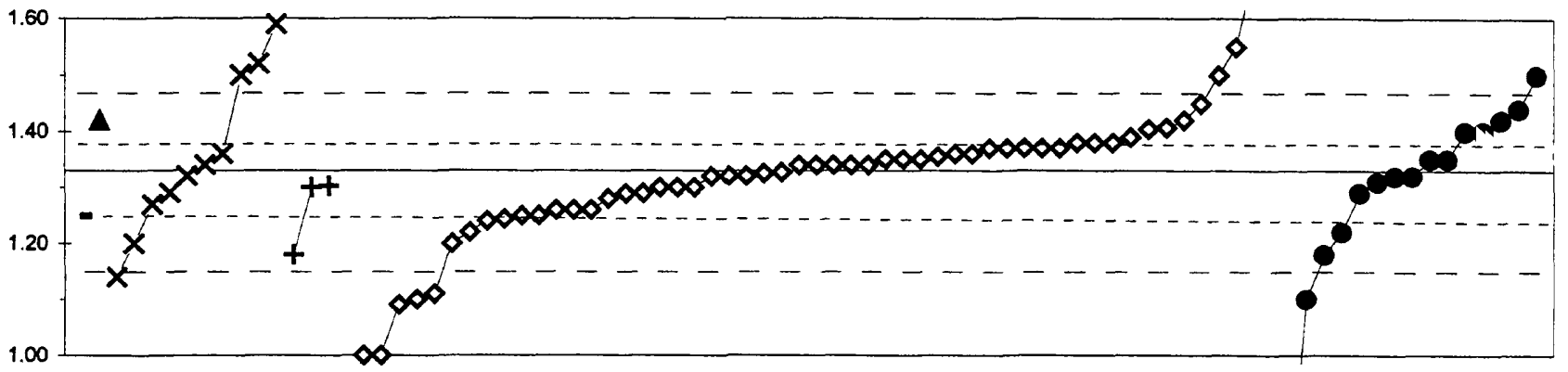

$$
\rightarrow 0-7-x-22+22 \mathrm{~N}-\diamond-22 \mathrm{p}-40
$$

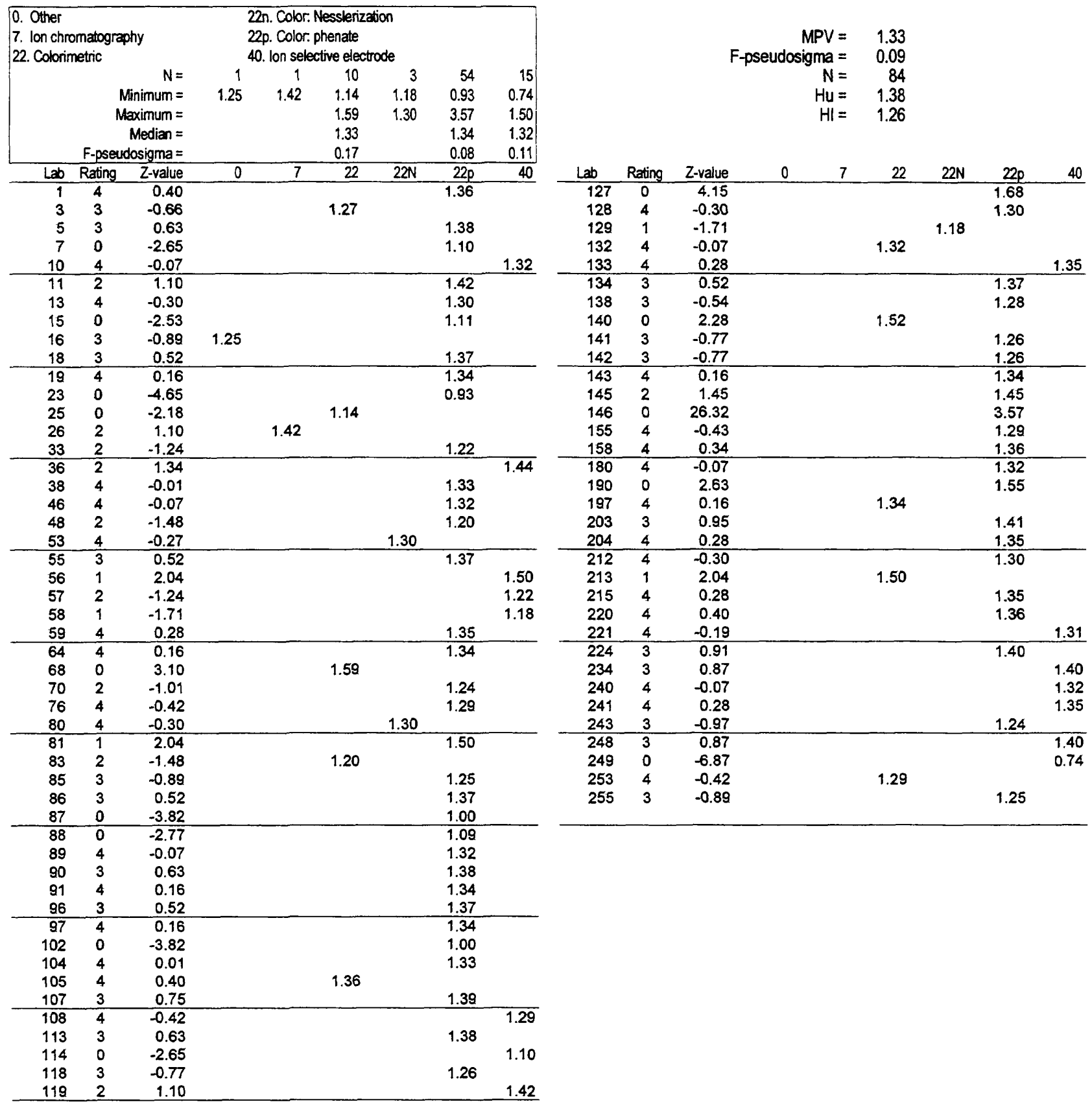


Table 16. Statistical summary of reported data for standard reference water sample N-52 (nutrient constituents)-Continued $\mathrm{NH}_{3}+\operatorname{Org~N}$ as $\mathrm{N}$ (Ammonia + Organic nitrogen as nitrogen) $\mathrm{mg} / \mathrm{L}$

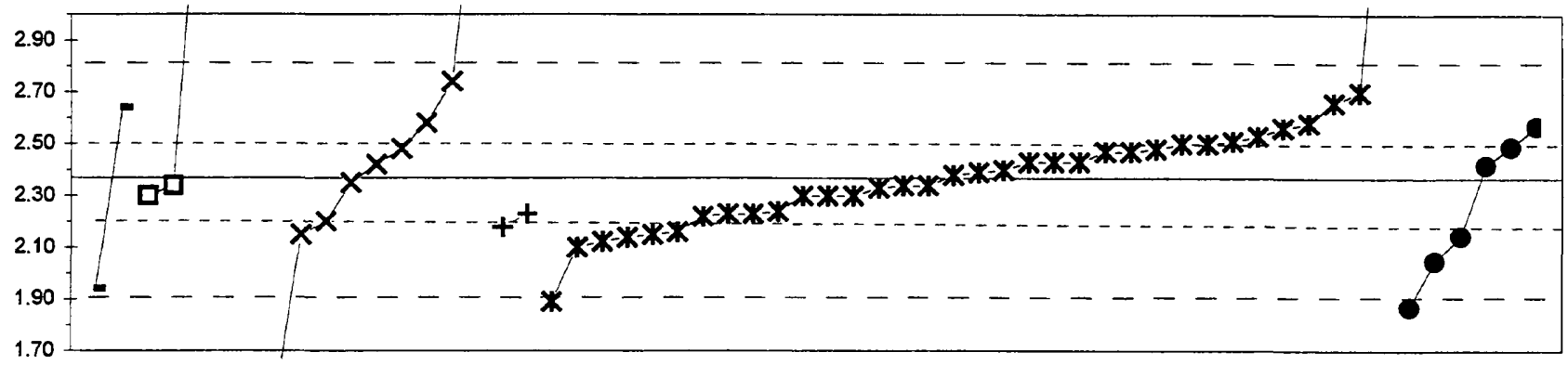

$=-0-\square-20-X-22+22 \mathrm{~N}-X-22 \mathrm{p}-0-40$

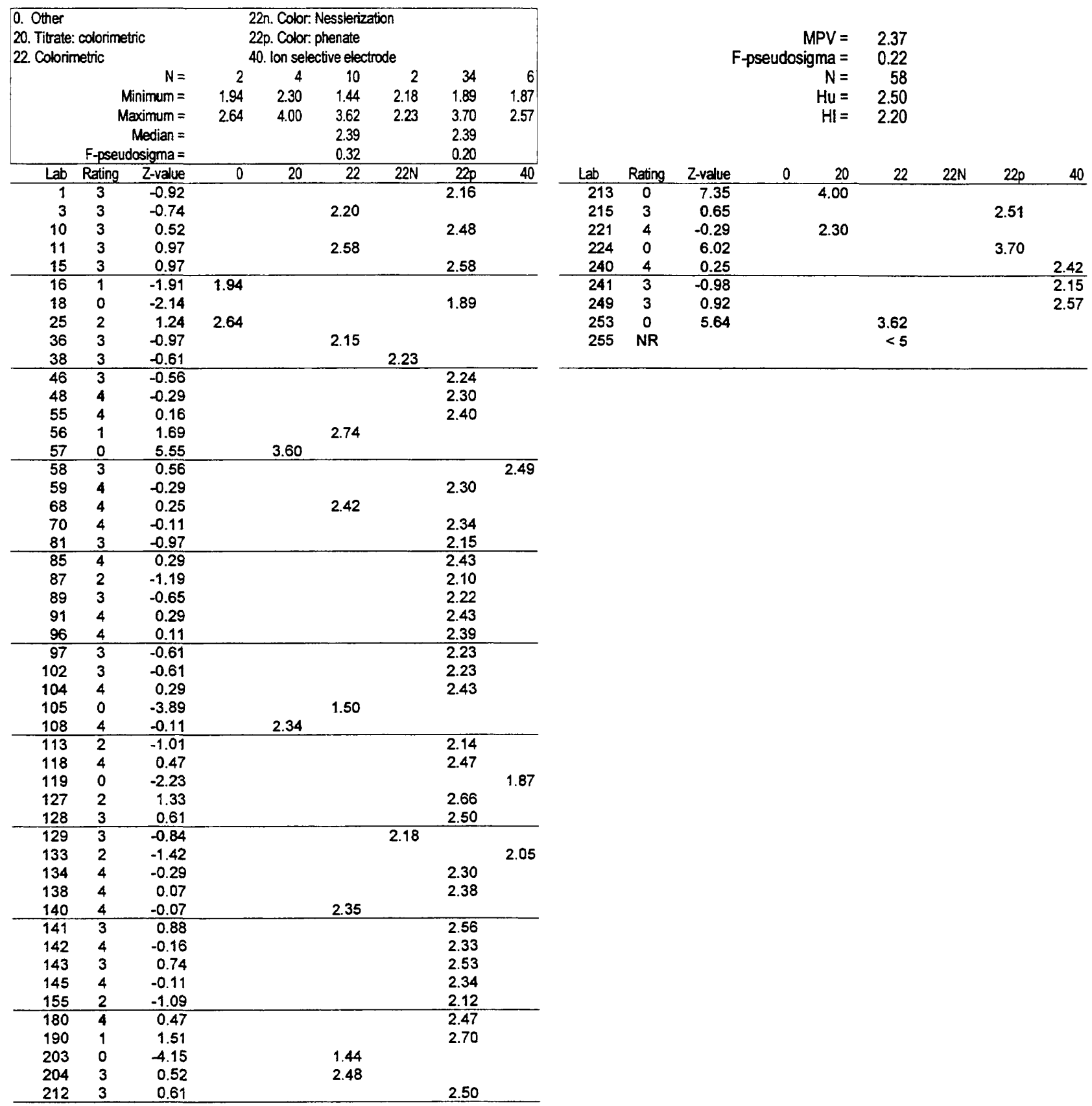


Table 16. Statistical summary of reported data for standard reference water sample N-52 (nutrient constituents)-Continued $\mathrm{NO}_{3}+\mathrm{NO}_{2}$ as $\mathrm{N}$ (Nitrate + nitrite as nitrogen) $\mathrm{mgh}$

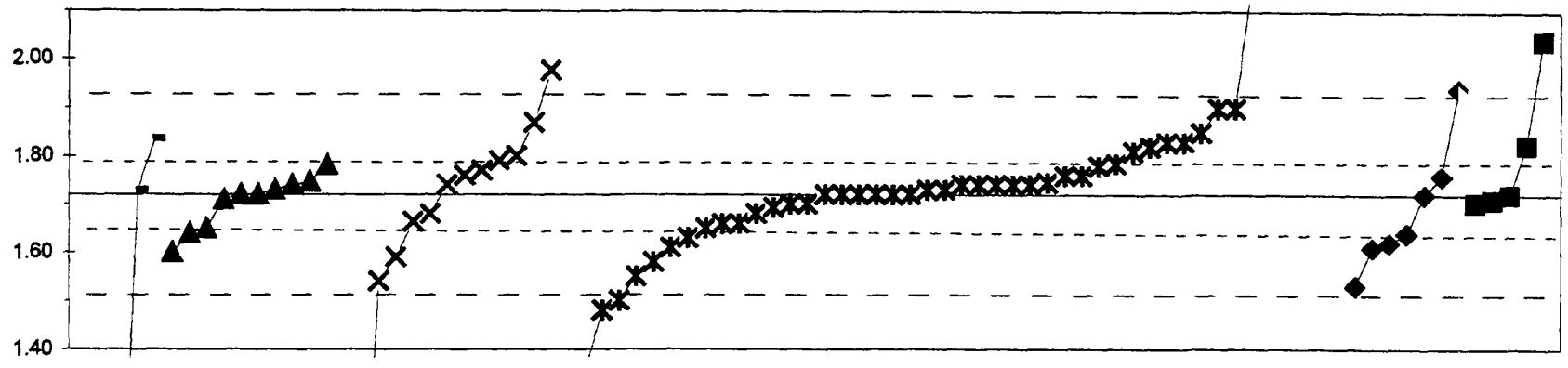

$-0-1-7-22-x-220 d-22 h$

22sulf

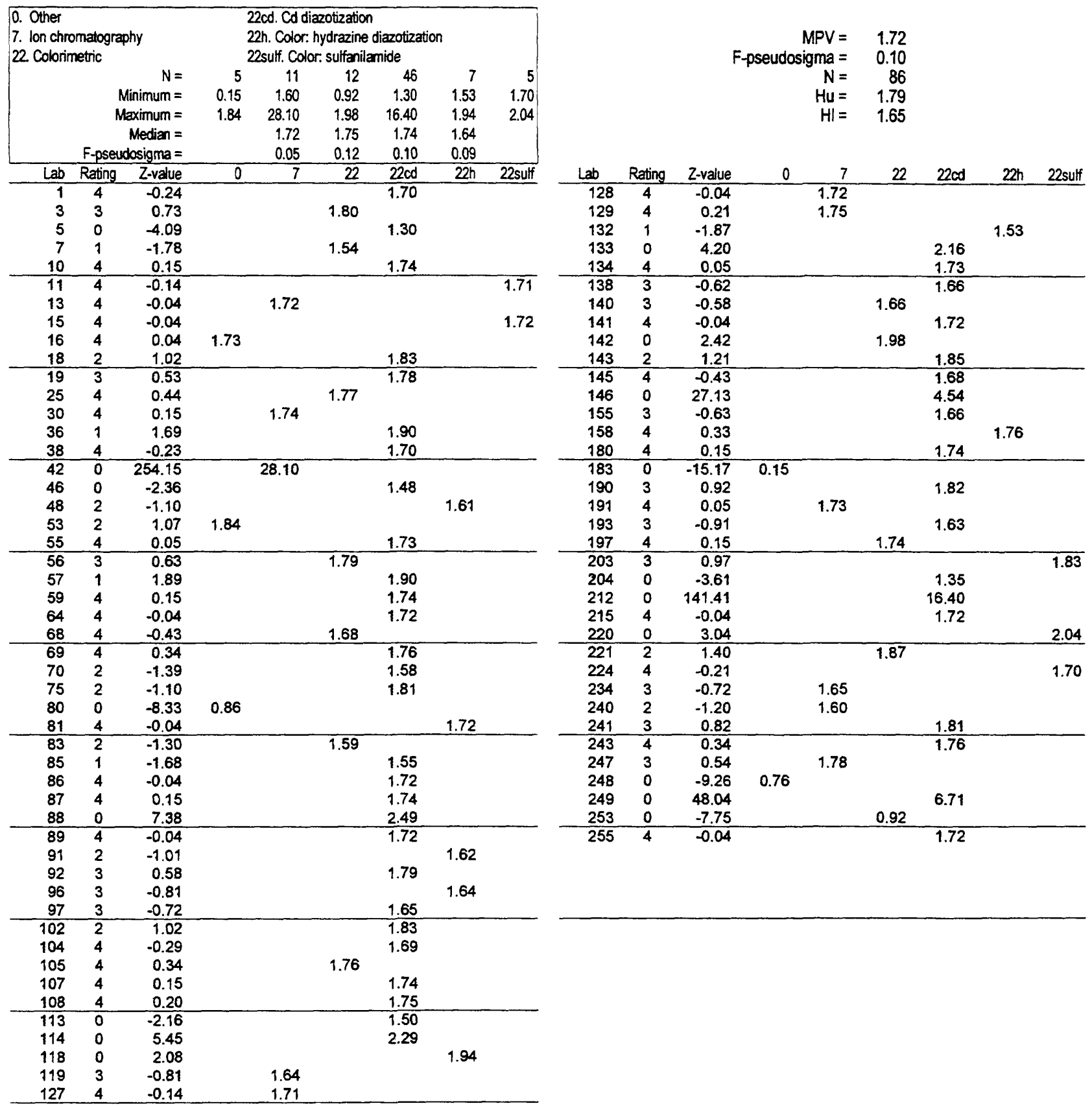


Table 16. Statistical summary of reported data for standard reference water sample N-52 (nutrient constituents)-Continued total $\mathrm{P}$ as $\mathrm{P}$ (toral Phorphorus as phosphorus) $\mathrm{mg} / \mathrm{L}$

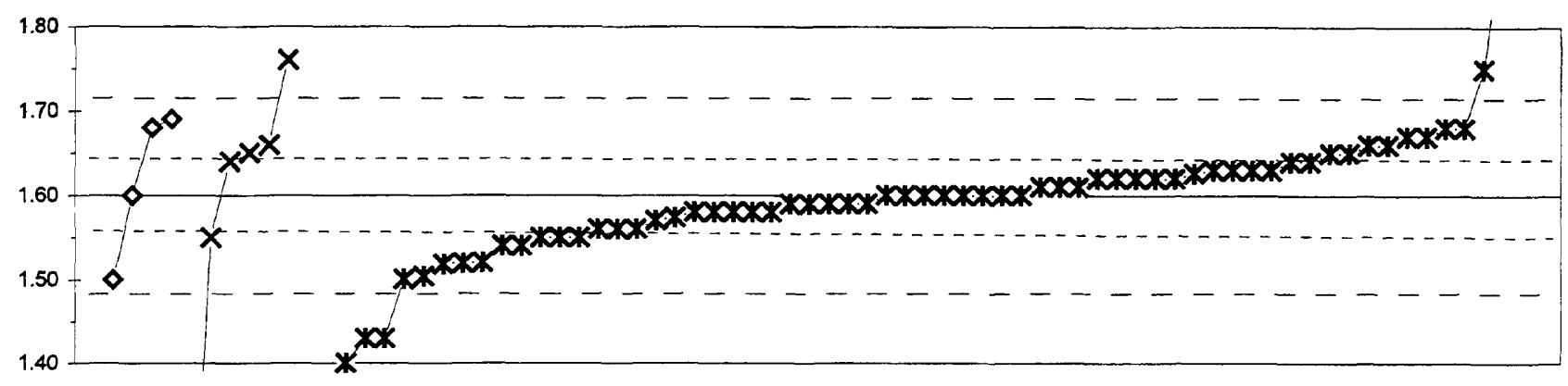

$\rightarrow 0-4-x-22-x-22 m$

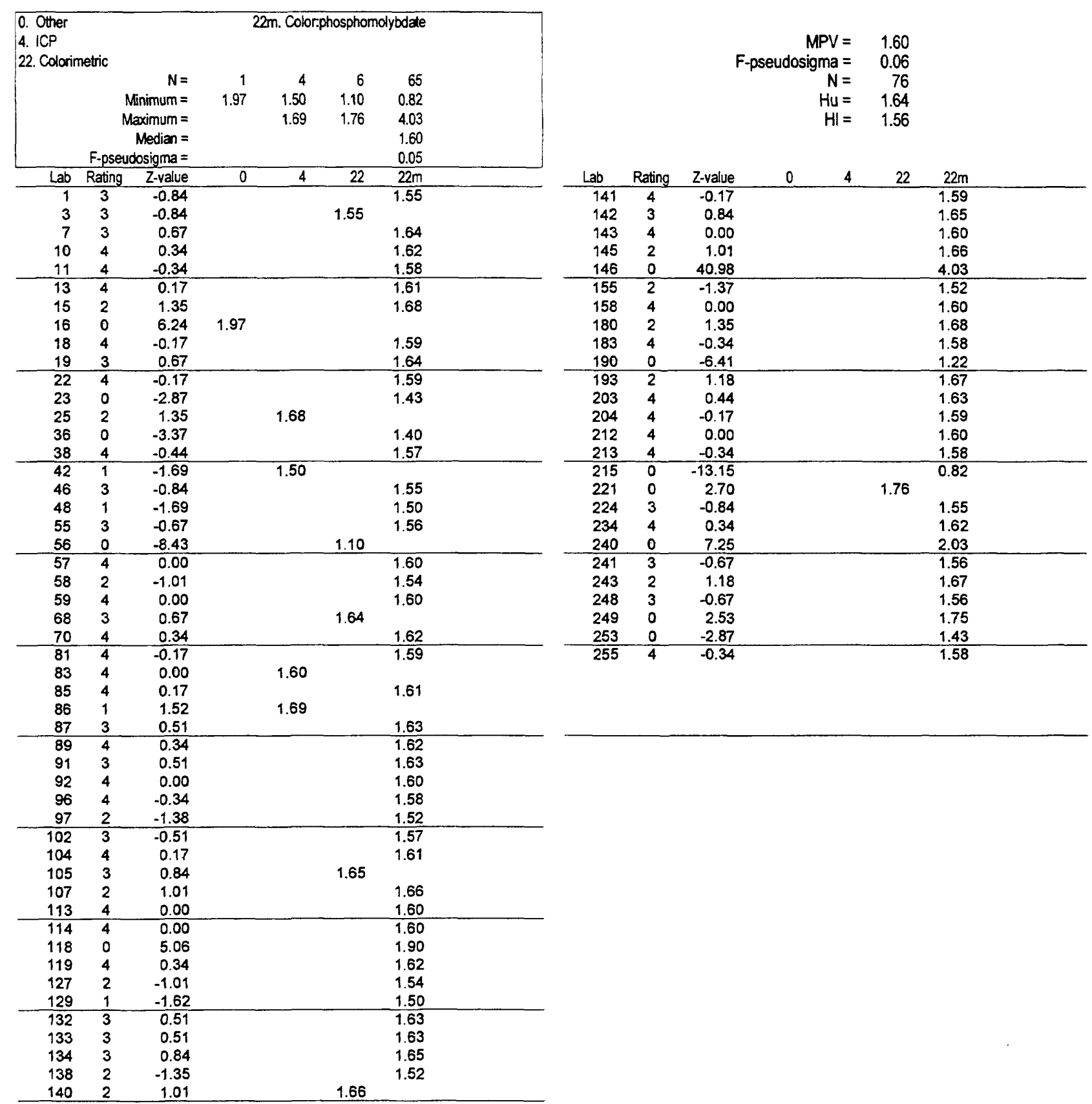


Table 16. Statistical summary of reponted data for standard reference water sample N-52 (nutrient constituents)-Continued $\mathrm{PO}_{4}$ as $\mathrm{P}$ (orthophosphate as phosphorus) $\mathrm{mg} / \mathrm{L}$

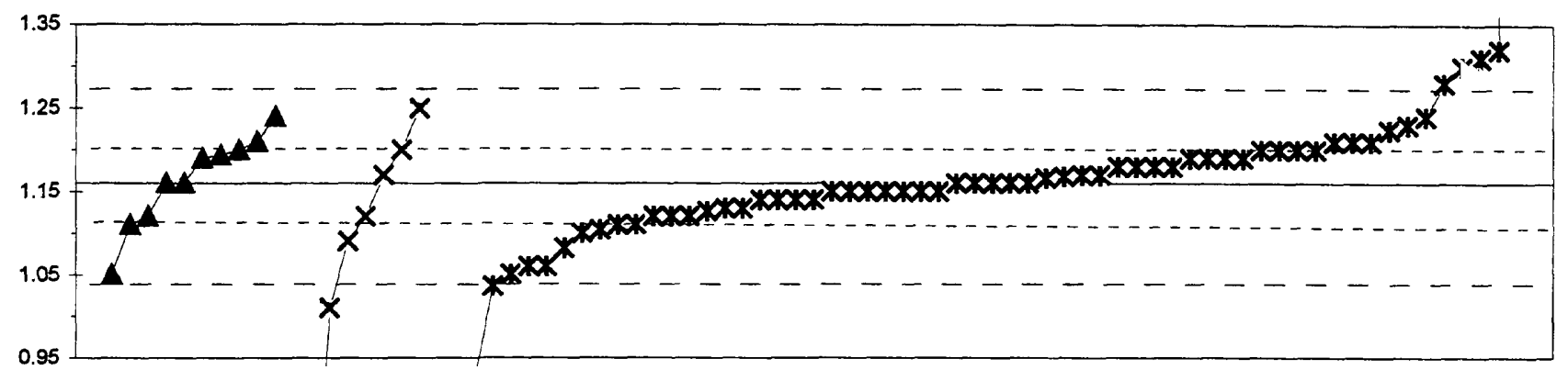

$-0-7-x-22-x-22 m$

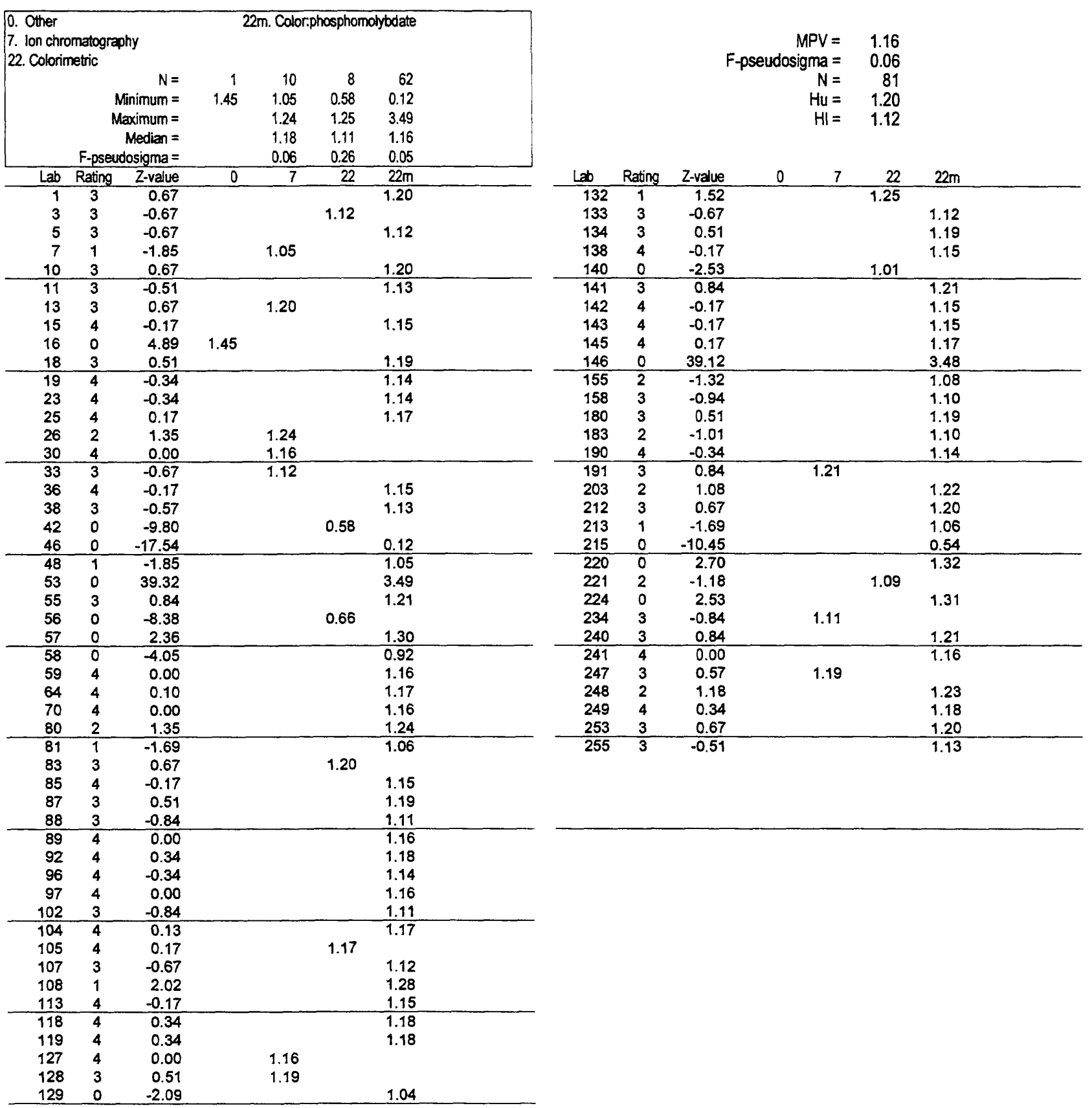


Table 17. Statistical summary of reported data for standard reference water sample P-27 (low ionic strength constituents)

\section{Definition of analytical methods, abbreviations, and symbols}

Analytical methods

0 Other/Not reported

1 AA: direct, air

$2 \mathrm{AA}$ : direct, $\mathrm{N}_{2} \mathrm{O}$

3 AA: graphite fumace

4 ICP

5 DCP

6 ICPMS

7 IC

12 Flame emission

20 Titrate: color

21 Titrate: electro

22 Color:

40 Ion electrode

41 Electro

50 Gravimetric

51 Turbidimetric

= atomic absorption: direct,air

$=$ atomic absorption: direct, nitrous oxide

= atomic absorption: graphite fumace

$=$ inductively coupled plasma

= direct current plasma

= mass spectrometry/inductively coupled plasma

= ion chromatography

= flame emission

= titration: colorimetric [color reagent specified]

$=$ titration: electrometric

= colorimetric [color reagent specified]

$=\quad$ ion selective electrode

= electrometric: [type meter specified]

= gravimetric: [precipitate specified]

$=$ turbidimetric: [precipitate specified]

Abbreviations and symbols

\begin{tabular}{|c|c|}
\hline$N=$ & number of samples \\
\hline MPV $=$ & most probable value \\
\hline F-pseudosigma $=$ & nonparametric statistic deviation \\
\hline $\mathrm{Hu}=$ & upper hinge value \\
\hline$H I=$ & lower hinge value \\
\hline $\mathrm{mg} / \mathrm{L}=$ & milligrams per liter \\
\hline$\mu \mathrm{S} / \mathrm{cm}=$ & microsiemens per centimeter at $25^{\circ} \mathrm{C}$ \\
\hline Lab $=$ & laboratory code number \\
\hline $\begin{aligned} N R & = \\
< & =\end{aligned}$ & not rated, less than value reported \\
\hline
\end{tabular}

Constituent

Acid Acidity as $\mathrm{CaCO}_{3}$

$\mathrm{Ca}$ Calcium 133

Cl Chloride 134

F Fluoride 135

K Potassium 136

Mg Magnesium 137

$\mathrm{Na}$ Sodium 138

$\mathrm{pH} \quad 139$

$\mathrm{PO}_{4}$ as $\mathrm{P}$ Orthophosphate as Phosphorus 140

$\mathrm{SO}_{4}$ Sulfate 141

Sp Cond Specific Conductance $\quad 142$ 
Table 17. Statistical summary of reported data for standard reference water sample P-27 (low ionic strength constituents)-Continued Acidity $\left(\mathrm{as}^{\left.\mathrm{CaCO}_{3}\right)}\right.$ $\mathrm{mg} / \mathrm{L}$

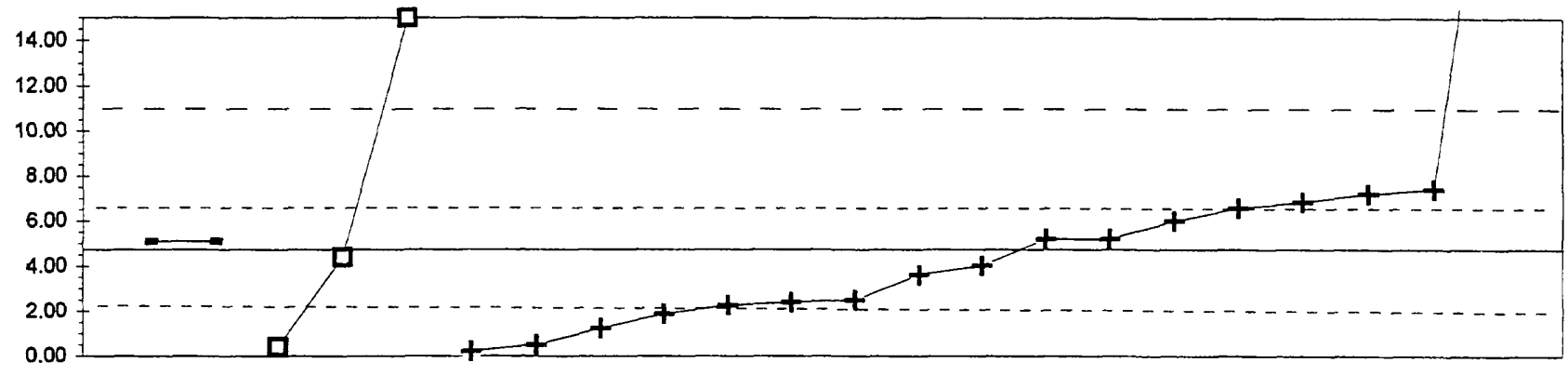

$=0-\square-20+-21$

\begin{tabular}{|c|c|c|c|c|c|}
\hline \multicolumn{6}{|c|}{$\begin{array}{l}\text { 0. Other } \\
\text { 20. Titrate: colorimetric } \\
\text { 21. Titrate: electrometric }\end{array}$} \\
\hline \multirow{2}{*}{\multicolumn{3}{|c|}{$\begin{array}{r}N= \\
\text { Minimum }=\end{array}$}} & 2 & 3 & 17 \\
\hline & & & 5.08 & 0.42 & 0.20 \\
\hline \multicolumn{3}{|c|}{ Maximum = } & 5.10 & 15.00 & 27.00 \\
\hline \multicolumn{3}{|c|}{ Median $=$} & & & 4.01 \\
\hline \multicolumn{3}{|c|}{ F-pseudosigma = } & & & 3.19 \\
\hline Lab & Rating & Z-value & 0 & 20 & 21 \\
\hline 1 & 3 & -0.70 & & & 2.50 \\
\hline 3 & NR & & & & $<10$ \\
\hline 5 & 3 & 0.84 & & & 7.42 \\
\hline 7 & 4 & 0.14 & & & 5.20 \\
\hline 15 & NR & & & & $<2$ \\
\hline 25 & 0 & 6.97 & & & 27.00 \\
\hline 38 & 3 & 0.66 & & & 6.85 \\
\hline 81 & 2 & -1.33 & & & 0.50 \\
\hline 83 & 4 & 0.11 & 5.10 & & \\
\hline 89 & 3 & -0.78 & & & 2.26 \\
\hline 105 & 2 & -1.35 & & 0.42 & \\
\hline 109 & 3 & 0.57 & & & 6.57 \\
\hline 132 & 4 & 0.11 & 5.08 & & \\
\hline 141 & 3 & -0.73 & & & 2.40 \\
\hline 146 & NR & & & & $<10$ \\
\hline 190 & 4 & 0.39 & & & 6.00 \\
\hline 215 & 4 & -0.36 & & & 3.60 \\
\hline 220 & 3 & 0.77 & & & 7.21 \\
\hline 224 & 4 & -0.23 & & & 4.01 \\
\hline 240 & 3 & -0.90 & & & 1.88 \\
\hline 247 & 2 & -1.42 & & & 0.20 \\
\hline 257 & 2 & -1.09 & & & 1.25 \\
\hline 272 & 0 & 3.21 & & 15.00 & \\
\hline 273 & 4 & 0.14 & & & 5.20 \\
\hline 276 & 4 & -0.11 & & 4.40 & \\
\hline
\end{tabular}


Table 17. Statistical summary of reported data for standard reference water sample P-27 (low ionic strength constituents)-Contin'led $\mathrm{Ca}$ (Calcium) $\mathrm{mg} / \mathrm{L}$

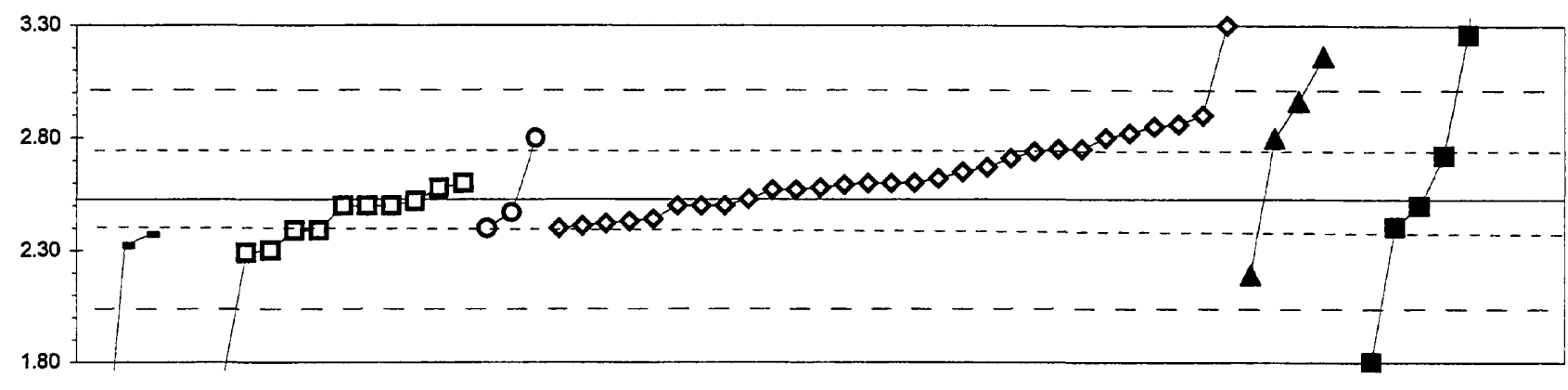

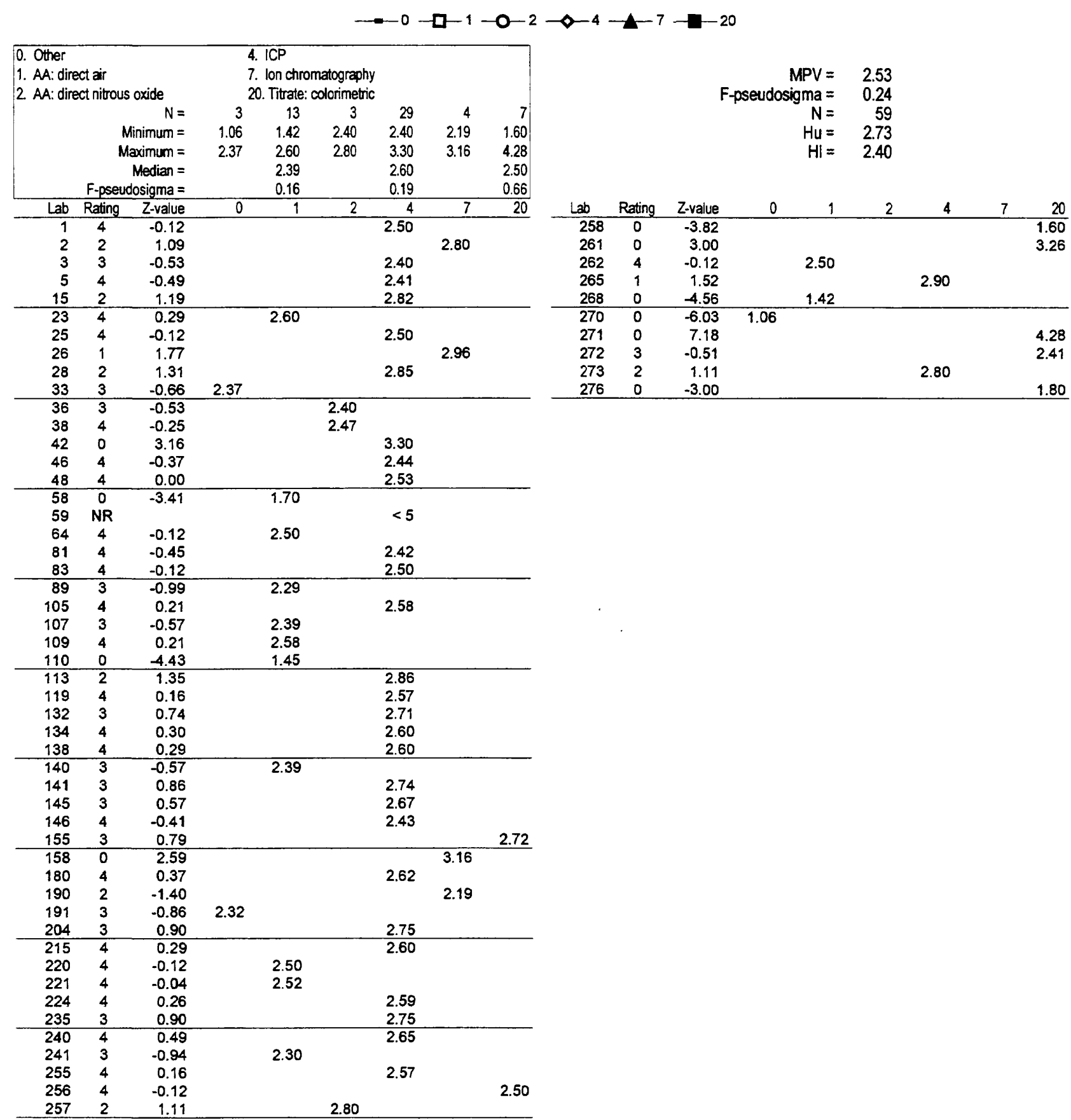


Table 17. Statistical summary of reported data for standard reference water sample P-27 (low ionic strength constituents)-Continued $\mathrm{Cl}$ (Chloride) $\mathrm{mg} / \mathrm{L}$

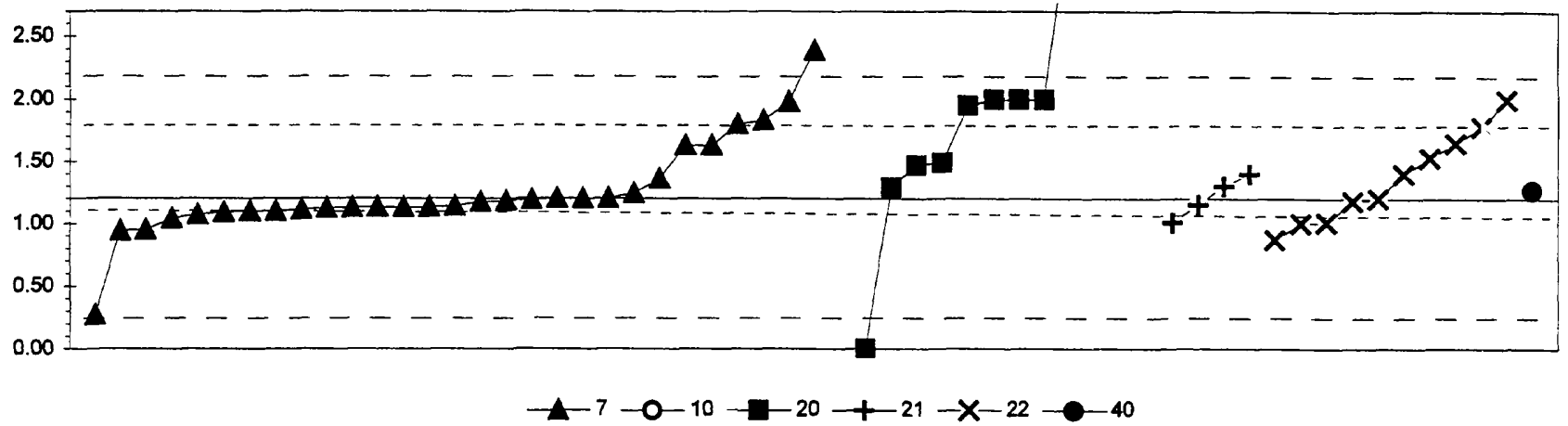

\begin{tabular}{|c|c|c|c|c|c|c|c|c|}
\hline \multicolumn{3}{|c|}{$\begin{array}{l}\text { 7. Ion chromatography } \\
\text { 8. AA: cold vapor } \\
\text { 20. Titrate: colorimetric }\end{array}$} & \multicolumn{4}{|c|}{$\begin{array}{l}\text { 21. Titrate: electrometric } \\
\text { 22. Colorimetric } \\
\text { 40. Ion selective electrode }\end{array}$} & \multirow[b]{2}{*}{10} & \multirow{6}{*}{$\begin{array}{r}1 \\
1.27\end{array}$} \\
\hline & & $N=$ & 29 & 1 & 12 & 4 & & \\
\hline & & nimum = & 0.27 & 13.00 & 0.00 & 1.01 & 0.87 & \\
\hline & & ximum = & 2.39 & & 8.40 & 1.40 & 2.00 & \\
\hline & & Vedian = & 1.14 & & 2.00 & & 1.30 & \\
\hline & F-pse & sigma $=$ & 0.10 & & 1.50 & & 0.47 & \\
\hline Lab & Rating & Z-value & 7 & 10 & 20 & 21 & 22 & 40 \\
\hline 1 & 4 & 0.00 & 1.20 & & & & & \\
\hline 2 & 4 & -0.15 & 1.13 & & & & & \\
\hline 3 & 2 & 1.19 & & & & & 1.78 & \\
\hline 5 & 0 & 2.45 & 2.39 & & & & & \\
\hline 7 & 4 & -0.14 & 1.13 & & & & & \\
\hline 15 & 3 & 0.89 & 1.63 & & & & & \\
\hline 23 & 4 & 0.14 & & & & & & 1.27 \\
\hline 25 & 4 & 0.00 & 1.20 & & & & & \\
\hline 26 & 4 & -0.14 & 1.13 & & & & & \\
\hline 33 & 4 & 0.08 & 1.24 & & & & & \\
\hline 36 & NR & & & & $<5$ & & & \\
\hline 39 & 1 & 1.65 & & & 2.00 & & & \\
\hline 42 & 4 & -0.21 & 1.10 & & & & & \\
\hline 46 & 3 & 0.92 & & & & & 1.65 & \\
\hline 48 & 1 & 1.65 & & & & & 2.00 & \\
\hline 58 & 3 & 0.60 & & & 1.49 & & & \\
\hline 59 & 4 & -0.21 & 1.10 & & & & & \\
\hline 64 & 4 & 0.00 & 1.20 & & & & & \\
\hline 81 & 4 & -0.10 & & & & 1.15 & & \\
\hline 83 & NR & & & & & $<5$ & & \\
\hline 89 & 4 & 0.19 & & & 1.29 & & & \\
\hline 105 & 4 & -0.12 & 1.14 & & & & & \\
\hline 107 & NR & & & & & $<0.6$ & & \\
\hline 109 & 4 & -0.39 & & & & 1.01 & & \\
\hline 110 & 4 & -0.15 & 1.13 & & & & & \\
\hline 113 & 1 & 1.61 & 1.98 & & & & & \\
\hline 119 & 4 & -0.04 & 1.18 & & & & & \\
\hline 134 & 4 & -0.05 & 1.17 & & & & & \\
\hline 138 & 4 & -0.27 & 1.07 & & & & & \\
\hline 140 & 3 & -0.68 & & & & & 0.87 & \\
\hline 141 & 4 & 0.41 & & & & & 1.40 & \\
\hline 143 & 4 & 0.00 & & & & & 1.20 & \\
\hline 145 & 4 & -0.33 & 1.04 & & & & & \\
\hline 146 & 3 & 0.68 & & & & & 1.53 & \\
\hline 158 & 1 & -1.92 & 0.27 & & & & & \\
\hline 180 & 4 & -0.41 & & & & & 1.00 & \\
\hline 183 & 4 & -0.39 & & & & & 1.01 & \\
\hline 190 & 4 & -0.23 & 1.09 & & & & & \\
\hline 191 & 2 & 1.30 & 1.83 & & & & & \\
\hline 196 & 4 & -0.02 & 1.19 & & & & & \\
\hline 197 & 3 & -0.54 & 0.94 & & & & & \\
\hline 203 & NR & & & & & $<2$ & & \\
\hline 204 & 4 & 0.41 & & & & 1.40 & & \\
\hline 215 & 1 & 1.65 & & & 2.00 & & & \\
\hline 220 & 4 & -0.04 & & & & & 1.18 & \\
\hline 221 & 3 & 0.56 & & & 1.47 & & & \\
\hline 224 & 4 & 0.33 & 1.36 & & & & & \\
\hline 240 & 4 & -0.14 & 113 & & & & & \\
\hline 241 & 0 & 4.74 & & & 3.50 & & & \\
\hline 247 & 2 & 1.24 & 1.80 & & & & & \\
\hline
\end{tabular}

\begin{tabular}{|c|c|c|c|c|c|c|c|c|}
\hline Lab & Rating & Z-value & 7 & 10 & 20 & 21 & 22 & 40 \\
\hline 255 & NR & & & & & & $<5$ & \\
\hline 256 & 1 & 1.54 & & & 1.95 & & & \\
\hline 257 & 3 & 0.89 & 1.63 & & & & & \\
\hline 258 & 0 & 14.83 & & & 8.40 & & & \\
\hline 261 & 0 & -2.47 & & & 0.00 & & & \\
\hline 262 & 4 & 0.21 & & & & 1.30 & & \\
\hline 265 & 3 & -0.51 & 0.95 & & & & & \\
\hline 268 & 4 & -0.19 & 1.11 & & & & & \\
\hline 271 & 1 & 1.65 & & & 2.00 & & & \\
\hline 272 & 0 & 12.13 & & & 7.09 & & & \\
\hline 273 & 0 & 24.30 & & 3.00 & & & & \\
\hline 276 & 0 & 4.74 & & & 3.50 & & & \\
\hline
\end{tabular}


Table 17. Statistical summary of reported data for standard reference water sample P-27 (low ionic strength constituents)-Contin'red $F$ (Fluoride) $\mathrm{mg} / \mathrm{L}$

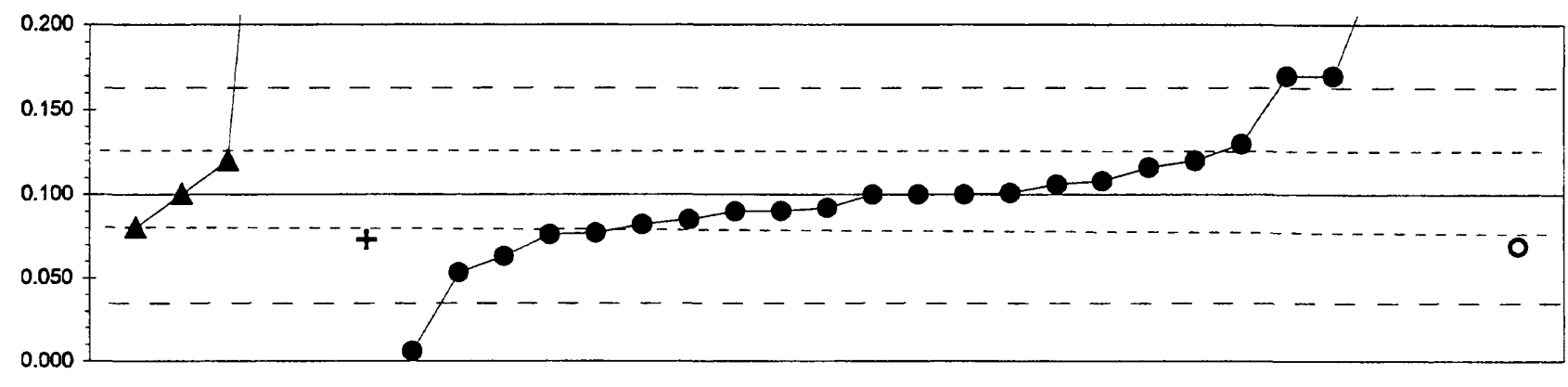

$-7-0-10+-21-40-0-50$

\begin{tabular}{|c|c|c|c|c|c|c|c|}
\hline \multicolumn{3}{|c|}{$\begin{array}{l}\text { 7. Ion chromatography } \\
\text { 8. AA: cold vapor } \\
\text { 21. Titrate: electrometric }\end{array}$} & \multicolumn{4}{|c|}{$\begin{array}{l}\text { 40. Ion selective electrode } \\
\text { 50. Gravimetric }\end{array}$} & \multirow[b]{2}{*}{1} \\
\hline & & $N=$ & 4 & 1 & 1 & 24 & \\
\hline \multicolumn{3}{|c|}{ Minimum = } & 0.08 & 0.40 & 0.07 & 0.01 & 0.07 \\
\hline \multicolumn{3}{|c|}{ Maximum = } & 0.45 & & & 0.54 & \\
\hline \multicolumn{3}{|c|}{ Median $=$} & & & & 0.10 & \\
\hline \multicolumn{3}{|c|}{ F-pseudosigma $=$} & & & & 0.03 & \\
\hline $\mathrm{Lab}$ & Rating & Z-value & 7 & 10 & 21 & 40 & 50 \\
\hline 1 & NR & & & & & $<0.1$ & \\
\hline 3 & 2 & -1.13 & & & & 0.063 & \\
\hline 7 & NR & & $<0.5$ & & & & \\
\hline $\begin{array}{l}15 \\
23\end{array}$ & $\begin{array}{c}4 \\
\text { NR }\end{array}$ & 0.25 & & & & $\begin{array}{l}0.108 \\
<0.1\end{array}$ & \\
\hline 25 & 4 & 0.00 & & & & 0.100 & \\
\hline 26 & 3 & 0.61 & 0.120 & & & & \\
\hline 36 & NR & & & & & $<0.1$ & \\
\hline 39 & 0 & -2.88 & & & & 0.006 & \\
\hline 48 & 0 & 4.29 & & & & 0.240 & \\
\hline 58 & 4 & -0.25 & & & & 0.092 & \\
\hline 59 & NR & & & & & $<0.2$ & \\
\hline 81 & 3 & -0.95 & & & & & 0.069 \\
\hline 83 & 0 & 2.15 & & & & 0.170 & \\
\hline 89 & 0 & 13.40 & & & & 0.537 & \\
\hline 105 & NR & & $<0.2$ & & & & \\
\hline 107 & 3 & -0.71 & & & & 0.077 & \\
\hline 109 & 4 & -0.31 & & & & 0.090 & \\
\hline 113 & 4 & -0.30 & & & & 0.090 & \\
\hline 119 & 4 & 0.00 & & & & 0.100 & \\
\hline 134 & 3 & 0.61 & & & & 0.120 & \\
\hline 138 & 4 & 0.03 & & & & 0.101 & \\
\hline 140 & 2 & -1.44 & & & & 0.053 & \\
\hline 141 & 3 & 0.92 & & & & 0.130 & \\
\hline 145 & 3 & -0.61 & 0.080 & & & & \\
\hline 146 & NR & & & & & $<0.2$ & \\
\hline 190 & 3 & -0.83 & & & 0.073 & & \\
\hline 196 & 4 & 0.18 & & & & 0.106 & \\
\hline 215 & 4 & 0.00 & & & & 0.100 & \\
\hline 224 & 0 & 10.73 & 0.450 & & & & \\
\hline 240 & 3 & -0.74 & & & & 0.076 & \\
\hline 241 & 4 & 0.49 & & & & 0.116 & \\
\hline 247 & 4 & 0.00 & 0.100 & & & & \\
\hline 255 & NR & & & & & $<0.2$ & \\
\hline 257 & 4 & -0.46 & & & & 0.085 & \\
\hline 258 & 0 & 2.15 & & & & 0.170 & \\
\hline 262 & 3 & -0.55 & & & & 0.082 & \\
\hline 265 & NR & & & & & $<0.1$ & \\
\hline 272 & 0 & 9.35 & & & & 0.405 & \\
\hline 273 & 0 & 9.20 & & 0.400 & & & \\
\hline
\end{tabular}


Table 17. Statistical summary of reported data for standard reference water sample P-27 (Iow ionic strength constituents)-Continued $\mathrm{K}$ (Potassium) $\mathrm{mg} / \mathrm{L}$

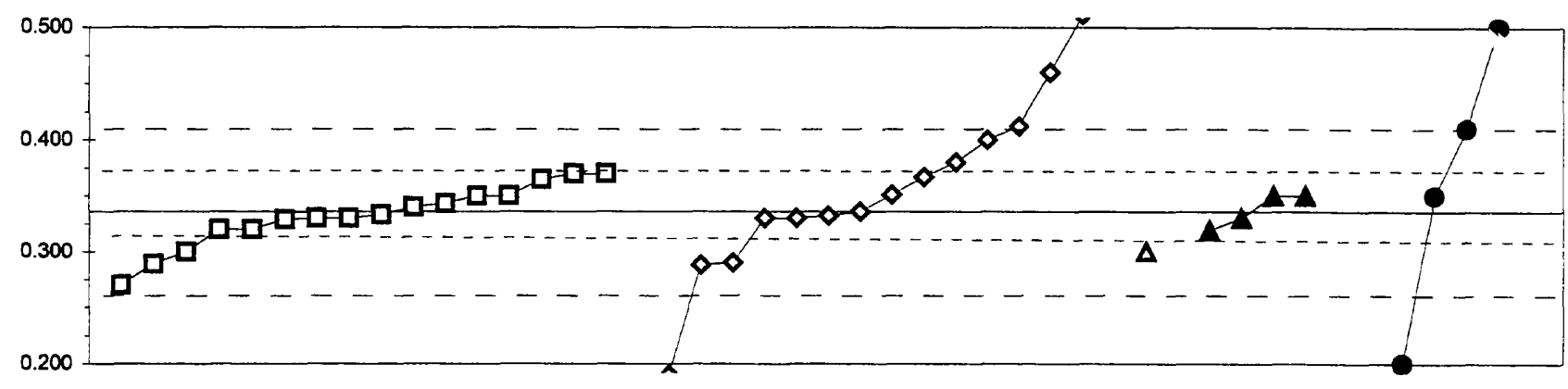

$-\square-1 \multimap-4-\Delta 5-6 \neg-7-12$

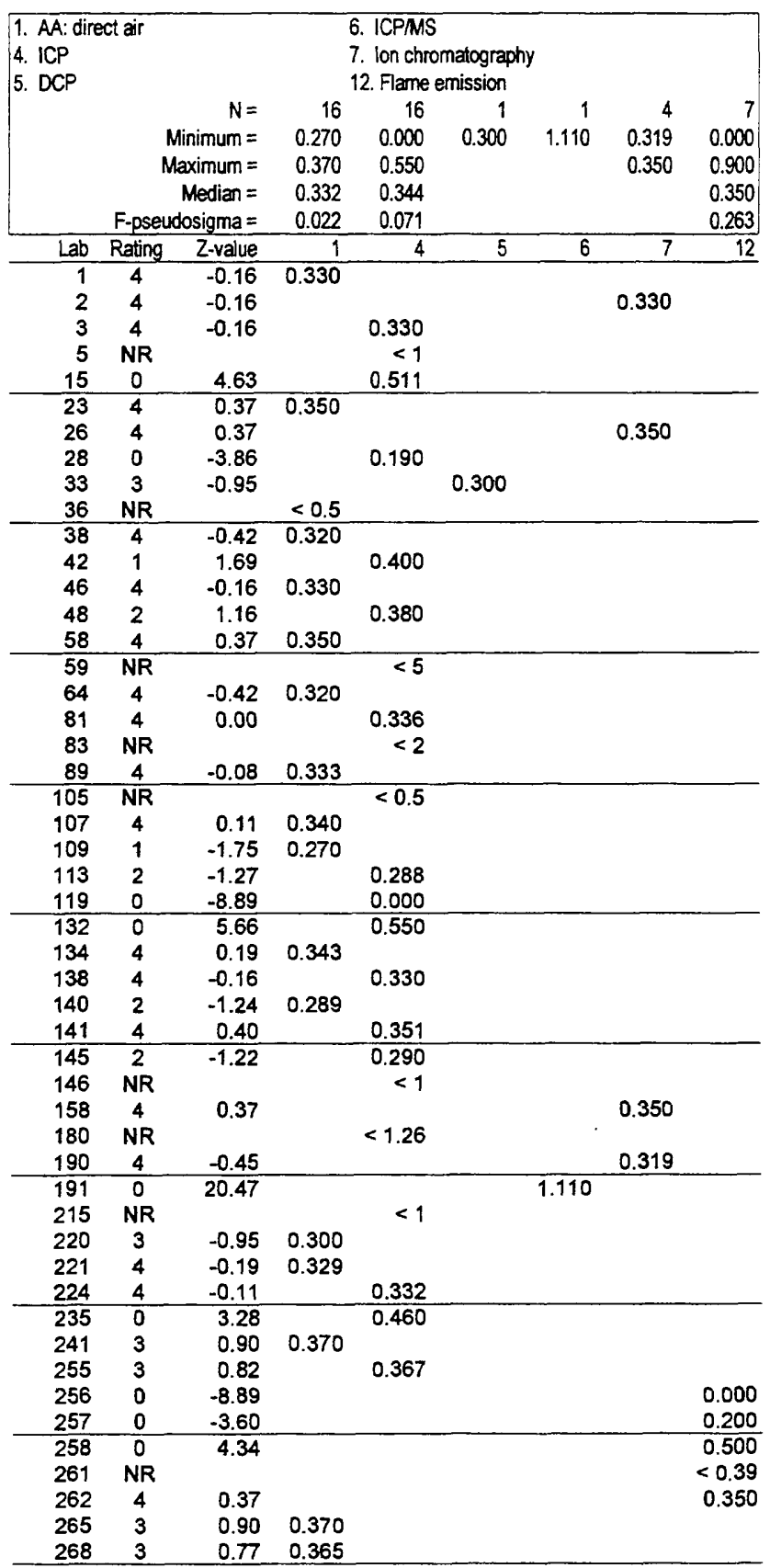

MPV $=0.336$

F-pseudosigma $=0.038$

$\mathrm{N}=45$

$\mathrm{Hu}=0.370$

$\mathrm{HI}=0.319$

\begin{tabular}{crrrrrrr} 
Lab & Rating & Z-value & 1 & 4 & 5 & 6 & 7 \\
\hline 270 & 1 & 1.96 & & & & & 12 \\
271 & 0 & 14.92 & & & & & 0.410 \\
272 & 0 & -8.89 & & & & & 0.900 \\
273 & 1 & 2.01 & 0.412 & & & \\
\hline
\end{tabular}


Table 17. Statistical summary of reported data for standard reference water sample P-27 (low ionic strength constituents)-Continued $\mathrm{Mg}$ (Magnesium) $\mathrm{mg} / \mathrm{L}$

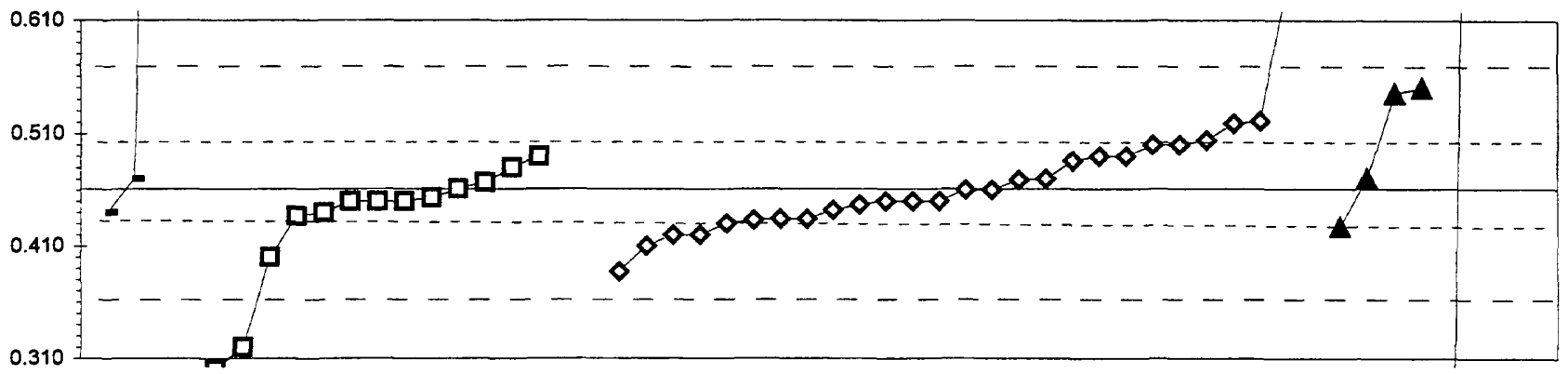

$-0-\square-1-0-2 \rightarrow-4-\Delta-7-20$

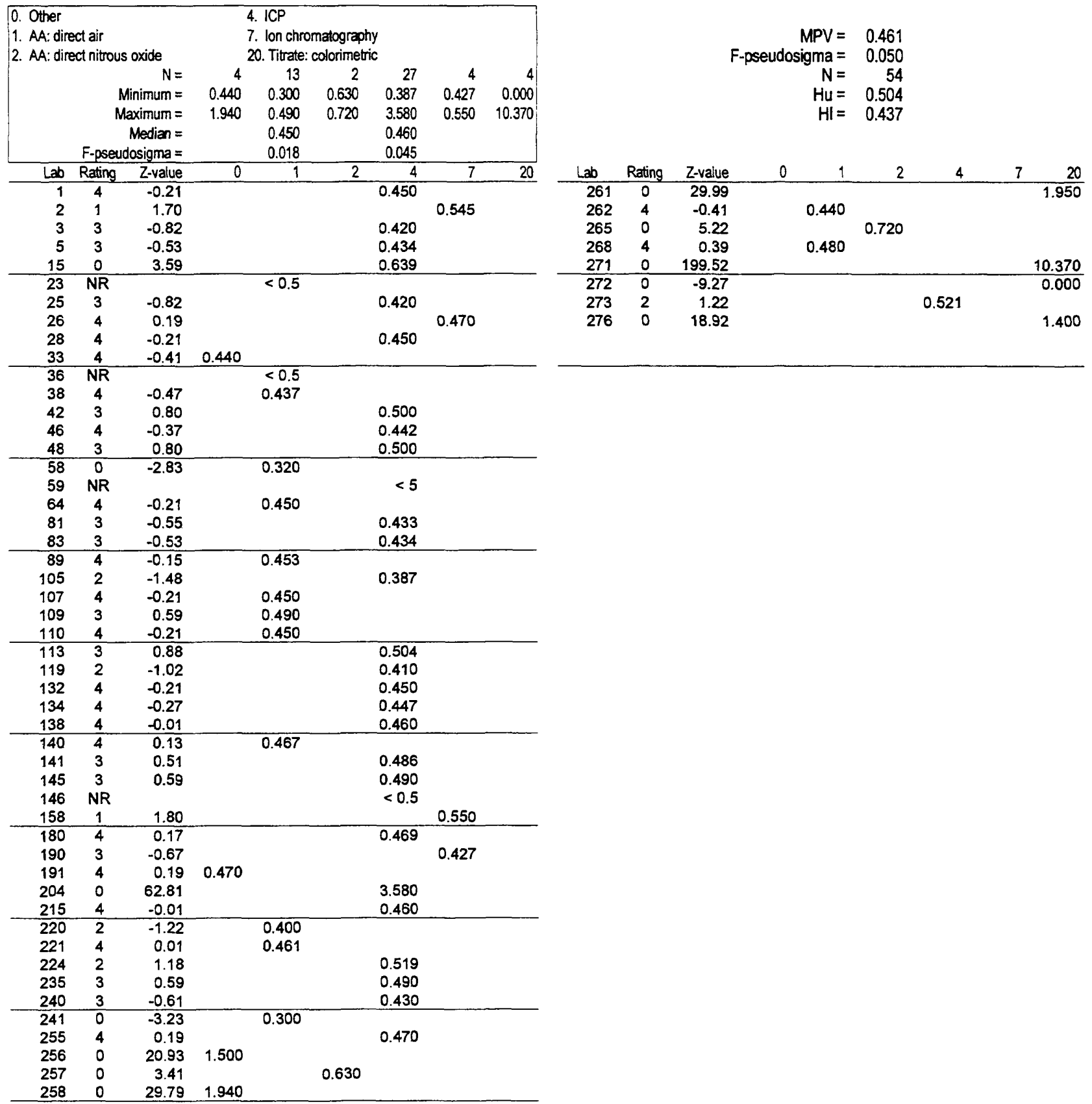


Table 17. Statistical summary of reported data for standard reference water sample P-27 (low ionic strength constituents)-Continued $\mathrm{Na}$ (Sodium) $\mathrm{mg} / \mathrm{L}$

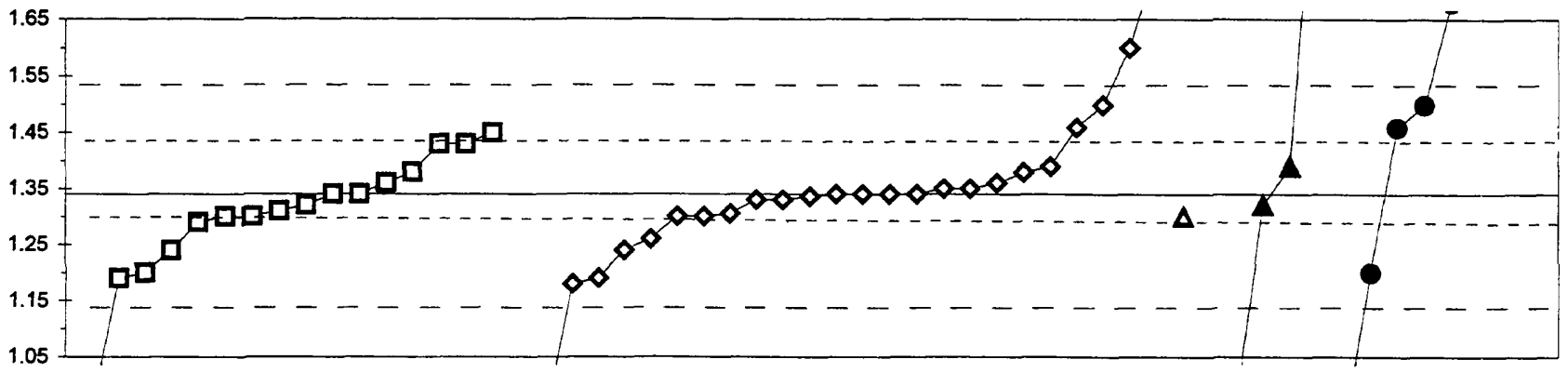

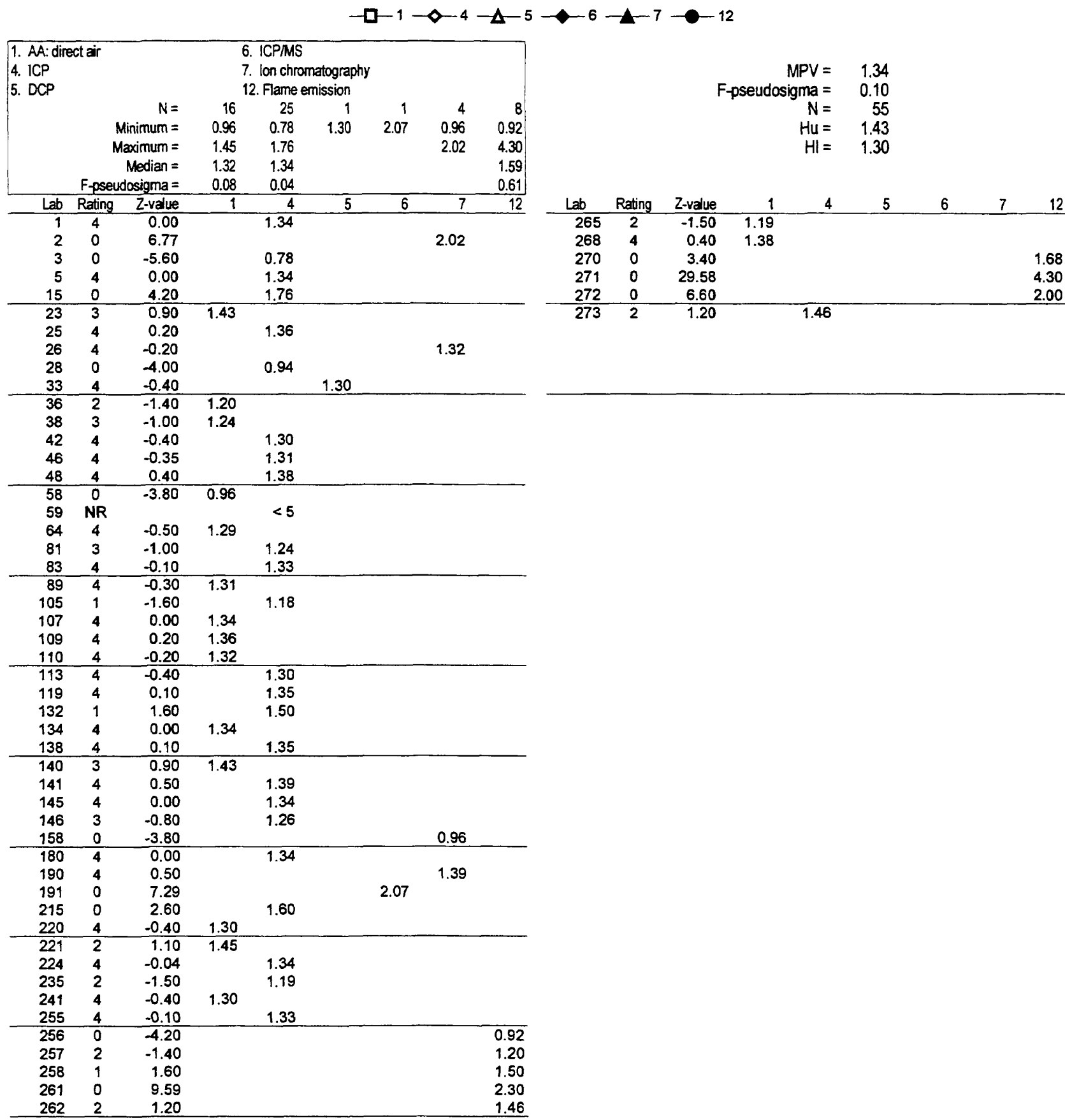


Table 17. Statistical summary of reported data for standard reference water sample P-27 (low ionic strength constituents)-Continued $\mathrm{pH}$

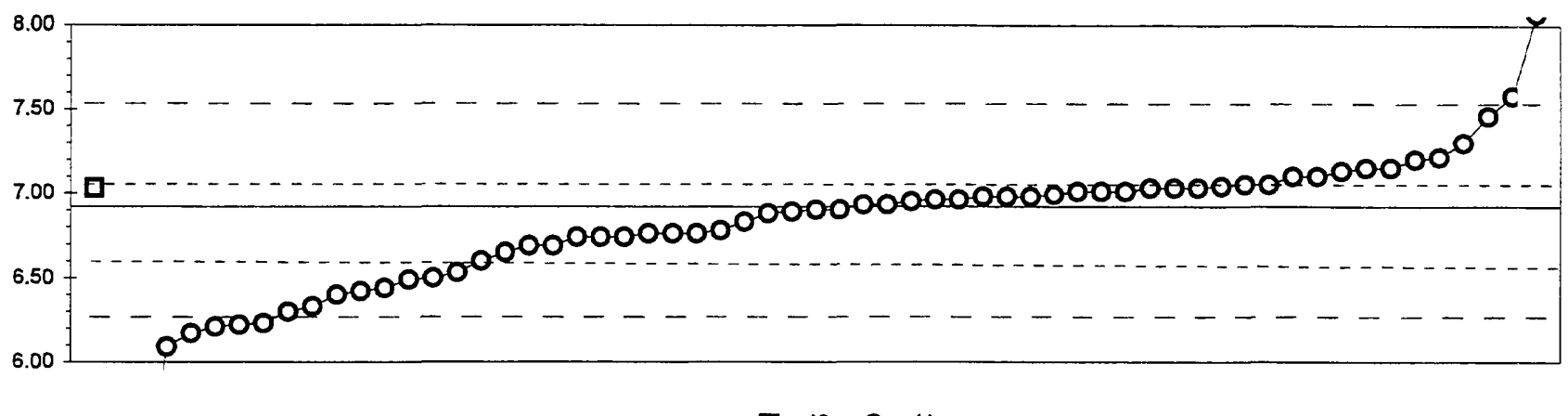

$-\square-10-0-41$

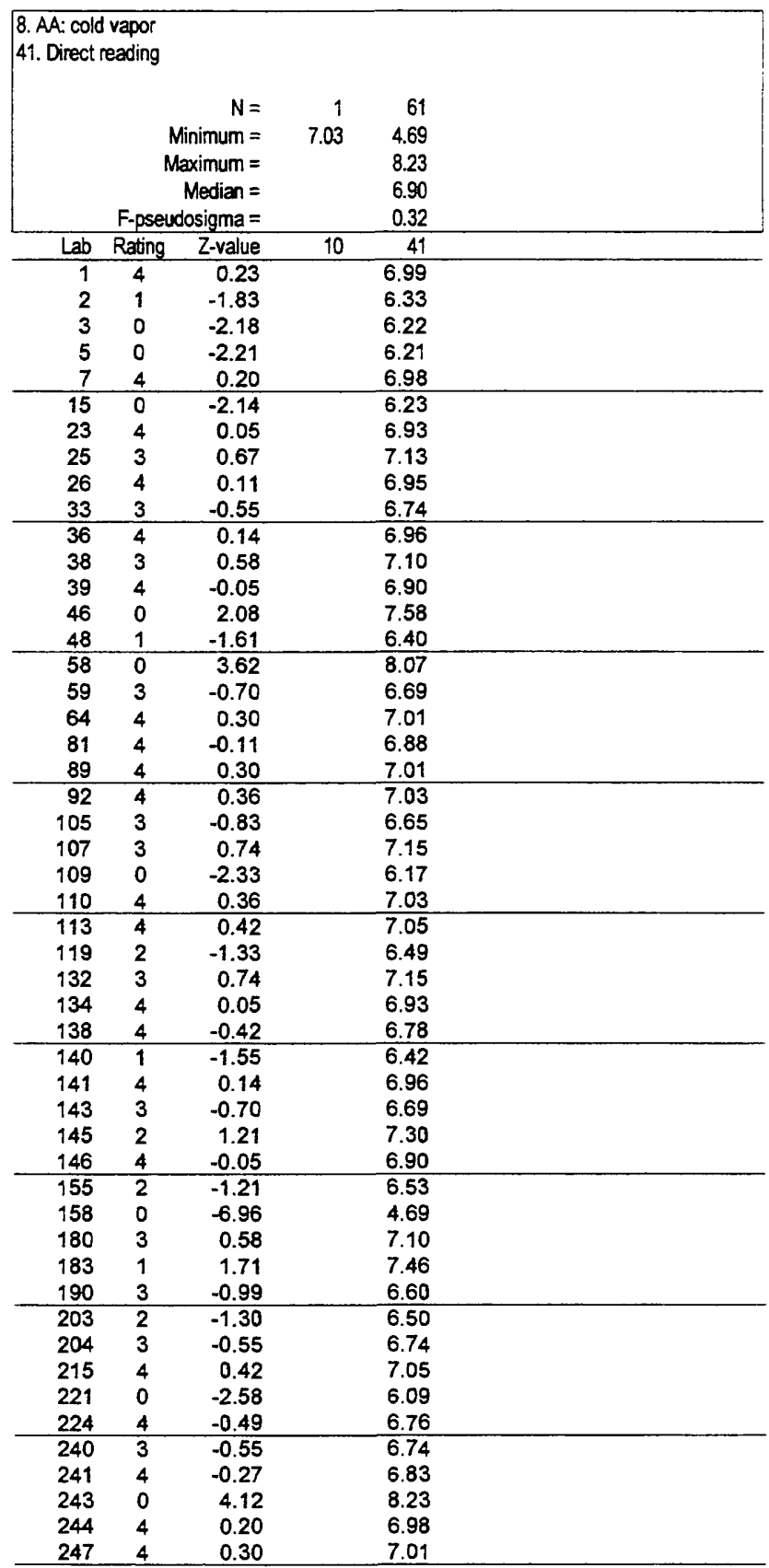

\begin{tabular}{ccrcc} 
Lab & Rating & Z-value & 10 & 41 \\
\hline 255 & 3 & 0.89 & & 7.20 \\
256 & 4 & 0.39 & 7.04 \\
257 & 4 & 0.36 & 7.03 \\
258 & 1 & -1.92 & 6.30 \\
261 & 2 & -1.49 & 6.44 \\
\hline 262 & 0 & -5.43 & 5.18 \\
265 & 4 & -0.49 & 6.76 \\
268 & 4 & -0.49 & 6.76 \\
271 & 4 & 0.20 & 6.98 \\
272 & 3 & 0.93 & 7.21 \\
\hline 273 & 4 & 0.36 & 7.03 & \\
276 & 4 & -0.08 & & 6.89
\end{tabular}


Table 17. Statistical summary of reported data for standard reference water sample P-27 (low ionic strength constituents)-Continued $\mathrm{PO}_{4}$ as $\mathrm{P}$ (orthophosphate as phosphorus) $\mathrm{mg} / \mathrm{L}$

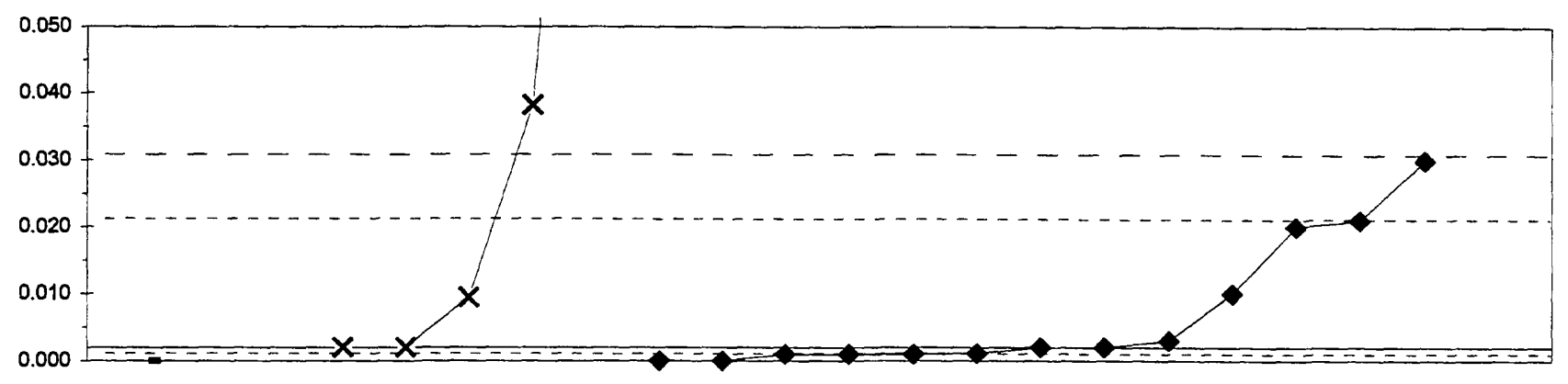

$\rightarrow 0 \multimap-4-7-x-22 \multimap-22 m-x-22$ snd

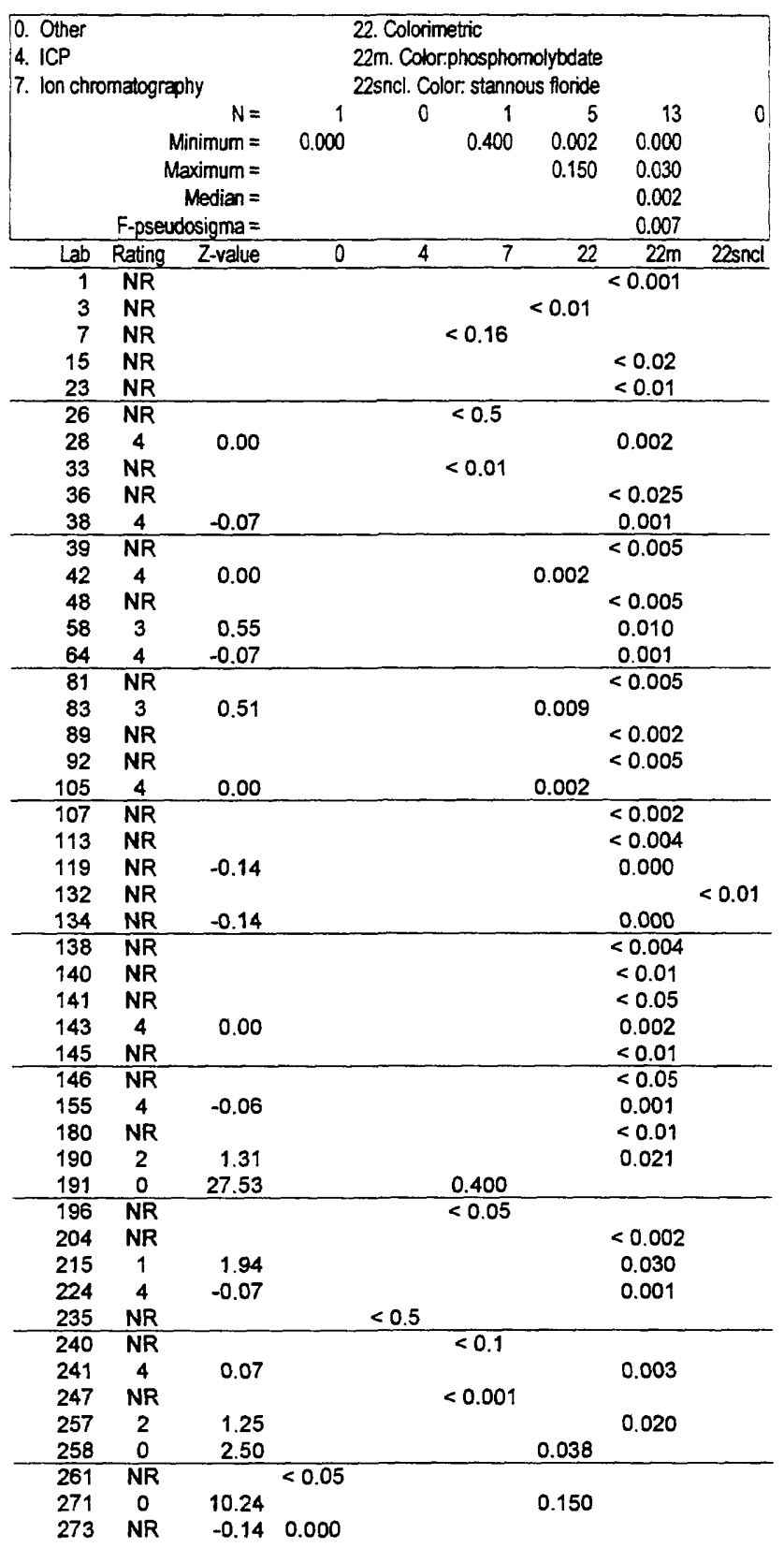


Table 17. Statistical summary of reported data for standard reference water sample P-27 (low ionic strength constituents)-Conitinued $\mathrm{SO}_{4}$ (Sulfate) $\mathrm{mg} / \mathrm{L}$
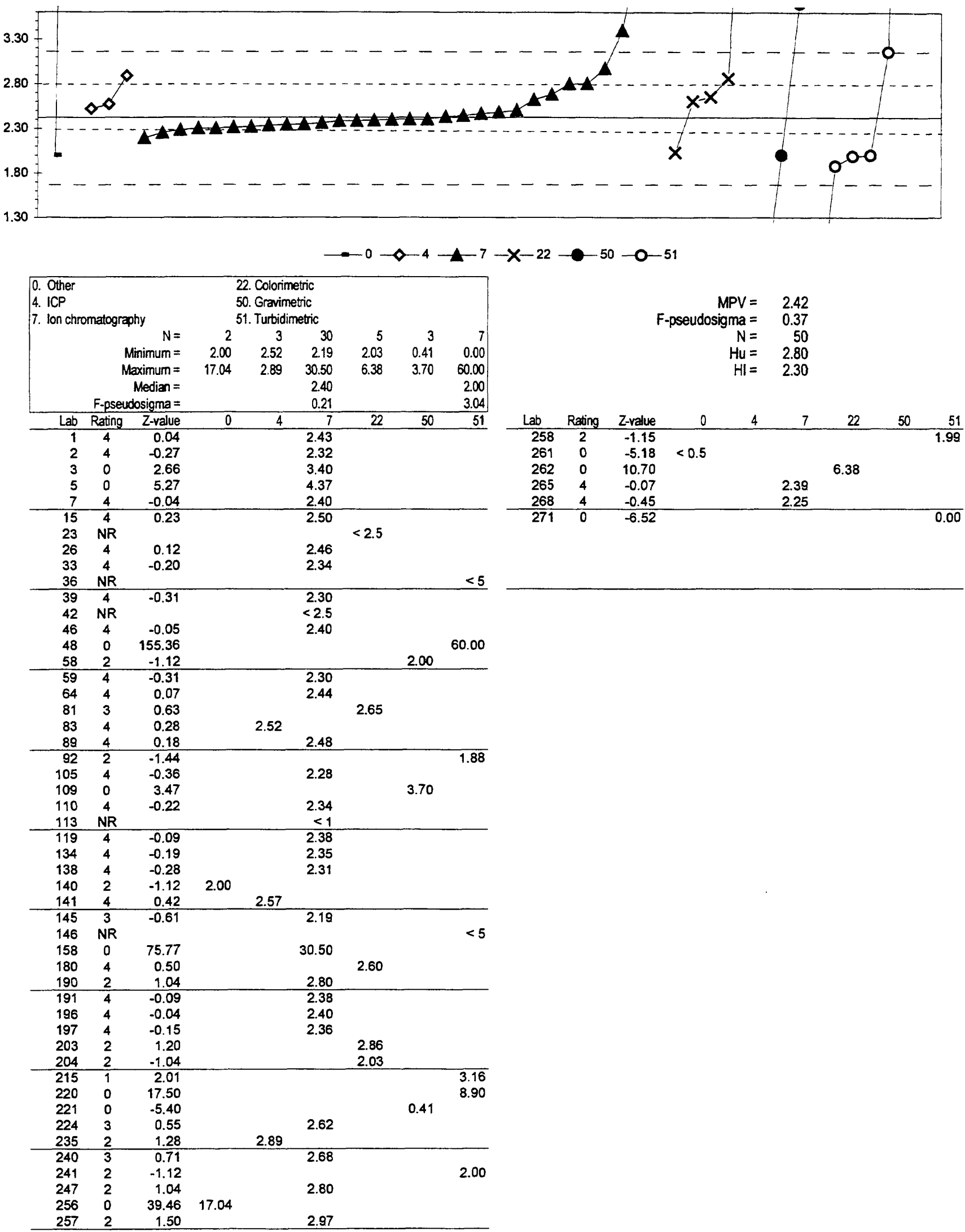
Table 17. Statistical summary of reported data for standard reference water sample P-27 (low ionic strength constituents)-Continued Sp Cond (Specific Conductance) $\mu \mathrm{S} / \mathrm{cm}$
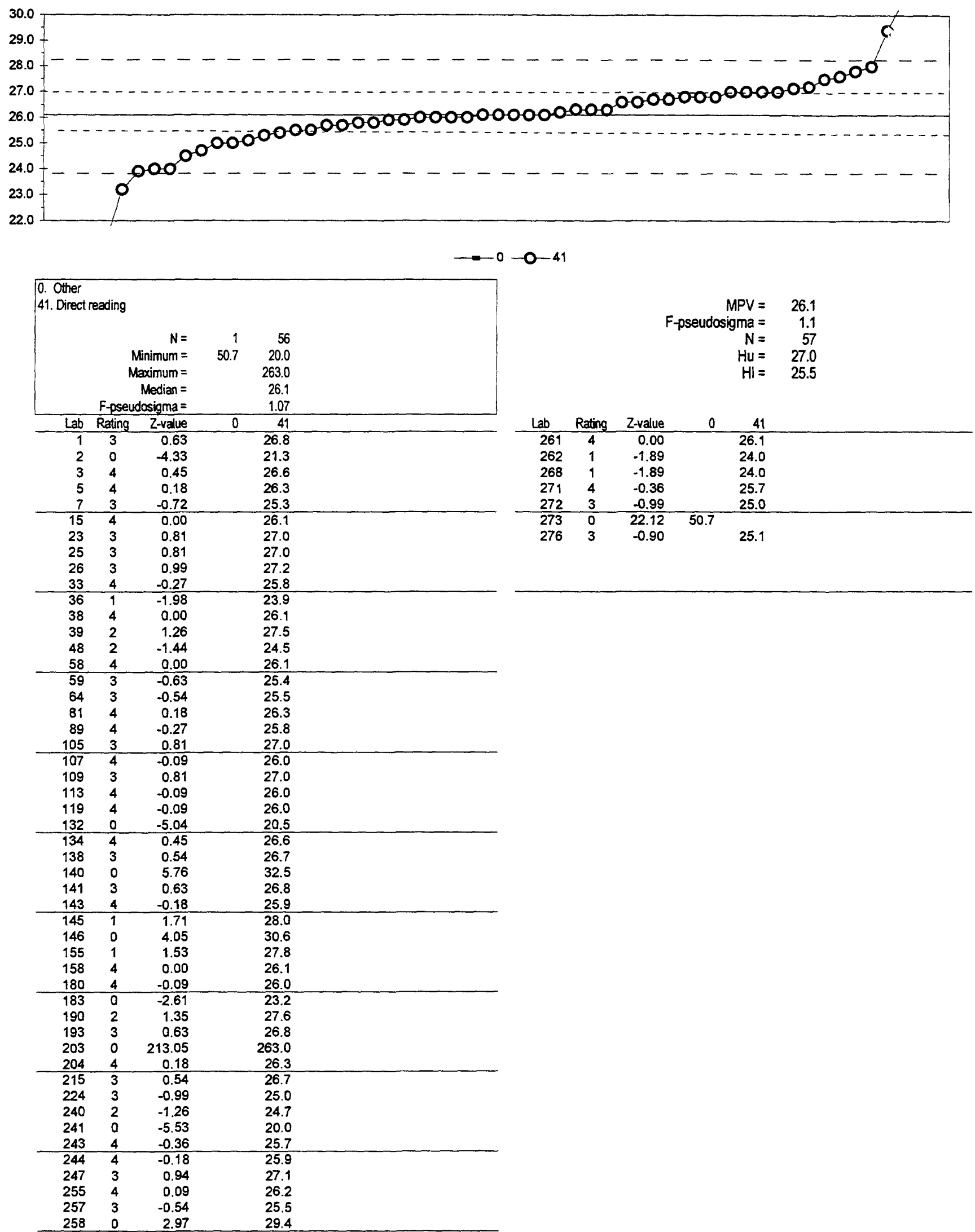
Table 18. Statistical summary of reported data for standard reference water sample Hg-23 (mercury)

Definition of analytical methods, abbreviations, and symbols

Analytical methods

0 Other/Not reported

6 ICPMS = mass spectrometry/inductively coupled plasma

8 AA: cold vapor $=$ atomic absorption: cold vapor

$\begin{array}{rlrl}\text { Abbreviations and symbols } & & \\ \mathrm{N} & = & & \text { number of samples } \\ \text { MPV } & = & & \text { most probable value } \\ \text { F-pseudosigma } & = & & \text { nonparametric statistic deviation } \\ H u & = & & \text { upper hinge value } \\ H I & = & & \text { lower hinge value } \\ \mu \mathrm{g} / \mathrm{L} & = & & \text { micrograms per liter } \\ \mathrm{Lab} & = & & \text { laboratory code number } \\ \mathrm{NR} & = & & \text { not rated, less than value reported } \\ < & = & & \text { less than }\end{array}$


Table 18. Statistical summary of reported data for standard reference water sample Hg-23 (mercury)--Continued $\mathrm{Hg}$ (Mercury) $\mu \mathrm{gg} / \mathrm{L}$
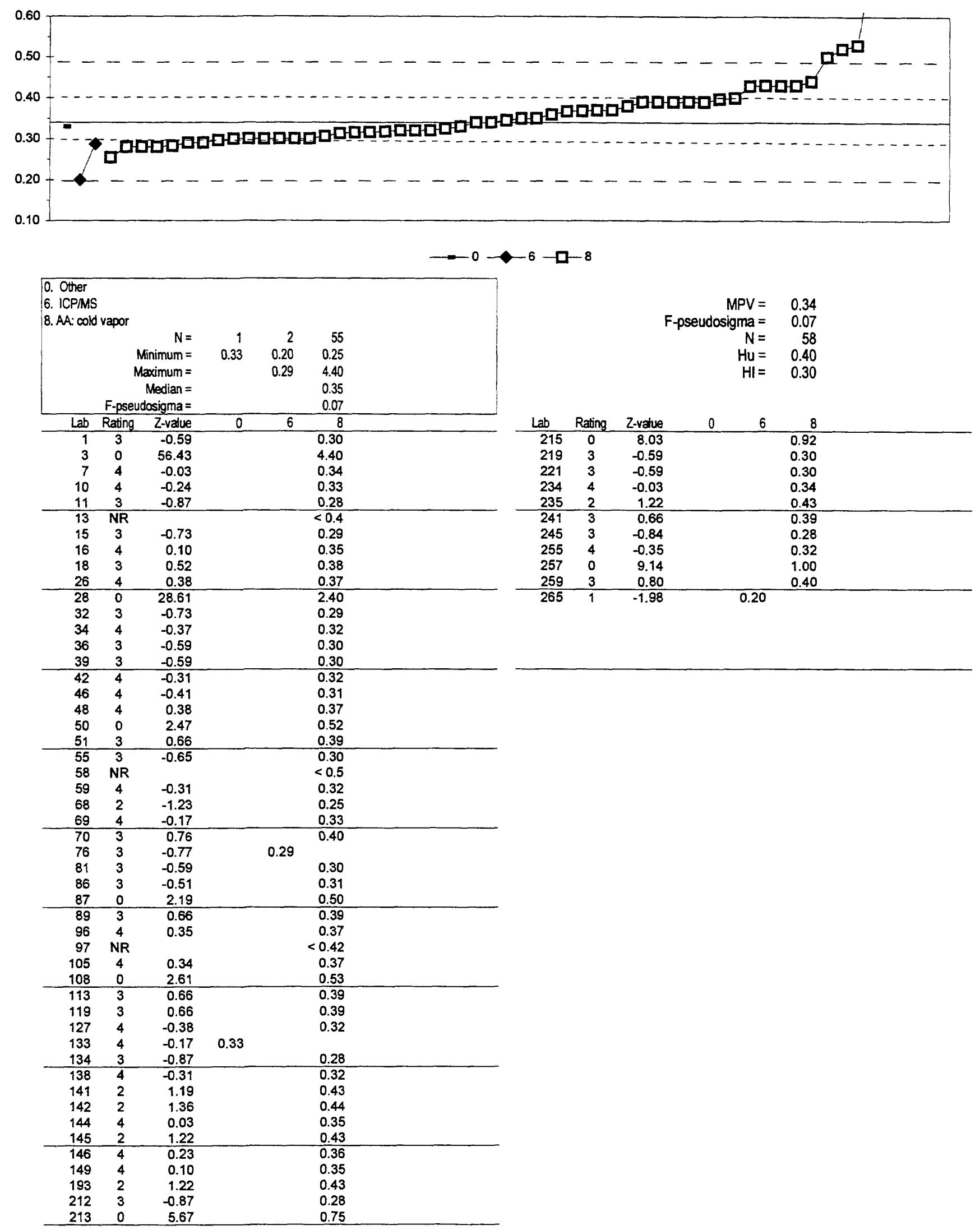
Table 19. Most probable values for constituents and properties in standard reference samples distributed in September 1996

[MPV, most probable value; ug $h$, microgram per liter, $\mathrm{mg} / \mathrm{L}$, milligram per liter, uS/cm, microsiemen per centimeter at 25 degrees Celsius]

\begin{tabular}{|c|c|c|c|c|c|c|c|}
\hline \multicolumn{8}{|c|}{ T-143 (trace constituents) } \\
\hline Analute & MPV & F-pseudosigma & $\underline{\mathbf{N}}$ & Analute & MPV & F-pseudosigma & $\underline{N}$ \\
\hline $\mathrm{Ag}$ & $\overline{19.8} \mu \mathrm{g} / \mathrm{L}$ & 1.4 & $6 \overline{5}$ & $\mathrm{Mg}$ & $\overline{10.4} \mathrm{mg} \Omega$ & 0.5 & 88 \\
\hline Al & $22.1 \mu \mathrm{g} / \mathrm{L}$ & 8.3 & 50 & $\mathrm{Mn}$ & $18.2 \mu \mathrm{g} / \mathrm{h}$ & 1.9 & 86 \\
\hline As & $15.2 \mu \mathrm{g} / \mathrm{L}$ & 1.2 & 66 & Mo & $36.1 \mu \mathrm{g} / \mathrm{L}$ & 4.3 & 54 \\
\hline B & $35.0 \mu \mathrm{g} / \mathrm{L}$ & 5.2 & 35 & $\mathrm{Na}$ & $34.0 \mathrm{mg} / \mathrm{L}$ & 1.6 & 88 \\
\hline $\mathrm{Ba}$ & $81.9 \mu \mathrm{g} / \mathrm{L}$ & 4.5 & 68 & $\mathrm{Ni}$ & $71.0 \mu \mathrm{g} / \mathrm{L}$ & 5.0 & 81 \\
\hline $\mathrm{Be}$ & $8.50 \mu g / L$ & 0.66 & 61 & $\mathrm{~Pb}$ & $83.4 \mu \mathrm{g} / \mathrm{L}$ & 7.1 & 84 \\
\hline $\mathrm{Ca}$ & $53.7 \mathrm{mg} \Omega$ & 2.2 & 86 & $\mathbf{S b}$ & $16.6 \mu \mathrm{g} / \mathrm{L}$ & 1.5 & 46 \\
\hline $\mathrm{Cd}$ & $19.1 \mu \mathrm{g} / \mathrm{L}$ & 1.5 & 81 & $\mathrm{Se}$ & $9.63 \mu \mathrm{g} / \mathrm{L}$ & 1.64 & 60 \\
\hline Co & $17.0 \mu \mathrm{g} / \mathrm{L}$ & 1.2 & 53 & $\mathrm{SiO}_{2}$ & $23.4 \mathrm{mg} / \mathrm{L}$ & 1.7 & 56 \\
\hline $\mathrm{Cr}$ & $37.0 \mu \mathrm{g} / \mathrm{L}$ & 2.6 & 79 & $\mathrm{Sr}$ & $306 \mu \mathrm{g} / \mathrm{L}$ & 15 & 45 \\
\hline $\mathrm{Cu}$ & $22.3 \mu \mathrm{g} / \mathrm{L}$ & 1.9 & 90 & $T I$ & $10.0 \mu \mathrm{g} / \mathrm{L}$ & 1.0 & 33 \\
\hline $\mathrm{Fe}$ & $222 \mu \mathrm{g} / \mathrm{L}$ & 14 & 93 & $u$ & $12.0 \mu \mathrm{g} / \mathrm{L}$ & 0.9 & 9 \\
\hline $\mathrm{K}$ & $2.50 \mathrm{mg} / \mathrm{L}$ & 0.21 & 84 & $v$ & $30.0 \mu \mathrm{g} / \mathrm{L}$ & 3.0 & 54 \\
\hline Li & $18.0 \mu \mathrm{g} / \mathrm{L}$ & 2.1 & 34 & $\mathrm{Zn}$ & $20.0 \mu \mathrm{g} / \mathrm{L}$ & 2.2 & 75 \\
\hline T-145 (tr & nstituen & ts) & & & & & \\
\hline Analute & MPV & F-pseudosigma & $\underline{\mathbf{N}}$ & Analyte & MPV & E-pseudosigma & $\underline{\mathbf{N}}$ \\
\hline $\mathrm{Ag}$ & $\overline{7.55} \mu \mathrm{g} / \mathrm{L}$ & 0.92 & 61 & $\mathrm{Mg}$ & $\overline{8.68} \mathrm{mg} /$ & 0.45 & $8 \overline{5}$ \\
\hline Al & $67.6 \mu \mathrm{g} / \mathrm{L}$ & 11.0 & 64 & $\mathrm{Mn}$ & $20.9 \mu g^{\prime} L$ & 1.5 & 85 \\
\hline As & $9.88 \mu \mathrm{g} / \mathrm{L}$ & 1.04 & 66 & Mo & $9.23 \mu \mathrm{g} / \mathrm{L}$ & 1.29 & 43 \\
\hline B & $45.6 \mu \mathrm{g} / \mathrm{L}$ & 5.8 & 36 & $\mathrm{Na}$ & $41.2 \mathrm{mg} / \mathrm{L}$ & 1.9 & 84 \\
\hline $\mathrm{Ba}$ & $37.1 \mu \mathrm{g} / \mathrm{L}$ & 1.9 & 63 & $\mathrm{Ni}$ & $11.0 \mu \mathrm{g} / \mathrm{L}$ & 1.3 & 66 \\
\hline $\mathrm{Be}$ & $9.04 \mu \mathrm{g} / \mathrm{L}$ & 0.70 & 62 & $\mathrm{~Pb}$ & $12.7 \mu \mathrm{g} / \mathrm{L}$ & 1.2 & 80 \\
\hline $\mathrm{Ca}$ & $30.7 \mathrm{mg} / \mathrm{L}$ & 1.3 & 84 & $\mathbf{S b}$ & $8.80 \mu \mathrm{g} / \mathrm{L}$ & 0.96 & 39 \\
\hline $\mathrm{Cd}$ & $9.33 \mu \mathrm{g} / \mathrm{L}$ & 0.82 & 80 & Se & $10.1 \mu \mathrm{g} / \mathrm{L}$ & 1.3 & 60 \\
\hline Co & $10.0 \mu \mathrm{g} / \mathrm{L}$ & 0.9 & 53 & $\mathrm{SiO}_{2}$ & $11.3 \mathrm{mg} / \mathrm{L}$ & 0.7 & 53 \\
\hline $\mathrm{Cr}$ & $15.3 \mu \mathrm{g} / \mathrm{L}$ & 1.4 & 78 & $\mathrm{sr}$ & $203 \mu \mathrm{g} / \mathrm{L}$ & 9 & 44 \\
\hline $\mathrm{Cu}$ & $11.0 \mu \mathrm{g} / \mathrm{L}$ & 1.4 & 84 & $\pi$ & $15.3 \mu g /$ & 2.7 & 37 \\
\hline $\mathrm{Fe}$ & $101 \mu \mathrm{g} / \mathrm{L}$ & 8 & 89 & $u$ & $1.10 \mu \mathrm{g} / \mathrm{L}$ & 0.10 & 9 \\
\hline $\mathrm{K}$ & $2.13 \mathrm{mg} /$ & 0.16 & 83 & v & $11.7 \mu \mathrm{g} / \mathrm{L}$ & 1.7 & 46 \\
\hline $\mathrm{Li}$ & $27.3 \mu \mathrm{g} / \mathrm{L}$ & 2.5 & 36 & $\mathrm{Zn}$ & $10.0 \mu \mathrm{g} / \mathrm{L}$ & 2.4 & 70 \\
\hline$M-140(m$ & onstitue & nts) & & & & & \\
\hline Analyte & MPV & F-pseudosigma & $\underline{N}$ & Analvte & MPV & F-pseudosigma & $\underline{\mathbf{N}}$ \\
\hline Alkalinity & $114 \mathrm{mg} / \mathrm{L}$ & 3 & 103 & $\mathrm{Na}$ & $39.0 \mathrm{mg} / \mathrm{L}$ & 1.9 & 92 \\
\hline B & $41.6 \mathrm{mg} / \mathrm{L}$ & 5.2 & 40 & total $\mathbf{P}$ & $0.032 \mathrm{mg} / \mathrm{L}$ & 0.011 & 57 \\
\hline $\mathrm{Ca}$ & $60.7 \mathrm{mg} \Omega$ & 3.1 & 96 & $\mathrm{pH}$ & 8.28 units & 0.17 & 108 \\
\hline $\mathrm{Cl}$ & $25.8 \mathrm{mg} / \mathrm{L}$ & 1.4 & 104 & $\mathrm{SiO}_{2}$ & $7.35 \mathrm{mg} / \mathrm{L}$ & 0.46 & 66 \\
\hline DSRD & $382 \mathrm{mg} / \mathrm{L}$ & 16 & 67 & $\mathrm{SO}_{4}$ & $150 \mathrm{mg} /$ & 7 & 98 \\
\hline$F$ & $0.530 \mathrm{mg} / \mathrm{L}$ & 0.037 & 71 & Sp Cond & $600 \mu \mathrm{s} / \mathrm{cm}$ & 19 & 100 \\
\hline $\mathrm{K}$ & $2.58 \mathrm{mg} / \mathrm{L}$ & 0.14 & 87 & $\mathrm{Sr}$ & $671 \mu \mathrm{g} / \mathrm{L}$ & 31 & 43 \\
\hline $\mathrm{Mg}$ & $18.0 \mathrm{mg} / \mathrm{L}$ & 1.0 & 96 & v & $3.42 \mu \mathrm{g} / \mathrm{L}$ & 3.01 & 19 \\
\hline
\end{tabular}

\section{N-51 (nutrients)}

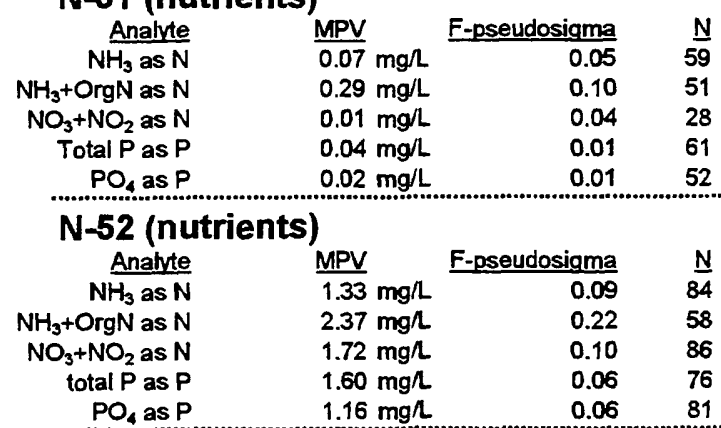

P-27 (low ionic strength constituents)

\begin{tabular}{|c|c|c|c|c|c|c|c|}
\hline Analite & MPV & F-pseudosigma & $\underline{\mathbf{N}}$ & Analyte & MPV & F-pseudosigma & $\underline{N}$ \\
\hline$\overline{\text { Acidity }}$ & $\overline{4.74} \mathrm{mg} /$ & 3.19 & $2 \overline{2}$ & $\mathrm{Na}$ & $1.34 \mathrm{mg} / \mathrm{L}$ & 0.10 & $5 \overline{5}$ \\
\hline $\mathrm{Ca}$ & $2.53 \mathrm{mg} / \mathrm{L}$ & 0.24 & 59 & $\mathrm{pH}$ & 6.92 units & 0.32 & 62 \\
\hline $\mathrm{Cl}$ & $1.20 \mathrm{mg} / \mathrm{L}$ & 0.49 & 57 & $\mathrm{PO}_{4}$ as $\mathrm{P}$ & $0.002 \mathrm{mg} / \mathrm{L}$ & 0.014 & 20 \\
\hline$F$ & $0.100 \mathrm{mg} / \mathrm{L}$ & 0.033 & 31 & $\mathrm{SO}_{4}$ & $2.42 \mathrm{mg} / \mathrm{L}$ & 0.37 & 50 \\
\hline $\mathrm{K}$ & $0.336 \mathrm{mg} / \mathrm{L}$ & 0.038 & 45 & Sp Cond & $26.1 \mu \mathrm{S} / \mathrm{cm}$ & 1.1 & 57 \\
\hline $\mathbf{M g}$ & $0.461 \mathrm{mg} / \mathrm{L}$ & 0.050 & 54 & & & & \\
\hline
\end{tabular}

Hg-23 (mercury)

\begin{tabular}{llrl} 
Analyte & MPV & F-pseudosigma & $\frac{N}{0.07}$ \\
\hline $\mathrm{Hg}$ & $0.34 \mathrm{gg} /$ &
\end{tabular}

